WSRC-TR-99-00012

Part I

\title{
F-Area Hazardous Waste Management Facility Correction Action Report, Third and Fourth Quarter 1998, Volumes I and II
}

by

J. Chase

RECORDS ADMINISTRATION

Westinghouse Savannah River Company

R0124635

Savannah River Site

Aiken, South Carolina 29808

DOE Contract No. DE-AC09-96SR18500

This paper was prepared in connection with work done under the above contract number with the U. S.

Department of Energy. By acceptance of this paper, the publisher and/or recipient acknowledges the U. S.

Government's right to retain a nonexclusive, royalty-free license in and to any copyright covering this paper, along with the right to reproduce and to authorize others to reproduce all or part of the copyrighted paper. 


\title{
F-AREA HAZARDOUS WASTE MANAGEMENT FACILITY CORRECTIVE ACTION REPORT (U)
}

\section{THIRD AND FOURTH QUARTER 1998}

\author{
Volume I
}

Publication Date: March 1999

Authorized Derivative Classifier and Reviewing Official:

C.QReered, RERO. UNCLASSIFIED

Does Not Contain Unclassified Controlled Nuclear Information

Westinghouse Savannah River Company

Savannah River Site

Aiken, SC 29808 
WSRC-TR-99-00012

Unclassified

\section{F-AREA HAZARDOUS WASTE MANAGEMENT FACILITY CORRECTIVE ACTION REPORT (U)}

THIRD AND FOURTH QUARTER 1998

Volume I

Publication Date: March 1999

Authorized Derivative Classifier and Reviewing Official:

UNCLASSIFIED

Does Not Contain Unclassified

Controlled Nuclear Information

Westinghouse Savannah River Company

Savannah River Site

Aiken, SC 29808

Prepared for the U.S. Department of Energy under Control Contract No. DE-AC09-96SR18500 


\section{DISCLAIMER}

This report was prepared as an account of work sponsored by an agency of the United States Government. Neither the United States Government nor any agency thereof, nor any of their employees, makes any warranty, express or implied, or assumes any legal liability or responsibility for the accuracy, completeness, or usefulness of any information, apparatus, product, or process disclosed, or represents that its use would not infringe privately owned rights. Reference herein to any specific commercial product, process, or service by trade name, trademark, manufacturer, or otherwise does not necessarily constitute or imply its endorsement, recommendation, or favoring by the United States Government or any agency thereof. The views and opinions of authors expressed herein do not necessarily state or reflect those of the United States Government or any agency thereof.

This report has been reproduced directly from the best available copy.

Available to DOE and DOE contractors from the Office of Scientific and Technical Information, P.O. Box 62, Oak Ridge, TN 37831; prices available from (615) 576-8401.

Available to the public from the National Technical Information Service, U.S. Department of Commerce, 5285 Port Royal Road, Springfield, VA 22161. 


\section{Contents}

Volume I

Page

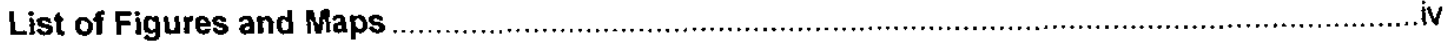

List of Tables

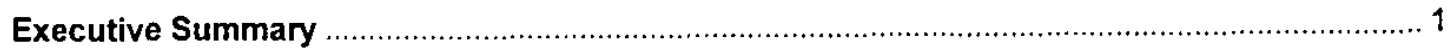

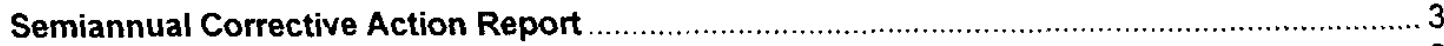

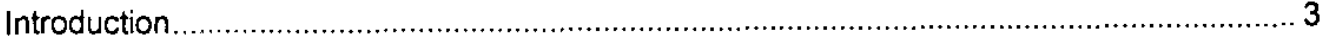

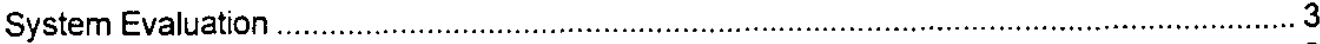

Water Quality and Elevation Data Tables (IIIC.H.11.C.i) .................................................... 3

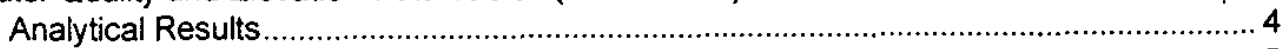

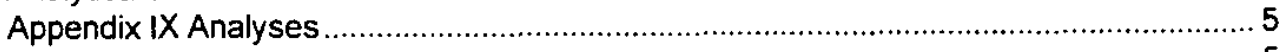

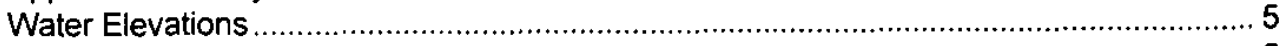

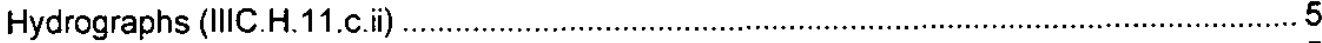

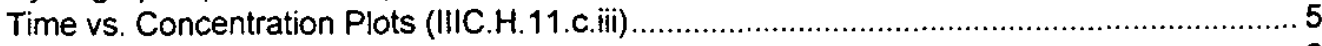

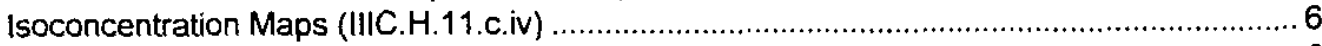

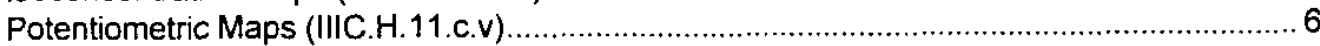

Potentiometric Cross-Sections and Isoconcentration Cross-Sections (IIIC.H.11.C.vi) ...........6 6

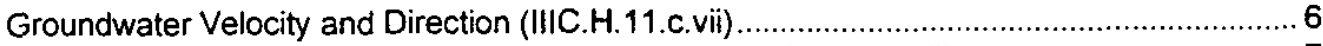

Extent and Severity of Groundwater Contamination (IIIC.H.11.c.viii) .............................. 7

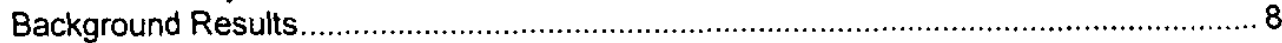

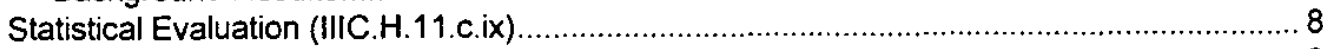

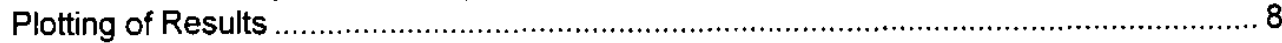

Evaluation of Water Quality and Water Elevation Data (IIIC.H.11.C.X) ................................. 9

Volume and Rates of Groundwater Pumped (IIIC.H.11.C.xi) ............................................. 9

Volume and Rates of Groundwater Injected (IIIC.H.11.c.xii) ............................................. 9

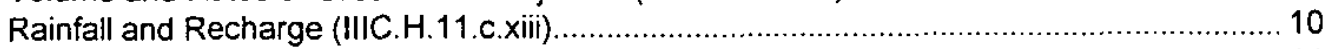

Summary of System Downtime (IIIC.H.11.c.xiv) …....................................................... 11

Minor Modifications to the System (IIIC.H.11.C.XV) ......................................................... 11

Effectiveness of the Corrective Action System (IIIC.H.11.c.xvi) ....................................... 12

Underground Injection Control (UIC) Sampling Results................................................. 12

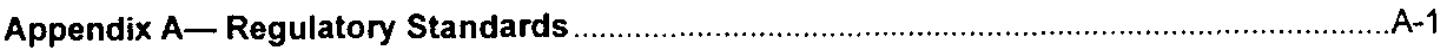

Appendix B-Figures

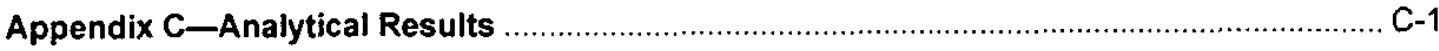

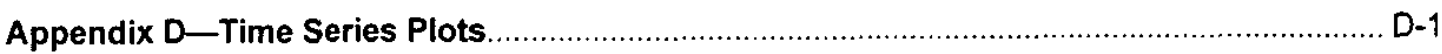

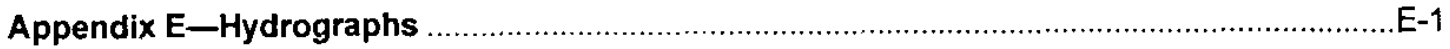

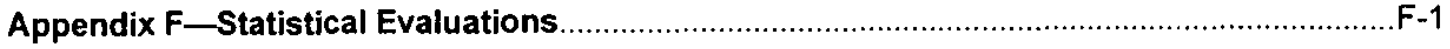

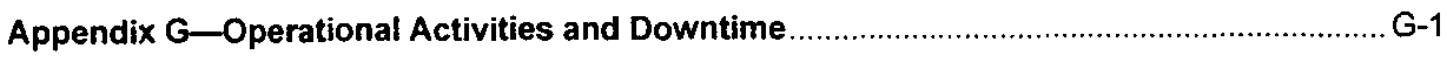

Volume II

Appendix $\mathrm{H}-$ General Separations Area Maps for the F-and H-Area Hazardous Waste Management Facilities Semiannual Corrective Action Reports (U) 


\section{List of Figures and Maps}

Volume I

1. Rainfall Data for F Area During Third and Fourth Quarter 1998

\section{Appendix B-Figures}

1. Location of the F-Area HWMF at the Savannah River Site

2. Location of the Groundwater Monitoring Wells at the F-Area HWMF.

3. Location of Aquifer Zone $\|_{1} B_{2}$ (Water Table) Monitoring Wells at the F-Area HWMF...

4. Location of Aquifer Zone $\mathrm{IB}_{1}$ (Barnwell/McBean) Monitoring Wells at the F-Area HWMF .......B-6

5. Location of Aquifer Unit IIA (Congaree) Monitoring Wells at the F-Area HWMF.

6. Hydrogeologic Cross-Section Showing Potentiometric Surfaces at the F-Area HWMF, Fourth Quarter 1998.

7. Hydrogeologic Cross-Section Showing Cadmium Concentrations at the F-Area HWMF, Fourth Quarter 1998.

8. Hydrogeologic Cross-Section Showing Lead Concentrations at the F-Area HWMF, Fourth Quarter 1998.

9. Hydrogeologic Cross-Section Showing Nitrate Concentrations at the F-Area HWMF. Fourth Quarter 1998.

10. Hydrogeologic Cross-Section Showing Tritium Activities at the F-Area HWMF, Fourth Quarter 1998.

11. Hydrogeologic Cross-Section Showing Uranium-233/234 Activities at the F-Area HWMF, Third Quarter 1998

12. Hydrogeologic Cross-Section Showing Uranium-238 Activities at the F-Area HWMF, Third Quarter 1998

13. Hydrogeologic Cross-Section Showing Strontium-90 Activities at the F-Area HWMF, Third Quarter 1998

14. Hydrogeologic Cross-Section Showing Technetium-99 Activities at the F-Area HWMF, Third Quarter 1998

15. Hydrogeologic Cross-Section Showing Gross Alpha Activities at the F-Area HWMF, Fourth Quarter 1998.

16. Hydrogeologic Cross-Section Showing Nonvolatile Beta Activities at the F-Area HWMF, Fourth Quarter 1998

\section{Volume II}

Appendix H-Maps

Page

1. Cadmium Concentrations in Aquifer Zone $\| \mathrm{B}_{2}$ (Water Table) at the General Separations Area, Fourth Quarter 1998

2. Cadmium Concentrations in Aquifer Zone IIB, (Barnwell/McBean) at the General Separations Area, Fourth Quarter 1998. 
3. Cadmium Concentrations in Aquifer Unit IIA (Congaree) at the General Separations Area, Fourth Quarter 1998

4. Gross Alpha Activities in Aquifer Zone $1 / \mathrm{B}_{2}$ (Water Table) at the General Separations Area, Fourth Quarter 1998

5. Gross Alpha Activities in Aquifer Zone IIB, (Barnwell/McBean) at the General Separations Area, Fourth Quarter 1998

6. Gross Alpha Activities in Aquifer Unit IIA (Congaree) at the General Separations Area, Fourth Quarter 1998

7. Iodine-129 Activities in Aquifer Zone $\|_{2}$ (Water Table) at the General Separations Area, Third Quarter 1998

8. lodine-129 Activities in Aquifer Zone IB $_{1}$ (Barnwell/McBean) at the General Separations Area, Third Quarter 1998

9. Iodine-129 Activities in Aquifer Unit IIA (Congaree) at the General Separations Area, Third Quarter 1998

10. Lead Concentrations in Aquifer Zone $\| B_{2}$ (Water Table) at the General Separations Area, Fourth Quarter 1998

11. Lead Concentrations in Aquifer Zone IIB, (Barnwell/McBean) at the General Separations Area, Fourth Quarter 1998

12. Lead Concentrations in Aquifer Unit IIA (Congaree) at the General Separations Area, Fourth Quarter 1998

13. Mercury Concentrations in Aquifer Zone $\| 1_{2}$ (Water Table) at the General Separations Area, Fourth Quarter 1998

14. Mercury Concentrations in Aquifer Zone $11 B_{1}$ (Barnwell/McBean) at the General Separations Area, Fourth Quarter 1998

15. Mercury Concentrations in Aquifer Unit IIA (Congaree) at the General Separations Area, Fourth Quarter 1998

16. Nitrate Concentrations in Aquifer Zone $\|_{2}$ (Water Table) at the General Separations Area, Fourth Quarter 1998

17. Nitrate Concentrations in Aquifer Zone IIB, (Barnwell/McBean) at the General Separations Area, Fourth Quarter 1998

18. Nitrate Concentrations in Aquifer Unit IIA (Congaree) at the General Separations Area, Fourth Quarter 1998

19. Nonvolatile Beta Activities in Aquifer Zone $\| \mathrm{B}_{2}$ (Water Table) at the General Separations Area, Fourth Quarter 1998

20. Nonvolatile Beta Activities in Aquifer Zone $\| l_{1} B_{1}$ Barnwell/McBean) at the General Separations Area, Fourth Quarter 1998

21. Nonvolatile Beta Activities in Aquifer Unit IIA (Congaree) at the General Separations Area, Fourth Quarter 1998.

22. Strontium-90 Activities in Aquifer Zone $\mathrm{IIB}_{2}$ (Water Table) at the General Separations Area, Third Quarter 1998

23. Strontium-90 Activities in Aquifer Zone $11 B_{1}$ (Barnwell/McBean) at the General Separations Area, Third Quarter 1998 
24. Strontium-90 Activities in Aquifer Unit IIA (Congaree) at the General Separations Area, Third Quarter 1998.

25. Technetium-99 Activities in Aquifer Zone $\|_{1} B_{2}$ (Water Table) at the General Separations Area, Third Quarter 1998

26. Technetium-99 Activities in Aquifer Zone IIB, (Barnwell/McBean) at the General Separations Area, Third Quarter 1998

27. Technetium-99 Activities in Aquifer Unit IIA (Congaree) at the General Separations Area, Third Quarter 1998

28. Tritium Activities in Aquifer Zone $\| B_{2}$ (Water Table) at the General Separations Area, Fourth Quarter 1998

29. Tritium Activities in Aquifer Zone $\|_{1}$ (Barnwell/McBean) at the General Separations Area, Fourth Quarter 1998

30. Tritium Activities in Aquifer Unit IIA (Congaree) at the General Separations Area, Fourth Quarter 1998

31. Uranium-233/234 Activities in Aquifer Zone $1 \mathrm{~B}_{2}$ (Water Table) at the General Separations Area, Third Quarter 1998

32. Uranium-233/234 Activities in Aquifer Zone IIB (Barnwell/McBean) at the General Separations Area, Third Quarter 1998

33. Uranium-233/234 Activities in Aquifer Unit IIA (Congaree) at the General Separations Area, Third Quarter 1998

34. Uranium-238 Activities in Aquifer Zone $\| B_{2}$ (Water Table) at the General Separations Area, Third Quarter 1998

35. Uranium-238 Activities in Aquifer Zone IIB, (Barnwell/McBean) at the General Separations Area, Third Quarter 1998

36. Uranium-238 Activities in Aquifer Unit IIA (Congaree) at the General Separations Area, Third Quarter 1998.

37. Potentiometric Surface Map of Aquifer Zone $\| \mathrm{B}_{2}$ (Water Table) at the General Separations Area, Third Quarter 1998

38. Potentiometric Surface Map of Aquifer Zone $\mathrm{IIB}_{2}$ (Water Table) at the General Separations Area, Fourth Quarter 1998

39. Potentiometric Surface Map of Aquifer Zone IIB, (Barnwell/McBean) at the General Separations Area, Third Quarter 1998

40. Potentiometric Surface Map of Aquifer Zone IIB 1 (Barnwell/McBean) at the General Separations Area, Fourth Quarter 1998

41. Potentiometric Surface Map of Aquifer Unit IIA (Congaree) at the General Separations Area, Third Quarter 1998.

42. Potentiometric Surface Map of Aquifer Unit IIA (Congaree) at the General Separations Area, Fourth Quarter 1998 


\section{List of Tables}

In-text

Page

1. Horizontal Groundwater Velocities (ft/year) in the Hydrostratigraphic Units beneath the F-Area HWMF...

7

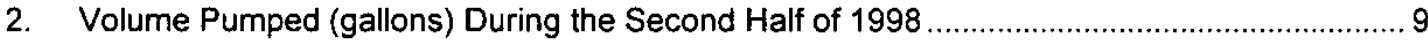

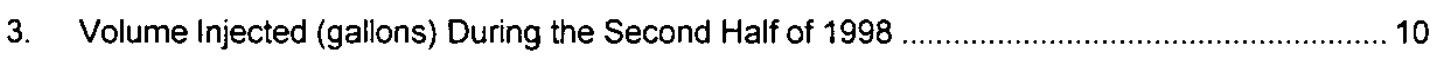

Appendix A Page

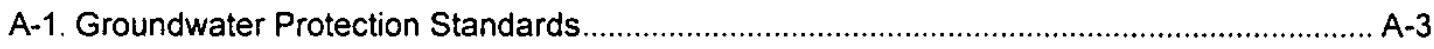

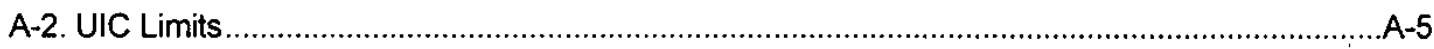

Appendix C Page

C-1. Groundwater Monitoring Results for Individual Wells ............................................................ C-7

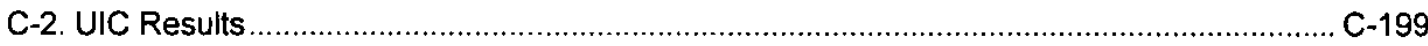


WSRC-TR-99-00012

Unclassified

THIS PAGE LEFT BLANK INTENTIONALLY 


\section{Executive Summary}

The groundwater in the uppermost aquifer beneath the F-Area Hazardous Waste Management Facility (HWMF), also known as the F-Area Seepage Basins, at the Savannah River Site (SRS) is monitored periodically for selected hazardous and radioactive constituents as required by Module III. Section C, of the 1995 Resource Conservation and Recovery Act (RCRA) Renewal Permit (South Carolina Hazardous and Mixed Waste Permit SC1-890-008-989), effective October 5 , 1995 (hereafter referred to as the RCRA permit). Currently, the F-Area HWMF monitoring network consists of 86 wells of the FSB well series, 9 wells of the FSL series, and well HSB 85A, each of which is screened in one of the three hydrostratigraphic units that make up the uppermost aquifer beneath the F-Area HWMF (Figures 2-5, Appendix B). This report presents the results of the required groundwater monitoring program as identified in provision IIIC.H.11.c of the RCRA permit and Section $C$ of the Underground Injection Control permit application (hereafter referred to as the UIC application).

As shown in Table C-1 (Appendix C), the following constituents or summations exceeded the Groundwater Protection Standard (GWPS) during the second half of 1998 in samples from monitoring wells at the F-Area HWMF: beta dose, cadmium, cobalt, lead, mercury, nickel, nitratenitrite (as $\mathrm{N}$ ), vanadium, zinc, bis(2-ethylhexyl) phthalate, trichloroethylene,

trichlorofluoromethane, gross alpha, nonvolatile beta, sum of alpha emitters, sum of beta emitters, total radium, and tritium.

In addition, the following radionuclides individually exceeded the standard for either sum of alpha emitters or sum of beta emitters: americium-241, cesium-137, curium-243/244, iodine-129, radium-226, radium-228, strontium-90, technetium-99, uranium-233/234, uranium-235, and uranium-238.

Tritium was the primary constituent detected above the GWPS; it was elevated in $68(72 \%)$ of the wells. Cadmium, lead, and cobalt were the metals that exceeded the GWPS in the largest number of wells during the second half of 1998.

Isoconcentration maps included in Volume $\|$ of this report illustrate the concentration or activity of key constituents and the extent of contamination during the second half of 1998 in each of the three hydrostratigraphic units.

Tritium, the radionuclide most frequently above the GWPS at the F-Area HWMF, had a maximum activity of $15,000 \mathrm{pCi} / \mathrm{mL}$ in Water Table well FSB 79 during third quarter 1998. Maximum activities for other key indicator radionuclides were as follows: gross alpha at $1,500 \mathrm{pCi} / \mathrm{L}$ in Water Table well FSB 79 during third quarter 1998 , nonvolatile beta at $3,100 \mathrm{pCi} / \mathrm{L}$ in Barnwell/MCBean well FSB 95CR during third quarter 1998, and strontium-90 at $880 \mathrm{pCi} / \mathrm{L}$ in Barnwell/MCBean well FSB 95CR during third quarter 1998.

Uranium-233/234 had a maximum activity of $420 \mathrm{pCi} / \mathrm{L}$ in Barnwell/McBean well FSB $95 \mathrm{CR}$ during third quarter 1998, and uranium-238 had a maximum activity of $560 \mathrm{pCi} / \mathrm{L}$ in Barnwell/McBean well FSB $95 C R$ during third quarter 1998 . Technetium-99 had a maximum activity of $320 \mathrm{pCi} / \mathrm{L}$ in Barnwell/McBean well FSB112C during third quarter 1998.

Cadmium occurred at a maximum concentration of $36 \mu \mathrm{g} / \mathrm{L}$ in Barnwell/McBean well FSB112C during third and fourth quarter 1998. Lead was found at a maximum concentration of $230 \mu \mathrm{g} / \mathrm{L}$ in Barnwell/McBean well FSB $94 \mathrm{C}$ during fourth quarter 1998, and nitrate-nitrite (as N) exceeded the GWPS in numerous wells during the second half of 1998, with a maximum concentration of $320,000 \mu \mathrm{g} / \mathrm{L}$ in Water Table well FSB 79 during third quarter 1998. 
Water-level maps indicate that the groundwater velocities and directions at the F-Area HWMF have remained relatively constant since the basins ceased operation in 1988. Groundwater flow in Aquifer Zone $\|_{B_{2}}$ (Water Table) and Aquifer Zone $\| \mathrm{B}_{1}$ (Barnwell/McBean) is generally south or southeast toward Fourmile Branch (using UTM coordinates). Flow in Aquifer Unit IIA (Congaree) is generally northwest toward Upper Three Runs Creek.

The estimated maximum groundwater velocity in Aquifer Zone $\|_{2} \mathrm{~B}_{2}$ (Water Table) during third quarter 1998 ranged from approximately 360 to 470 ftyear and during fourth quarter 1998 from

approximately 340 to $470 \mathrm{ft} / \mathrm{yr}$. The velocity estimate in Aquifer Zone $\mathrm{IIB}_{1}$ (Barnwell/McBean) ranged from approximately 110 to $240 \mathrm{ft} /$ year during third quarter 1998 and approximately 120 to $190 \mathrm{ft} /$ year during fourth quarter 1998. The velocity in Aquifer Unit IIA (Congaree) was estimated at $660 \mathrm{ft} /$ year during third quarter 1998 and 730 ftyear during fourth quarter 1998.

The F-Area Groundwater Remediation Wastewater Treatment Units (WTU) facility operating permit was received on April 18, 1997, and shakedown activities began upon receipt of RCRA approval on April 22, 1997. The WTUs operated in a shakedown mode through fourth quarter 1998. 


\section{Semiannual Corrective Action Report}

\section{Introduction}

SRS monitors groundwater quality at the F-Area Hazardous Waste Management Facility (HWMF) and provides results of this monitoring to the South Carolina Department of Health and Environmental Control (SCDHEC) semiannually as required by the Resource Conservation and Recovery Act (RCRA) permit. SRS also performs monthly sampling of the WTU effluent in accordance with Section C of the Underground Injection Control (UIC) application.

The F-Area HWMF is described in the introduction to Module III, Section C, of the RCRA permit. The F-Area HWMF well network monitors three distinct hydrostratigraphic units in the uppermost aquifer beneath the facility. The hydrostratigraphy at the F-Area HWMF is described in permit Section IIIC.H.2, and the groundwater monitoring system is described in IIIC.H.4 and Appendix IIIC-B. A detailed description of the uppermost aquifer is included in the RCRA Part B postclosure care permit renewal application for the F-Area HWMF submitted to SCDHEC in October 1993.

Sampling and analysis are conducted as required by Section IIIC.H.6 for the constituents and at the intervals specified in Appendix IIIC-D of the RCRA permit. Groundwater quality is compared to the Groundwater Protection Standards (GWPS) list in Appendix IIIC-A of the RCRA permit.

\section{System Evaluation}

The F-Area Wastewater Treatment Unit (WTU) continued in a shakedown mode during third and fourth quarter 1998. Approximately 12.8 million gallons of groundwater were treated and injected during this period. Consistent flow rates were limited due to ongoing system testing. Full-scale operation was not initiated due to various problems with the WTU. SRS has initiated a significant effort to identify and correct the problems that have been encountered during shakedown operations.

A statistical evaluation of water quality and water level data was performed and is included in this report (Section IIIC.H.11.C.ix and Appendix F). Shewhart-CUSUM charts (Appendix F) are provided in accordance with the RCRA permit application. Due to limited operations during 1998, no significant remediation system operation effects were identified. However, several trends in water quality and water levels were identified and are discussed in Section IIIC.H.11.c.ix.

\section{Water Quality and Elevation Data Tables (IIIC.H.11.c.i)}

Water quality and water elevation data for all F-Area HWMF wells during the second half of 1998 are presented in Table C-1 (Appendix C). Table C-1 identifies field parameters and modifiers that define laboratory accuracy and precision. Definitions of the abbreviations and the modifiers used in Table C-1, as well as descriptions of holding times, data rounding, and data qualification practices, are provided in Appendix C. Analytical results in Table C-1 are rounded to two significant digits. Constituents that exceeded either the GWPS or United States Environmental Protection Agency (US EPA)-approved holding times are denoted by symbols in columns headed ST and $H$, respectively.

SRS implemented a data filtering process during third and fourth quarter 1998 to better evaluate the data collected. This process qualifies certain data ("L", "R", "U", and "J") for the purposes of regulatory decision making. The data tables in Appendix $C$ include both a column for all data collected and a column for filtered data for comparison purposes. Permission to implement the data 
filtering process was requested from SCDHEC (Cook to Taylor; November 25, 1997) and granted (Taylor to Cook; April 21, 1998).

Variable-speed pumps have been installed in wells with a history of elevated analytical results for metals. Samples from these wells are collected at a slower rate to minimize turbidity, which has been associated with elevated metals levels. The chart below identifies those wells that currently have variable-speed pumps as well as those with single-speed pumps.

\begin{tabular}{|l|l|}
\hline \multicolumn{1}{|c|}{ Variable-Speed Pumps } & Single-Speed Pumps \\
\hline FSB 77, 78, 78C, 79, 79C, 88D, & All remaining FSB wells and well HSB 85A \\
$89 D, 90 D, 91 D, 92 C, 92 D, 93 D$, & have single-speed pumps \\
94C, 97C, 97D,98C,98D, 102C, & \\
104D, 105C, 105DR, 106C, 107D, & \\
and 110D & \\
FSL 1D, 2D, 3D, 4D, 5D, 6D,7D, \\
$8 D$, and 9D
\end{tabular}

\section{Analytical Results}

All data received from laboratories are validated and verified in accordance with Savannah River Site (SRS) and US EPA guidelines. Due to analytical limitations, curium-245/246 activities are reported as an upper limit of curium-246 activities, and uranium-233/234 activities are reported as an upper limit of uranium-234.

Data received from the laboratories for the following radionuclide analyses were rejected during third quarter 1998 due to matrix interference:

\begin{tabular}{|l|l|}
\hline Constituent & Well Name \\
\hline Cesium-137 & FSB 88C, FSB 90C, FSB 90D, FSB 99D, FSB107C \\
\hline Cobalt-60 & FSB104D, FSB109D \\
\hline lodine-129 & FSB 89C, FSB118D \\
\hline Plutonium-238 & FSB 78C, FSB 97C, FSB110D \\
\hline Radium-228 & FSB 95CR, FSB107C, FSB110C \\
\hline Thorium-228 & FSB 93D, FSB 94C, FSB 98D, FSB107C, FSB112C, FSB112D \\
\hline
\end{tabular}

Data received from the laboratories for the following radionuclide analyses were rejected during third quarter 1998 because surrogate or tracer spike recovery results were not within the control limits:

\begin{tabular}{|l|l|}
\hline Well Name & Constituents \\
\hline FSB 78C & $\begin{array}{l}\text { Americium-241, Curium-242, Curium-243/244, and } \\
\text { Curium-245/246. }\end{array}$ \\
\hline FSB 92C & $\begin{array}{l}\text { Americium-241, Curium-242, Curium-243/244, and } \\
\text { Curium-245/246. }\end{array}$ \\
\hline
\end{tabular}


The following wells had maintenance problems for the first sampling event; maintenance was performed, and the wells were successfully sampled on the second date listed, with no loss of permit-required data.

\begin{tabular}{|l|l|l|}
\hline Well Name & Dates of Failed Sampling Attempts & Dates of Successful Sampling Attempts \\
\hline FSB 95CR & $7 / 9 / 98 ; 8 / 4 / 98$ & $8 / 25 / 98$ \\
\hline FSB 98C & $7 / 9 / 98$ & $8 / 25 / 98$ \\
\hline FSL 2D & $7 / 9 / 98$ & $7 / 20 / 98$ \\
\hline FSL 3D & $7 / 9 / 98$ & $7 / 20 / 98$ \\
\hline FSB 87D & $10 / 27 / 98$ & $12 / 7 / 98$ \\
\hline FSB 91D & $10 / 9 / 98$ & $12 / 21 / 98$ \\
\hline FSL 1D & $10 / 28 / 98$ & $12 / 30 / 98$ \\
\hline
\end{tabular}

Wells FSL 3D and FSL 8D were purged through the sample port to lower turbidity for fourth quarter 1998 sampling.

\section{Appendix IX Analyses}

During third quarter 1998, Appendix IX analyses were performed as required by the RCRA permit. Beryllium in wells FSB 94C and 95CR was the only constituent not already listed on the RCRA permit's GWPS list that was reported above detection limit in F Area. Confirmation sampling was performed during January 1999, and laboratory results are pending.

\section{Water Elevations}

Synchronous water-level elevations were measured during third quarter and fourth quarter 1998 in compliance with Section IIIC.H.7 of the RCRA permit. Potentiometric maps are provided in Volume II. A significant water level increase observed in most wells during the spring of 1998 is likely due to increased precipitation and associated recharge during this time period. Compared to the uppermost aquifer zone, the lower aquifer zones generally have smaller, yet more gradual increases, as would be expected from recharge effects.

\section{Hydrographs (IIIC.H.11.c.ii)}

Hydrographs showing the water elevations for the F-Area HWMF are provided in Appendix E. Hydrograph data include synchronous water level measurements and water level measurements taken during sampling. Clustered wells are shown on a single graph.

A significant water level increase in most wells in the spring of 1998 is likely due to increased precipitation and associated recharge during this time period. See section IIIC.H.11.C.xiii. Compared to the uppermost aquifer zone, the lower aquifer zones generally have smaller, more gradual increases as would be expected from recharge effects.

\section{Time vs. Concentration Plots (IIIC.H.11.c.iii)}

Appendix D contains time series plots for cadmium, lead, nitrate (or nitrate/nitrite), gross alpha, nonvolatile beta, strontium-90, technetium-99, tritium, uranium-233/234, and uranium-238 for selected wells. The wells were selected to delineate the extent of the constituent distribution, to monitor the migration of the constituents, and to follow trends of constituents present at high levels. Constituents reported as below detection are not plotted. 


\section{Isoconcentration Maps (IIIC.H.11.c.iv)}

Isoconcentration maps for strontium-90, technetium-99, uranium-233/234, and uranium-238 in the three hydrostratigraphic units during third quarter and for cadmium, lead, nitrate (or nitrate-nitrite), gross alpha, nonvolatile beta, and tritium during fourth quarter 1998 are presented in Volume II.

\section{Potentiometric Maps (IIIC.H.11.c.v)}

Piezometric and potentiometric surface maps for the monitored water-bearing units during third and fourth quarters 1998 are located in Volume II. The maps illustrate groundwater flow patterns beneath the F-Area HWMF for third and fourth quarter 1998.

\section{Potentiometric Cross-Sections and Isoconcentration Cross-Sections (IIIC.H.11.c.vi)}

Potentiometric and isoconcentration cross-section figures for permit-required constituents are located in Appendix B.

\section{Groundwater Velocity and Direction (IIIC.H.11.c.vii)}

Horizontal groundwater velocity calculations are used to estimate the transport rate of constituents originating from the F-Area HWMF. Velocities in Aquifer Zone $\|_{2} B_{2}$ (Water Table) and Aquifer Zone $\mathrm{IIB}_{1}$ (Barnwell/McBean) are calculated along two flow paths. The flow paths, designated fiow paths $A$ and $B$, characterize the approximate maximum and minimum groundwater velocity within these units in areas associated with the basins. The velocity in Aquifer Unit IIA (Congaree) is calculated along a single flow path because the velocity within this unit is generally more uniform than in the overtying units.

Groundwater velocity is estimated using the following equation:

$$
\text { Flow }\left(\mathrm{ft} / \text { day) }=\frac{\text { Hydraulic Conductivity }(\mathrm{ft} / \text { day) }}{\text { Porosity }} \times \frac{d h(\mathrm{ft})}{d l(\mathrm{ft})}\right.
$$

The value $d h$ is the difference in head, and $d l$ is the length of the flow path. Flow path length is calculated to the nearest $50 \mathrm{ft}$. Maximum velocity per day is calculated to two significant digits, then multiplied by 365 and rounded to two significant digits for the velocity per year. Velocity estimates vary depending on the vertical gradient between wells, the size of the area under consideration, the number of data points, and the length and location of the flow path. Because these calculations are based on inferred or estimated parameters, groundwater velocity estimates are accurate to an order of magnitude only.

Maximum horizontal velocity estimates for the hydrostratigraphic units during third and fourth quarter are provided in Table 1. 
Table 1. Horizontal Groundwater Velocities (ft/year) in the Hydrostratigraphic Units beneath the F-Area HWMF

\begin{tabular}{|lccc|}
\hline & $\begin{array}{c}\text { Aquifer Zone IIB } \\
\text { (Water Table) }\end{array}$ & $\begin{array}{c}\text { Aquifer Zone IIB } \\
\text { (Barnwell/McBean) }\end{array}$ & $\begin{array}{c}\text { Aquifer Unit IIA } \\
\text { (Congaree) }\end{array}$ \\
\hline $\mathrm{K}_{\mathrm{n}}$ (ft/day) & 40 & 10.4 & 65 \\
Effective porosity & $20 \%$ & $20 \%$ & $20 \%$ \\
$\mathrm{dh} / \mathrm{dl}$ & Varies & varies & varies \\
Velocity (3Q98) & $360-470$ & $110-240$ & 660 \\
Velocity (4Q98) & $340-470$ & $120-190$ & 730 \\
\hline
\end{tabular}

The hydraulic conductivity $\left(K_{h}\right)$ values represent regional average hydraulic conductivities and may vary locally.

Flow in Aquifer Zone $\mathrm{IIB}_{2}$ (Water Table) and Aquifer Zone IIB, (Barnwell/McBean) was south along both paths during third and fourth quarter 1998. Flow in Aquifer Unit IIA (Congaree) was west during third quarter 1998 and northwest toward Upper Three Runs Creek during fourth quarter.

\section{Extent and Severity of Groundwater Contamination (IIIC.H.11.c.viii)}

Constituents that exceeded the GWPS (Appendix A) during third and fourth quarter 1998 in the FArea HWMF wells are denoted in Table C-1 (Appendix $C$ ).

As shown in Table $\mathrm{C}-1$, the following constituents or summations exceeded the GWPS during the second half of 1998 in samples from monitoring wells at the F-Area HWMF: beta dose, cadmium, cobalt, lead, mercury, nickel, nitrate-nitrite (as N), vanadium, zinc, bis(2-ethylhexyl) phthalate, trichloroethylene, trichlorofluoromethane, gross alpha, nonvolatile beta, sum of alpha emitters, sum of beta emitters, total radium, and tritium.

In addition, the following radionuclides individually exceeded the standard for either total alpha emitters or total beta emitters: americium-241, cesium-137, curium-243/244, iodine-129, radium226, racium-228, strontium-90, technetium-99, uranium-233/234, uranium-235, and uranium-238.

The extent and severity of groundwater contamination is delineated by the contours above the GWPS on the isoconcentration maps (Volume II). Tritium is the most widespread contaminant and exceeds the GWPS in all three aquifers (Maps 28-30 in Appendix H). The highest activities are downgradient of the basins in the Water Table and the Barnwell/McBean aquifer zones. Tritium is also present in the Water Table aquifer zone along the F-Area Inactive Process Sewer Line to the basins. Tritium is elevated in some Congaree wells between the basins and Fourmile Branch.

The extent of the nitrate-nitrite (as N) plume (Maps 16-18) is very similar to the tritium plume. The cadmium, gross alpha, and nonvolatile beta plumes (Maps 1-6 and 19-21) in the Water Table and Barnwell/McBean are similar in shape but less extensive than the tritium plume.

Individual radionuclides are reported in the Water Table and Barnwell/McBean aquifer zones between the basins and Fourmile Branch. These include strontium-90, technetium-99, uranium233/234, and uranium-238 (Maps 22-27 and 31-36). Uranium-233/234 and uranium-238 are both reported as elevated in one Congaree well south of the basins. 
Lead is elevated in several Water Table and Barnwell/McBean wells (Maps 10-12) near the basins. There is no clear pattern to the distribution of elevated lead.

\section{Background Results}

Wells FSB 76C and 108D and well HSB $85 \mathrm{~A}$ are the background wells for the F-Area HWMF. These wells are screened in the Barnwell-McBean, Water Table, and Upper Congaree aquifer zones, respectively. Although vanadium appears to have exceeded GWPS in background well HSB 85A during third quarter 1998, the detection is not considered valid. The well is a background well screened in the Congaree aquifer, there has been no trend of vanadium contamination in the well, the laboratory blank was contaminated, and the sample had to be diluted.

\section{Statistical Evaluation (IIIC.H.11.c.ix)}

The F-Area RCRA Permit requires an annual statistical evaluation of water quality and water level data to assess significant changes or impacts associated with operation of the F-Area WTU. Constituents listed in Appendix IIIC-A of the RCRA permit must be evaluated in all Point of Compliance (POC) wells and in a representative number of plume assessment wells.

Control charts have historically been used in industry and laboratories to monitor processes to determine whether the monitoring data shows a constituent either "in control" or "out of control." For groundwater monitoring, control charts can be used to monitor water quality data and to flag anomalous results. The statistical procedure used by SRS to assess the effectiveness of corrective action is the Shewhart-CUSUM control chart, detailed in Appendix F. The chart will be used to detect both increasing and decreasing trends and to identify either sudden incursions (contaminant slugs) or steady drifts (changes in plume concentration or size). Water level data will be evaluated similarly.

The selected wells for this statistical analysis include all POC wells (as listed in Table IIIC-B of the RCRA permit) and a representative number of plume wells, as follows: FSB102C, 104C, 104D, $110 \mathrm{C}, 110 \mathrm{D}, 112 \mathrm{C}, 112 \mathrm{D}, 117 \mathrm{D}, 121 \mathrm{C}$, and 121D.

For this evaluation, background samples consisted of all samples from the first quarter of 1991 through the last quarter of 1995 . The end of 1995 was chosen as a background cutoff date to provide adequate background sample quantities and to provide control plot points for at least one year prior to startup of remediation operations.

\section{Plotting of Results}

Shewhart-CUSUM control plots are given in Appendix $F$ for those constituent/well pairings that made it through the data qualification and treatment process described above. To maintain clarity and uniformity in all the control plots, the $y$-axis ranges have been set between -6 and +6 standardized units.

Due to the limited operation of the WTU and the minimal amount of available trend data since the background time period, the analysis of the control chart statistics cannot be extensive, but some general comments can be made concerning the indications of gradual increases/decreases, sudden breakthroughs, or sudden drops.

In general, the charts do not show any effect from the shakedown operations. There are a number of wells that show statistically significant decreases of tritium and nitrate-nitrite (as N), but at the same time, there are also a number of wells that show statistically significant increases of these same constituents. There is no spatial correlation evident to these increases or decreases. 


\section{Evaluation of Water Quality and Water Elevation Data (IIIC.H.11.c.x)}

Due to the limited remediation operations, zone of capture and drawdown have not yet been determined. No significant trends or changes have been attributed to the operation of the WTU.

\section{Volume and Rates of Groundwater Pumped (IIIC.H.11.c.xi)}

Table 2 summarizes the pump rates, monthly volumes, and total volumes pumped from each extraction well during third and fourth quarter 1998. The pump rates represent the average pump rate for each well. The individual well volumes (and consequently, the average flow rates) are estimated due to instrument errors. The well totals are based on recorded well performance (PLC) and extraction totals. The total volumes extracted and injected are based on the totalizers on the extraction and injection tanks. Data are collected daily from the totalizers.

Table 2. Volume Pumped (gallons) During the Second Half of 1998

\begin{tabular}{|c|c|c|c|c|c|c|c|c|}
\hline Well & $\begin{array}{l}\text { Rate } \\
\text { (gpm) }\end{array}$ & $\begin{array}{l}\text { July } \\
1998 \\
\end{array}$ & $\begin{array}{c}\text { August } \\
1998\end{array}$ & $\begin{array}{c}\text { September } \\
1998\end{array}$ & $\begin{array}{c}\text { October } \\
1998\end{array}$ & $\begin{array}{c}\text { November } \\
1998\end{array}$ & $\begin{array}{c}\text { December } \\
1998\end{array}$ & $\begin{array}{l}\text { 6-Month } \\
\text { Total }\end{array}$ \\
\hline FEX 1 & 8.4 & 48,938 & 331,845 & 222,466 & 450.807 & 136,178 & 122,640 & $1,312,874$ \\
\hline FEX 2 & 10.7 & 164,651 & 397,407 & 215,922 & 271,842 & 275.811 & 162,448 & $1,488,081$ \\
\hline FEX 3 & 10.7 & 176,628 & 434,270 & 274,811 & 282,174 & 156,891 & 153,640 & $1,478,414$ \\
\hline FEX 4 & 9.2 & 114,867 & 331,285 & 268,267 & 275,274 & 153,936 & 154,840 & $1,298,469$ \\
\hline FEX 5 & 4.8 & 41,172 & 102,426 & 176,664 & 185,828 & 97,681 & 101,740 & 705,511 \\
\hline FEX 6 & 9.0 & 152,042 & 315,466 & 196,293 & 258,078 & 162,827 & 158,740 & $1,243,446$ \\
\hline FEX 7 & 8.3 & 102,823 & 307,277 & 202,836 & 309,887 & 153,936 & 123,140 & $1,199,899$ \\
\hline FEX 8 & 6.7 & 69,912 & 204,852 & 183,575 & 295,530 & 130,656 & 110,040 & 994,565 \\
\hline FEX 9 & 5.4 & 16,442 & 221,230 & 189,750 & 137,637 & 156,891 & 85,140 & 807,090 \\
\hline FEX 10 & 8.6 & 127,500 & 413,785 & 255,181 & 378,742 & 124,331 & - & $1,299,539$ \\
\hline FEX 11 & 9.2 & 198,425 & 409,703 & 261.724 & 289.573 & 130,242 & - & $1,289,667$ \\
\hline Total & & $1,213,400$ & $3,469,546$ & $2,447,489$ & $3,135,372$ & $1,679,380$ & $1,172,368$ & $13,117,555$ \\
\hline
\end{tabular}

\section{Volume and Rates of Groundwater Injected (IIIC.H.11.c.xii)}

Table 3 summarizes the pump rates, monthly volumes, and total volumes pumped toeach injection well during third and fourth quarter 1998. The pump rates represent the average pump rate for each well. The individual well volumes (and consequently, the average flow rates) are estimated due to instrument errors. The well totals are based on recorded well performance (PLC) and injection totals. The total volumes extracted and injected are based on the totalizers on the extraction and injection tanks. Data are collected daily from the totalizers. The difference in total volume extracted (Table 2) and total volume injected (Table 3 ) is due to the time delay between collecting extraction and injection readings as well as water retention in the WTU. 
WSRC-TR-99-00012

Unclassified

Table 3. Volume Injected (gallons) During the Second Half of 1998

\begin{tabular}{|c|c|c|c|c|c|c|c|c|}
\hline Well & $\begin{array}{l}\text { Rate } \\
\text { (gpm) }\end{array}$ & $\begin{array}{l}\text { July } \\
1998\end{array}$ & $\begin{array}{c}\text { August } \\
1998\end{array}$ & $\begin{array}{c}\text { September } \\
1998\end{array}$ & $\begin{array}{c}\text { October } \\
1998\end{array}$ & $\begin{array}{c}\text { November } \\
1998\end{array}$ & $\begin{array}{c}\text { December } \\
1998\end{array}$ & $\begin{array}{l}\text { 6-Month } \\
\text { Total }\end{array}$ \\
\hline FIN 1 & 12.3 & 129,356 & 507,114 & 348,066 & 435,420 & 237,497 & 145,119 & $1,802,572$ \\
\hline FIN 2 & 9.3 & 115,739 & 332,247 & 257,187 & 335,710 & 180,225 & 122,517 & $1,343,625$ \\
\hline FIN 3 & 9.1 & 113,546 & 342,729 & 238,322 & 313,650 & 187,434 & 118,817 & $1,314,498$ \\
\hline FIN 4 & 12.2 & 125,952 & 489,628 & 377,916 & 418,660 & 237,197 & 140,717 & $1,790,070$ \\
\hline FIN 5 & 8.2 & 119,144 & 167,812 & 231,636 & 324,305 & 201,852 & 122,517 & $1,167,266$ \\
\hline FIN 6 & 10.9 & 130,760 & 447,498 & 232,636 & 440,800 & 223,179 & 135,617 & $1,610,490$ \\
\hline FIN 7 & 9.0 & 105,527 & 360,327 & 281,784 & 276,750 & 158,598 & 109,317 & $1,292,303$ \\
\hline FIN 8 & 7.0 & 124,952 & 209,639 & 186,264 & 206,640 & 151,389 & 90,217 & 969,101 \\
\hline FIN 9 & 5.3 & 119,050 & 132,788 & 143,280 & 199,260 & 50,395 & 76,817 & 721,590 \\
\hline FIN 10 & 5.0 & 61,274 & 251,718 & 175,578 & 191,880 & 79,299 & - & 759,749 \\
\hline Total & & $1,145,300$ & $3,241,500$ & $2,472,669$ & $3,143,075$ & $1,707,065$ & $1,061,655$ & $12,771,264$ \\
\hline
\end{tabular}

\section{Rainfall and Recharge (IIIC.H.11.c.xiii)}

Figure 1 illustrates the rainfall data for $F$ Area during third and fourth quarter 1998. Recharge to groundwater is estimated to be approximately $30 \%$ of rainfall in the General Separations Area.

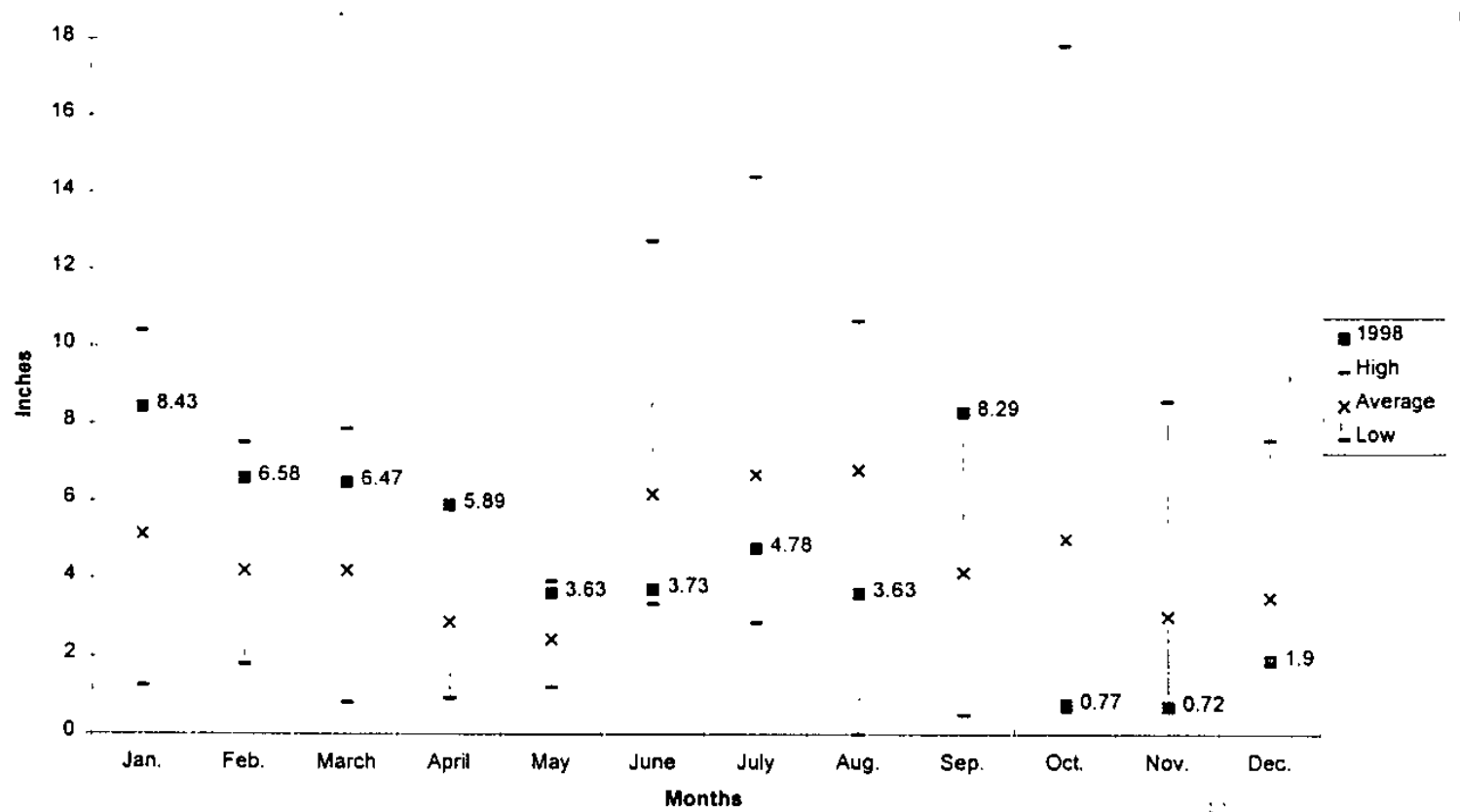

Figure 1. Rainfall Data for F Area During Third and Fourth Quarter 1998 


\section{Summary of System Downtime (IIIC.H.11.c.xiv)}

Significant downtime to the facility was incurred because of lightning strikes in the well fields or in the vicinity of the control room programmable logic controller (PLC). The electrical surges through the system resulted in damage to well instrumentation, PLC input/output modules, and miscellaneous controls. Initial modifications consisted of the addition of in-line fuses to help protect electrical components. In addition, SRS is currently designing an improved lightning protection system. The additional fuses have been effective in reducing much of the electrical damage of lightning strikes. The design is complete and scheduled for installation during second quarter 1999.

Vibration problems that have been identified during this time period will be resolved in the near future. The first area of concern is the vibration induced into the system by the positive displacement, piston-type, reverse osmosis $(R O)$ pumps. To date, no failures or problems have been directly attributed to this vibration in $\mathrm{F}$ Area. A vibration analysis is being conducted and will be followed by recommendations for design modifications, as applicable. The modifications are scheduled for completion in third quarter 1999. The second area of concern is the vibration caused by the air-driven diaphragm pumps used for sludge transfer from the sludge collection tanks (flocculation tank, clarifier, or filtrate return tank) to the filter press. This vibration has contributed to a failure of the surge protector for the large diaphragm pump in the sludge transfer system. SRS is currently designing modifications to the sludge transfer systems to reduce vibration and improve reliability. The estimated schedule for completion of this modification is second quarter 1999.

The F-Area facility was limited to one-RO operation due to problems associated with the clarification and sludge removal systems. Most operations and maintenance downtime problems were due to one of the following causes:

- Clogging, pump, or flow problems with the polymer feed system

- Clogging, pump, or flow problems with the ferric chloride feed system

- Clogging, pump, or flow problems with the caustic $(\mathrm{NaOH})$ feed system

- Excessive carryover of the flocculant and precipitants through the clarifier due to incomplete chemical reaction and/or settling

- $\mathrm{pH}$ probe coating or calibration problems

- Removal of process solids caking on top of the medium in the roughing filters

- Fouling or platting of the flow control valve in the chemical feed line due to iron settling out of solution during low-flow or no-flow operating conditions.

SRS assumed operation of the WTU from the subcontractor on January 1,1999. Upon transition to SRS operation, several modifications to improve process efficiency and reliability are anticipated.

Appendix G summarizes the operations activities and downtime for third and fourth quarter 1998.

\section{Minor Modifications to the System (IIIC.H.11.c.xv)}

Subsequent to receiving the wastewater permit to operate the modified treatment system on June 24,1998 , it was determined that numerous process changes would be required to correct the problems associated with the effective operation of the treatment system. These approved temporary process changes included: installation of additional roughing filters, new PolyBlend addition system, and numerous piping reconfigurations. A permit application modification for the 
F-Area WTU, which incorporated these and other temporary changes, was submitted to SCDHEC on September 30,1998. A permit to construct was issued on November 10, 1998.

Implementation of the permanent changes will commence upon receipt of the permanent roughing filters.

Permanent access platforms for the ion exchangers were also fabricated and installed.

\section{Effectiveness of the Corrective Action System (IIIC.H.11.c.xvi)}

The F-Area WTU operated in a shakedown mode during the third and fourth quarter 1998 but has not commenced full-scale operation. A statistical analysis was completed on the POC wells and a representative number of plume assessment wells, and this information will serve as a baseline for future reports. Due to the limited operations thus far, no direct impact to groundwater from operation of the WTU has been established. The statistical analysis identified groundwater trends for a few constituents discussed in Section IIIC.H.11.b.ix.

\section{Underground Injection Control (UIC) Sampling Results}

SRS collected samples to determine compliance with UIC standards as required by the operating permit issued on April 17, 1997. The results of these samples are presented in Appendix C.

The F-Area WTU was sampled on July 30 , August 13 , September 29 , October 19 , November 17 , and December 21, 1998, for the suite of contaminants specified in the current UIC permit. Results were reported to SCDHEC both by electronic submittal and by letter. Whenever an exceedence was confirmed, SRS immediately notified SCDHEC. In each case, SCDHEC granted approval to restart/continue operating the unit based on proposed corrective actions.

Starting with the October 1998 samples, a change was implemented in the reporting format for UIC sampling results. SRS replaced the AN95 reporting format with the AN98, the most significant change being the implementation of a sample-specific quantitation limit (SSEQL) for radionuclide results. Radionuclides were previously reported using the method detection limit as the quantitation limit. Reported detection limits that appear higher for October, November, and December may be attributable to this change. The UIC permit divides the compliance suite of constituents into four sections: Section I, Inorganics; Section II, Organics; Section III, Radionuclides; and Section IV, Radionuclides. Section IV includes speciated radionuclide results, and Section III reports results for gross alpha, gross (nonvolatile) beta, and total radium.

None of the Section I or Section II constituents exceeded their respective regulatory standards during third and fourth quarter 1998. Only barium, cobalt, and copper regularly exceeded their respective quantitation limits during the last half of 1998.

Gross alpha exceeded its limit of $15 \mathrm{pCi} / \mathrm{L}$ in November, and although the reported quantitation limit was also higher than $15 \mathrm{pCi} / \mathrm{L}$ in December, the raw sample result of $9.56 \mathrm{pCi} / \mathrm{L}$ indicated that there was no actual exceedence. Gross (nonvolatile) beta and total radium were below their respective compliance limits of $50 \mathrm{pCi} / \mathrm{L}$ and $5 \mathrm{pCi} / \mathrm{L}$ for all six months.

The alpha-emitting radionuclides, curium-242, curium-243/244, curium-246, plutonium-238, plutonium-239/240, thorium-230, and thorium-232, were all below their respective detection limits during third and fourth quarter 1998. Americium-241 exceeded its detection limit in July 1998 and November 1998 but was well below the sum of alphas compliance limit of $15 \mathrm{pCi} / \mathrm{L}$ in both of those months. Radium-226 exceeded its detection limit during July and August but was less than the compliance limit of $5 \mathrm{pCi} / \mathrm{L}$ for total radium in both of those months. Thorium-228 exceeded its detection limit during September but remained well below the compliance standard for the sum of alpha contributors. Uranium-235 exceeded its detection limit in August, September, and November but remained well below the compliance limit for the sum of alphas. Uranium-233/234 
and uranium-238 exceeded their respective detection limits during the last half of 1998, but both remained below their compliance limit for the sum of alpha contributors. Consequently, the sum of alpha contributors exceeded the compliance limit in November at $24.08 \mathrm{pCi} / \mathrm{L}$ but remained below the $15 \mathrm{pCi} / \mathrm{L}$ standard in each of the other five months. Overall, the sum of alpha contributors decreased or remained steady at acceptable levels through October, increased in November, and decreased in December below the compliance limit.

Results for the beta-emitting radionuclides cesium- 137 and cobalt- 60 were both below their respective detection limits during the last half of 1998 . Carbon-14 exceeded its detection limit in September, October, and December. The October result was $60.1 \mathrm{pCi} / \mathrm{L}$, above the compliance limit of $50 \mathrm{pCi} / \mathrm{L}$ for the sum of beta contributors. However, results during the following months were below the detection limit; therefore, no exceedence of carbon-14 was confirmed during third and fourth quarters. Radium-228 exceeded its detection limit only during July but was below its compliance limit of $5 \mathrm{pCi} / \mathrm{L}$ for total radium. Technetium- 99 exceeded its detection limit during four of the six months (the October result was rejected), but did not exceed the compliance limit of 50 $\mathrm{pCi} / \mathrm{L}$ for the sum of betas. lodine-129 was detected in all six months, and was elevated above the $50 \mathrm{pCi} / \mathrm{L}$ compliance limit for the sum of beta contributors during August, September, and November. The September result was a confirmed exceedence. Strontium-90 exceeded its detection limit in July, August, September, and December. It exceeded its compliance limit of 8 $\mathrm{pCi} / \mathrm{L}$ during July, August, and September but remained below that fimit in December. Elevated results in each of the first three months represented confirmed exceedences. However, modifications performed on the F-Area WTU resulted in significantly lower strontium-90 results during fourth quarter 1998 . The sum of beta contributors exceeded the compliance limit in each month during the last half of 1998 , and every month was a confirmed exceedence. All results ranged between 70 and $100 \mathrm{pCi/L}$. The most common contributors to that sum were carbon-14, iodine-129, technetium- 99 , and strontium- 90 . Overall, the sum of the beta contributors remained elevated over the $50 \mathrm{pCi} / \mathrm{L}$ standard during the last half of 1998, with iodine-129, carbon-14, and technetium-99 replacing each other as the major contributor in any given month. Based on reported levels, difficulity of treatment, and health risk, the most problematic constituent at present is iodine-129. Carbon-14 and technetium-99 are lower risks to human health and appear elevated relative to other constituents because of their high detection limits. Modifications to the unit designed to control strontium-90 appear to have been successful relative to the limited operations through the end of 1998. 
WSRC-TR-99-00012 Unclassified

THIS PAGE LEFT BLANK INTENTIONALLY. 


\section{Appendix A}

\section{Regulatory Standards}




\section{WSRC-TR-99-00012}

Unclassified

THIS PAGE LEFT BLANK INTENTIONALLY. 
WSRC-TR-99-00012

Unclassified

Table A-1. Groundwater Protection Standard

Established for the F-Area Hazardous Waste Management Facility by the 1995 RCRA Renewal Permit (SCDHEC, 1995).

\begin{tabular}{|c|c|c|}
\hline \multirow[b]{2}{*}{ Analyte } & \multicolumn{2}{|l|}{ Concentration/ } \\
\hline & Activity Limit & Unit \\
\hline \multicolumn{3}{|l|}{ Inorganic Constituents } \\
\hline Antimony & 6 & $\mu g$ L \\
\hline Arsenic & 50 & $\mu g / L$ \\
\hline Barium & 2,000 & $\mu g / L$ \\
\hline Cadmium & 5 & $\mu g / L$ \\
\hline Chromium & 100 & $\mu g / L$ \\
\hline Cobalt & $3^{a}$ & $\mu g / L$ \\
\hline Copper & 1,300 & $\mu g / L$ \\
\hline Cyanide & $20^{b}$ & $\mu g / L$ \\
\hline Lead & 15 & $\mu g / L$ \\
\hline Mercury & 2 & $\mu g / L$ \\
\hline Nickel & 100 & $\mu g / L$ \\
\hline Nitrate & 10,000 & $\mu g / L$ \\
\hline Selenium & 50 & $\mu g / L$ \\
\hline Silver & 50 & $\mu g / L$ \\
\hline Thallium & 2 & $\mu g / L$ \\
\hline Vanadium & $3.5^{a}$ & $\mu g / L$ \\
\hline Zinc & 5,000 & $\mu g / L$ \\
\hline \multicolumn{3}{|l|}{ Organic Constituents } \\
\hline Benzene & 5 & $\mu g / L$ \\
\hline Bis(2-ethylhexyl) phthalate & $10 \mathrm{C}$ & $\mu g / L$ \\
\hline Dichloromethane (methylene chloride) & 5 & $\mu g / L$ \\
\hline Phenols & $2^{d}$ & $\mu g / L$ \\
\hline Tetrachloroethylene & 5 & $\mu g / L$ \\
\hline Trichloroethylene & 5 & $\mu g / L$ \\
\hline Trichlorofluoromethane & $5 e$ & $\mu g / L$ \\
\hline \multicolumn{3}{|l|}{ Radionuclide Constituents } \\
\hline Gross alpha & 15 & pCi/l. \\
\hline Nonvolatile beta & $50 f$ & $\mathrm{pCi} / \mathrm{L}$ \\
\hline Tritium & 20,000 & $\mathrm{pCi} / \mathrm{L}$ \\
\hline Americium-241 & Sum of alphas $<15 \mathrm{pCi} / \mathrm{L}$ & $\mathrm{pCi} / \mathrm{L}$ \\
\hline Cesium-137 & $\begin{array}{l}\text { Sum of beta dose }<4 \text { mrem/yr and } \\
<50 \mathrm{pCi} / \mathrm{L}\end{array}$ & $\mathrm{pCi} / \mathrm{L}$ \\
\hline Cobalt-60 & $\begin{array}{l}\text { Sum of beta dose }<4 \mathrm{mrem} / \mathrm{yr} \text { and } \\
<50 \mathrm{pCi} / \mathrm{L}\end{array}$ & $\mathrm{pCi} / \mathrm{L}$ \\
\hline Curium-242 & Sum of alphas $<15 \mathrm{pCi} / \mathrm{L}$ & $\mathrm{pCi} / \mathrm{L}$ \\
\hline Curium-243/244 & Sum of alphas $<15 \mathrm{pCi} / \mathrm{L}$ & $\mathrm{pCi} / \mathrm{L}$ \\
\hline Curium-245/246 & Sum of alphas $<15 \mathrm{pCi} / \mathrm{L}$ & pCi/L \\
\hline lodine-129 & $\begin{array}{l}\text { Sum of beta dose }<4 \text { mrem/yr and } \\
<50 \mathrm{pCi} / L\end{array}$ & $\mathrm{pCi} / \mathrm{L}$ \\
\hline
\end{tabular}


Analyte

Plutonium-238

Plutonium-239/240

Radium-226

Radium-228

Strontium-90

Technetium-99

Thorium-228

Thorium-230

Thorium-232

Total alpha-emitting radiumg

Total radium (radium-226 plus radium-

228)

Uranium-233/234

Uranium-234

Uranium-235

Uranium-238

\section{Concentration/}

Activity Limit

Sum of alphas $<15 \mathrm{pCi} / \mathrm{L}$

Unit

Sum of alphas $<15 \mathrm{pCi} / \mathrm{L}$

Total radium $<5 \mathrm{pCi} / \mathrm{L}$

Sum of beta dose $<4$ mrem/yr and $<50 \mathrm{pCi} / \mathrm{L}$ and total radium $<5$

pCill

Sum of beta dose $<4 \mathrm{mrem} / \mathrm{yr}$ and $<50 \mathrm{pCi} / \mathrm{L}$ and strontium- $90<8$ $\mathrm{pCi} / \mathrm{L}$

Sum of beta dose $<4$ mrem/yr and $\quad \mathrm{pCi} / \mathrm{L}$ $<50 \mathrm{pCi} / \mathrm{L}$

Sum of alphas $<15 \mathrm{pCi} / \mathrm{L}$

Sum of alphas $<15 \mathrm{pCi} / L$

Sum of alphas $<15 \mathrm{pCi} /$

5

5

Sum of alphas $<15 \mathrm{pCi} / \mathrm{L}$

Sum of alphas $<15 \mathrm{pCi} / \mathrm{L}$

Sum of alphas $<15 \mathrm{pCi} /$

Sum of alphas $<15 \mathrm{pCi} / \mathrm{L}$
$\mathrm{pCi} / \mathrm{L}$

$\mathrm{pCi} / L$

$\mathrm{pCi} / \mathrm{L}$

pCi/L

$\mathrm{pCi} / \mathrm{L}$

pCi/L

$\mathrm{pCi} i$

$\mathrm{pCi} / \Omega$

pCill

$\mathrm{pCi}$ /

pCi/L

pCi/L

$\mathrm{pCi} / 2$

$\mathrm{pCi} / \mathrm{L}$

a Concentrations are observed background levels.

b Concentration is the practical quantitation limit (PQL) for EPA Method 335.2 (used by WA) and 335.3 (Used by GE).

c Concentration is the PQL for EPA Method 8270 as published in 40CFR Part 264, Appendix IX.

d Concentration is the PQL for EPA Method 420.1 (used by GE) and 420.2 (used by WA).

e Concentration is the Appendix IX PQL for EPA Method 8240.

$f \quad$ This is the screening level above which providers of public drinking water should perform analyses for specific manmade radionuclides. The standard for the total dose equivalent from all such radionuclides is 4 mrem/yr.

g Results reported by this analysis, which does not distinguish among radium-223, radium-224, and radium-226, are assumed to be primarily radium-226 and are compared to the GWPs for total radium. 
Table A-2. Regulatory Limits for UIC Permitted Constituents

\begin{tabular}{|c|c|c|}
\hline \multicolumn{3}{|l|}{ Section I, Inorganics } \\
\hline Arsenic & $\mu g / L$ & 50 \\
\hline Barium & $\mu g / L$ & 2,000 \\
\hline Cadmium & $\mu g / L$ & 5 \\
\hline Chromium & $\mu g / L$ & 100 \\
\hline Lead & $\mu g / L$ & 50 \\
\hline Mercury & $\mu g / L$ & 2 \\
\hline Selenium & $\mu g / L$ & 50 \\
\hline Silver & $\mu g / L$ & 50 \\
\hline \multicolumn{3}{|l|}{ Section II, Organics } \\
\hline Antimony & $\mu g / L$ & 6 \\
\hline Cobalt & $\mu g / L$ & 140 \\
\hline Copper & $\mu g / L$ & 1,300 \\
\hline Cyanide & $\mu g / L$ & 200,000 \\
\hline Benzene & $\mu g / L$ & 5 \\
\hline Bis(2-ethylhexyl) phthalate & $\mu g / L$ & 140 \\
\hline $\begin{array}{l}\text { Methylene chloride } \\
\text { (Dichloromethane) }\end{array}$ & $\mu g / L$ & 5 \\
\hline Nickel & $\mu g / L$ & 100 \\
\hline Phenol & $\mu g / L$ & 10 \\
\hline Tetrachloroethylene & $\mu g / L$ & 5 \\
\hline Thallium & $\mu g / L$ & 2 \\
\hline Tin & $\mu g / L$ & 50 \\
\hline Trichloroethylene & $\mu g / L$ & 5 \\
\hline Trichlorofluoromethane & $\mu g / L$ & 100 \\
\hline Vanadium & $\mu g / L$ & 49 \\
\hline Zinc & $\mu g / L$ & 5,000 \\
\hline \multicolumn{3}{|l|}{ Section III, Radionuclides } \\
\hline Gross alpha & $\mathrm{pCi} / \mathrm{L}$ & 15 \\
\hline Gross beta & $\mathrm{pCi} / \mathrm{L}$ & 50 \\
\hline Total radium $(226+228)$ & $\mathrm{pCi} / \mathrm{L}$ & 5 \\
\hline \multicolumn{3}{|l|}{ Section IV, Radionuclides } \\
\hline Americium-241 & $\mathrm{pCi} / \mathrm{L}$ & SOA \\
\hline Cesium-137 & $\mathrm{pCin}$ & SOB \\
\hline Curium-242 & $\mathrm{pCi} / \mathrm{L}$ & SOA \\
\hline
\end{tabular}


Constituent

Curium-243/244

Curium-246

Carbon-14

Cobalt-60

lodine-129

Plutonium-238

Plutonium-239/240

Nickel-63

Radium-226

Radium-228

Strontium-90

Technetium-99

Thorium-228

Thorium-230

Thorium-232

Uranium-233/234

Uranium-234

Uranium-235

Uranium-238
Reg. Limit

Unit

$\mathrm{pCi/L}$

pCi/L

pCi/L

$\mathrm{pCi} / \mathrm{L}$

$\mathrm{pCi} / \mathrm{L}$

$\mathrm{pCi} / \mathrm{L}$

$\mathrm{pCi} / \mathrm{L}$

pCill

$\mathrm{pCi} / \mathrm{L}$

$\mathrm{pCi} / \mathrm{L}$

$\mathrm{pCi} / \mathrm{L}$

$\mathrm{pCi} / \mathrm{L}$

pCi/L

pCi/L

pCi/L

pCill

pCíl

pCi/L

pCill
SOA

SOA

SOB

SOB

SOB

SOA

SOA

SOB

SOR

SOR

SOB

SOB

SOA

SOA

SOA

SOA

SOA

SOA

SOA 
WSRC-TR-99-00012

Unclassified

\section{Appendix B}

Figures 
WSRC-TR-99-00012

Unclassified

THIS PAGE LEFT BLANK INTENTIONALLY. 


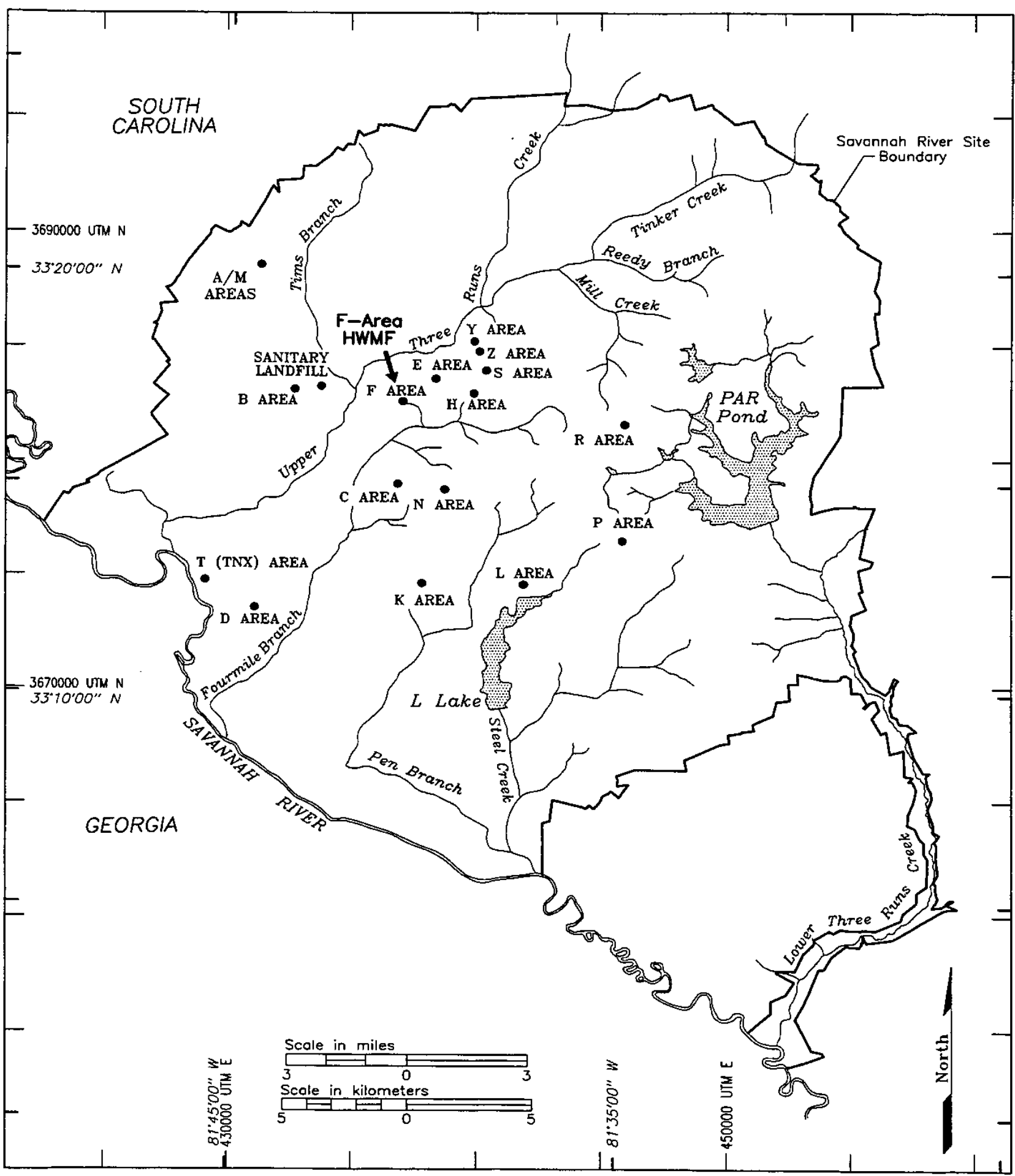

Figure 1. Location of the F-Area HWMF at the Savannah River Site 


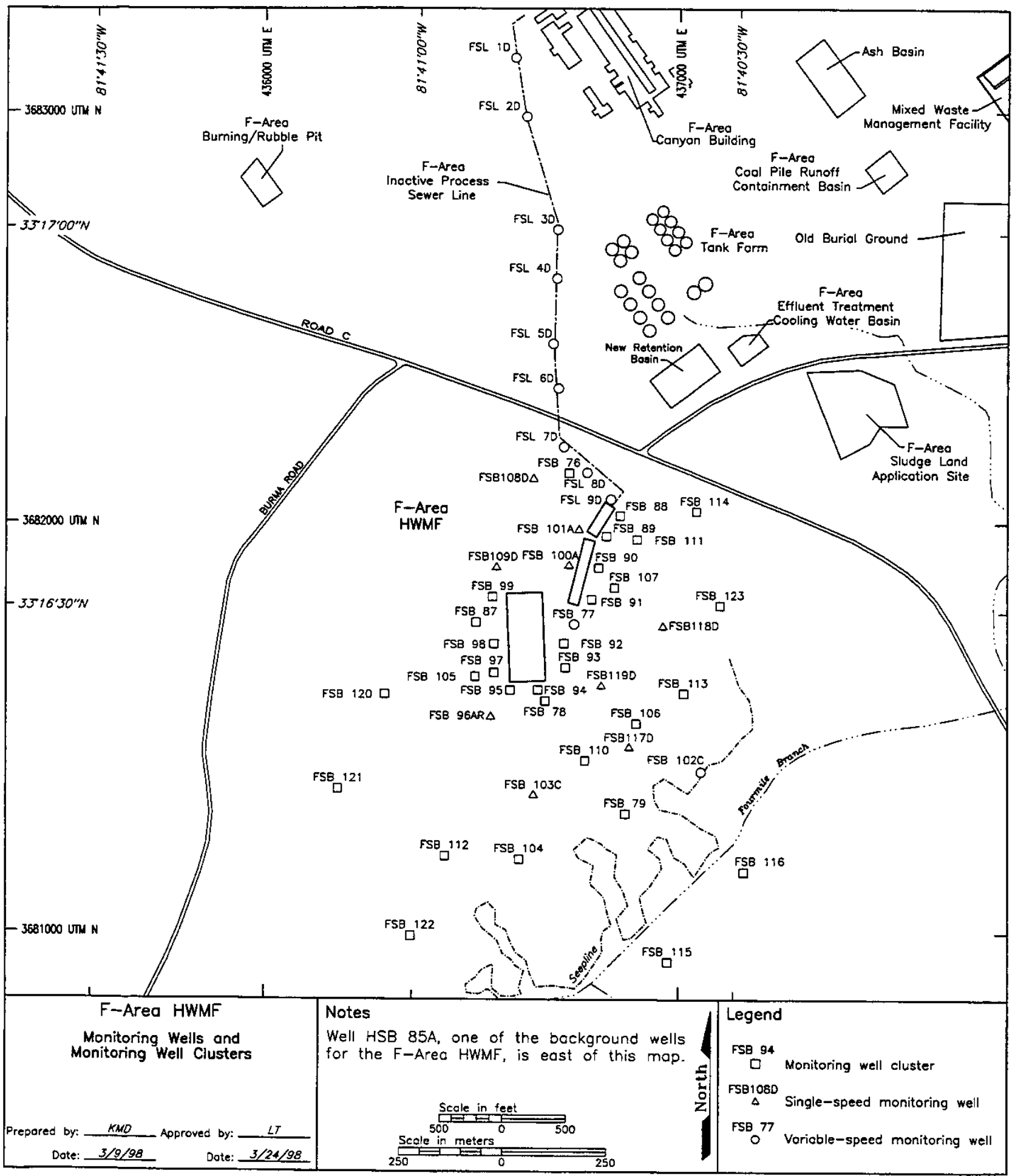

Figure 2. Location of the Groundwater Monitoring Wells at the F-Area HWMF 


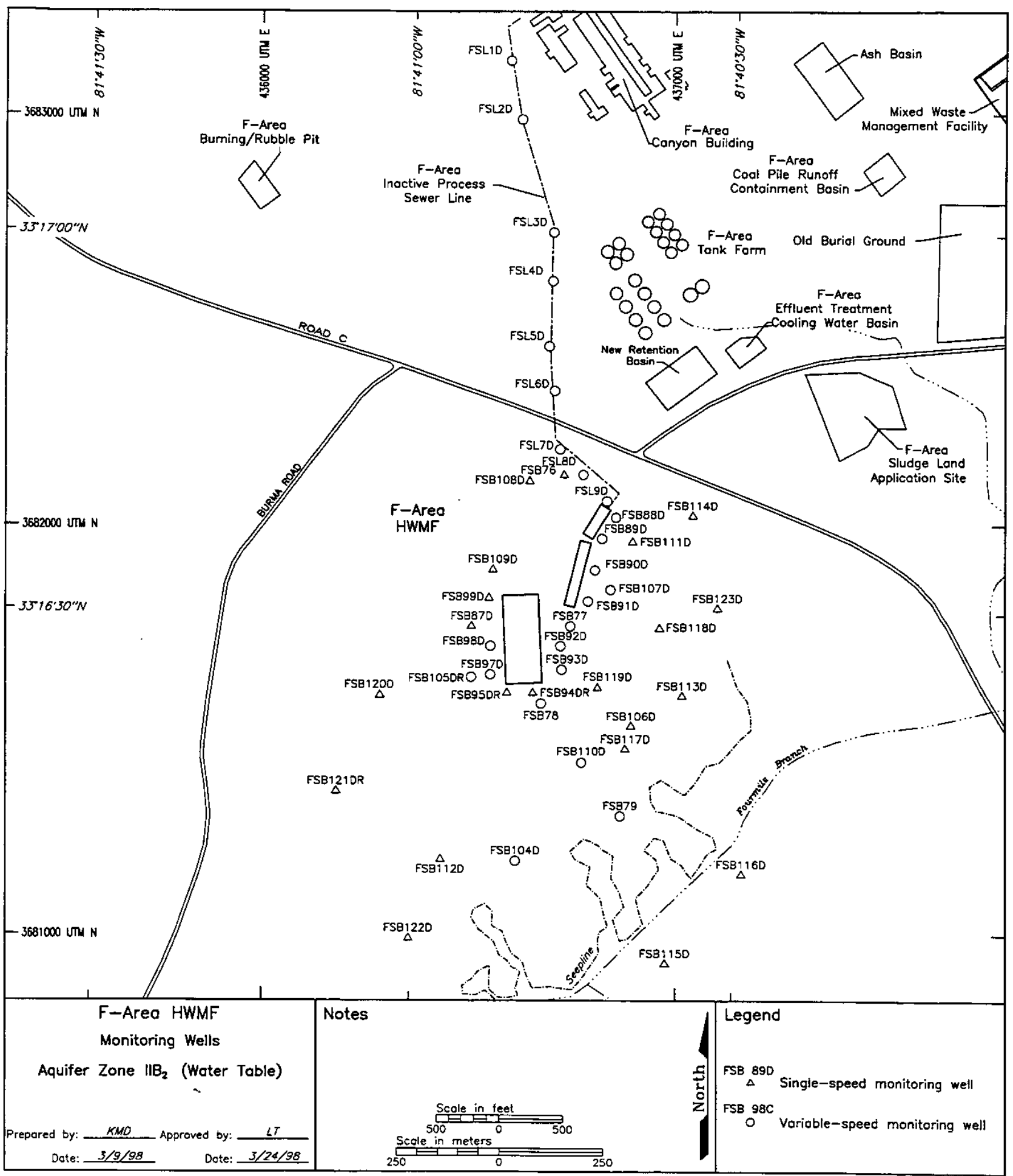

Figure 3. Location of Aquifer Zone $\mathrm{IIB}_{2}$ (Water Table) Monitoring Wells at the F-Area HWMF 


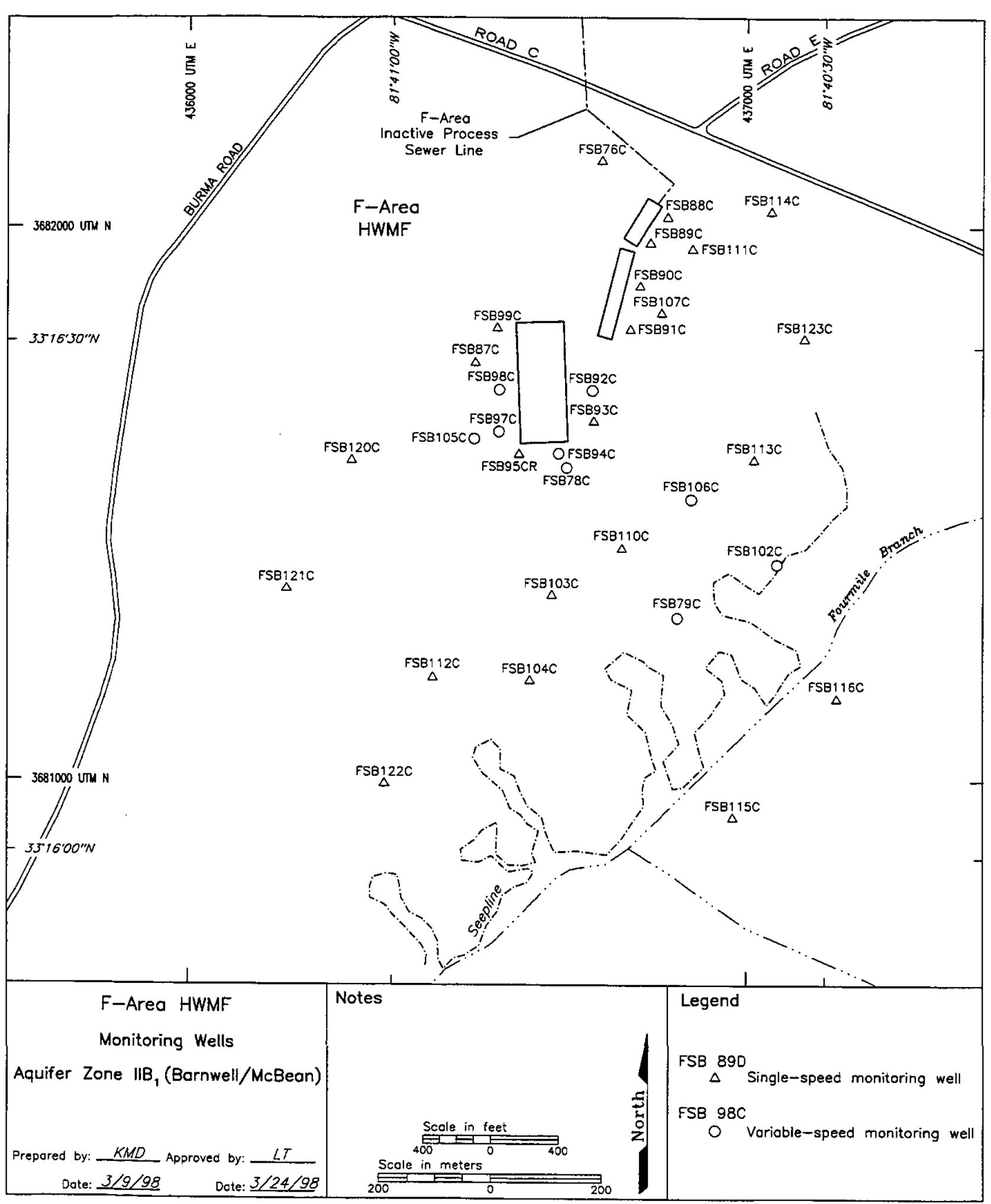

Figure 4. Location of Aquifer Zone IIB, (Barnwell/McBean) Monitoring Wells at the F-Area HWMF 


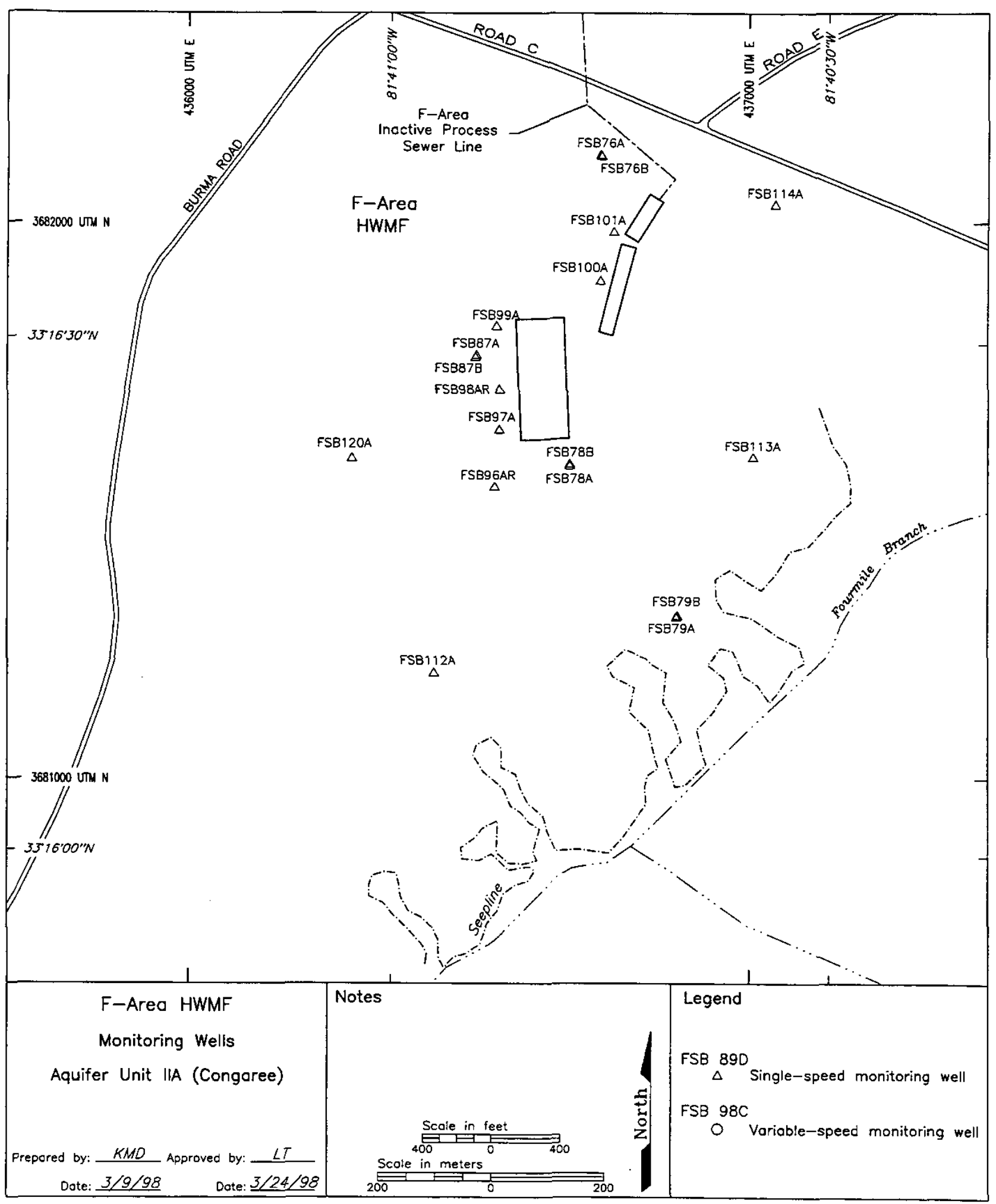

Figure 5. Location of Aquifer Unit IIA (Congaree) Monitoring Wells at the F-Area HWMF 

Figure 6. Hydrogeologic Crose-section Showing Potentiometric Surfaces at the F-Area HMmF,
Fourth Quarter 1998 Figure 7. Hydrogeologic Croas-Soction Showing Cadmium Concentrations at the F-Area HMmF,
Fourth Quarter 1958 


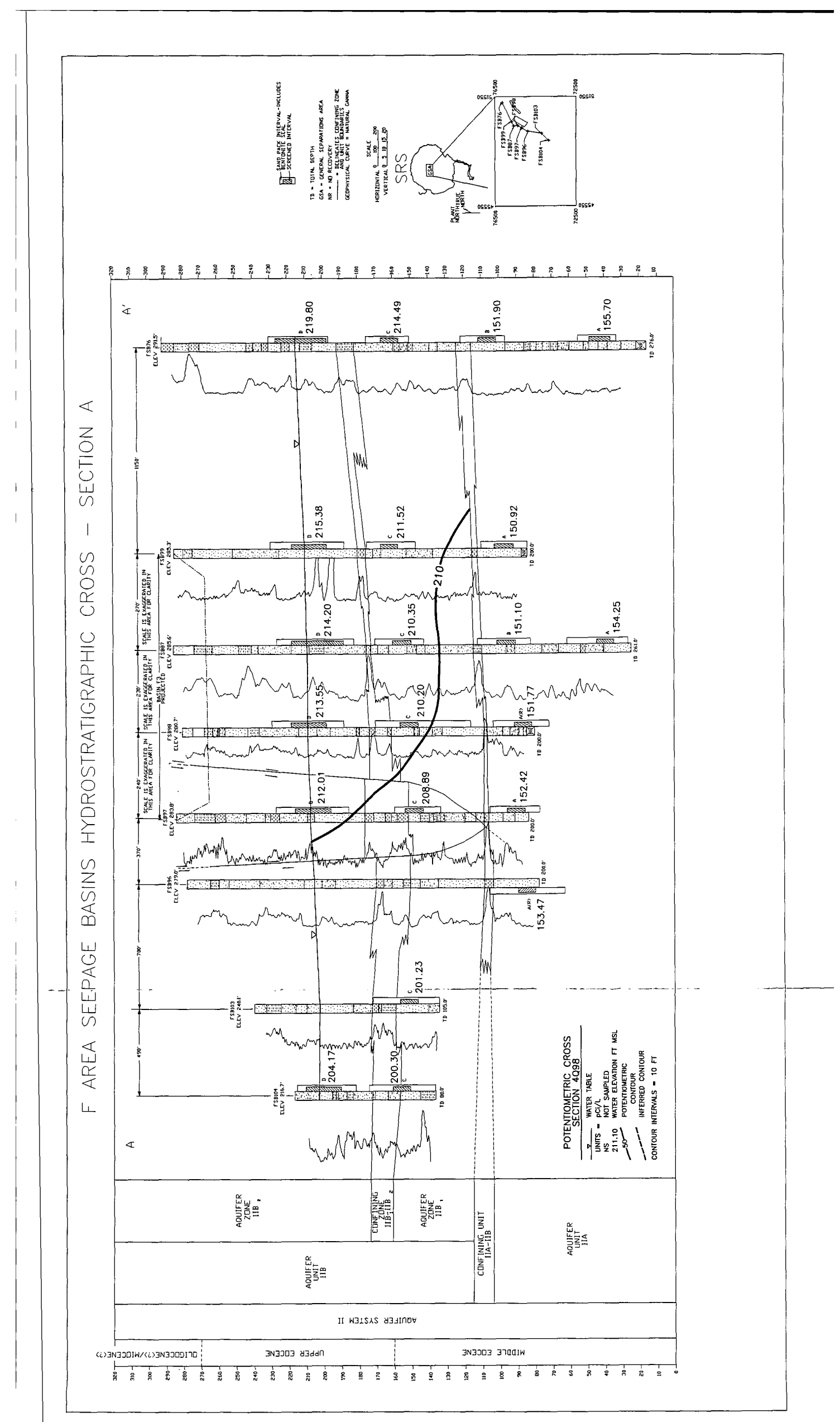




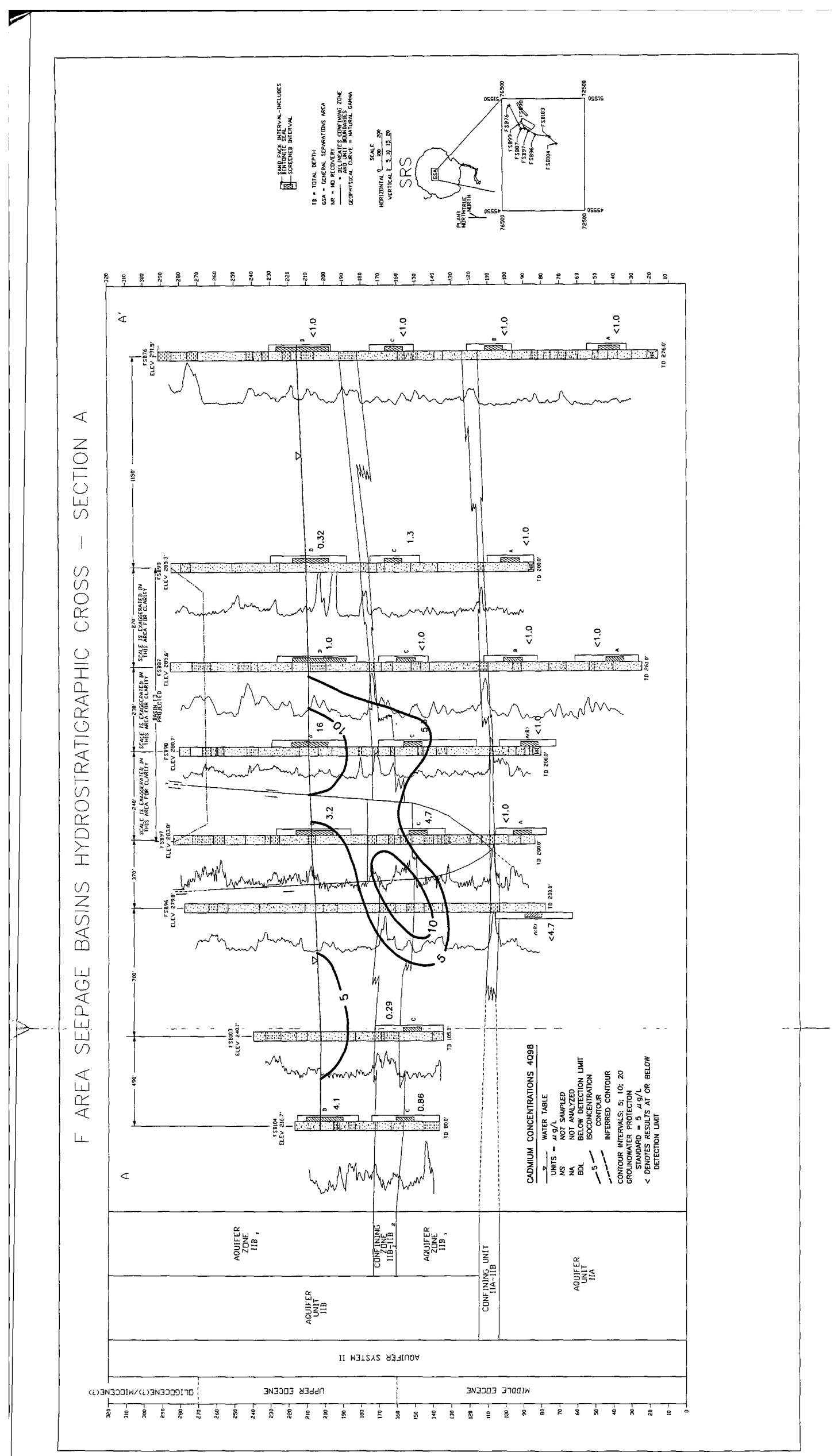


Figure 8. Hydrogeologic Cross-Section Showing Lead Concentrations at the F-Area HWMF, Fourth Quarter 1998

Figure 8. Hydrogeologic Crose-Section Showing Nitrate Activities at the F-Area HWMF, Fourth Quarter 1998 


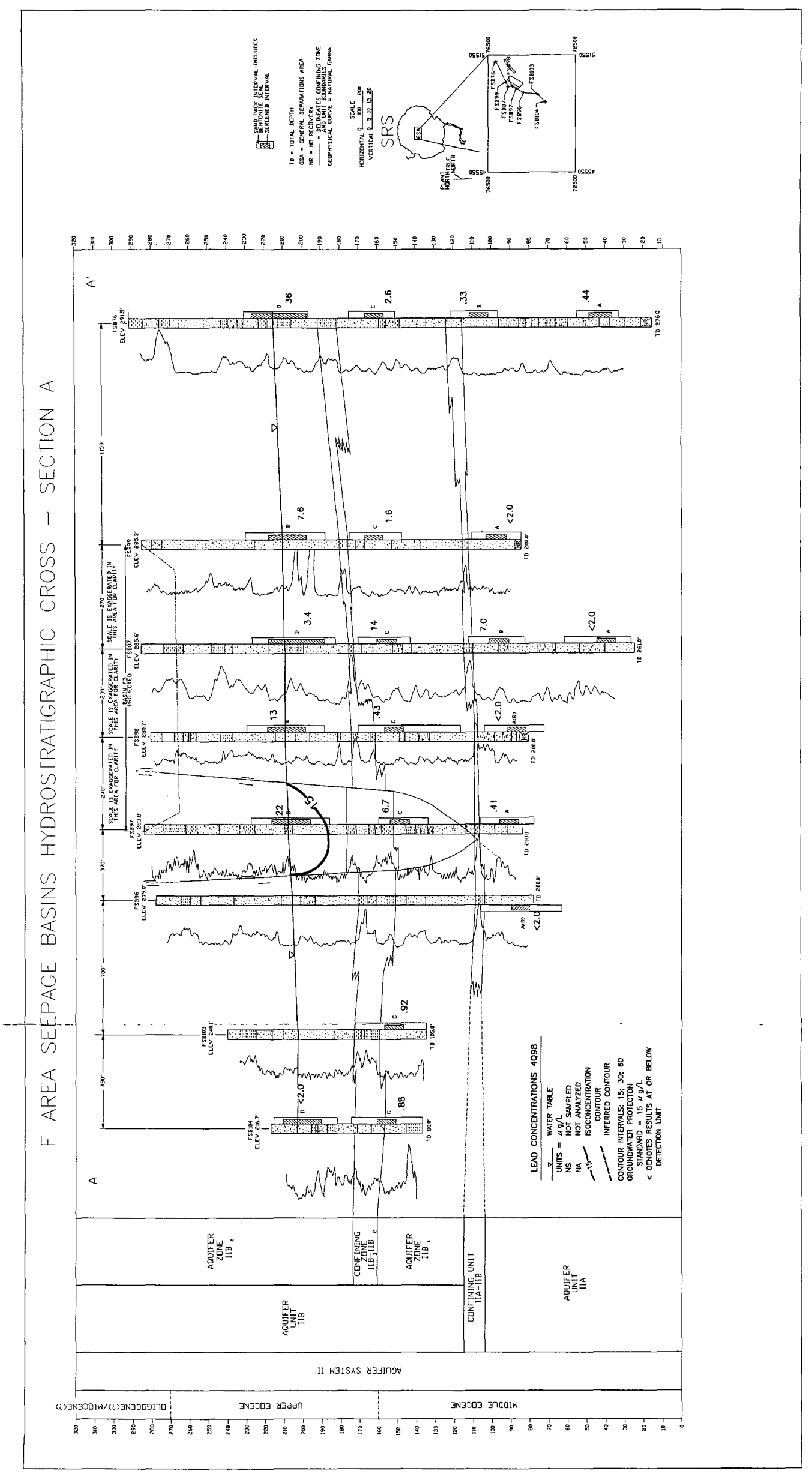




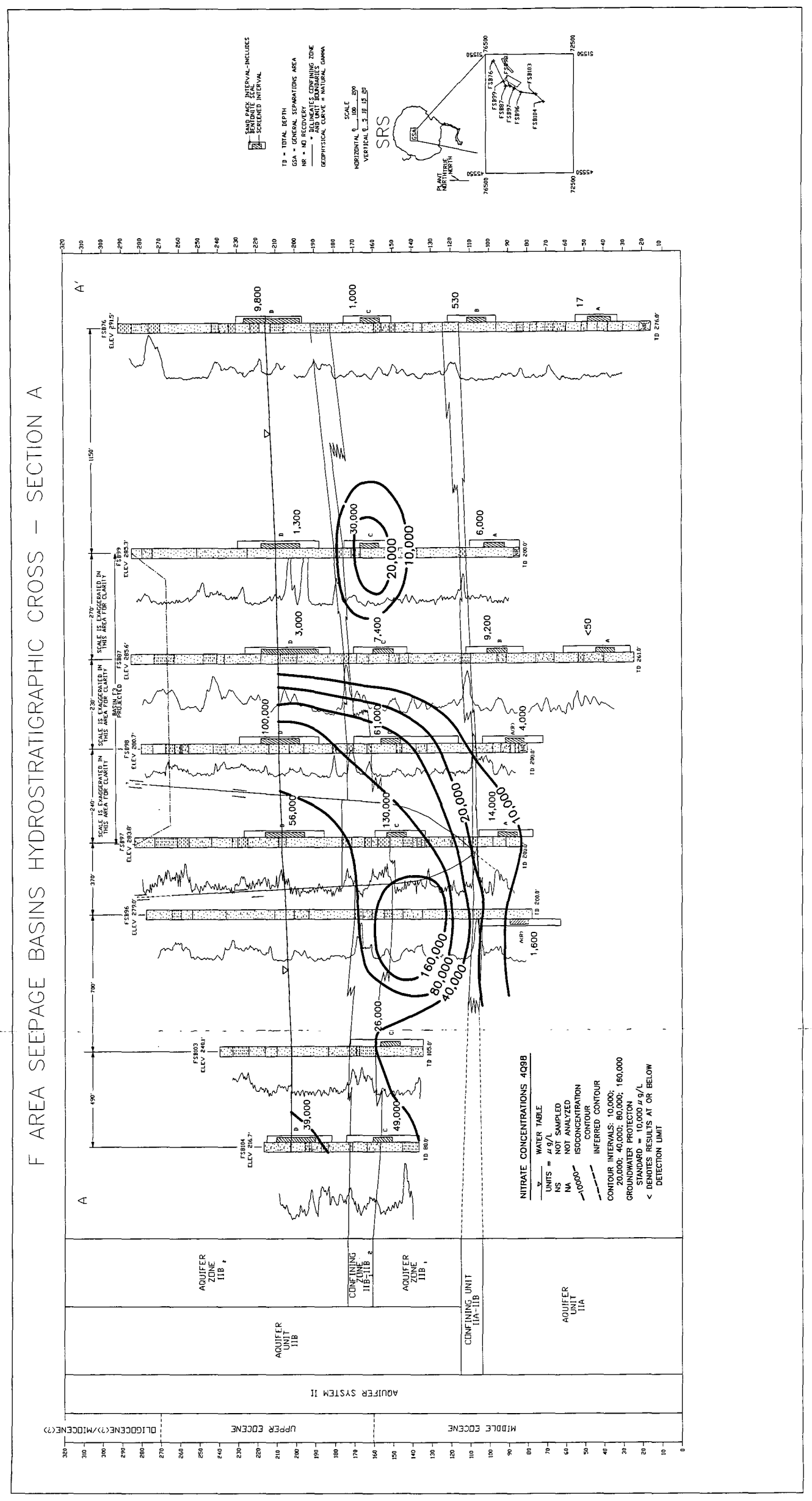


Figure 10. Hydrogeologic Cross-Section Showing Trtium Activities at the F-Area HWMF, Fourth
Quarter 1998 Figure 11. Hydrogeologic Cross-Section Showing Uranium-233/234 Activities at the F-Area HMmF,
Third Quarter 1998 


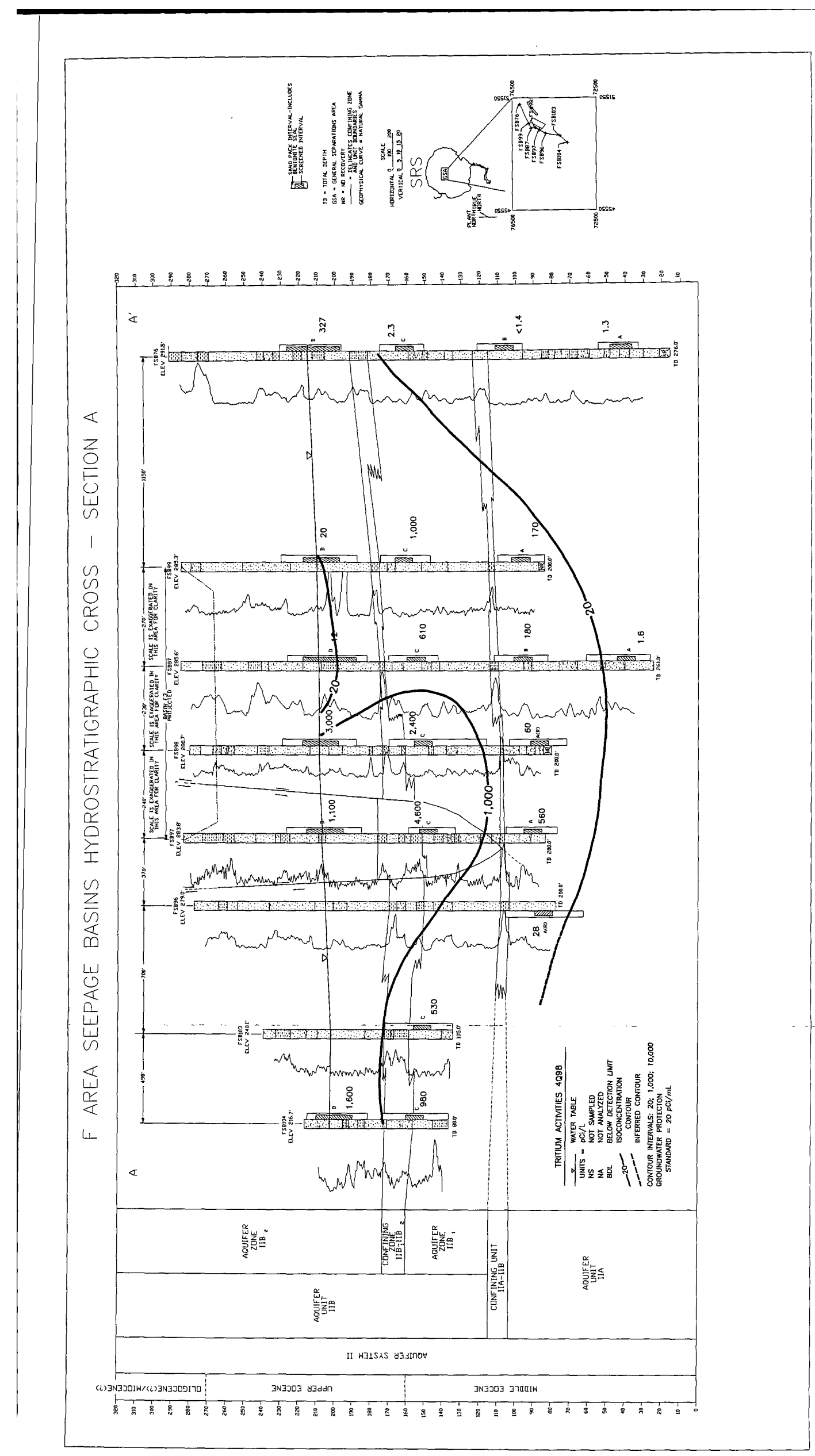




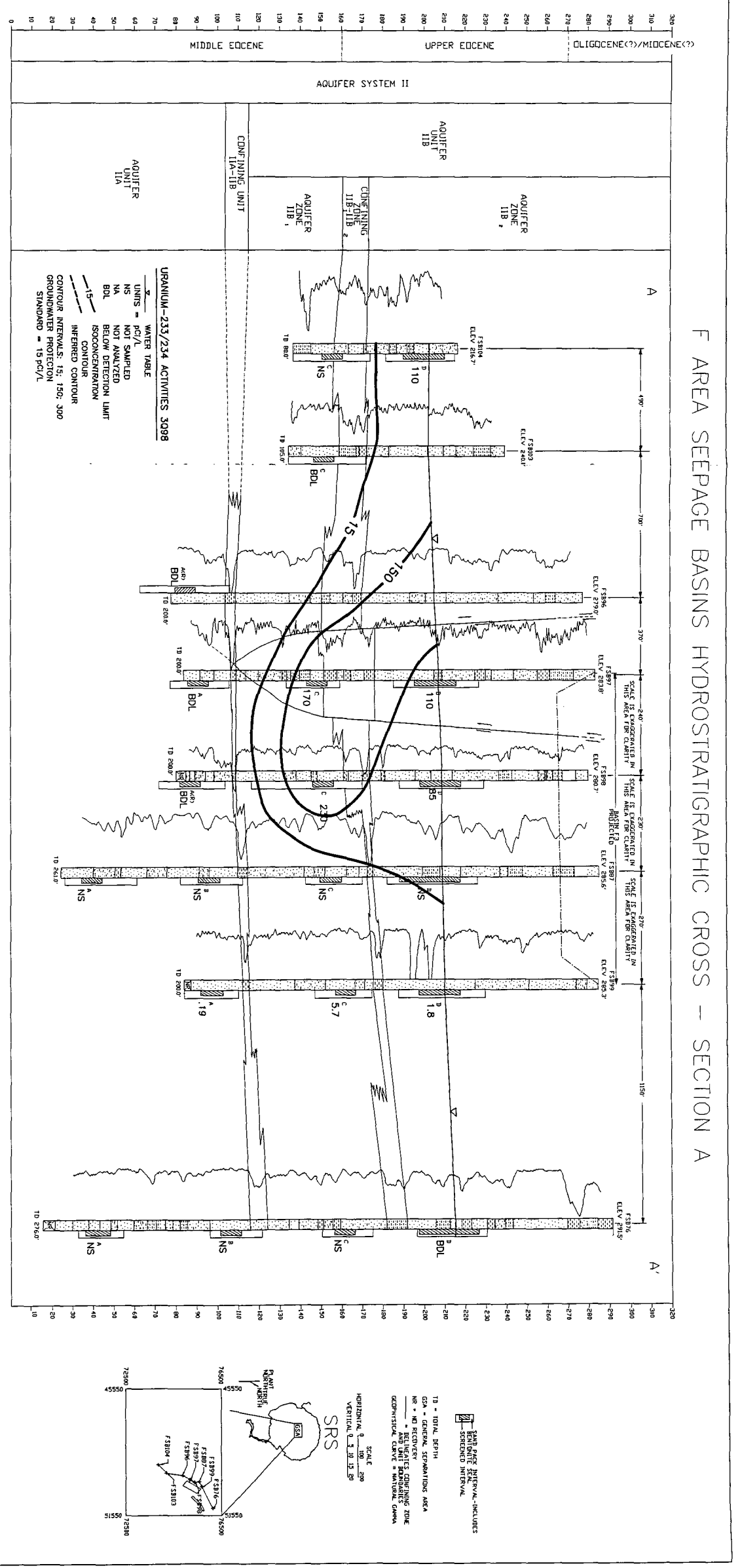


Figure 12. Hydrogeologic Cross-Section Showing Uranium-238 Activities at the F-Area HWMF, Third Quarter 1988

Figure 13. Hydrogeologic Cross-Section Showing Strontium-90 Activities at the F-Area HWMF, Third Quarter 1988 


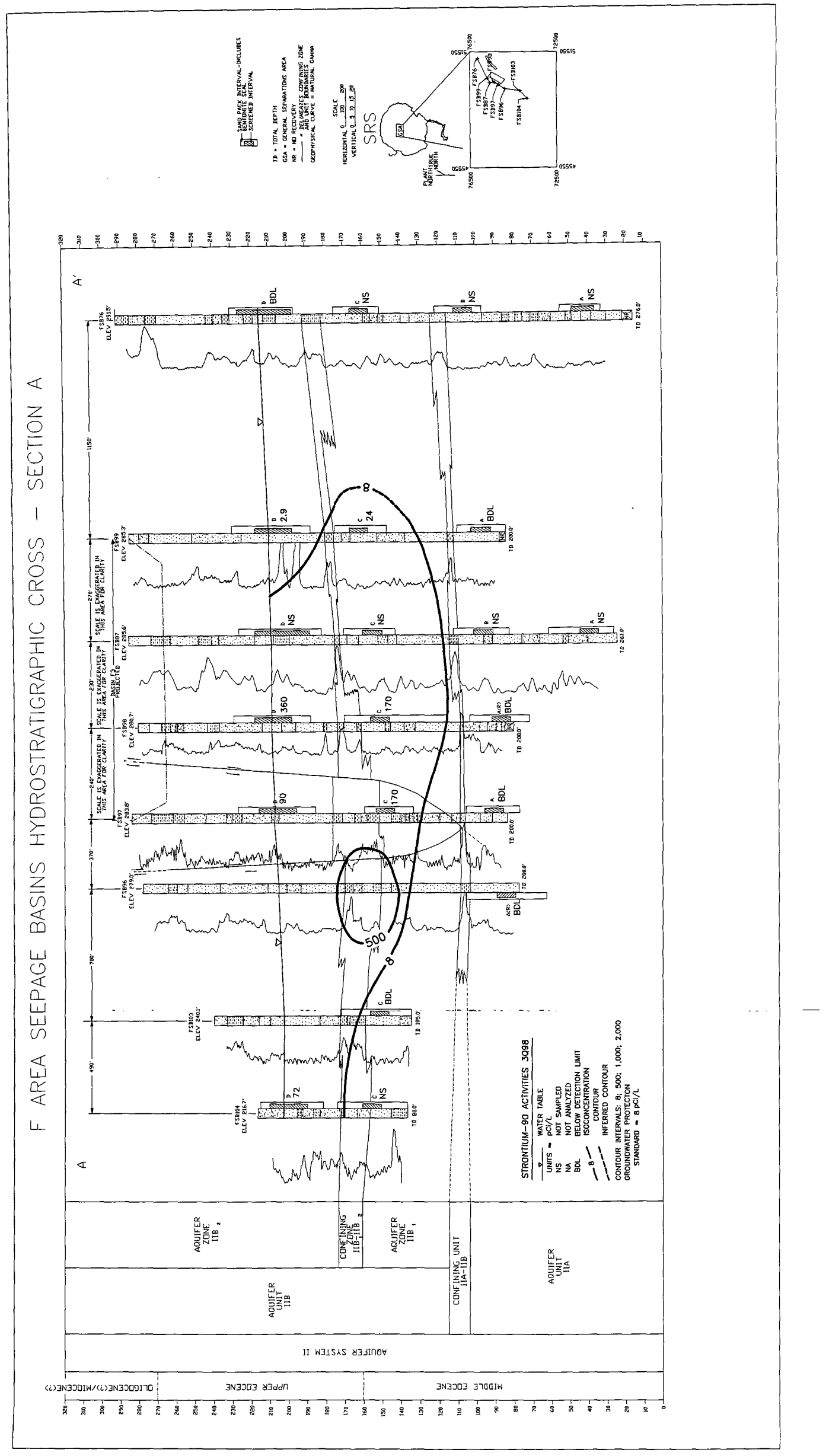




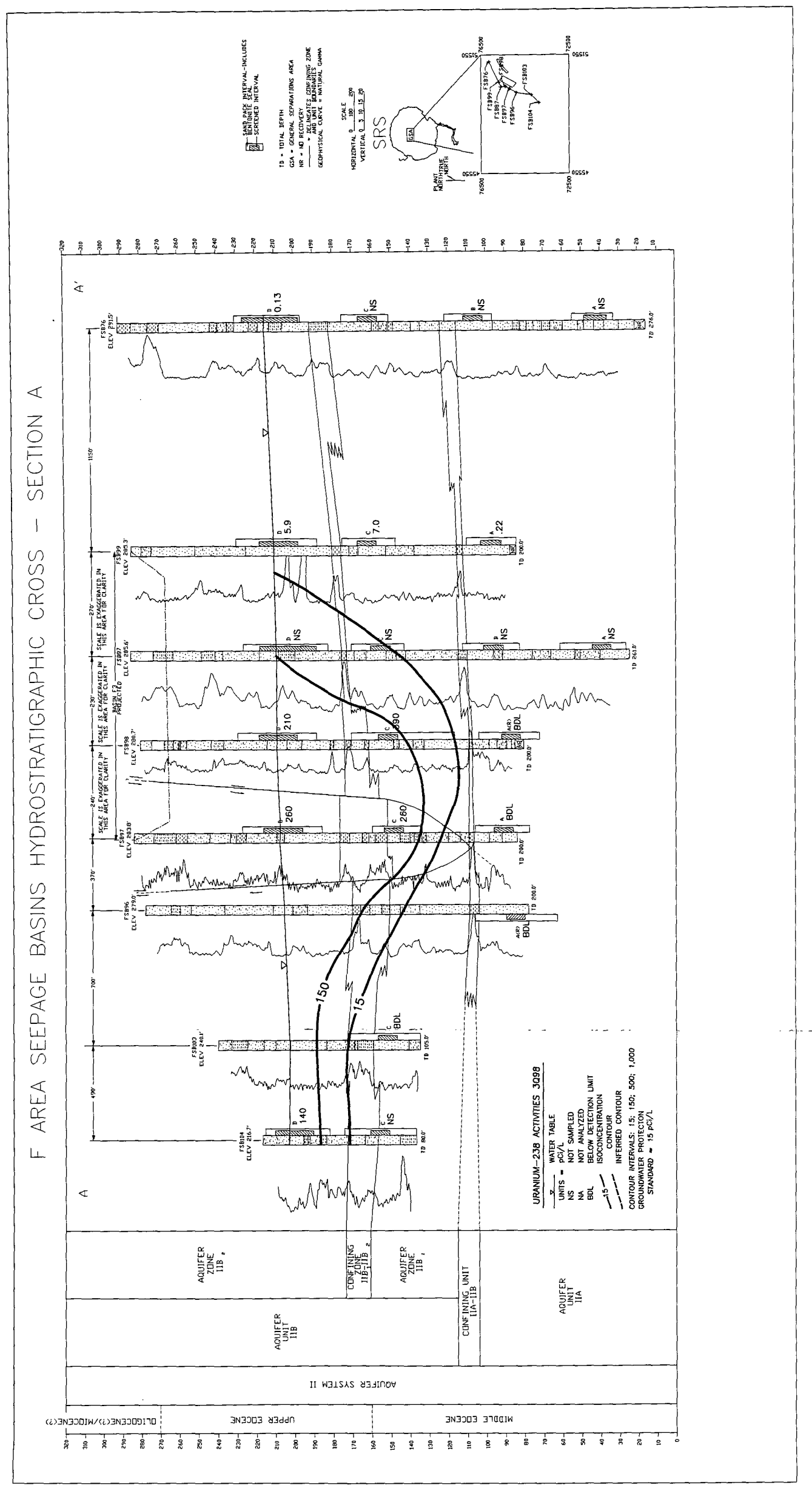


Figure 14. Hydrogeologic Cross-section Showing Technetium-99 Activities at the F-Area HWMF, Third Quarter 1908

Figure 15. Hydrogeologic Crose-section Showing Gross Alpha Activities at the F-Area HWMF, Fourth Quartor 1 (E) 


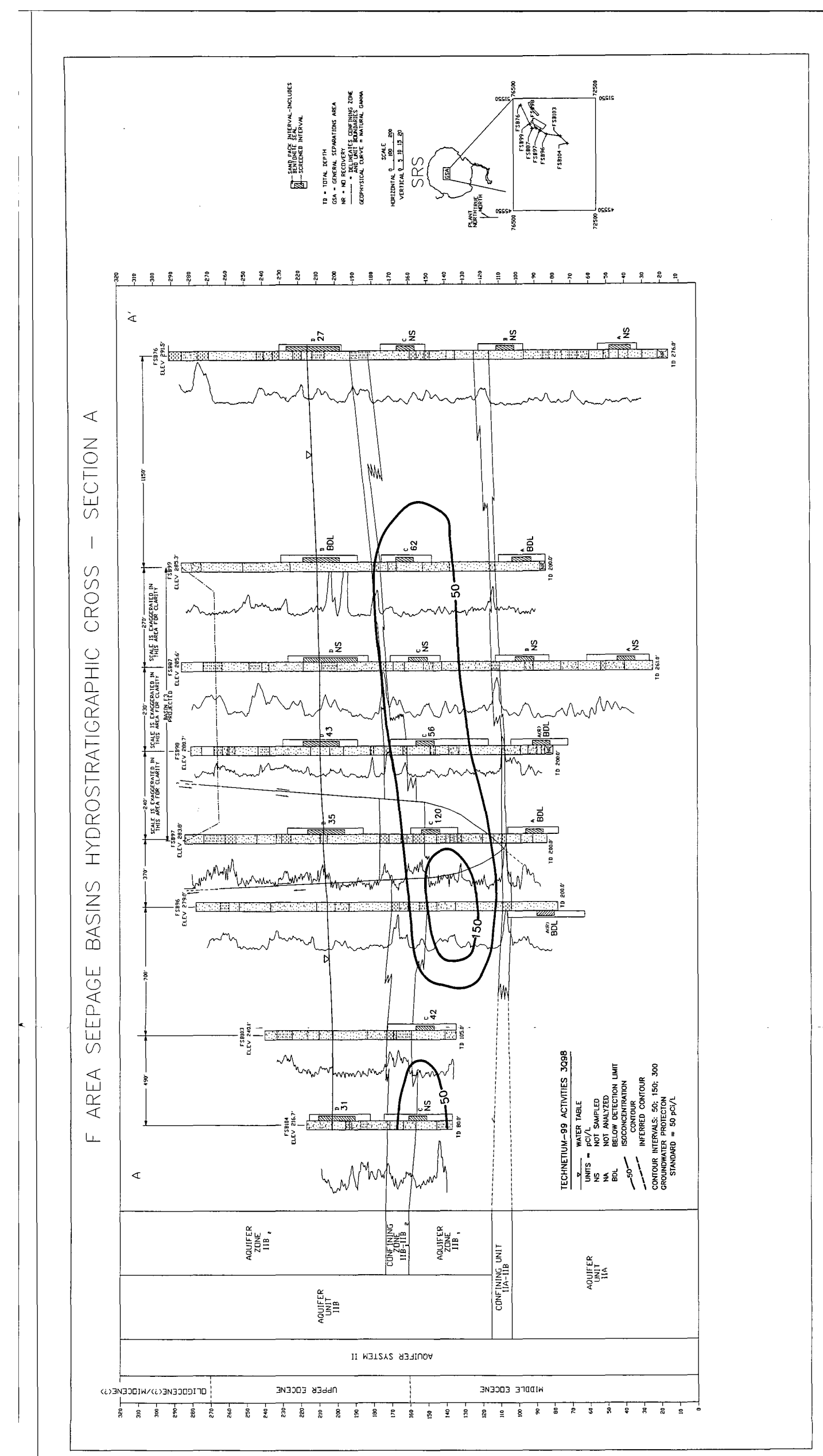



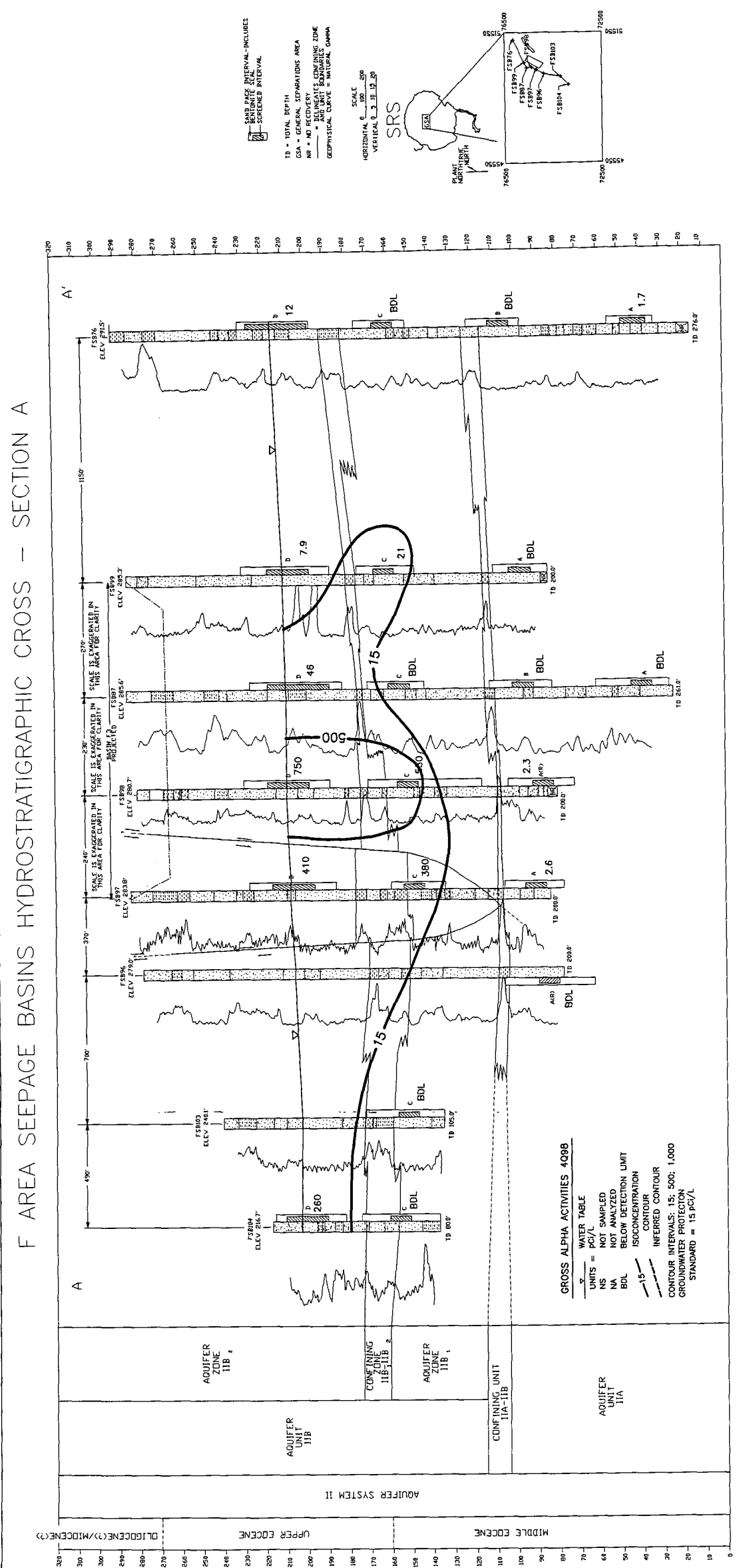
Figure 16. Hydrogeologic Cross-Section Showing Nonvolatile Beta Activities at the F-Area HWmF, Fourth Quarter 1988 

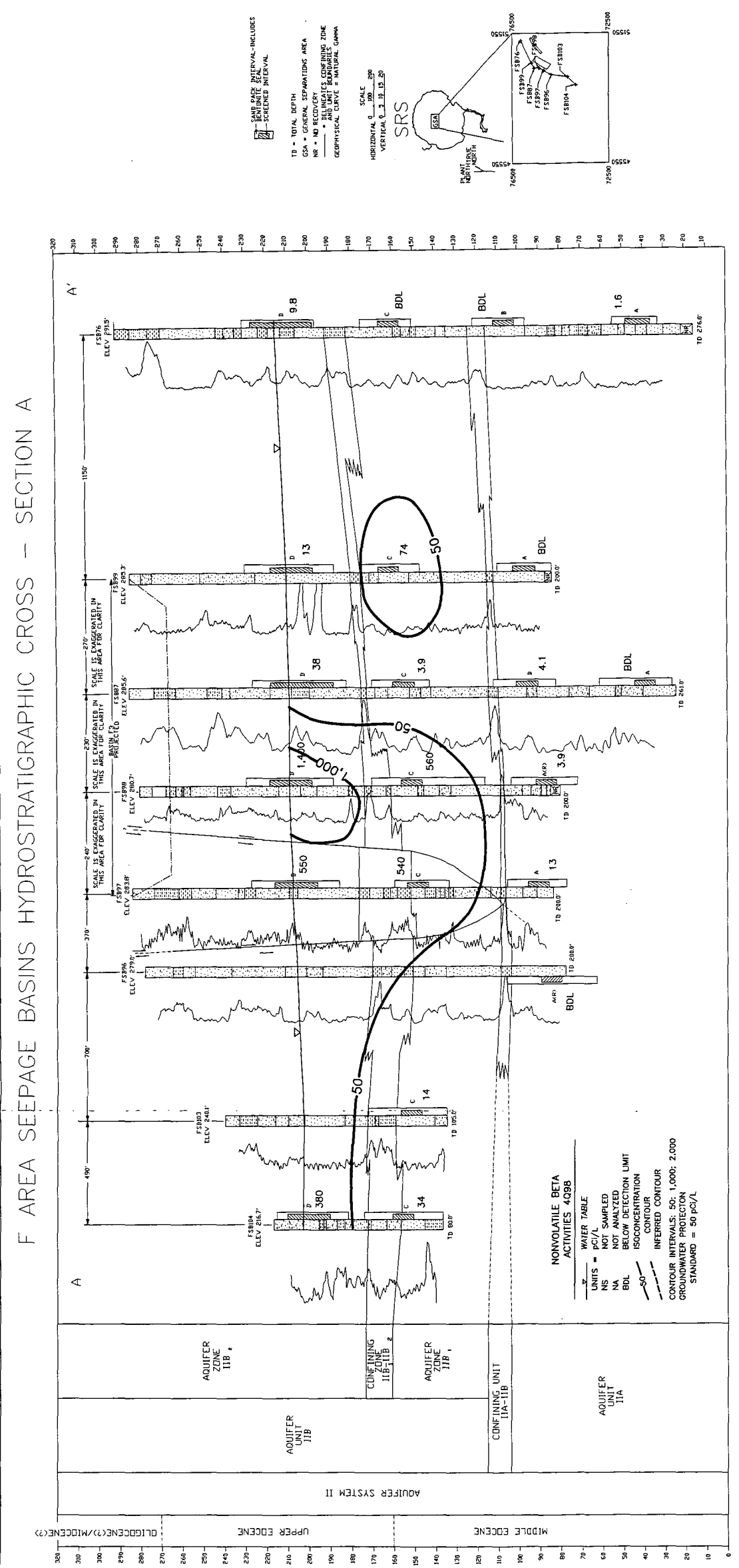
WSRC-TR-99.00012

Unclassified

\section{Appendix C}

Analytical Results 
WSRC-TR-99-00012

Unclassified

THIS PAGE LEFT BLANK INTENTIONALLY. 


\section{Key to Reading the Tables}

The following abbreviations may appear in the data tables:

\section{Constituents}

Sp. conductance

Specific conductance

\section{Laboratories}

EM

Environmental Protection Department/Environmental Monitoring Section (EPD/EMS) Laboratory

GE and GP

General Engineering Laboratories, Inc.

TM

WA

Thermo NUtech

Recra LabNet Philadelphia (was Roy F. Weston, Inc., until June 1997)

\section{Sampling Codes}

A
B
C
D
I
L
N
P
S
$T$
W
X

Units

$\mathrm{mg} / \mathrm{L}$

msl

NTU

$\mathrm{pCi} / \mathrm{L}$

$\mathrm{pCi} / \mathrm{mL}$

$\mathrm{pH}$

$\mu g / L$

$\mu \mathrm{S} / \mathrm{cm}$
Pump was surging excessively; aerated

Blank sample was collected

Well was pumping continuously

Well was dry

Well went dry during sampling; insufficient water to collect all samples

Well went dry before sampling began; only depth to water can be determined

Well was not stabilized before sampling began

Inaccessibility or mechanical failure prevented sample collection and field analysis of the water

No water in standpipe; for water-level events only

Samples were collected, but some samples were not sent to the laboratory due to high turbidity

Unable to sample well because of stabilization or sampling equipment failure; water-level measurements were obtained

Well went dry during purging; samples were collected after well recovered

milligrams per liter

mean sea level

nephelometric turbidity unit

picocuries per liter

picocuries per milliliter

pH units

micrograms per liter

microsiemens per centimeter 


\section{Data Table Column Headings}

$\begin{array}{ll}\text { DF } & \text { dilution factor } \\ \text { H } & \text { exceeded the holding time } \\ \text { Mod } & \text { modifier } \\ \text { ST } & \text { exceeded the GWPS }\end{array}$

Other

$\begin{array}{ll}\text { CS } & \text { carbon steel } \\ \mathrm{E} & \text { exponential notation }\left(\text { e.g., } 1.1 \mathrm{E}-09=1.1 \times 10^{-9}=0.0000000011\right) \\ \text { PVC } & \begin{array}{l}\text { polyvinyl chloride } \\ \text { top of casing }\end{array}\end{array}$

\section{Data Qualification}

The contract laboratories submit sample- or batch-specific quality assurance/quality control information either at the same time as analytical results or in a quarterly summary. Properly defined and used data modifiers (also referred to as qualifiers) can be a key component in assessing data usability. These modifiers appear in the data tables under the column Mod. Data for third quarter 1998 were reported by the laboratories in data format AN95, while data for fourth quarter 1998 were reported in AN98. One major difference between the two formats is the modifiers used. In the data tables, three possible fields for modifiers for each quarter are separated by slashes. For AN95 data, result qualifiers (RQ) may appear before the first slash, analysis qualifiers $(A Q)$ between the two slashes, and bias modifiers $(B)$ after the second. For AN98 data, functional guidance qualifiers (FG) may appear before the first slash, STORET codes (SC) between the two slashes, and EMS codes (EC) after the second slash. For further information on modifiers and their definitions, contact EMS.

\section{AN95 Modifiers}

(Blank) Data are not qualified. Numbers should be interpreted exactly as reported. control samples did not meet specifications. (RQ)

Value is off-scale high. The actual value is not known but is known to be greater than the value shown. (RQ)

R

Result was rejected because performance requirements in the sample analysis or associated quality control analyses were not met. (RQ)

Material was analyzed for but not detected. Analytical result reported is less than the sample quantitation limit. (RQ)

$E$

The detected result is between the sample-specific EQL and the method detection limit. (AQ)

The value in the result field is the instrument reading, not the sample quantitation limit. Always used with the result qualifier $U$. (AQ)

Surrogate or tracer spike recovery is out of specification. (AQ)

Analyte was detected in an associated method blank. (AQ)

Result was obtained from an unpreserved or improperly preserved sample. Data may not be accurate. (AQ)

Matrix interference. Value cannot be determined. Used with RQR. (AQ) 


\author{
AN98 Modifiers \\ (Blank) Data are not qualified. Numbers should be interpreted exactly as reported. \\ $\mathrm{J}$ \\ R \\ U \\ C \\ The analyte was positively identified; the associated numerical value is an \\ estimated concentration of the analyte in the sample. (FG) \\ The sample results are rejected due to serious deficiencies in the ability to \\ analyze the sample and meet quality control criteria. The presence or absence \\ of the analyte cannot be verified. (FG) \\ Material was analyzed for, but not detected. The analyte concentration is <SSEQL. \\ (SC) \\ The result is calculated. (SC) \\ The result is less than the SSEQL, but equal to or greater than the MDL. (SC) \\ The actual concentration is known to be less than the reported result. (SC) \\ The actual concentration is known to be greater than the reported result. (SC) \\ The sample was held beyond the normal holding time prior to analysis. (SC) \\ The analyte was detected in both the method blank and the sample. (SC) \\ The result is from an unpreserved or incorrectly preserved sample; the data may \\ not be accurate. (SC) \\ Compound identification criteria were not met. (EC) \\ LCS or BS criteria were not met. (EC) \\ ICP serial dilution criteria were not met. (EC) \\ Internal standard criteria were not met when the IS was used for quantitation. \\ (EC) \\ Matrix spike recovery was not within the control limits. (EC) \\ A tentatively identified compound is a suspected aldol-condensation product. \\ (EC) \\ Initial or continuing calibration criteria were not met. (EC) \\ Surrogate or tracer spike recovery is out of specification. (EC) \\ Graphite furnace atomic absorption QC \\ a. Duplicate injection criteria were not met. \\ b. Post-digestion spike recovery was not within control limits and the sample \\ absorbance is $>50 \%$ of the post-digestion spike absorbance. (EC) \\ The sample was analyzed by the method of standard additions. (EC) \\ Graphite furnace atomic absorption QC: the post-digestion spike recovery is not \\ within control limits and the sample absorbance is $<50 \%$ of the post-digestion \\ spike absorbance. (EC) \\ The laboratory duplicate RPD or MS/MSD RPD was not within control limits. (EC) \\ Matrix interference is present. (EC) \\ Matrix spike concentration was $<0.25 \times$ the sample concentration, and the \\ percent recovery cannot be determined. (EC) \\ The analyte was detected in both the sample and associated field blank. (EC) \\ The analyte was detected in both the sample and associated trip blank. (EC) \\ The field duplicate RPD was not within control limits. (EC)
}




\section{Results below Detection}

For radiological analyses, the analytical result field contains the result recorded on the analytical instrument and reported by the laboratory, even if it is negative. For nonradiological analyses, if the analyte is not detected, the sample-specific estimated quantitation limit (EQL) is entered into the result field and is reported with a less than $[<]$ sign. The EQL is defined as the lowest concentration that can be achieved reliably within specified limits of precision and accuracy during routine laboratory operating conditions. The sample-specific EQL is modified for sample concentration or dilution or unusual aliquot size that affects analytical sensitivity.

\section{Data Filtering}

Data in the column headed Filt. have been filtered to clarify their usability. In this column, Rej. is used to indicate rejected data.

For nondetects, this column contains the less-than symbol and the sample-specific method detection limit or sample-specific minimum detectable activity. For chemical analyses, this is the same numeric value as appears in the unfiltered result column with a few exceptions, generally data that were qualified as nondetects during validation because of contamination in an associated blank.

For data qualified with the result qualifier (third quarter 1998) or field guidance code (fourth quarter 1998) $\mathrm{J}$, indicating an estimated quantity, the Filt. column contains the letters NDD, meaning that these data are considered not "decision" data.

\section{Holding Times}

Standard analytical methods include a limit, called holding time, on the maximum elapsed time between sample collection and extraction or analysis by the laboratory. In the data tables, a large bullet $(\cdot)$ in the $H$ (holding time) column indicates that holding time was exceeded. Analyses performed beyond holding times may not yield valid results.

The laboratory procedure used for the determination of specific conductance allows one day to elapse between sampling and analysis. Thus, laboratory specific conductance measurements may exceed the holding time criterion.

\section{Data Rounding}

A constituent result in the analytical results tables that is not marked in the ST column but appears to equal an Appendix A standard is below the standard in the database. Values stored in the database contain more significant digits than the reported results. Apparent discrepancies in the tables are due to the rounding of reported results.

\section{Sampling Dates}

Samples for field data are colfected once each quarter, but samples for analytical data may be collected more than once each quarter. Because the results tables present the highest analytical results for each quarter, the date of collection for reported analytical results may not coincide with the date of collection for field data. 
Table C-1. Groundwater Monitoring Results for Individual Wells

WELLL FSB 76

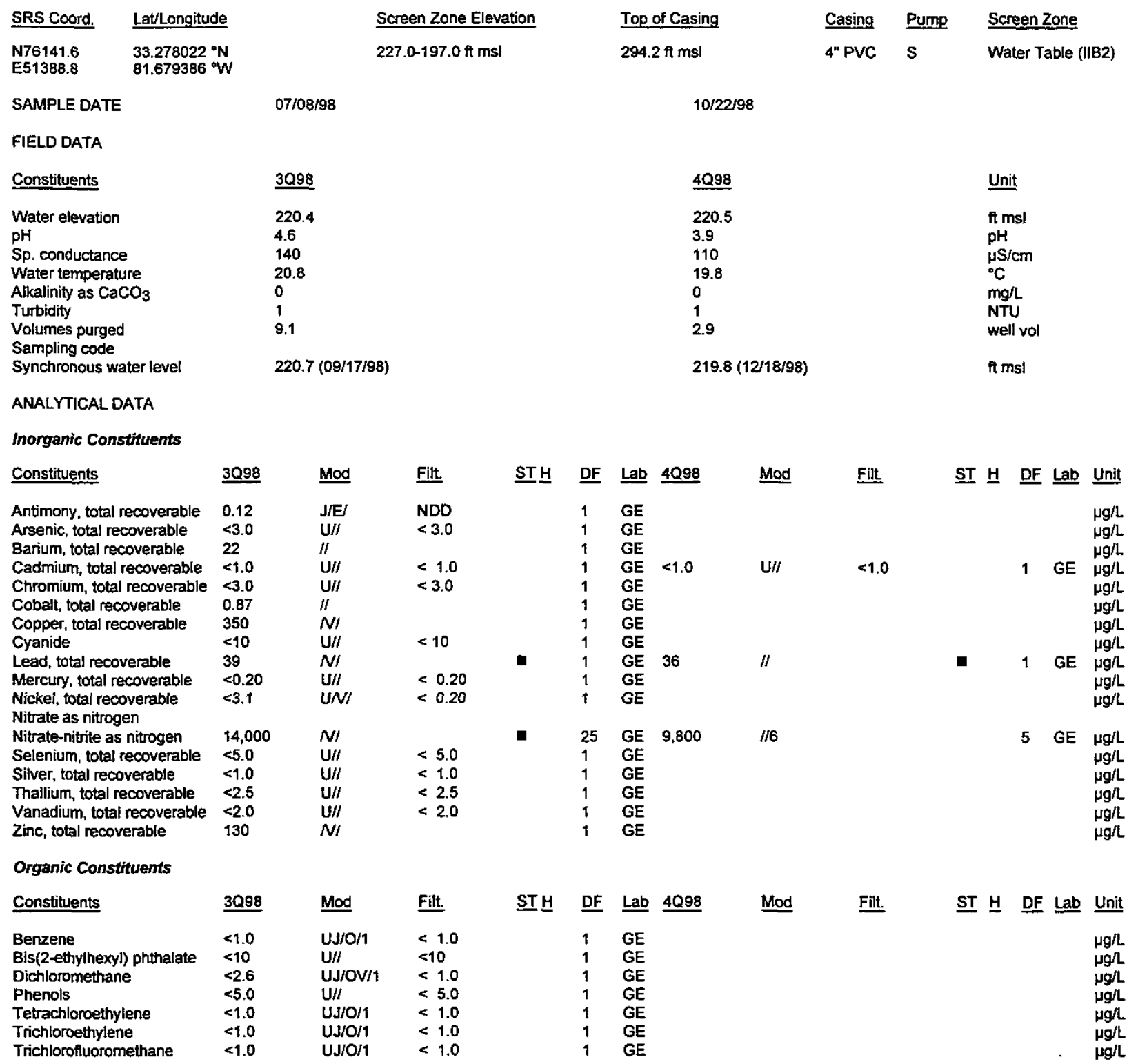

Notes:

- exceeded holding time

w = exceeded grounowater protection or monitoring constituent standard (See Appendix A.) 
WELL FSB 76 (cont.)

Radioactive Constituents

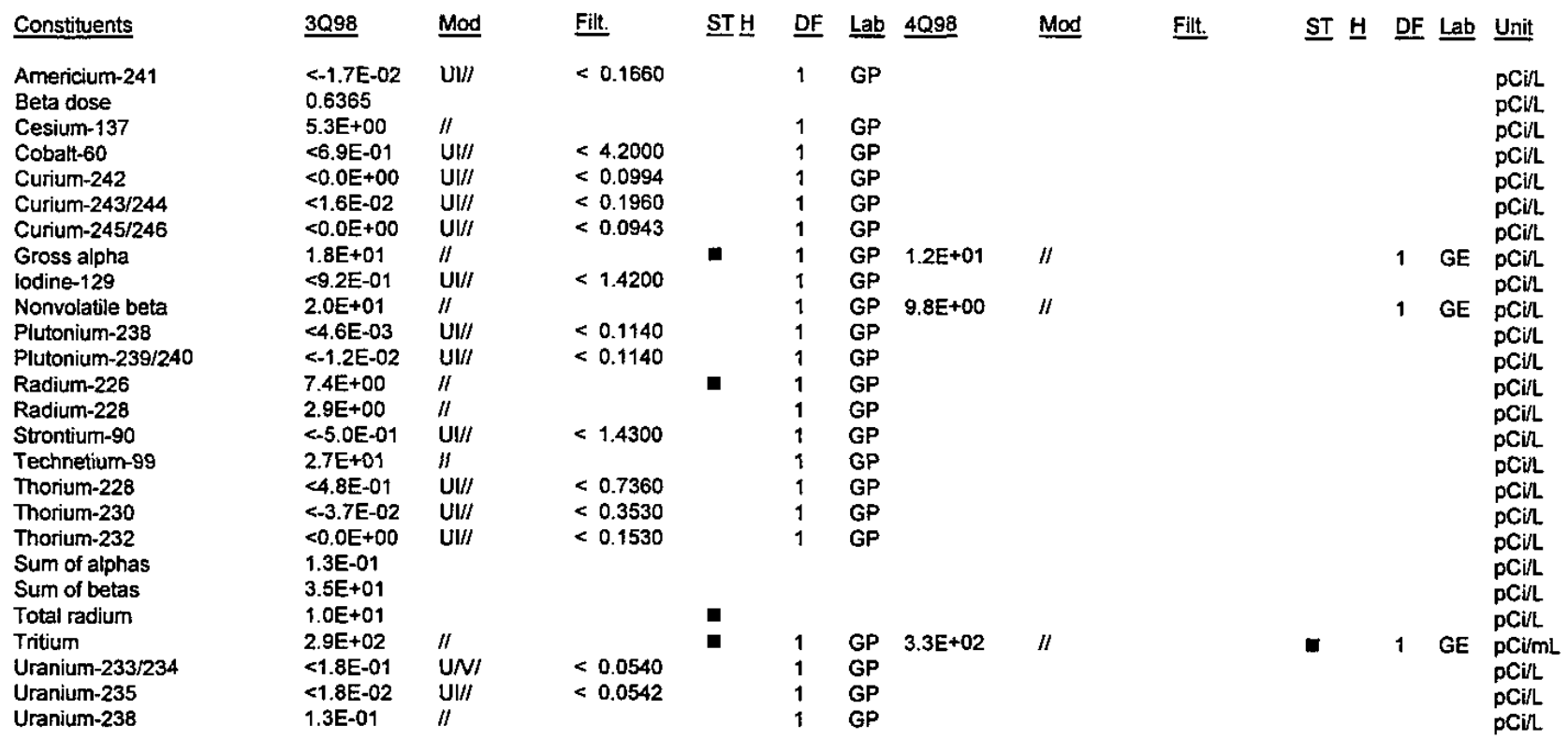

Notes:

- = exceeded holding time

w = exceeded groundwater protection or monitoring constituent standard (See Appendix A.) 


\section{WELL FSB 76A}

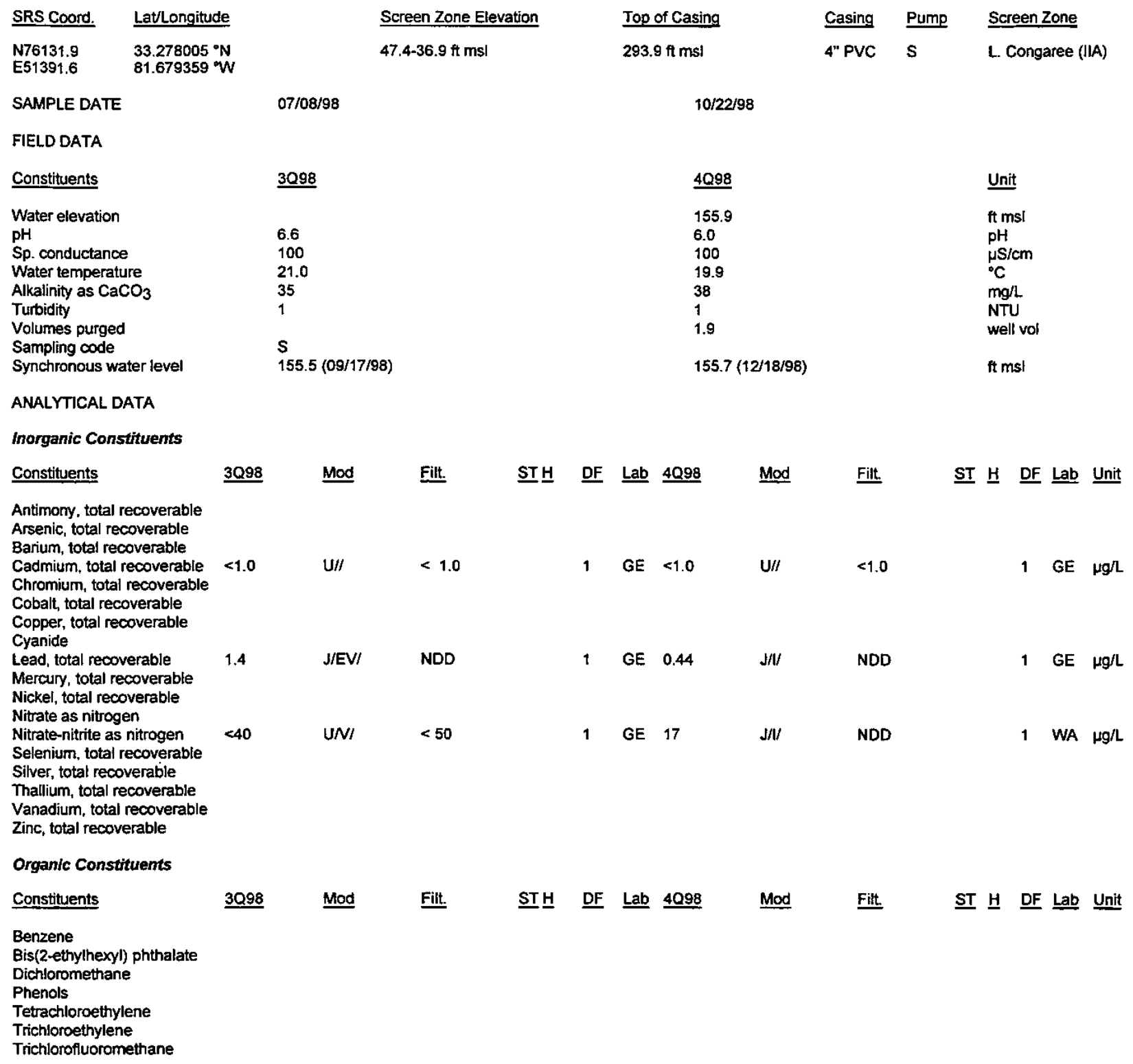

Notes:

- = exceeded holding time

E = exceeded groundwater protection or monitoring constituent standard (See Appendix A.) 
WELL FSB 76A (cont.)

Radioactive Constituents

Constituents

Americium-241

Cesium-137

Cobalt-60

Curium-242

Curium-243/244

Curium-245/246

Gross alpha

lodine-129

Nonvolatile beta

Plutonium-238

Plutonium-239/240

Radium-226

Radium-228

Strontium-90

Technetium-99

Thorium-228

Thorium-230

Thorium-232

Sum of alphas

Sum of betas

Tritium

Uranium-233/234

Uranium-235

Uranium-238
3Q98 $\quad$ Mod

$1.1 \mathrm{E}+00 \quad l$

$<7.9 \mathrm{E}-01 \quad \mathrm{UI} / \mathrm{I}$

$<1.2200$

1 GP $1.7 E+00 \quad S / /$

NDD

1 GP $1.6 \mathrm{E}+00 \quad \mathrm{~J} / /$

NDD

$1 \mathrm{TM} \mathrm{pCi} / \mathrm{L}$

$1 \mathrm{TM}$ pCil

<-2.2E-01 UI/I

$<6.6 E-01$

1 GP $<-9.2 E-02 \quad \mathrm{U} / 1$

$<<6.5 E-01$

1 GE pCi/mL

Notes:

- exceeded holding time

= exceeded groundwater protection or monitoring constituent standard (See Appendix A.) 
WELL FSB 76B

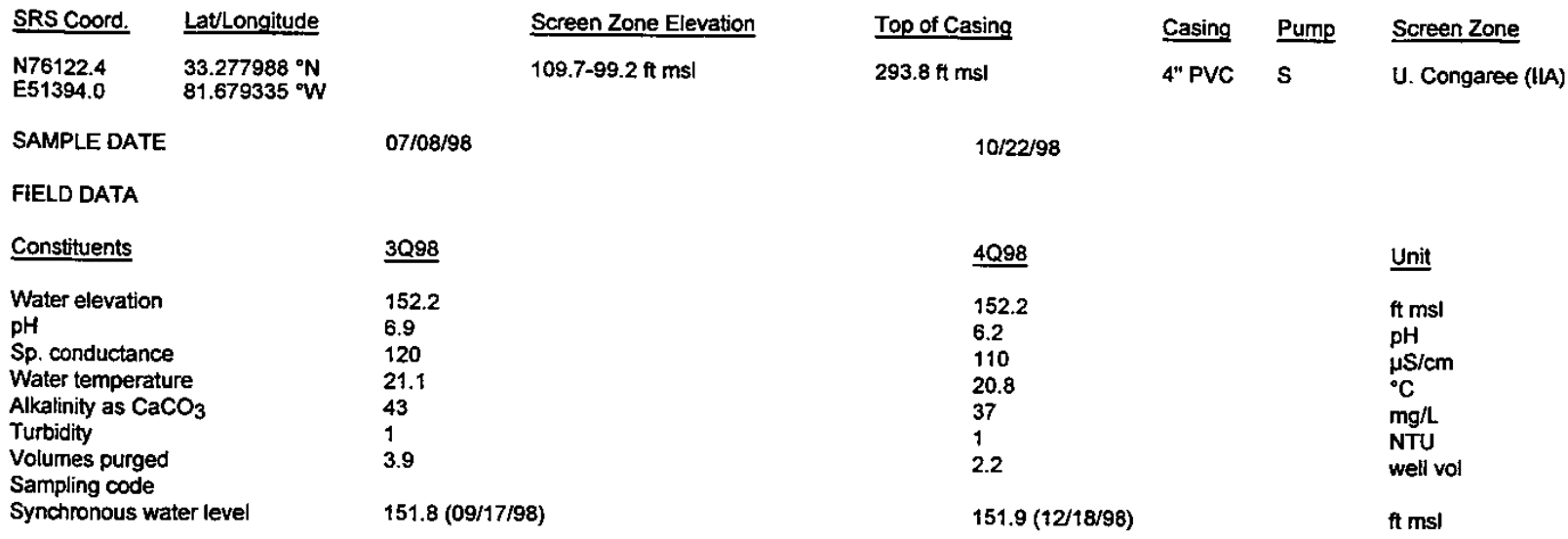

ANALYTICAL DATA

Inorganic Constituents

Constituents

Antimony, total recoverable

Arsenic, total recoverable

Barium, total recoverable

Cadmium, total recoverable

Chromium, total recoverable

Cobalt, total recoverable

Copper, total recoverable

Cyanide

Lead, total recoverable

Mercury, total recoverable

Nickel, total recoverable

Nitrate as nitrogen

Nitrate-nitrite as nitrogen

Selenium, total recoverable

Silver, total recoverable

Thallium, total recoverable

Vanadium, total recoverable

Zinc, total recoverable

Organic Constituents

Constituents

$<0.27$

UNI

$<2.0$

1 GE 530

Benzene

Bis(2-thylhexyl) phthalate

Dichloromethane

Phenols

Tetrachloroethylene

Trichloroethylene

Trichlorofluoromethane
$N$

510

Mod

Filt.

ST브
$1 / 6$

GE $<1.0$

U/I

NDD

1 GE $\mu g / L$

Mod

Filt.

Notes:

- exceeded holding time

- exceeded groundwater protection or monitoring constituent standard (See Appendix A.) 


\section{WELL FSB 76B (cont.)}

Radioactive Constituents

Constituents

Americium-241

Cesium-137

Cobalt-60

Curium-242

Curium-243/244

Curium-245/246

Gross alpha

lodine-129

Nonvolatile beta

Plutonium-238

Plutonium-239/240

Radium-226

Radium-228

Strontium-90

Technetium-99

Thorium-228

Thorium-230

Thorium-232

Sum of alphas

Sum of betas

Tritium

Uranium-233/234

Uranium-235

Uranium-238
3099

Mod

7.7E-01 $/ /$

$<5.3 \mathrm{E}-01 \quad \mathrm{U} \mathrm{l} / \mathrm{I}$

$<1.0900$

$<2.9 E-01 \quad \mathrm{Ul} / /$

$<6.6 \mathrm{E}-01$

1

GP <-1.3E-01 U/I

1 GP $<3.2 \mathrm{E}-01 \quad \mathrm{U} / \prime$

GP

8.6E-01

$\mathrm{U} / /$

$<$

$<6.4 E-01$
1 GE $\mathrm{pCi} / \mathrm{L}$

1 GE pCill
$\underline{S} \underline{\mathrm{H} F}$ Lab $\underline{\text { Unit }}$ 
WSRC-TR-99-00012

Unclassiffed

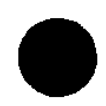

WELL FSB 76C

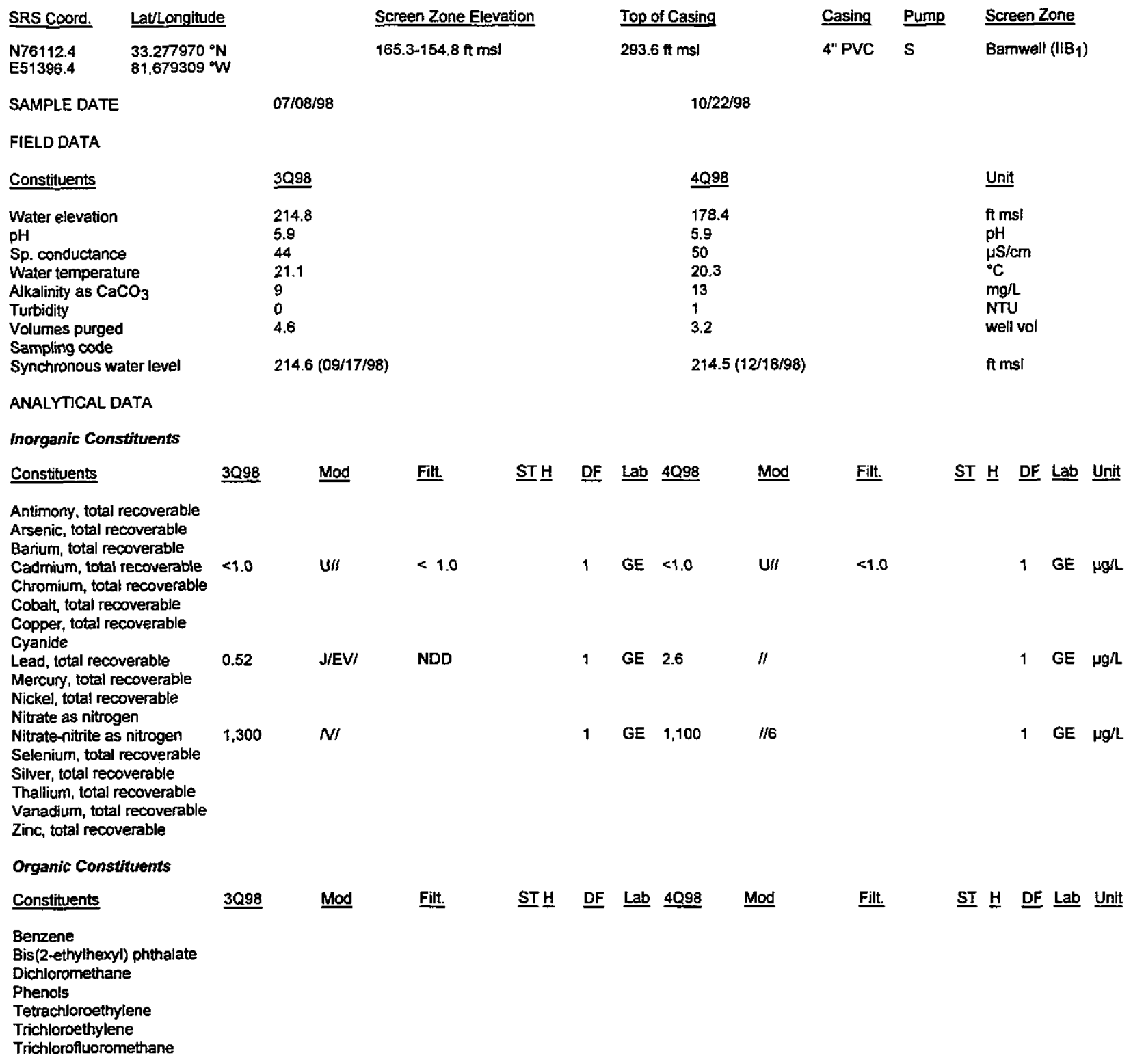

Notes:

- = exceeded holding time

- exceeded groundwater protection or monitoring constituent standard (See Appendix A.) 
WELL FSB 76C (cont.)

Radioactive Constituents

Constituents

Americium-241

Cesium-137

Cobalt-60

Curium-242

Curium-243/244

Curium-245/246

Gross alpha

lodine-129

Nonvolatile beta

Plutonium-238

Plutonium-239/240

Radium-226

Radium-228

Strontium-90

Technetium-99

Thorium-228

Thorium-230

Thorium-232

Sum of alphas

Sum of betas

Tritium

Uranium-233/234

Uranium-235

Uranium-238

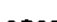

3Q9

Mod

Fit.

ST브 DF Lab 4098

Mod

Filt

ST $\underline{H}$ DF Lab Unit

$<4.3 \mathrm{E}-01 \quad \mathrm{UI} / \mathrm{I}$

$<0.6480$

$<5.7 \mathrm{E}-01 \quad \mathrm{UI} / \prime$

$<1.1800$

$1 \quad G P<2.0 E-01$

U/I

1 GP $<6.4 \mathrm{E}-01 \quad \mathrm{U} / \mathrm{l}$

$<$

$<$

1 GE pCill

1 GE pCill

$2.3 \mathrm{E}+00 \quad / /$

1 GP $23 E+00$

/I

1 GE $\mathrm{pCi} / \mathrm{mL}$

Notes:

= exceeded holding time

I = exceeded groundwater protection or monitoring constituent standard (See Appendix A.) 


\section{WELL FSB 77}

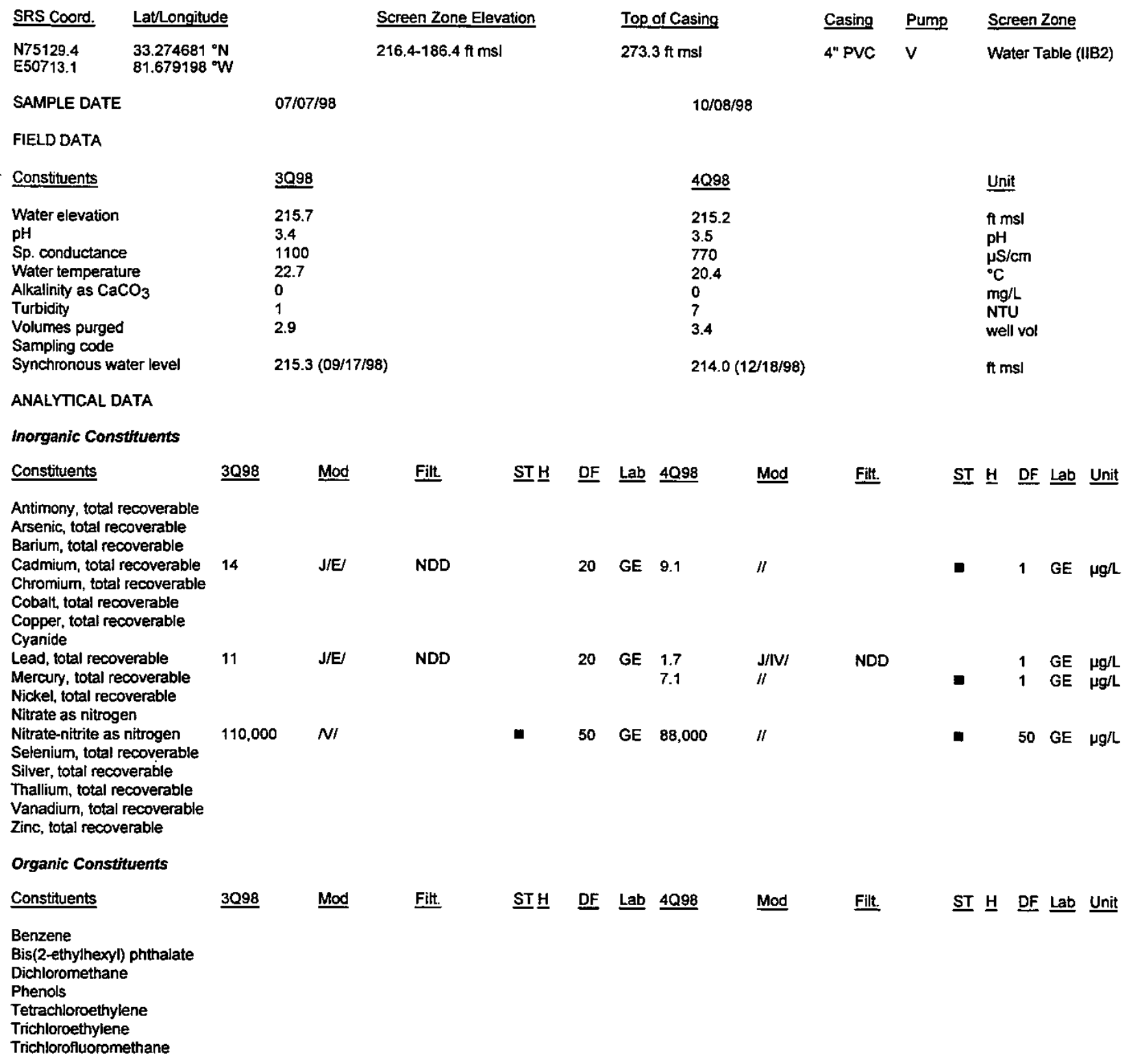

Notes:

- = exceeded holding time

- exceeded groundwater protection or monitoring constituent standard (See Appendix A.) 


\section{WELL FSB 77 (cont.)}

Radioactive Constituents

Constituents

Americium-241

Cesium-137

Cobalt-60

Curium-242

Curium-243/244

Curium-245/246

Gross alpha

lodine-129

Nonvolatile beta

Plutonium-238

Plutonium-239/240

Radium-226

Radium-228

Strontium-90

Technetium-99

Thorium-228

Thorium-230

Thorium-232

Sum of alphas

Sum of betas

Tritium

Uranium-233/234

Uranium-235

Uranium-238
3Q98

Mod

Filt.

STH DF Lab 4098

$1.0 \mathrm{E}+03 \quad \mathrm{~J} / \mathrm{N} / 1$

NDD

1.8E+03

J/IV/1

NDD

6.3E+02 J/LI

1 GP $1.3 E+03$ /

NDD

Filt.

ST 브 DF Lab Unit

บ1

-

1

GP 2.7E+03
GE PCil

-

1 GE pCill

- 1 GE DCímL

Notes:

- exceeded holding time

= exceeded groundwater protection or monitoring constituent standard (See Appendix A.) 
WELL FSB 78

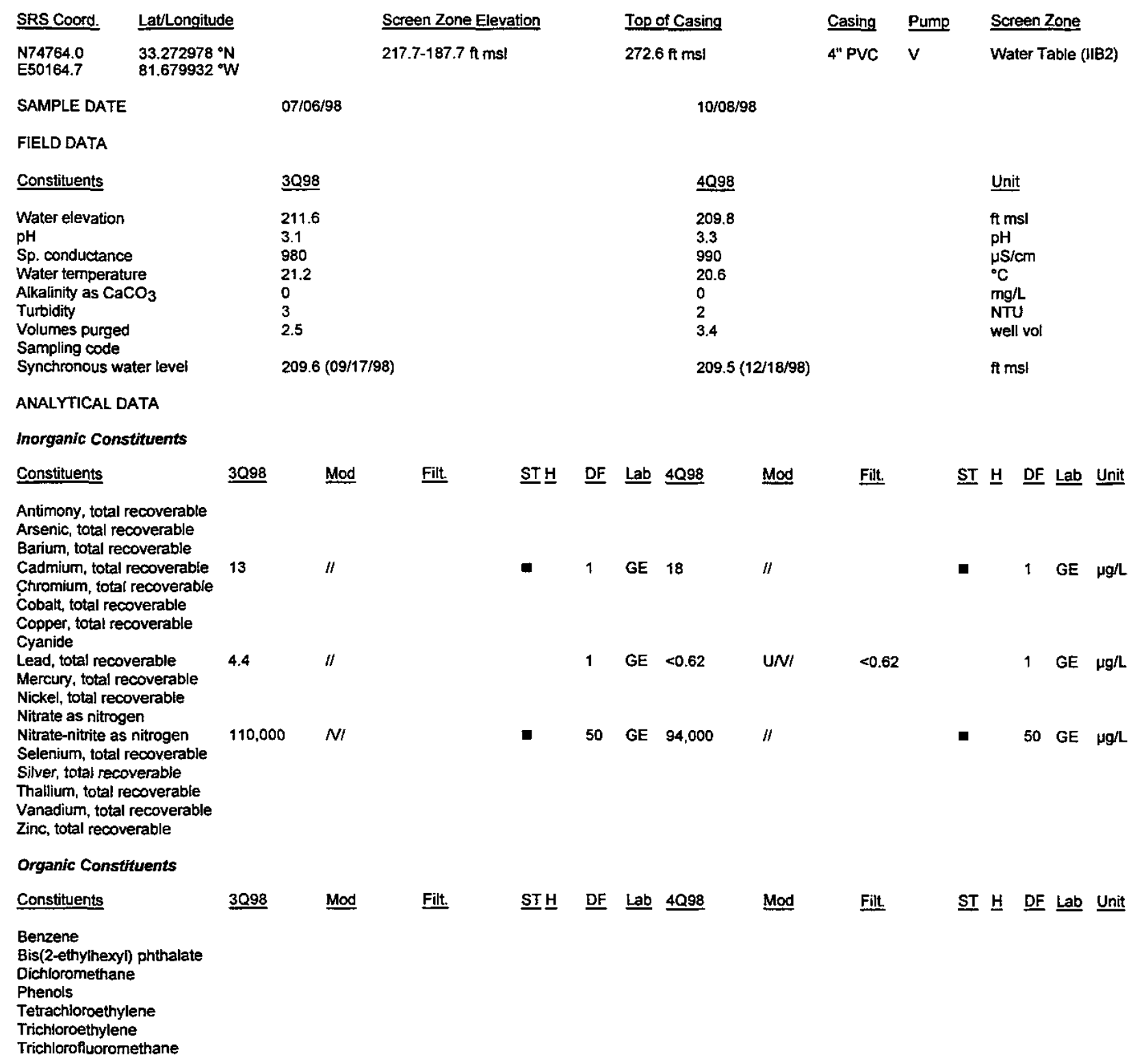

Notes:

- exceeded holding time

I = exceeded groundwater protection or monitoring constituent standard (See Appendix A.) 
WSRC-TR-99-00012

Unclassified

WELL FSB 78 (cont.)

Radioactive Constituents

Constituents

Americium-241

Cesium-137

Cobalt-60

Curium-242

Curium-243/244

Curium-245/246

Gross alpha

lodine-129

Nonvolatile beta

Plutonium-238

Plutonium-239/240

Radium-226

Radium-228

Strontium-90

Technetium-99

Thorium-228

Thorium-230

Thorium-232

Sum of alphas

Sum of betas

Tritium

Uranium-233/234

Uranium-235

Uranium-238
8.5E+02 J/1/1 NDD

$1.8 \mathrm{E}+03 \quad \mathrm{~J} / \mathrm{IV} / 1 \quad \mathrm{NDD}$

1 GP $9.3 E+02$

$\mathrm{J} / \mathrm{LI}$

1

GP 2.4E+03 //

4.5E+03 $/ /$

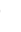

1 GP $2.4 E+03 \quad / 1$
Filt.

ST H DF Lab Unit

NDD

1 GE pCil

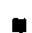

1 GE PCin

Notes:

- = exceeded holding time

- exceeded groundwater protection or monitoring constituent standard (See Appendix A.)

F-Area HWMF

C-18

Third and Fourth Quarter 1998 
WELL FSB 78A

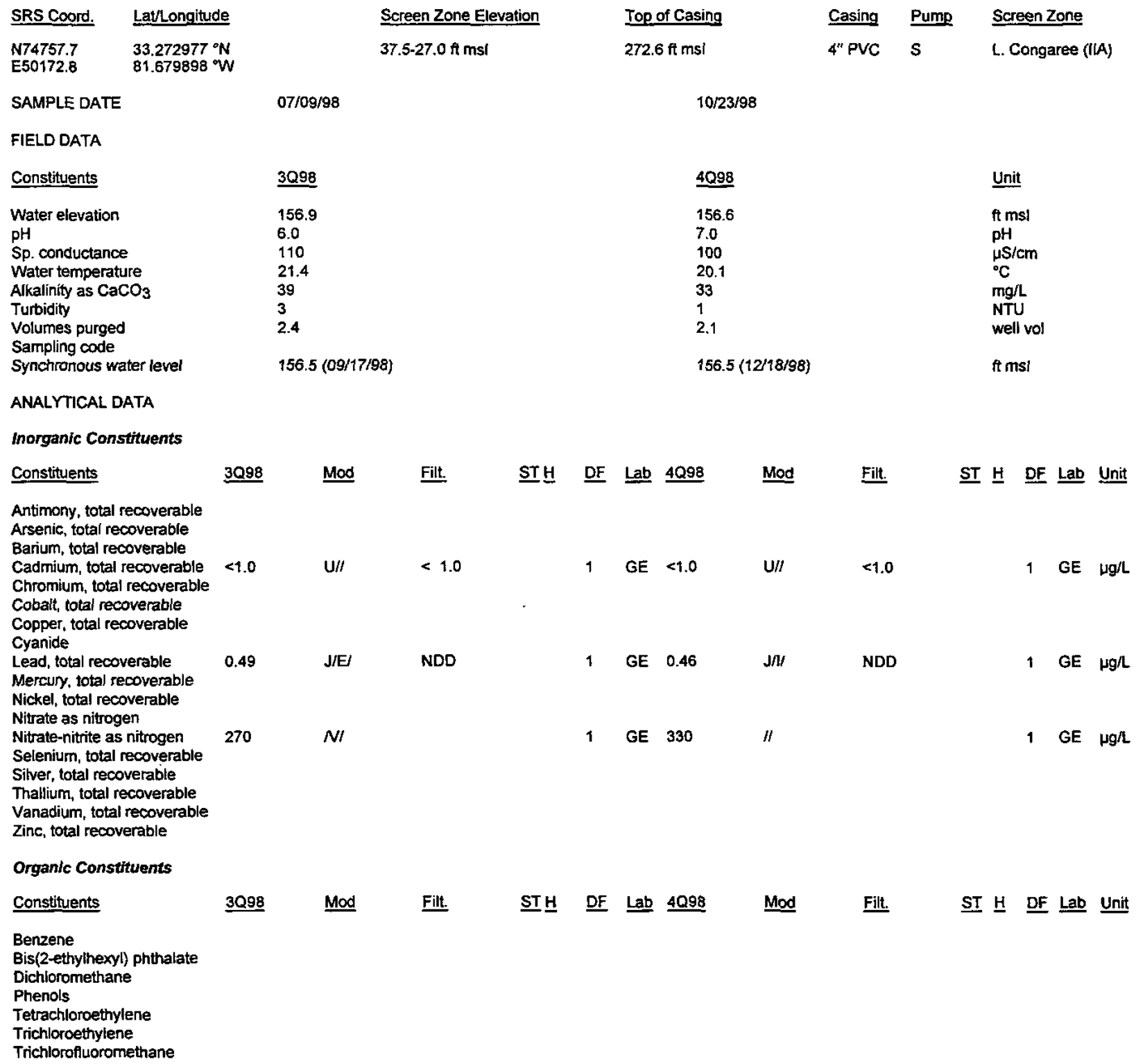

Notes:

- exceeded holding time

- = exceeded groundwater protection or monitoring constituent standard (See Appendix A.) 
WELL FSB 78A (cont.)

Radioactive Constituents

Constituents

Americium-241

Cesium- 137

Cobalt-60

Curium-242

Curium-243/244

Curium-245/246

Gross alpha

lodine-129

Nonvolatile beta

Plutonium-238

Plutonium-239/240

Radium-226

Radium-228

Strontium-90

Technetium-99

Thorium-228

Thorium-230

Thorium-232

Sum of alphas

Sum of betas

Tritium

Uranium-233/234

Uranium-235

Uranium-238
$1.3 E+00$

$1.5 \mathrm{E}+00 \quad / /$

1 GP $<1.1 E+00$

U/I

$<1.3 E+00 \quad U / 1$

$<8.7 \mathrm{E}-01$

$1 \mathrm{GE} \mathrm{pCin}$

1

$<1.5 \mathrm{E}+00$

1 GE pCil
$1 \quad \mathrm{GP} \quad 5.4 \mathrm{E}+00 \quad / /$

1 GE pCi/mL

Notes:

- = exceeded holding time

- = exceeded groundwater protection or monitoring constituent standard (See Appendix A.) 
WSRC-TR-99-00012

Unclassified

WELL FSB 78B

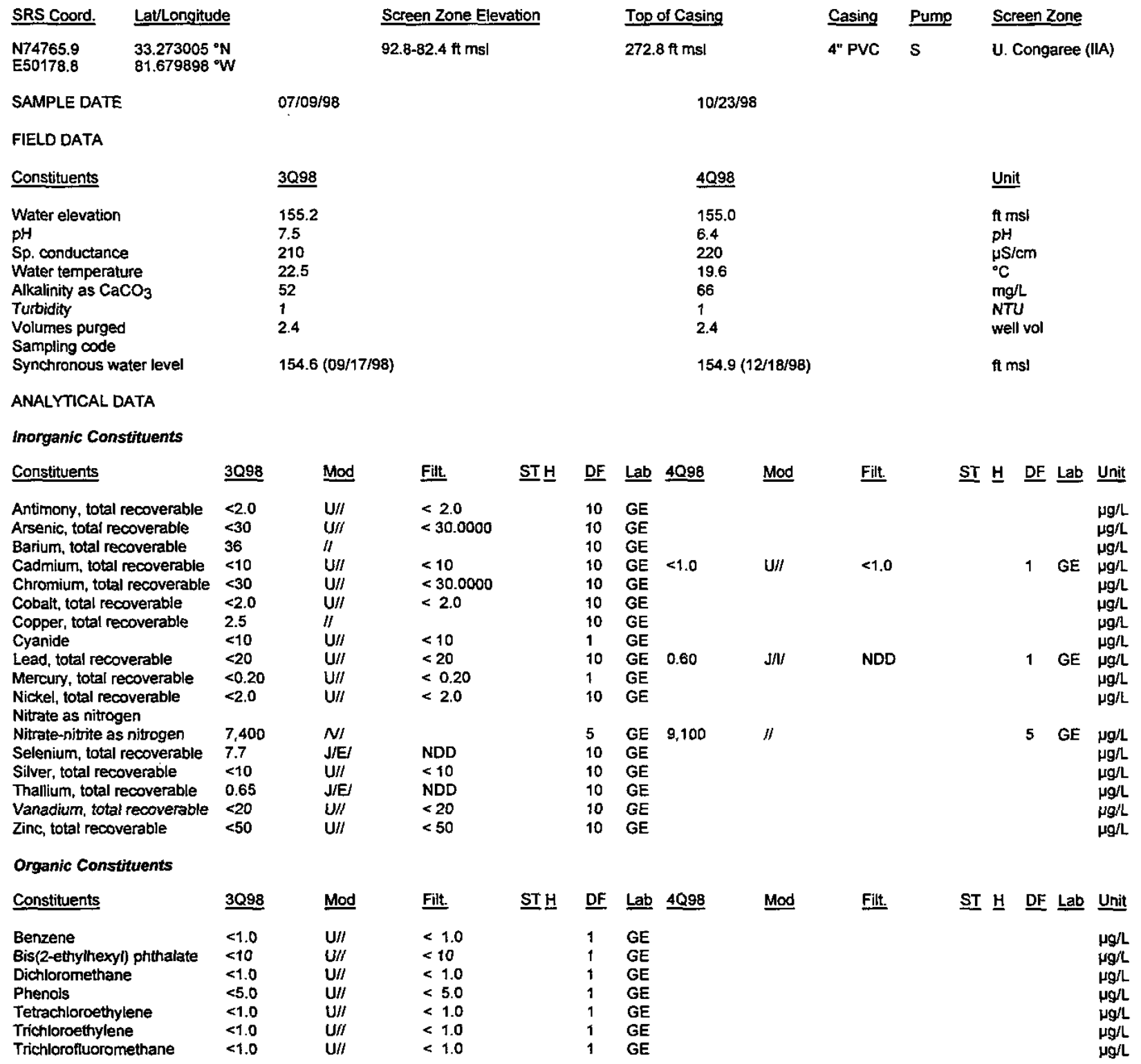

Notes:

- exceeded holding time

= exceeded groundwater protection or monitoring constituent standard (See Appendix A.)

F-Area HWMF

C-21

Third and Fourth Quarter 1998 
WELL FSB 78B (cont.)

Radioactive Constituents

\begin{tabular}{|c|c|c|c|c|c|c|c|c|c|c|c|c|c|}
\hline Constituents & $\underline{3 Q 98}$ & Mod & Filt. & STH & $\underline{\mathrm{DF}}$ & $\underline{\text { Lab }}$ & 4098 & Mod & Filt. & $\underline{\text { ST }} \underline{H}$ & DF & $\underline{\text { Lab }}$ & $\underline{\text { Unit }}$ \\
\hline Americium-241 & $<-1.9 E-02$ & UIII & $<0.1210$ & & 1 & GP & & & & & & & $\mathrm{pCill}$ \\
\hline Cesium-137 & $<1.9 E+00$ & $U \mathrm{UI}$ & $<3.8500$ & & 1 & GP & & & & & & & pCin \\
\hline Cobalt -60 & $<-1.8 \mathrm{E}-01$ & $\mathrm{U} 1 / / \mathrm{I}$ & $<2.7800$ & & 1 & GP & & & & & & & $\mathrm{pCil}$ \\
\hline Curium-242 & $<-9.9 E-03$ & UiI/ & $<0.1290$ & & 1 & GP & & & & & & & pCirl \\
\hline Curium-243/244 & $<4.9 \mathrm{E}-02$ & $\mathrm{U} 1 / /$ & $<0.1210$ & & 1 & GP & & & & & & & pCill \\
\hline Curium-245/246 & $<0.0 E+00$ & $U I I I$ & $<0.0582$ & & 1 & GP & & & & & & & $\mathrm{pCin}$ \\
\hline Gross alpha & $1.4 E+00$ & $n$ & & & 1 & GP & $<1.5 \mathrm{E}+00$ & $\mathrm{u} / 1$ & $<9.9 E-01$ & & 1 & GE & pcin \\
\hline lodine-129 & $<3.7 \mathrm{E}-01$ & $\mathrm{UI} / /$ & $<0.5390$ & & 1 & GP & & & & & & & pCill \\
\hline Nonvolatile beta & $3.4 \mathrm{E}+00$ & "/ & & & 1 & GP & $<1.4 E+00$ & $\mathrm{U} / / \mathrm{I}$ & $<1.3 E+\infty 0$ & & 1 & GE & PCil \\
\hline Plutonium-238 & $<-4.2 E-03$ & UI/I & $<0.0912$ & & 1 & GP & & & & & & & pCin \\
\hline Plutonium-239/240 & $<-1.2 \mathrm{E}-02$ & $\mathrm{UI} / /$ & $<0.1200$ & & 1 & $G P$ & & & & & & & pCill \\
\hline Radium-226 & $<4.8 E-01$ & $\mathrm{U} / / \mathrm{I}$ & $<0.5600$ & & 1 & GP & & & & & & & pCin \\
\hline Radium-228 & $<4.3 \mathrm{E}-01$ & UIII & $<1.2900$ & & 1 & GP & & & & & & & $\mathrm{pC}$ C $\Omega$ \\
\hline Strontium-90 & $<2.9 \mathrm{E}-01$ & UIJ/N & & & 1 & GP & & & & & & & $\mathrm{pCil}$ \\
\hline Technetium-99 & $<0.0 \mathrm{E}+00$ & $\mathrm{UI} / /$ & $<24.3000$ & & 1 & GP & & & & & & & PCill \\
\hline Thorium-228 & $<2.2 \mathrm{E}-01$ & $U \mathrm{UI} / \mathrm{I}$ & $<0.2550$ & & 1 & GP & & & & & & & pCill \\
\hline Thorium-230 & $<3.2 E-02$ & $\mathrm{U} \mid \prime \prime$ & $<0.0946$ & & 1 & GP & & & & & & & pCil \\
\hline Thorium-232 & $<-1.2 E-02$ & $\mathrm{UI} / / \mathrm{I}$ & $<0.1840$ & & 1 & GP & & & & & & & pCill. \\
\hline $\begin{array}{l}\text { Sum of alphas } \\
\text { Sum of betas }\end{array}$ & & & & & & & & & & & & & \\
\hline $\begin{array}{l}\text { Tritium } \\
\text { Pas }\end{array}$ & & $/ 1$ & & a & 1 & GP & $2.2 E+02$ & $\|$ & & घ & 1 & GE & \\
\hline Uranium-233/234 & $<3.7 \mathrm{E}-01$ & UN/ & $<0.1050$ & & 1 & GP & & & & & & & $\mathrm{pC} / \mathrm{il}$ \\
\hline Uranium-235 & $<9.5 \mathrm{E}-02$ & $\mathrm{U} \mathbf{I} / /$ & $<0.1050$ & & 1 & GP & & & & & & & pCill \\
\hline Uranium-238 & $<7.5 \mathrm{E}-02$ & $U \mathrm{U} / \mathrm{I}$ & $<0.1050$ & & 1 & GP & & & & & & & $\mathrm{pCin}$ \\
\hline
\end{tabular}

Notes:

- = exceeded holding time

- exceeded groundwater protection or monitoring constituent standard (See Appendix A.) 
WELL FSB 78C

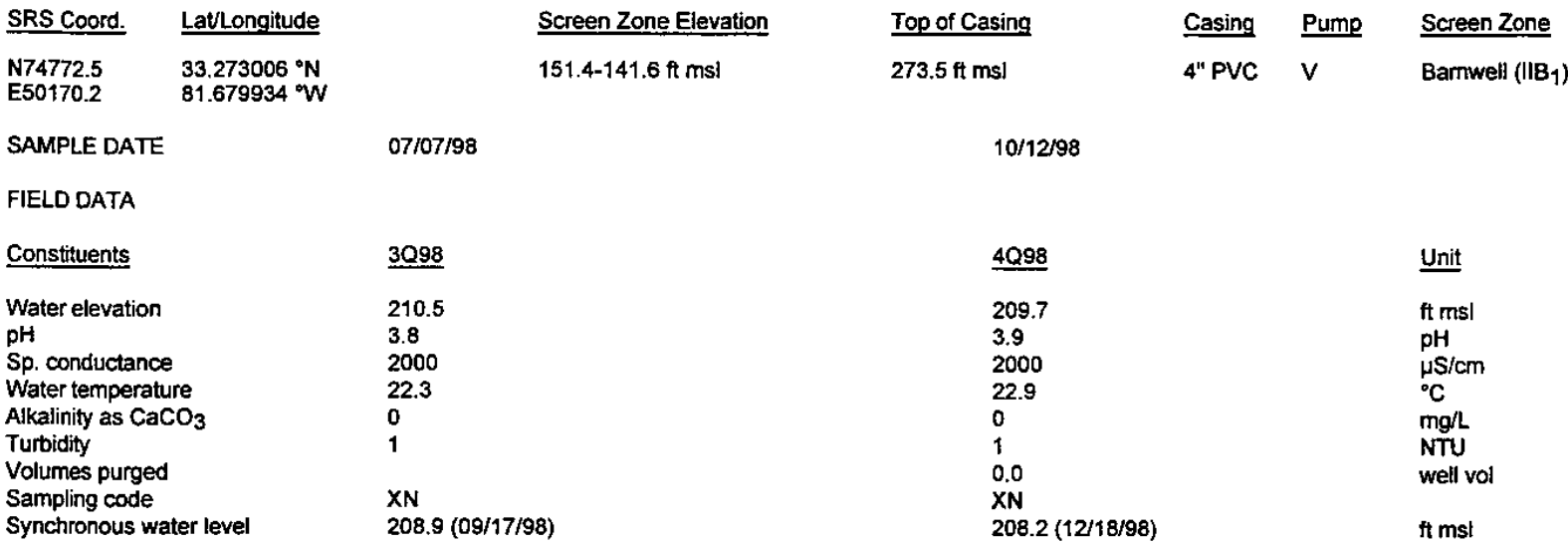

ANALYTICAL DATA

Inonganic Constituents

\begin{tabular}{|c|c|c|c|c|c|c|c|c|c|c|c|c|c|}
\hline Constituents & 3Q98 & Mod & Filt. & ST브 & $\underline{\mathrm{DF}}$ & $\underline{\text { Lab }}$ & 4Q998 & Mod & Filt. & $\underline{\text { ST }} \underline{H}$ & $\underline{\mathrm{DF}}$ & Lab & Unit \\
\hline Antimony, total recoverable & $<0.20$ & UNI & $<0.20$ & & 1 & GE & & & & & & & $\mu g / L$ \\
\hline Arsenic, total recoverable & $<150$ & $\mathrm{U} / / \mathrm{I}$ & $<150$ & & 50 & GE & & & & & & & $\mu g / L$ \\
\hline Barium, total recoverable & 650 & $N /$ & & & 1 & GE & & & & & & & $\mu g / L$ \\
\hline Cadmium, total recoverable & 16 & $\|$ & & $\mathbf{\square}$ & 1 & GE & 17 & $\|$ & & $\mathbf{0}$ & 1 & GE & $\mu g / L$ \\
\hline Chromium, total recoverable & $<3.1$ & $\mathrm{UN} /$ & $<3.0$ & & 1 & GE & & & & & & & $\mu g / L$. \\
\hline Cobalt, total recoverable & 250 & $\| 1$ & & - & 1 & GE & & & & & & & $\mu g / L$ \\
\hline Copper, total recoverable & 290 & $N I$ & & & 1 & GE & & & & & & & $\mu g / L$ \\
\hline Cyanide & $<10$ & $\mathrm{U} / /$ & $<10$ & & 1 & GE & & & & & & & $\mu g / L$ \\
\hline Lead, total recoverable & 210 & $N /$ & & $\mathbf{\square}$ & 1 & GE & 24 & /I & & 口 & 1 & GE & $\mu \mathrm{g} / \mathrm{L}$ \\
\hline Mercury, total recoverable & $<0.20$ & $\mathrm{U} / /$ & $<0.20$ & & 1 & GE & & & & & & & $\mu g / L$ \\
\hline $\begin{array}{l}\text { Nickel, total recoverable } \\
\text { Nitrate as nitrogen }\end{array}$ & $<120$ & UNI & $<0.20$ & & 1 & GE & & & & & & & $\mu g / h$ \\
\hline Nitrate-nitrite as nitrogen & 270,000 & $\|$ & & a & 100 & GE & 290,000 & $\|$ & & घ & 150 & GE & $\mu g / L$ \\
\hline Selenium, total recoverable & $<250$ & $\mathrm{U} / /$ & $<250$ & & 50 & GE & & & & & & & $\mu g / L$ \\
\hline Silver, total recoverable & $<0.28$ & UNI & $<1.0$ & & 1 & GE & & & & & & & $\mu g / h$ \\
\hline Thalijum, total recoverable & 0.79 & $\mathrm{~J} / \mathrm{E} /$ & NDD & & 1 & GE & & & & & & & $\mu g / L$ \\
\hline Vanadium, total recoverable & $<2.0$ & $\mathrm{U} / 1$ & $<2.0$ & & 1 & GE & & & & & & & $\mu g / L$ \\
\hline Zinc, total recoverable & 620 & $N /$ & & & 1 & GE & & & & & & & $\mu g / L$ \\
\hline \multicolumn{14}{|l|}{ Organic Constituents } \\
\hline Constituents & $\underline{3098}$ & Mod & Filt. & $\underline{\text { ST }} \underline{H}$ & OF & $\underline{\text { Lab }}$ & $\underline{4 Q 98}$ & Mod & Filt. & $\underline{\mathrm{ST}} \underline{\mathrm{H}}$ & DF & Lab & Unit \\
\hline Benzene & $<1.0$ & $\mathrm{U} / \prime$ & $<1.0$ & & 1 & GE & & & & & & & $\mu g / h$ \\
\hline Bis(2-ethylhexyl) phthalate & $<10$ & $\mathrm{U} / /$ & $<13$ & & 1 & GE & & & & & & & $\mu g / l$ \\
\hline Dichloromethane & $<3.8$ & UN8I & $<1.0$ & & 1 & GE & & & & & & & $\mu g / \mathrm{L}$ \\
\hline Phenols & $<5.0$ & $\mathrm{U} / 1$ & $<5.0$ & & 1 & $\overline{G E}$ & & & & & & & $\mu g / L$ \\
\hline Tetrachloroethylene & $<1.0$ & $\mathrm{U} / /$ & $<1.0$ & & 1 & GE & & & & & & & $\mu g / L$ \\
\hline Trichloroethylene & $<1.0$ & $\mathrm{U} / \mathrm{I}$ & $<1.0$ & & 1 & GE & & & & & & & $\mu g / L$ \\
\hline Trichlorofluoromethane & $<1.0$ & $\mathrm{U} / /$ & $<1.0$ & & 1 & GE & & & & & & & $\mu g / L$ \\
\hline
\end{tabular}

Notes:

- exceeded holding time

- exceeded groundwater protection or monitoring constituent standard (See Appendix A.) 


\section{WELL FSB 78C (cont.)}

Radioactive Constifuents

\begin{tabular}{|c|c|c|c|c|c|c|c|c|c|c|c|c|c|}
\hline Constituents & $\underline{3098}$ & Mod & Filt. & $\underline{\text { ST}} \underline{\underline{H}}$ & DF & Lab & 4Q98 & Mod & Filt. & $\underline{S T} \underline{H}$ & $\underline{D F}$ & Lab & Unit \\
\hline Americium-241 & 3.5E-01 & R/OI & Rej & & 1 & GP & & & & & & & pCill \\
\hline Beta dose & 165.3000 & & & - & & & & & & & & & pCill \\
\hline Cesium-137 & $<1.7 \mathrm{E}+00$ & $\mathrm{UI} / /$ & $<4.1700$ & & 1 & GP & & & & & & & pCill \\
\hline Cobalt-60 & $<4.0 \mathrm{E}-01$ & $\mathrm{UI} / /$ & $<4.6800$ & & 1 & GP & & & & & & & pCill \\
\hline Curium-242 & $-1.1 \mathrm{E}-01$ & $\mathrm{R} / \mathrm{O} /$ & Rej & & 1 & GP & & & & & & & pCíl \\
\hline Curium-243/244 & 3.7E-01 & R/OI & Rej & & 1 & GP & & & & & & & $\mathrm{pCi} / \mathrm{L}$ \\
\hline Curium-245/246 & $0.0 \bar{E}+00$ & RIOI & $\operatorname{Rej}$ & & 1 & GP & & & & & & & $\mathrm{pCilL}$ \\
\hline Gross alpha & $1.6 \mathrm{E}+02$ & $\mathrm{~J} / / 1 / 1$ & NDD & & 1 & GP & $2.8 \mathrm{E}+02$ & J/L & NDD & & 1 & GE & pCill \\
\hline Nonvolatile beta & $9.7 E+02$ & $\mathrm{~J} / \mathrm{IV} / 1$ & NDD & & 1 & GP & $1.2 \mathrm{E}+03$ & $\|$ & & a & 1 & GE & pCill \\
\hline Plutonium-238 & $1.4 \mathrm{E}+00$ & $\mathrm{R} / 4 /$ & Rej & & 1 & GP & & & & & & & $\mathrm{pCi} / \mathrm{L}$ \\
\hline Plutonium-239/240 & $<1,5 \mathrm{E}-01$ & $\mathrm{UIJ} / \mathrm{Cl}$ & & & 1 & GP & & & & & & & pCill \\
\hline Radium-226 & 4.3E+01 & $/ 1$ & & 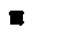 & 1 & GP & & & & & & & pCill \\
\hline Radium-228 & $<-3.3 E-01$ & $\mathrm{UI} / /$ & $<1.4000$ & & 1 & GP & & & & & & & PCill \\
\hline Strontium-90 & $4.4 E+02$ & $/ /$ & & $\mathbf{E}$ & 1 & GP & & & & & & & pCíl \\
\hline Technetium-99 & $2.7 E+02$ & $\|$ & & a & 1 & GP & & & & & & & pCill \\
\hline Thorium-228 & $<4.5 \mathrm{E}-01$ & $\mathrm{UI} / /$ & $<0.7230$ & & 1 & GP & & & & & & & pCill \\
\hline Thorium-230 & $<6.4 \mathrm{E}-02$ & $\mathrm{UI} / /$ & $<0.2640$ & & 1 & GP & & & & & & & pCill \\
\hline Sum of atphas & 1.1E+02 & & & - & & & & & & & & & pCin \\
\hline Sum of betas & $8.2 E+02$ & & & $\bar{z}$ & & & & & & & & & pCill \\
\hline Total radium & $4.3 E+01$ & & & 들 & & & & & & & & & pCíl \\
\hline Tritium & $7.8 E+03$ & $\|$ & & E & 1 & GP & $9.7 E+03$ & $/ /$ & & E & 1 & GE & pCímL \\
\hline Uranium-233/234 & $4.8 E+01$ & $/ /$ & & - & 1 & GP & & & & & & & pCill \\
\hline Uranium-235 & $3.5 E+00$ & $/ /$ & & & 1 & GP & & & & & & & pCill \\
\hline Uranium-238 & $5.5 E+01$ & $/ /$ & & $\mathbf{E}$ & 1 & GP & & & & & & & pCill \\
\hline
\end{tabular}

\section{Notes:}

- = exceeded holding time

E = exceeded groundwater protection or monitoring constituent standard (See Appendix A.) 


\section{WELL FSB 79}

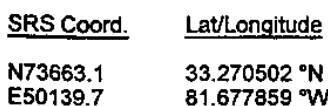

SAMPLE DATE

FIELD DATA

Constituents

Water elevation

$\mathrm{pH}$

Sp. conductance

Water temperature

Alkalinity as $\mathrm{CaCO}_{3}$

Turbidity

Volumes purged

Sampling code

Synchronous water level

ANALYTICAL DATA

\section{Screen Zone Elevation}

204.1-174.1 ft msl

$07 / 10 / 98$

$\underline{3098}$

190.6

3.6

2020

22.4

1
2.4

$201.8(09 / 17 / 98)$

\section{Top of Casing}

$217.8 \mathrm{ft} \mathrm{ms}$

$10 / 05 / 98$

$\underline{4098}$
202.4
3.6
700
21.9
0
1
3.6

$190.6(12 / 18 / 98)$
Casing Pump

4" PVC V
Screen Zone

Water Table (IIB2)

Inorganic Constituents

\begin{tabular}{|c|c|c|c|c|c|c|c|c|c|c|c|c|c|}
\hline Constituents & 3098 & Mod & Filt. & $\underline{\text { ST }} \underline{\text { H }}$ & DF & Lab & 4Q98 & Mod & Filt. & ST $\underline{H}$ & DF & $\underline{L a b}$ & Unit \\
\hline Antimony, total recoverable & $<0.77$ & UNI & $<2.0$ & & 10 & GE & & & & & & & $\mu g / L$ \\
\hline Arsenic, total recoverable & $<300$ & $\mathrm{U} / /$ & $<300.0000$ & & 100 & GE & & & & & & & $\mu \mathrm{g} / \mathrm{L}$ \\
\hline Barium, total recoverable & 750 & $/ /$ & & & 10 & GE & & & & & & & Holl \\
\hline Cadmium, total recoverable & 5.4 & $\mathrm{~J} / \mathrm{E} /$ & NDD & & 10 & GE & 4.6 & $\|$ & & & 1 & GE & $\mu g / L$ \\
\hline Chromium, total recoverable & 29 & J/EV/ & NDD & . & 10 & GE & & & & & 1 & ( & $\mu g / L$ \\
\hline Cobalt, total recoverable & 580 & $/ /$ & & $\mathbf{E}$ & 10 & $\overline{G E}$ & & & & & & & Mg/L \\
\hline Copper, total recoverable & 90 & $/ /$ & & & 10 & GE & & & & & & & u $/ \mathrm{L}$ \\
\hline Cyanide & $<10$ & $\mathrm{u} / /$ & $<10$ & & 1 & GE & & & & & & & $\mu g / L$ \\
\hline Lead, total recoverable & 19 & $\mathbf{J} / \mathbf{E}$ & NDD & & 10 & $\mathbf{G E}$ & 0.57 & $\mathrm{~J} / / /$ & NDD & & 1 & GE & $\mu g / L$ \\
\hline Mercury, total recoverable & 3.7 & $/ /$ & & च & 1 & GE & & & & & & & $\mu g / L$ \\
\hline $\begin{array}{l}\text { Nickel, total recoverable } \\
\text { Nitrate as nitrogen }\end{array}$ & 74 & $\|$ & & & 10 & $\overline{\mathrm{GE}}$ & & & & & & & $\mu g / L$ \\
\hline Nitrate-nitrite as nitrogen & 320,000 & $\|$ & & $\mathbf{\square}$ & 200 & GE & 80,000 & $\|$ & & - & 50 & GE & $\mu g / L$ \\
\hline Selenium, total recoverable & $<500$ & $\mathrm{U} / /$ & $<500.0000$ & & 100 & GE & & & & & & & $\mu g / L$ \\
\hline Silver, total recoverable & 1.7 & J/CEVI & NDD & & 10 & GE & & & & & & & $\mu g / 2$ \\
\hline Thallium, total recoverable & 5.9 & $\mathrm{~J} / \mathrm{E} /$ & NDD & & 10 & GE & & & & & & & $\mu g^{\prime} l$ \\
\hline Vanadium, total recoverable & $<20$ & $\mathrm{U} / /$ & $<20$ & & 10 & GE & & & & & & & $\mu g / l$ \\
\hline Zinc, total recoverable & 160 & $\|$ & & & 10 & GE & & & & & & & $\mu g / L$ \\
\hline \multicolumn{14}{|l|}{ Organic Constituents } \\
\hline Constituents & 3098 & Mod & Filt. & $\underline{\text { ST }} \underline{H}$ & DF & Lab & 4Q98 & Mod & Filt. & $\underline{\text { ST }} \underline{H}$ & DF & $\underline{L a b}$ & Unit \\
\hline Benzene & $<1.0$ & $\mathrm{UJ} / \mathrm{O} / 1$ & $<1.0$ & 1 & & GE & & & & & & & $\mathrm{jg} / \mathrm{L}$ \\
\hline Bis(2-ethylhexyl) phthalate & $<11$ & $\mathrm{U} / /$ & $<11$ & & 1 & $\overline{G E}$ & & & & & & & ug/L \\
\hline Dichloromethane & $<2.3$ & UJNO8/1 & $<1.0$ & 1 & & $\mathrm{GE}$ & & & & & & & ugl \\
\hline Phenols & $<5.0$ & $\mathrm{U} / /$ & $<5.0$ & & 1 & GE & & & & & & & doll \\
\hline Tetrachloroethylene & 0.81 & $\mathrm{~J} / \mathrm{EO} / 1$ & NDD & & 1 & GE & & & & & & & $\mathrm{dg} / \mathrm{L}$ \\
\hline Trichloroethylene & $<1.0$ & $\mathrm{U} \mathrm{J} / \mathrm{O} / 1$ & $<1.0$ & & 1 & GE & & & & & & & a \\
\hline Trichlorofluoromethane & $<1.0$ & $\mathrm{UJ} / \mathrm{O} / 1$ & $<1.0$ & & 1 & GE & & & & & & & \\
\hline
\end{tabular}

Notes:

- exceeded holding time

= exceeded groundwater protection or monitoring constituent standard (See Appendix A.)

F-Area HWMF

Third and Fourth Quarter 1998 
WELL FSB 79 (cont.)

Radioactive Constituents

\begin{tabular}{|c|c|c|c|c|c|c|c|c|c|c|c|c|c|}
\hline Constituents & 3098 & Mod & Filt. & STT브 & $\underline{\mathrm{DF}}$ & Lab & 4098 & Mod & Filt. & $\underline{\text { ST }} \underline{H}$ & DF & $\underline{L a b}$ & Unit \\
\hline Americium-241 & $4.7 E+01$ & $\|$ & & घ & 1 & GP & & & & & & & pCill \\
\hline Beta dose & 166.3611 & & & - & & & & & & & & & $\mathrm{pCill}$ \\
\hline Cesium-137 & $<-7.9 E-01$ & $\mathrm{UI} / / \mathrm{I}$ & $<4.1600$ & & 1 & GP & & & & & & & $\mathrm{pCi} / \mathrm{L}$ \\
\hline Cobalt -60 & $<3.5 E+00$ & $\mathrm{UI} / / \mathrm{I}$ & $<5.6400$ & & 1 & GP & & & & & & & $\mathrm{pCi}$ \\
\hline Curium-242 & $<1.7 \mathrm{E}-01$ & $\mathrm{UI} / /$ & $<0.5100$ & & 1 & GP & & & & & & & $\mathrm{pCi} / \mathrm{L}$ \\
\hline Curium-243/244 & $9.2 E+01$ & $\|$ & & a & 1 & GP & & & & & & & $\mathrm{pCi} / \mathrm{L}$ \\
\hline Curium-245/246 & $<5.2 \mathrm{E}-01$ & $\mathrm{UI} / /$ & $<0.9370$ & & 1 & $\mathrm{GP}$ & & & & & & & $\mathrm{pCi} / \mathrm{L}$ \\
\hline Gross alpha & $1.5 E+03$ & $\|$ & & m & 1 & GP & $4.0 \mathrm{E}+02$ & $\|$ & & - & 1 & GE & $\mathrm{pCi} / \mathrm{L}$ \\
\hline lodine-129 & $1.3 E+02$ & $\| \prime$ & & घ & 1 & GP & & & & & & & $\mathrm{pCi} / \mathrm{L}$ \\
\hline Nonvolatile beta & $1.6 \mathrm{E}+03$ & $\mathrm{~J} / \mathrm{C} /$ & NDD & & 1 & GP & $6.5 E+02$ & $\|$ & & - & 1 & GE & $\mathrm{pCi} / \mathrm{L}$ \\
\hline Plutonium-238 & $<1.5 E-02$ & UI/I & $<0.1030$ & & 1 & GP & & & & & & & $\mathrm{pCi} / \mathrm{L}$ \\
\hline Plutonium-239/240 & $<0.0 E+00$ & UIII & $<0.0587$ & & 1 & GP & & & & & & & $\mathrm{pCi} / \mathrm{L}$ \\
\hline Radium-226 & $2.5 E+01$ & $\| l$ & & - & 1 & GP & & & & & & & pCill \\
\hline Radium-228 & $<-4.7 E-01$ & $\mathrm{UI} / /$ & $<1.2100$ & & 1 & GP & & & & & & & $\mathrm{pCil}$ \\
\hline Strontium-90 & $2.9 E+02$ & $\|$ & & 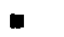 & 1 & GP & & & & & & & pCir \\
\hline Technetium-99 & $1.0 E+02$ & $\|$ & & 口 & 1 & GP & & & & & & & pCir \\
\hline Thorium-228 & $1.4 E+00$ & $\|$ & & & 1 & GP & & & & & & & pCill \\
\hline Thorium-230 & $4.9 E-01$ & $\mathrm{~J} / \mathrm{C} /$ & NDD & & 1 & GP & & & & & & & pCill \\
\hline Thorium-232 & $<7.2 E-02$ & $\mathrm{UI} / /$ & $<0.2150$ & & 1 & GP & & & & & & & pCill \\
\hline Sum of alphas & $7.4 \mathrm{E}+02$ & & & $\mathbf{\square}$ & & & & & & & & & pCill \\
\hline Sum of betas & $5.2 E+02$ & & & 口 & & & & & & & & & $\mathrm{pCi \Omega}$ \\
\hline Total radium & $2.5 E+01$ & & & $\bar{a}$ & & & & & & & & & $\mathrm{pCil}$ \\
\hline Tritium & $1.5 E+04$ & $\|$ & & 曰 & 1 & GP & $9.7 E+03$ & $\|$ & & a & 1 & GE & pCilmL \\
\hline Uranium-233/234 & $2.0 E+02$ & $1 /$ & & - & 1 & GP & & & & & & & $\mathrm{pCi} \mathrm{Cl}$ \\
\hline Uranium-235 & 2.1E+01 & $\|$ & & 口 & 1 & GP & & & & & & & pCill \\
\hline Uranium-238 & $3.8 \mathrm{E}+02$ & $\|$ & & 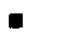 & 1 & GP & & & & & & & pCill \\
\hline
\end{tabular}

Notes:

- exceeded holding time

a = exceeded groundwater protection or monitoring constituent standard (See Appendix A.) 


\section{WELL FSB 79A}

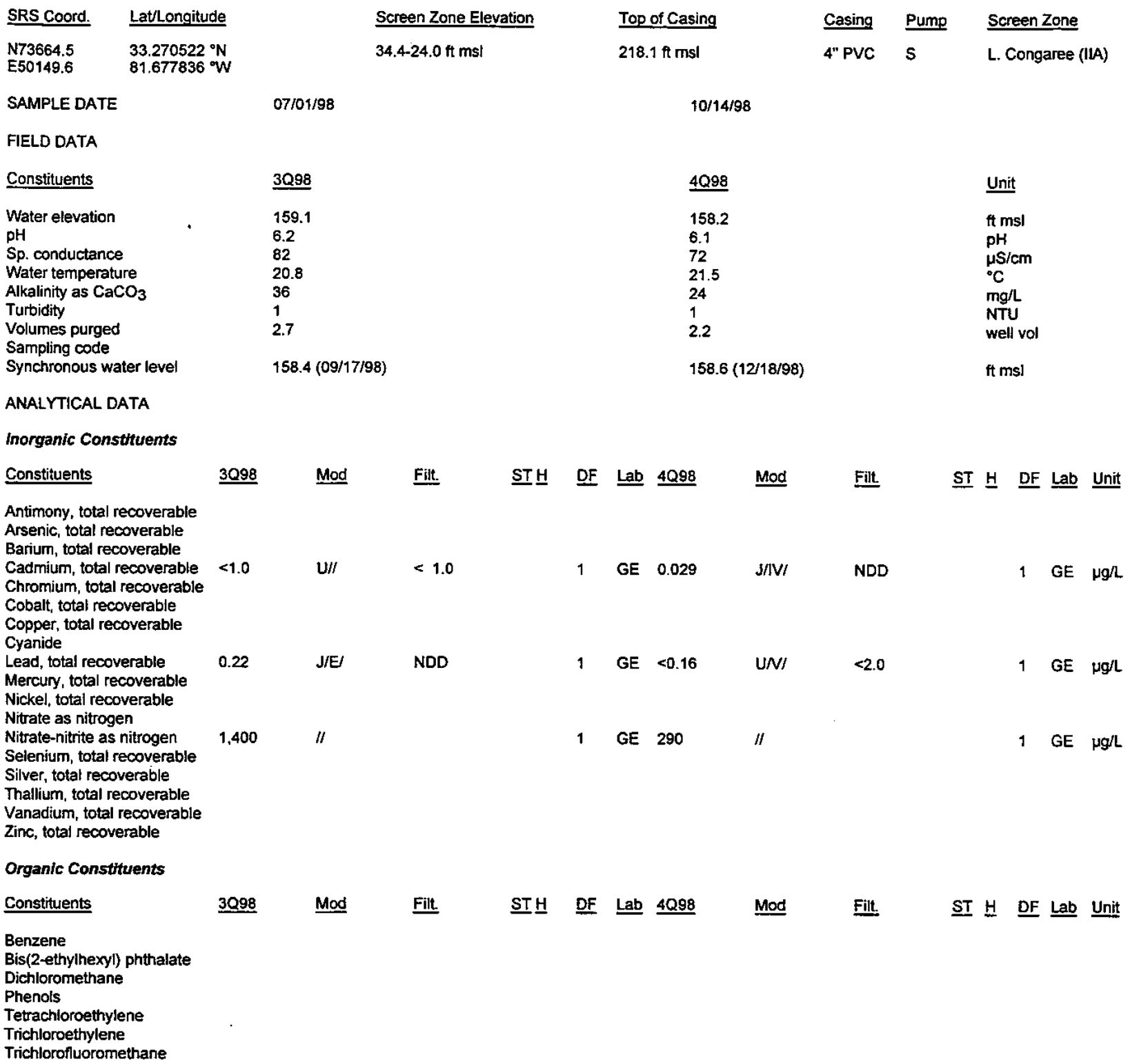

Notes:

- = exceeded holding time

च = exceeded groundwater protection or monitoring constituent standard (See Appendix A.) 
WELL FSB 79A (cont.)

Radloactive Constituents

Constituents

Americium-241

Cesium-137

Cobalt-60

Curium-242

Curium-243/244

Curium-245/246

Gross atpha

lodine-129

Nonvolatile beta

Plutonium-238

Plutonium-239/240

Radium-226

Radium-228

Strontium-90

Technetium-99

Thorium-228

Thorium-230

Thorium-232

Sum of alphas

Sum of betas

Tritium

Uranium-233/234

Uranium-235

Uranium-23B
$3 \mathrm{Q} 9$

UIII

$1.8 E+00$

ll

3.4E+01 /

Mod

Filt.

ST브

DF Lab $\underline{4098}$

Mod

$1 \quad$ GP $<9.2 E-01 \quad U / \prime$

$1 \quad \mathrm{GP}<2.0 \mathrm{E}+00 \quad \mathrm{U} / 1$

$<6.1 \mathrm{E}-01$

$<1.2 E+00$

$1 \mathrm{GE} \mathrm{pCi} / \mathrm{L}$

$\uparrow$ GE pCill

1 GE $\mathrm{pCi} / \mathrm{mL}$

Notes:

- = exceeded holding time

E = exceeded groundwater protection or monitoring constituent standard (See Appendix A.) 


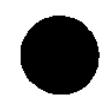

WELL FSB 79B

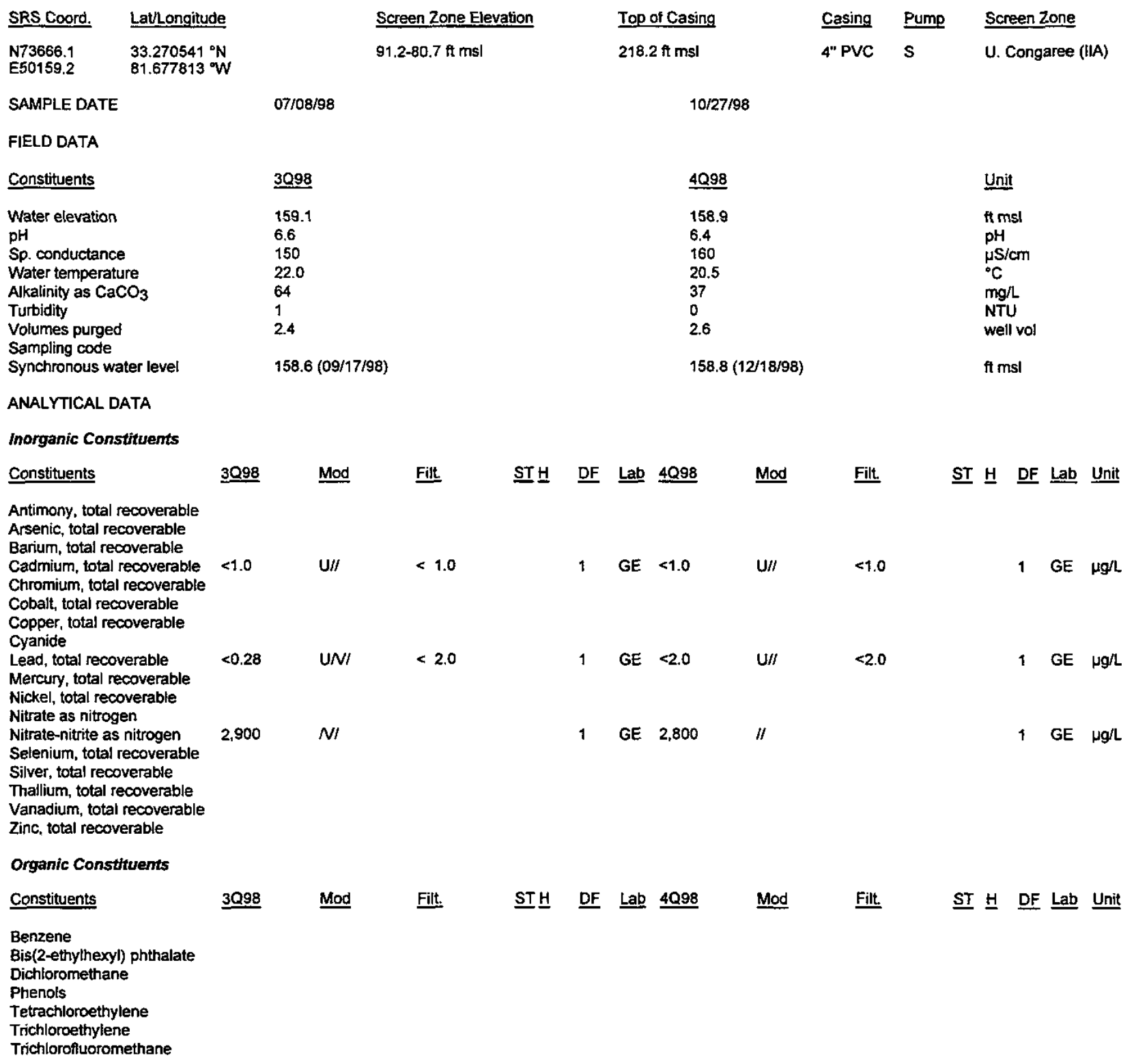

Notes:

- = exceeded holding time

- = exceeded groundwater protection or monitoring constituent standard (See Appendix A.)

F-Area HWMF 
WELL FSB 79B (cont.)

Radioactive Constituents

Constituents

3098

Mod

Filt.

STH DF Lab 4098 Mod

Filt.

ST 브 DF Lab Unit

Americium-241

Cesium-137

Cobalt-60

Curium-242

Curium-243/244

Curium-245/246

Gross alpha

lodine-129

Nonvolatile beta

Plutonium-238

Plutonium-239/240

Radium-226

Radium-228

Strontium-90

Technetium-99

Thorium-228

Thorium-230

Thorium-232

Sum of alphas

Sum of betas

Tritium

Uranium-233/234

$1.2 E+\infty \quad / /$

1 GP $<4.5 E-01 \quad U / 1$

$<9.9 E-01$

$1 \quad \mathrm{GP} \quad<1.5 \mathrm{E}+00 \quad \mathrm{U} / /$

$<1.3 E+00$

1 GE pCilL

$2.2 E+00$

1

(1)

1 GE pCill

Uranium-235

Uranium-238

Notes:

- exceeded holding time

- exceeded groundwater protection or monitoring constituent standard (See Appendix A.)

F-Area HWMF

C-30

Third and Fourth Quarter 1998 
WELL FSB 79C

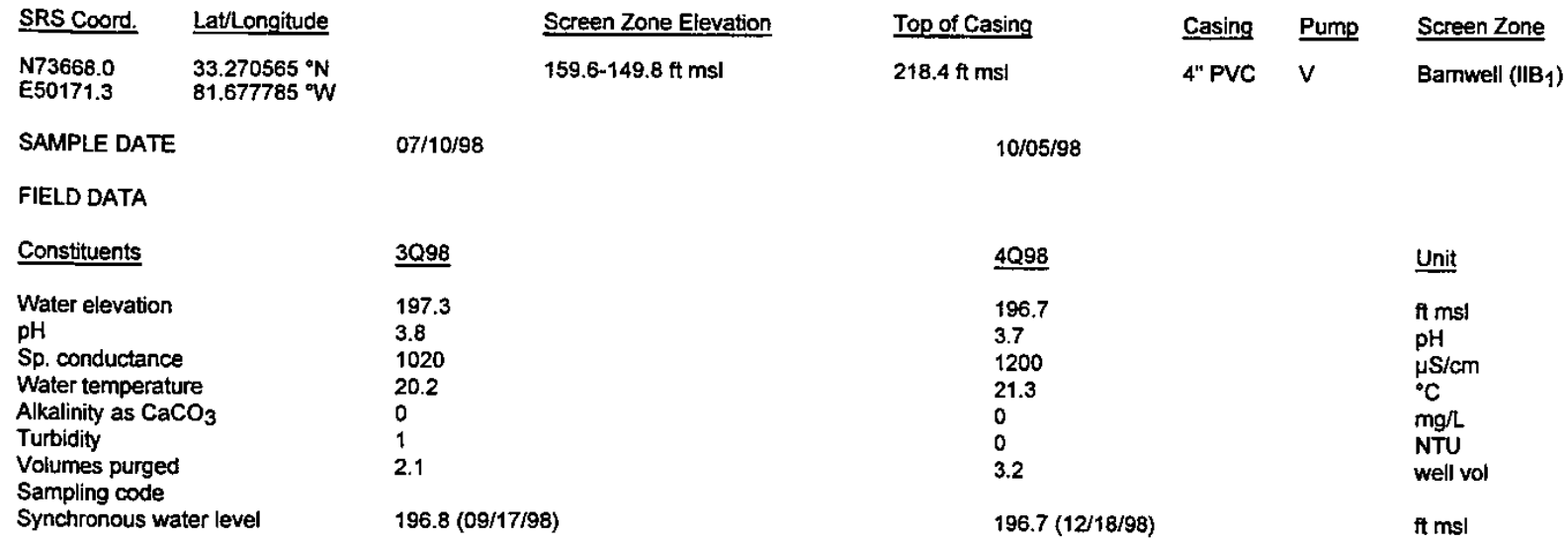

ANALYTICAL DATA

Inorganic Constituemts

\begin{tabular}{|c|c|c|c|c|c|c|c|c|c|c|c|c|c|}
\hline Constituents & $3 Q 98$ & Mod & Filt. & $\underline{\text { ST }} \underline{H}$ & $\underline{\text { DF }}$ & $\underline{\text { Lab }}$ & 4098 & Mod & Filt. & $\underline{\text { ST }} \underline{H}$ & $\underline{D F}$ & $\underline{\text { Lab }}$ & $\underline{\text { Unit }}$ \\
\hline Antimony, total recoverable & $<4.0$ & $\mathrm{U} / /$ & $<4.0000$ & & 20 & GE & & & & & & & $\mu g / L$ \\
\hline Arsenic, total recoverable & $<300$ & $\mathrm{U} / I$ & $<300.0000$ & & 100 & $\mathrm{GE}$ & & & & & & & $\mu g / L$ \\
\hline Barium, total recoverable & 530 & $\| l$ & & & 20 & GE & & & & & & & $\mu g /$ \\
\hline Cadmium, total recoverable & 15 & $\mathrm{~J} / \mathrm{E} /$ & NDD & & 20 & GE & 14 & $\|$ & & $\mathbf{\square}$ & 1 & GE & $\mu g / L$ \\
\hline Chromium, total recoverable & 39 & J/EV/ & NDD & & 20 & GE & & & & & & & $\mu g / L$ \\
\hline Cobalt, total recoverable & 120 & $/ 1$ & & a & 20 & GE & & & & & & & $\mu g / L$ \\
\hline Copper, total recoverable & 55 & /I & & & 20 & GE & & & & & & & $\mu g / L$ \\
\hline Cyanide & $<10$ & $\mathrm{U} / /$ & $<10$ & & 1 & GE & & & & & & & $\mu g / L$ \\
\hline Lead, total recoverable & 6.4 & $\mathrm{~J} / \mathrm{E} /$ & NDD & & 20 & GE & $<2.0$ & $\mathrm{U} / /$ & $<2.0$ & & 1 & GE & $\mu g / L$ \\
\hline Mercury, total recoverable & 1.9 & $/ I$ & & & 1 & GE & & & & & & & $\mu g / L$ \\
\hline $\begin{array}{l}\text { Nickel, total recoverable } \\
\text { Nitrate as nitrogen }\end{array}$ & 40 & $/ 1$ & & & 20 & GE & & & & & & & $\mu g / L$ \\
\hline Nitrate-nitrite as nitrogen & 170,000 & $/ 1$ & & - & 150 & GE & 170,000 & $\|$ & & - & 100 & GE & $\mu g / L$ \\
\hline Selenium, total recoverable & $<500$ & $\mathrm{U} / /$ & $<500.0000$ & & 100 & GE & & & & & & & $\mu g / L$ \\
\hline Silver, total recoverable & 1.4 & J/CEV/ & NDD & & 20 & GE & & & & & & & $\mu g / L$ \\
\hline Thallium, total recoverable & 3.4 & $\mathrm{~J} / \mathrm{E} /$ & NDD & & 20 & GE & & & & & & & $\mu g / L$ \\
\hline Vanadium, total recoverable & $<40$ & $\mathrm{U} / I$ & $<40.0000$ & & 20 & GE & & & & & & & $\mu g / L$ \\
\hline Zinc, total recoverable & 130 & $\|$ & & & 20 & GE & & & & & & & \\
\hline \multicolumn{14}{|l|}{ Organic Constituents } \\
\hline Constituents & $\underline{3 Q 98}$ & Mod & Filt. & $\underline{\text { ST }} \underline{H}$ & $\underline{\text { DF }}$ & Lab & $\underline{4 Q 98}$ & Mod & Filt. & $\underline{\text { ST }} \underline{\mathrm{H}}$ & $\underline{\mathrm{DF}}$ & $\underline{\text { Lab }}$ & Unit \\
\hline Benzene & $<1.0$ & $\mathrm{UJ} / \mathrm{O} / 1$ & $<1.0$ & & 1 & GE & & & & & & & \\
\hline Bis(2-ethylhexyl) phthalate & $<10$ & $\mathrm{U} / 1$ & $<10$ & & 1 & GE & & & & & & & $\mu g / L$ \\
\hline Dichloromethane & $<2.6$ & UJNO8/1 & $<1.0$ & & 1 & GE & & & & & & & $\mu g / L$ \\
\hline Phenols & 3.6 & J/E $/$ & NDD & & 1 & GE & & & & & & & $\mu g / L$ \\
\hline Tetrachloroethylene & 0.76 & $\mathrm{~J} / \mathrm{EO} / 1$ & NDD & & 1 & GE & & & & & & & $\mu g / L$ \\
\hline Trichloroethylene & $<1.0$ & $\mathrm{UJ} / \mathrm{O} / 1$ & $<1.0$ & & 1 & GE & & & & & & & $\mu g / L$ \\
\hline Trichlorofluoromethane & $<1.0$ & UJ/O/1 & $<1.0$ & & 1 & GE & & & & & & & \\
\hline
\end{tabular}

Notes:

- = exceeded holding time

- exceeded groundwater protection or monitoring constituent standard (See Appendix A.)

F-Area HWMF

C-31

Third and Fourth Quarter 1998 
WELL FSB 79C (cont.)

Radioactive Constituents

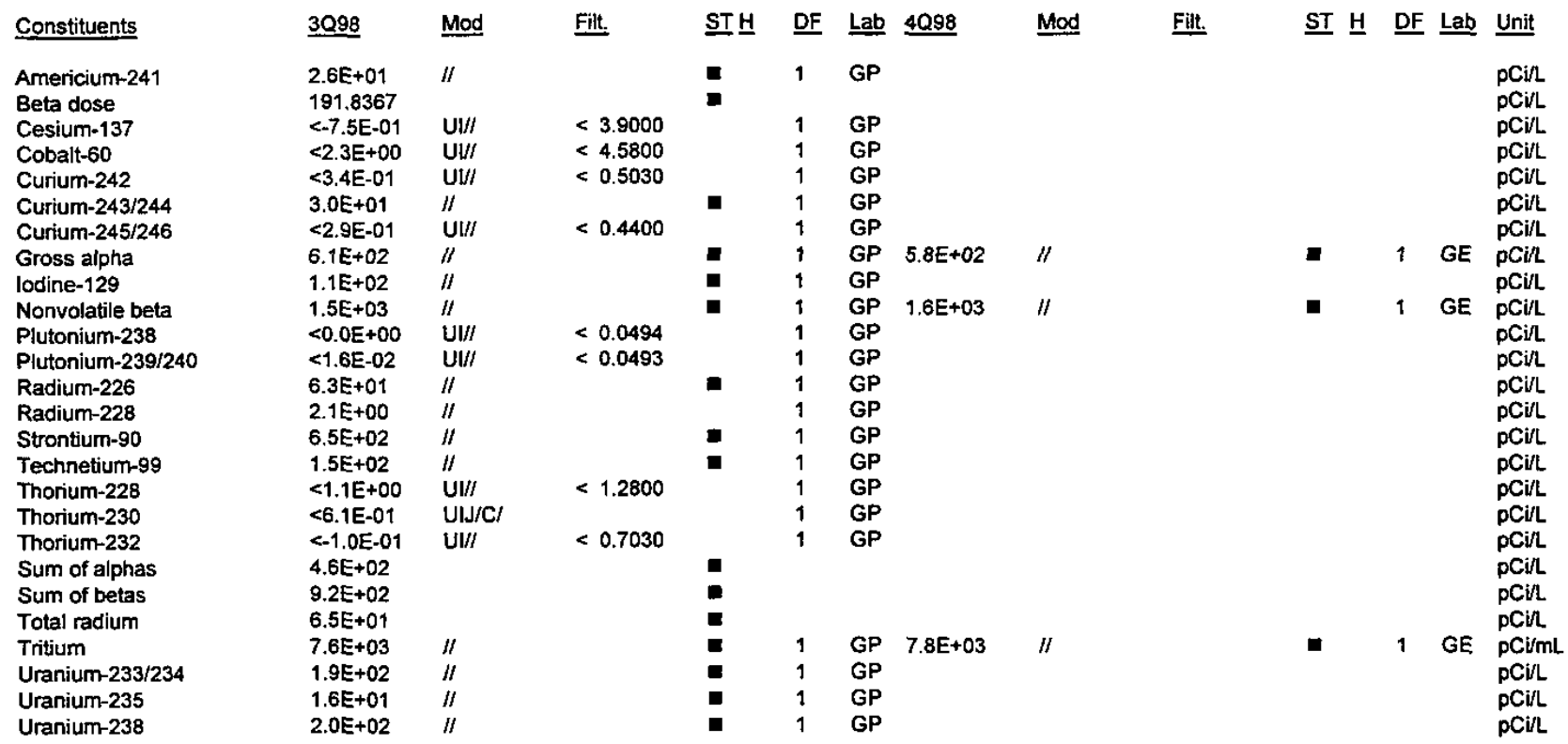

Notes:

- =xceeded holding time

- = exceeded groundwater protection or monitoring constituent standard (See Appendix A.) 


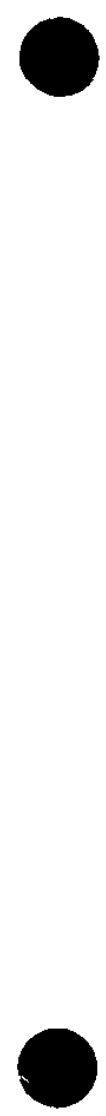

WELL FSB 87A

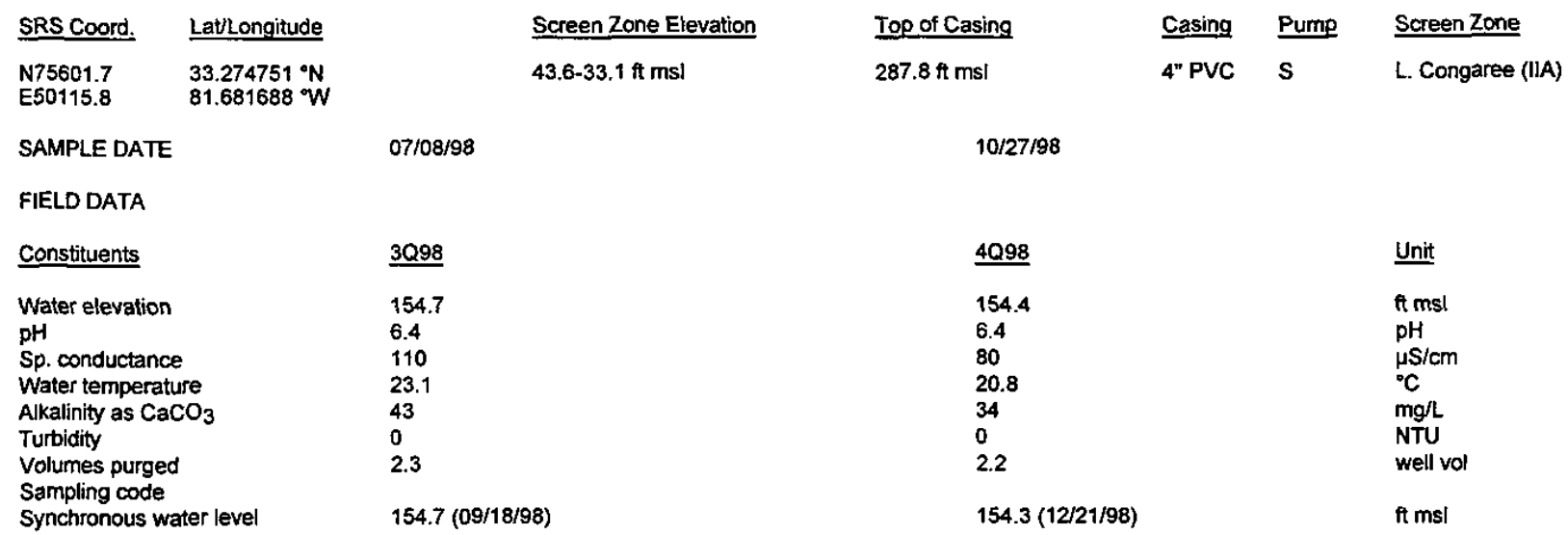

ANALYTICAL DATA

Inorganic Constituents

Constituents

Antimony, total recoverable

Arsenic, total recoverable

Barium, total recoverable

Cadmium, total recoverable

Chromium, total recoverable

$<1.0$

$\mathrm{U} / 1$

$<1.0$

1 GE $<1.0$

U/I

$<1.0$

$1 \mathrm{GE} \mu g / \mathrm{L}$

Cobalt, total recoverable

Copper, total recoverable

Cyanide

Lead, total recoverable

$<0.17 \quad$ UN $/ \quad<2.0$

1 GE $<2.0$

$\mathrm{U} / /$

$<2.0$

1 GE $\mu g / L$

Mercury, total recoverable

Nickel, total recoverable

Nitrate as nitrogen

Nitrate-nitrite as nitrogen

Selenium, total recoverable

Silver, total recoverable

Thallium, total recoverable

Vanadium, total recoverable

Zinc, total recoverable

Organic Constituents

Constituents

Benzene

Bis (2-ethylhexyl) phthalate

Dichloromethane

Phenols

Tetrachloroethylene

Trichloroethylene

Trichlorofluoromethane

Notes:

- = exceeded holding time

= exceeded groundwater protection or monitoring constituent standard (See Appendix A.) 
WELL FSB 87A (cont.)

Radioactive Constituents

Constituents

Americium-241

Cesium-137

Cobalt-60

Curium-242

Curium-243/244

Curium-245/246

Gross alpha

lodine-129

Nonvolatile beta

Plutonium-238

Plutonium-239/240

Radium-226

Radium-228

Strontium-90

Technetium-99

Thorium-228

Thorium-230

Thorium-232

Sum of alphas

Sum of betas

Tritium

Uranium-233/234

Uranium-235

Uranium-238 $\underline{3098} \quad \underline{\text { Mod }} \quad \underline{\text { Filt. }} \quad \underline{\text { STㅂ }} \quad \underline{\text { DF }} \underline{\text { Lab }} \underline{4098} \quad \underline{\text { Mod }} \quad \underline{\text { Filt. }} \quad \underline{\text { ST }} \underline{\text { DF }} \underline{\underline{H}} \underline{\text { Unit }}$

8.6E-01 $\quad / I$

GP $<8.0 E-01 \quad U / /$

$<1.0 E+00$

1 GE $p C i / L$

$1.6 \mathrm{E}+00 \quad / 1$

1

$<1.3 E+00$

1 GE PCi/l

1 GP $1.7 \mathrm{E}+00 \quad / /$

1 GE $\mathrm{pCi} / \mathrm{mL}$

\section{Notes:}

$\bullet=$ exceeded holding time

a = exceeded groundwater protection or monitoring constituent standard (See Appendix A.) 
WELL FSB 87B

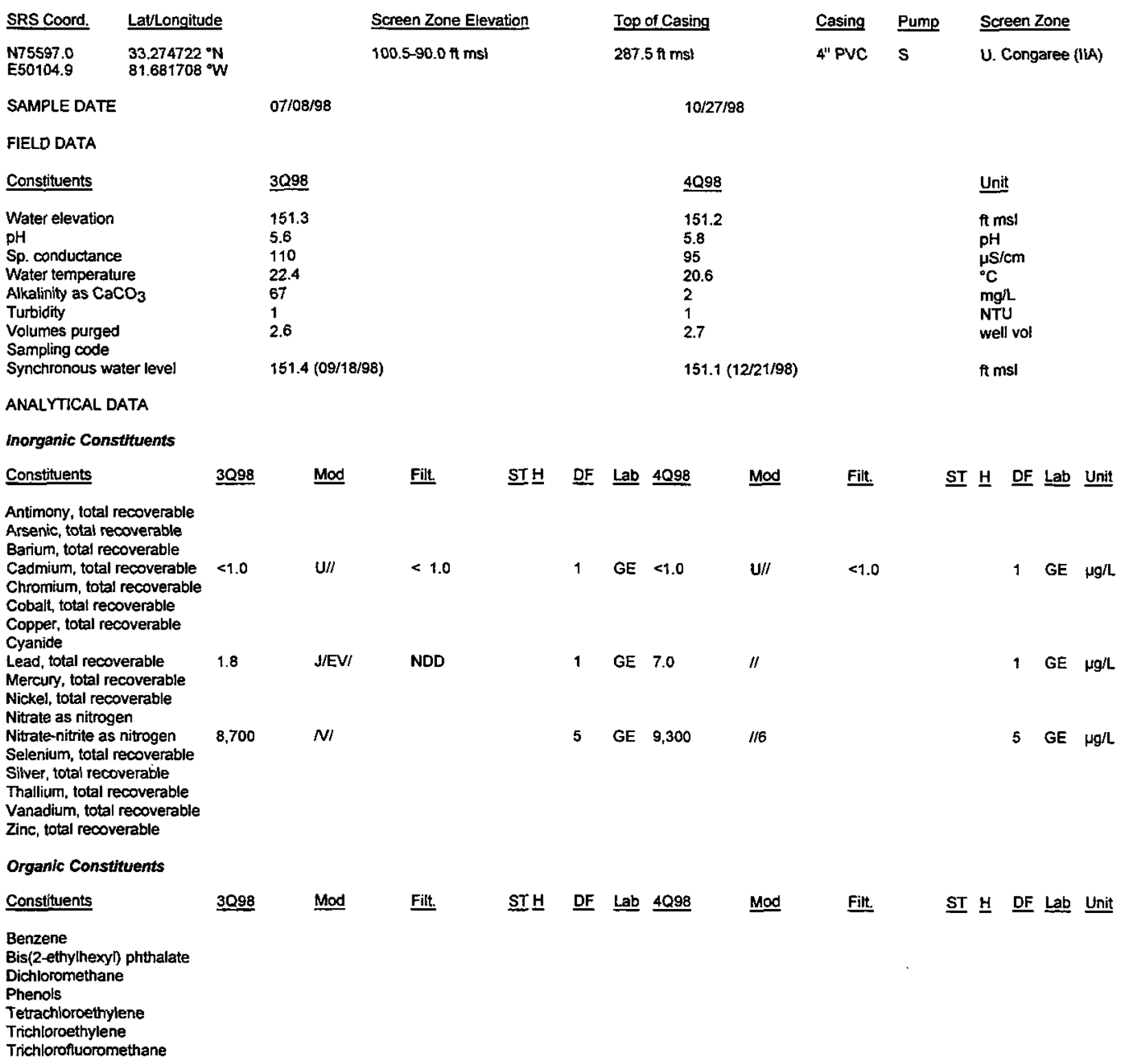

Notes:

- exceeded hoiding time

- exceeded groundwater protection or monitoring constituent standard (See Appendix A.) 
WELL FSB 87B (cont.)

Radioactive Constituents

Constituents

Americium-241

Cesium-137

Cobalt-60

Curium-242

Curium-243/244

Curium-245/246

Gross alpha

lodine-129

Nonvolatile beta

Plutonium-238

Plutonium-239/240

Radium-226

Radium-228

Strontium-90

Technetium-99

Thorium-228

Thorium-230

Thorium-232

Sum of alphas

Sum of betas

Tritium

Uranium-233/234

Uranium-235

Uranium-238
$3 Q 98$

$\underline{M o d}$

$<2.6 \mathrm{E}-01 \quad \mathrm{U}$ U/

$<0.5800$

$5.1 E+00$

II

$1.3 \mathrm{E}+02$

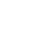

$x^{2}$

DF Lab 4Q98

Filt.

$\underline{\text { ST }} \underline{H}$ DF Lab Unit
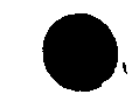

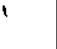


WELL FSB 87C

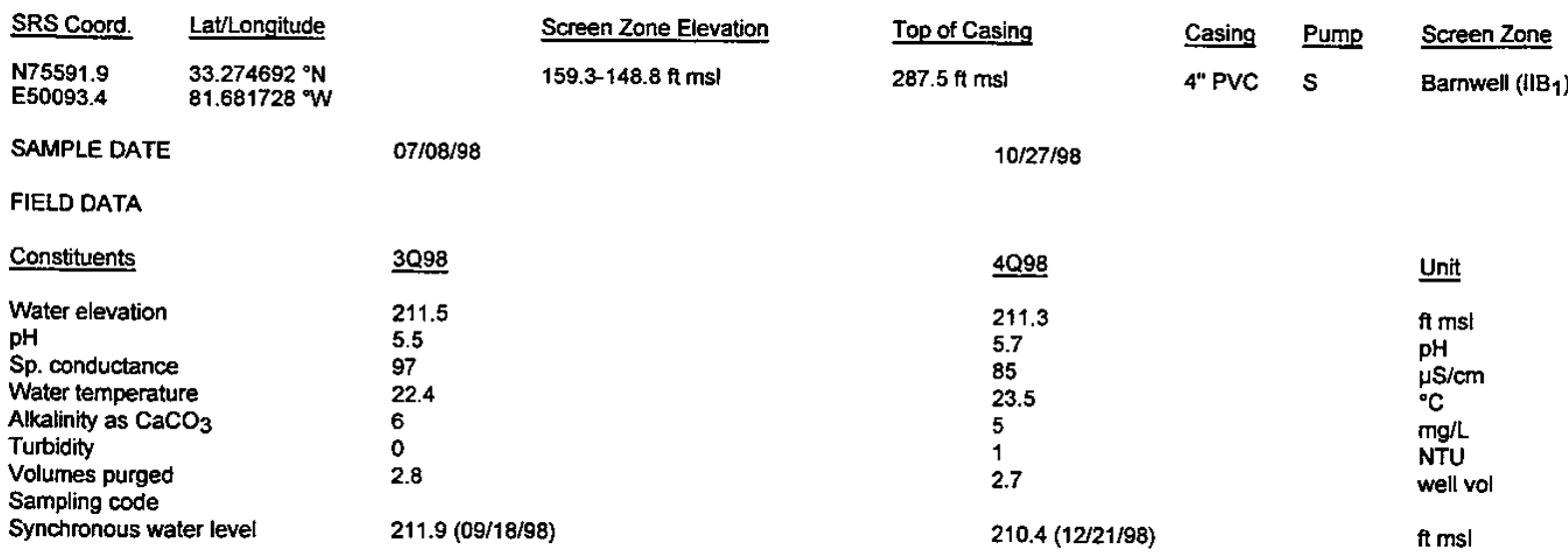

\section{ANALYTICAL DATA}

inorganic Constituents

Constituents

Antimony, total recoverable

Arsenic, total recoverable

Barium, total recoverable

Cadmium, total recoverable

Chromium, total recoverable

Cobalt, total recoverable

Copper, total recoverable

Cyanide

Lead, total recoverable

Mercury, total recoverable

Nickel, total recoverable

Nitrate as nitrogen

Nitrate-ritrite as nitrogen

Selenium, total recoverable

Silver, total recoverable

Thallium, total recoverable

Vanadium, total recoverable

Zinc, total recoverable

Organic Constituemts

Constituents

3098

Benzene

Bis(2-ethylhexyl) phthalate

Dichloromethane

Phenols

Tetrachloroethylene

Trichloroethylene

Trichlorofluoromethane

3098

$<1.0$

9.3
NI

$N \prime$

Filt.

ST브

DF Lab 4 QQ98

Mod

1 GE $<10$

$\mathrm{U} / 1$

1 GE 14

/I

5 GE $7,400 \quad / / 6$

Mod

Fill.

ST브
DF L ab 4 Q998

Filt.

ST $\underline{H}$ DF Lab Unit

Notes:

- exceeded holding time

* = exceeded groundwater protection or monitoring constituent standard (See Appendix A.)

F-Area HWMF 


\section{WELLL FSB 87C (cont.)}

Radioactive Constituents

Constituents

Americium-241

Cesium-137

Cobalt -60

Curium-242

Curium-243/244

Curium-245/246

Gross alpha

lodine-129

Nonvolatile beta

Plutonium-238

Plutonium-239/240

Radium-226

Radium-228

Strontium-90

Technetium-99

Thorium-228

Thorium-230

Thorium-232

Sum of alphas

Sum of betas

Tritium

Uranium-233/234

Uranium-235

Uranium-238
309

$1.2 \mathrm{E}+00$

$2.8 \mathrm{E}+00 \quad /$

$4.4 \mathrm{E}+02 \quad / /$
$1 \quad \mathrm{GP} \quad<8.0 \mathrm{E}-01$

1 GP $3.9 E+00 \quad / /$

- 1 GP $6.1 \mathrm{E}+02 \quad / /$
Filt.

$\underline{\text { ST }} \underline{\mathrm{H}}$ DF Lab Unit

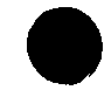

Mod

$1 \mathrm{GE} \mathrm{PCill}$

1 GE PCilL

- 1 GE pCirmL

Notes:

- exceeded holding time

- exceeded groundwater protection or monitoring constituent standard (See Appendix A.) 
WELL FSB 87D

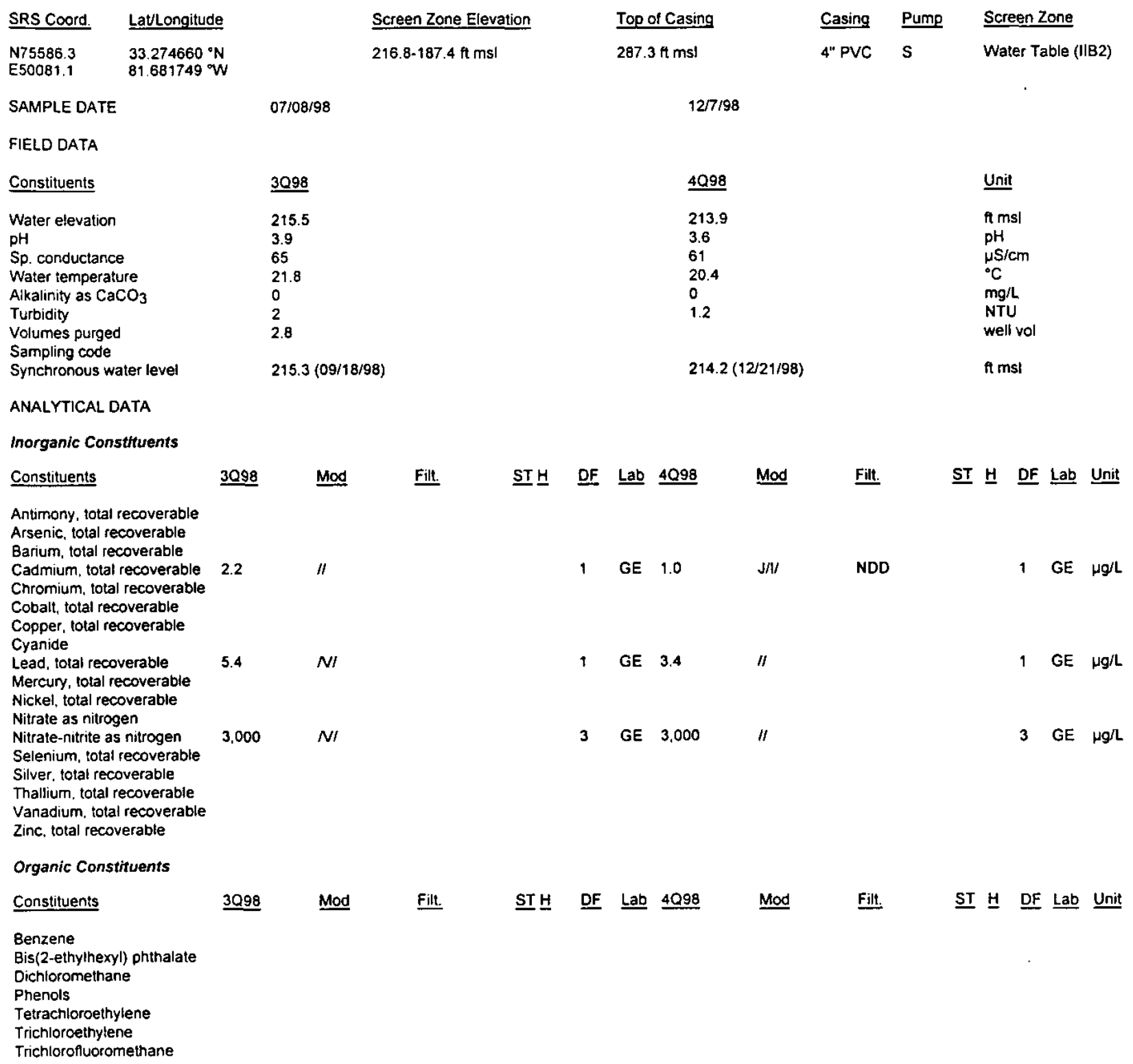


WSRC-TR-99-00012

Unclassified

\section{WELL FSB 87D (cont.)}

Radloactive Consthuents

Constituents

Americium-241

Cesium-137

Cobalt- 60

Curium-242

Curium-243/244

Curium-245/246

Gross alpha

lodine-129

Nonvolatile beta

Plutonium-238

Plutonium-239/240

Radium-226

Radium-228

Strontium-90

Technetium-99

Thorium-228

Thorium-230

Thorium-232

Sum of alphas

Sum of betas

Tritium

Uranium-233/234

Uranium-235

Uranium-238
3098

8. $2 E+01$

$5.8 \mathrm{E}+01 \quad / /$

Fill.

ST브 DF Lab $\underline{4098}$

Mod

Filt.

ST $\underline{H}$, DF Lab Unit

-

- 1 GP $4.6 E+01 \quad / 1$

a 1 GP $\quad 3.8 \mathrm{E}+01$

1 GE $\mathrm{pCi} / \mathrm{mL}$ 
WELL FSB 88C

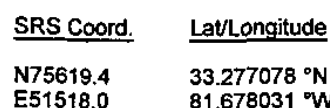

E51518.0

$81.678031^{\circ}$

SAMPLE DATE

FIELD DATA

Constituents

Water elevation

pH

Sp. conductance

Water temperature

Alkalinity as $\mathrm{CaCO}_{3}$

Turbidity

Volumes purged

Sampling code

Synchronous water level

3Q98

214.8

5.4

21.2

2.2

$214.9(09 / 17 / 98)$
Screen Zone Elevation

168.4-158.4 ft msl

Top of Casing

$283 \mathrm{ft}$ msl

$10 / 27 / 98$

4Q98

215.0

5.1

42

5.3

$214.3(12 / 18 / 98)$
Casing Pump Screen Zone

4" PVC S

ANALYTICAL DATA

Inorganic Constituents

Constituents

3Q98

Mod Filt. $\quad \underline{\text { ST }}$

DF Lab 4Q98

GE

Lead, total recoverable $\quad 0.29$

Nitrate-nitrite as nitrogen $\quad 4,800$

GE

GE

1 GE

1 GE

1 GE
Antimony, total recoverable $<0.20$

Arsenic, total recoverable $<3.0$

Barium, total recoverable $\quad 12$

Cadmium, total recoverable $<1.0$

Chromium, total recoverable 0.71

Cobalt total recoverable $\quad 0.093$

Copper, total recoverable $\quad 2.4$

Cyanide

Mercury, total recoverable $\quad<0.20$

Nickel total re

Nitrate as nitrogen

Selenium, total recoverable $<0.89$

Silver, total recoverable $\quad 0.15$

Thallium, total recoverable $\quad 0.22$

Vanadium, total recoverable $<2.0$

Zinc, total recoverable

UII $<0.20$

II

$\mathrm{U} / 1<1.0$

J/E NDD

J/E $\quad$ NDD

II

U/I $\quad<10$

$\mathrm{J} / \mathrm{E} / \mathrm{NDD}$

UII $<0.20$

II

II

UN

J/EJ NDD

$\mathrm{J} / \mathrm{E} /$ NDD

$\mathrm{U} / /<2$.

II

Organic Constituents

Constituents $\quad 3098$

Benzene

Bis(2-ethylhexyl) phthalate

Dichloromethane

Phenols

Tetrachloroethylene

Trienterosthijlente

Trichlorofluoromethane
$<1.0$

$<10$

$<2.0$

4.2

$<1.8$

0.59

$<1.8$
Mod

U.J/O/1

$\mathrm{U} / /$

UJNO8/1

$\mathrm{J} / \mathrm{E} /$

UJ/O/1

J/EO/1

$\mathrm{UJ} / \mathrm{O} / 1$
Filt.

$<1.0$

$<10$

$<1.0$

NDD

$<1.0$

NDD

$<1.0$

Mod

Filt.

$\underline{\text { ST }} \underline{H}$ DF Lab Unit

ft msl

pH

$\mu S / \mathrm{cm}$

$\mathrm{mg} / \mathrm{L}$

NTU

well vol

ft msl

msl
/cm
msl

$0.35 \mathrm{~J} / \mathrm{J}$

$\mu g / L$

$\mu g / L$

$\mu g^{\prime L}$

1 GE $\mu g / L$

$\mu g / L$

$\mu g / L$

$\mu \mathrm{g} / \mathrm{L}$

$\mu g / L$

1 GE $\mu g / L$

$\mu g / L$

$\mu g /$

1 GE $\mu g / L$

$\mu g / L$

Hgll

$\mu g / L$

$\mu g / L$

$\mu g / L$

$\underline{\text { ST }} \underline{\underline{H}}$

$\begin{array}{ll}\text { DF } & \text { Lab } \\ 1 & \text { GE } \\ 1 & \text { GE } \\ 1 & \text { GE } \\ 1 & \text { GE } \\ 1 & \text { GE } \\ 1 & \text { GE } \\ 1 & \text { GE }\end{array}$

Mod Filt.

ST 브 DF Lab Unit

$\mu g / L$

$\mu g / L$

$\mu g /$.

$\mu g / L$

$\mu \mathrm{g} / \mathrm{L}$

$\mu g / L$.

Notes:

- exceeded holding time

= exceeded groundwater protection or monitoring constituent standard (See Appendix A.)

F-Area HWMF

C-41

Third and Fourth Quarter 1998 
WELL FSB $88 \mathrm{C}$ (cont.)

Radioactive Constituents

\begin{tabular}{|c|c|c|c|c|c|c|c|c|c|c|c|c|c|}
\hline Constituents & 3098 & Mod & Filt. & $\underline{\text { STH}}$ & $\underline{D F}$ & Lab & 4098 & Mod & Filt. & $\underline{\text { ST }} \underline{H}$ & $\underline{\mathrm{DF}}$ & Lab & Unit \\
\hline Americium-241 & $<6.2 \mathrm{E}-02$ & $\mathrm{UI} / /$ & $<0.1660$ & & 1 & GP & & & & & & & pCir \\
\hline Cesium-137 & $7.4 E+00$ & $\mathrm{R} / 4 /$ & Rej & & 1 & GP & & & & & & & $\mathrm{pCi} / \mathrm{L}$ \\
\hline Cobalt- 60 & $<-9.8 \mathrm{E}-02$ & $\mathrm{UI} / /$ & $<3.4700$ & & 1 & GP & & & & & & & $\mathrm{pCi} / \mathrm{L}$ \\
\hline Curium-242 & $<-2.7 E-02$ & $\mathrm{UI} / \mathrm{I}$ & $<0.1790$ & & 1 & GP & & & & & & & $\mathrm{pCi} / \mathrm{L}$ \\
\hline Curium-243/244 & $<2.0 \mathrm{E}-02$ & $\mathrm{U} \mathrm{y} / /$ & $<0,1460$ & & 1 & GP & & & & & & & $\mathrm{pCill}$ \\
\hline Curium-245/246 & $<0.0 E+00$ & $\mathrm{U} 1 / /$ & $<0.0579$ & & 1 & GP & & & & & & & $\mathrm{pCj} / \mathrm{L}$ \\
\hline Gross alpha & $<6.0 \mathrm{E}-01$ & $\mathrm{U} 1 / /$ & $<0.6320$ & & 1 & GP & $<2.1 E+00$ & $\mathrm{U} / /$ & $<2.1 E+00$ & & 1 & GE & $\mathrm{pCi} / \mathrm{L}$ \\
\hline lodine-129 & $<1.0 E+00$ & $\mathrm{Ut} / /$ & $<1.3600$ & & 1 & GP & & & & & & & $\mathrm{pCi} / \mathrm{L}$ \\
\hline Plutonium-238 & $<0.0 E+00$ & UL// & $<0.0629$ & & 1 & GP & & & & & & & PCi/L \\
\hline Plutonium-239/240 & $<0.0 E+00$ & $\mathrm{UI} / /$ & $<0.0628$ & & 1 & GP & & & & & & & pCill \\
\hline Radium-226 & $<3.1 E-01$ & UI/I & $<0.5750$ & & 1 & GP & & & & & & & $\mathrm{pCi} / \mathrm{L}$ \\
\hline Radium-228 & $<5.8 \mathrm{E}-01$ & UI/I & $<1.1500$ & & 1 & $G P$ & & & & & & & $\mathrm{pCi} / \mathrm{L}$ \\
\hline Strontium-90 & $<5.1 E-01$ & $\mathrm{Ul} / /$ & $<1.0$ & & 1 & GP & & & & & & & oCi/L \\
\hline Technetiurn-99 & $<-6.0 E+00$ & บU/I & $<23.5000$ & & 1 & GP & & & & & & & pCi/L \\
\hline Thorium-228 & $<1.2 \mathrm{E}-01$ & UNI & $<0.0702$ & & 1 & GP & & & & & & & pCill \\
\hline Thorium-230 & $<4.0 \mathrm{E}-02$ & UUII & $<0.1210$ & & 1 & GP & & & & & & & pCir \\
\hline Thorium-232 & $<-5.5 E-03$ & $U I I I$ & $<0.1210$ & & 1 & GP & & & & & & & pCir \\
\hline Tritium & $6.9 E+01$ & $\|$ & & - & 1 & GP & $1.5 E+01$ & $/ 1$ & & & 1 & GE & $\mathrm{pCi} / \mathrm{mL}$ \\
\hline Uranium-233/234 & $2.2 E-01$ & $\|$ & & & 1 & $G P$ & & & & & & & pCill \\
\hline Uranium-235 & $<7.2 \mathrm{E}-02$ & $\mathrm{U} \mid / /$ & $<0.1370$ & & 1 & GP & & & & & & & $\mathrm{pCi} / \mathrm{L}$ \\
\hline Uranium-238 & $1.1 E+00$ & $\|$ & & & 1 & GP & & & & & & & $\mathrm{pCi} / \mathrm{L}$ \\
\hline
\end{tabular}

Notes:

- exceeded holding time

- exceeded groundwater protection or monitoring constituent standard (See Appendix A.) 
WSRC-TR-99-00012

Unclassified

WELL FSB 88D

\begin{tabular}{|c|c|c|c|c|c|}
\hline LatlLongitude & Screen Zone Elevation & Top of Casing & Casing & Pump & Screen Zone \\
\hline $\begin{array}{l}33.277098^{\circ} \mathrm{N} \\
81.678012^{\circ} \mathrm{W}\end{array}$ & $222.1-202.1 \mathrm{ft} \mathrm{ms!}$ & $282.4 \mathrm{ft} \mathrm{msl}$ & 4" PVC & $v$ & Water Table (IIB2) \\
\hline SAMPLE DATE & $07 / 07 / 98$ & $10 / 07 / 98$ & & & \\
\hline \multicolumn{6}{|l|}{ FIELD DATA } \\
\hline Constituents & $\underline{3098}$ & 4Q98 & & & $\underline{\text { Unit }}$ \\
\hline $\begin{array}{l}\text { Water elevation } \\
\text { pH } \\
\text { Sp. conductance } \\
\text { Water temperature } \\
\text { Alkalinity as } \mathrm{CaCO}_{3} \\
\text { Turtidity } \\
\text { Volumes purged } \\
\text { Sampling code } \\
\text { Synchronous water level }\end{array}$ & $\begin{array}{l}219.1 \\
3.8 \\
220 \\
20.8 \\
0 \\
2 \\
0.18 \\
X N \\
219.2(09 / 17 / 98)\end{array}$ & $\begin{array}{l}219.1 \\
3.9 \\
210 \\
21.0 \\
0 \\
2 \\
2.4 \\
218.0(12 / 18 / 98)\end{array}$ & & & $\begin{array}{l}\mathrm{ft} \text { msl } \\
\mathrm{pH} \\
\mu \mathrm{S} / \mathrm{cm} \\
{ }^{\circ} \mathrm{C} \\
\mathrm{mg} / \mathrm{L} \\
\mathrm{NTU} \\
\text { well vol } \\
\mathrm{ft} \mathrm{msl}\end{array}$ \\
\hline
\end{tabular}

ANALYTICAL DATA

Inorganic Constituents

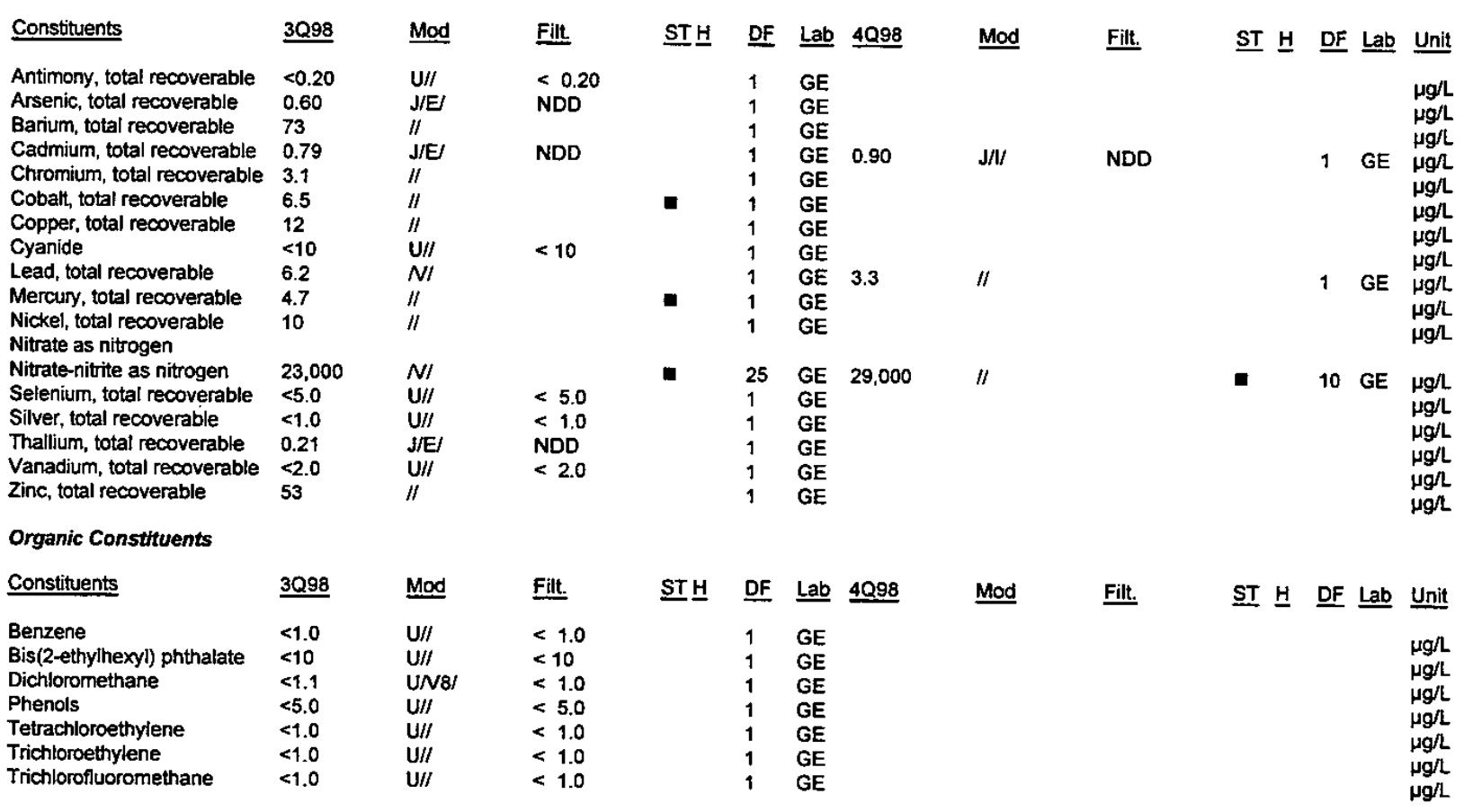

Notes:

- = exceeded holding time

- = exceeded groundwater protection or monitoring constituent standard (See Appendix A.)

F-Area HWMF

C-43

Third and Fourth Quarter 1998 


\section{WELL FSB 88D (cont.)}

Radioactive Constituents

\begin{tabular}{|c|c|c|c|c|c|c|c|c|}
\hline Constituents & 3098 & Mod & Filt. & ST $\underline{H}$ & DF & Lab & 4Q98 & Mo \\
\hline Americium-241 & $7.0 E+00$ & $\|$ & & & 1 & GP & & \\
\hline Beta dose & 73.9789 & & & $\mathbf{\square}$ & & & & \\
\hline Cesium-137 & $<7.0 E-01$ & $\mathrm{UI} / /$ & $<4.4700$ & & 1 & GP & & \\
\hline Cobalt -60 & $<3.8 \mathrm{E}+00$ & U: $/ /$ & $<5.4000$ & & 1 & GP & & \\
\hline Curium-242 & $<7.3 E-03$ & $\mathrm{UI} / \mathrm{I}$ & $<0.0868$ & & 1 & GP & & \\
\hline Curium-243/244 & $1.9 E+01$ & $\|$ & & $\mathbf{\square}$ & 1 & GP & & \\
\hline Curium-245/246 & $<2.4 E-01$ & UNI & $<0.0396$ & & 1 & GP & & \\
\hline Gross alpha & $2.8 E+02$ & $\|$ & & a & 1 & GP & $2.5 E+02$ & $/ /$ \\
\hline lodine-129 & $6.3 E+01$ & $N \prime$ & & $\boldsymbol{\sigma}$ & 1 & $G P$ & & \\
\hline Nonvolatile beta & $2.2 E+02$ & $/ /$ & & $=$ & 1 & GP & $3.0 \mathrm{E}+02$ & $\|$ \\
\hline Plutonium-238 & $<3.3 E-03$ & $\mathrm{UI} / /$ & $<0.2180$ & & 1 & GP & & \\
\hline Plutonium-239/240 & $<-3.3 E-03$ & $\mathrm{UI} / I$ & $<0.1340$ & & 1 & GP & & \\
\hline Radium-226 & $1.6 E+00$ & $/ /$ & & & 1 & GP & & \\
\hline Radium-228 & $9.7 E+00$ & /I & & - & 1 & GP & & \\
\hline Strontium-90 & $7.2 E+01$ & /I & & $\mathbf{a}$ & 1 & GP & & \\
\hline Technetium-99 & $3.5 E+01$ & $\|$ & & & 1 & GP & & \\
\hline Thorium-228 & $3.9 \mathrm{E}-01$ & $\|$ & & & 1 & GP & & \\
\hline Thorium-230 & $<3.2 \mathrm{E}-02$ & $\mathrm{UI} / /$ & $<0.0967$ & & 1 & GP & & \\
\hline Thorium-232 & $<0.0 E+00$ & UIII & $<0.0967$ & & 1 & GP & & \\
\hline Sum of alphas & $2.3 E+02$ & & & 口 & & & & \\
\hline Sum of betas & $1.8 \mathrm{E}+02$ & & & $\overline{\mathbf{a}}$ & & & & \\
\hline Total radium & 1.1E+01 & & & $\bar{a}$ & & & & \\
\hline Tritium & $3.8 \mathrm{E}+02$ & /I & & a & 1 & GP & $4.7 E+02$ & /I \\
\hline Uranium-233/234 & $8.6 E+01$ & $\|$ & & 口 & 1 & GP & & \\
\hline Uranium-235 & $6.9 E+00$ & /I & & & 1 & GP & & \\
\hline Uranium-238 & $1.1 E+02$ & /I & & n & 1 & GP & & \\
\hline
\end{tabular}

Mod Filt. $\quad \underline{\text { ST }} \underline{\mathrm{H}} \underline{\mathrm{DF}} \underline{\underline{L}} \underline{\underline{a b}} \underline{\text { Unit }}$

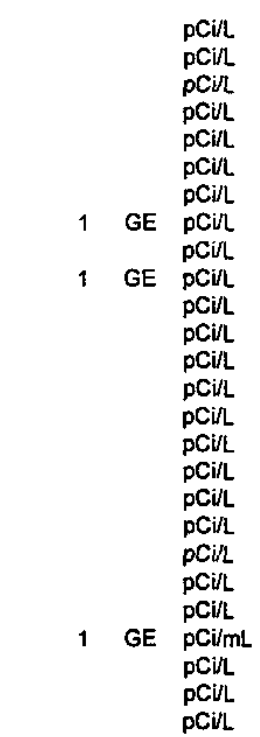

Notes:

- = exceeded holding time

E = exceeded groundwater protection or monitoring constituent standard (See Appendix A.) 
WELL FSB 89C

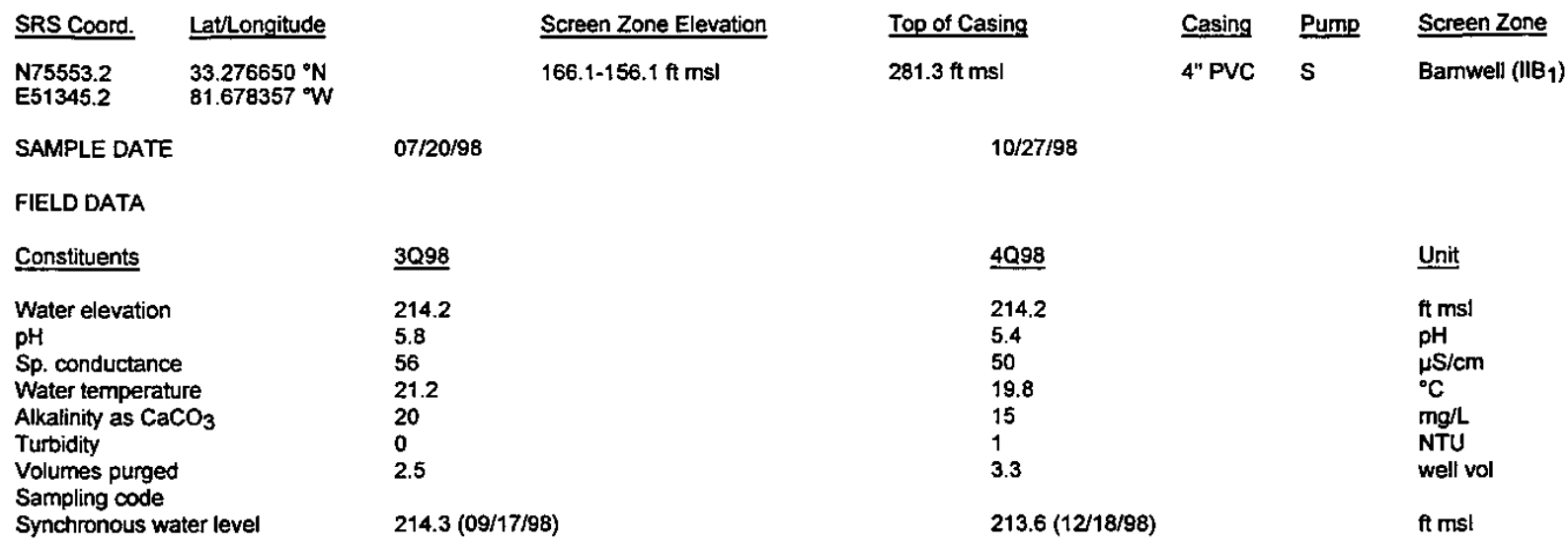

ANALYTICAL DATA

Inorganic Constituents

\begin{tabular}{|c|c|c|c|c|c|c|c|c|c|c|c|c|c|}
\hline Constituents & 3098 & Mod & Filt. & ST브 & $\underline{\text { DF }}$ & $\underline{\text { Lab }}$ & 4Q98 & Mod & Filt. & $\underline{\mathrm{ST}} \underline{\mathrm{H}}$ & $\underline{\mathrm{DF}}$ & Lab & $\underline{\text { Unit }}$ \\
\hline Antimony, total recoverable & $<0.20$ & $U / /$ & $<0.20$ & & 1 & $\mathrm{GE}$ & & & & & & & $\mu g / L$ \\
\hline Arsenic, total recoverable & $<3.0$ & $\mathrm{U} / /$ & $<3.0$ & & 1 & GE & & & & & & & $\mu g / L$ \\
\hline Barium, total recoverable & 12 & $/ 1$ & & & 1 & GE & & & & & & & $\mu g / L$ \\
\hline Cadmium, total recoverable & $<1.0$ & $\mathrm{U} / /$ & $<1.0$ & & 1 & GE & $<1.0$ & $\mathbf{U} / /$ & $<1.0$ & & 1 & GE & $\mu g / L$ \\
\hline Chromium, total recoverable & 0.84 & $\mathrm{~J} / \mathrm{E} /$ & NDD & & 1 & GE & & & & & & & $\mu g / L$ \\
\hline Cobalt, total recoverable & $<0.20$ & $\mathrm{U} / /$ & $<0.20$ & & 1 & GE & & & & & & & $\mu g / L$ \\
\hline Copper, total recoverable & 2.1 & $/ /$ & & & 1 & GE & & & & & & & $\mu g / L$ \\
\hline Cyanide & $<10$ & $\mathrm{U} / /$ & $<10$ & & 1 & GE & & & & & & & $\mu g / L$ \\
\hline Lead, total recoverabie & 0.75 & $\mathrm{~J} / \mathrm{E} J$ & NDD & & 1 & $\overrightarrow{G E}$ & 1.4 & $\mathrm{~J} / \mathrm{I} /$ & NDD & & 1 & GE & $\mu g / L$ \\
\hline Mercury, total recoverable & $<0.20$ & $\mathrm{U} / /$ & $<0.20$ & & 1 & GE & & & & & & & $\mu g / L$ \\
\hline $\begin{array}{l}\text { Nickel, total recoverable } \\
\text { Nitrate as nitrogen }\end{array}$ & 1.1 & $\|$ & & & 1 & GE & & & & & & & $\mu g / L$ \\
\hline Nitrate-nitrite as nitrogen & 1,800 & $\|$ & & & 1 & GE & 1,800 & $\|$ & & & 1 & GE & $\mu g / L$ \\
\hline Selenium, total recoverable & $<0.85$ & UNI & $<5.0$ & & 1 & GE & & & & & & & $\mu g / L$ \\
\hline Silver, total recoverable & $<1.0$ & $\mathrm{U} / /$ & $<1.0$ & & 1 & GE & & & & & & & $\mu g / L$ \\
\hline Thallium, total recoverable & 0.065 & $\mathrm{~J} / \mathrm{E} /$ & NDD & & 1 & GE & & & & & & & $\mu g / L$ \\
\hline Vanadium, total recoverable & $<2.0$ & $\mathrm{U} / \mathrm{I}$ & $<2.0$ & & 1 & GE & & & & & & & $\mu g / L$ \\
\hline Zinc, total recoverable & 18 & $/ /$ & & & 1 & $\mathrm{GE}$ & & & & & & & ر \\
\hline
\end{tabular}

Organic Constituents

\begin{tabular}{|c|c|c|c|c|c|c|c|c|c|c|c|c|}
\hline Constituents & $3 Q 98$ & Mod & Filt. & ST브 & $\underline{\mathrm{DF}}$ & $\underline{\text { Lab }}$ & 4Q98 & Mod & Filt. & $\underline{\text { ST }} \underline{\text { ㅂ}}$ & $\underline{D F} \underline{L a b}$ & Unit \\
\hline Benzene & $<1.0$ & $\mathrm{UJ} / \mathrm{O} / 1$ & $<1.0$ & & 1 & GE & & & & & & $\mu g /$ \\
\hline Bis(2-ethylhexyl) phthalate & $<9.9$ & $\mathrm{U} / I$ & $<9.9$ & & 1 & GE & & & & & & $\mu g / L$ \\
\hline Dichloromethane & $<1.9$ & UJNO8/1 & $<1.0$ & & 1 & GE & & & & & & $\mu g / 2$ \\
\hline Phenols & $<5.0$ & $\mathrm{U} / 1$ & $<5.0$ & & 1 & $\overline{G E}$ & & & & & & $\mu g / \mathrm{L}$ \\
\hline Tetrachloroethylene & $<1.4$ & UJ/O/1 & $<1.0$ & & $i$ & $\overline{G E}$ & & & & & & $\mu g /$ \\
\hline Trichloroethylene & $<1.0$ & US/O/1 & $<1.0$ & & 1 & GE & & & & & & $\mu g / L$ \\
\hline Trichlorofluoromethane & $<1.0$ & $\mathrm{UJ} / \mathrm{O} / 1$ & $<1.0$ & & 1 & GE & & & & & & $\mu g /$ \\
\hline
\end{tabular}

Notes:

- = exceeded holding time

= exceeded groundwater protection or monitoring constituent standard (See Appendix A.) 
WELL FSB $89 \mathrm{C}$ (cont.)

Radioactive Constituents

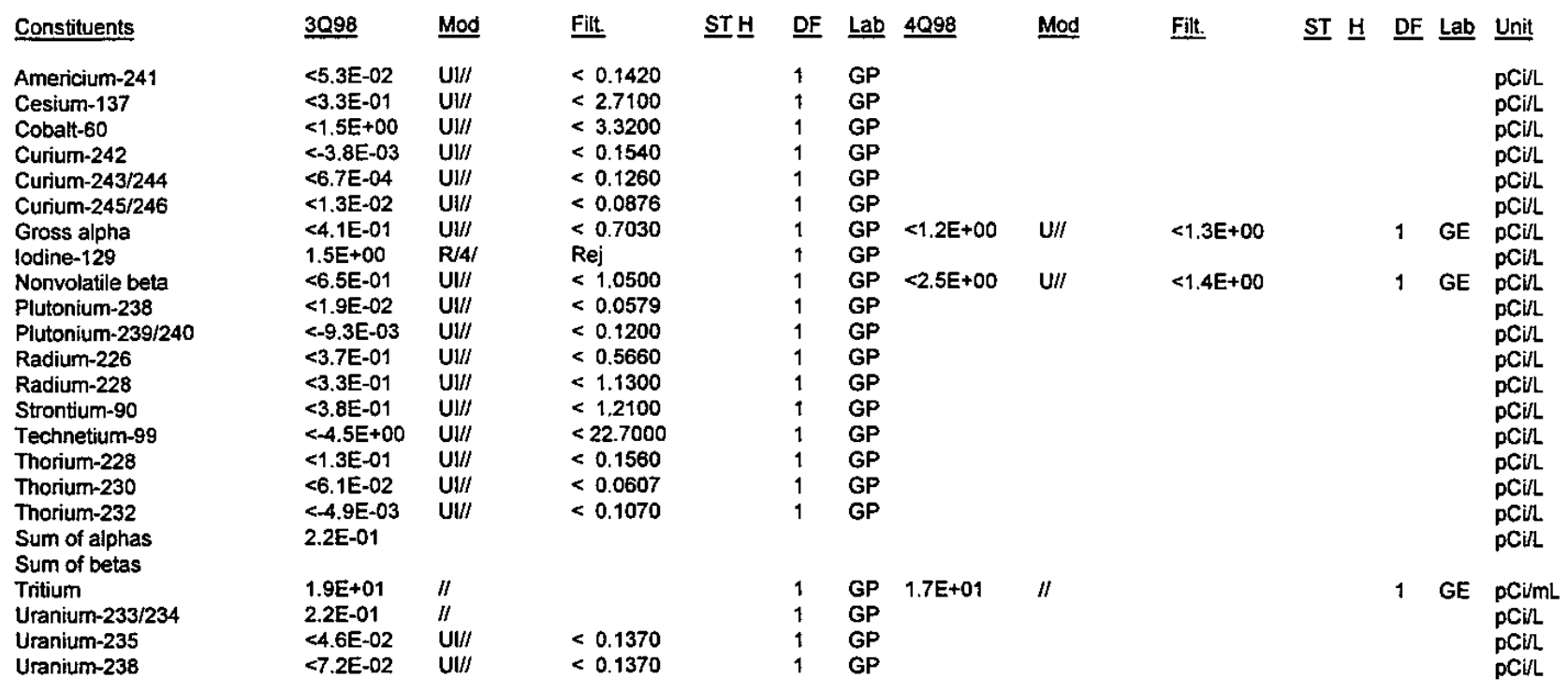

Notes:

- = exceeded holding time

= exceeded groundwater protection or monitoring constituent standard (See Appendix A.) 


\section{WELL FSB 89D}

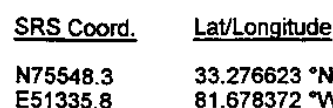

E51335.8

$33.276623^{\circ} \mathrm{N}$

SAMPLE DATE

Screen Zone Elevation

221.9-201.9 ft ms
Top of Casing

$281.2 \mathrm{ft} \mathrm{msi}$
Casing Pum

4" PVC V
Screen Zone

Water Table (IIB2)

FIELD DATA

Constituents

Water elevation

pH

Sp. conductance

Water temperature

Alkalinity as $\mathrm{CaCO}_{3}$

Turbidity

Volumes purged

Sampling code

Synchronous water leve

ANALYTICAL DATA

Inorganic Constituents $\underline{3098}$

218.5

4.0

160

20.9

0

5.2

$218.5(09 / 17 / 98)$
$10 / 09 / 98$

4Q98

218.4

3.8

220

20.4

0

3.9

$217.3(12 / 18 / 98)$
Unit

ft msl

$\mathrm{pH}$

ision

${ }^{\circ} \mathrm{C}$

$\mathrm{mg} / \mathrm{L}$

well vol

$\mathrm{ft}$ msl

\begin{tabular}{|c|c|c|c|c|c|c|c|c|c|c|c|c|c|}
\hline Constituents & $3 Q 98$ & Mod & Filt. & $\underline{\text { ST }} \underline{\underline{H}}$ & $\underline{\text { DF }}$ & $\underline{L a b}$ & 4Q98 & Mod & $\underline{\text { Filt. }}$ & $\underline{\text { ST }} \underline{\text { H}}$ & $\underline{D F}$ & Lab & $\underline{\text { Unit }}$ \\
\hline Antimony, total recoverable & $<0.20$ & $U / /$ & $<0.20$ & & 1 & GE & & & & & & & $\mu g / L$ \\
\hline Arsenic, total recoverable & $<3.0$ & $\mathrm{U} / /$ & $<3.0$ & & 1 & GE & & & & & & & $\mu g / L$ \\
\hline Barium, total recoverable & 42 & $\|$ & & & 1 & GE & & & & & & & $\mu g / L$ \\
\hline Cadmium, total recoverable & 1.4 & $\|$ & & & 1 & GE & 1.4 & $\|$ & & & 1 & GE & $\mu g \Omega$ \\
\hline Chromium, total recoverable & 1.8 & $J / E$ & NDD & & 1 & GE & & & & & & & $\mu g / \mathrm{h}$ \\
\hline Cobalt, total recoverable & 16 & $\|$ & & - & 1 & GE & & & & & & & $\mu g / L$ \\
\hline Copper, total recoverable & 18 & $\|$ & & & 1 & GE & & & & & & & $\mu g / L$ \\
\hline Cyanide & $<10$ & $\mathrm{U} / I$ & $<10$ & & 1 & GE & & & & & & & $\mu g / L$ \\
\hline Lead, total recoverable & 2.6 & $/ /$ & & & 1 & GE & 2.4 & $/ /$ & & & 1 & GE & $\mu g / L$ \\
\hline Mercury, total recoverable & 2.5 & $\|$ & & $\mathbf{\square}$ & 1 & GE & & & & & & & $\mu g / L$ \\
\hline $\begin{array}{l}\text { Nickel, total recoverable } \\
\text { Nitrate as nitrogen }\end{array}$ & 6.3 & $\|$ & & & 1 & $\overline{\mathrm{GE}}$ & & & & & & & $\mu g^{\prime}$ \\
\hline Nitrate-nitrite as nitrogen & 19,000 & $\mathrm{~J} / \mathrm{V} / 1$ & NDD & & 10 & GE & 22,000 & $\|$ & & $\mathbf{E}$ & 25 & GE & $\mu g / L$ \\
\hline Selenium, total recoverable & $<5.0$ & $\mathrm{U} / 1$ & $<5.0$ & & 1 & GE & & & & & & & $\mu g / L$ \\
\hline Silver, total recoverable & $<1.0$ & $\mathrm{U} / l$ & $<1.0$ & & 1 & GE & & & & & & & $\mu g / L$ \\
\hline Thallium, total recoverable & $<2.5$ & $\mathbf{U} / /$ & $<2.5$ & & 1 & GE & & & & & & & $\mu g / L$ \\
\hline Vanadium, total recoverable & $<2.0$ & $\mathrm{U} / /$ & $<2.0$ & & 1 & GE & & & & & & & $\mu g / L$ \\
\hline Zinc, total recoverable & 45 & $\|$ & & & 1 & GE & & & & & & & $\mu g / L$ \\
\hline \multicolumn{14}{|l|}{ Organic Constituents } \\
\hline Constituents & $\underline{3098}$ & Mod & Filt. & $\underline{\text { ST }} \underline{H}$ & $\underline{\text { DF }}$ & $\underline{\text { Lab }}$ & $\underline{4 Q 98}$ & Mod & Fill. & $\underline{\text { ST }} \underline{H}$ & $\underline{D F}$ & Lab & Unit \\
\hline Benzene & $<1.0$ & $\mathrm{UJ} / \mathrm{O} / 1$ & $<1.0$ & & 1 & GE & & & & & & & $\mu g / L$ \\
\hline Bis(2-ethylhexyl) phthalate & $<9.8$ & $U \mathrm{U} / \mathrm{Q} /$ & $<9.8$ & $\bullet$ & 1 & $\mathrm{GE}$ & . & & & & & & $\mu g / L$ \\
\hline Dichloromethane & $<3.8$ & UJ/OB/1 & $<1.0$ & & 1 & GE & & & & & & & $\mu g / L$ \\
\hline Phenols & $<5.0$ & $\mathrm{U} / \mathrm{I}$ & $<5.0$ & & 1 & GE & & & & & & & $\mu g / L$ \\
\hline Tetrachloroethylene & $<1.0$ & $\mathrm{UJ} / \mathrm{O} / 1$ & $<1.0$ & & 1 & GE & & & & & & & $\mu g / L$ \\
\hline Trichloroethylene & $<1.0$ & UJ/O/1 & $<1.0$ & & 1 & $\mathrm{GE}$ & & & & & & & $\mu g / L$ \\
\hline Trichtorofluoromethane & 3.1 & $\mathrm{~J} / \mathrm{O} / 1$ & NDD & & 1 & GE & & & & & & & \\
\hline
\end{tabular}

Notes:

- = exceeded holding time

- exceeded groundwater protection or monitoring constituent standard (See Appendix A.)

F-Area HWMF

$C-47$

Third and Fourth Quarter 1998 
WELL FSB 89D (cont.)

Radioactive Constituents

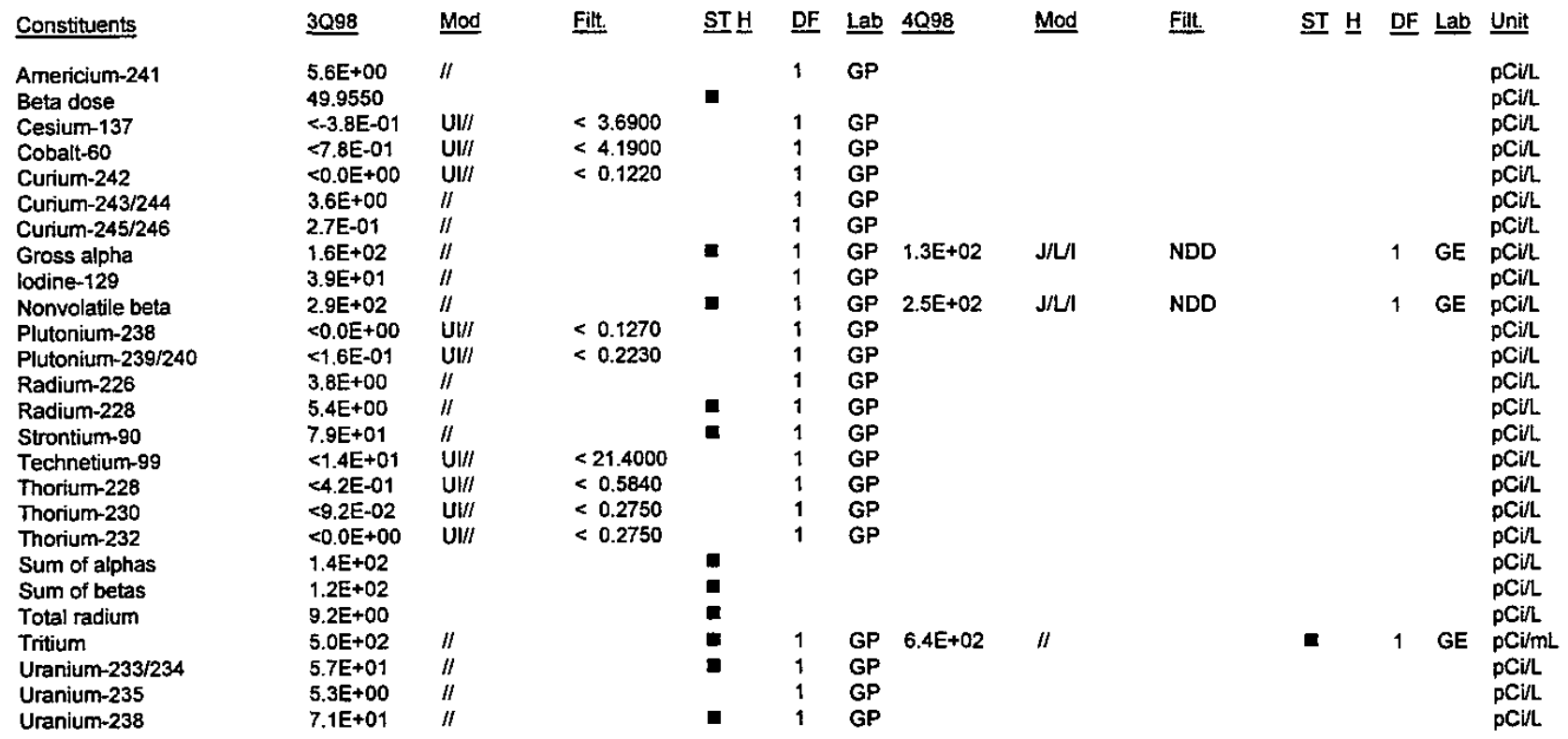

Notes:

- exceeded holding time

w = exceeded groundwater protection or monitoring constituent standard (See Appendix A.) 


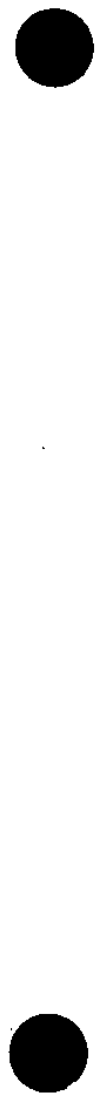

\section{WELL FSB 90C}

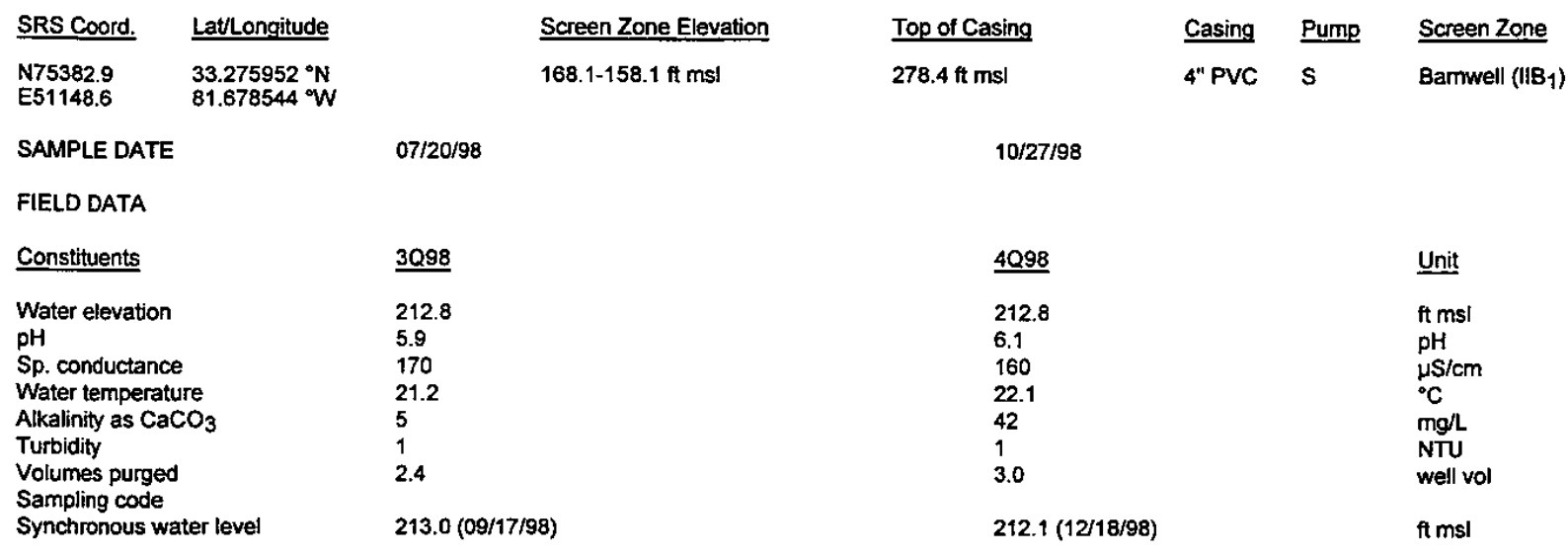

ANALYTICAL DATA

Inorganic Constituemts

\begin{tabular}{|c|c|c|c|c|c|c|c|c|c|c|c|c|c|}
\hline Constituents & 3098 & Mod & Filt. & ST브 & $\underline{\text { DF }}$ & $\underline{\text { Lab }}$ & 4098 & Mod & Filt. & $\underline{\text { ST }} \underline{\mathrm{H}}$ & $\underline{\text { DF }}$ & $\underline{\text { Lab }}$ & Unit \\
\hline Antimony, total recoverable & $<0.20$ & $\mathrm{U} / I$ & $<0.20$ & & 1 & GE & & & & & & & $\mu g / L$ \\
\hline Arsenic, total recoverable & $<3.0$ & $\mathrm{U} / 1$ & $<3.0$ & & 1 & GE & & & & & & & $\mu g / L$ \\
\hline Barium, total recoverable & 40 & $/ 1$ & & & 1 & GE & & & & & & & $\mu g / \mathrm{L}$ \\
\hline Cadmium, total recoverable & $<1.0$ & $\mathrm{U} / /$ & $<1.0$ & & 1 & GE & $<1.0$ & $\mathrm{U} / 1$ & $<1.0$ & & 1 & GE & $\mu g h$ \\
\hline Chromium, total recoverable & 1.3 & $\mathrm{~J} / \mathrm{E} /$ & NDD & & 1 & $\mathrm{GE}$ & & & & & & & $\mu g / L$ \\
\hline Cobalt, total recoverable & 1.1 & $\|$ & & & 1 & GE & & & & & & & $\mu g / L$ \\
\hline Copper, total recoverable & 0.85 & $/ 1$ & & & 1 & GE & & & & & & & $\mu g / L$ \\
\hline Cyanide & $<10$ & $\mathrm{U} / /$ & $<10$ & & 1 & GE & & & & & & & $\mu g / L$ \\
\hline Lead, total recoverable & $<2.0$ & $\mathrm{U} / I$ & $<2.0$ & & 1 & GE & $<2.0$ & $\mathrm{U} / /$ & $<2.0$ & & 1 & GE & $\mu g$ 几 \\
\hline Mercury, total recoverable & $<0.20$ & $\mathrm{U} / I$ & $<0.20$ & & 1 & GE & & & & & & & $\mu g /$ \\
\hline $\begin{array}{l}\text { Nickel, total recoverable } \\
\text { Nitrate as nitrogen }\end{array}$ & 4.9 & $/ l$ & & & 1 & GE & & & & & & & $\mu g / \mathrm{L}$ \\
\hline Nitrate-nitrite as nitrogen & 13,000 & $\|$ & & - & 5 & GE & 12,000 & $\|$ & & 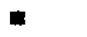 & 25 & GE & $\mu g / L$ \\
\hline Selenium, total recoverable & $<0.95$ & UNI & $<5.0$ & & 1 & GE & & & & & & & $g / 2$ \\
\hline Silver, total recoverable & $<1.0$ & $\mathrm{U} / /$ & $<1.0$ & & 1 & GE & & & & & & & $\mu g / L$ \\
\hline Thallium, total recoverable & $<2.5$ & $\mathrm{U} / /$ & $<2.5$ & & 1 & GE & & & & & & & $\mu g /$ \\
\hline Vanadium, total recoverable & $<2.0$ & $\mathrm{U} / I$ & $<2.0$ & & 1 & GE & & & & & & & $\mu g / L$ \\
\hline Zinc, total recoverable & 21 & $\|$ & & & 1 & GE & & & & & & & $\mu g /$ \\
\hline \multicolumn{14}{|l|}{ Organic Constituents } \\
\hline Constituents & 3098 & Mod & Filt. & ST브 & $\underline{\text { DF }}$ & $\underline{\text { Lab }}$ & 4Q98 & Mod & Filt. & $\underline{\text { ST }} \underline{\mathrm{H}}$ & $\underline{\mathrm{DF}}$ & $\underline{\text { Lab }}$ & $\underline{\text { Unit }}$ \\
\hline Benzene & $<1.0$ & $\mathrm{UJ} / \mathrm{O} / 1$ & $<1.0$ & & 1 & GE & & & & & & & I \\
\hline Bis(2-ethylhexyl) phthalate & $<9.9$ & $\mathrm{U} / /$ & $<9.9$ & & 1 & GE & & & & & & & $\mu g /$ \\
\hline Dichtoromethane & $<2.1$ & UJNO8/1 & $<1.0$ & & 1 & GE & & & & & & & $\lg \Omega$ \\
\hline Phenols & 4.1 & $\mathrm{~J} / \mathrm{E} /$ & NDD & & 1 & GE & & & & & & & $\mu g / L$ \\
\hline Tetrachloroethylene & $<1,1$ & $\mathrm{UJ} / \mathrm{O} / 1$ & $<1.0$ & & 1 & $\mathrm{GE}$ & & & & & & & $\mu g /$ \\
\hline Trichloroethylene & $<1.1$ & $\mathrm{UJ} / \mathrm{O} / 1$ & $<1.0$ & & 1 & GE & & & & & & & $\mu g / L$ \\
\hline Trichlorofluoromethane & $<1.0$ & UJ/O/1 & $<1.0$ & & 1 & GE & & & & & & & $g / L$ \\
\hline
\end{tabular}

Notes:

- exceeded holding time

- = exceeded groundwater protection or monitoring constituent standard (See Appendix A.) 
WELL FSB 90C (cont.)

Radloactive Constituents

\begin{tabular}{|c|c|c|c|c|c|c|c|c|c|c|c|c|c|}
\hline Constituents & 3Q98 & Mod & Filt. & $\underline{\text { ST }} \underline{H}$ & DF & $\underline{\text { Lab }}$ & $\underline{4 Q 98}$ & Mod & Filt. & $\underline{\text { ST }} \underline{H}$ & DF & $\underline{\text { Lab }}$ & $\underline{\text { Unit }}$ \\
\hline Americium-241 & $<3.5 \mathrm{E}-02$ & UII/ & $<0.1760$ & & 1 & GP & & & & & & & pCill \\
\hline Beta dose & 4.3125 & & & $\mathbf{\square}$ & & & & & & & & & $\mathrm{pCi} / \mathrm{L}$ \\
\hline Cesium-137 & 1.1E+01 & $\mathbf{R} / 4$ & Rej & & 1 & GP & & & & & & & $\mathrm{pCi} / \mathrm{L}$ \\
\hline Cobalt-60 & $<2.6 \mathrm{E}-01$ & $U: / /$ & $<2.2900$ & & 1 & GP & & & & & & & $\mathrm{pCi} / \mathrm{L}$ \\
\hline Curium-242 & $<-5.1 E-02$ & UII/ & $<0.2310$ & & 1 & GP & & & & & & & $\mathrm{pCi} / \mathrm{L}$ \\
\hline Curium-243/244 & $<3.6 \mathrm{E}-02$ & $\mathrm{U} 1 / / /$ & $<0.1090$ & & 1 & GP & & & & & & & $\mathrm{pCi} L$ \\
\hline Curium-245/246 & $<0.0 E+00$ & $\mathrm{U}: / / /$ & $<0.0616$ & & 1 & GP & & & & & & & $\mathrm{pCi} / \mathrm{L}$ \\
\hline Gross alpha & $4.6 E+00$ & $\|$ & & & 1 & GP & $<1.3 E+00$ & $\mathrm{U} / l$ & $<9.9 E-01$ & & 1 & GE & $\mathrm{pCi} / \mathrm{L}$ \\
\hline lodine-129 & $3.9 E+00$ & $\|$ & & & 1 & GP & & & & & & & $\mathrm{pCi} / \mathrm{L}$ \\
\hline Nonvolatile beta & $1.9 E+01$ & $\|$ & & & 1 & GP & $1.8 E+01$ & $\|$ & & & 1 & GE & $\mathrm{pCi} / \mathrm{L}$ \\
\hline Plutonium-238 & $<0.0 \mathrm{E}+00$ & UIII & $<0.0546$ & & 1 & GP & & & & & & & $\mathrm{pCi} / \mathrm{L}$ \\
\hline Plutonium-239/240 & $<0.0 E+00$ & $\mathrm{UI} / /$ & $<0.0545$ & & 1 & GP & & & & & & & $\mathrm{pCi} / \mathrm{L}$ \\
\hline Radium-226 & $<4.3 E-01$ & $\mathrm{UI} / /$ & $<0.6610$ & & 1 & GP & & & & & & & $\mathrm{pCi} / \mathrm{L}$ \\
\hline Radium-228 & $<8.0 E-01$ & $\mathrm{UI} / /$ & $<0.9550$ & & 1 & GP & & & & & & & $\mathrm{pCi} / \mathrm{L}$ \\
\hline Strontium-90 & $3.3 E+00$ & 11 & & & 1 & GP & & & & & & & pcill \\
\hline Technetium-99 & $<1.7 \mathrm{E}+01$ & $\mathrm{UI} / \mathrm{I}$ & $<21.7000$ & & 1 & GP & & & & & & & pCiAL \\
\hline Thorium-228 & $<1.2 E-01$ & $\mathrm{U} I / /$ & $<0.2020$ & & 1 & GP & & & & & & & $\mathrm{pCi} / \Omega$ \\
\hline Thorium-230 & $<1.5 E-02$ & $\mathrm{UI} / /$ & $<0.1060$ & & 1 & GP & & & & & & & pcin \\
\hline Thorium-232 & $<-9.6 \mathrm{E}-03$ & $\mathrm{UI} / /$ & $<0.1250$ & & 1 & GP & & & & & & & pCill \\
\hline Sum of alphas & 2.3E-01 & & & & & & & & & & & & pCill \\
\hline Sum of betas & $7.2 E+00$ & & & & & & & & & & & & $\mathrm{pCi} / \mathrm{L}$ \\
\hline Tritium & $4.1 E+02$ & /I & & घ & 1 & GP & $3.9 E+02$ & $\|$ & & $\mathbf{\square}$ & 1 & GE & $\mathrm{Ci} / \mathrm{ml}$ \\
\hline Uranium-233/234 & $<2.3 E-02$ & UII/ & $<0.1560$ & & 1 & GP & & & & & & & $\mathrm{Ci} / \mathrm{L}$ \\
\hline Uranium-235 & $<-7.1 E-03$ & $\mathrm{UI} / I$ & $<0.1570$ & & 1 & $G P$ & & & & & & & $\mathrm{Ci} / \mathrm{L}$ \\
\hline Uranium-238 & $2.3 E-01$ & $\|$ & & & 1 & GP & & & & & & & $\mathrm{pCi} / \mathrm{L}$ \\
\hline
\end{tabular}

Notes:

- exceeded holding time

- = exceeded groundwater protection or monitoring constituent standard (See Appendix A.) 
WELL FSB 90D

\begin{tabular}{|c|c|}
\hline is coord. & Lat/Longitude \\
\hline $\begin{array}{l}753 \\
\mathbf{5 1 1}\end{array}$ & 81 \\
\hline
\end{tabular}

SAMPLE DATE

FIELD DATA

Water elevation

$\mathrm{pH}$

Sp. conductance

Water temperature

Alkalinity as $\mathrm{CaCO}_{3}$

Turbidity

Volumes purged

Sampling code

Synchronous water leve
Constituents

\section{Screen Zone Elevation}

225.1-205.1 ft ms

$09 / 24 / 98$

3Q98

217.2

3.4

570

20.6

0

0.25

$\mathrm{XN}$

$217.4(09 / 17 / 98)$
Top of Casing

$278.6 \mathrm{ft} \mathrm{ms}$

$10 / 07 / 98$

$4 Q 98$
217.2
3.5
720
20.8
0
2
0.0
$\times N$
$216.1(12 / 18 / 98)$

ANALYTICAL DATA

Inorganic Constituents

Mod

Filt.

ST

DF Lab 4Q98

$\underline{\operatorname{Mod}}$

Antimony, total recoverable

Arsenic, total recoverable

Barium, total recoverable

$<0.073$

UN/

Chromium, total recoverable 12

Cobalt, total recoverable

Copper, total recoverable

Cyanide

Lead, total recoverable

Mercury, total recoverable

Nickel, total recoverable

Nitrate as nitrogen

Nitrate-nitrite as nitrogen $\quad 53,000$

Selenium, total recoverable $<100$

Silver, total recoverable $\quad<0.069$

Thallium, total recoverable $<2.5$

Vanadium, total recoverable $<2.0$

Zinc, total recoverable

$<5$

Organic Constituents

Constituents

Benzene

Bis(2-ethylhexyl) phthalate

Dichloromethane

Phenols

Tetrachloroethylene

Trichloroethylene

Trichlorofluoromethane

3098
$<1.0$
$<10$
$<9.5$
$<5.0$
$<1.0$
$<1.0$
$<1.0$

0.20

$\mathrm{U} / 1$

II

$\begin{array}{ll}\text { Mod } & \text { Filt. } \\ \text { U/I } & <1.0 \\ \text { U/I } & <10 \\ \text { UN8/ } & <1.0 \\ U / I & <5.0 \\ U / I & <1.0 \\ U \prime \prime & <1.0 \\ U / I & <1.0\end{array}$

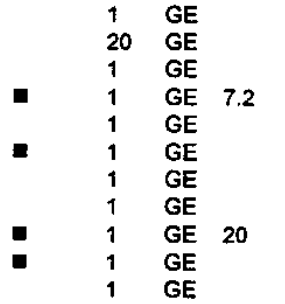

อ

$\mathrm{U} / / \quad<100$

$\mathrm{UN} /$

$\mathrm{U} / 1$

$<2.5$

$<2.0$

Filt.

$<1.0$

5.0

$<1.0$

$<1.0$ $\underline{\text { ST}} \underline{H}$

$\begin{array}{ll}\text { DF } & \text { Lab } \\ 1 & \text { GE } \\ 1 & \text { GE } \\ 1 & \text { GE } \\ 1 & \text { GE } \\ 1 & \text { GE } \\ 1 & \text { GE } \\ 1 & \text { GE }\end{array}$

$\begin{array}{lll}\text { Casing } & \text { Pump } & \frac{\text { Screen Zone }}{4^{n} \text { PVC }} \\ V & \text { Water Table (IIB2) }\end{array}$

$\underline{\text { Unit }}$

ft msl

pH

$\mu \mathrm{S} / \mathrm{cm}$

${ }^{\circ} \mathrm{C}$

$\mathrm{mg} / \mathrm{L}$

well vol

ft msl 
WELL FSB 90D (cont.)

Radioactive Constituents

\section{Constituents}

Americium-241

Beta dose

Cesium-137

Cobalt-60

Curium-242

Curium-243/244

Curium-245/246

Gross alpha

lodine-129

Nonvolatile beta

Plutonium-238

Plutonium-239/240

Radium-226

Radium-228

Strontium-90

Technetium-99

Thorium-228

Thorium-230

Thorium-232

Sum of aiphas

Sum of betas

Total radium

Tritium

Uranium-233/234

Uranium-235

Uranium-238

\begin{tabular}{|c|c|c|c|c|c|c|c|}
\hline 3Q98 & Mod & Filt. & ST브 & $\underline{D F}$ & $\underline{\text { Lab }}$ & 4098 & Mod \\
\hline $\begin{array}{l}6.0 \mathrm{E}+00 \\
171.3300\end{array}$ & /I & & $\mathbf{m}$ & 1 & GP & & \\
\hline $1.4 \mathrm{E}+01$ & $R / 4$ I & Rej & & 1 & GP & & \\
\hline$<1.4 E+00$ & $\mathrm{U!} / /$ & $<3.2100$ & & 1 & GP & & \\
\hline$<-2.3 E-03$ & $\mathrm{UV} / /$ & $<0.2330$ & & 1 & GP & & \\
\hline $2.6 E+\infty 0$ & /l & & & 1 & GP & & \\
\hline 4.6E-01 & /I & & & 1 & GP & & \\
\hline $3.1 E+02$ & $\mathrm{~J} / / / 1$ & NDD & & 1 & GP & $5.8 E+02$ & /I \\
\hline $1.2 \mathrm{E}+02$ & II & & $\boldsymbol{E}$ & 1 & GP & & \\
\hline $9.1 \mathrm{E}+02$ & $\mathrm{~J} / \mathrm{IV} / 1$ & NDD & & 1 & GP & $1.3 E+03$ & $\|$ \\
\hline$<5.4 E-01$ & $\mathrm{U} N /$ & $<0.1650$ & & 1 & GP & & \\
\hline$<6.5 \mathrm{E}-02$ & UIJ/C/ & & & 1 & $G P$ & & \\
\hline $1.2 E+01$ & II & & $\boldsymbol{\square}$ & 1 & GP & & \\
\hline $3.3 E+00$ & $\mathrm{~J} / / / 2$ & NDD & & 1 & GP & & \\
\hline 4.1E+02 & "l & & $\boldsymbol{D}$ & 1 & $G P$ & & \\
\hline $7.2 E+01$ & "l & & 口 & 1 & $G P$ & & \\
\hline$<6.4 E-01$ & $\mathrm{UI} / /$ & $<0.8140$ & & 1 & GP & & \\
\hline$<4.2 \mathrm{E}-02$ & $v \mathrm{u} / \mathrm{I}$ & $<0.3700$ & & 1 & GP & & \\
\hline$<-5.5 E-02$ & UII/ & $<0.3990$ & & 1 & $G P$ & & \\
\hline $3.3 E+02$ & & & - & & & & \\
\hline $\begin{array}{l}6.0 E+02 \\
1.5 E+01\end{array}$ & & & 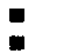 & & & & \\
\hline $1.8 E+03$ & "I & & 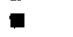 & 1 & GP & $3.3 E+03$ & /I \\
\hline $1.4 E+02$ & II & & a & 1 & GP & & \\
\hline 1.1E+01 & 11 & & & 1 & GP & & \\
\hline $1.8 E+02$ & 11 & & 口 & 1 & GP & & \\
\hline
\end{tabular}

Filt.

ST 브 DF Lab Unit

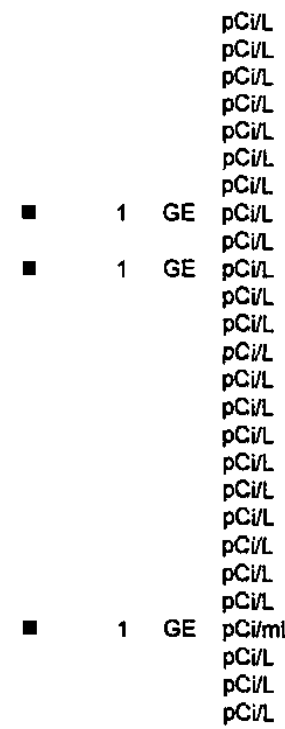

Notes: 
WELL FSB 91C

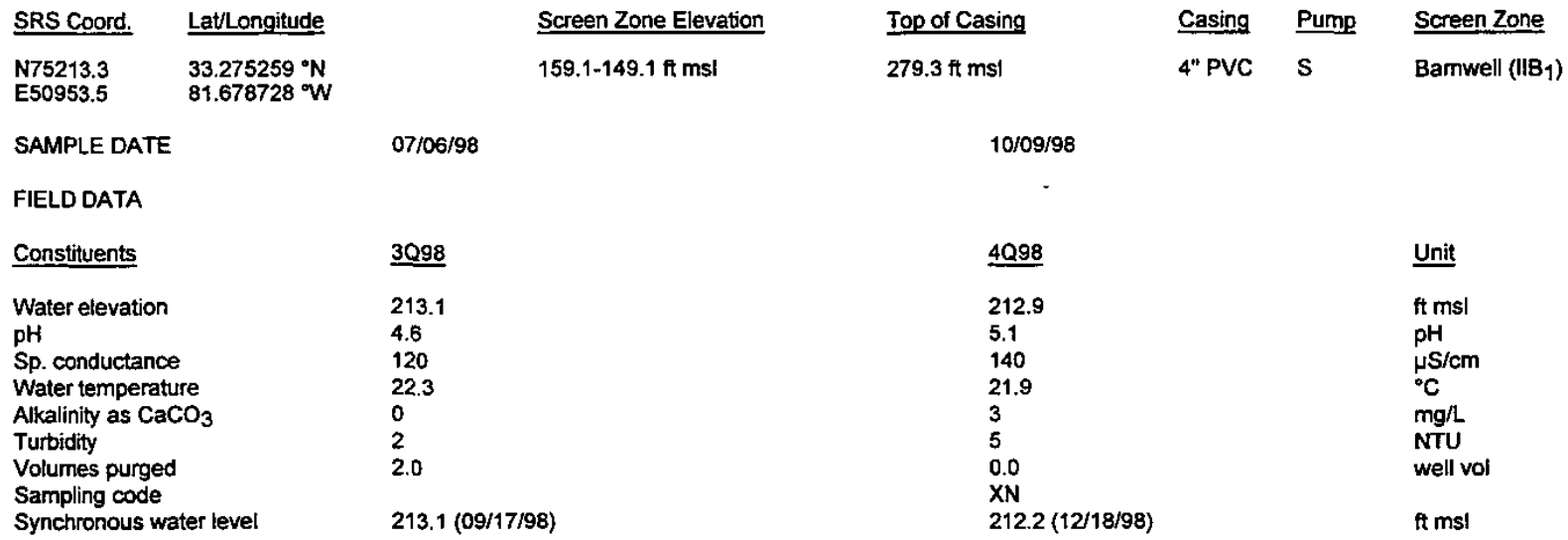

ANALYTICAL DATA

Inorganic Constituents

\begin{tabular}{|c|c|c|c|c|c|c|c|c|c|c|c|c|c|}
\hline Constituents & $\underline{3 Q 98}$ & Mod & Filt. & ST브 & $\underline{\text { DF }}$ & LLab & $\underline{4 Q 98}$ & Mod & Filt. & $\underline{\text { ST }} \underline{H}$ & $\underline{\text { DF }}$ & $\underline{\text { Lab }}$ & Unit \\
\hline Antimony, total recoverable & $<1.0$ & $\mathrm{U} / /$ & $<1.0$ & & 5 & GE & & & & & & & $\mu g / L$ \\
\hline Arsenic, total recoverable & $<15$ & $\mathrm{U} / /$ & $<15.0$ & & 5 & GE & & & & & & & $\mu g / L$ \\
\hline Barium, total recoverable & 52 & $\|$ & & & 5 & GE & & & & & & & $\mu g /$ \\
\hline Cadmium, total recoverable & 2.1 & $\mathrm{~J} / \mathrm{E} J$ & NDD & & 5 & GE & 1.7 & /I & & & 1 & GE & $\mu g /$ \\
\hline Chromium, total recoverable & $<15$ & $\mathrm{U} / /$ & $<15.0$ & & 5 & GE & & & & & & & $\mu g h$ \\
\hline Cobalt, total recoverable & 7.3 & $\|$ & & घ & 5 & GE & & & & & & & $\mu g / L$ \\
\hline Copper, total recoverable & 8.9 & $N /$ & & & 5 & GE & & & & & & & $\mu g / L$ \\
\hline Cyanide & $<10$ & $\mathrm{U} / /$ & $<10$ & & 1 & $\mathrm{GE}$ & & & & & & & $\mu \mathrm{g} / \mathrm{L}$ \\
\hline Lead, total recoverable & 3.2 & J/E $/$ & NDD & & 5 & $\mathrm{GE}$ & 1.8 & $\mathrm{~J} / / /$ & NDD & & 1 & GE & $\mu g / L$ \\
\hline Mercury, total recoverable & $<0.20$ & UJ/1/1 & $<0.20$ & 1 & GE & & & & & & & & $\mu g / L$ \\
\hline $\begin{array}{l}\text { Nickel, total recoverable } \\
\text { Nitrate as nitrogen }\end{array}$ & 2.8 & $/ /$ & & & 5 & GE & & & & & & & $\mu g / L$ \\
\hline Nitrate-nitrite as nitrogen & 13,000 & $N I$ & & = & 5 & GE & 13,000 & /l & & ш & 5 & $\mathrm{GE}$ & $\mu g / L$ \\
\hline Selenium, total recoverable & $<25$ & $\mathrm{U} / I$ & $<25.0$ & & 5 & GE & & & & & & & $\mu g / L$ \\
\hline Silver, total recoverable & 1.7 & $\mathrm{~J} / \mathrm{E} /$ & NDD & & 5 & GE & & & & & & & $\mu g / L$ \\
\hline Thallium, total recoverable & 0.72 & $\mathrm{~J} / \mathrm{E} J$ & NDD & & 5 & GE & & & & & & & $\mu g / L$ \\
\hline Vanadium, total recoverable & $<10$ & $\mathrm{U} / I$ & $<10$ & & 5 & GE & & & & & & & $\mu g / \mathrm{c}$ \\
\hline Zinc, total recoverable & 18 & J/EI & NDD & & 5 & GE & & & & & & & $\mu g / L$ \\
\hline \multicolumn{14}{|l|}{ Organic Constituents } \\
\hline Constituents & 3098 & Mod & Filt. & ST브 & DF & Lab & 4Q98 & Mod & Filt. & $\underline{\mathrm{ST}} \underline{\mathrm{H}}$ & $\underline{\text { DF }}$ & $\underline{L a b}$ & $\underline{\text { Unit }}$ \\
\hline Benzene & $<1.0$ & $\mathrm{UJ} / \mathrm{O} / 1$ & $<1.0$ & & 1 & GE & & & & & & & $\mu g / L$ \\
\hline Bis(2-ethylhexyl) phthalate & $<9.8$ & $\mathrm{U} / /$ & $<9.8$ & & 1 & GE & & & & & & & $\mu \mathrm{g} / \mathrm{L}$ \\
\hline Dichloromethane & $<2.3$ & UJNO8/1 & $<1.0$ & & 1 & $\overline{G E}$ & & & & & & & $\mu g / L$ \\
\hline Phenols & $<5.0$ & $\mathrm{U} / /$ & $<5.0$ & & 1 & $\overline{G E}$ & & & & & & & $\mu g / L$ \\
\hline Tetrachloroethylene & $<1.0$ & $\mathrm{UJ} / \mathrm{O} / 1$ & $<1.0$ & & 1 & $\overline{G E}$ & & & & & & & $\mu g /$ \\
\hline Trichloroethylene & 1.8 & $\|$ & & & 1 & GE & & & & & & & $\mu g / L$ \\
\hline Trichlorofluoromethane & 2.3 & $\mathrm{~J} / \mathrm{O} / 1$ & NDD & & 1 & GE & & & & & & & $g / L$ \\
\hline
\end{tabular}

Notes:

- exceeded holding time

- exceeded groundwater protection or monitoring constituent standard (See Appendix A.) 
WELL FSB 91C (cont.)

Radioactive Constituents

\begin{tabular}{|c|c|c|c|c|c|c|c|c|c|c|c|c|c|}
\hline Constituents & 3Q98 & Mod & Filt. & ST브 & $\underline{D F}$ & Lab & 4Q98 & Mod & Filt. & $\underline{\text { ST }} \underline{H}$ & $\underline{D F}$ & Lab & Unit \\
\hline Americium-241 & $6.5 E+00$ & $/ /$ & & & 1 & GP & & & & & & & pCill \\
\hline Beta dose & 15.6200 & & & $\mathbf{0}$ & & & & & & & & & $\mathrm{pCi}$ \\
\hline Cesium-137 & $<-7.6 E-01$ & $\mathrm{UV} / /$ & $<4.33$ & & 1 & GP & & & & & & & pCil \\
\hline Cobalt-60 & $<3.1 \mathrm{E}-01$ & $\mathrm{U}: / /$ & $<4.96$ & & 1 & GP & & & & & & & pCin \\
\hline Curium-242 & $<-6.8 E-03$ & $\mathrm{U} / \prime$ & $<0.133$ & & 1 & $\mathrm{GP}$ & & & & & & & pCir \\
\hline Curium-243/244 & 2.1E+01 & $/ 1$ & & घ & 1 & GP & & & & & & & $\mathrm{pCi} / \mathrm{L}$ \\
\hline Curium-245/246 & $<3.3 E-01$ & UN/ & $<0.132$ & & 1 & GP & & & & & & & pCill \\
\hline $\begin{array}{l}\text { Gross alpha } \\
\text { lodine-129 }\end{array}$ & $\begin{array}{l}2.0 E+01 \\
1.4 E+01\end{array}$ & $/ 1$ & & a & $\begin{array}{l}1 \\
1\end{array}$ & $\begin{array}{l}\text { GP } \\
\text { GP }\end{array}$ & $1.5 \mathrm{E}+01$ & $J / L /$ & NDD & & 1 & GE & pCi/l \\
\hline Nonvolatile beta & $2.1 E+02$ & $\|$ & & - & 1 & GP & $2.1 E+02$ & $\mathrm{~J} / \mathrm{L} /$ & NDD & & 1 & GE & $\mathrm{pCi} / \mathrm{L}$ \\
\hline Plutonium-238 & $<-2.9 E-02$ & $\mathrm{U} \mathrm{L} / \mathrm{I}$ & $<0.295$ & & 1 & GP & & & & & & & pCil \\
\hline Plutonium-239/240 & $<-3.8 E-02$ & $\mathrm{U} ! / l$ & $<0.188$ & & 1 & GP & & & & & & & pCill \\
\hline Radium-226 & $1.0 \mathrm{E}+01$ & $/ /$ & & $\boldsymbol{\square}$ & 1 & GP & & & & & & & $\mathrm{pCi} / \mathrm{L}$ \\
\hline Radium-228 & 8.1E+00 & $\|$ & & $\mathbf{\square}$ & 1 & GP & & & & & & & $\mathrm{pCil}$ \\
\hline Strontium-90 & $8.7 E+01$ & $\|$ & & - & 1 & GP & & & & & & & pCil \\
\hline Technetium-99 & $<1.8 E+01$ & $\mathrm{Uu} / /$ & $<20.6000$ & & 1 & GP & & & & & & & pCilL \\
\hline Thorium-228 & $<1.2 E-01$ & Uul/ & $<0.3000$ & & 1 & GP & & & & & & & pCilL \\
\hline Thorium-230 & $<-9.3 E-03$ & $\mathrm{UI} / /$ & $<0.2050$ & & 1 & GP & & & & & & & $\mathrm{pCi} / \mathrm{L}$ \\
\hline Sum of alphas & $3.0 \mathrm{E}+01$ & & & $\mathbf{0}$ & & & & & & & & & pCill \\
\hline Sum of betas & $1.1 E+02$ & & & n & & & & & & & & & pCill \\
\hline Total radium & $1.9 E+01$ & & & 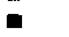 & & & & & & & & & pCill \\
\hline Tritium & $2.2 E+02$ & $n$ & & $\mathbf{E}$ & 1 & GP & 2.1E+02 & / & & 曰 & 1 & GE & $\mathrm{pCi} / \mathrm{mL}$ \\
\hline Uranium-233/234 & $1.2 E+\infty 0$ & $\|$ & & & 1 & GP & & & & & & & pCir \\
\hline Uranium-235 & $1.4 E-01$ & $\|$ & & & 1 & GP & & & & & & & $\mathrm{pCi} / \mathrm{L}$ \\
\hline Uranium-238 & 8.3E-01 & $\|$ & & & 1 & GP & & & & & & & pCill \\
\hline
\end{tabular}

Notes:

- exceeded holding time

$\omega$ = exceeded groundwater protection or monitoring constituent standard (See Appendix A.)

F-Area HWMF

C-54 
WELL FSB 91D

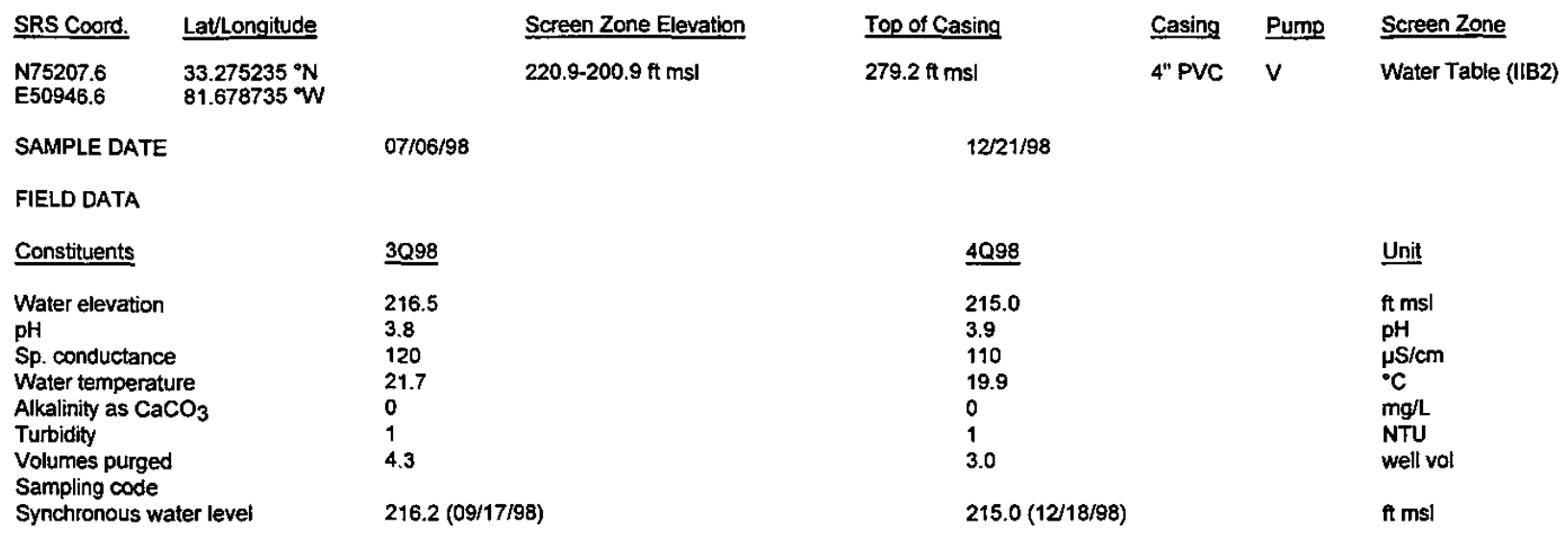

ANALYTICAL DATA

Inorganic Constituents

\begin{tabular}{|c|c|c|c|c|c|c|c|c|c|c|c|c|c|}
\hline Constituents & 3098 & Mod & Filt. & $\underline{\text { ST브 }}$ & DF & $\underline{\text { Lab }}$ & 4Q98 & Mod & Filt. & $\underline{\text { ST }} \underline{H}$ & DF & $\underline{\text { Lab }}$ & $\underline{\text { Unit }}$ \\
\hline Antimony, total recoverable & $<0.20$ & $\mathrm{U} / /$ & $<0.20$ & & 1 & GE & & & & & & & $\mu g / L$ \\
\hline Arsenic, total recoverable & $<3.0$ & $\mathrm{U} / /$ & $<3.0$ & & 1 & GE & & & & & & & $\mu g / L$ \\
\hline Barium, total recoverable & 38 & $\|$ & & & 1 & GE & & & & & & & $\mu g \Lambda$ \\
\hline Cadmium, total recoverable & 0.95 & $J / E /$ & NDD & & 1 & GE & 0.48 & $\mathrm{~J} / N$ & NDD & & 1 & $\mathrm{GE}$ & $\mu g / L$ \\
\hline Chromium, total recoverable & 0.76 & $\mathrm{~J} / \mathrm{E} J$ & NDD & & 1 & GE & & & & & & & $\mu g / L$ \\
\hline Cobalt, total recoverable & 0.96 & /I & & & 1 & $\overline{G E}$ & & & & & & & $\mu g / L$ \\
\hline Copper, total recoverable & 5.8 & $N /$ & & & 1 & GE & & & & & & & $\mu g / L$ \\
\hline Cyanide & $<10$ & $\mathrm{U} / /$ & $<10$ & & 1 & $\overline{G E}$ & & & & & & & $\mu g / \mathrm{L}$ \\
\hline Lead, total recoverable & 1.5 & $\mathrm{~J} / \mathrm{E} /$ & NDD & & 1 & GE & 1,1 & $\mathrm{~J} / \mathrm{H}$ & NDD & & 1 & GE & $\mu g / L$ \\
\hline Mercury, total recoverable & $<0.12$ & UJ/IV/1 & $<0.20$ & & 1 & $\overline{G E}$ & & & & & & & $\mu g /$ \\
\hline $\begin{array}{l}\text { Nickel, total recoverable } \\
\text { Nitrate as nitrogen }\end{array}$ & 2.0 & $/ /$ & & & 1 & GE & & & & & & & $\mu g^{\prime} / \mathrm{L}$ \\
\hline Nitrate-nitrite as nitrogen & 11,000 & $N I$ & & $\mathbf{E}$ & 10 & GE & 13,000 & $/ I$ & & $\mathbf{E}$ & 15 & GE & $\mu g / L$ \\
\hline Selenium, total recoverable & $<5.0$ & $\mathrm{u} / 1$ & $<5.0$ & & 1 & GE & & & & & & & $\mu g / h$ \\
\hline Silver, total recoverable & $<1.0$ & $\mathrm{U} / \prime$ & $<1.0$ & & 1 & $\overline{\mathrm{GE}}$ & & & & & & & $\mu g / L$ \\
\hline Thallium, total recoverable & $<2.5$ & $\mathrm{U} / 1$ & $<2.5$ & & 1 & GE & & & & & & & $\mu g^{\prime}$ \\
\hline Vanadium, total recoverable & $<2.0$ & UII & $<2.0$ & & 1 & $\overrightarrow{G E}$ & & & & & & & $\mu g / L$ \\
\hline Zinc, total recoverable & 8.0 & $\|$ & & & 1 & $\mathrm{GE}$ & & & & & & & $g / L$ \\
\hline
\end{tabular}

Organic Constituemts

\begin{tabular}{|c|c|c|c|c|c|c|c|c|c|c|c|}
\hline Constituents & 3098 & Mod & Filt & $\underline{\text { ST }} \underline{H}$ & DF & $\underline{\text { Lab }}$ & $\underline{4 Q 98}$ & Mod & Filt. & $\underline{\text { ST }} \underline{H}$ DF Lab & Unit \\
\hline Benzene & $<1.0$ & $\mathrm{UJ} / \mathrm{O} / 1$ & $<1.0$ & & 1 & GE & & & & & $\mu g / L$ \\
\hline Bis(2-ethylhexyl) phthalate & $<9.8$ & $U / /$ & $<9.8$ & & 1 & GE & & & & & $\mu g h$ \\
\hline Dichloromethane & $<2.5$ & UJNO8/1 & $<1.0$ & & 1 & GE & & & & & $\mu g^{\prime} L$ \\
\hline Phenols & $<5.0$ & $\mathrm{U} / 1$ & $<5.0$ & & 1 & GE & & & & & $\mu g / L$ \\
\hline Tetrachloroethylene & $<1.0$ & UJ/O/1 & $<1.0$ & & 1 & GE & & & & & $\mu \mathrm{g} / \mathrm{L}$ \\
\hline Trichloroethylene & $<1.0$ & $\mathrm{UJ} / \mathrm{O} / 1$ & $<1.0$ & & 1 & GE & & & & & $\mu g / \mathrm{L}$ \\
\hline Trichlorofluoromethane & $<1.0$ & UJ/O/1 & $<1.0$ & & 1 & GE & & & & & $g / L$ \\
\hline
\end{tabular}

Notes:

- = exceeded holding time

- exceeded groundwater protection or monitoring constituent standard (See Appendix A.) 
WELL FSB 91D (cont.)

Radioactive Constituents

\begin{tabular}{|c|c|c|c|c|c|c|c|c|c|c|c|c|c|}
\hline Constituents & $3 Q 98$ & Mod & Filt. & ST보 & $\underline{\text { DF }}$ & Lab & 4Q98 & Mod & Filt. & $\underline{\text { ST }} \underline{H}$ & DF & Lab & Unit \\
\hline Americium-241 & $1.2 E+\infty$ & $\|$ & & & 1 & GP & & & & & & & pCill \\
\hline Beta dose & 16.1250 & & & घ & & & & & & & & & $\mathrm{pCi} / \mathrm{L}$ \\
\hline Cesium-137 & $<1.0 \mathrm{E}+00$ & $\mathrm{U} I / !$ & $<5.0$ & & 1 & GP & & & & & & & pCill \\
\hline Cobalt- 60 & $<6.4 E-01$ & $\mathrm{U} / / /$ & $<5.4000$ & & 1 & GP & & & & & & & pCirl \\
\hline Curium-242 & $<-5.3 \mathrm{E}-02$ & UIII & $<0.1420$ & & 1 & GP & & & & & & & pCill \\
\hline Curium-243/244 & 7.6E-01 & ll & & & 1 & GP & & & & & & & pCill \\
\hline Curium-245/246 & $<5.0 \mathrm{E}-02$ & UNI & $<0.0248$ & & 1 & GP & & & & & & & $\mathrm{pCi} / \mathrm{L}$ \\
\hline Gross alpha & $8.2 E+01$ & $/ 1$ & & घ & 1 & GP & $1.3 E+02$ & $\|$ & & $\mathbf{n}$ & 1 & GE & $\mathrm{pCi} / \mathrm{L}$ \\
\hline lodine-129 & 1.3E+01 & $/ 1$ & & & 1 & GP & & & & & & & pCill \\
\hline Nonvolatile beta & $7.3 E+01$ & $\|$ & & $\mathbf{n}$ & 1 & GP & $8.2 E+01$ & $/ /$ & & घ & 1 & GE & $\mathrm{pCil}$ \\
\hline Plutonium-238 & $<2.7 \mathrm{E}-02$ & $\mathrm{U} ! / /$ & $<0.2720$ & & 1 & $\mathrm{GP}$ & & & & & & & pCill \\
\hline Plutonium-239/240 & $<-7.6 \mathrm{E}-03$ & $\mathrm{UI} / /$ & $<0.1490$ & & 1 & GP & & & & & & & pCill \\
\hline Radium-226 & $2.7 E+00$ & $\|$ & & & 1 & GP & & & & & & & pCin \\
\hline Radium-228 & $5.0 E+00$ & $\|$ & & & 1 & GP & & & & & & & $\mathrm{pCi} / \mathrm{L}$ \\
\hline Strontium-90 & 1.7E+01 & $\|$ & & - & 1 & GP & & & & & & & $\mathrm{pCi} / \mathrm{L}$ \\
\hline Technetium-99 & $<1.8 \mathrm{E}+01$ & $\mathrm{UI} / /$ & $<19.1000$ & & 1 & GP & & & & & & & $\mathrm{pCi} / \mathrm{L}$ \\
\hline Thorium-228 & $<2.0 \mathrm{E}-01$ & UNI & $<0.1470$ & & 1 & GP & & & & & & & $\mathrm{pCi} / \mathrm{L}$ \\
\hline Thorium-230 & $<6.7 E-02$ & $\mathrm{UI} / /$ & $<0.1180$ & & 1 & GP & & & & & & & $\mathrm{pCi} / \mathrm{L}$ \\
\hline Sum of alphas & $7.2 E+01$ & & & $\mathbf{v}$ & & & & & & & & & $\mathrm{pCil}$ \\
\hline Sum of betas & $3.4 E+01$ & & & & & & & & & & & & pCir \\
\hline Total radium & 7.7E+00 & & & घ & & & & & & & & & $\mathrm{pCi} / \mathrm{L}$ \\
\hline Tritium & $1.5 E+02$ & $\|$ & & घ & 1 & GP & $1.3 E+02$ & $/ /$ & & $\mathbf{n}$ & 1 & GE & pCi/mL \\
\hline Uranium-233/234 & 2.7E+01 & $\|$ & & $\mathbf{\square}$ & 1 & GP & & & & & & & pCill \\
\hline Uranium-235 & $2.1 E+00$ & $\|$ & & & 1 & GP & & & & & & & pCil \\
\hline Uranium-238 & 4.1E+01 & $\|$ & & $\mathbf{0}$ & 1 & $\mathrm{GP}$ & & & & & & & $\mathrm{pCi} / \mathrm{L}$ \\
\hline
\end{tabular}

Notes:

- = exceeded holding time

- = exceeded groundwater protection or monitoring constituent standard (See Appendix A.) 
WELLL FSB 92C

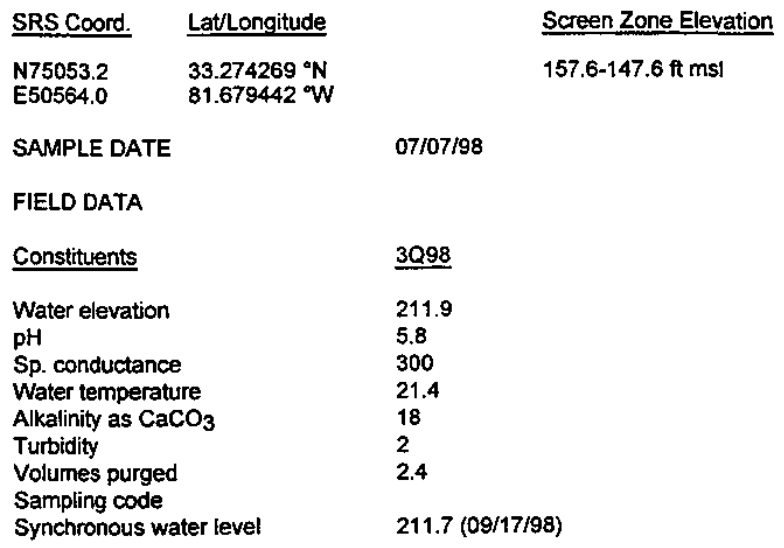

$\begin{array}{llll}\text { Top of Casing } & \text { Casing } & \text { Pump } & \text { Screen Zone } \\ 275.7 \mathrm{ft} \mathrm{msl} & 4^{n} \text { PVC } & \mathrm{V} & \text { Bamwell (IIB1) }\end{array}$

$10 / 09 / 98$

\begin{tabular}{ll}
\hline 4098 & Unit \\
211.6 & $\mathrm{ft} \mathrm{ms!}$ \\
5.1 & $\mathrm{pH}$ \\
290 & $\mu \mathrm{S} / \mathrm{cm}$ \\
21.0 & ${ }^{\circ} \mathrm{C}$ \\
1 & $\mathrm{mg} / \mathrm{L}$ \\
0 & $\mathrm{NTU}$ \\
2.2 & well vol \\
$210.7(12 / 18 / 98)$ & \\
& $\mathrm{ft} \mathrm{msl}$
\end{tabular}

ANALYTICAL DATA

Inorganic Constituents

3Q98

Mod

Filt.

ST브

DF Lab 4098

Mod

Filt.

ST $\underline{\text { H }}$ DF Lab Unit

Antimony, total recoverable $<1$.

Arsenic, total tecoverable $<15$

Barium, total recoverable $\quad 150$

Cadmium, total recoverable 3.9

Chromium, total recoverable $<15$

Cobalt, total recoverable

Copper, total recoverable

Cyanide

$\begin{array}{ll}U / I & <1.0 \\ U / I & <15.0\end{array}$

II

J/EI NDD

UII $<\$ 5.0$

Lead, total recoverable

Mercury, total recoverable $\quad<0.20$

Nickel, total recoverable

Nitrate as nitrogen

Nitrate-nitrite as nitrogen $\quad 33,000$

Selenium, total recoverable $<25$

Silver, total recoverable $<5.0$

Thallium, total recoverable $\quad 0.29$

Vanadium, total recoverable $<10$

Zinc, total recoverable

43

Organic Constituents

Constituents

3Q98

Mod
$\mathrm{U} / \prime$
$\mathrm{U} / \prime$
$\mathrm{U} N 8 /$
$\mathrm{U} / \prime$
$\mathrm{U} / \prime$
$\prime \prime$
$\prime \prime$

Bis(2-ethylhexyl) phthatate

Dichloromethane

Phenols

$<1.0$
$<9.8$

$<0.89$

Tetrachloroethylene

Trichloroethylene

$<5.0$

Trichlorofluoromethane
Filt.

$<1.0$

"II

$\mathrm{U} / \textrm{ }<10$

J/EV/ NDD

$\mathrm{U} / I \quad<0.20$

$\begin{array}{ll}N / & \\ \mathrm{U} / I & <25.0 \\ \mathrm{U} / & <5.0\end{array}$

$\mathrm{J} / \mathrm{E} /$ NDD

Uil $<10$

$<9.8$

$<1.0$

$<5.0$

$<1.0$

ST브

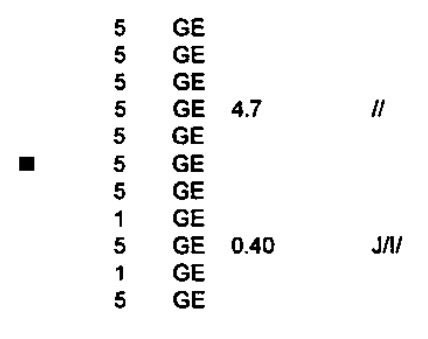

- 25 GE $33,000 \quad / 1$

5 GE

5 GE

5 GE

$\begin{array}{ccc}\text { ST브 } & \text { DF } & \text { Lab } \\ & 1 & \text { GE } \\ 1 & \text { GE } \\ 1 & \text { GE } \\ 1 & \text { GE } \\ 1 & \text { GE } \\ 1 & \text { GE } \\ 1 & \text { GE }\end{array}$
5 GE

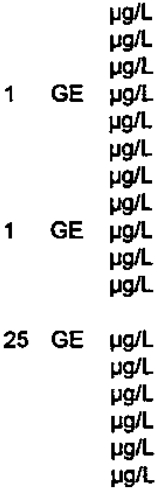

Filt.

ST 보 DF Lab Unit

$\mu g / L$
$\mu g / L$
$\mu g / L$
$\mu g / L$
$\mu g / L$
$\mu g / L$
$\mu g / L$

\section{Notes:}

- = exceeded holding time

= exceeded groundwater protection or monitoring constituent standard (See Appendix A.) 
WELL FSB 92C (cont.)

Radioactive Constituents

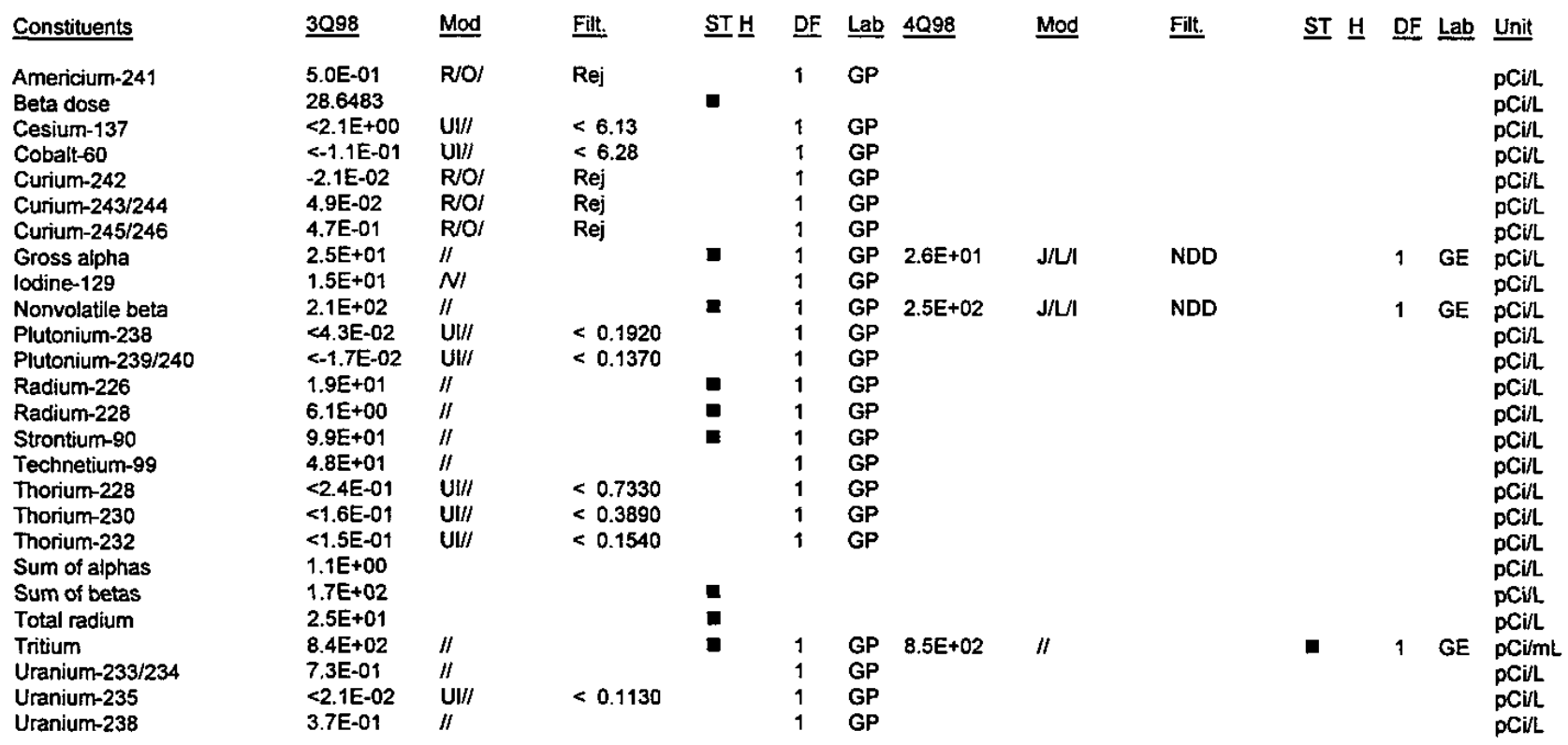

\section{Notes:}

$\checkmark=$ exceeded holding time

- = exceeded groundwater protection or monitoring constituent standard (See Appendix A.) 


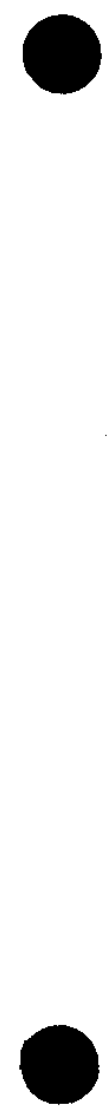

WELL FSB 92D

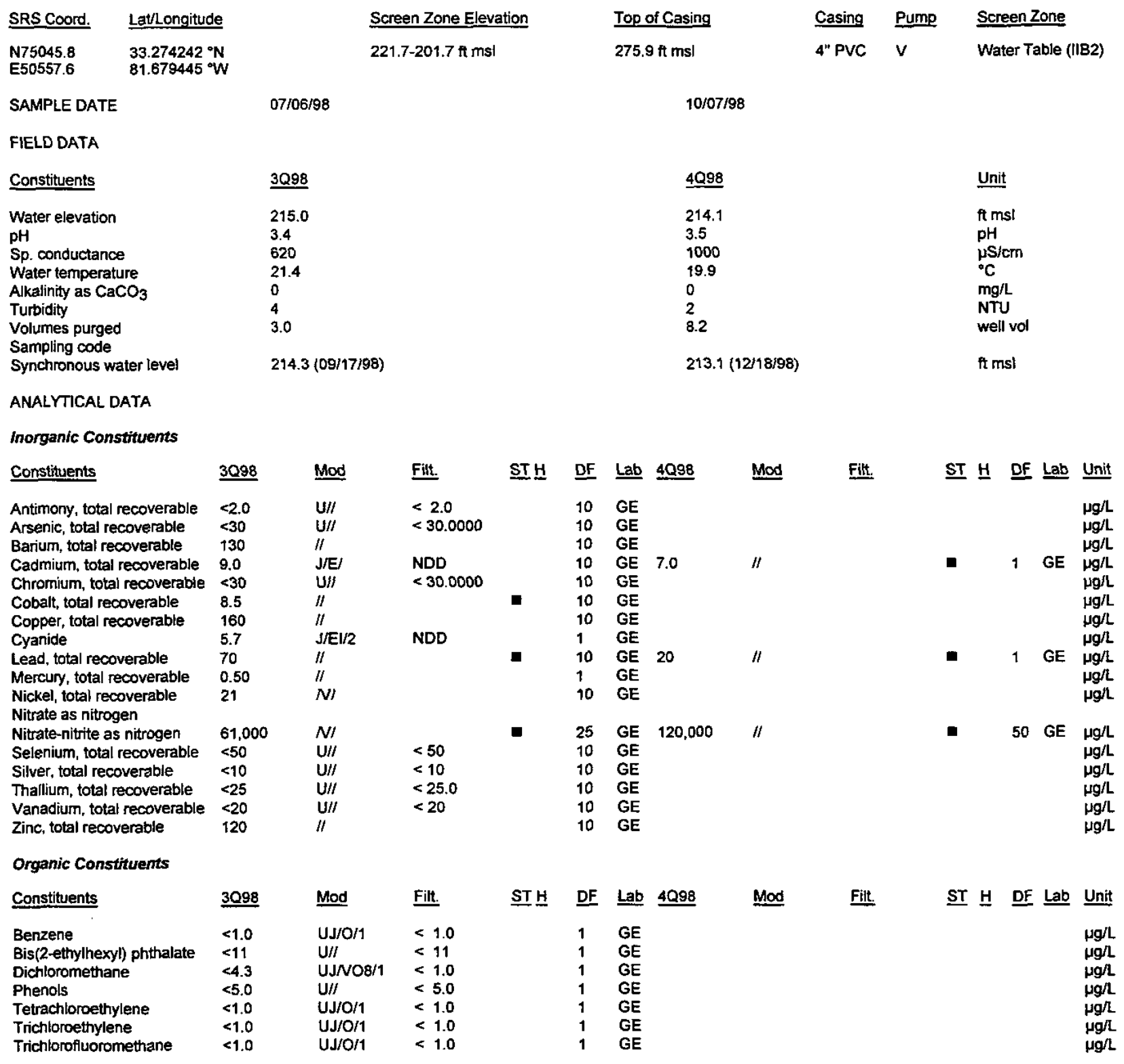

Notes:

- exceeded holding time

= exceeded groundwater protection or monitoring constituent standard (See Appendix A.) 
WELL FSB 92D (cont.)

Radioactive Constituents

\begin{tabular}{|c|c|c|c|c|c|c|c|c|c|c|c|c|c|}
\hline Constituents & 3098 & Mod & Filt. & $\underline{\text { ST}} \underline{\text { H }}$ & $\underline{D F}$ & Lab & 4Q98 & Mod & Filt. & $\underline{\text { ST }} \underline{\text { H }}$ & $\underline{D F}$ & $\underline{\text { Lab }}$ & $\underline{\text { Unit }}$ \\
\hline Americium-241 & $1.6 \mathrm{E}+01$ & $\|$ & & घ & 1 & GP & & & & & & & pCill \\
\hline Beta dose & 47.0422 & & & घ & & & & & & & & & $\mathrm{pCi} / \mathrm{L}$ \\
\hline Cesium-137 & $<-9.0 E-01$ & $\mathrm{UI} / /$ & $<4.1300$ & & 1 & GP & & & & & & & $\mathrm{pCill}$ \\
\hline Cobalt-60 & $<-7.7 E-01$ & UIII & $<4.1600$ & & 1 & GP & & & & & & & $\mathrm{pCi} /$ \\
\hline Curium-242 & $<-3.0 E-02$ & $\mathrm{UI} / /$ & $<0.1950$ & & 1 & GP & & & & & & & $\mathrm{pCi} / \mathrm{L}$ \\
\hline Curium-243/244 & $1.0 \mathrm{E}+01$ & $\|$ & & & 1 & GP & & & & & & & pCíl \\
\hline Curium-245/246 & 1.1E+00 & $\|$ & & & 1 & GP & & & & & & & pCíl \\
\hline Gross alpha & $4.4 \mathrm{E}+02$ & $\mathrm{~J} / / / 1$ & NDD & & 1 & $\mathrm{GP}$ & $6.6 E+02$ & $\|$ & & - & 1 & GE & $\mathrm{pCill}$ \\
\hline lodine-129 & 4.7E+01 & /I & & & 1 & GP & & & & & & & $\mathrm{pCi} / \mathrm{L}$ \\
\hline Nonvolatile beta & $8.3 E+02$ & $\mathrm{~J} / \mathrm{IV} / 1$ & NDD & & 1 & GP & $2.2 E+03$ & $\|$ & & 口 & 1 & GE & pCill \\
\hline Plutonium-238 & $<3.9 E-02$ & $\mathrm{UV} / /$ & $<0.1910$ & & 1 & GP & & & & & & & $\mathrm{pCi} / \mathrm{L}$ \\
\hline Piutonium-239/240 & $<4.0 \mathrm{E}-02$ & $\mathrm{UI} / /$ & $<0.0595$ & & 1 & GP & & & & & & & $\mathrm{pCi} / \mathrm{L}$ \\
\hline Radium-226 & $2.2 E+01$ & /I & & $\mathbf{E}$ & 1 & GP & & & & & & & pCill \\
\hline Radium-228 & $6.5 E+00$ & $\mathrm{~J} / / / 2$ & NDD & & 1 & GP & & & & & & & pCill \\
\hline Strontium-90 & $2.0 E+02$ & $J / U$ & NDD & & 1 & $G P$ & & & & & & & pCuL \\
\hline Technetium-99 & $3.8 E+01$ & $\|$ & & & 1 & GP & & & & & & & pCill \\
\hline Thorium-228 & $<6.0 \mathrm{E}-01$ & UU/ & $<0.7340$ & & 1 & GP & & & & & & & pCill \\
\hline Thorium-230 & $<2.1 E-01$ & $\mathrm{UI} / /$ & $<0.3390$ & & 1 & GP & & & & & & & $\mathrm{pCin}$ \\
\hline Thorium-232 & $<-1.8 \mathrm{E}-02$ & $\mathrm{UI} / /$ & $<0.3080$ & & 1 & GP & & & & & & & $\mathrm{pCi} / \mathrm{L}$ \\
\hline Sum of alphas & $4.7 E+02$ & & & a & & & & & & & & & $\mathrm{pCil}$ \\
\hline Sum of betas & $2.9 E+02$ & & & 0 & & & & & & & & & pCirl \\
\hline Total radium & $2.8 \mathrm{E}+01$ & & & 口 & & & & & & & & & pCill \\
\hline Tritium & $9.6 \mathrm{E}+02$ & /I & & $\mathbf{0}$ & 1 & GP & $3.4 E+03$ & $\|$ & & $\mathbf{n}$ & 1 & GE & pCi/mL \\
\hline Uranium-233/234 & $1.9 E+02$ & $1 /$ & & 口 & 1 & GP & & & & & & & pCill \\
\hline Uranium-235 & $1.4 E+01$ & $\|$ & & & 1 & GP & & & & & & & $\mathrm{pCin}$ \\
\hline Uranium-238 & $2.4 E+02$ & $/ /$ & & $\mathbf{\square}$ & 1 & GP & & & & & & & $\mathrm{pCi} / \mathrm{L}$ \\
\hline
\end{tabular}

Notes:

= exceeded holding time

= exceeded groundwater protection or monitoring constituent standard (See Appendix A.) 
WELL FSB 93C

\begin{tabular}{|c|c|}
\hline SRS Coord. & Lat/Longitude \\
\hline $\begin{array}{l}N 74897.3 \\
\text { E50458.3 }\end{array}$ & $\begin{array}{l}33.273752^{\circ} \mathrm{N} \\
81.679418^{\circ} \mathrm{V}\end{array}$ \\
\hline
\end{tabular}

SAMPLE DATE

FIELD DATA

Constituents

Water elevation

pH

Sp. conductance

Water temperature

Alkalinity as $\mathrm{CaCO}_{3}$

Turbidity

Volumes purged

Sampling code

Synchronous water level

ANALYTICAL DATA

Inorganic Constituents

Constituents

\section{Q98}

Antimony, total recoverable $<0.20$

Arsenic, total recoverable

Barium, total recoverable

Cadmium, total recoverable

Chromium, total recoverable 0.64

Cobalt, total recoverable $\quad 2.4$

Copper, total recoverable $\quad 7.8$

Cyanide

Lead, total recoverable $\quad 3.4$

Mercury, total recoverable $\quad<0.20$

Nickel, total recoverable

Nitrate as nitrogen

Nitrate-nitrite as nitrogen $\quad 33,000$

Selenium, total recoverable $<5.0$

Silver, total recoverable $\quad 0.55$

Thallium, total recoverable $\quad 0.57$

Vanadium, total recoverable $<2.0$

Zinc, total recoverable
3098

211.0

5.1

300

21.4

2.2

$210.6(09 / 17 / 98)$
Screen Zone Elevation

$152.0-142.0 \mathrm{ft} \mathrm{msl}$
Top of Casing

$276.2 \mathrm{ft} \mathrm{msl}$

$10 / 27 / 98$

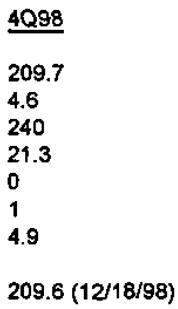

Unit

ft ms!

$\mathrm{pH}$

${ }^{\circ} \mathrm{C} / \mathrm{cm}$

$\mathrm{mg} / \mathrm{L}$

NTU

well vol

ft msl

Organic Constituents

$\begin{array}{llll}\text { Constituents } & 3 Q 98 & \text { Mod } & \text { Filt. } \\ \text { Benzene } & <1.0 & \text { UJ/O/1 } & <1.0 \\ \text { Bis(2-ethylhexyl) phthalate } & <10 & \text { U/I } & <10 \\ \text { Dichloromethane } & <2.2 & \text { UJNO8/1 } & <1.0 \\ \text { Phenols } & 4.5 & \text { J/E } & \text { NDD } \\ \text { Tetrachloroethylene } & <1.0 & \text { UJ/O/1 } & <1.0 \\ \text { Trichloroethylene } & <2.5 & \text { UJ/O/1 } & <1.0 \\ \text { Trichlorofluoromethane } & <1.0 & \text { UJ/O/1 } & <1.0\end{array}$

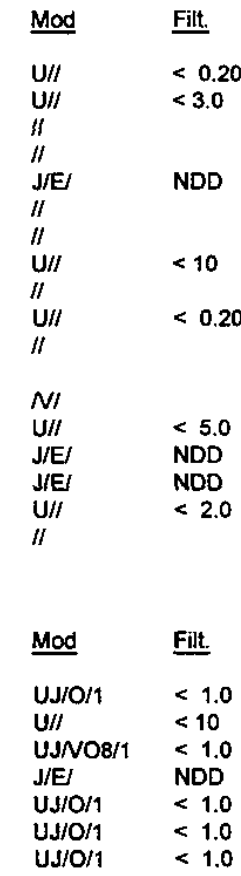

STH

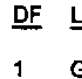

409

GE

GE

1 GE

1 GE

$\begin{array}{llll}\text { GE } & 0.79 & \text { J/ } / & \text { NDD }\end{array}$

1 GE

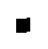

$\begin{array}{ll}25 & \text { GE } \\ 1 & \text { GE } \\ 1 & \text { GE } \\ 1 & \text { GE } \\ 1 & \text { GE } \\ 1 & \text { GE }\end{array}$

31,000

NDD

NOD

$<1.0$
STH

$\begin{array}{ll}\text { DF } & \text { Lab } \\ 1 & \text { GE } \\ 1 & \text { GE } \\ 1 & \text { GE } \\ 1 & \text { GE } \\ 1 & \text { GE } \\ 1 & \text { GE } \\ 1 & \text { GE }\end{array}$

Mod

II

II




\section{WELL FSB 93C (cont.)}

Radioactive Constituents

\begin{tabular}{|c|c|c|c|c|c|c|c|c|c|c|c|c|c|}
\hline Constituents & $\underline{3 Q 98}$ & Mod & Filt. & $\underline{\mathrm{ST}} \underline{\mathrm{H}}$ & $\underline{D F}$ & $\underline{\mathrm{Lab}}$ & 4098 & $\underline{\operatorname{Mod}}$ & Filt. & $\underline{\text { ST }} \underline{H}$ & $\underline{D F}$ & Lab & Unit \\
\hline Americium-241 & $<-1.6 E-02$ & $\mathrm{UV} / /$ & $<0.1800$ & & 1 & GP & & & & & & & pCill \\
\hline Beta dose & 2.8717 & & & - & & & & & & & & & pCirl \\
\hline Cesium-137 & $<-7.5 E-01$ & UIII & $<3.7100$ & & 1 & GP & & & & & & & $\mathrm{pCi} / \mathrm{L}$ \\
\hline Cobalt-60 & $<8.0 E-01$ & $\mathrm{UI} / I$ & $<4.0800$ & & 1 & GP & & & & & & & pCi/L \\
\hline Curium-242 & $<-5.2 E-02$ & UIII & $<0.2360$ & & 1 & GP & & & & & & & $\mathrm{pCi} / \mathrm{L}$ \\
\hline Curium-243/244 & $<-1.9 E-02$ & UII/ & $<0.1990$ & & 1 & $\mathrm{GP}$ & & & & & & & $\mathrm{pCi} / \mathrm{L}$ \\
\hline Curium-245/246 & $<0.0 E+00$ & UIII & $<0.0630$ & & 1 & GP & & & & & & & pCirl \\
\hline Gross alpha & $3.7 \mathrm{E}+00$ & $\|$ & & & 1 & GP & $<3.0 \mathrm{E}+00$ & $\mathrm{U} / I$ & $<1.1 E+00$ & & 1 & GE & pCill \\
\hline Nonvolatile beta & $4.7 E+01$ & $\| l$ & & & 1 & GP & $5.7 E+01$ & $\|$ & & $\mathbf{\square}$ & 1 & GE & pCill. \\
\hline Plutonium-238 & $<1.7 \mathrm{E}-02$ & $\mathrm{UI} / /$ & $<0.0495$ & & 1 & GP & & & & & & & $\mathrm{pCi} / \mathrm{L}$ \\
\hline Plutonium-239/240 & $<-4.0 \mathrm{E}-03$ & $\mathrm{UI} / /$ & $<0.0869$ & & 1 & GP & & & & & & & $\mathrm{pCill}$ \\
\hline Radium-226 & $2.4 E+00$ & $/ l$ & & & 1 & GP & & & & & & & pCill \\
\hline Radium-228 & 9.5E-01 & /I & & & 1 & GP & & & & & & & $\mathrm{pCi} / \mathrm{L}$ \\
\hline Strontium-90 & $2.1 E+01$ & $\|$ & & a & 1 & GP & & & & & & & $\mathrm{pCi} / \mathrm{L}$ \\
\hline Technetium-99 & $5.1 E+01$ & $\|$ & & 口 & 1 & GP & & & & & & & pCill \\
\hline Thorium-228 & $<1,2 E-01$ & $\mathrm{U} U /$ & $<0.1460$ & & 1 & GP & & & & & & & pCin \\
\hline Thorium-230 & $<8.0 \mathrm{E}-02$ & Uw/ & $<0.1100$ & & 1 & GP & & & & & & & $\mathrm{pCi} / \mathrm{L}$ \\
\hline Sum of aiphas & 2.2E-01 & & & & & & & & & & & & $\mathrm{pCi} / \mathrm{L}$ \\
\hline Sum of betas & $7.3 E+01$ & & & $\mathbf{\square}$ & & & & & & & & & pCill \\
\hline Total radium & $3.3 \mathrm{E}+00$ & & & & & & & & & & & & pCill \\
\hline Tritium & $9.9 \mathrm{E}+02$ & $/ /$ & & 口 & 1 & GP & $9.2 \mathrm{E}+02$ & $/ /$ & & $\mathbf{a}$ & 1 & GE & $\mathrm{pCi} / \mathrm{mL}$ \\
\hline Uranium-233/234 & $<1.7 E-01$ & $\mathrm{U} \mathrm{U} / /$ & $<0.1910$ & & 1 & GP & & & & & & & $\mathrm{pCi}$ \\
\hline Uranium-235 & $<0.0 E+00$ & $\mathrm{UH} / /$ & $<0.0926$ & & 1 & GP & & & & & & & pCill \\
\hline Uranium-238 & 2.2E-01 & $/ 1$ & & & 1 & GP & & & & & & & $\mathrm{pCi} / \mathrm{L}$ \\
\hline
\end{tabular}

Notes:

- exceeded holding time

- = exceeded groundwater protection or monitoring constituent standard (See Appendix A.) 
WELL FSB 93D

\begin{tabular}{|c|c|}
\hline RS Coord. & Latllongitu \\
\hline $\begin{array}{l}\text { N74 } \\
\text { E50 }\end{array}$ & 81.679416 \\
\hline
\end{tabular}

SAMPLE DATE

FIELD DATA

Constituents

Water elevation

$\mathrm{pH}$

Sp. conductance

Water temperature

Akalinity as $\mathrm{CaCO}_{3}$

Turbidity

Volumes purged

Sampling code

Synchronous water leve

ANALYTICAL DATA

Inorganic Constituents

\begin{tabular}{ll} 
Constituents & $3 Q 98$ \\
\hline & \\
Antimony, total recoverable & $<0.20$ \\
Arsenic, total recoverable & $<30$ \\
Barium, total recoverable & 300 \\
Cadmium, total recoverable & 9.7 \\
Chromium, total recoverable & $<3.0$ \\
Cobalt, total recoverable & 7.5 \\
Copper, total recoverable & 61 \\
Cyanide & $<10$ \\
Lead, total recoverable & 39 \\
Mercury, total recoverable & 0.20 \\
Nickel, total recoverable & 15 \\
Nitrate as nitrogen & \\
Nitrate-nitrite as nitrogen & $<10$ \\
Selenium, total recoverable & $<50$ \\
Silver, total recoverable & $<1.0$ \\
Thallium, total recoverable & 0.20 \\
Vanadium, total recoverable & $<2.0$ \\
Zinc, total recoverable & 78
\end{tabular}

$\underline{3098}$

213.4

3.8

680

20.0

0

0.099

XN

$212.6(09 / 17 / 98)$

\section{Screen Zone Elevation}

217.9-197.9 ft ms

$7 / 21 / 98$

\section{Top of Casing}

$276.1 \mathrm{ft} \mathrm{msl}$

$10 / 12 / 98$
Casing Pump Screen Zone

4" PVC V Water Table (IIB2)

Organic Constituents

$\begin{array}{lllllll}\text { Constituents } & \text { 3Q98 } & \text { Mod } & \text { Filt } & \text { ST } \underline{\text { H }} & \underline{\text { DF }} & \text { Lab } \\ \text { Benzene } & <1.0 & \text { UJ/O/1 } & <1.0 & & 1 & \text { GE } \\ \text { Bis(2-ethylhexyl) phthalate } & <10 & \text { UJJ/Q/ } & <10 & & 1 & \text { GE } \\ \text { Dichloromethane } & <1.6 & \text { UJ/O8/1 } & <1.0 & & 1 & \text { GE } \\ \text { Phenols } & <5.0 & \text { U/I } & <5.0 & & 1 & \text { GE } \\ \text { Tetrachloroethylene } & <1.0 & \text { UJ/O/1 } & <1.0 & & 1 & \text { GE } \\ \text { Trichloroethylene } & <1.0 & \text { UJ/O/1 } & <1.0 & & 1 & \text { GE } \\ \text { Trichlorofluoromethane } & <1.0 & \text { UJ/O/1 } & <1.0 & & 1 & \text { GE }\end{array}$

\begin{tabular}{ll}
\hline 4Q98 & Unit \\
212.3 & $\mathrm{ft} \mathrm{msl}$ \\
3.7 & $\mathrm{pH}$ \\
650 & $\mu \mathrm{S} / \mathrm{cm}$ \\
22.6 & ${ }^{\circ} \mathrm{C}$ \\
0 & $\mathrm{mg} / \mathrm{L}$ \\
1 & $\mathrm{NTU}$ \\
0.0 & well vol \\
XN & \\
$211.6(12 / 18 / 98)$ & $\mathrm{ft} \mathrm{msl}$
\end{tabular}

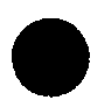

\begin{tabular}{|c|c|c|c|c|c|c|}
\hline Mod & Filt. & ST브 & $\underline{\text { DF }}$ & $\underline{\text { Lab }}$ & 4Q98 & Mod \\
\hline$U / I$ & $<0.20$ & & 1 & GE & & \\
\hline $\mathrm{U} / /$ & $<30.0000$ & & 10 & GE & & \\
\hline II & & & 1 & GE & & \\
\hline /I & & a & 1 & GE & 8.5 & $\|$ \\
\hline $\mathrm{U} / /$ & $<3.0$ & & 1 & GE & & \\
\hline$\|$ & & 口 & 10 & GE & & \\
\hline /I & & & 1 & GE & & \\
\hline $\mathrm{U} / / /$ & $<10$ & & 1 & GE & & \\
\hline$N /$ & & - & 1 & GE & 14 & $\|$ \\
\hline "l & & & 1 & GE & & \\
\hline$\|$ & & & 1 & GE & & \\
\hline UNI & $<50$ & & 1 & GE & 83,000 & $/ 1$ \\
\hline $\mathrm{U} / /$ & $<50$ & & 10 & GE & & \\
\hline $\mathrm{U} / /$ & $<1.0$ & & 1 & GE & & \\
\hline $\mathrm{J} / \mathrm{E} /$ & NDD & & 1 & GE & & \\
\hline $\mathrm{U} / \prime$ & $<2.0$ & & 1 & GE & & \\
\hline$\|$ & & & 1 & GE & & \\
\hline
\end{tabular}

Filt.

ST H DF Lab Unit

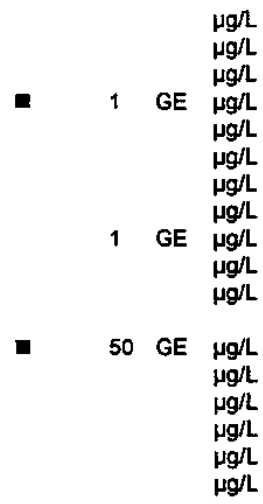

$<1.0$

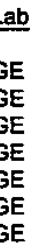

$\mu g / L$
$\mu g / h$
$\mu g / L$
$\mu g / L$
$\mu g / L$
$\mu g / L$
$\mu g / L$

Notes:

- exceeded holding time

- exceeded groundwater protection or monitoring constituent standard (See Appendix A.)

F-Area HWMF

C-63

Third and Fourth Quarter 1998 
WELL FSB 93D (cont.)

Radioactive Constituents

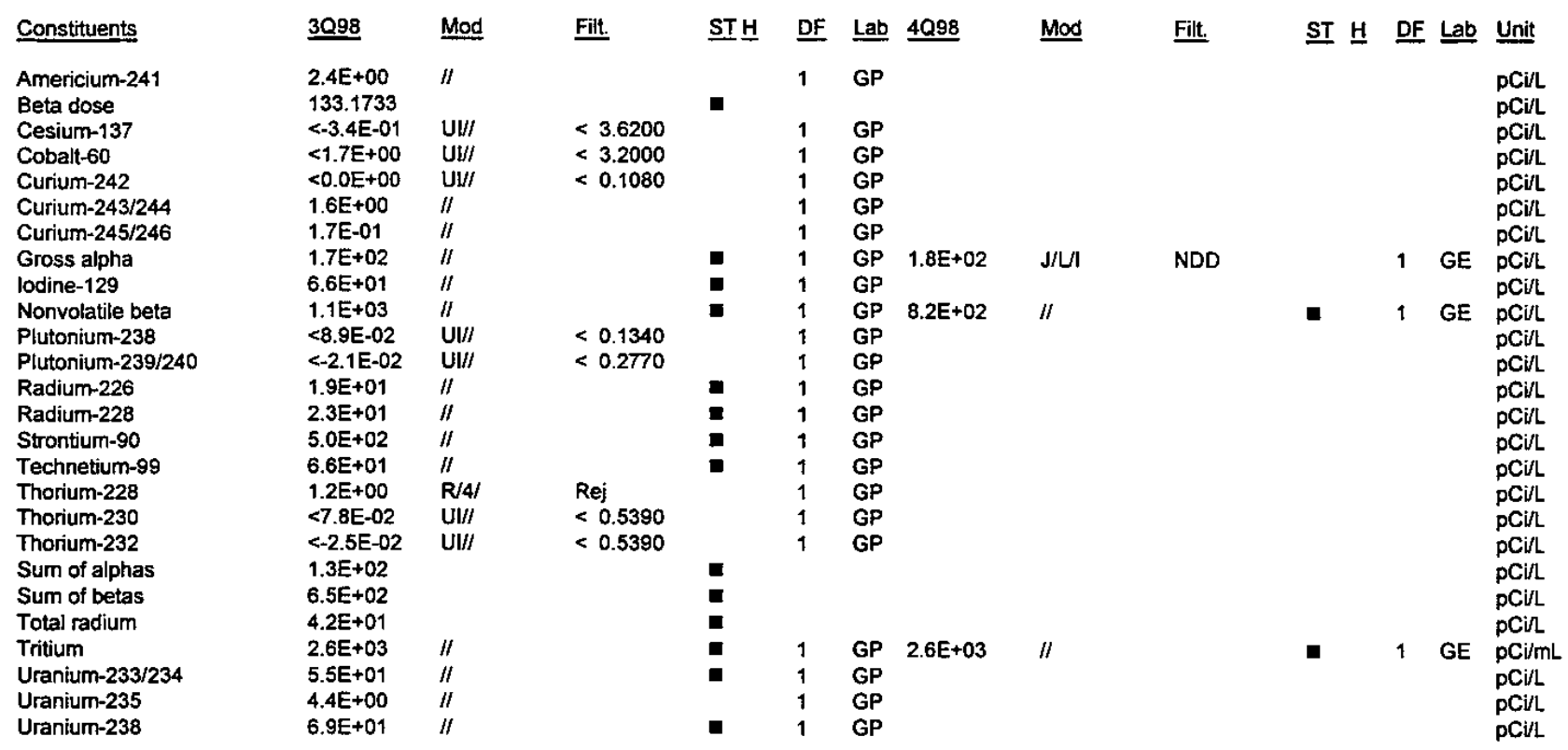

Notes:

- = exceeded holding time

- = exceeded groundwater protection or monitoring constituent standard (See Appendix A.) 
WELL FSB 94C

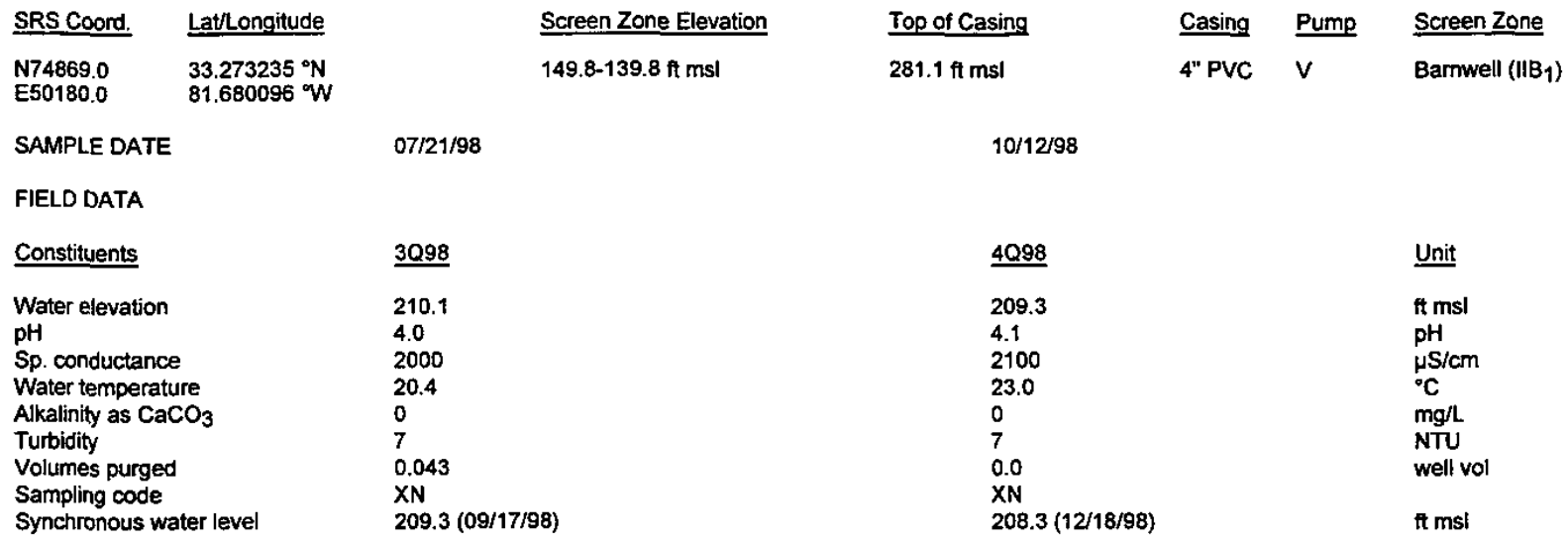

ANALYTICAL DATA

Inorganic Constituemts

Constituents

Filt.

$<0.20$

Antimony, total recoverable $<0.20$

$\begin{array}{ll}\text { Arsenic, total recoverable } & <150 \\ \text { Barium, total recoverable } & 790\end{array}$

Cadmium, total recoverable 24

Chromium, total recoverable $<3.0$

Cobalt, total recoverable $\quad 440$

Copper, total recoverable

Cyanide

Lead, total

Mercury, total recoverable $<0.20$

Nickel, total recoverable $\quad 160$

Nitrate as nitrogen

Nitrate-nitrite as nitrogen $\quad 83,000$

Selenium, total recoverable $<250$

Silver, total recoverable $<1.0$

Thallium, total recoverable $<2.5$

Vanadium, total recoverable $<2.0$

Zinc, total recoverable

290

Organic Consthtuents

Benzene

$\underline{3098}$

Bis(2-ethylhexyl) phthalate

Dichloromethane

Phenols

Tetrachloroethylene

Trichloroethylene

Trichiorofluoromethane

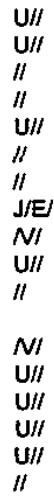

$\underline{M o d}$

$\mathrm{U} / \mathrm{O} / \mathrm{I}$

$\mathrm{UJ} / \mathrm{O} / \mathrm{O}$

$\mathrm{UJ} / \mathrm{O} / 1$

$\mathrm{J} / \mathrm{E} /$

US/O/1

UJ/O/1

UJ/O/1
$<150$

$<3.0$

NDD

$<0.20$

$<250$

$<1.0$

$<2.5$

$<2.0$

Filt.

$<1.0$

$<9.8$

$<1.0$

NDD

$<1.0$

$<1.0$

$<1.0$ $\underline{\text { ST}}$

DF Lab 4Q998

Mod

$1 \quad G E$

$50 \mathrm{GE}$

1 GE

$\begin{array}{ll}1 & \mathrm{GE} \\ - & 1 \\ \mathrm{GE}\end{array}$

24

"I

$\begin{array}{ll}50 & \mathrm{GE} \\ 1 & \mathrm{GE}\end{array}$

$\mathrm{GE}$

GE 230

$1 \mathrm{GE}$
$-\quad 1 \mathrm{GE}$

- $\quad \begin{array}{rl}50 & \mathrm{GE} \\ 50 & \mathrm{GE}\end{array}$

1 GE

$1 \mathrm{GE}$

1 GE

300,000
ST H DF Lab Unit

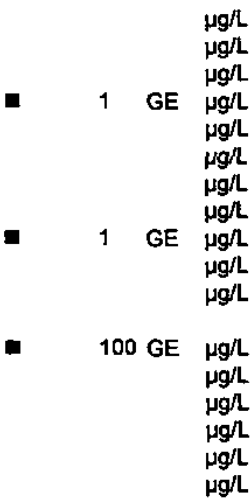

\begin{tabular}{|c|c|c|}
\hline ST브 & $\underline{\mathrm{DF}}$ & $\underline{\text { Lab }}$ \\
\hline & 1 & $\mathbf{G}$ \\
\hline & $\begin{array}{l}1 \\
1\end{array}$ & $\begin{array}{l}G \\
G\end{array}$ \\
\hline & 1 & $\mathrm{GE}$ \\
\hline & 1 & GE \\
\hline & $\begin{array}{l}1 \\
1\end{array}$ & \\
\hline
\end{tabular}

Mod

Notes:

- exceeded holding time

- = exceeded groundwater protection or monitoring constituent standard (See Appendix A.)

F-Area HWMF

C.65

Third and Fourth Quarter 1998 
WELL FSB 94C (cont.)

Radioactive Constituents

\begin{tabular}{|c|c|c|c|c|c|c|c|c|c|c|c|c|c|}
\hline Constituents & $\underline{3 Q 98}$ & Mod & Filt. & ST브 & $\underline{\text { DF }}$ & $\underline{\text { Lab }}$ & 4Q98 & Mod & Filt. & $\underline{\text { ST }} \underline{H}$ & $\underline{D F}$ & $\underline{\mathrm{Lab}}$ & Unit \\
\hline Americium-241 & $4.8 E-01$ & $/ 1$ & & & 1 & GP & & & & & & & pCil \\
\hline Beta dose & 191.7778 & & & $\boldsymbol{w}$ & & & & & & & & & pCill \\
\hline Cesium-137 & $8.0 \mathrm{E}+00$ & $/ 1$ & & & 1 & GP & & & & & & & pCill \\
\hline Cobalt-60 & $<3.2 \mathrm{E}+00$ & UIII & $<3.9600$ & & 1 & GP & & & & & & & pCirl \\
\hline Curium-242 & $<0.0 \mathrm{E}+00$ & $U \mid I I$ & $<0.1800$ & & 1 & GP & & & & & & & $\mathrm{pCilL}$ \\
\hline Curium-243/244 & $<8.5 \mathrm{E}-02$ & UVII & $<0.3470$ & & 1 & GP & & & & & & & pCilL \\
\hline Curium-245/246 & $<0.0 \mathrm{E}+00$ & $\mathrm{U} V /$ & $<0.1420$ & & 1 & GP & & & & & & & pCill \\
\hline Gross alpha & $1.5 \mathrm{E}+02$ & $/ 1$ & & a & 1 & GP & $1,0 E+02$ & $/ /$ & & घ & 1 & $\mathrm{GE}$ & pCirl \\
\hline lodine-129 & $1.2 E+02$ & $\|$ & & a & 1 & GP & & & & & & & pCill \\
\hline Nonvolatile beta & $2.2 E+03$ & $/ /$ & & a & 1 & GP & $1.8 E+03$ & $/ /$ & & $\omega$ & 1 & GE & pCilL \\
\hline Plutonium-238 & $<2.5 \mathrm{E}-02$ & $U \mathrm{II} /$ & $<0.2990$ & & 1 & GP & & & & & & & pCilL \\
\hline Plutonium-239/240 & $<4.8 \mathrm{E}-02$ & ull/ & $<0.1440$ & & 1 & GP & & & & & & & $\mathrm{pCi} / \mathrm{L}$ \\
\hline Radjum-226 & $6.5 \mathrm{E}+01$ & $\|$ & & - & 1 & GP & & & & & & & $\mathrm{pCi} / \mathrm{L}$ \\
\hline Radium-228 & $2.0 E+01$ & $/ /$ & & $\mathbf{\square}$ & 1 & GP & & & & & & & pCirl \\
\hline Strontium-90 & $5.4 \mathrm{E}+02$ & $\|$ & & $\mathbf{a}$ & 1 & GP & & & & & & & pCill \\
\hline Technetium-99 & $2.5 E+02$ & $/ 1$ & & च & 1 & GP & & & & & & & $\mathrm{pCi} / \mathrm{L}$ \\
\hline Thorium-228 & $1.8 \mathrm{E}+00$ & $R / 4 /$ & Rej & & 1 & GP & & & & & & & pCill \\
\hline Thorium-230 & $<-1.1 E-01$ & $\mathrm{UU} / \mathrm{I}$ & $<0.8830$ & & 1 & GP & & & & & & & $\mathrm{pCi} / \mathrm{L}$ \\
\hline Sum of alphas & $5.0 E+01$ & & & $\boldsymbol{\square}$ & & & & & & & & & pCill \\
\hline Sum of betas & $9.3 E+02$ & & & $\mathbf{\square}$ & & & & & & & & & pCin \\
\hline Total radium & $8.5 E+01$ & & & a & & & & & & & & & $\mathrm{pCil}$ \\
\hline Tritium & $1.0 \mathrm{E}+04$ & $\|$ & & $\mathbf{\square}$ & 1 & GP & 1. $\{E+04$ & $/ /$ & & $\mathbf{n}$ & 1 & GE & pCiumL \\
\hline Uranium-233/234 & $2.5 E+01$ & $\|$ & & $\mathbf{a}$ & 1 & GP & & & & & & & pCil \\
\hline Uranium-235 & $1.8 E+00$ & $/ /$ & & & 1 & GP & & & & & & & pCill \\
\hline Uranium-238 & $2.3 E+01$ & $/ 1$ & & च & 1 & GP & & & & & & & $\mathrm{pCi} / \mathrm{L}$ \\
\hline
\end{tabular}

Notes:

- = exceeded holding time

= exceeded groundwater protection or monitoring constituent standard (See Appendix A.) 
WELL FSB 94DR

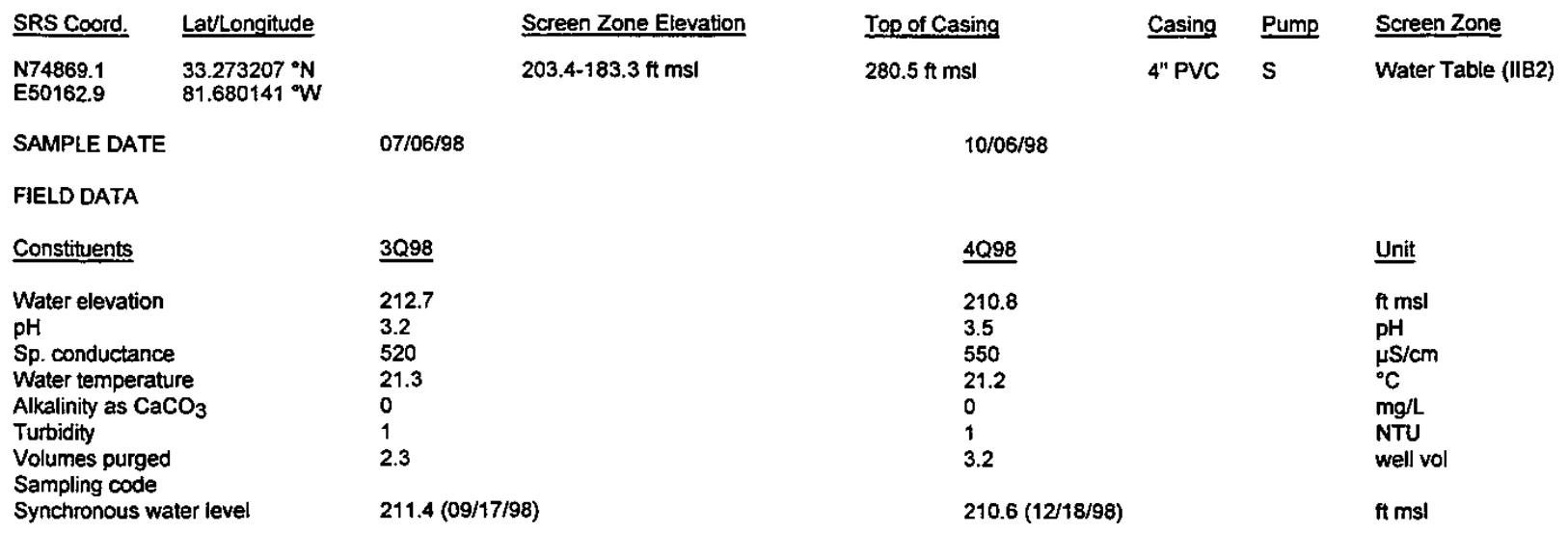

ANALYTICAL DATA

Inorganic Constituents

\begin{tabular}{|c|c|c|c|c|c|c|c|c|c|c|c|c|c|}
\hline Constituents & 3098 & Mod & Filt. & $\underline{\text { ST }} \underline{\mathrm{H}}$ & $\underline{\mathrm{DF}}$ & $\underline{\text { Lab }}$ & 4Q98 & Mod & Filt & $\underline{\text { ST }} \underline{H}$ & DF & $\underline{\operatorname{Lab}}$ & Unit \\
\hline Antimony, total recoverable & $<2.0$ & $\mathrm{U} / /$ & $<2.0$ & & 10 & GE & & & & & & & $\mu g / L$ \\
\hline Arsenic, total recoverable & $<30$ & $\mathrm{U} / 1$ & $<30.0000$ & & 10 & GE & & & & & & & $\mu g / L$ \\
\hline Barium, total recoverable & 280 & $/ /$ & & & 10 & GE & & & & & & & 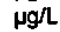 \\
\hline Cadmium, total recoverable & 1.6 & $\mathrm{~J} / \mathrm{E} /$ & NDD & & 10 & GE & 7.2 & $\|$ & & $\mathbf{n}$ & 1 & GE & $\mu g / L$ \\
\hline Chromium, total recoverable & $<30$ & $\mathrm{U} / l$ & $<30.0000$ & & 10 & GE & & & & & & & $\mu g / L$ \\
\hline Cobalt, total recoverable & 9.9 & $\|$ & & $\mathbf{\square}$ & 10 & GE & & & & & & & $\mu g / L$ \\
\hline Copper, total recoverable & 200 & II & & & 10 & GE & & & & & & & $\mu g / L$ \\
\hline Cyanide & 6.0 & $\mathrm{~J} / \mathrm{E} \mathrm{t} / 2$ & NDD & & 1 & GE & & & & & & & $\mu g / L$ \\
\hline Lead, total recoverable & 12 & $\mathrm{~J} / \mathrm{E} /$ & NDD & & 10 & GE & 20 & $/ 1$ & & $\boldsymbol{\square}$ & 1 & GE & $\mu g / L$ \\
\hline Mercury, total recoverable & $<0.20$ & $\mathrm{U} / /$ & $<0.20$ & & 1 & GE & & & & & & & $\mu g / L$ \\
\hline $\begin{array}{l}\text { Nickel, total recoverable } \\
\text { Nitrate as nitrogen }\end{array}$ & 11 & $N \prime$ & & & 10 & GE & & & & & & & $\mu g / L$ \\
\hline Nitrate-nitrite as nitrogen & 38,000 & $N I$ & & - & 25 & GE & 61,000 & $/ /$ & & $\mathbf{\square}$ & 50 & GE & $\mu g / L$ \\
\hline Selenium, total recoverable & 8.7 & $\mathrm{~J} / \mathrm{E} /$ & NDD & & 10 & GE & & & & & & & $\mu g / \mathrm{L}$ \\
\hline Silver, total recoverable & $<10$ & $\mathrm{U} / /$ & $<10$ & & 10 & $\overrightarrow{G E}$ & & & & & & & $\mu g / L$ \\
\hline Thallium, total recoverable & $<25$ & $\mathrm{U} / I$ & $<25.0$ & & 10 & GE & & & & & & & $\mu g / h$ \\
\hline Vanadium, total recoverable & $<20$ & $\mathrm{U} / /$ & $<20$ & & 10 & GE & & & & & & & $\mu g / L$ \\
\hline Zinc, total recoverable & 87 & /I & & & 10 & GE & & & & & & & $g / L$ \\
\hline
\end{tabular}

Organic Constituents

\begin{tabular}{|c|c|c|c|c|c|c|c|c|c|c|}
\hline Constituents & 3098 & Mod & Filt. & $\underline{\text { ST }} \underline{\text { H }}$ & DF & $\underline{\text { Lab }} \underline{4098}$ & Mod & Filt. & $\underline{\text { ST }} \underline{H}$ DF Lab & $\underline{\text { Unit }}$ \\
\hline Benzene & $<1.0$ & $\mathrm{U} / /$ & $<1.0$ & & 1 & GE & & & & $\mu g / L$ \\
\hline Bis(2-ethylhexyl) phthalate & 160 & $\|$ & & $\mathbf{0}$ & 2 & GE & & & & $\mu g / L$ \\
\hline Dichloromethane & $<1.4$ & UN8I & $<1.0$ & & 1 & GE & & & & $\mu g / L$ \\
\hline Phenols & $<5.0$ & $\mathrm{U} / \prime$ & $<5.0$ & & 1 & GE & & & & $\mu g / L$ \\
\hline Tetrachloroethylene & $<1.0$ & $\mathrm{U} / \prime$ & $<1.0$ & & 1 & GE & & & & $\mu g / L$ \\
\hline Trichloroethylene & 3.5 & $\|$ & & & 1 & GE & & & & $\mu g / L$ \\
\hline Trichlorofluoromethane & $<1.0$ & $\mathrm{U} / 1$ & $<1.0$ & & 1 & GE & & & & $\mu g / L$ \\
\hline
\end{tabular}

Notes:

- exceeded holding time

- exceeded groundwater protection or monitoring constituent standard (See Appendix A.)

F-Area HWMF 
WELL FSB 94DR (cont.)

Radioactive Construents

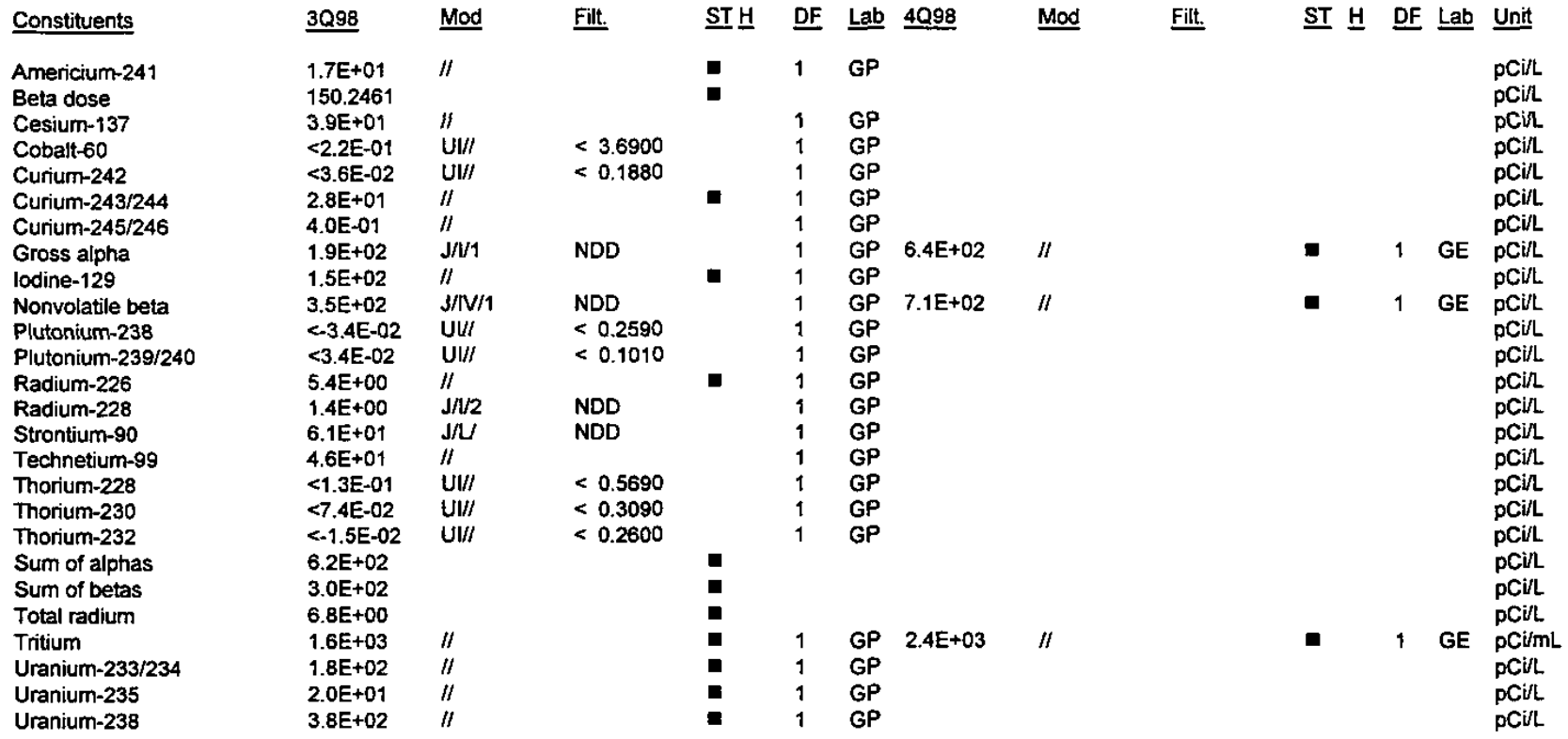

Notes:

- exceeded holding time

- exceeded groundwater protection or monitoring constituent standard (See Appendix A.) 
WELL FSB 95CR

\begin{tabular}{|c|c|}
\hline SRS Coord. & LatlLongitude \\
\hline $\begin{array}{l}\text { N75001.9 } \\
\text { E49987.8 }\end{array}$ & $\begin{array}{l}33.273215^{\circ} \mathrm{N} \\
81.680860^{\circ} \mathrm{V}\end{array}$ \\
\hline
\end{tabular}

SAMPLE DATE

FIELLO DATA

Constituents

Water elevation

$\mathrm{pH}$

Sp. conductance

Water temperature

Alkalinity as $\mathrm{CaCO}_{3}$

Turbidity

Volumes purged

Sampling code

Synchronous water level

ANALYMICAL DATA

Inorganic Constituents

Constituents

Antimony, total recoverable Arsenic, total recoverable Barium, total recoverable 1,100

Cadmium, total recoverable 18

Chromium, total recoverable 1.4

Cobalt, total recoverable $\quad 490$

Copper, total recoverable 63

Cyanide

Lead, total recoverable

Mercury, total recoverable

Nickel, total recoverable $\quad 110$

Nitrate as nitrogen

Nitrate-nitrite as nitrogen $\quad 290,000$

Selenium, total recoverable

Silver, total recoverable

Thallium, total recoverable

Zinc, total recoverable

320

Organic Constituents

\begin{tabular}{ll} 
Constituents & $\underline{3098}$ \\
\hline & $<1.0$ \\
Benzene & $<10$ \\
Bis(2-ethylhexyl) phthalate & $<5.0$ \\
Dichloromethane & $<5.0$ \\
Phenols & $<1.0$ \\
Tetrachloroethylene & $<1.0$ \\
Trichloroethylene & $<5.0$ \\
Trichlorofluoromethane &
\end{tabular}

Screen Zone Elevation

161.9-151.9 ft msi

08/25/98

$\underline{3098}$

209.2

3.5

2200

21.3

0

3.6

$207.7(09 / 18 / 98)$
Top of Casing

$284 \mathrm{ft} \mathrm{ms}$

$10 / 05 / 98$

4098

207.4

3.8

22.8

1

2.5

$206.9(12 / 18 / 98)$
Casing Pump

$4^{\text {" PVC }} 5$

Screen Zone

Barnwell (IIB 1$)$
Unit

ft msl

$\mathrm{pH}$

$\mu \mathrm{S} / \mathrm{cm}$

${ }^{\circ} \mathrm{C}$

mg/l

well vol

$\mathrm{ft} \mathrm{msl}$

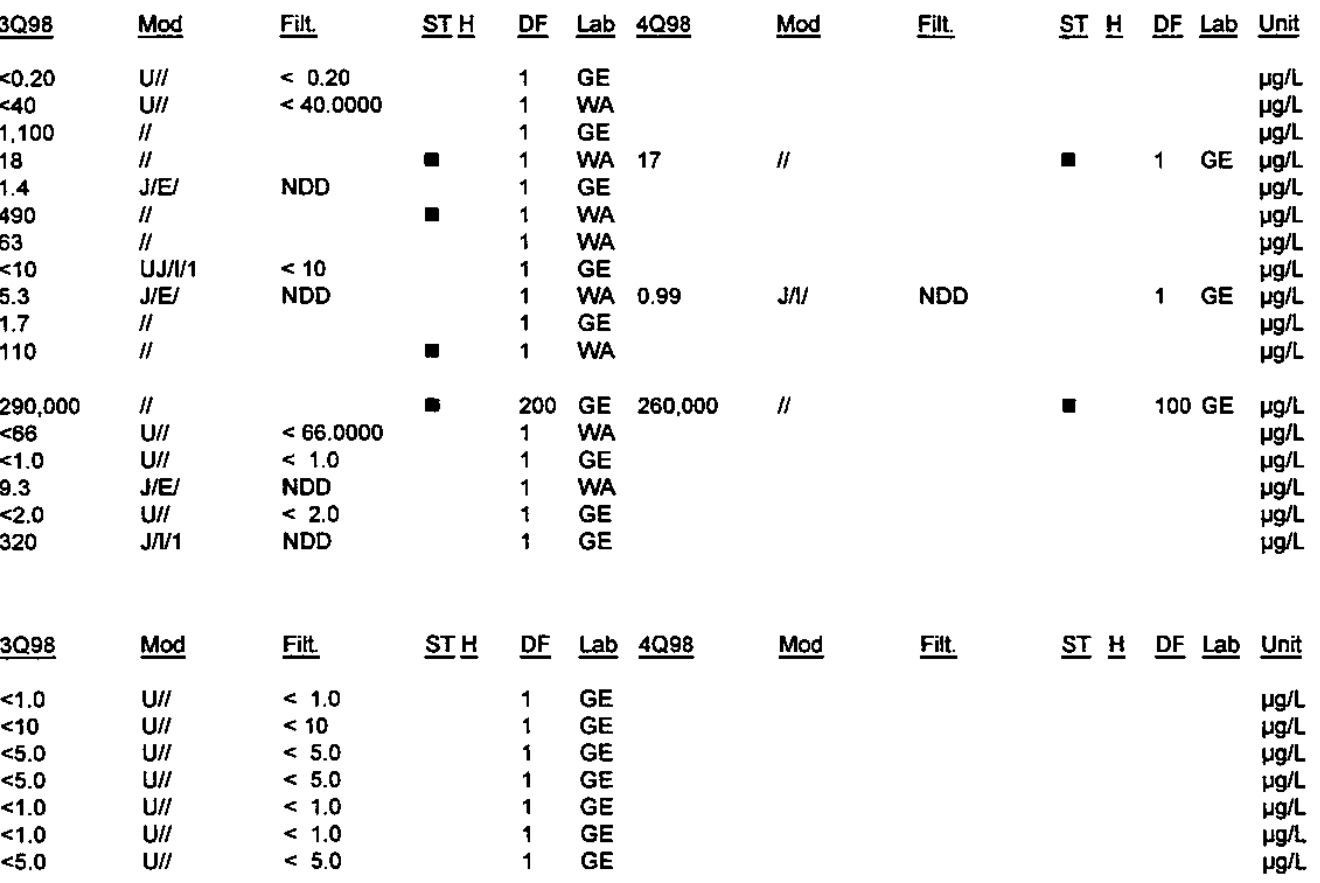

Notes:

- exceeded holding time

- exceeded groundwater protection or monitoring constituent standard (See Appendix A.)

F-Area HWMF 
WELL FSB 95CR (cont.)

Radioactive Constituents

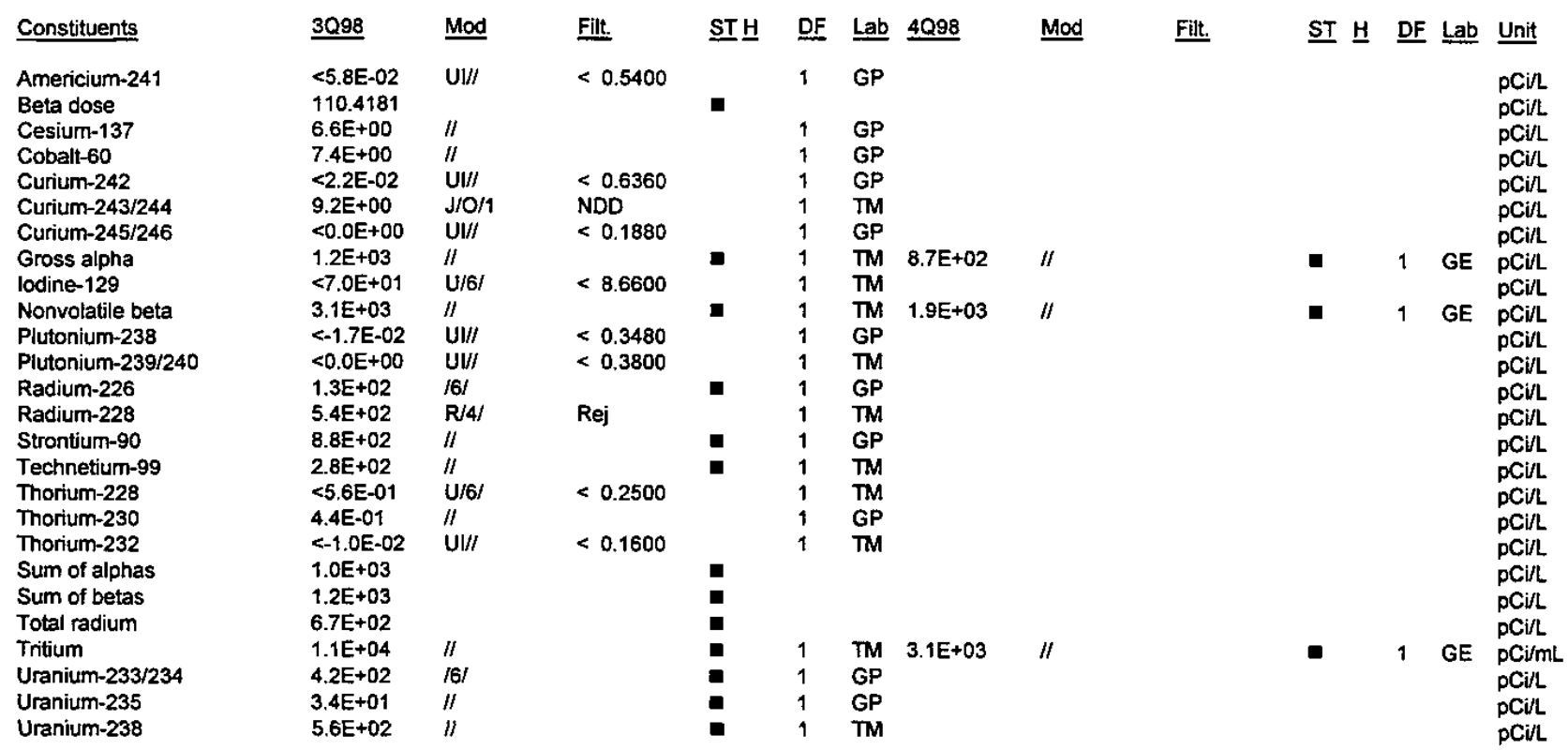

Notes:

- = exceeded holding time

- = exceeded groundwater protection or monitoring constituent standard (See Appendix A.) 
WELL FSB 95DR

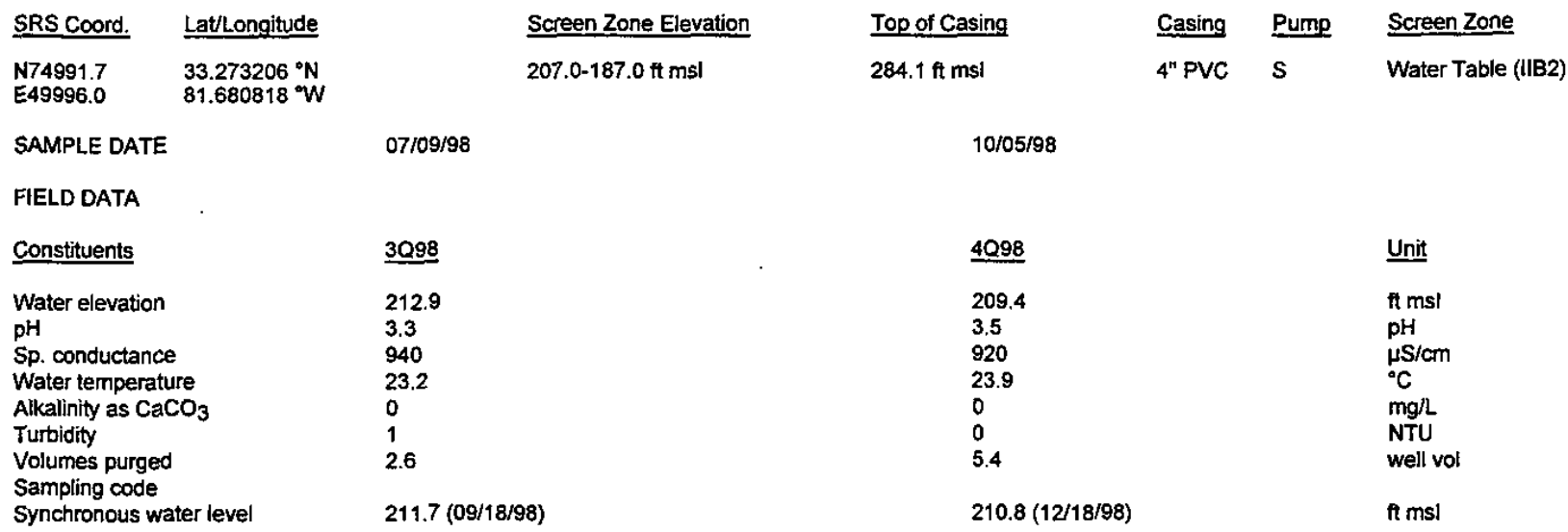

ANALYTICAL DATA

inorganic Consthuents

\begin{tabular}{|c|c|c|c|c|c|c|c|c|c|c|c|c|c|}
\hline Constituents & 3098 & Mod & Filt. & $\underline{\text { ST }} \underline{H}$ & $\underline{D F}$ & $\underline{L a b}$ & 4098 & Mod & Filt. & $\underline{\text { ST }} \underline{\mathrm{H}}$ & DF & Lab & Unit \\
\hline Antimony, total recoverable & $<0.20$ & $\mathbf{U} / /$ & $<0.20$ & & 1 & GE & & & & & & & $\mu g / 2$ \\
\hline Arsenic, total recoverable & $<3.0$ & $\mathrm{U} / 1$ & $<3.0$ & & 1 & GE & & & & & & & $\mu g /$ \\
\hline Barium, total recoverable & 290 & $\|$ & & & 1 & GE & & & & & & & $\mu g / L$ \\
\hline Cadmium, total recoverable & 4.8 & $/ /$ & & & 1 & $\overline{G E}$ & 4.5 & /l & & & 1 & GE & $\mu g / L$ \\
\hline Chromium, total recoverable & $<2.3$ & UNI & $<3.0$ & & 1 & $\overline{G E}$ & & & & & & & $\mu \mathrm{g} / \mathrm{L}$ \\
\hline Cobalt, total recoverable & 10 & $\|$ & & - & 1 & GE & & & & & & & $\mu g / L$ \\
\hline Copper, total recoverable & 66 & $\|$ & & & 1 & GE & & & & & & & $\mu g / 2$ \\
\hline Cyanide & $<10$ & $\mathrm{U} / I$ & $<10$ & & 1 & GE & & & & & & & $\mu g / \mathrm{L}$ \\
\hline Lead, total recoverable & 15 & $\| l$ & & 口 & 1 & GE & 9.0 & $/ /$ & & & 1 & GE & $\mu g /$ \\
\hline Mercury, total recoverable & $<0.20$ & $\mathrm{U} / /$ & $<0.20$ & & 1 & GE & & & & & & & $\mu g / L$ \\
\hline $\begin{array}{l}\text { Nickel, total recoverable } \\
\text { Nitrate as nitrogen }\end{array}$ & 18 & $\|$ & & & 1 & GE & & & & & & & $\mu g / L$ \\
\hline Nitrate-nitrite as nitrogen & 94.000 & $\|$ & & $\mathbf{\square}$ & 50 & GE & 110,000 & $\|$ & & 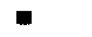 & 50 & GE & $\mu g / L$ \\
\hline Selenium, total recoverable & 0.74 & $\mathrm{~J} / \mathrm{E} /$ & NDD & & 1 & GE & & & & & & & $\mu g / L$ \\
\hline Silver, total recoverable & $<0.086$ & $\mathrm{UJ} / \mathrm{CV} /$ & $<1.0$ & & 1 & GE & & & & & & & $\mu g / L$ \\
\hline Thallium, total recoverable & 0.13 & $\mathrm{~J} / \mathrm{E} /$ & NDD & & 1 & GE & & & & & & & $\mu g / L$ \\
\hline Vanadium, total recoverable & $<2.0$ & $\mathrm{U} / I$ & $<2.0$ & & 1 & GE & & & & & & & $\mathrm{Jg} / \mathrm{h}$ \\
\hline Zinc, total recoverable & 74 & $/ /$ & & & 1 & $\overline{G E}$ & & & & & & & \\
\hline
\end{tabular}

Organic Constituents

\begin{tabular}{|c|c|c|c|c|c|c|c|c|c|c|}
\hline Constituents & 3098 & Mod & Filt. & $\underline{\text { ST}} \underline{\mathrm{H}}$ & $\underline{\mathrm{DF}}$ & $\underline{\text { Lab }} \underline{4 Q 98}$ & Mad & Filt. & ST $\underline{H}$ DF Lab & Unit \\
\hline Benzene & $<1.0$ & UJ/O/1 & $<1.0$ & & 1 & GE & & & & $\mu g / L$ \\
\hline Bis(2-ethylhexyl) phthalate & $<11$ & Unl & $<0.0108$ & & 1 & GE & & & & $\mu g / L$ \\
\hline Dichloromethane & $<2.5$ & UJNO8/1 & $<1.0$ & & 1 & GE & & & & $\mu g / L$ \\
\hline Phenols & $<5,0$ & $\mathrm{U} / /$ & $<5.0$ & & 1 & GE & & & & $\mu g / L$ \\
\hline Tetrachioroethylene & $<1.0$ & UJ/O/1 & $<1.0$ & & 1 & GE & & & & $\mu g / h$ \\
\hline Trichioroethylene & $<1.0$ & UJ/O/1 & $<1.0$ & & 1 & GE & & & & $\mu g / L$ \\
\hline Trichlorofluoromethane & $<1.0$ & US/O/1 & $<1.0$ & & 1 & GE & & & & gn \\
\hline
\end{tabular}

Notes:

- exceeded holding time

- exceeded groundwater protection or monitoring constituent standard (See Appendix A.) 
WELL FSB 95DR (cont.)

Radioactive Constituents

\begin{tabular}{|c|c|c|c|c|c|c|c|c|c|c|c|c|c|}
\hline Constituents & 3098 & Mod & Filt. & ST브 & $\underline{\mathrm{DF}}$ & Lab & 4098 & Mod & Filt. & $\underline{\mathrm{ST}} \underline{\mathrm{H}}$ & DF & Lab & Unit \\
\hline $\begin{array}{l}\text { Americium-241 } \\
\text { Beta dose }\end{array}$ & $\begin{array}{l}2.9 E+01 \\
826.8222\end{array}$ & $\prime \prime$ & & a & 1 & $G P$ & & & & & & & pCir \\
\hline Cesium-137 & $2.5 E+02$ & $\|$ & & ט & 1 & GP & & & & & & & DCil \\
\hline Cobalt-60 & $<8.4 E-01$ & UI/I & $<3.6700$ & & 1 & GP & & & & & & & pCill \\
\hline Curium-242 & $<1.7 E-01$ & $\mathrm{U} \mathbf{l} / /$ & $<0.5000$ & & 1 & GP & & & & & & & pCin \\
\hline Curium-243/244 & $2.0 E+01$ & $\|$ & & घ & 1 & GP & & & & & & & $\mathrm{pCi} / \mathrm{L}$ \\
\hline Curium-245/246 & $<6.5 \mathrm{E}-01$ & $\mathrm{U} \mathbf{Y} /$ & $<0.9140$ & & 1 & GP & & & & & & & $\mathrm{pCi} / \mathrm{L}$ \\
\hline Gross alpha & $7.4 E+02$ & $\| !$ & & 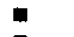 & 1 & GP & $5.8 E+02$ & /I & & घ & 1 & GE & $\mathrm{pCi} / \mathrm{L}$ \\
\hline lodine-129 & 8.1E+02 & $\| 1$ & & $\mathbf{0}$ & 1 & GP & & & & & & & $\mathrm{pCi} / \mathrm{L}$ \\
\hline Nonvolatile beta & $8.4 \mathrm{E}+02$ & $\|$ & & $\mathbf{\square}$ & 1 & GP & $8.5 E+02$ & /I & & घ & 1 & GE & $\mathrm{pCi} / \mathrm{L}$ \\
\hline Plutonium-238 & $<9.4 E-03$ & $\mathrm{UI} / /$ & $<0.1120$ & & 1 & GP & & & & & & & $\mathrm{pCi}$ \\
\hline Plutonium-239/240 & $<1.4 E-02$ & $\mathrm{UI} / I$ & $<0.0952$ & & 1 & GP & & & & & & & $\mathrm{pCin}$ \\
\hline Radium-226 & $<3.1 E-01$ & $\mathrm{UI} / I$ & $<0.6570$ & & 1 & GP & & & & & & & pCill \\
\hline Radium-228 & $2.4 E+\infty 0$ & $\|$ & & & 1 & GP & & & & & & & $\mathrm{pCi} / \mathrm{L}$ \\
\hline Strontium-90 & $1.2 \mathrm{E}+02$ & $\|$ & & $\mathbf{a}$ & 1 & GP & & & & & & & pCill \\
\hline Technetium-99 & $8.3 E+01$ & $\| t$ & & $\mathbf{a}$ & 1 & GP & & & & & & & $\mathrm{pCil}$ \\
\hline Thorium-228 & $<5.7 \mathrm{E}-01$ & $\mathrm{U} \boldsymbol{y} /$ & $<1.1900$ & & 1 & GP & & & & & & & pCill \\
\hline Thorium-230 & $<1.7 E-01$ & $\mathrm{UIJ} / \mathrm{Cl}$ & & & 1 & GP & & & & & & & pCir \\
\hline Sum of alphas & $7.9 E+02$ & & & ש & & & & & & & & & $\mathrm{pCi} \Omega$ \\
\hline Sum of betas & 1.3E+03 & & & ש & & & & & & & & & $\mathrm{pCill}$ \\
\hline Total radium & $2.4 E+00$ & & & & & & & & & & & & $\mathrm{pCi} / \mathrm{L}$ \\
\hline Tritium & $3.5 E+03$ & $\prime \prime$ & & 曰 & 1 & GP & $3.6 E+03$ & $\|$ & & ט & 1 & GE & $\mathrm{pCimL}$ \\
\hline Uranium-233/234 & $1.8 E+02$ & 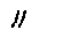 & & $\mathbf{\square}$ & 1 & GP & & & & & & & pCill \\
\hline Uranium-235 & $2.7 E+01$ & $\|$ & & $\mathbf{\square}$ & 1 & $\mathrm{GP}$ & & & & & & & $\mathrm{pCi} / \mathrm{h}$ \\
\hline Uranium-238 & $5.3 E+02$ & $/ /$ & & घ & 1 & GP & & & & & & & $\mathrm{pCi} / \mathrm{L}$ \\
\hline
\end{tabular}

Notes:

- = exceeded holding time

- = exceeded groundwater protection or monitoring constituent standard (See Appendix A.) 
WELL FSB 96AR

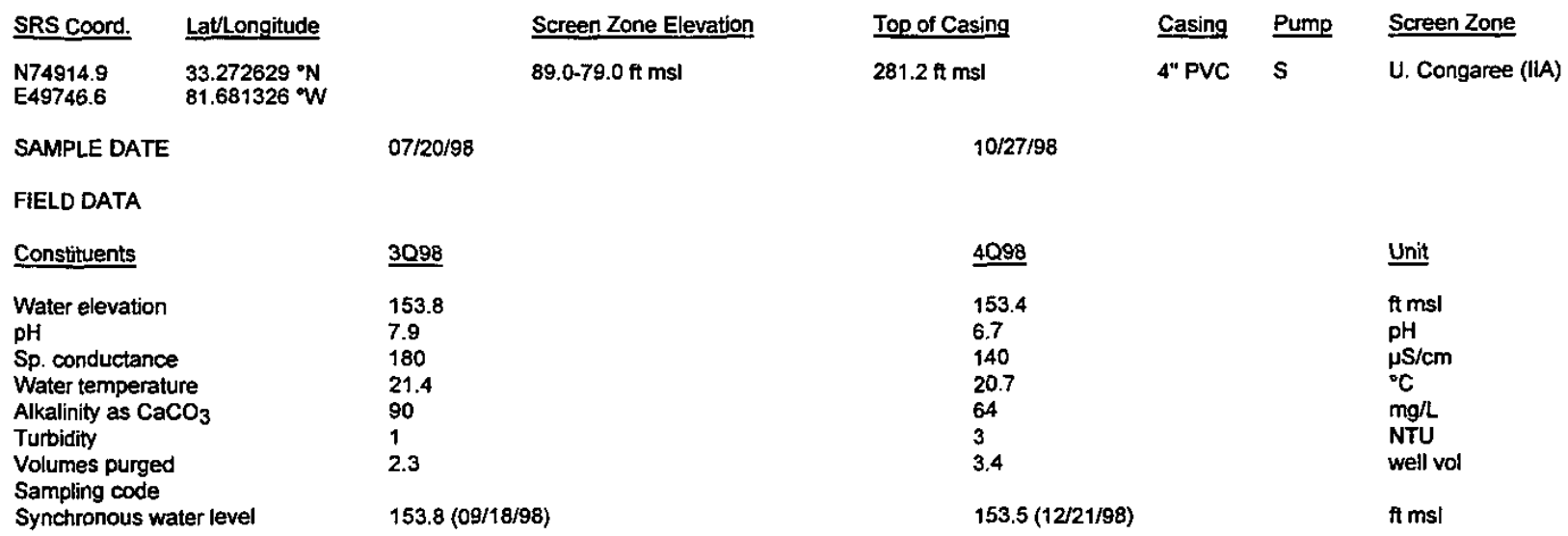

\section{ANALYTICAL DATA}

Inorganic Constituents

\begin{tabular}{|c|c|c|c|c|c|c|c|c|c|c|c|c|c|}
\hline Constituents & $\underline{3 Q 98}$ & Mod & Filt. & ST브 & $\underline{\mathrm{DF}}$ & Lab & 4Q98 & Mod & Fill. & ST $\underline{H}$ & $\underline{\mathrm{DF}}$ & Lab & Unit \\
\hline Antimony, total recoverable & $<0.20$ & $\mathrm{U} / /$ & $<0.20$ & & 1 & GE & & & & & & & $\mu g / L$ \\
\hline Arsenic, total recoverable & 0.63 & J/E & NDD & & 1 & GE & & & & & & & $\mu g / t$ \\
\hline Barium, total recoverable & 42 & "1) & & & 1 & GE & & & & & & & $\mu g h$ \\
\hline Cadmium, total recoverable & $<1.0$ & $\mathrm{U} / /$ & $<1.0$ & & 1 & GE & $<1.0$ & $\mathbf{U} / /$ & $<1.0$ & & 1 & GE & $\mu g / L$ \\
\hline Chromium, total recoverable & 1.6 & $\mathrm{~J} / \mathrm{E} J$ & NDD & & 1 & GE & & & & & & & $\mu g / L$ \\
\hline Cobalt, total recoverable & $<0.20$ & $\mathrm{U} / /$ & $<0.20$ & & 1 & GE & & & & & & & $\mu g / L$ \\
\hline Copper, total recoverable & 0.32 & $\|$ & & & 1 & GE & & & & & & & $\mu g / L$ \\
\hline Cyanide & $<10$ & $U / !$ & $<10$ & & 1 & GE & & & & & & & $\mu g h$ \\
\hline Lead, total recoverable. & $<2.0$ & $\mathrm{U} / /$ & $<2.0$ & & 1 & GE & $<2.0$ & $\mathrm{U} / /$ & $<2.0$ & & 1 & GE & $\mu g /$ \\
\hline Mercury, total recoverable & $<0.20$ & $\mathrm{U} / /$ & $<0.20$ & & 1 & GE & & & & & & & $\mu g / L$ \\
\hline $\begin{array}{l}\text { Nickel, total recoverable } \\
\text { Nitrate as nitrogen }\end{array}$ & 0.79 & $\|$ & & & 1 & GE & & & & & & & $\mu g / L$ \\
\hline Nitrate-nitrite as nitrogen & 6,000 & $\|$ & & & 3 & GE & 1,600 & $/ /$ & & & 1 & GE & 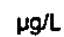 \\
\hline Selenium, total recoverable & $<0.84$ & UNI & $<5.0$ & & 1 & GE & & & & & & & $\mathrm{g} / \mathrm{L}$ \\
\hline Silver, total recoverable & $<1.0$ & $\mathrm{u} / I$ & $<1.0$ & & 1 & GE & & & & & & & gll \\
\hline Thallium, total recoverable & $<2.5$ & $\mathrm{U} / I$ & $<2.5$ & & 1 & $\overrightarrow{G E}$ & & & & & & & $g / L$ \\
\hline Vanadium, total recoverable & $<2.0$ & $\mathrm{U} / 1$ & $<2.0$ & & 1 & GE & & & & & & & $g / 2$ \\
\hline Zinc, total recoverable & 2.7 & $\mathrm{~J} / \mathrm{E}$ & NDD & & 1 & $\overrightarrow{\mathrm{GE}}$ & & & & & & & \\
\hline
\end{tabular}

Organic Constituents

\begin{tabular}{|c|c|c|c|c|c|}
\hline Constituents & 3Q98 & Mod & Filt & ST븐 & $\underline{\mathrm{DF}}$ \\
\hline Benzene & $<1.0$ & $\mathrm{UJ} / \mathrm{O} / 1$ & $<1.0$ & & 1 \\
\hline Bis(2-ethylhexyl) phthalate & $<10$ & $\mathrm{U} / \mathrm{I}$ & $<10$ & & 1 \\
\hline Dichloromethane & $<1.9$ & UJNO8/1 & $<1.0$ & & 1 \\
\hline Phenols & $<5.0$ & U/I & $<5.0$ & & 1 \\
\hline Tetrachloroethylene & $<1.0$ & $\mathrm{UJ} / \mathrm{O} / \mathrm{A}$ & $<1.0$ & & 1 \\
\hline Trichloroethylene & $<1.0$ & $\mathrm{UJ} / \mathrm{O} / \mathrm{t}$ & $<1.0$ & & 1 \\
\hline Trichlorofluoromethane & $<1.0$ & $\mathrm{UJ} / \mathrm{O} / 1$ & $<1.0$ & & 1 \\
\hline
\end{tabular}

Notes:

= exceeded holding time

- exceeded groundwater protection or monitoring constituent standard (See Appendix A.) 
WELL FSB 96AR (cont.)

Radioactive Constituents

\begin{tabular}{|c|c|c|c|c|c|c|c|c|c|c|c|c|c|}
\hline Constituents & 3098 & Mod & Filt. & ST브 & DF & Lab & 4Q98 & Mod & Filt. & $\underline{\text { ST }} \underline{H}$ & DF & $\underline{L a b}$ & Unit \\
\hline Americium-241 & $\begin{array}{l}<2.9 E-03 \\
29000\end{array}$ & $\mathrm{UI} / /$ & $<0.1440$ & a & 1 & GP & & & & & & & pCin \\
\hline $\begin{array}{l}\text { Beta dose } \\
\text { Cesium-137 }\end{array}$ & $\begin{array}{l}2.9000 \\
<1.3 E+00\end{array}$ & $\mathrm{UI} / /$ & $<4.5200$ & 0 & 1 & GP & & & & & & & $\begin{array}{l}\text { pCiLL } \\
\text { pCill }\end{array}$ \\
\hline Cobalt-60 & $<1.5 \mathrm{E}+00$ & $\mathrm{UN} / /$ & $<4.0000$ & & 1 & GP & & & & & & & pCir \\
\hline Curium-242 & $<7.7 \mathrm{E}-04$ & $\mathrm{UL} / /$ & $<0.1460$ & & 1 & GP & & & & & & & pCill \\
\hline Curium-243/244 & $<7.2 \mathrm{E}-02$ & $\mathrm{UL} / \mathrm{I}$ & $<0.1170$ & & 1 & GP & & & & & & & pCir \\
\hline Curium-245/246 & $<1.7 \mathrm{E}-02$ & $\mathrm{UI} / /$ & $<0.0505$ & & 1 & GP & & & & & & & pCill \\
\hline Gross alpha & $1.5 E+00$ & $/ 1$ & & & 1 & GP & $3.1 E+00$ & $\mathrm{~J} / \mathrm{I} /$ & NDD & & 1 & $\mathbf{T M}$ & pCil \\
\hline lodine-129 & $2.9 E+00$ & $\|$ & & & 1 & GP & & & & & & & $\mathrm{pCi} / \mathrm{L}$ \\
\hline Nonvolatile beta & $<9.3 E-01$ & $\mathrm{UI} / /$ & $<1.0800$ & & 4 & GP & $9.4 E+00$ & $/ 1$ & & & 1 & $\mathrm{TM}$ & pCil \\
\hline Plutonium-238 & $<0.0 E+00$ & $\mathrm{U} \mathrm{I} / \mathrm{I}$ & $<0.0554$ & & 1 & GP & & & & & & & pCil \\
\hline Plutonium-239/240 & $<1.8 \mathrm{E}-02$ & U1// & $<0.0553$ & & 1 & GP & & & & & & & pCill \\
\hline Radium-226 & 4.7E-01 & 11 & & & 1 & GP & & & & & & & $\mathrm{pCin}$ \\
\hline Radium-228 & $<1.2 \mathrm{E}+00$ & $\mathrm{U} \mathbf{V} /$ & $<1.4400$ & & 1 & GP & & & & & & & pCill \\
\hline Strontium-90 & $<3.1 E-01$ & $\mathbf{U} / /$ & $<1.5500$ & & 1 & GP & & & & & & & $\mathrm{pCill}$ \\
\hline Technetium-99 & $<-1.9 E+01$ & $\mathrm{UIII}$ & $<27.4000$ & & 1 & GP & & & & & & & $\mathrm{pCi} / \mathrm{L}$ \\
\hline Thorium-228 & $2.0 E-01$ & /I & & & 1 & GP & & & & & & & pCill \\
\hline Thorium-230 & $1.6 \mathrm{E}-01$ & $\|$ & & & 1 & GP & & & & & & & pCill \\
\hline Thorium-232 & $\begin{array}{l}<0.0 \mathrm{E}+00 \\
3.6 \mathrm{E}-01\end{array}$ & $\mathrm{UI} / \mathrm{I}$ & $<0.0695$ & & 1 & GP & & & & & & & $\begin{array}{l}\text { pCill } \\
\text { pCill }\end{array}$ \\
\hline $\begin{array}{l}\text { Sum of alphas } \\
\text { Sum of betas }\end{array}$ & $\begin{array}{l}3.6 E-01 \\
2.9 E+00\end{array}$ & & & & & & & & & & & & pCill \\
\hline Total radium & 4.7E-01 & & & & & & & & & & & & pCirl \\
\hline Tritium & $1.2 E+02$ & $\|$ & & 口 & 1 & GP & $2.8 E+01$ & $/ 1$ & & - & 1 & GE & pCímL \\
\hline Uranium-233/234 & $<6.9 E-02$ & $\mathrm{UL} / /$ & $<0.1690$ & & 1 & GP & & & & & & & $\mathrm{pCi} / \mathrm{L}$ \\
\hline Uranium-235 & $<5.5 E-02$ & $\mathrm{UI} / /$ & $<0.0819$ & & 1 & GP & & & & & & & pCill \\
\hline Uranium-238 & $<6.9 \mathrm{E}-02$ & $\mathrm{UU} / I$ & $<0.1690$ & & 1 & GP & & & & & & & Cill \\
\hline
\end{tabular}

Notes:

- exceeded holding time

= exceeded groundwater protection or monitoring constituent standard (See Appendix A.) 
WELL FSB 97A

\begin{tabular}{|c|c|c|c|c|c|c|c|}
\hline SRS Coord. & \multicolumn{2}{|l|}{ Lat/Longitude } & Screen Zone Elevation & Top of Casing & Casing & Pump & Screen Zone \\
\hline $\begin{array}{l}N 75171.2 \\
\text { E49965.7 }\end{array}$ & $\begin{array}{l}33.273554^{\circ} \mathrm{N} \\
81.681247^{\circ} \mathrm{W}\end{array}$ & & $95.8-85.8 \mathrm{ft} \mathrm{msl}$ & $286.1 \mathrm{ft} \mathrm{msl}$ & 4" PVC & $s$ & U. Congaree (IIA) \\
\hline \multicolumn{2}{|c|}{ SAMPLE DATE } & \multicolumn{2}{|l|}{$07 / 21 / 98$} & \multicolumn{2}{|c|}{$10 / 29 / 98$} & & \\
\hline \multicolumn{8}{|l|}{ FIELD DATA } \\
\hline \multicolumn{2}{|l|}{ Constituents } & \multicolumn{2}{|l|}{ 3Q98 } & $\underline{4 Q 98}$ & & & Unit \\
\hline \multicolumn{2}{|c|}{ Water elevation } & \multicolumn{2}{|l|}{152.9} & \multirow{2}{*}{\multicolumn{2}{|c|}{152.6}} & & \multirow{2}{*}{$\begin{array}{l}\mathrm{ft} \text { msl } \\
\mathrm{pH}\end{array}$} \\
\hline \multirow{2}{*}{\multicolumn{2}{|c|}{$\begin{array}{l}\text { pH } \\
\text { Sp. conductance }\end{array}$}} & \multirow{2}{*}{\multicolumn{2}{|c|}{$\begin{array}{l}7.2 \\
250\end{array}$}} & & & & \\
\hline & & & & \multicolumn{2}{|l|}{$\begin{array}{l}6.9 \\
235\end{array}$} & & $\mu \mathrm{S} / \mathrm{cm}$ \\
\hline \multicolumn{2}{|c|}{ Water temperature } & \multicolumn{2}{|l|}{21.5} & \multicolumn{2}{|c|}{20.5} & & ${ }^{\circ} \mathrm{C}$ \\
\hline \multicolumn{2}{|c|}{ Alkalinity as $\mathrm{CaCO}_{3}$} & \multicolumn{2}{|l|}{66} & \multicolumn{2}{|l|}{68} & & $\mathrm{mg} / \mathrm{l}$ \\
\hline \multicolumn{2}{|c|}{ Turbidity } & \multicolumn{2}{|l|}{1} & \multicolumn{2}{|l|}{1} & & NTU \\
\hline \multicolumn{2}{|c|}{$\begin{array}{l}\text { Volumes purged } \\
\text { Sampling code }\end{array}$} & \multicolumn{2}{|l|}{2.2} & \multicolumn{2}{|l|}{2.4} & & well vol \\
\hline \multicolumn{2}{|c|}{ Synchronous water level } & \multicolumn{2}{|c|}{$152.8(09 / 18 / 98)$} & \multicolumn{2}{|c|}{$152.4(12 / 18 / 98)$} & & ft mst \\
\hline
\end{tabular}

\section{ANALYTICAL DATA}

Inorganic Constituents

\begin{tabular}{|c|c|c|c|c|c|c|c|c|c|c|c|c|c|}
\hline Constituents & 3098 & Mod & Filt. & ST브 & $\underline{\text { DF }}$ & $\underline{\mathrm{Lab}}$ & $\underline{4098}$ & Mod & Fill. & $\underline{\text { ST }} \underline{H}$ & $\underline{D F}$ & Lab & Unit \\
\hline Antimony, total recoverable & $<0.20$ & $\mathbf{U} / /$ & $<0.20$ & & 1 & GE & & & & & & & $\mu g / L$ \\
\hline Arsenic, total recoverable & $<3.0$ & $\mathrm{U} / /$ & $<3.0$ & & 1 & GE & & & & & & & $\mu g / L$ \\
\hline Barium, total recoverable & 40 & /I & & & 1 & GE & & & & & & & $\mu g / L$ \\
\hline Cadmium, total recoverable & 0.92 & $\mathrm{~J} / \mathrm{E} /$ & NDD & & 1 & GE & $<1.0$ & $\mathrm{U} / \mathrm{I}$ & $<1.0$ & & 1 & GE & $\mu g / L$ \\
\hline Chromium, total recoverable & 2.0 & $\mathrm{~J} / \mathrm{E} /$ & NDD & & 1 & GE & & & & & & & $\mu g / 2$ \\
\hline Cobalt, total recoverable & $<0.20$ & $\mathbf{U} / /$ & $<0.20$ & & 1 & GE & & & & & & & $\mu g / 2$ \\
\hline Copper, total recoverable & 2.9 & $\| \prime$ & & & 1 & GE & & & & & & & $\mu g /$ \\
\hline Cyanide & $<10$ & $\mathrm{U} / 1$ & $<10$ & & 1 & GE & & & & & & & $\mu g / L$ \\
\hline Lead, total recoverable & 0.57 & $\mathrm{~J} / \mathrm{E}$ & NDD & & 1 & GE & 0.41 & $\mathrm{~J} / \mathrm{I} /$ & NDD & & 1 & GE & $\mu g / L$ \\
\hline Mercury, total recoverable & $<0.20$ & $\mathrm{U} / /$ & $<0.20$ & & 1 & GE & & & & & & & $\mu g / L$ \\
\hline $\begin{array}{l}\text { Nickel, total recoverable } \\
\text { Nitrate as nitrogen }\end{array}$ & 1.1 & $\|$ & & & 1 & GE & & & & & & & $\mu g /$ \\
\hline Nitrate-nitrite as nitrogen & 12,000 & $N I$ & & a & 5 & GE & 14,000 & $\|$ & & घ & 5 & GE & $\mu g / L$ \\
\hline Selenium, total recoverable & $<5.0$ & $\mathbf{U} / /$ & $<5.0$ & & 1 & GE & & & & & & & $\mu g / L$ \\
\hline Silver, total recoverable & $<1.0$ & $\mathrm{U} / /$ & $<1.0$ & & 1 & GE & & & & & & & $\mu g / L$ \\
\hline Thallium, total recoverable & 0.20 & $\mathrm{~J} / \mathrm{E}$ & NDD & & 1 & GE & & & & & & & $\mu g / L$ \\
\hline Vanadium, total recoverable & $<2.0$ & $\mathrm{U} / /$ & $<2.0$ & & 1 & GE & & & & & & & $\mu g / L$ \\
\hline Zinc, total recoverable & $<5.0$ & $\mathrm{U} / /$ & $<5.0$ & & 1 & GE & & & & & & & $\mu g / L$ \\
\hline \multicolumn{14}{|l|}{ Organic Constituents } \\
\hline Constituents & $\underline{3098}$ & Mod & Filt, & ST브 & $\underline{\text { DF }}$ & Lab & 4Q998 & Mod & Filt. & $\underline{\text { ST }} \underline{H}$ & $\underline{\mathrm{DF}}$ & 느모 & Unit \\
\hline Benzene & $<1.0$ & UJ/O/1 & $<1.0$ & & 1 & GE & & & & & & & $\mu g /$ \\
\hline Bis(2-ethylhexyl) phthalate & $<10$ & $\mathrm{U} / I$ & $<10$ & & 1 & GE & & & & & & & $\mu g / L$ \\
\hline Dichloromethane & $<1.9$ & UJNO8/1 & $<1.0$ & & 1 & GE & & & & & & & $\mu g / L$ \\
\hline Phenols & $<5.0$ & $\mathrm{U} / /$ & $<5.0$ & & 1 & GE & & & & & & & $\mu \mathrm{g} / \mathrm{L}$ \\
\hline Tetrachloroethylene & $<1.0$ & $\mathrm{UJ} / \mathrm{O} / 1$ & $<1.0$ & & 1 & GE & & & & & & & $\mu g / L$ \\
\hline Trichloroethylene & $<1.0$ & UJ/O/1 & $<1.0$ & & 1 & GE & & & & & & & $\mu g / L$ \\
\hline Trichlorofluoromethane & $<1.0$ & $\mathrm{UJ} / \mathrm{O} / 1$ & $<1.0$ & & 1 & GE & & & & & & & $\mu g / L$ \\
\hline
\end{tabular}

Notes:

- = exceeded holding time

- exceeded groundwater protection or monitoring constituent standard (See Appendix A.) 
WELL FSB $97 A$ (cont.)

Radioactive Constituents

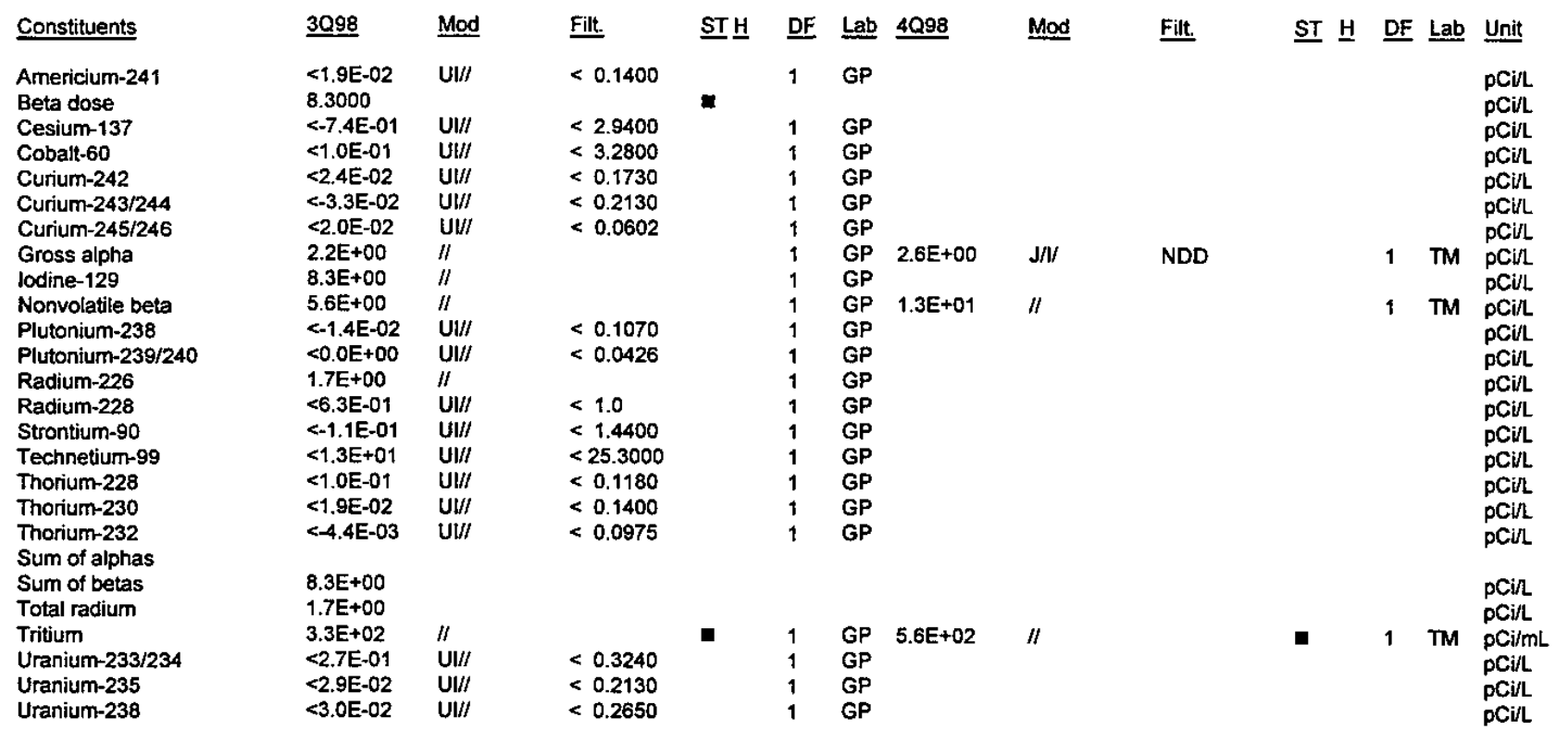

Notes:

- exceeded holding time

w = exceeded groundwater protection or monitoring constituent standard (See Appendix A.) 
WELL FSB 97C

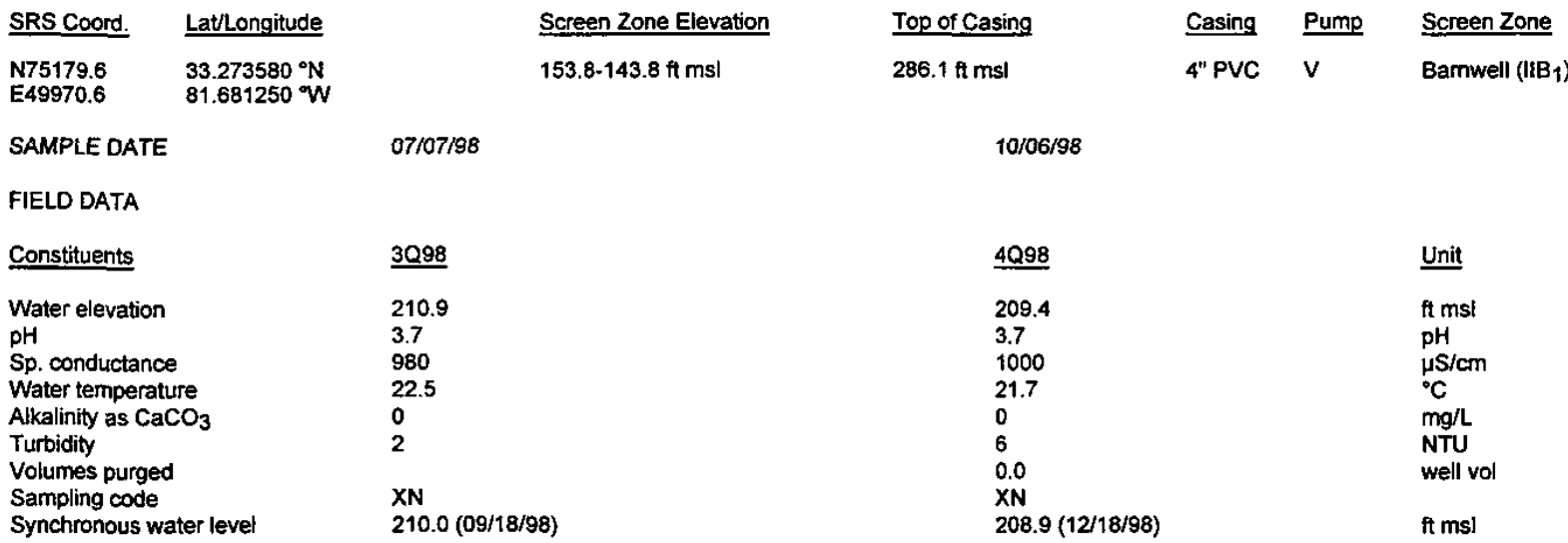

ANALYTICAL DATA

inorganic Constituents

\begin{tabular}{|c|c|c|c|c|c|c|c|c|c|c|c|c|c|}
\hline Constituents & $\underline{3 Q 98}$ & Mod & Filt. & ST브 & $\underline{\text { DF }}$ & $\underline{\text { Lab }}$ & 4Q98 & Mod & Filt. & $\underline{\mathrm{ST}} \underline{\mathrm{H}}$ & $\underline{\text { DF }}$ & $\underline{\text { Lab }}$ & Unit \\
\hline Antimony, total recoverable & $<0.078$ & UNI & $<0.20$ & & 1 & GE & & & & & & & $\mu g / L$ \\
\hline Arsenic, total recoverable & $<150$ & $\mathrm{U} / /$ & $<150$ & & 50 & GE & & & & & & & $\mu g / L$ \\
\hline Barium, total recoverable & 260 & $N /$ & & & 1 & GE & & & & & & & $\mu g / L$ \\
\hline Cadmium, total recoverable & 5.0 & $\|$ & & & 1 & GE & 4.7 & $\mathrm{~J} / / /$ & NDD & & 10 & GE & $\mu g^{\prime} \mathrm{L}$ \\
\hline Chromium, total recoverable & $<2.3$ & UNI & $<3.0$ & & $i$ & $\overrightarrow{G E}$ & & & & & & & $\mu g / L$ \\
\hline Cobalt, total recoverable & 100 & $/ /$ & & - & 1 & GE & & & & & & & $\mu g / L$ \\
\hline Copper, total recoverable & 60 & $N /$ & & & 1 & GE & & & & & & & $\mu g / L$ \\
\hline Cyanide & $<10$ & $\mathrm{U} / \prime$ & $<10$ & & 1 & $\overline{G E}$ & & & & & & & $\mu g / L$ \\
\hline Lead, total recoverable & 10 & NI & & & 1 & $\overline{G E}$ & 6.7 & $\mathrm{~J} / \mathrm{l} /$ & NDD & & 10 & GE & $\mu g / L$ \\
\hline Mercury, total recoverable & $<0.20$ & $\mathrm{U} / /$ & $<0.20$ & & 1 & GE & & & & & & & $\mu g / L$ \\
\hline $\begin{array}{l}\text { Nickel, total recoverable } \\
\text { Nitrate as nitrogen }\end{array}$ & $<35$ & UNI & $<0.20$ & & 1 & GE & & & & & & & $\mu g / l$ \\
\hline Nitrate-nitrite as nitrogen & 110,000 & $\|$ & & $\mathbf{0}$ & 75 & GE & 130,000 & $\|$ & & ש & 50 & GE & $\mu g / L$ \\
\hline Selenium, total recoverable & $<250$ & $\mathbf{U} / /$ & $<250$ & & 50 & GE & & & & & & & $\mu g / L$ \\
\hline Silver, total recoverable & $<1.0$ & $\mathrm{u} / \prime$ & $<1.0$ & & 1 & $\overline{G E}$ & & & & & & & $\mu$ gll \\
\hline Thallium, total recoverable & 0.28 & $\mathrm{~J} / \mathrm{E} /$ & NDD & & 1 & GE & & & & & & & Hg/L \\
\hline Vanadium, total recoverable & $<2.0$ & $\mathrm{U} / /$ & $<2.0$ & & 1 & GE & & & & & & & $\mu g / L$ \\
\hline Zinc, total recoverable & 120 & $N \prime$ & & & 1 & GE & & & & & & & $\mu g / L$ \\
\hline \multicolumn{14}{|l|}{ Organic Constituents } \\
\hline Constituents & 3098 & $\underline{\text { Mod }}$ & Filt. & ST브 & $\underline{\mathrm{DF}}$ & $\underline{\text { Lab }}$ & 4Q98 & Mod & Filt. & $\underline{\mathrm{ST}} \underline{\mathrm{H}}$ & $\underline{D F}$ & Lab & Unit \\
\hline Benzene & $<1.0$ & $\mathrm{U} / /$ & $<1.0$ & & 1 & GE & & & & & & & $\mu g / L$ \\
\hline Bis(2-ettylhexyl) phthalate & $<10$ & $\mathrm{U} / /$ & $<10$ & & 1 & GE & & & & & & & $\mu g / L$ \\
\hline Dichloromethane & $<3.8$ & UN8I & $<1.0$ & & 1 & GE & & & & & & & $\mu \mathrm{g} / \mathrm{L}$ \\
\hline Phenols & $<5.0$ & 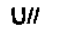 & $<5.0$ & & 1 & GE & & & & & & & $\mu g / L$ \\
\hline Tetrachloroethylene & $<1.0$ & $\mathrm{U} / /$ & $<1.0$ & & 1 & GE & & & & & & & $\mu g / L$ \\
\hline Trichloroethylene & 33 & $/ /$ & & $\mathbf{E}$ & 1 & GE & & & & & & & $\mu g / L$ \\
\hline Trichlorofluoromethane & $<1.0$ & $\mathrm{U} / /$ & $<1.0$ & & 1 & GE & & & & & & & $\mu g / L$ \\
\hline
\end{tabular}

Notes:

- exceeded holding time

= exceeded groundwater protection or monitoring constituent standard (See Appendix A.)

F-Area HWMF 
WELL FSB $97 \mathrm{C}$ (cont.)

Radioactive Constituents

\begin{tabular}{|c|c|c|c|c|c|c|c|c|c|c|c|c|c|}
\hline Constituents & $\underline{3098}$ & Mod & Filt. & $\underline{\text { ST}} \underline{H}$ & DF & $\underline{\text { Lab }}$ & $\underline{4 Q 98}$ & Mod & Filt. & $\underline{\text { ST }} \underline{H}$ & DF & $\underline{\text { Lab }}$ & Unit \\
\hline Americium-241 & $1.4 \mathrm{E}+01$ & $\|$ & & & 1 & GP & & & & & & & $\mathrm{pCi} /$ \\
\hline Beta dose & 58.3833 & & & a & & & & & & & & & $\mathrm{pCil}$ \\
\hline Cesium-137 & $<1.7 \mathrm{E}+\infty 0$ & $\mathrm{U} \mid \mathrm{I} /$ & $<2.8700$ & & 1 & GP & & & & & & & $\mathrm{pCil}$ \\
\hline Cobalt-60 & $<1.2 \mathrm{E}+00$ & $\mathrm{UI} / \mathrm{I}$ & $<3.1600$ & & 1 & $G P$ & & & & & & & $\mathrm{pCil}$ \\
\hline Curium-242 & $<3.7 \mathrm{E}-02$ & $U \mathrm{~W} / \mathrm{I}$ & $<0.1610$ & & 1 & GP & & & & & & & pCill \\
\hline Curium-243/244 & 1.1E+01 & $\|$ & & & 1 & GP & & & & & & & pCill \\
\hline Curium-245/246 & $1.1 \mathrm{E}+00$ & $\|$ & & & 1 & GP & & & & & & & $\mathrm{pCIL}$ \\
\hline Gross alpha & $6.0 E+02$ & $\mathrm{~J} / / / 1$ & NDD & & 1 & $G P$ & $3.8 \mathrm{E}+02$ & $\|$ & & ш & 1 & $\mathrm{GE}$ & $\mathrm{pCin}$ \\
\hline lodine-129 & $3.7 E+01$ & $\|$ & & & 1 & $G P$ & & & & & & & $\mathrm{pCin}$ \\
\hline Nonvolatile beta & $6.1 \mathrm{E}+02$ & $\mathrm{~J} / \mathrm{IV} / 1$ & NDD & & 1 & GP & $5.4 \mathrm{E}+02$ & "I & & m & 1 & GE & pCilL \\
\hline Plutonium-238 & 1.1 $1 E+\infty 0$ & R/4i & $\operatorname{Rej}$ & & $i$ & GP & & & & & & & $\mathrm{pCil}$ \\
\hline Plutonium-239/240 & $<2.6 \mathrm{E}-01$ & $\mathrm{UJ} / \mathrm{CV} /$ & $<0.0713 \mathrm{NDD}$ & & 1 & GP & & & & & & & $\mathrm{pCilL}$ \\
\hline Radium-226 & $3.7 \mathrm{E}+01$ & "I & & ш & 1 & GP & & & & & & & $\mathrm{pCin}$ \\
\hline Radium-228 & $1.6 \mathrm{E}+01$ & $\mathrm{~J} / 1 / 2$ & NDD & & 1 & GP & & & & & & & $\mathrm{PCi} / \mathrm{L}$ \\
\hline Strontium-90 & $1.7 E+02$ & 11 & & - & 1 & GP & & & & & & & $\mathrm{pCin}$ \\
\hline Technetium-99 & $1.2 E+02$ & $\|$ & & a & 1 & $G P$ & & & & & & & pCill \\
\hline Thorium-228 & $<4.0 E-01$ & Uw/ & $<1.0900$ & & 1 & GP & & & & & & & pCil \\
\hline Thorium-230 & $3.6 \mathrm{E}-01$ & 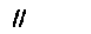 & & & 1 & $G P$ & & & & & & & $\mathrm{pCil}$ \\
\hline Thorium-232 & $<-1.6 \mathrm{E}-01$ & UI// & $<0.6760$ & & 1 & GP & & & & & & & pCin \\
\hline Sum of alphas & $4.8 \mathrm{E}+02$ & & & - & & & & & & & & & $\mathrm{pCilL}$ \\
\hline Sum of betas & $3.4 \mathrm{E}+02$ & & & - & & & & & & & & & pCilL \\
\hline Total radium & $5.4 E+01$ & & & - & & & & & & & & & $\mathrm{pCi} / \mathrm{L}$ \\
\hline Tritium & $3.8 E+03$ & $\|$ & & $=$ & 1 & GP & $4.6 E+03$ & "I & & $=$ & 1 & GE & pCimL \\
\hline Uranium-233/234 & $1.7 \mathrm{E}+02$ & $\|$ & & - & 1 & GP & & & & & & & $\mathrm{pCilL}$ \\
\hline Uranium-235 & $2.1 E+01$ & "l & & - & 1 & GP & & & & & & & $\mathrm{pCilL}$ \\
\hline Uranium-238 & $2.6 E+02$ & /I & & 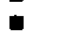 & 1 & GP & & & & & & & $\mathrm{pCi} / \mathrm{L}$ \\
\hline
\end{tabular}

Notes:

- exceeded holding time

- exceeded groundwater protection or monitoring constituent standard (See Appendix A.) 
WELL FSB 97D

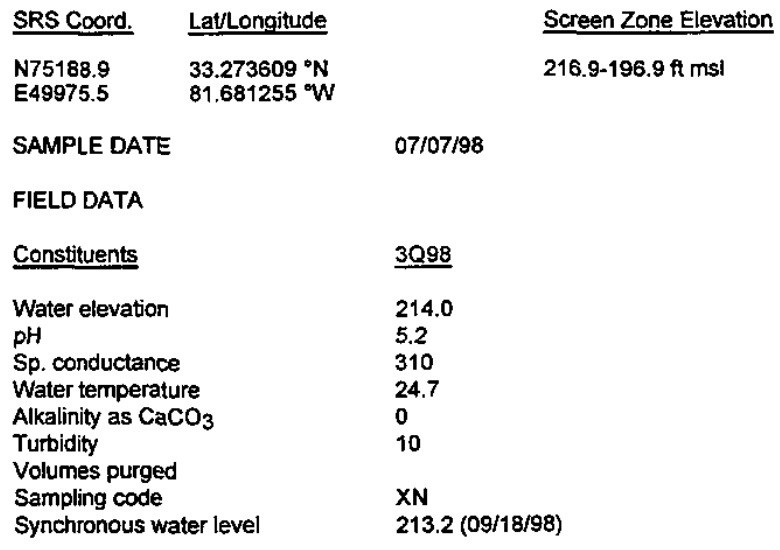

\begin{tabular}{|c|c|c|c|}
\hline Top of Casing & Casing & Pump & Screen Zone \\
\hline $286 \mathrm{ft} \mathrm{msl}$ & 4" PVC & v & Water Table (IIB2) \\
\hline
\end{tabular}

$10 / 06 / 98$

ANALYTICAL DATA

Inorganic Constituents

3098

Mod Filt. ST

ST브

DF Lab 4Q98

Mod

Filt.

ST 브 DF Lab Unit

Antimony, total recoverable $<0.14$

Arsenic, total recoverable $<3.0$

Barium, total recoverable 230

Cadmium, total recoverable 2.6

Chromium, total recoverable 7.6

Cobalt, total recoverable $\quad 8.5$

Copper, total recoverable $\quad 33$

Cyanide

Lead, total recoverable

Mercury, total recoverable $\quad<0.20$

Nickel, total recoverable $<14$

UNI $<0.20$

UII $\quad<3.0$

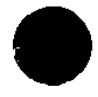

Nitrate as nitrogen

Nitrate-nitrite as nitrogen $\quad 45,000$

Selenium, total recoverable $<5.0$

Silver, total recoverable

Thallium, total recoverable $<2.5$

Vanadium, total recoverable $<2.0$

Zinc, total recoverable

58

NI

$<3.0$

Organic Constituents

Constituents

$\underline{3098}$

Mod

Filt.

GE

$N 1$

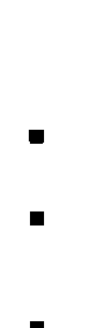

GE

GE

(1)

4Q98

$\underline{\text { Unit }}$

212.9
4.2

430

21.6

5

0.0

$212.0(12 / 18 / 98)$

ft mst

$\mathrm{pH}$

$\mu \mathrm{S} / \mathrm{cm}$

${ }^{\circ} \mathrm{C}$

mg/L

well vol

ft msl

Benzene

$<1.0$

Bis(2-ethylhexyl) phthalate

Dichloromethane

Phenols

Tetrachloroethylene

Trichloroethylene

$<10$
$<1.0$

$<1.0$
$<5.0$

Trichlorofluoromethane

$<1.0$

$<1.0$

$\begin{array}{ll}U / I & <1.0 \\ U J / O / & <10 \\ U / I & <1.0 \\ U I I & <5.0 \\ U I I & <1.0 \\ U / I & <1.0 \\ U I I & <1.0\end{array}$

ST브

$\begin{array}{llll}\text { DF } & \text { Lab } & \text { 4Q998 } & \text { Mod } \\ 1 & \text { GE } & \\ 1 & \text { GE } & \\ 1 & \text { GE } & \\ 1 & \text { GE } & \\ 1 & \text { GE } & \\ 1 & \text { GE } & \\ 1 & \text { GE } & \end{array}$

Filt.

SI H DF Lab Unit

$\mu g / L$
$\mu g / L$
$\mu g / L$
$\mu g / L$
$\mu g / L$
$\mu g / L$
$\mu g / L$

Notes:

- = exceeded holding time

= exceeded groundwater protection or monitoring constituent standard (See Appendix A.)

F-Area HWMF 
WELL FSB 97D (cont.)

Radioactive Constituents

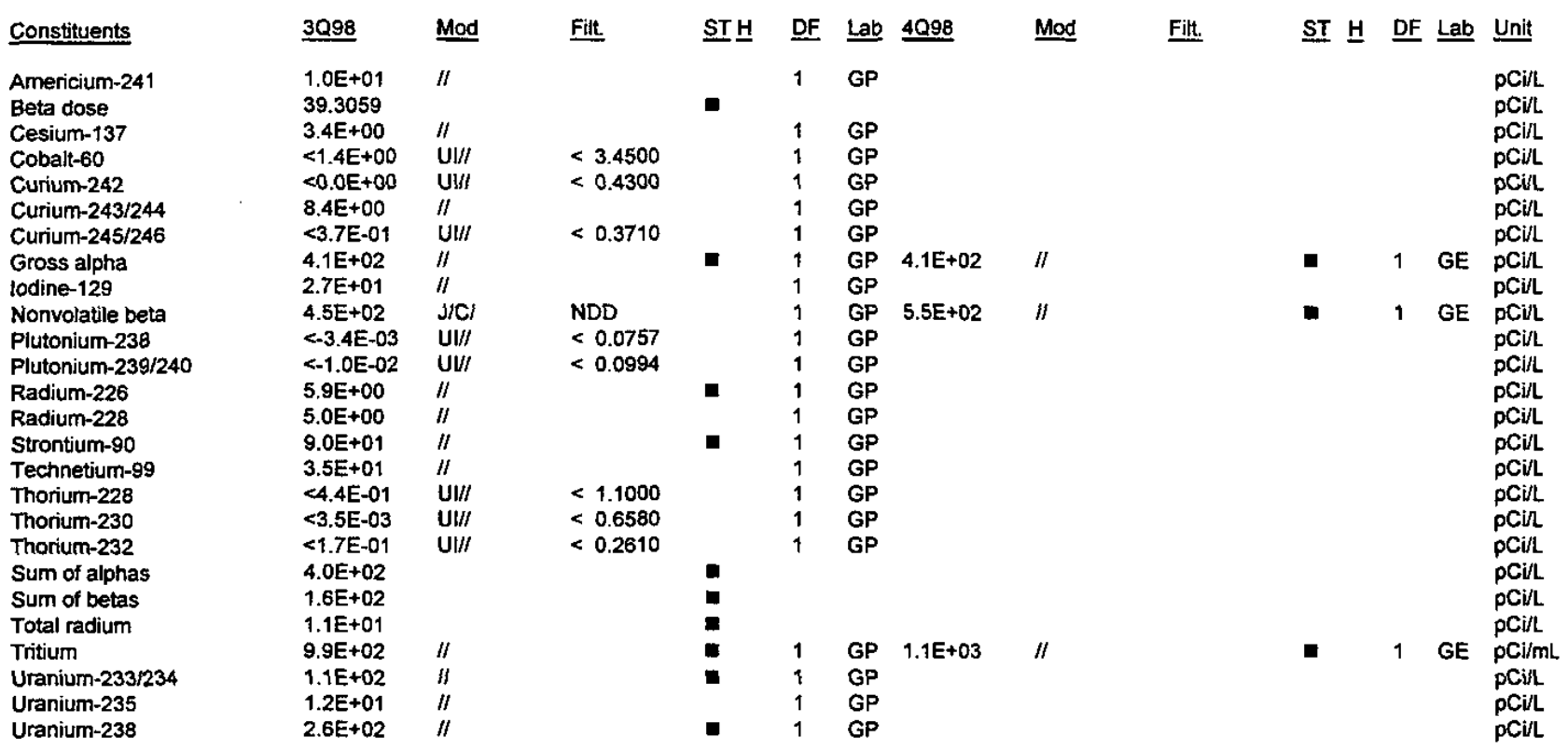

Notes:

- = exceeded holding time

- = exceeded groundwater protection or monitoring constituent standard (See Appendix A.) 
WELL FSB 98AR

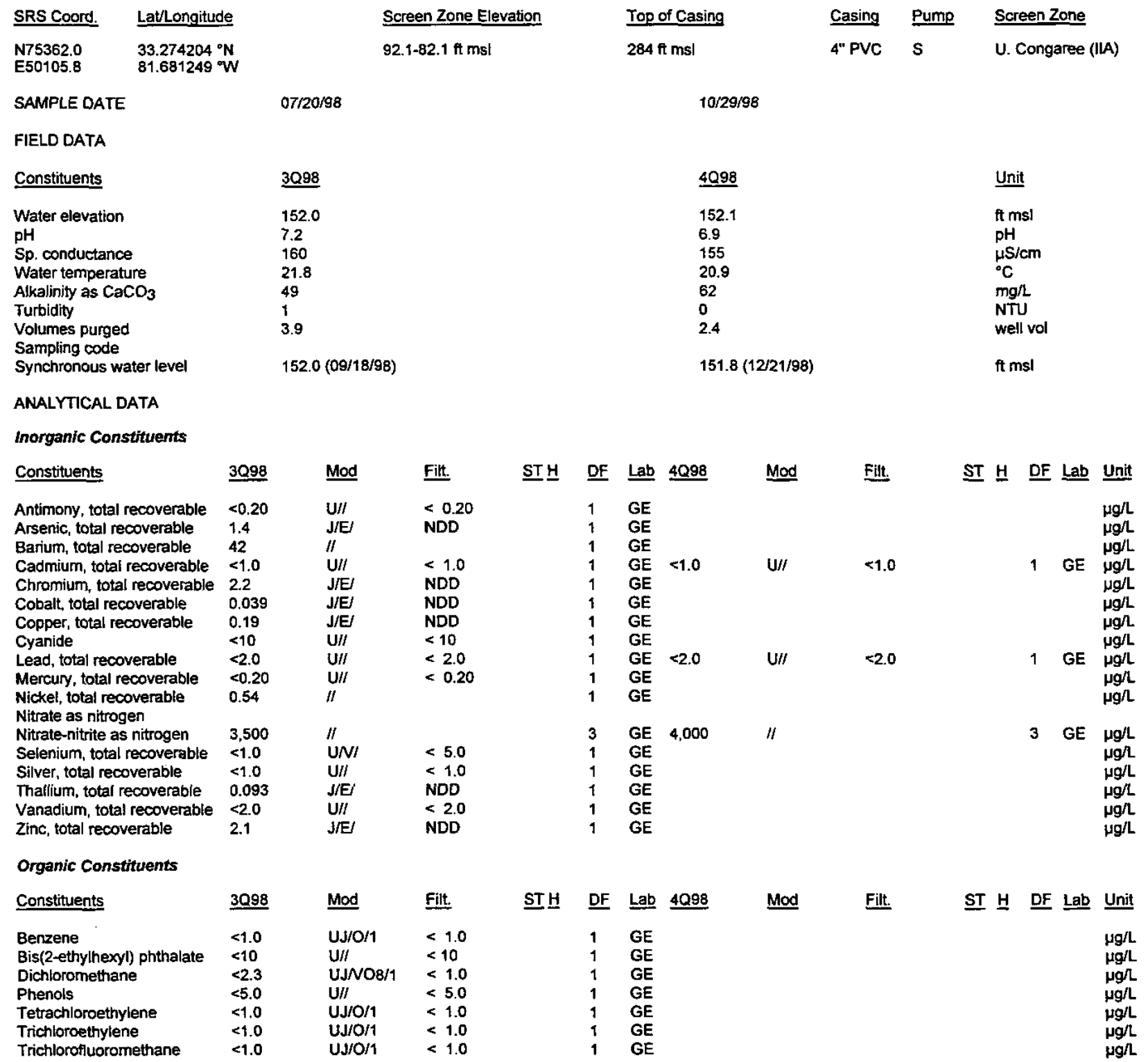

Notes:

- exceeded holding time

- exceeded groundwater protection or monitoring constituent standard (See Appendix A.) 
WELL FSB 98AR (cont.)

Radioactive Constituents

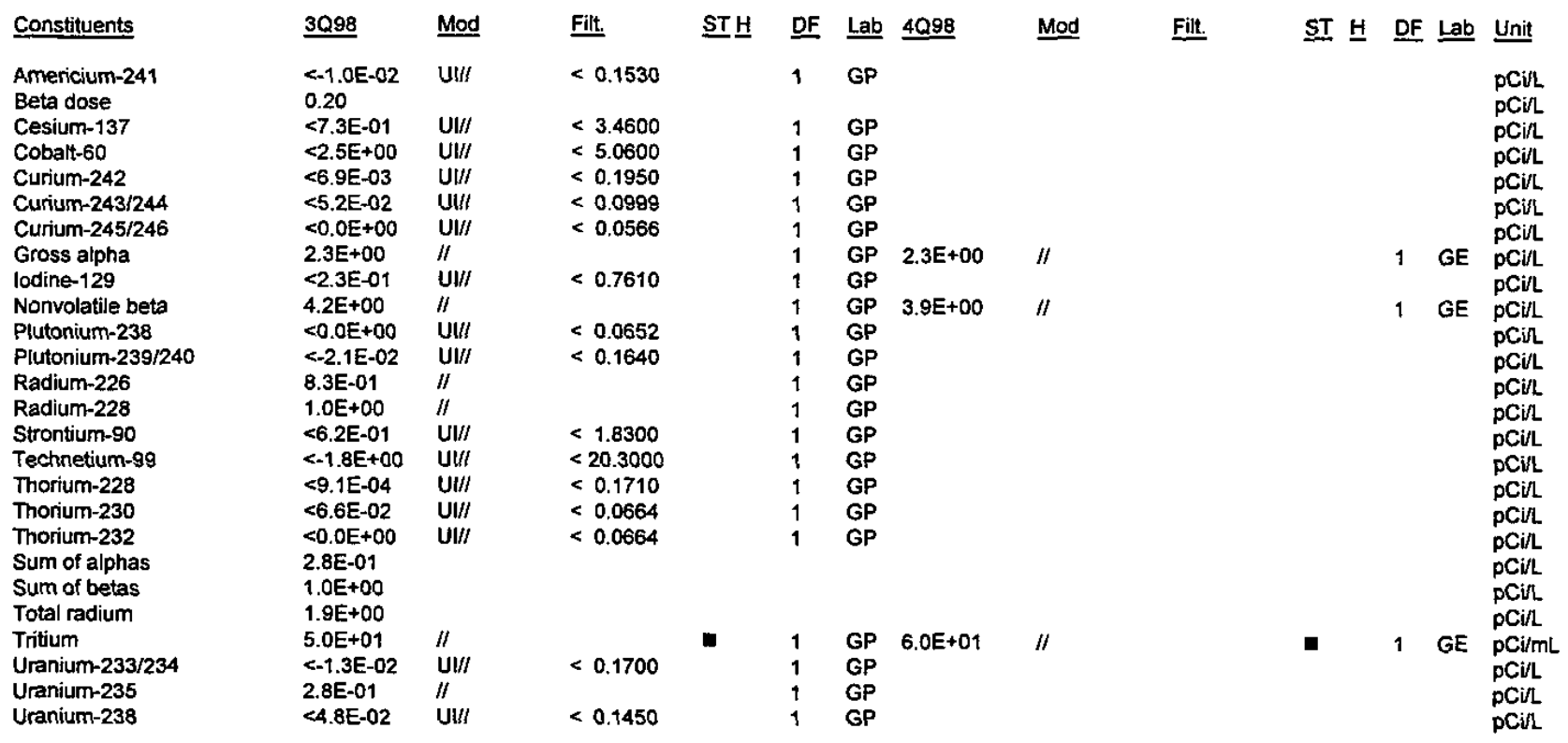

Notes:

- = exceeded holding time

- = exceeded groundwater protection or monitoring constituent standard (See Appendix A.)

F-Area HWMF

C-82 
WELL FSB 98C

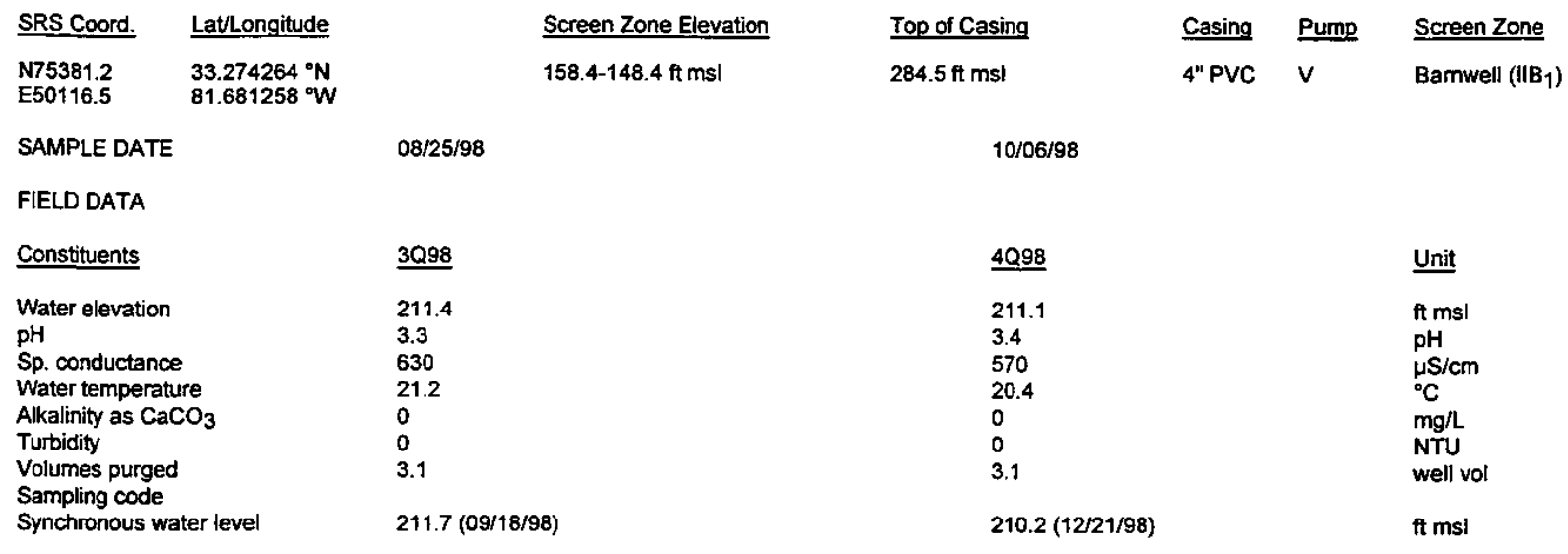

ANALYTICAL DATA

Inonganic Constituents

\begin{tabular}{|c|c|c|c|c|c|c|c|c|c|c|c|c|c|}
\hline Constituents & $\underline{3098}$ & Mod & Eilt. & $\underline{\text { ST }} \underline{H}$ & DF & $\underline{\text { Lab }}$ & 4Q98 & Mod & Filt. & $\underline{\mathrm{ST}} \underline{\mathrm{H}}$ & $\underline{D F}$ & Lab & Unit \\
\hline Antimony, total recoverable & $<0.20$ & $\mathbf{U} / l$ & $<0.20$ & & 1 & GE & & & & & & & $\mu g / L$ \\
\hline Arsenic, total recoverable & $<75$ & UJ/I/1 & $<75.0$ & & 25 & $\overline{G E}$ & & & & & & & $\mu g / h$ \\
\hline Barium, total recoverable & 200 & $\|$ & & & 1 & $\overline{G E}$ & & & & & & & $\mu g / \mathrm{h}$ \\
\hline Cadmium, total recoverable & 5.3 & $\|$ & & $\mathbf{\square}$ & 1 & GE & 5.3 & $/ 1$ & & - & 1 & GE & $\mu g / L$ \\
\hline Chromium, total recoverable & 0.71 & $\mathrm{~J} / \mathrm{E} /$ & NDD & & 1 & $G E$ & & & & & & & $\mu g / 2$ \\
\hline Cobalt, total recoverable & 120 & 11 & & 口 & 1 & GE & & & & & & & $\mu g / L$ \\
\hline Copper, total recoverable & 26 & 11 & & & 1 & GE & & & & & & & $\mu g / L$ \\
\hline Cyanide & 4.2 & $\mathrm{~J} / \mathrm{El} / 1$ & NDD & & 1 & GE & & & & & & & $\mu g / L$ \\
\hline Lead, total recoverable & 0.42 & $\mathrm{~J} / \mathrm{E} /$ & NDD & & 1 & GE & 0.43 & $\mathrm{~J} / \mathrm{N}$ & NOD & & 1 & GE & $\mu \mathrm{g} / \mathrm{L}$ \\
\hline Mercury, total recoverable & 0.14 & $\mathrm{~J} / \mathrm{E} /$ & NDD & & 1 & GE & & & & & & & $\mu g / L$ \\
\hline $\begin{array}{l}\text { Nickel, total recoverable } \\
\text { Nitrate as nitrogen }\end{array}$ & 19 & $\|$ & & & 1 & $\mathrm{GE}$ & & & & & & & $\mu g / L$ \\
\hline Nitrate-nitrite as nitrogen & 62,000 & $\|$ & & $\boldsymbol{\square}$ & 25 & GE & 61,000 & $/ I$ & & घ & 50 & GE & $\mu g / L$ \\
\hline Selenium, total recoverable & $<130$ & $\mathrm{UJ} / \mathrm{I} / 1$ & $<125.0$ & & 25 & GE & & & & & & & $\mu g / L$ \\
\hline Silver, total recoverable & $<1.0$ & $\mathrm{U} / /$ & $<1.0$ & & 1 & GE & & & & & & & $\mu g / L$ \\
\hline Thallium, total recoverable & 1.0 & $\mathrm{~J} / \mathrm{E} /$ & NDD & & 1 & GE & & & & & & & Holl \\
\hline Vanadium, total recoverable & $<2.0$ & $\mathrm{U} / \prime$ & $<2.0$ & & 1 & $\overline{G E}$ & & & & & & & $\mu g / L$ \\
\hline Zinc, total recoverable & 52 & $\mathrm{~J} / / / 1$ & NDD & & 1 & GE & & & & & & & $\mu g / L$ \\
\hline \multicolumn{14}{|l|}{ Organic Constituerrts } \\
\hline Constituents & $\underline{3098}$ & Mod & Filt. & $\underline{\text { ST }} \underline{H}$ & $\underline{\mathrm{DF}}$ & Lab & 4Q98 & Mod & Filt. & $\underline{\text { ST }} \underline{H}$ & DF & Lab & Unit \\
\hline Benzene & $<1.0$ & $\mathrm{U} / /$ & $<1.0$ & & 1 & GE & & & & & & & $\mu g / L$ \\
\hline Bis(2-ethylhexyl) phthalate & $<10$ & $\mathrm{U} / I$ & $<10$ & & 1 & GE & & & & & & & $\mu g / L$ \\
\hline Dichloromethane & $<5.0$ & $\mathrm{U} / I$ & $<5.0$ & & 1 & GE & & & & & & & $\mu g / L$ \\
\hline Phenols & $<5.0$ & $\mathrm{U} / I$ & $<5.0$ & & 1 & GE & & & & & & & $\mu g / L$ \\
\hline Tetrachloroethylene & $<1.0$ & $\mathrm{U} / /$ & $<1.0$ & & 1 & $\overline{G E}$ & & & & & & & $\mu g /$ \\
\hline Trichloroethylene & 44 & $/ /$ & & $\mathbf{a}$ & 1 & GE & & & & & & & $\mu g / L$ \\
\hline Trichlorofluoromethane & $<5.0$ & $\mathrm{U} / I$ & $<5.0$ & & 1 & $\mathrm{GE}$ & & & & & & & $\mu g /$ \\
\hline
\end{tabular}

Notes:

- = exceeded holding time

- = exceeded groundwater protection or monitoring constituent standard (See Appendix A.) 


\section{WELL FSB 98C (cont.)}

Radioactive Constituents

\begin{tabular}{|c|c|c|c|c|c|c|c|c|c|c|c|c|c|}
\hline Constituents & 3098 & Mod & Filt. & $\underline{\mathrm{ST}} \underline{\mathrm{H}}$ & $\underline{\mathrm{DF}}$ & Lab & 4Q98 & Mod & Filt. & ST $\underline{H}$ & DF & $\underline{\underline{L a b}}$ & Unit \\
\hline Americium-241 & $7.9 E+00$ & $\|$ & & & 1 & GP & & & & & & & $\mathrm{pCil}$ \\
\hline Beta dose & 52.3122 & & & 口 & & & & & & & & & pCill \\
\hline Cesium-137 & $<1.8 \mathrm{E}+00$ & $\mathrm{UI} /$ & $<2.8100$ & & 1 & GP & & & & & & & $\mathrm{pCi} / \mathrm{L}$ \\
\hline Cobalt-60 & $<-4.9 E-01$ & $\mathrm{UI} / /$ & $<2.9500$ & & 1 & GP & & & & & & & $\mathrm{pCi} / \mathrm{L}$ \\
\hline Curium-242 & $<-9.5 E-02$ & $\mathrm{UII}$ & $<0.6150$ & & 1 & GP & & & & & & & $\mathrm{pCi} / \mathrm{L}$ \\
\hline Curium-243/244 & $5.5 E+00$ & 11 & & & 1 & GP & & & & & & & $\mathrm{pCi} / \mathrm{L}$ \\
\hline Curium-245/246 & $<2.6 \mathrm{E}-01$ & $\mathrm{U} ! \prime \prime$ & $<0.1970$ & & 1 & GP & & & & & & & pCill \\
\hline Gross alpha & $6.0 \mathrm{E}+02$ & $\| 1$ & & $\boldsymbol{\square}$ & 1 & GP & $5.3 E+02$ & $\|$ & & ט & 1 & GE & pCill \\
\hline lodine-129 & $3.1 E+01$ & $/ 1$ & & & 1 & GP & & & & & & & $\mathrm{pCi} / \mathrm{L}$ \\
\hline Nonvolatile beta & $6.2 \mathrm{E}+02$ & $\mathrm{~J} / \mathrm{C} /$ & NDD & & 1 & GP & $5.6 \mathrm{E}+02$ & $/ 1$ & & ש & 1 & GE & $\mathrm{pCi} / \mathrm{L}$ \\
\hline Piutonium-238 & $<4.9 \mathrm{E}-02$ & UWI & $<0.3480$ & & 1 & GP & & & & & & & pCill \\
\hline Plutonium-239/240 & $<0.0 \mathrm{E}+00$ & $\mathrm{UU} /$ & $<0.1950$ & & 1 & GP & & & & & & & $\mathrm{pCil}$ \\
\hline Radium-226 & $2.3 E+01$ & $\|$ & & $=$ & 1 & GP & & & & & & & $\mathrm{pCil}$ \\
\hline Radium-228 & $<-5.9 E-01$ & UII/ & $<1.0900$ & & 1 & GP & & & & & & & $\mathrm{pCi} / \mathrm{L}$ \\
\hline Strontium-90 & $1.7 E+02$ & /I & & - & 1 & GP & & & & & & & $\mathrm{pCil}$ \\
\hline Technetium-99 & $5.6 \mathrm{E}+01$ & /I & & $m$ & 1 & GP & & & & & & & $\mathrm{pCi} / \mathrm{L}$ \\
\hline Thorium-228 & $<6.6 \mathrm{E}-01$ & $\mathrm{UU} /$ & $<0.6900$ & & 1 & GP & & & & & & & $\mathrm{pCin}$ \\
\hline Thorium-230 & $<3.0 \mathrm{E}-01$ & $\mathrm{UI} / /$ & $<0.4920$ & & 1 & GP & & & & & & & pCill \\
\hline Thorium-232 & $<3.7 E-02$ & $U I / /$ & $<0.4410$ & & 1 & GP & & & & & & & pCin \\
\hline Sum of alphas & $6.6 E+02$ & & & $\boldsymbol{\square}$ & & & & & & & & & $\mathrm{pCi} / \mathrm{L}$ \\
\hline Sum of betas & $2.6 E+02$ & & & $\mathbf{\square}$ & & & & & & & & & pCill \\
\hline Total radium & $2.3 E+01$ & & & $\overline{\mathbf{a}}$ & & & & & & & & & pCill \\
\hline Tritium & $2.6 E+03$ & $/ /$ & & च & 1 & GP & $2.4 E+03$ & $/ /$ & & $\mathbf{a}$ & 1 & GE & pCi/mL \\
\hline Uranium-233/234 & $2.3 E+02$ & $/ 1$ & & - & 1 & GP & & & & & & & $\mathrm{pCi} / \mathrm{L}$ \\
\hline Uranium-235 & $2.2 \mathrm{E}+01$ & $/ /$ & & $\mathbf{a}$ & 1 & GP & & & & & & & pCill. \\
\hline Uranium-238 & $3.9 E+02$ & $/ 1$ & & $\mathbf{\square}$ & 1 & $G P$ & & & & & & & pCill \\
\hline
\end{tabular}

Notes:

= exceeded holding time

= exceeded groundwater protection or monitoring constituent standard (See Appendix A.) 
WELL FSB 98D

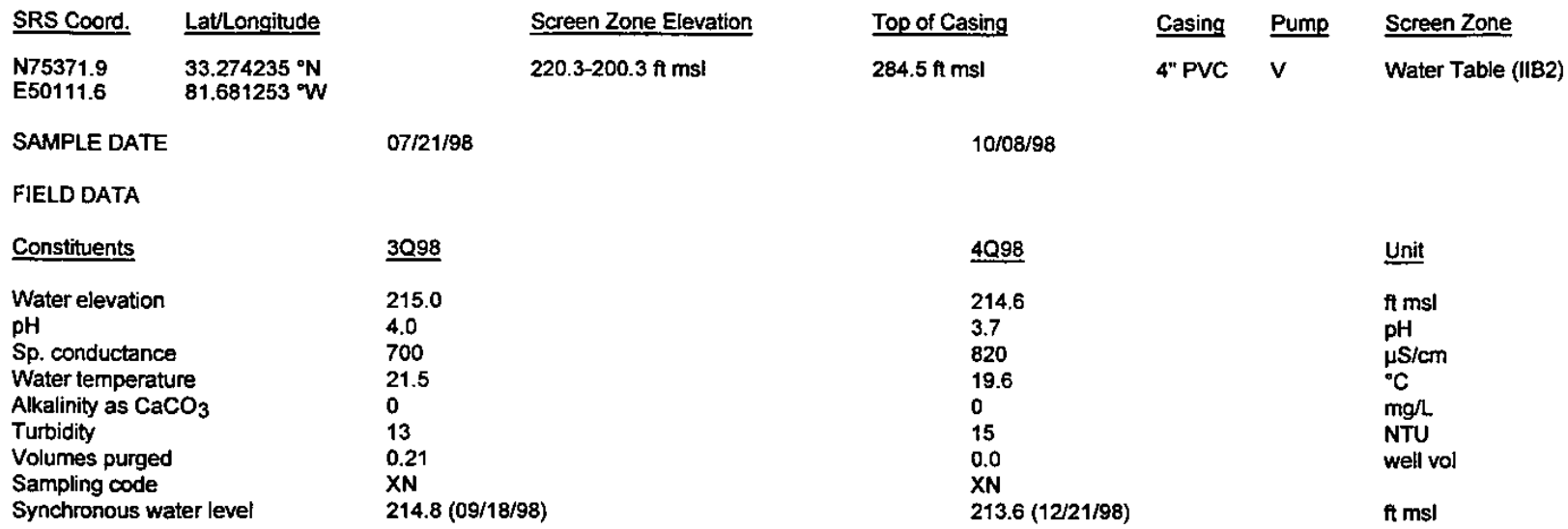

ANALYTICAL DATA

Inorganic Constituents

\begin{tabular}{|c|c|c|c|c|c|c|c|c|c|c|c|c|c|}
\hline Constituents & $3 Q 98$ & Mod & Filt. & ST $\underline{H}$ & $\underline{\text { DF }}$ & Lab & 4Q98 & Mod & Filt. & ST $\underline{H}$ & DF & Lab & Unit \\
\hline Antimony, total recoverable & $<0.20$ & $u / \prime$ & $<0.20$ & & 1 & GE & & & & & & & $\mu g / L$ \\
\hline Arsenic, total recoverable & $<3.0$ & $U / I$ & $<3.0$ & & 1 & GE & & & & & & & rgl \\
\hline Barium, total recoverable & 450 & $\|$ & & & 1 & GE & & & & & & & $\mu g / L$ \\
\hline Cadmium, total recoverable & 9.7 & "I & & च & 1 & GE & 16 & /I & & ש & 1 & GE & $\mu g / L$ \\
\hline Chromium, total recoverable & 3.1 & $\|$ & & & 1 & GE & & & & & & & $\mu g / L$ \\
\hline Cobalt, total recoverable & 15 & $\|$ & & - & 1 & GE & & & & & & & $\mu g / L$ \\
\hline Copper, total recoverable & 21 & /I & & & 1 & GE & & & & & & & $\mu g / L$ \\
\hline Cyanide & 2.2 & $\mathrm{~J} / \mathrm{E} /$ & NDD & & 1 & GE & & & & & & & $\mu g / L$ \\
\hline Lead, total recoverable & 2.3 & $N I$ & & & 1 & GE & 13 & /I & & & 1 & GE & $\mu g / L$ \\
\hline Mercury, total recoverable & 0.061 & $\mathrm{~J} / \mathrm{E} /$ & NDD & & 1 & GE & & & & & & & $\mu g / L$ \\
\hline $\begin{array}{l}\text { Nickel, total recoverable } \\
\text { Nitrate as nitrogen }\end{array}$ & 15 & $\|$ & & & 1 & $\overline{G E}$ & & & & & & & $\mu g / L$ \\
\hline Nitrate-nitrite as nitrogen & 94,000 & $N \prime$ & & $\mathbf{v}$ & 50 & GE & 100,000 & $\|$ & & $\boldsymbol{\square}$ & 50 & GE & $\mu g / L$. \\
\hline Selenium, total recoverable & $<5.0$ & $U / I$ & $<5.0$ & & 1 & GE & & & & & & & $\mu \mathrm{g} / \mathrm{L}$ \\
\hline Silver, total recoverable & $<1.0$ & $u / I$ & $<1.0$ & & 1 & GE & & & & & & & $\mu g / L$ \\
\hline Thallium, total recoverable & 0.28 & $\mathrm{~J} / \mathrm{E} /$ & NDD & & 1 & $\mathrm{GE}$ & & & & & & & (L \\
\hline Vanadium, total recoverable & $<2.0$ & $\mathrm{U} / /$ & $<2.0$ & & 1 & GE & & & & & & & $\mathrm{g} / \mathrm{L}$ \\
\hline Zinc, total recoverable & 43 & $\|$ & & & 1 & GE & & & & & & & $g / L$ \\
\hline
\end{tabular}

Organic Constituents

\begin{tabular}{|c|c|c|c|c|c|c|c|c|c|c|c|}
\hline Constituents & $\underline{3098}$ & Mod & Filt. & ST브 & $\underline{\text { DF }}$ & $\underline{\text { Lab }}$ & 4Q98 & Mod & Filt. & $\underline{\text { ST }} \underline{\mathrm{H}} \underline{\mathrm{DF}}$ Lab & Unit \\
\hline Benzene & $<1.0$ & $\mathrm{UJ} / \mathrm{O} / 1$ & $<1.0$ & & 1 & GE & & & & & $\mu g / \mathrm{L}$ \\
\hline Bis(2-ethylhexyl) phthalate & $<10$ & $\mathrm{UJ} / \mathrm{Q} /$ & $<10$ & $\bullet$ & 1 & GE & & & & & $\mu g / 2$ \\
\hline Dichloromethane & $<1.0$ & $\mathrm{UJ} / \mathrm{O} / 1$ & $<1.0$ & & 1 & GE & & & & & $\mu g / L$ \\
\hline Phenois & $<5.0$ & $U / l$ & $<5.0$ & & 1 & GE & & & & & $\mu g / L$ \\
\hline Tetrachloroethylene & $<1.0$ & UJ/O/1 & $<1.0$ & & 1 & GE & & & & & $\mu g h$ \\
\hline Trichloroethylene & $<1.0$ & $\mathrm{UJ} / \mathrm{O} / 1$ & $<1.0$ & & 1 & GE & & & & & $\mu g /$ \\
\hline Trichlorofluoromethane & $<1.0$ & $\mathrm{UJ} / \mathrm{O} / 1$ & $<1.0$ & & 1 & GE & & & & & $\mathrm{ug} /$ \\
\hline
\end{tabular}

Notes:

- = exceeded holding time

= exceeded groundwater protection or monitoring constituent standard (See Appendix A.) 


\section{WELL FSB 98D (cont.)}

Radioactive Constituents

\begin{tabular}{|c|c|c|c|c|c|c|c|c|}
\hline Constituents & 3098 & Mod & Filit. & ST브 & DF & $\underline{\text { Lab }}$ & 4Q98 & Mod \\
\hline Americium-241 & $1.3 E+01$ & $\|$ & & & 1 & GP & & \\
\hline Beta dose & 94.4478 & & & a & & & & \\
\hline Cesium-137 & $<8.6 \mathrm{E}-01$ & $\mathrm{UI} / /$ & $<3.3700$ & & 1 & GP & & \\
\hline Cobalt-60 & $<4.2 E-01$ & $\mathrm{UV} / /$ & $<4.3400$ & & 1 & GP & & \\
\hline Curium-242 & $<0.0 E+00$ & $\mathrm{UI} / /$ & $<0.0971$ & & 1 & GP & & \\
\hline Curium-243/244 & $1.1 E+01$ & $\|$ & & & 1 & GP & & \\
\hline Curium-245/246 & $5.4 \mathrm{E}-01$ & $n$ & & & 1 & GP & & \\
\hline Gross alpha & $2.7 \mathrm{E}+02$ & /1 & & - & 1 & GP & $7.5 E+02$ & $\|$ \\
\hline lodine-129 & $4.5 \mathrm{E}+01$ & "I & & & 1 & GP & & \\
\hline Nonvolatile beta & $9.1 E+02$ & II & & $\boldsymbol{u}$ & 1 & GP & $1.4 E+03$ & $\|$ \\
\hline Plutonium-238 & $<0.0 \mathrm{E}+00$ & $\mathrm{UV} / /$ & $<0.1380$ & & 1 & GP & & \\
\hline Plutonium-239/240 & $<9.2 \mathrm{E}-02$ & UIII & $<0.1380$ & & 1 & GP & & \\
\hline Radium-226 & $1.3 E+01$ & 11 & & 口 & 1 & GP & & \\
\hline Radium-228 & $2.2 E+01$ & /I & & $\bar{\square}$ & 1 & GP & & \\
\hline Strontium-90 & $3.6 E+02$ & /I & & 붕 & 1 & GP & & \\
\hline Technetium-99 & $4.3 \bar{E}+01$ & /I & & & 1 & GP & & \\
\hline Thorium-228 & $1.4 E+00$ & $\mathrm{R} / 4 /$ & Rej & & 1 & GP & & \\
\hline Thorium-230 & $<1.5 \mathrm{E}-01$ & $\mathrm{UI} / /$ & $<0.3610$ & & 1 & GP & & \\
\hline Thorium-232 & $<4.4 \mathrm{E}-02$ & $\mathrm{UII} /$ & $<0.3070$ & & 1 & GP & & \\
\hline Sum of alphas & $3.3 E+02$ & & & $\mathbf{E}$ & & & & \\
\hline Sum of betas & $4.7 E+02$ & & & $\mathbf{a}$ & & & & \\
\hline Total radium & $3.5 E+01$ & & & $\mathbf{E}$ & & & & \\
\hline Tritium & $3.0 E+03$ & $\|$ & & 口 & 1 & GP & $3.0 E+03$ & $\|$ \\
\hline Uranium-233/234 & $8.5 E+01$ & $\|$ & & $\overline{0}$ & 1 & $G P$ & & \\
\hline Uranium-235 & $9.9 E+00$ & II & & & 1 & GP & & \\
\hline Uranium-238 & 2.1E+02 & // & & జ & 1 & GP & & \\
\hline
\end{tabular}

Mod Filt. $\quad \underline{\text { ST }} \underline{H}$ DF Lab Unit

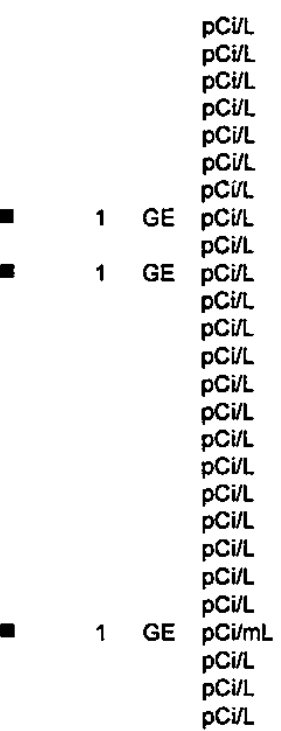




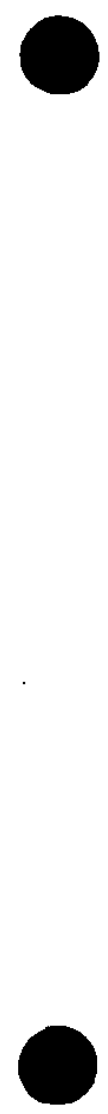

WELL FSB 99A

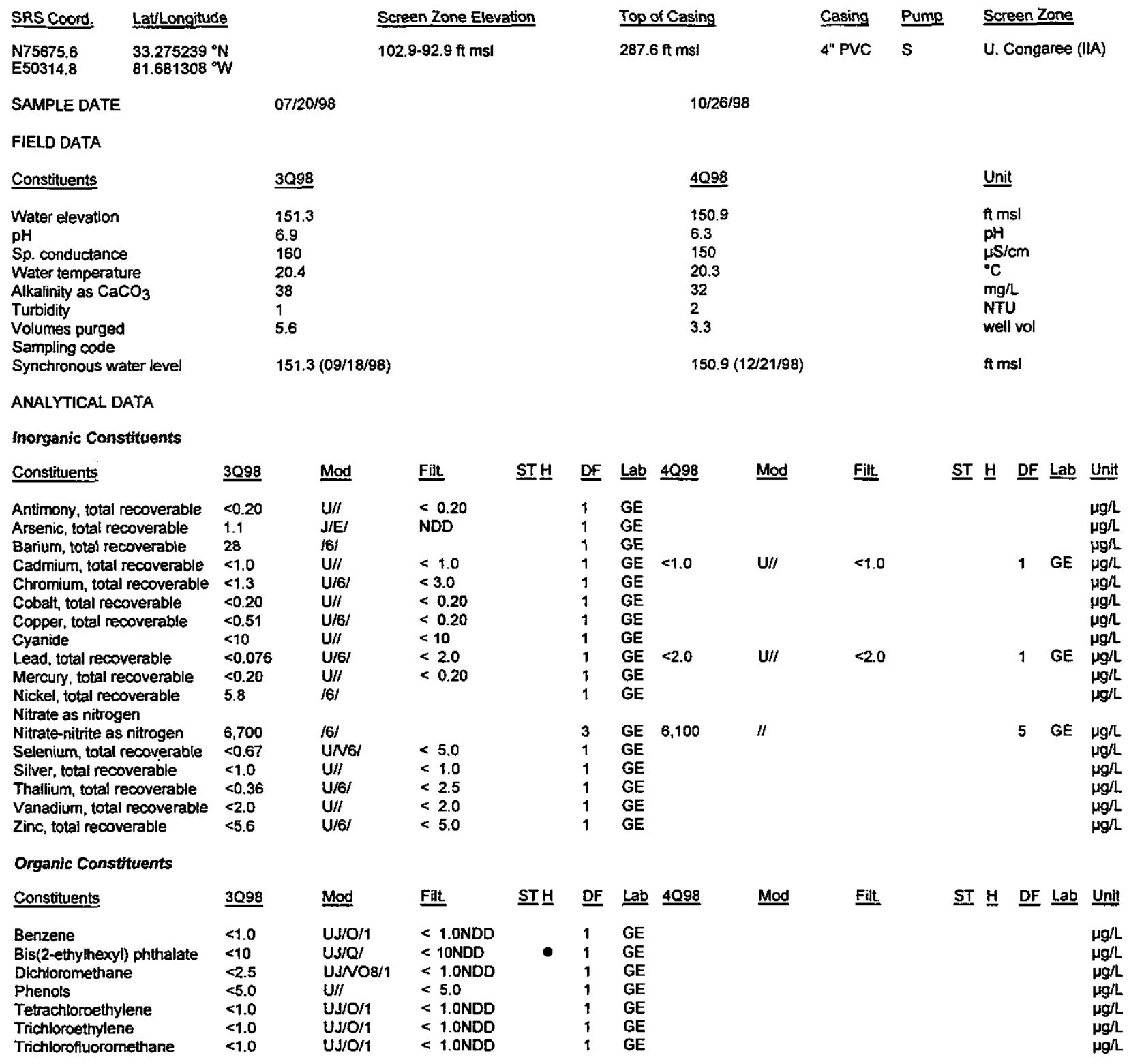

Notes:

- exceeded holding time

w = exceeded groundwater protection or monitoring constituent standard (See Appendix A.) 
WELL FSB 99A (cont.)

Radjoactive Constituents

\begin{tabular}{|c|c|c|c|c|c|c|c|c|c|c|c|c|c|}
\hline Constituents & 3098 & Mod & Filt. & ST보 & DF & Lab & 4Q98 & Mod & Fill. & $\underline{\mathrm{ST}} \underline{\mathrm{H}}$ & DF & Lab & Unit \\
\hline Americium-241 & $<8.8 \mathrm{E}-02$ & $\mathrm{UI} / /$ & $<0.1190$ & & 1 & GP & & & & & & & $\mathrm{pCi} / \mathrm{L}$ \\
\hline Cesium-137 & $<-1.2 \mathrm{E}+00$ & $\mathrm{UI} / /$ & $<2.9400$ & & 1 & GP & & & & & & & $\mathrm{pCi} / \mathrm{L}$ \\
\hline Cobalt -60 & $<-2.5 \mathrm{E}-01$ & $\mathrm{UI} / 1$ & $<3.5300$ & & 1 & GP & & & & & & & $\mathrm{pCi} / \mathrm{L}$ \\
\hline Curium-242 & $<7.2 \mathrm{E}-04$ & Uul & $<0.1360$ & & 1 & GP & & & & & & & $\mathrm{pCi} / \mathrm{L}$ \\
\hline Curium-243/244 & 4.1E-01 & $\|$ & & & 1 & GP & & & & & & & $\mathrm{pCi} / \mathrm{L}$ \\
\hline Curium-245/246 & $<1.6 \mathrm{E}-02$ & $\mathrm{UII} /$ & $<0.0471$ & & 1 & GP & & & & & & & $\mathrm{pCin}$ \\
\hline Gross alpha & $1.8 E+00$ & $/ /$ & & & 1 & GP & $<8.6 \mathrm{E}-01$ & $\mathrm{U} / /$ & $<6.3 E-01$ & & 1 & GE & $\mathrm{pCi} / \mathrm{L}$ \\
\hline lodine-129 & $<1.1 E+00$ & UIII & $<1.3800$ & & 1 & GP & & & & & & & $\mathrm{pCi} / \mathrm{L}$ \\
\hline Nonvolatile beta & $1.9 E+00$ & $\|$ & & & 1 & GP & $<2.2 \mathrm{E}+00$ & $\mathrm{U} / \mathrm{I}$ & $<1.1 \mathrm{E}+00$ & & 1 & GE & $\mathrm{pCi} / \mathrm{L}$ \\
\hline Plutonium-238 & $<-4.1 \mathrm{E}-03$ & $\mathrm{UI} / /$ & $<0.0894$ & & 1 & GP & & & & & & & $\mathrm{pCi} / \mathrm{L}$ \\
\hline Plutonium-239/240 & $<-8.1 E-03$ & $\mathrm{UI} / /$ & $<0.1050$ & & 1 & GP & & & & & & & $\mathrm{pCi} / \mathrm{L}$ \\
\hline Radium-226 & $<4.1 \mathrm{E}-01$ & $\mathrm{UI} / /$ & $<0.6330$ & & $i$ & GP & & & & & & & pCill \\
\hline Radium-228 & $<7.7 E-01$ & $\mathrm{UI} / I$ & $<0.9940$ & & 1 & GP & & & & & & & $\mathrm{pCi} / \mathrm{L}$ \\
\hline Strontium-90 & $<-1.4 \mathrm{E}-01$ & $\mathrm{UI} / 1$ & $<1.4200$ & & 1 & GP & & & & & & & $\mathrm{pCil}$ \\
\hline Technetium-99 & $<-1.1 E+01$ & $\mathrm{UI} /$ & $<22.0$ & & 1 & GP & & & & & & & $\mathrm{pCi} / \mathrm{L}$ \\
\hline Thorium-228 & $1.6 \mathrm{E}-01$ & // & & & 1 & GP & & & & & & & $\mathrm{pCi} \Omega$ \\
\hline Thorium-230 & $<5.8 \mathrm{E}-02$ & $\mathrm{Ut} / 1$ & $<0.0581$ & & 1 & GP & & & & & & & $\mathrm{pCi} / \mathrm{L}$ \\
\hline Thorium-232 & $<0.0 E+00$ & Uy/ & $<0.0581$ & & 1 & GP & & & & & & & $\mathrm{pCi} \Omega$ \\
\hline $\begin{array}{l}\text { Sum of alphas } \\
\text { Sum of betas }\end{array}$ & $9.9 E-01$ & & & & & & & & & & & & $\mathrm{pCi} / \mathrm{L}$ \\
\hline Tritium & 1.7E+02 & $/ /$ & & $\mathbf{a}$ & 1 & GP & 1.7E+02 & $/ /$ & & $\mathbf{E}$ & 1 & GE & $\mathrm{pCi} / \mathrm{mL}$ \\
\hline Uranium-233/234 & 1.9E-01 & /I & & & 1 & GP & & & & & & & $\mathrm{pCi} / \mathrm{L}$ \\
\hline Uranium-235 & $<8.6 \mathrm{E}-02$ & $\mathrm{UU} /$ & $<0.0858$ & & 1 & GP & & & & & & & pCill \\
\hline Uranium-238 & $2.2 E-01$ & $\|$ & & & 1 & GP & & & & & & & $\mathrm{pCi} / \mathrm{L}$ \\
\hline
\end{tabular}

\section{Notes:}


WELL FSB 99C

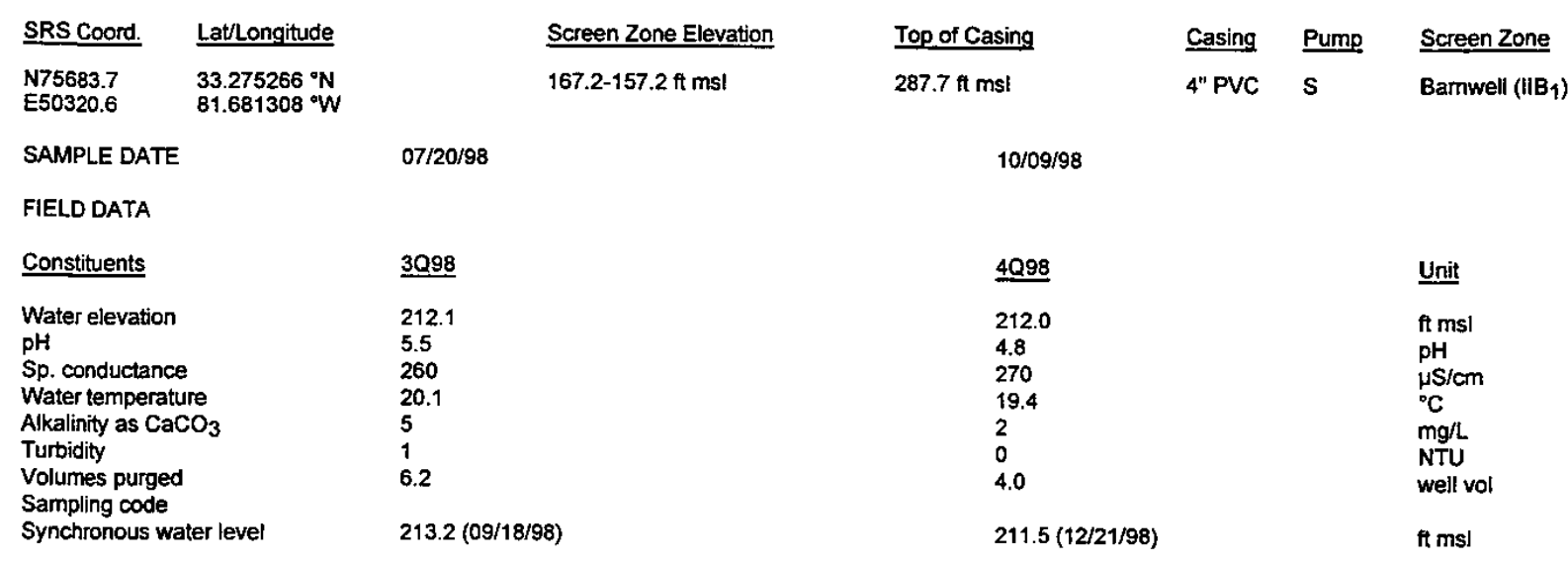

ANALYTICAL DATA

Inorganic Constituents

\begin{tabular}{|c|c|c|c|c|c|c|c|c|c|}
\hline Constituents & 3098 & Mod & Filt. & ST브 & DF & $\underline{L a b}$ & $4 Q 98$ & Mod & Filt. \\
\hline Antimony, total recoverable & $<0.088$ & UN6I & $<0.20$ & & 1 & GE & & & \\
\hline Arsenic, total recoverable & $<3.0$ & $\mathrm{U} / /$ & $<3.0$ & & 1 & GE & & & \\
\hline Barium, total recoverable & 70 & $16 /$ & & & 1 & GE & & & \\
\hline Cadmium, total recoverable & 1.6 & $/ /$ & & & 1 & GE & 1.3 & /l & \\
\hline Chromium, total recoverable & $<0.76$ & $\mathrm{U} / 6 /$ & $<3.0$ & & 1 & GE & & & \\
\hline Cobalt, total recoverable & 14 & N6/ & & $m$ & 1 & WA & & & \\
\hline Copper, total recoverable & $<1.9$ & $U / 6 /$ & $<0.20$ & & 1 & GE & & & \\
\hline Cyanide & $<10$ & $\mathrm{U} / I$ & $<10$ & & 1 & GE & & & \\
\hline Lead, total recoverable & 1.5 & J/E6/ & NDD & & 1 & GE & 1.6 & $\mathrm{~J} / \mathrm{l} /$ & NDD \\
\hline Mercury, total recoverable & 0.14 & $\mathrm{~J} / \mathrm{E} /$ & NDD & & 1 & GE & & & \\
\hline $\begin{array}{l}\text { Nickel, total recoverable } \\
\text { Nitrate as nitrogen }\end{array}$ & 8.1 & J/E6/ & NDD & & $i$ & WA & & & \\
\hline Nitrate-nitrite as nitrogen & 31,000 & $16 /$ & & ฮ & 15 & GE & 30,000 & $\|$ & \\
\hline Selenium, total recoverable & $<0.88$ & UN6/ & $<5.0$ & & 1 & GE & & & \\
\hline Silver, total recoverable & $<1.0$ & $\mathrm{U} / I$ & $<1.0$ & & 1 & GE & & & \\
\hline Thallium, total recoverable & $<0.11$ & $\mathrm{U} / 6 /$ & $<2.5$ & & 1 & GE & & & \\
\hline Vanadium, total recoverable & $<2.0$ & $\mathrm{U} / /$ & $<2.0$ & & 1 & ב & & & \\
\hline Zinc, total recoverable & $<17$ & $\mathrm{U} / 6 /$ & $<53.0000$ & & 1 & & & & \\
\hline
\end{tabular}

Organic Constituents

\begin{tabular}{|c|c|c|c|c|c|c|c|c|c|c|c|c|}
\hline Constituents & 3098 & Mod & Filt. & $\underline{\mathrm{ST}} \underline{\mathrm{H}}$ & DF & Lab & 4Q988 & Mod & Filt. & $\underline{\mathrm{ST}} \underline{\mathrm{H}}$ & DF Lab & Unit \\
\hline Benzene & $<1.0$ & UJ/O/1 & $<1.0$ & & 1 & GE & & & & & & $\mu g^{\prime} /$ \\
\hline Bis(2-ethylhexyl) phthalate & $<1.7$ & UNI & $<10$ & & 1 & WA & & & & & & $\mu g / L$ \\
\hline Dichloromethane & $<2.0$ & UJNO8/1 & $<1.0$ & & 1 & $\mathrm{GE}$ & & & & & & pgl \\
\hline Phenols & $<5.0$ & $\mathrm{U} / /$ & $<5.0$ & & 1 & GE & & & & & & uall \\
\hline Tetrachloroethylene & $<1.0$ & $\mathrm{UJ} / \mathrm{O} / 1$ & $<1.0$ & & 1 & GE & & & & & & yoll \\
\hline Trichloroethylene & 75 & $/ /$ & & $\mathbf{0}$ & 1 & WA & & & & & & ualt \\
\hline Trichlorofluoromethane & 3.0 & $\|$ & & & 1 & WA & & & & & & 1 \\
\hline
\end{tabular}

Notes:

- exceeded holding time

- exceeded groundwater protection or monitoring constituent standard (See Appendix A.)

F-Area HWMF

C-89

Third and Fourth Quarter 1998 
WELL FSB 99C (cont.)

Radioactive Constituents

\begin{tabular}{|c|c|c|c|c|c|c|c|c|c|c|c|c|c|}
\hline Constituents & $3 Q 98$ & Mod & Filt. & $\underline{\text { ST }} \underline{H}$ & DF & Lab & $\underline{4098}$ & Mod & Filt. & ST $\underline{H}$ & DF & $\underline{L a b}$ & Unit \\
\hline Americium-241 & $<6.5 E-02$ & $\mathrm{UI} / /$ & $<0.1260$ & & 1 & GP & & & & & & & pCi/L \\
\hline Beta dose & 18.8689 & & & $\mathbf{0}$ & & & & & & & & & $\mathrm{pCill}$ \\
\hline Cesium-137 & $<-2.1 E-01$ & $\mathrm{UI} / /$ & $<3.4200$ & & 1 & GP & & & & & & & pCill \\
\hline Curium-242 & $<-2.1 E-02$ & UIII & $<0.2200$ & & 1 & GP & & & & & & & pCill \\
\hline Curium-243/244 & $<-9.8 E-03$ & UIII & $<0.1270$ & & 1 & $G P$ & & & & & & & $\mathrm{pCi} / \mathrm{L}$ \\
\hline Curium-245/246 & $<0.0 E+00$ & U: $/ /$ & $<0.0902$ & & 1 & GP & & & & & & & pCill \\
\hline Gross alpha & $2.9 E+01$ & $/ /$ & & - & 1 & GP & $2.4 E+01$ & J/I & NDD & & 1 & GE & $\mathrm{pCi} / \mathrm{L}$ \\
\hline Nonvolatile beta & $1.2 E+02$ & /I & & a & 1 & $T M$ & $7.4 E+01$ & $\mathrm{~J} / \mathrm{LI}$ & NDD & & 1 & GE & $\mathrm{pCi} / \mathrm{L}$ \\
\hline Plutonium-238 & $<-4.4 E-03$ & $\mathrm{UI} / /$ & $<0.0974$ & & 1 & GP & & & & & & & $\mathrm{pCi} / \mathrm{L}$ \\
\hline Plutonium-239/240 & $<-1.0 E-02$ & UU/I & $<0.2100$ & & 1 & TM & & & & & & & $\mathrm{pCi} / \mathrm{L}$ \\
\hline Radium-226 & $<4.5 E+00$ & $\mathrm{U} / 6 /$ & $<0.1600$ & & 1 & $\mathrm{TM}$ & & & & & & & $\mathrm{pCi} / \mathrm{L}$ \\
\hline Radium-228 & $1.4 E+01$ & $/ I$ & & $\mathbf{\square}$ & 1 & $\mathrm{TM}$ & & & & & & & $\mathrm{pCill}$ \\
\hline Strontium-90 & $2.4 E+01$ & $\|$ & & 口 & 1 & GP & & & & & & & $\mathrm{PCil}$ \\
\hline Technetium-99 & $6.2 E+01$ & $\|$ & & $\bar{a}$ & 1 & GP & & & & & & & pCill \\
\hline Thorium-228 & $<0.0 \mathrm{E}+00$ & $\mathrm{UI} / /$ & $<0.6000$ & & 1 & TM & & & & & & & pCill \\
\hline Thorium-230 & $<0.0 \mathrm{E}+00$ & $\mathrm{UI} / /$ & $<0.0729$ & & 1 & GP & & & & & & & $\mathrm{pCi}$ \\
\hline Sum of aiphas & $1.3 E+01$ & & & & & & & & & & & & $\mathrm{pCi} / \mathrm{L}$ \\
\hline Sum of betas & $1.1 E+02$ & & & - & & & & & & & & & pCill \\
\hline Total radium & $1.4 E+01$ & & & $\overline{\mathbf{a}}$ & & & & & & & & & PCil \\
\hline Tritium & $1.1 E+03$ & $\|$ & & $\square$ & 1 & $T M$ & $1.0 E+03$ & $\|$ & & $\mathbf{E}$ & 1 & GE & $\mathrm{pCi} / \mathrm{mL}$ \\
\hline Uranium-233/234 & $5.7 E+00$ & $\|$ & & & 1 & GP & & & & & & & pCill \\
\hline Uranium-235 & $6.9 E-01$ & $\|$ & & & 1 & $\mathrm{rM}$ & & & & & & & pCill \\
\hline Uranium-238 & $7.0 \mathrm{E}+00$ & $\|$ & & & $i$ & $\pi M$ & & & & & & & $\mathrm{pCilL}$ \\
\hline
\end{tabular}

Notes:

= exceeded holding time

- exceeded groundwater protection or monitoring constituent standard (See Appendix A.) 


\section{WELL FSB 99D}

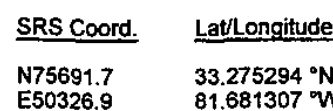

SAMPLE DATE

FIELD DATA

Constituents

Water elevation

pH

Sp. conductance

Water temperature

Alkalinity as $\mathrm{CaCO}_{3}$

Turbidity

Volumes purged

Sampling code

Synchronous water level

ANALYTICAL DATA

Inorganic Constituents

Constituents

Antimony, total recoverable $<0.20$

Arsenic, total recoverable $\quad<3.0$

Barium, total recoverable $\quad 7.1$

Cadmium, total recoverable $<1.0$

Chromium, total recoverable $<3.0$

Cobalt total recoverable $\quad<0.084$

Copper,

Cyanide

lead, total recoverable 6.0

Mercury, total recoverable $\quad<0.20$

Nickel, total recoverable $\quad<0.85$

Nitrate as nitrogen

Nitrate-nitrite as nitrogen $\quad 1,600$

Selenium, total recoverable $<5.0$

Silver, total recoverable $<1.0$

Thallium, total recoverable $<2.5$

Vanadium, total recoverable $<2$.

Zinc, total recoverable

$<30$

Organic Constituents

$\begin{array}{ll}\text { Constituents } & 3098 \\ \text { Benzene } & <1.0 \\ \text { Bis(2-ethylhexyl) phthalate } & 18 \\ \text { Dichloromethane } & <2.1 \\ \text { Phenols } & <5.0 \\ \text { Tetrachloroethylene } & <1.0 \\ \text { Trichloroethylene } & <1.0 \\ \text { Trichlorofluoromethane } & <1.0\end{array}$

3098

216.2

5.4

34

21.8

4.7

$216.6(09 / 18 / 98)$

Trichlorofluoromethane $<1.0$
Screen Zone Elevation

218.1-198.1 ft msl

Top of Casing

$287.6 \mathrm{ft} \mathrm{msl}$

$10 / 26 / 98$

$\underline{4098}$

216.5

5.4

70
22.9

3

0.083

XN

$215.4(12 / 21 / 98)$
Casing Pump Screen Zone

$4^{n}$ PVC 5
Water Table (IIB2)

Unit

ft msl

pH

${ }^{\circ \mathrm{CS} / \mathrm{cm}}$

$\mathrm{mg} / \mathrm{L}$

NTU

well vol

ft msl

\begin{tabular}{|c|c|}
\hline Mod & Filt. \\
\hline$U / I$ & $<0.20$ \\
\hline $\mathrm{U} / /$ & $<3.0$ \\
\hline 161 & \\
\hline $\mathrm{U} / /$ & $<1.0$ \\
\hline $\mathrm{U} / /$ & $<3.0$ \\
\hline U/6/ & $<0.20$ \\
\hline $16 /$ & \\
\hline $\mathrm{U} / /$ & $<10$ \\
\hline $16 /$ & \\
\hline $\mathrm{U} / /$ & $<0.20$ \\
\hline$U / 6 /$ & $<0.20$ \\
\hline$N 6 /$ & \\
\hline $\mathrm{U} / \prime$ & $<5.0$ \\
\hline$U / I$ & $<1.0$ \\
\hline $\mathrm{U} / 1$ & $<2.5$ \\
\hline $\mathrm{U} / /$ & $<2.0$ \\
\hline$U / 6 /$ & $<5.0$ \\
\hline
\end{tabular}

ST브

$$
\begin{aligned}
& \text { D } \\
& 1 \\
& 1 \\
& 1 \\
& 1 \\
& 1 \\
& 1 \\
& 1 \\
& 1 \\
& 1 \\
& 1 \\
& 1 \\
& 1 \\
& 1 \\
& 1 \\
& 1 \\
& 1 \\
& 1
\end{aligned}
$$

DF
1
1
1

Lab 4098

Mod

Filt.

ST 브 DF Lab Unit

0.32

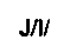

NDD

$\mu g /$

1 GE $\mu g / L$

$\mu g / L$

$\mu g / 2$

1 GE $\begin{aligned} & \mu g / L \\ & \mu g / L\end{aligned}$

$7.6 \quad / 1$

GE

1,300

/I

GE

GE

GE

$\mu g /$

$\mu g /$

1 GE $\mu g / L$

$\mu g /$

$\mu g / L$

$\mu g /$

$\mu g / L$

$\mu g h$

$\begin{array}{lllll}\text { Mod } & \text { Filt. } & \text { ST브 } & \underline{\text { DF }} & \underline{\text { Lab }} \\ \text { UJ/O/1 } & <1.0 & & 1 & \text { GE } \\ \| & & & 1 & \text { GE } \\ U J / N O 8 / 1 & <1.0 & & 1 & \text { GE } \\ U / I & <5.0 & & 1 & \text { GE } \\ U J / O / 1 & <1.0 & & 1 & \text { GE } \\ \text { UJ/O/1 } & <1.0 & & 1 & \text { GE } \\ \text { UJ/O/1 } & <1.0 & & 1 & \text { GE }\end{array}$

Mod $\quad$ Filt.
ST $H$ DF Lab Unit

$\mu g / L$
$\mu g / L$
$\mu g / L$
$\mu g / L$
$\mu g / L$
$\mu g / L$

Notes:

- exceeded holding time

= exceeded groundwater protection or monitoring constituent standard (See Appendix A.)

F-Area HWMF 
WELL FSB 99D (cont.)

Radioactive Constituents

\begin{tabular}{|c|c|c|c|c|c|c|c|c|}
\hline Constituents & 3Q98 & Mod & Filt. & STH & DF & $\underline{\mathrm{Lab}}$ & 4Q98 & Mod \\
\hline $\begin{array}{l}\text { Americium-241 } \\
\text { Beta dose }\end{array}$ & $\begin{array}{l}<9.6 E-02 \\
0.3625\end{array}$ & $\mathrm{UII} / /$ & $<0.1900$ & & 1 & GP & & \\
\hline Cesium-137 & $5.8 E+00$ & $\mathrm{R} / 4 /$ & Rej & & 1 & GP & & \\
\hline Cobalt-60 & $<-9.0 \mathrm{E}-01$ & UII/ & $<2.6300$ & & 1 & GP & & \\
\hline Curium-242 & $<1.2 \mathrm{E}-02$ & $\mathrm{UI} / /$ & $<0.1440$ & & $i$ & GP & & \\
\hline Curium-243/244 & 1.5E-01 & $/ 1$ & & & 1 & GP & & \\
\hline Curium-245/246 & $<0.0 E+00$ & $\mathrm{UI} / /$ & $<0.0605$ & & 1 & GP & & \\
\hline Gross alpha & $7.2 E+00$ & $\|$ & & & 1 & GP & $7.9 E+00$ & $\|$ \\
\hline lodine-129 & $<1.8 E-01$ & $\mathrm{UI} /$ & $<0.8190$ & & 4 & GP & & \\
\hline Nonvolatile beta & $7.9 \mathrm{E}+00$ & $\|$ & & & 1 & GP & $1.3 E+01$ & $\|$ \\
\hline Plutonium-238 & $<-1.6 \mathrm{E}-02$ & $\mathrm{UI} / /$ & $<0.1240$ & & 1 & GP & & \\
\hline Plutonium-239/240 & $<1.3 \mathrm{E}-02$ & $\mathrm{UI} / /$ & $<0.0868$ & & 1 & GP & & \\
\hline Radium-226 & $<4.0 E-01$ & UIII & $<0.5330$ & & 1 & GP & & \\
\hline Radium-228 & $<5.0 \mathrm{E}-01$ & $\mathrm{UV} / /$ & $<1.1900$ & & 1 & GP & & \\
\hline Strontium-90 & $2.9 E+00$ & $\|$ & & & 1 & GP & & \\
\hline Technetium-99 & $<-1,0 \mathrm{E}+01$ & $\mathrm{U} U /$ & $<20.5000$ & & 1 & GP & & \\
\hline Thorium-228 & $<1.7 E-01$ & UV/ & $<0.2610$ & & 1 & GP & & \\
\hline Thorium-230 & $<5.4 E-02$ & $\mathrm{UI} / /$ & $<0.0812$ & & 1 & GP & & \\
\hline Thorium-232 & $<4.8 E-02$ & UVI & $<0.1430$ & & 1 & GP & & \\
\hline Sum of alphas & $7.8 \mathrm{E}+00$ & & & & & & & \\
\hline Sum of betas & $2.9 E+00$ & & & & & & & \\
\hline Tritium & $1.4 E+01$ & $/ /$ & & & 1 & GP & $2.0 \mathrm{E}+01$ & $\|$ \\
\hline Uranium-233/234 & $1.8 \mathrm{E}+00$ & $\|$ & & & 1 & GP & & \\
\hline Uranium-235 & $<1.6 \mathrm{E}-01$ & UV/ & $<0.1770$ & & 1 & GP & & \\
\hline Uranium-238 & $5.9 E+00$ & $/ 1$ & & & 1 & GP & & \\
\hline
\end{tabular}

Filt. $\quad \underline{\text { ST }} \underline{\mathrm{H}}$ DF Lab Unit

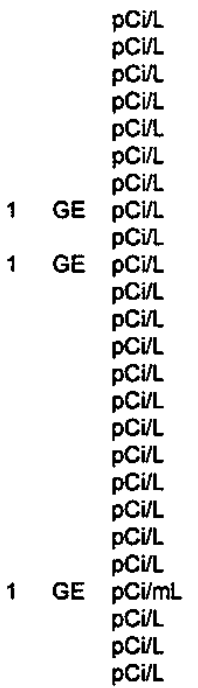

Notes: 
WELL FSB100A

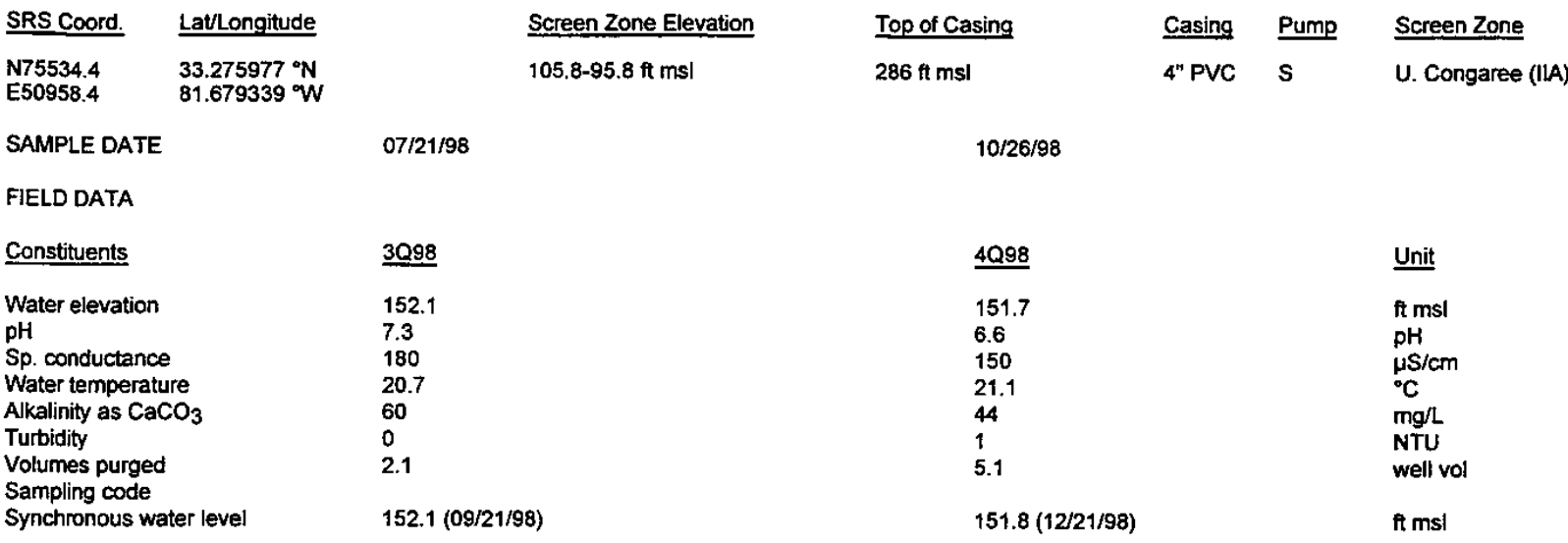

ANALYTICAL DATA

Inorganic Constituents

\begin{tabular}{|c|c|c|c|c|c|c|c|c|c|c|c|c|c|}
\hline Constituents & 3098 & Mod & Filt. & ST브 & DF & $\underline{\text { Lab }}$ & 4Q98 & Mod & Filt. & $\underline{\mathrm{ST}} \underline{\mathrm{H}}$ & $\underline{\mathrm{DF}}$ & $\underline{\text { Lab }}$ & Unit \\
\hline Antimony, total recoverable & $<0.20$ & $\mathrm{U} / /$ & $<0.20$ & & 1 & GE & & & & & & & $\mu g / L$ \\
\hline Arsenic, total recoverable & 1.1 & $\mathrm{~J} / \mathrm{E} /$ & NDD & & 1 & $\mathbf{G E}$ & & & & & & & $\mu g / 2$ \\
\hline Barium, total recoverable & 39 & $16 /$ & & & 1 & $\overline{G E}$ & & & & & & & $\mu g / L$ \\
\hline Cadmium, total recoverable & $<1.0$ & $\mathrm{U} / /$ & $<1.0$ & & 1 & GE & $<1.0$ & $\mathrm{u} / \prime$ & $<1.0$ & & 1 & GE & $\mu g / L$. \\
\hline Chromium, total recoverable & 0.82 & $\mathrm{~J} / \mathrm{E} /$ & NDD & & 1 & GE & & & & & & & $\mu g / L$ \\
\hline Cobalt, total recoverable & 8.8 & $N \prime$ & & a & 1 & WA & & & & & & & $\mu g /$ \\
\hline Copper, total recoverable & $<0.63$ & $\mathrm{U} / 6 /$ & $<0.20$ & & 1 & GE & & & & & & & $\mu g / L$ \\
\hline Cyanide & $<10$ & $\mathrm{U} / /$ & $<10$ & & 1 & GE & & & & & & & $\mu g / L$ \\
\hline Lead, total recoverable & $<0.47$ & $\mathrm{U} / 6 /$ & $<2.0$ & & 1 & GE & $<2.0$ & $\mathrm{U} / /$ & $<2.0$ & & 1 & GE & $\mu g / L$ \\
\hline Mercury, total recoverable & $<0.20$ & $\mathrm{U} / /$ & $<0.20$ & & 1 & GE & & & & & & & $\mu g / L$ \\
\hline $\begin{array}{l}\text { Nickel, total recoverable } \\
\text { Nitrate as nitrogen }\end{array}$ & 5.6 & $\mathrm{~J} / \mathrm{E} /$ & NDD & & 1 & WA & & & & & & & $\mu g / L$ \\
\hline Nitrate-nitrite as nitrogen & 4,100 & N6/ & & & 2 & GE & 3,800 & $\|$ & & & 5 & GE & $\mu \mathrm{g} / \mathrm{L}$ \\
\hline Selenium, total recoverable & $<5.0$ & $\mathrm{U} / I$ & $<5.0$ & & 1 & $\overline{G E}$ & & & & & & & $\mu g / L$ \\
\hline Silver, total recoverable & $<1.0$ & $\mathrm{U} / \prime$ & $<1.0$ & & 1 & GE & & & & & & & $\mu g / L$ \\
\hline Thallium, total recoverable & 0.39 & J/E6/ & NDD & & 1 & GE & & & & & & & $\mu g / L$ \\
\hline Vanadium, total recoverable & $<2.0$ & $\mathrm{U} / /$ & $<2.0$ & & 1 & GE & & & & & & & $\mu g / L$ \\
\hline Zinc, total recoverable & 16 & $\mathrm{~J} / \mathrm{E} /$ & NDD & & 1 & WA & & & & & & & $\mu g / L$ \\
\hline \multicolumn{14}{|l|}{ Organic Constituents } \\
\hline Constituents & $\underline{3098}$ & Mod & Filt. & ST브 & $\underline{\text { DF }}$ & $\underline{\text { Lab }}$ & 4Q98 & Mod & Filt. & $\underline{\text { ST }} \underline{H}$ & DF & $\underline{\text { Lab }}$ & Unit \\
\hline Benzene & $<1.0$ & Us/O/1 & $<1.0$ & & 1 & GE & & & & & & & $\mu g /$ \\
\hline Bis(2-ethythexyl) phthalate & $<0.85$ & UNI & $<5.1$ & & 1 & WA & & & & & & & $\mu g / h$ \\
\hline Dichloromethane & $<2.0$ & USNO8/1 & $<1.0$ & & 1 & GE & & & & & & & $\mu g / \mathrm{h}$ \\
\hline Phenols & 1.8 & $\mathrm{~J} / \mathrm{E} /$ & NDD & & 1 & GE & & & & & & & $\mu \mathrm{g} / \mathrm{h}$ \\
\hline Tetrachloroethylene & $<1.0$ & UN/O/1 & $<1.0$ & & 1 & $\overline{G E}$ & & & & & & & $\mu g / L$. \\
\hline Trichloroethylene & $<1.0$ & $\mathrm{U} / \mathrm{O} / \mathrm{A}$ & $<1.0$ & & 1 & GE & & & & & & & $\mu g / L$ \\
\hline Trichlorofluorornethane & $<1.0$ & $\mathrm{UJ} / \mathrm{O} / 1$ & $<1.0$ & & 1 & GE & & & & & & & $\mu g / l$ \\
\hline
\end{tabular}

Notes:

- exceeded holding time

- exceeded groundwater protection or monitoring constituent standard (See Appendix A.)

F-Area HWMF 


\section{WELL FSB100A (cont.)}

Radioactive Constituents

\begin{tabular}{|c|c|c|c|c|c|c|c|c|c|c|c|c|c|}
\hline Constituents & 3098 & Mod & Filt. & $\underline{\text { ST브 }}$ & DF & $\underline{\mathrm{Lab}}$ & 4Q98 & Mod & Filt. & SI $\underline{H}$ & $\underline{D F}$ & $\underline{\text { Lab }}$ & Unit \\
\hline Americium-241 & $<-1,2 \mathrm{E}-02$ & Ui:/ & $<0.1110$ & & 1 & GP & & & & & & & pCill. \\
\hline Cesium-137 & $<2.9 E-02$ & U1/ & $<3.2900$ & & 1 & GP & & & & & & & $\mathrm{pCil}$ \\
\hline Beta dose & 0.38 & & & & & & & & & & & & \\
\hline Cobalt-60 & $<-4.0 E-02$ & $\mathrm{U} \mathbf{3} / /$ & $<2.5800$ & & 1 & GP & & & & & & & pCill \\
\hline Curium-242 & $<0.0 E+00$ & $\mathrm{UV} / /$ & $<0.0717$ & & 1 & GP & & & & & & & pCill \\
\hline Curium-243/244 & $<-1.0 E-02$ & $\mathrm{U} / 1 /$ & $<1.3500$ & & 1 & $T M$ & & & & & & & pCill \\
\hline Curium-245/246 & $<0.0 \mathrm{E}+00$ & UI/I & $<0.0580$ & & $i$ & GP & & & & & & & $\mathrm{pCil}$ \\
\hline Gross alpha & $<1.9 E+00$ & $\mathrm{U} / 6 /$ & $<0.6700$ & & 1 & $\pi M$ & $<5.9 E-01$ & $\mathrm{U} / /$ & $<6.1 E-01$ & & 1 & GE & pCill \\
\hline lodine-129 & $<1.2 \mathrm{E}+00$ & UIII & $<1.2300$ & & 1 & GP & & & & & & & $\mathrm{pCil}$ \\
\hline Nonvolatile beta & $4.5 E+00$ & $/ l$ & & & 1 & TM & $<2.3 E+00$ & $\mathrm{U} / \prime$ & $<1.1 E+\infty 0$ & & 1 & GE & $\mathrm{pCi} / \mathrm{L}$ \\
\hline Plutonium-238 & $<5.2 E-03$ & $\mathrm{UIII}$ & $<0.1460$ & & 1 & GP & & & & & & & pCitt \\
\hline Plutonium-239/240 & $<-7.7 E-03$ & UIII & $<0.1000$ & & 1 & GP & & & & & & & pCill \\
\hline Radium-226 & $<4.6 \mathrm{E}-01$ & $\mathrm{U} / 6 /$ & $<0.2400$ & & 1 & TM & & & & & & & pCill \\
\hline Radium-228 & $1.9 E+00$ & $\|$ & & & 1 & GP & & & & & & & pCin \\
\hline Strontium-90 & $<-2.0 \mathrm{E}-01$ & บบ/ & $<1.6500$ & & 1 & GP & & & & & & & $\mathrm{pCi} / \mathrm{L}$ \\
\hline Technetium-99 & $<-5.4 E+00$ & $\mathbf{U I} / /$ & $<23.5000$ & & 1 & GP & & & & & & & pCill \\
\hline Thorium-228 & $<7.7 E-02$ & $\mathrm{UI} / /$ & $<0.1430$ & & 1 & GP & & & & & & & $\mathrm{pCi} / \mathrm{L}$ \\
\hline Thorium-230 & $1.2 E-01$ & $/ /$ & & & 1 & GP & & & & & & & pCill \\
\hline Thorium-232 & $<-1.4 \mathrm{E}-02$ & $\mathrm{UI} / /$ & $<0.1300$ & & 1 & GP & & & & & & & pCill \\
\hline Sum of alphas & 8.9E-01 & & & & & & & & & & & & pCill \\
\hline Sum of betas & $1.9 E+00$ & & & & & & & & & & & & pCir \\
\hline Total radium & $1.9 E+00$ & & & & & & & & & & & & pcin \\
\hline Tritium & $7.3 E+01$ & $\|$ & & - & 1 & $\mathrm{TM}$ & $6.7 E+01$ & $\|$ & & $\mathbf{a}$ & 1 & GE & pCi/mL \\
\hline Uranium-233/234 & $2.4 E-01$ & $\|$ & & & 1 & GP & & & & & & & pCin \\
\hline Uranium-235 & $<-2.0 \mathrm{E}-02$ & UIII & $<0.3800$ & & 1 & TM & & & & & & & pCill \\
\hline Uranium-238 & $5.2 E-01$ & $/ /$ & & & 1 & $T M$ & & & & & & & $\mathrm{Cil}$ \\
\hline
\end{tabular}

Notes:

- exceeded holding time

- exceeded groundwater protection or monitoring constituent standard (See Appendix A.) 
WELL FSB101A

\begin{tabular}{|c|c|c|c|}
\hline SRS Coord. & \multicolumn{2}{|l|}{ Lat/Longitude } & Screen Zone Elevation \\
\hline $\begin{array}{l}\text { N75719.0 } \\
\text { E51191.3 }\end{array}$ & \multicolumn{2}{|l|}{$\begin{array}{l}33.276765^{\circ} \mathrm{N} \\
81.679085^{\circ} \mathrm{W}\end{array}$} & $102.9-92.9 \mathrm{ft} \mathrm{msl}$ \\
\hline \multicolumn{2}{|c|}{ SAMPLE DATE } & \multicolumn{2}{|l|}{$07 / 06 / 98$} \\
\hline \multicolumn{4}{|l|}{ FIELD DATA } \\
\hline \multicolumn{2}{|l|}{ Constituents } & \multicolumn{2}{|l|}{ 3Q998 } \\
\hline \multirow{2}{*}{\multicolumn{2}{|c|}{$\begin{array}{l}\text { Water elevation } \\
\mathrm{pH}\end{array}$}} & \multicolumn{2}{|l|}{152.3} \\
\hline \multirow{2}{*}{\multicolumn{2}{|c|}{$\begin{array}{l}\text { pH } \\
\text { Sp. conductance }\end{array}$}} & \multicolumn{2}{|l|}{7.1} \\
\hline & & \multicolumn{2}{|l|}{140} \\
\hline \multicolumn{2}{|c|}{ Water temperature } & \multicolumn{2}{|l|}{20.6} \\
\hline \multicolumn{2}{|c|}{ Alkalinity as $\mathrm{CaCO}_{3}$} & \multicolumn{2}{|l|}{32} \\
\hline \multicolumn{2}{|c|}{ Turbidity } & \multicolumn{2}{|l|}{2} \\
\hline \multicolumn{2}{|c|}{ Volumes purged } & \multicolumn{2}{|l|}{5.0} \\
\hline & Sampling code & \multicolumn{2}{|c|}{$152.3(09 / 21 / 98)$} \\
\hline
\end{tabular}

$\begin{array}{llll}\text { Top of Casing } & \text { Casing } & \text { Pump } & \text { Screen Zone } \\ 285.2 \mathrm{ft} \mathrm{msl} & 4 " \text { PVC } & \text { S } & \text { U. Congaree (IIA) }\end{array}$

ANALYTICAL DATA

Inorganic Constituents

\begin{tabular}{|c|c|c|c|c|c|c|c|c|c|c|c|c|c|}
\hline Constituents & $\underline{3 Q 98}$ & Mod & Filt. & $\underline{\text { ST }} \underline{H}$ & DF & $\underline{\text { Lab }}$ & 4Q98 & Mod & Filt. & $\underline{\text { ST }} \underline{H}$ & $\underline{\mathrm{DF}}$ & $\underline{\text { Lab }}$ & Unit \\
\hline Antimony, total recoverable & $<0.20$ & $\mathrm{U} / /$ & $<0.20$ & & 1 & GE & & & & & & & $\mu g / L$. \\
\hline Arsenic, total recoverable & 0.70 & $\mathrm{~J} / \mathrm{E} /$ & NDD & & 1 & GE & & & & & & & $\mu g / L$ \\
\hline Barium, total recoverable & 34 & $/ /$ & & & 1 & GE & & & & & & & $\mu g / L$ \\
\hline Cadmium, total recoverable & $<1.0$ & $\mathrm{U} / /$ & $<1.0$ & & 1 & $\mathrm{GE}$ & $<1.0$ & $\mathrm{U} / /$ & $<1.0$ & & 1 & GE & $\mu g / L$ \\
\hline Chromium, total recoverable & 1.9 & $\mathrm{~J} / \mathrm{E} /$ & NDD & & 1 & GE & & & & & & & $\mu g / \mathrm{L}$ \\
\hline Cobalt, total recoverable & 0.083 & $\mathrm{~J} / \mathrm{E} /$ & NDD & . & 1 & GE & & & & & & & رg/L \\
\hline Copper, total recoverable & $<0.88$ & UNI & $<0.20$ & & 1 & GE & & & & & & & $\mu g / L$ \\
\hline Cyanide & $<10$ & $\mathrm{U} / /$ & $<10$ & & 1 & $\mathrm{GE}$ & & & & & & & $\mu g / L$ \\
\hline Lead, total recoverable & 0.11 & $\mathrm{~J} / \mathrm{E} /$ & NDD & & 1 & GE & $<2.0$ & $\mathrm{U} / /$ & $<2.0$ & & 1 & GE & $\mu g / L$ \\
\hline Mercury, total recoverable & $<0.20$ & $\mathrm{UJ} / \mathrm{l} / 1$ & $<0.20$ & & 1 & $\overline{G E}$ & & & & & & & $\mu g /$. \\
\hline $\begin{array}{l}\text { Nickel, total recoverable } \\
\text { Nitrate as nitrogen }\end{array}$ & 0.77 & $\|$ & & & 1 & $\mathrm{GE}$ & & & & & & & $\mu \mathrm{g} / \mathrm{L}$ \\
\hline Nitrate-nitrite as nitrogen & 1,500 & $N /$ & & & 1 & $\mathrm{GE}$ & 1,500 & $/ /$ & & & 1 & GE & $\mu \mathrm{g} / \mathrm{h}$ \\
\hline Selenium, total recoverable & 0.48 & $\mathrm{~J} / \mathrm{E} /$ & NDD & & 1 & GE & & & & & & & $\mu g / L$ \\
\hline Silver, total recoverable & $<1.0$ & $\mathrm{U} / /$ & $<1.0$ & & 1 & $\overline{G E}$ & & & & & & & $\mu g / \mathrm{L}$ \\
\hline Thallium, total recoverable & 0.093 & $\mathrm{~J} / \mathrm{E} /$ & NDD & & 1 & $\overrightarrow{\mathrm{GE}}$ & & & & & & & $\mu g / L$ \\
\hline Vanadium, total recoverable & $<2.0$ & $\mathrm{U} / /$ & $<2.0$ & & 1 & $G E$ & & & & & & & $\mu g / L$ \\
\hline Zinc, total recoverable & 5.1 & $\|$ & & & 1 & $\overline{G E}$ & & & & & & & $g / L$ \\
\hline
\end{tabular}

Organic Constituents

\begin{tabular}{|c|c|c|c|c|c|c|c|c|c|c|c|}
\hline Constituents & 3Q98 & Mod & Filt. & $\underline{\text { STㅂ}}$ & DF & Lab & $4 Q 98$ & Mod & Filt. & $\underline{\text { ST }} \underline{H}$ DF Lab & Unit \\
\hline Benzene & $<1.0$ & $\mathrm{UJ} / \mathrm{O} / 1$ & $<1.0$ & & 1 & GE & & & & & $\mu و /$ \\
\hline Bis(2-ethylhexyl) phthalate & $<10$ & $\mathrm{U} / 1$ & $<10$ & & 1 & $\overline{G E}$ & & • & & & $\mu g / L$ \\
\hline Dichloromethane & $<2.6$ & UJNO8/1 & $<1.0$ & & 1 & $\overline{G E}$ & & & & & H \\
\hline Phenols & $<5.0$ & $\mathrm{U} / \mathrm{l}$ & $<5.0$ & & 1 & GE & & & & & $\mu g / L$ \\
\hline Tetrachloroethylene & $<1.0$ & $\mathrm{UJ} / \mathrm{O} / 1$ & $<1.0$ & & 1 & GE & & & & & $\mu g / L$ \\
\hline Trichloroethylene & $<1.0$ & $\mathrm{UJ} / \mathrm{O} / 1$ & $<1.0$ & & 1 & GE & & & & & $\mu g / L$ \\
\hline Trichlorofluoromethane & $<1.0$ & UJ/O/1 & $<1.0$ & & 1 & GE & & & & & $\mu g / L$ \\
\hline
\end{tabular}

Notes:

- exceeded holding time

E = exceeded groundwater protection or monitoring constituent standard (See Appendix A.) 
WELL FSB101A (cont.)

Radioactive Constituemts

\begin{tabular}{|c|c|c|c|c|c|c|c|c|c|c|c|c|c|}
\hline Constituents & 3098 & Mod & Filt. & ST브 & $\underline{D F}$ & $\underline{\text { Lab }}$ & 4Q988 & Mod & Filt. & $\underline{\text { ST }} \underline{H}$ & DF & $\underline{\text { Lab }}$ & Unit \\
\hline Americium-241 & $<1.9 E-03$ & $\mathrm{UI} / /$ & $<0.2020$ & & 1 & GP & & & & & & & pCi/l \\
\hline Beta dose & 1.2500 & & & 口 & & & & & & & & & $\mathrm{pCi} / \mathrm{L}$ \\
\hline Cesium-137 & $<-4.6 \mathrm{E}-01$ & $\mathrm{UI} / \prime$ & $<3.5600$ & & 1 & GP & & & & & & & $\mathrm{pCi} / \mathrm{L}$ \\
\hline Cobalt-60 & $<-9.5 E-01$ & $\mathrm{UI} / /$ & $<3.1200$ & & 1 & GP & & & & & & & $\mathrm{pCi} / \mathrm{L}$ \\
\hline Curium-242 & $<2.7 \mathrm{E}-02$ & $\mathrm{UI} / /$ & $<0.1010$ & & 1 & GP & & & & & & & $\mathrm{pCi} / \mathrm{L}$ \\
\hline Curium-243/244 & $<2.9 E-02$ & $\mathrm{UI} / /$ & $<0.1930$ & & 1 & GP & & & & & & & pCin \\
\hline Curium-245/246 & $<2.1 E-02$ & $\mathrm{UI} / /$ & $<0.1000$ & & 1 & GP & & & & & & & pCill \\
\hline Gross alpha & $<5.7 \mathrm{E}-01$ & $\mathrm{UII/}$ & $<0.6870$ & & 1 & GP & $<5.7 E-01$ & $U / I$ & $<5.5 E-01$ & & 1 & GE & pCill \\
\hline lodine-129 & $<2.2 \mathrm{E}-01$ & $\mathrm{UI} / /$ & $<1.0$ & & 1 & GP & & & & & & & pCill. \\
\hline Nonvolatile beta & $1.5 E+00$ & $\|$ & & & 1 & GP & $<1.6 E+00$ & $U / I$ & $<9.9 E-01$ & & 1 & GE & $\mathrm{pCi} / \mathrm{L}$ \\
\hline Plutonium-238 & $<-8.8 E-03$ & UIII & $<0.3120$ & & 1 & GP & & & & & & & pCill \\
\hline Plutonium-239/240 & $<1.5 \mathrm{E}-02$ & $\mathrm{UI} / /$ & $<0.1050$ & & 1 & GP & & & & & & & pCirl \\
\hline Radium-226 & $<2.6 \mathrm{E}-01$ & UII/ & $<0.6080$ & & 1 & GP & & & & & & & $\mathrm{pCi} / \mathrm{L}$ \\
\hline Radium-228 & $<5.7 E-01$ & $\mathrm{UI} / /$ & $<0.8980$ & & 1 & GP & & & & & & & $\mathrm{pCi} / \mathrm{L}$ \\
\hline Strontium-90 & 1.DE+01 & $\|$ & & 口 & 1 & GP & & & & & & & $\mathrm{pCi} / \mathrm{L}$ \\
\hline Technetium-99 & $<9.8 E+00$ & $\mathrm{UI} / /$ & $<22.4000$ & & 1 & GP & & & & & & & $\mathrm{pCi} / \mathrm{L}$ \\
\hline Thorium-228 & 3.7E-01 & $\|$ & & & 1 & GP & & & & & & & $\mathrm{pCi} / \mathrm{L}$ \\
\hline Thorium-230 & $<3.1 E-02$ & $\mathrm{UI} / /$ & $<0.2240$ & & 1 & GP & & & & & & & $\mathrm{pCi} / \mathrm{L}$ \\
\hline Thorium-232 & $<2.3 \mathrm{E}-02$ & $\mathrm{UI} / /$ & $<0.1560$ & & 1 & GP & & & & & & & pCill \\
\hline Sum of alphas & 3.7E-01 & & & & & & & & & & & & $\mathrm{pCin}$ \\
\hline Sum of betas & $1.0 \mathrm{E}+01$ & & & & & & & & & & & & pCill \\
\hline Tritium & $<4.1 \mathrm{E}-01$ & $\mathrm{UII} /$ & $<5.6 \mathrm{E}-01$ & & 1 & GP & $<5.7 E-01$ & $\mathrm{U} / I$ & $<6.5 E-01$ & & 1 & GE & $\mathrm{pCi} / \mathrm{mL}$ \\
\hline Uranium-233/234 & $<1.1 E-01$ & $\mathrm{U} N I$ & $<0.0998$ & & 1 & GP & & & & & & & pCill \\
\hline Uranium-235 & $<8.4 \mathrm{E}-03$ & $\mathrm{U} I / /$ & $<0.1000$ & & 1 & GP & & & & & & & $\mathrm{pCi} / \mathrm{L}$ \\
\hline Uranium-238 & $<-3.9 \mathrm{E}-03$ & $U U / /$ & $<0.0847$ & & 1 & GP & & & & & & & $\mathrm{pCi}$ \\
\hline
\end{tabular}

Notes:

- exceeded holding time

= exceeded groundwater protection or monitoring constituent standard (See Appendix A.) 
WELL FSB102C

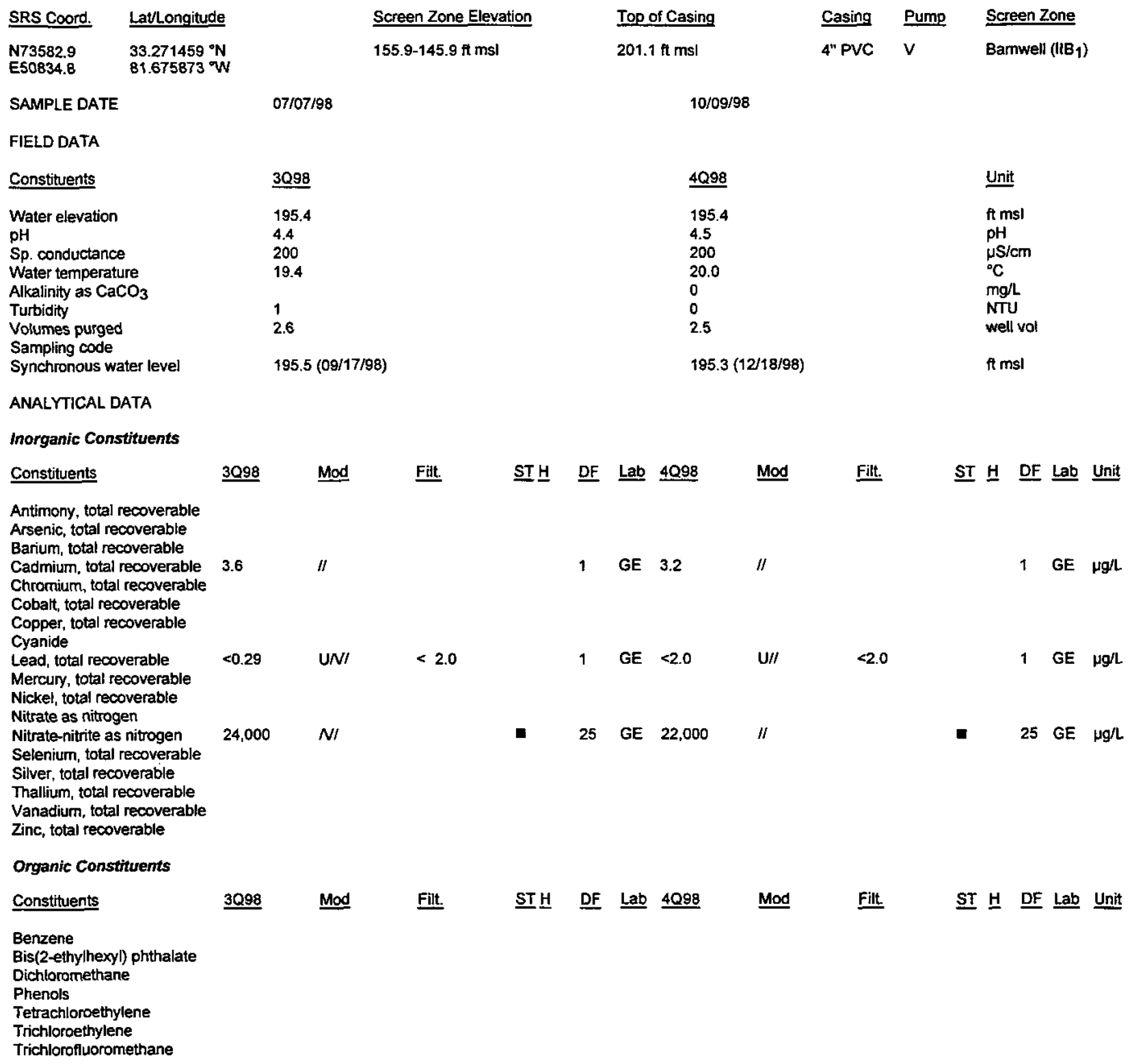

Notes:

- exceeded holding time

- exceeded groundwater protection or monitoring constituent standard (See Appendix A.)

F-Area HWMF 
WELL FSB102C (cont.)

Radioactive Constituents

Constituents

Americium-241

Cesium-137

Cobalt-60

Curium-242

Curium-243/244

Curium-245/246

Gross alpha

lodine-129

Nonvolatile beta

Plutonium-238

Plutonium-239/240

Radium-226

Radium-228

Strontium-90

Technetium-99

Thorium-228

Thorium-230

Thorium-232

Sum of alphas

Sum of betas

Tritium

Uranium-233/234

Uranium-235

Uranium-238
309

1.1E+01

$2.2 \mathrm{E}+02$

4.8E+02 /

1 GP $4.8 \mathrm{E}+02 \quad$ /I
SI $\underline{\text { DF }} \underline{\underline{L} \text { ba }}$ Unit
Filt.

1 GP $5.6 \mathrm{E}+00 \quad / 1$

-

1 GP $2.3 \mathrm{E}+02$ /
$1 \mathrm{GE} \mathrm{pCi} / \mathrm{l}$

- 1 GE pCill

- 1 GE pCi/mL

Notes:

- exceeded holding time

- exceeded groundwater protection or monitoring constituent standard (See Appendix A.) 


\section{WELL FSB103C}

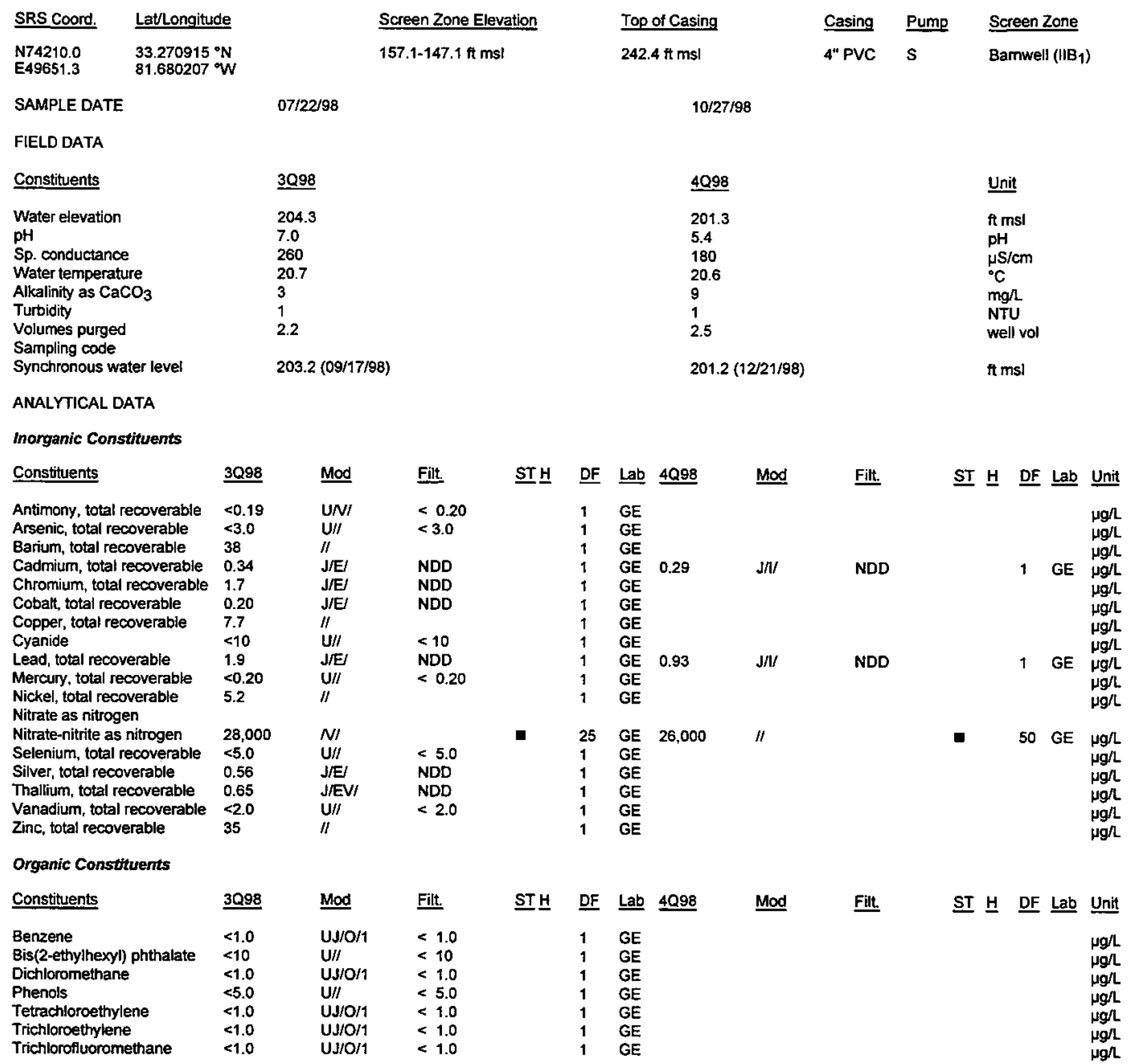

Notes:

- exceeded holding time

- exceeded groundwater protection or monitoring constituent standard (See Appendix A.)

F.Area HWMF 


\section{WELL FSB103C (cont.)}

Radioactive Constituents

\section{Constituents}

Americium-241

Beta dose

Cesium-137

Cobalt-60

Curium-242

Curium-243/244

Curium-245/246

Gross alpha

lodine-129

Nonvolatile beta

Plutonium-238

Plutonium-239/240

Radium-226

Radium-228

Strontium-90

Technetium-99

Thorium-228

Thorium-230

Thorium-232

Sum of alphas

Sum of betas

Tritium

Uranium-233/234

Uranium-235

Uranium-238

\begin{tabular}{|c|c|c|c|c|c|c|c|}
\hline$\underline{3098}$ & Mod & Fill. & ST $\underline{H}$ & $\underline{D F}$ & $\underline{\mathrm{Lab}}$ & 4Q98 & Mod \\
\hline$<5.7 E-02$ & $\mathrm{UI} / /$ & $<0.0632$ & & 1 & GP & & \\
\hline 2.0467 & & & $\boldsymbol{\square}$ & & & & \\
\hline$<2.8 E-01$ & $\mathrm{UI} / /$ & $<3.5500$ & & 1 & GP & & \\
\hline$<-9.9 E-01$ & $\mathrm{UI} / /$ & $<3.1000$ & & 1 & GP & & \\
\hline$<0.0 E+00$ & UVI/ & $<0.0719$ & & 1 & GP & & \\
\hline$<3.7 E-02$ & UI// & $<0.1120$ & & 1 & GP & & \\
\hline$<2.1 \bar{E}-02$ & $\mathrm{UH} /$ & $<0.0632$ & & 1 & $G P$ & & \\
\hline 9.3E-01 & $\|$ & & & 1 & $G P$ & $<6.3 E-01$ & $\mathrm{U} / /$ \\
\hline $2.0 E+00$ & /I & & & 1 & GP & & \\
\hline $6.6 E+00$ & /I & & & 1 & GP & $1.4 E+01$ & $/ /$ \\
\hline$<-6.9 E-03$ & $\mathrm{UI} / /$ & $<0.1350$ & & 1 & GP & & \\
\hline$<1.6 \mathrm{E}-02$ & $\mathrm{UI} / /$ & $<0.0471$ & & 1 & GP & & \\
\hline$<4,4 E-01$ & $\mathrm{U} \mid / /$ & $<0.5160$ & & 1 & GP & & \\
\hline$<9.0$ E-01 & $\mathrm{U} \mid / /$ & $<1.0200$ & & 1 & GP & & \\
\hline$<-4.2 E-02$ & $\mathrm{UI} / /$ & $<1.4600$ & & 1 & GP & & \\
\hline $4.2 E+01$ & /I & & & 1 & GP & & \\
\hline$<4.7 E-02$ & $\mathrm{UI} / /$ & $<0.1160$ & & 1 & $\mathrm{GP}$ & & \\
\hline$<2.8 E-02$ & $\mathrm{UV} / /$ & $<0.1510$ & & 1 & GP & & \\
\hline$<-1.0 \mathrm{E}-02$ & $\mathrm{UI} / I$ & $<0.1350$ & & 1 & GP & & \\
\hline $4.4 E+01$ & & & & & & & \\
\hline $5.2 E+02$ & $\|$ & & 口 & 1 & GP & $5.3 E+02$ & $/ /$ \\
\hline$<3.3 E-02$ & $\mathrm{UV} / /$ & $<0.0983$ & & 1 & GP & & \\
\hline$<0.0 E+00$ & $\mathrm{U} \mathrm{y} /$ & $<0.0848$ & & 1 & GP & & \\
\hline$<2.1 E-02$ & $\mathrm{UI} / /$ & $<0.1490$ & & 1 & $\mathrm{GP}$ & & \\
\hline
\end{tabular}

Filt

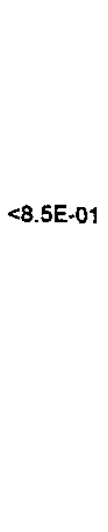

ST H DF Lab Unit

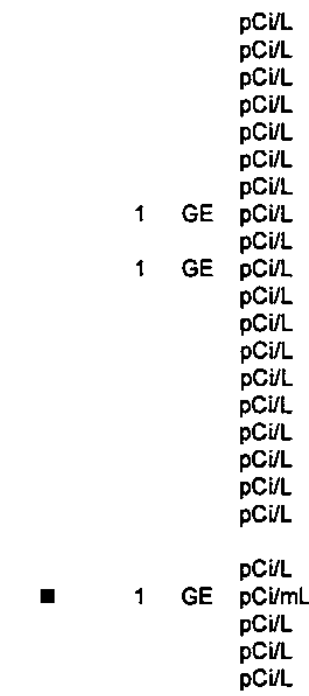

Notes:

- exceeded holding time

- exceeded groundwater protection or monitoring constituent standard (See Appendix A.) 


\section{WELL FSB104C}

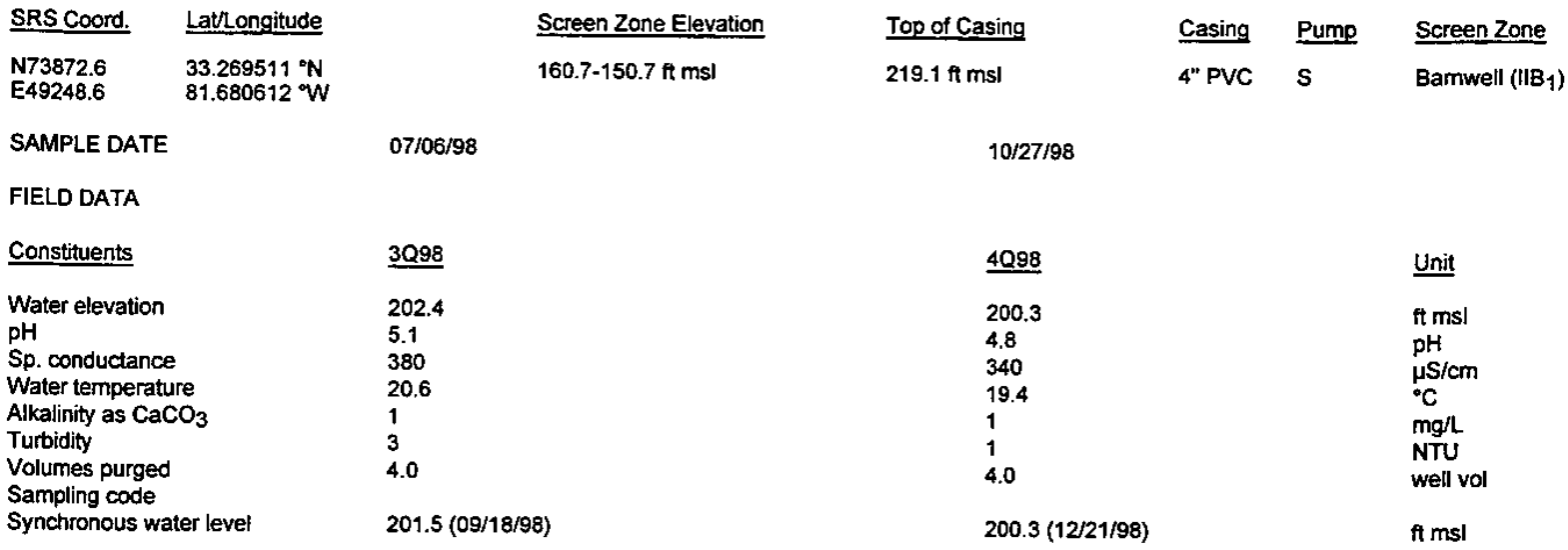

\section{ANALYTICAL DATA}

Inorganic Constituents

Constituents

Antimony, total recoverable Arsenic, total recoverable Barium, total recoverable Cadmium, total recoverable Chromium, total recoverable Cobalt, total recoverable Copper, total recoverable Cyanide

Lead, total recoverable

Mercury, total recoverable

Nickel, total recoverable

Nitrate as nitrogen

Nitrate-nitrite as nitrogen

Selenium, total recoverable

Silver, total recoverable

Thallium, total recoverable

Vanadium, total recoverable

Zinc, total recoverable

Organic Constituents

Constituents

Benzene

Bis(2-ethylhexyl) phthalate

Dichloromethane

Phenols

Tetrachloroethylene

Trichloroethylene

Trichlorofluoromethane $\underline{3098}$

1.0

0.78

$\mathrm{J} / \mathrm{E}$

NDD

47,000

NI

3Q98

Mod

$\mathrm{J} / \mathrm{E} /$

NDD
GE $\quad 0.86$

$1 \quad$ GE $\quad 0.88$

$\mathrm{J} / \mathrm{I} /$

a

25 GE 49,000

Filt.

Filt.

Notes:

- = exceeded holding time

- exceeded groundwater protection or monitoring constituent standard (See Appendix A.) 


\section{WELL. FSB104C (cont.)}

Radioactive Constifuents

Constituents

Americium-241

Cesium-137

Cobalt -60

Curium-242

Curium-243/244

Curium-245/246

Gross alpha

lodine-129

Nonvolatile beta

Plutonium-238

Plutonium-239/240

Radium-226

Radium-228

Strontium-90

Technetium-99

Thorium-228

Thorium-230

Thorium-232

Sum of alphas

Sum of betas

Tritium

Uranium-233/234

Uranium-235

Uranium-238
3098

$3.3+00$

$3.0 \mathrm{E}+01$

$9.3 \mathrm{E}+02$

$1 \quad \mathrm{GP}<1.8 \mathrm{E}+00 \quad \mathrm{U} / / \quad<9.6 \mathrm{E}-01$

1 GP $3.4 \mathrm{E}+01$ //

1 GE $p C i / L$

(n)

브 DF ㄴab Unit 


\section{WELL FSB104D}

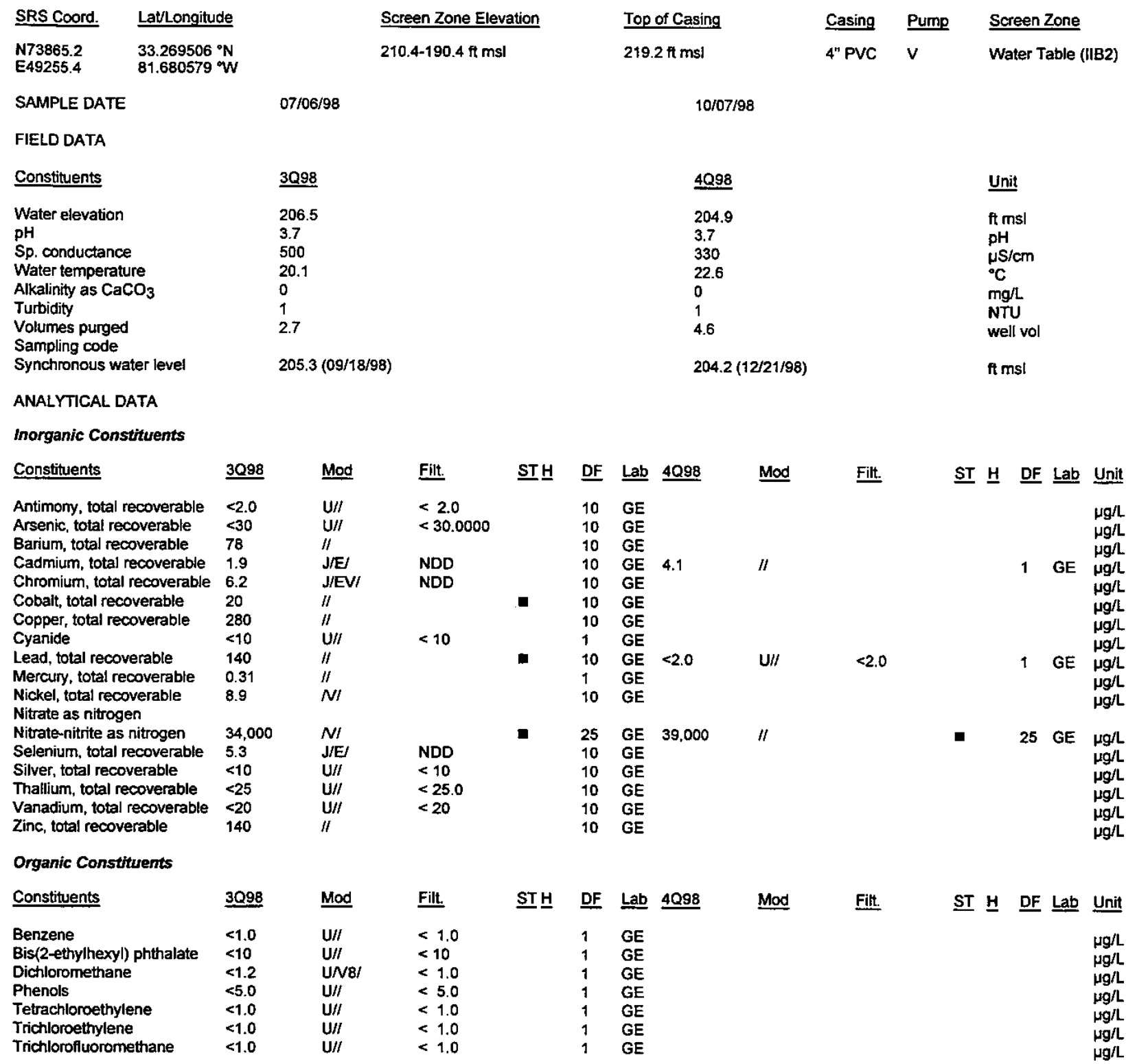

Notes:

- = exceeded holding time

- = exceeded groundwater protection or monitoring constituent standard (See Appendix A.) 
WELL FSB104D (cont.)

Radioactive Constituents

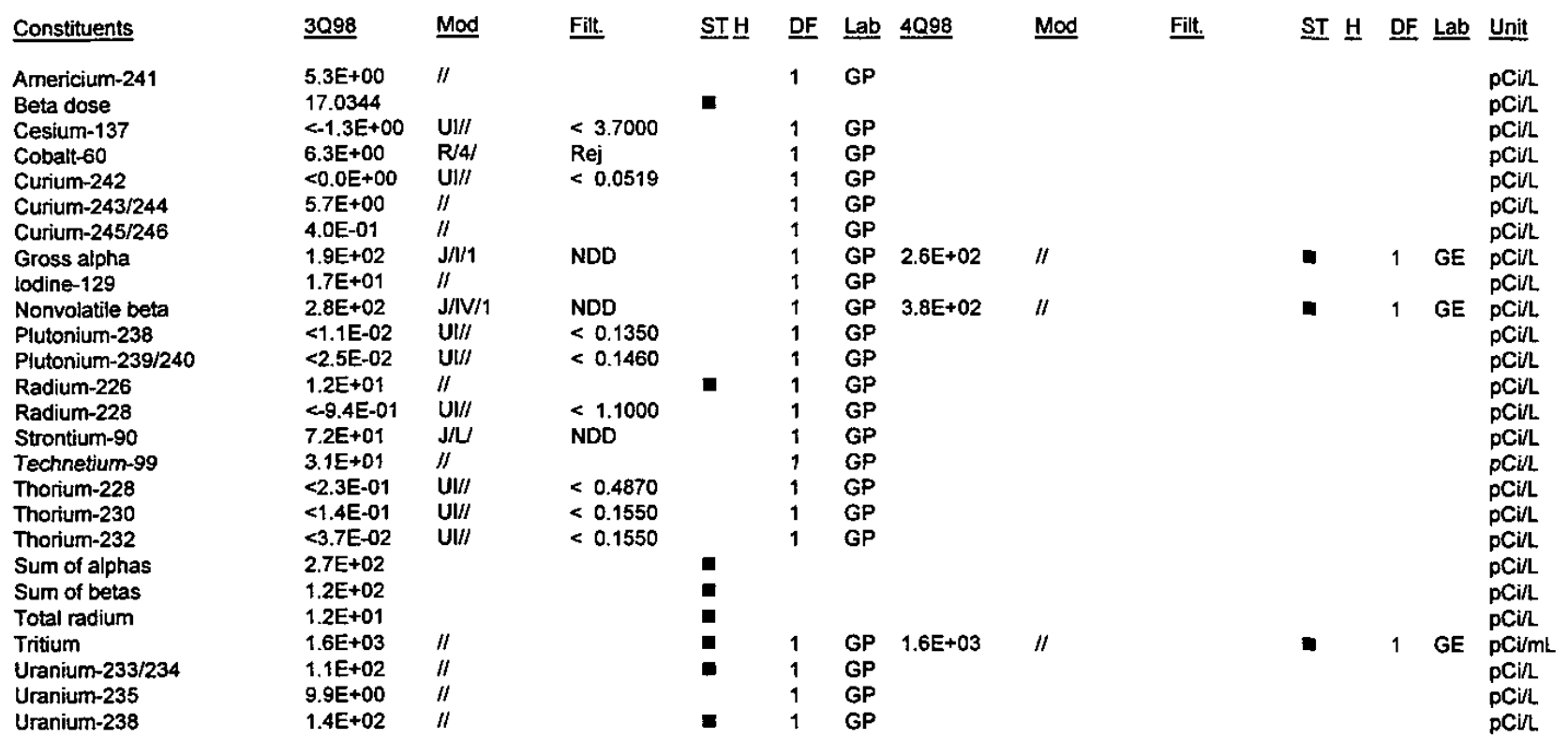

Notes:

- exceeded holding time

m = exceeded groundwater protection or monitoring constituent standard (See Appendix A.) 


\section{WELL FSB105C}

$\begin{array}{lll}\text { SRS Coord. } & & \text { LatlLongitude } \\ \text { N75234.2 } & & 33.273468^{\circ} \mathrm{N} \\ \text { E49828.0 } & 81.681732^{\circ} \mathrm{W}\end{array}$

SAMPLE DATE Screen Zone Elevation

151.5-141.5 ft mst

Top of Casing

$285.8 \mathrm{ft} \mathrm{msl}$

$10 / 05 / 98$

FIELD DATA

Constituents

3098

Water elevation

$\mathrm{pH}$

Sp. conductance

Water temperature

Alkalinity as $\mathrm{CaCO}_{3}$

Turbidity

Volumes purged

Sampling code

Synchronous water level

ANALYTICAL DATA

inorganic Constituents

Constituents

Antimony, total recoverable

Arsenic total recoverable

Barium, total recoverable

Cadmium, total recoverable 13

Chromium, total recoverable $\mathbf{4 9}$

Cobalt, total recoverable $\quad 330$

Copper, total recoverable 70

Cyanide

Lead, total recoverable

Mercury, total recoverable

Nickel, total recoverable

Nitrate as nitrogen

Nitrate-nitrite as nitrogen

Selenium, total recoverable $<500$

Silver, total recoverable

Thallium, total recoverable $\quad 1.8$

Vanadium, total recoverable $<40$

Zinc, total recoverabie

170

Organic Constituents

Constituents

Benzene

Bis(2-ethylhexyl) phthalate

Dichloromethane

Phenols

Tetrachioroethylene

Trichloroethylene

Trichlorofiuoromethane
3098

Mod

$\mathrm{U} / /$

J/E/

J/EV/

II

II

$\mathrm{U} / 1 / \quad<10$

$\mathrm{U} / I \quad<40.0000$

$\mathrm{J} / \mathrm{E} / \mathrm{NDD}$

U $/ /$
$\mathrm{UJ} / \mathrm{C} /$

UJ/CI

$\mathrm{U} / \mathrm{E} / /$

II

$\underline{3098}$

$<1.0$

$<10$

$<2.3$

4.2

$<1.0$

$<20$

$<1.0$

$\begin{array}{ll}\text { Mod } & \text { Filt. } \\ \text { UJ/O/1 } & <1.0 \\ \text { UJ/Q/ } & <10 \\ \text { UJNOO8/1 } & <1.0 \\ \text { J/E } & \text { NDD } \\ \text { UJ/O/1 } & <1.0 \\ \text { UJ/O/1 } & <1.0 \\ \text { UJ/O/1 } & <1.0\end{array}$

Filt.

$<4.0000$

$<300.0000$

ST브

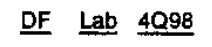

Mod

$20 \mathrm{GE}$

$100 \mathrm{GE}$

20 GE

20 GE

20 GE

20 GE

20 GE

1 GE

1 GE

$0.34 \mathrm{~J} / \mathrm{J}$

$\mathrm{J} / /$

GE

$<500.0000$

$<0$
NDD

$<40.0000$

\section{Q98}

208.2

1100

23.0

0

2.6

$207.5(12 / 21 / 98)$
Casing Pump Screen Zone

4" PVC V Barnwell (IIB $\underline{\text { Unit }}$

ft ms!

$\mathrm{pH}$

$\mu S / c m$

${ }^{\circ} \mathrm{C}$

MTU

well vol

$\mathrm{ft} \mathrm{ms}$ 


\section{WELL. FSB105C (cont.)}

\section{Radioactive Constituents}

\begin{tabular}{|c|c|c|c|c|c|c|c|c|c|c|c|c|c|}
\hline Constituents & 3098 & Mod & Filt. & $\underline{\text { ST브 }}$ & $\underline{\text { DF }}$ & $\underline{\text { Lab }}$ & 4098 & Mod & Filt. & $\underline{\mathrm{ST}} \underline{\mathrm{H}}$ & $\underline{\mathrm{DF}}$ & $\underline{\text { Lab }}$ & $\underline{\text { Unit }}$ \\
\hline Americium-241 & $1.9 \mathrm{E}+01$ & $\|$ & & a & 1 & GP & & & & & & & pCill \\
\hline Beta dose & 98.9744 & & & - & & & & & & & & & $\mathrm{pCi} / \mathrm{L}$ \\
\hline Cesium-137 & $<-7.7 \mathrm{E}-01$ & $\mathrm{UI} / /$ & $<3.6300$ & & 1 & GP & & & & & & & pCill \\
\hline Cobalt-60 & $<2.8 \mathrm{E}+00$ & $\mathrm{UI} / /$ & $<4.3700$ & & 1 & GP & & & & & & & $\mathrm{pCi} / \mathrm{L}$ \\
\hline Curium-242 & $<7.6 \mathrm{E}-02$ & $\mathrm{UI} / \mathrm{I}$ & $<0.9690$ & & 1 & GP & & & & & & & pCill \\
\hline Curium-243/244 & $1.4 E+01$ & $\|$ & & & 1 & GP & & & & & & & $\mathrm{pCi} / \mathrm{L}$ \\
\hline Curium-245/246 & $<2.7 E-01$ & $\mathrm{U} 1 / /$ & $<0.4010$ & & 1 & GP & & & & & & & $\mathrm{pCi} / \mathrm{L}$ \\
\hline Gross alpha & $6.4 E+02$ & $\|$ & & 口 & 1 & GP & $3.8 \mathrm{E}+02$ & $\|$ & & a & 1 & GE & $\mathrm{pCi} / \mathrm{L}$ \\
\hline lodine-129 & $5.2 \mathrm{E}+01$ & /I & & 口 & 1 & GP & & & & & & & $\mathrm{pCUL}$ \\
\hline Nonvolatile beta & $9.2 E+02$ & $\|$ & & a & 1 & GP & $8.4 E+02$ & $\|$ & & a & 1 & GE & $\mathrm{pCi} / \mathrm{L}$ \\
\hline Plutonium-238 & $<8.0 E-03$ & $\mathrm{UI} / I$ & $<0.0957$ & & 1 & GP & & & & & & & $\mathrm{pCi} / \mathrm{L}$ \\
\hline Plutonium-239/240 & $<-3.7 E-03$ & $\mathrm{UI} /$ & $<0.0811$ & & 1 & GP & & & & & & & $\mathrm{pCi} / \mathrm{L}$ \\
\hline Radium-226 & $7.9 \mathrm{E}+01$ & $\|$ & & $\approx$ & 1 & GP & & & & & & & $\mathrm{pCil}$ \\
\hline Radium-228 & $2.9 E+00$ & 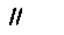 & & & 1 & GP & & & & & & & pCill \\
\hline Strontium-90 & $3.7 E+02$ & 11 & & च & 1 & GP & & & & & & & pCilL \\
\hline Technetium-99 & $1.3 E+02$ & $\|$ & & च & 1 & GP & & & & & & & pCill \\
\hline Thorium-228 & $9.0 \mathrm{E}-01$ & /1 & & & 1 & GP & & & & & & & pCill. \\
\hline Thorium-230 & $2.8 E-01$ & $\|$ & & & 1 & GP & & & & & & & $\mathrm{pCi} / \mathrm{L}$ \\
\hline Sum of alphas & 4.4E+02 & & & 田 & & & & & & & & & pCill \\
\hline Sum of betas & $5.5 E+02$ & & & m & & & & & & & & & pCirl \\
\hline Total radium & $8.1 E+01$ & & & 口 & & & & & & & & & pCill. \\
\hline Tritium & $6.3 E+03$ & $\|$ & & 田 & 1 & GP & $5.6 E+03$ & $\| I$ & & घ & 1 & GE & $\mathrm{pCi} / \mathrm{mL}$ \\
\hline Uranium-233/234 & $1.9 E+02$ & $\|$ & & $\mathbf{\square}$ & 1 & GP & & & & & & & $\mathrm{pCi} / \mathrm{L}$ \\
\hline Uranium-235 & $1.7 E+01$ & $\|$ & & - & 1 & GP & & & & & & & pCill \\
\hline Uranium-238 & 2.1E+02 & $\|$ & & $\mathbf{\square}$ & 1 & $\mathrm{GP}$ & & & & & & & pCill \\
\hline
\end{tabular}

Notes:

- = exceeded holding time

- exceeded groundwater protection or monitoring constituent standard (See Appendix A.) 
WELL FSB105DR

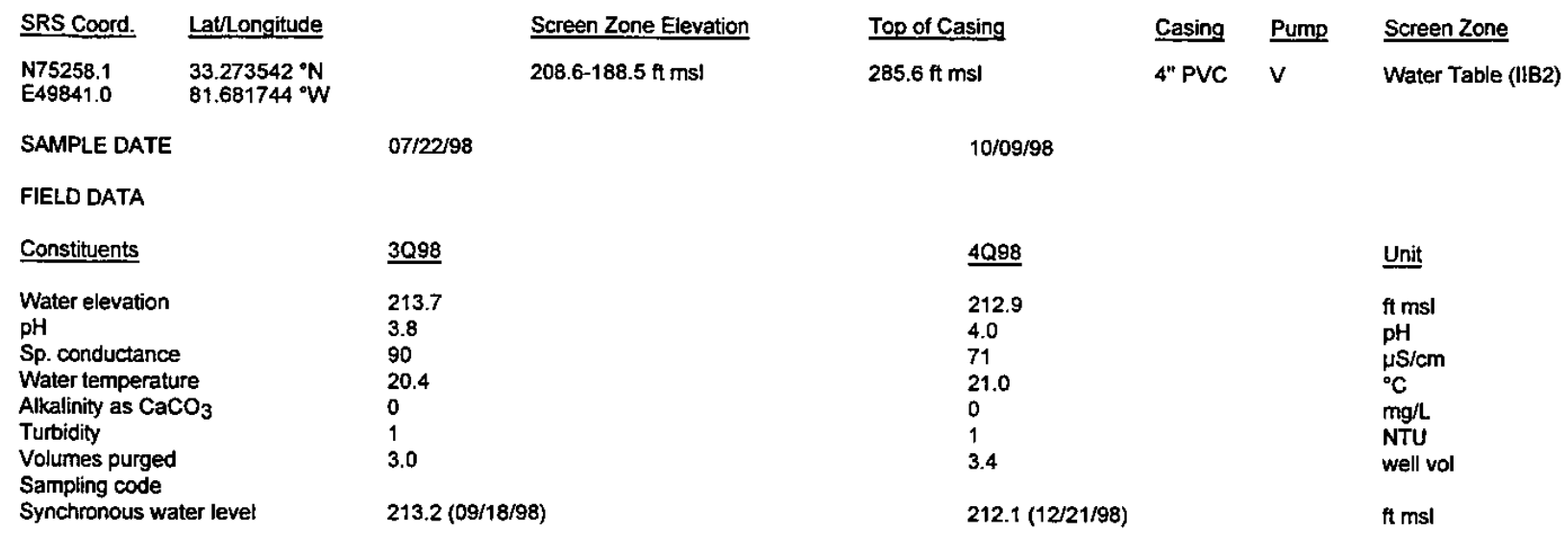

ANALYTICAL DATA

Inorganic Constituents

\begin{tabular}{|c|c|c|c|c|c|c|c|c|c|c|c|c|c|}
\hline Constituents & 3098 & Mod & Filt. & ST브 & DF & $\underline{\text { Lab }}$ & 4Q98 & Mod & Filt. & $\underline{S T} \underline{H}$ & $\underline{\text { DF }}$ & $\underline{\text { Lab }}$ & $\underline{\text { Unit }}$ \\
\hline Antimony, total recoverable & $<0.15$ & UNI & $<0.20$ & & 1 & GE & & & & & & & $\mu g / L$ \\
\hline Arsenic, total recoverable & $<3.0$ & $\mathrm{U} / I$ & $<3.0$ & & 1 & $\overrightarrow{G E}$ & & & & & & & $\mu g / L$ \\
\hline Barium, total recoverable & 94 & /I & & & 1 & GE & & & & & & & $\mu g / L$ \\
\hline Cadmium, total recoverable & $<1.0$ & $\mathrm{U} / /$ & $<1.0$ & & 1 & GE & $<1.0$ & $\mathrm{u} / /$ & $<1.0$ & & 1 & GE & $\mu g / L$ \\
\hline Chromium, total recoverable & 1.7 & $\mathrm{~J} / \mathrm{E} /$ & NDD & & 1 & GE & & & & & & & $\mu g / L$ \\
\hline Cobalt, total recoverable & 4.4 & $\|$ & & घ & 1 & GE & & & & & & & $\mu g / L$ \\
\hline Copper, total recoverable & 24 & /I & & & 1 & GE & & & & & & & $\mu g / L$ \\
\hline Cyanide & $<10$ & $\mathrm{U} / \prime$ & $<10$ & & 1 & GE & & & & & & & $\mu g / L$ \\
\hline Lead, total recoverable & 4.8 & $/ /$ & & & 1 & GE & 1.3 & $\mathrm{~J} / \mathrm{I} /$ & NDD & & 1 & GE & $\mu g / L$ \\
\hline Mercury, total recoverable & $<0.20$ & $\mathrm{U} / /$ & $<0.20$ & & 1 & $\overline{G E}$ & & & & & & & $\mu g / \mathrm{h}$ \\
\hline $\begin{array}{l}\text { Nickel, total recoverable } \\
\text { Nitrate as nitrogen }\end{array}$ & 2.4 & $/ /$ & & & 1 & GE & & & & & & & $\mu g / L$ \\
\hline Nitrate-nitrite as nitrogen & 4,100 & $N I$ & & & 3 & GE & 5,700 & /I & & & 5 & GE & $\mu g / L$ \\
\hline Selenium, total recoverable & $<5.0$ & $\mathbf{U} / /$ & $<5.0$ & & 1 & GE & & & & & & & $\mu g / L$ \\
\hline Silver, total recoverable & $<1.0$ & $\mathbf{U} / /$ & $<1.0$ & & 1 & GE & & & & & & & $\mu g / L$ \\
\hline Thallium, total recoverable & $<0.18$ & UNI & $<2.5$ & & 1 & GE & & & & & & & $\mu g / L$ \\
\hline Vanadium, total recoverable & $<2.0$ & $\mathbf{U} / /$ & $<2.0$ & & 1 & GE & & & & & & & $\mu g / L$ \\
\hline Zinc, total recoverable & 8.4 & $/ /$ & & & 1 & GE & & & & & & & $g$ \\
\hline
\end{tabular}

Organic Constituemts

\begin{tabular}{|c|c|c|c|c|c|c|c|c|c|c|}
\hline Constituents & $3 Q 98$ & Mod & Filt. & ST브 & DF & Lab 4Q98 & Mod & Filt. & ST $\underline{H}$ DF Lab & Unit \\
\hline Benzene & $<1.0$ & $\mathrm{UJ} / \mathrm{O} / 1$ & $<1.0$ & & 1 & GE & & & & $\mu g / L$ \\
\hline Bis(2-ethylhexyl) phthalate & $<10$ & $\mathrm{U} / 1$ & $<10$ & & 1 & GE & & & & $\mu g /$ \\
\hline Dichloromethane & $<1.0$ & $\mathrm{UJ} / \mathrm{O} / 1$ & $<1.0$ & & 1 & GE & & & & $\mu g / L$ \\
\hline Phenols & $<5.0$ & $\mathrm{U} / I$ & $<5.0$ & & 1 & $\overline{G E}$ & & & & $\mu g / L$ \\
\hline Tetrachloroethylene & $<1.0$ & $\mathrm{UJ} / \mathrm{O} / 1$ & $<1.0$ & & 1 & GE & & & & $\mu g / L$ \\
\hline Trichloroethylene & $<1.0$ & $\mathrm{UJ} / \mathrm{O} / \mathrm{1}$ & $<1.0$ & & 1 & $\mathrm{GE}$ & & & & $\mu g / L$ \\
\hline Trichlorofluoromethane & $<1.0$ & $\mathrm{UJ} / \mathrm{O} / \mathrm{t}$ & $<1.0$ & & 1 & GE & & & & $\mathrm{g} / \mathrm{L}$ \\
\hline
\end{tabular}

Notes:

- exceeded holding time

- exceeded groundwater protection or monitoring constituent standard (See Appendix A.)

F-Area HWMF

C-107

Third and Fourth Quarter 1998 


\section{WELL FSB105DR (cont.)}

Radioactive Constituents

\begin{tabular}{|c|c|c|c|c|c|c|c|}
\hline Constituents & $\underline{3 Q 98}$ & Mod & Filt. & ST브 & DF & Lab & $4 Q 98$ \\
\hline Americium-241 & $6.0 E-01$ & $/ 1$ & & & 1 & GP & \\
\hline Beta dose & 13.6250 & & & घ & & & \\
\hline Cesium-137 & $<6.6 E-01$ & UII/ & $<3.4000$ & & 1 & GP & \\
\hline Cobalt-60 & $<-6.6 \mathrm{E}-01$ & $\mathrm{UI} / /$ & $<3.2100$ & & 1 & GP & \\
\hline Curium-242 & $<1.8 \mathrm{E}-02$ & $\mathrm{UI} / /$ & $<0.1210$ & & 1 & $G P$ & \\
\hline Curium-243/244 & 6.4E-01 & $/ 1$ & & & 1 & GP & \\
\hline Curium-245/246 & $<0.0 E+00$ & UII/ & $<0.0606$ & & 1 & GP & \\
\hline Gross alpha & $1.5 E+02$ & $\|$ & & घ & 1 & GP & $7.8 \mathrm{E}+01$ \\
\hline lodine-129 & $1.2 \mathrm{E}+01$ & 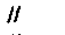 & & & 1 & GP & \\
\hline Nonvolatile beta & 7.1E+01 & /I & & $\mathbf{a}$ & 1 & GP & $7.5 \mathrm{E}+01$ \\
\hline Plutonium-238 & $<-2.2 \mathrm{E}-02$ & $\mathrm{U}: / /$ & $<0.1310$ & & 1 & GP & \\
\hline Piutonium-239/240 & $<0.0 E+00$ & Uy $/$ I & $<0.0457$ & & 1 & GP & \\
\hline Radium-226 & $3.8 E+00$ & /I & & & 1 & GP & \\
\hline Radium-228 & $<3.7 E+\infty 0$ & $\mathrm{UN} /$ & $<0.9810$ & & 1 & GP & \\
\hline Strontium-90 & $1.3 E+01$ & $\|$ & & $\boldsymbol{\square}$ & 1 & GP & \\
\hline Technetium-99 & $<-4.2 E+00$ & UII/ & $<25.0$ & & 1 & GP & \\
\hline Thorium-228 & $3.2 E-01$ & $/ /$ & & & 1 & $\mathrm{GP}$ & \\
\hline Thorium-230 & $<-3.2 E-02$ & $\mathrm{UI} / /$ & $<0.1910$ & & 1 & GP & \\
\hline Thorium-232 & $<1.2 \mathrm{E}-02$ & $\mathrm{U} U / /$ & $<0.1380$ & & 1 & GP & \\
\hline Sum of alphas & $1.5 \mathrm{E}+02$ & & & $\mathbf{a}$ & & & \\
\hline Sum of betas & $2.5 E+01$ & & & & & & \\
\hline Total radium & $3.8 E+00$ & & & & & & \\
\hline Tritium & 4.1E+01 & $/ /$ & & $\mathbf{\square}$ & 1 & GP & $4.6 E+01$ \\
\hline Uranium-233/234 & $3.6 \mathrm{E}+01$ & $\|$ & & $\mathbf{\square}$ & 1 & GP & \\
\hline Uranium-235 & $4.5 E+00$ & $/ /$ & & & 1 & GP & \\
\hline Uranium-238 & $1.0 E+02$ & // & & 口 & 1 & GP & \\
\hline
\end{tabular}

ST $\underline{H}$ DF Lab Unit

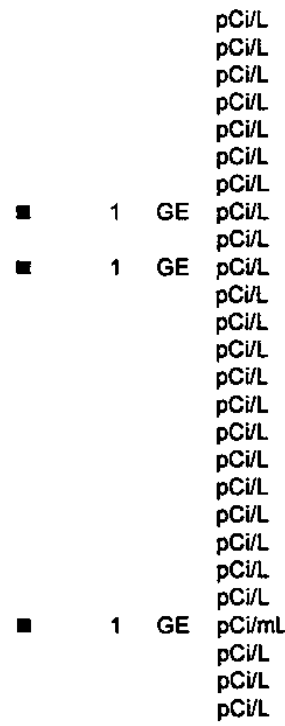

Notes:

- = exceeded holding time

= exceeded groundwater protection or monitoring constituent standard (See Appendix A.) 
WELL FSB106C

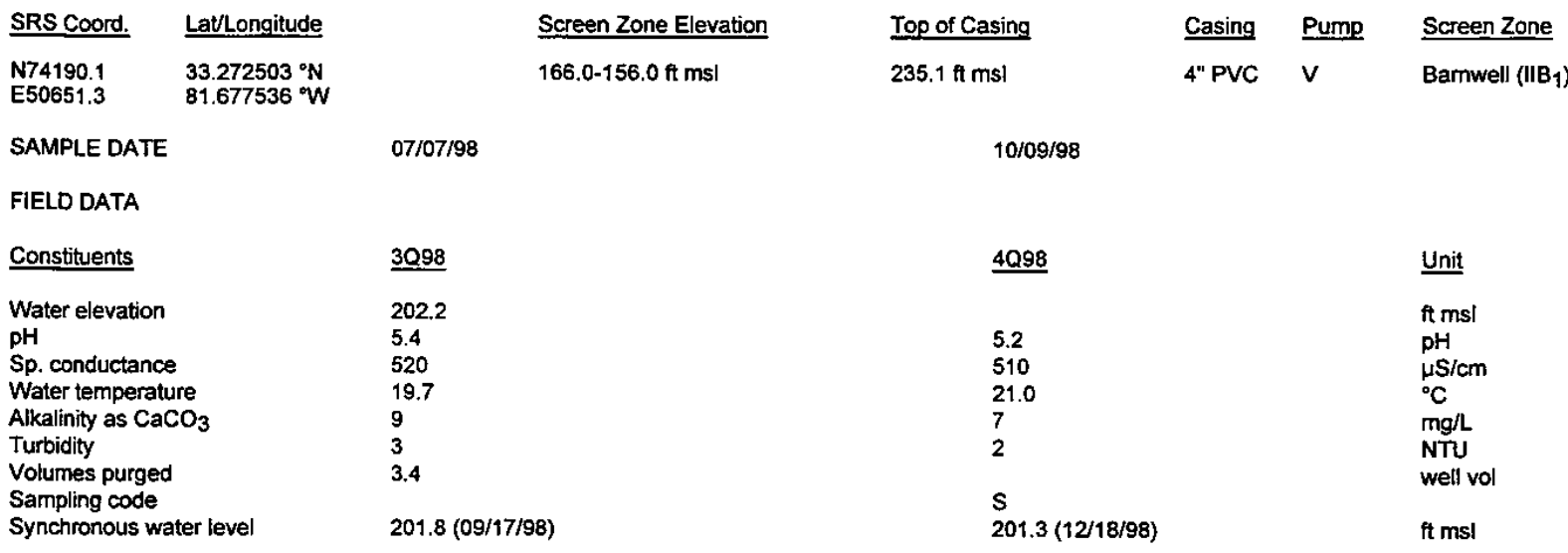

\section{ANALYTICAL DATA}

Inorganic Constituents

Constituents

Antimony, total recoverable

Arsenic, total recoverable

Barium, total recoverable

Cadmium, total recoverable

Chromium, total recoverable

Cobalt, total recoverable

Copper, total recoverable

Cyanide

Lead, total recoverable

Mercury, total recoverable

Nickel, total recoverable

Nitrate as nitrogen

Nitrate-nitrite as nitrogen

Selenium, total recoverable

Silver, total recoverable

Thallium, total recoverable

Vanadium, total recoverable

Zinc, total recoverable

Organic Constituents

Constituents

Benzene

Bis(2-ethylhexyl) phthalate

Dichloromethane

Phenols

Tetrachloroethylene

Trichloroethylene

Trichlorofluoromethane
309

7.0

$J / E$

NDD

25 GE 6.9

/I

J/EV $/ \quad$ NDD

$25 \mathrm{GE}<2.0$

U/I

$N$

50 GE 63,000

II

3098

Mod

Filt.

ST브

DF Lab

Filt.

ST $\underline{H}$ DF Lab Unit

\section{Notes:}

- = exceeded holding time

- exceeded groundwater protection or monitoring constituent standard (See Appendix A.) 
WELL FSB106C (cont.)

Radioactive Constituents

Constituents

3098

Mod

Filt.

ST브 므 ㄴab 4098

Mod

Filt.

ST $\underline{H}$ DF Lab Unit

Americium-241

Cesium-137

Cobalt-60

Curium-242

Curium-243/244

Curium-245/246

Gross alpha

4.3E+01

lodine-129

Nonvolatile beta

Plutonium-238

Plutonium-239/240

Radium-226

Radium-228

Strontium-90

Technetium-99

Thorium-228

Thorium-230

Thorium-232

Sum of alphas

Sum of betas

Tritium

Uranium-233/234

$5.6 \mathrm{E}+02 \quad / /$

-

GP $2.9 \mathrm{E}+01$

- 1 GP $6.0 \mathrm{E}+02 \quad / /$

Uranium-235

Uranium-238

Notes:

- exceeded holding time

- = exceeded groundwater protection or monitoring constituent standard (See Appendix A.) 
WELL FSB106D

\begin{tabular}{|c|c|}
\hline SRS coord. & Lat/Longituc \\
\hline $\begin{array}{l}N 74193.0 \\
\text { E50636.8 }\end{array}$ & 81.677580 व \\
\hline
\end{tabular}

SAMPLE DATE

FIELD DATA

Constituents

Water elevation

$\mathrm{pH}$

Sp. conductance

Water temperature

Alkalinity as $\mathrm{CaCO}_{3}$

Turbidity

Volumes purged

Sampling code

Synchronous water level

Screen Zone Elevation

222.9-202.9 ft ms

\section{Top of Casing}

$234.9 \mathrm{ft} \mathrm{msl}$
Casing Purmp

4" PVC S
Screen Zone

Water Table (II82)

ANALYTICAL DATA

Inorganic Constituents

\section{Constituents}

Antimony, total recoverable Arsenic, total recoverable

Barium, total recoverable

Cadmium, total recoverable

Chromium, total recoverable $\mathbf{5}$.7

Cobalt, total recoverable 1.8

Copper, total recoverable 38

Cyanide

Lead, total recoverable 220

Mercury, total recoverable $<0.20$

Nickel, total recoverat

Nitrate as nitrogen

Nitrate-nitrite as nitrogen $\quad 330$

Selenium, total recoverable $<25$

Silver, total recoverable $<5.0$

Thalium, total recoverable $<13$

Vanadium, total recoverable 17

Zinc, total recoverable

8,400

Organic Constituents

\begin{tabular}{ll} 
Constituents & 3098 \\
\hline & $<1.0$ \\
Benzene & $<10$ \\
Bis(2-ethylhexyl) phthalate & $<1.0$ \\
Dichloromethane & $<5.0$ \\
Phenols & $<1.0$ \\
Tetrachloroethylene & $<1.0$ \\
Trichloroethylene & $<1.0$ \\
Trichloroftuoromethane &
\end{tabular}

3Q98

207.6

6.6

120

24.7

0.0

$\mathrm{XN}$

$206.7(09 / 17 / 98)$
$10 / 30 / 98$

4Q98
206.0
5.9
80
14.5
18
17
0.49
$X N$
$205.9(12 / 18 / 98)$

Unit

ft ms!

$\mathrm{pH}$

$\mu \mathrm{S} / \mathrm{cm}$

mg/l

well vol

ft msl

\begin{tabular}{|c|c|c|}
\hline Mod & Filt. & $\underline{\mathrm{ST}} \underline{\mathrm{H}}$ \\
\hline \multicolumn{3}{|l|}{$N^{\prime}$} \\
\hline $\begin{array}{l}\mathrm{J} / \mathrm{E} / \\
/ /\end{array}$ & NDD & \\
\hline $\mathrm{J} / \mathrm{E} /$ & NDD & \\
\hline $\mathrm{J} / \mathrm{E} /$ & NDD & \\
\hline \multicolumn{3}{|l|}{ II } \\
\hline \multicolumn{3}{|l|}{ il } \\
\hline $\mathrm{U} / /$ & $<10$ & \\
\hline$N /$ & & $\mathbf{E}$ \\
\hline $\mathrm{U} / /$ & $<0.20$ & \\
\hline \multicolumn{3}{|l|}{$\| 1$} \\
\hline \multicolumn{3}{|l|}{$N I$} \\
\hline $\mathrm{U} / /$ & $<25.0$ & \\
\hline $\mathrm{U} / /$ & $<5.0$ & \\
\hline $\mathrm{U} / /$ & $<12.5$ & \\
\hline$N \prime$ & & ש \\
\hline$\|$ & & ש \\
\hline
\end{tabular}

DF Lab 4098

$\mathrm{GE}$
$\mathrm{GE}$
$\mathrm{GE}$
$\mathrm{GE}$
$\mathrm{GE}$
$\mathrm{GE}$
$\mathrm{GE}$
$\mathrm{GE}$
$\mathrm{GE}$
$\mathrm{GE}$
$\mathrm{GE}$
$\mathrm{GE}$
$\mathrm{GE}$
$\mathrm{GE}$
$\mathrm{GE}$
$\mathrm{GE}$

GE

EE

GE

$22 \quad / 1$

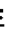

$1,400 \quad / /$

.

GE

GE

$\begin{array}{ll}\text { Mod } & \text { Filt. } \\ U / I & <1.0 \\ U / I & <10 \\ U / I & <1.0 \\ U / I & <5.0 \\ U / I & <1.0 \\ U / I & <1.0 \\ U / I & <1.0\end{array}$

ST브

\begin{tabular}{|c|c|c|c|}
\hline Ilt. & ST브 & $\underline{D F}$ & Lab \\
\hline 1.0 & & 1 & $G E$ \\
\hline 10 & & 1 & $\mathrm{GE}$ \\
\hline 1.0 & & 1 & $\mathrm{GE}$ \\
\hline 5.0 & & 1 & $\mathrm{GE}$ \\
\hline 1.0 & & 1 & $G E$ \\
\hline 1.0 & & 1 & GE \\
\hline 1. & & 1 & \\
\hline
\end{tabular}

Mod

Filt.

ST 브 DF Lab Unit

$\mu g / 1$

$\mu g / L$

$\mu g / L$

1 GE $\mu g / L$

$\mu g h$

$\mu g / L$

$\mu g / L$

1 GE $\mu g / L$

$\mu g / L$

$\mu g / L$

1 GE $\mu g / L$

$\mu g / L$

$\mu g / L$

$\mu g / L$

$\mu g / L$

$\mu g / L$

Mod

Filt.

ST 브 DF Lab Unit

$\mu g / L$

$\mu g / L$

$\mu g / L$

$\mu g / L$

$\mu g / L$

$\mu g / L$

$\mu g / L$

\section{Notes:}

- exceeded holding time

- exceeded groundwater protection or monitoring constituent standard (See Appendix A.)

F-Area HWMF

C-111

Third and Fourth Quarter 1998 


\section{WELL FSB106D (cont.)}

Radloactive Constituents

\begin{tabular}{|c|c|c|c|c|c|c|c|c|}
\hline Constituents & 3098 & Mod & Filt. & ST브 & DF & Lab & $4 Q 98$ & Mod \\
\hline Americium-241 & $\begin{array}{l}<-1.3 E-02 \\
0.4500\end{array}$ & $\mathrm{UI} / /$ & $<0.0868$ & & 1 & GP & & \\
\hline Beta dose & $\begin{array}{l}0.4500 \\
<2.2 \mathrm{E}-01\end{array}$ & & & & & & & \\
\hline Cesium-137 & & $\mathrm{UI} / /$ & $<3.5400$ & & 1 & GP & & \\
\hline Cobalt -60 & $<-1.8 E+00$ & UIII & $<3.0200$ & & 1 & GP & & \\
\hline Curium-242 & $<0.0 E+00$ & UIII & $<0.0522$ & & 1 & GP & & \\
\hline Curium-243/244 & $<8.2 \mathrm{E}-02$ & $\mathrm{UN} /$ & $<0.0494$ & & 1 & GP & & \\
\hline Curium-245/246 & $<3.3 E-02$ & $\mathrm{UI} / /$ & $<0.0493$ & & 1 & GP & & \\
\hline Gross alpha & $<1.3 E-01$ & UUII & $<0.8630$ & & 1 & GP & $2.6 \mathrm{E}+00$ & $\|$ \\
\hline lodine-129 & $<8.8 \mathrm{E}-01$ & $\mathrm{UI} / /$ & $<0.9910$ & & 1 & GP & & \\
\hline Nonvolatile beta & $4.3 E+00$ & "I & & & 1 & GP & $4.8 \mathrm{E}+00$ & $/ /$ \\
\hline Plutonium-238 & $<6.7 E-02$ & $\mathrm{U} \mathrm{U} / /$ & $<0.2360$ & & 1 & GP & & \\
\hline Plutonium-239/240 & $<-1.9 E-02$ & $\mathrm{Ut} / /$ & $<0.1270$ & & 1 & GP & & \\
\hline Radium-226 & $<3.6 \mathrm{E}-01$ & $\mathrm{UU} / /$ & $<0.6690$ & & 1 & GP & & \\
\hline Radium-228 & $<5.4 \mathrm{E}-01$ & $\mathrm{UI} / \mathrm{I}$ & $<0.7950$ & & 1 & GP & & \\
\hline Strontium-90 & $3.6 E+00$ & /I & & & 1 & GP & & \\
\hline Technetium-99 & $<-3.0 E+00$ & $\mathrm{UI} / /$ & $<21.6000$ & & 1 & GP & & \\
\hline Thorium-228 & $<3.2 \mathrm{E}-02$ & UIII & $<0.6000$ & & 1 & GP & & \\
\hline Thorium-230 & $<1.3 E-04$ & $\mathrm{Uu} / /$ & $<0.2440$ & & 1 & GP & & \\
\hline Thorium-232 & $<0.0 \mathrm{E}+00$ & $\mathrm{UI} / /$ & $<0.1390$ & & 1 & GP & & \\
\hline Sum of atphas & $1.2 \mathrm{E}-01$ & & & & & & & \\
\hline Sum of betas & $3.6 \mathrm{E}+00$ & & & & & & & \\
\hline Tritium & $1.2 \mathrm{E}+01$ & /I & & & 1 & GP & $1.2 E+01$ & $\|$ \\
\hline Uranium-233/234 & $<1.4 E-01$ & $\mathrm{U} N I$ & $<0.0916$ & & 1 & GP & & \\
\hline Uranium-235 & $<1.3 E-02$ & $\mathrm{U} \mid / /$ & $<0.0919$ & & 1 & GP & & \\
\hline Uranium-238 & $1.2 E-01$ & $/ /$ & & & 1 & GP & & \\
\hline
\end{tabular}

Filt. $\quad \underline{\text { ST }} \underline{\mathrm{H}} \underline{\mathrm{DF}} \underline{\underline{L} \mathrm{~b}} \underline{\text { Unit }}$

pCill

$\mathrm{pCi} / \mathrm{L}$

$\mathrm{pCil}$

PCilL

pCil

pCir

$\mathrm{pCi}$.

1 GE $\mathrm{pCi} / \mathrm{L}$

pCir

1 GE pCir

pCil

pCir

pcill

pCi/L

$\mathrm{pCi}$ il

pCill

pCil

pCil

pCil

pCill

pCir

1 GE pCimL

pCill

pCir

pCir

Notes:

- exceeded holding time

= exceeded groundwater protection or monitoring constituent standard (See Appendix A.) 


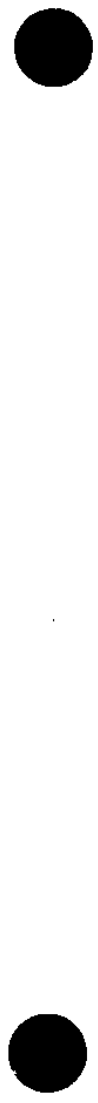

\section{WELL FSB107C}

\begin{tabular}{|c|c|c|c|c|c|c|c|}
\hline SRS Coord. & \multicolumn{2}{|l|}{ Lat/Longitude } & Screen Zone Elevation & Top of Casing & Casing & Pump & Screen Zone \\
\hline $\begin{array}{l}\text { N75184.0 } \\
\text { E51158.1 }\end{array}$ & $\begin{array}{l}33.275528^{\circ} \mathrm{N} \\
81.678133^{\circ} \mathrm{W}\end{array}$ & & $160.8-150.8 \mathrm{ft} \mathrm{msl}$ & $270.9 \mathrm{ft} \mathrm{msl}$ & 4" PVC & $\mathbf{s}$ & Bamwell (llB 1$)$ \\
\hline \multicolumn{2}{|c|}{ SAMPLE DATE } & \multicolumn{2}{|l|}{$07 / 22 / 98$} & \multicolumn{2}{|c|}{$10 / 27 / 98$} & & \\
\hline \multicolumn{8}{|l|}{ FIELD DATA } \\
\hline \multicolumn{2}{|l|}{ Constituents } & \multicolumn{2}{|l|}{3098} & \multicolumn{2}{|c|}{ 4Q98 } & & Unit \\
\hline \multicolumn{2}{|c|}{ Water elevation } & \multicolumn{2}{|l|}{213.0} & \multicolumn{2}{|c|}{212.6} & & $f t \mathrm{msl}$ \\
\hline \multirow{2}{*}{\multicolumn{2}{|c|}{$\mathrm{pH}$}} & \multicolumn{2}{|l|}{6.2} & \multicolumn{2}{|c|}{5.9} & & $\mathrm{pH}$ \\
\hline \multirow{2}{*}{\multicolumn{2}{|c|}{ Water temperature }} & \multicolumn{2}{|l|}{170} & \multicolumn{2}{|l|}{130} & & $\mu \mathrm{S} / \mathrm{cm}$ \\
\hline & & \multicolumn{2}{|l|}{21.2} & \multicolumn{2}{|l|}{21.2} & & ${ }^{\circ} \mathrm{C}$ \\
\hline \multicolumn{2}{|c|}{ Alkalinity as $\mathrm{CaCO}_{3}$} & \multicolumn{2}{|l|}{29} & \multicolumn{2}{|l|}{19} & & $\mathrm{mg} / \mathrm{l}$ \\
\hline \multicolumn{2}{|c|}{ Turbidity } & & \multicolumn{2}{|l|}{4} & & NTU \\
\hline \multicolumn{2}{|c|}{ Volumes purged } & & \multicolumn{2}{|l|}{2.4} & & well vol \\
\hline Synchronous & ter level & \multicolumn{2}{|c|}{$213.3(09 / 18 / 98)$} & \multicolumn{2}{|c|}{$212.0(12 / 18 / 98)$} & & $\mathrm{ft} \mathrm{ms}$ \\
\hline
\end{tabular}

ANALYTICAL DATA

Inorganic Constituents

\begin{tabular}{|c|c|c|c|c|c|c|c|c|c|c|c|c|c|}
\hline Constituents & 3Q98 & Mod & Filt. & $\underline{\text { ST }} \underline{H}$ & $\underline{\mathrm{DF}}$ & $\underline{\text { Lab }}$ & 4Q98 & Mod & Filt. & ST $\underline{H}$ & $\underline{\mathrm{DF}}$ & Lab & Unit \\
\hline Antimony, total recoverable & $<0.20$ & $v / l$ & $<0.20$ & & 1 & GE & & & & & & & $\mu g / L$ \\
\hline Arsenic, total recoverable & $<3.0$ & $\mathrm{U} / \mathrm{I}$ & $<\mathbf{3 . 0}$ & & 1 & GE & & & & & & & $\mu g / L$ \\
\hline Barium, total recoverable & 37 & $\|$ & & & 1 & GE & & & & & & & \\
\hline Cadmium, total recoverable & 0.56 & $\mathrm{~J} / \mathrm{E} /$ & NDD & & 1 & GE & 0.37 & $\mathrm{~J} / / /$ & NDD & & 1 & GE & \\
\hline Chromium, total recoverable & 1.3 & $\mathrm{~J} / \mathrm{E} /$ & NDD & & 1 & GE & & & & & & & \\
\hline Cobalt, total recoverable & 0.24 & $\|$ & & & 1 & GE & & & & & & & \\
\hline Copper, total recoverable & 4.0 & /I & & & 1 & GE & & & & & & & \\
\hline Cyanide & $<10$ & $\mathrm{U} / /$ & $<10$ & & 1 & GE & & & & & & & \\
\hline Lead, total recoverable & 0.88 & J/E $/$ & NDD & & 1 & GE & $<2.0$ & $\mathrm{U} / /$ & $<2.0$ & & 1 & GE & \\
\hline Mercury, total recoverable & $<0.20$ & $\mathbf{U} / /$ & $<0.20$ & & 1 & GE & & & & & & & \\
\hline Nickel, total recoverable & 3.2 & $/ 1$ & & & 1 & GE & & & & & & & \\
\hline Nitrate as nitrogen & & & & & & & & & & & & & \\
\hline Nitrate-nitrite as nitrogen & 8,700 & $N I$ & & & 100 & WA & 6,300 & /l & & & 5 & GE & \\
\hline Selenium, total recoverable & $<5.0$ & $\mathrm{U} / /$ & $<5.0$ & & 1 & GE & & & & & & & \\
\hline Silver, total recoverable & 0.62 & $\mathrm{~J} / \mathrm{E} /$ & NDD & & 1 & WA & & & & & & & \\
\hline Thallium, total recoverable & $<0.072$ & UN6/ & $<2.5$ & & 1 & GE & & & & & & & \\
\hline Vanadium, total recoverable & $<2.0$ & $\mathbf{U} / /$ & $<2.0$ & & 1 & GE & & & & & & & \\
\hline Zinc, total recoverable & 28 & $\|$ & & & 1 & GE & & & & & & & \\
\hline
\end{tabular}

Organic Constituents

\begin{tabular}{|c|c|c|c|c|c|c|c|c|c|c|c|}
\hline Constituents & $\underline{3098}$ & Mod & Filt. & ST $\underline{H}$ & OF & $\underline{\text { Lab }}$ & 4098 & $\underline{\text { Mod }}$ & Filt. & $\underline{\text { ST }} \underline{\text { H }}$ DF Lab & Unit \\
\hline Benzene & $<1.0$ & UJ/O/1 & $<1.0$ & & 1 & GE & & & & & $\mu g^{\prime}$ \\
\hline Bis(2-ethylhexyl) phthalate & $<1.7$ & $\mathrm{UN} /$ & $<5.1$ & & 1 & WA & & & & & $\mu g / L$ \\
\hline Dichloromethane & $<1.0$ & $\mathrm{UJ} / \mathrm{O} / 1$ & $<1.0$ & & 1 & GE & & & & & $\mu g / L$ \\
\hline Phenols & $<5.0$ & $\mathbf{U} / /$ & $<5.0$ & & 1 & GE & & & & & $\mu g / L$ \\
\hline Tetrachloroethylene & 1.3 & $\mathrm{~J} / \mathrm{E} /$ & NDD & & 1 & WA & & & & & $g / L$ \\
\hline Trichloroethylene & 1.4 & $\mathrm{~J} / \mathrm{E} /$ & NDD & & 1 & WA & & & & & L \\
\hline Trichlorofluoromethane & 5.2 & $\|$ & & 口 & 1 & WA & & & & & \\
\hline
\end{tabular}

Notes:

- = exceeded holding time

- = exceeded groundwater protection or monitoring constituent standard (See Appendix A.) 


\section{WELL FSB107C (cont.)}

Radioactive Constituents

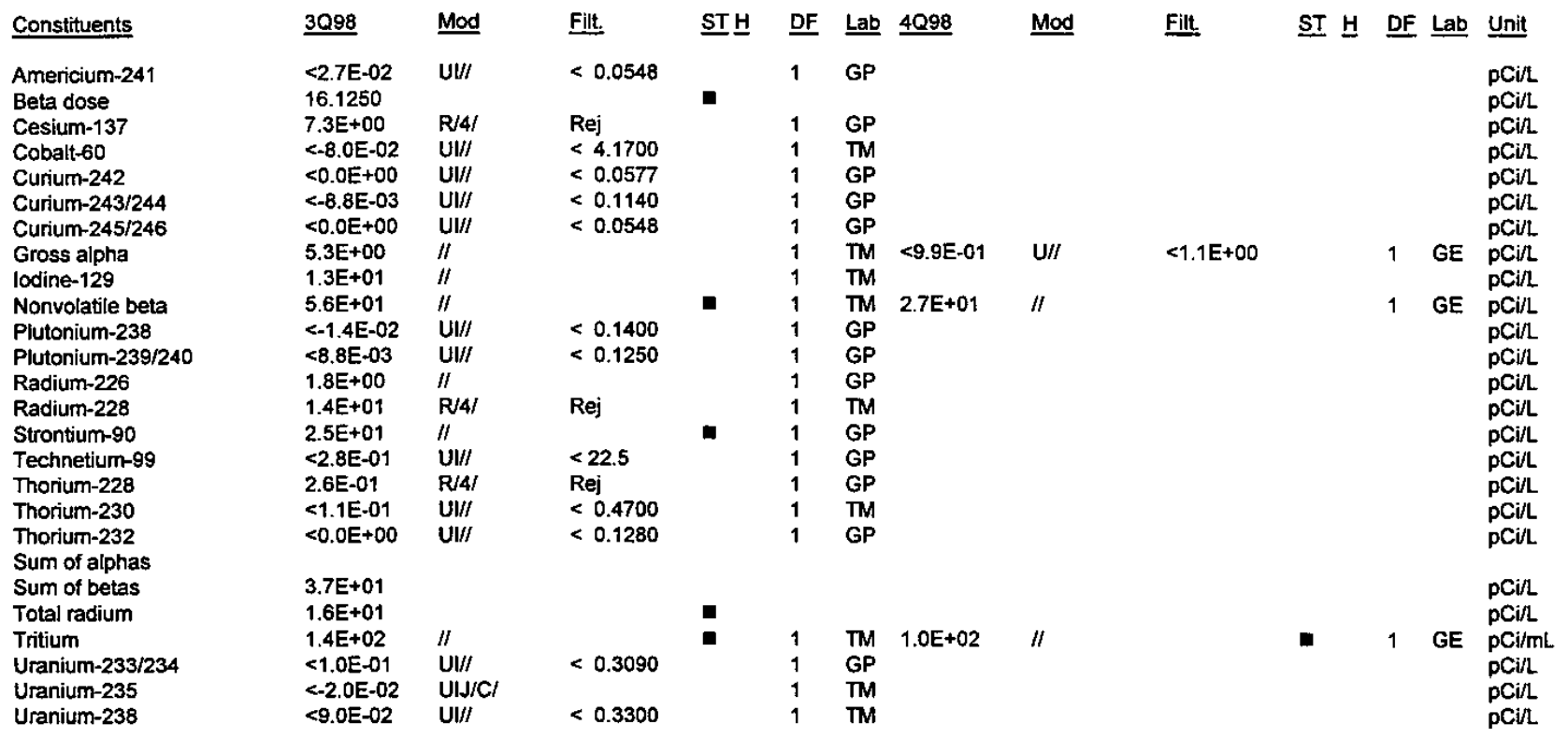

Notes:

- = exceeded holding time

- exceeded groundwater protection or monitoring constituent standard (See Appendix A.) 
WELL FSB107D

\begin{tabular}{|c|c|c|c|c|c|c|}
\hline SRS Coord. & Lat/Longitude & Screen Zone Elevation & Top of Casing & Casing & Pump & Screen Zone \\
\hline $\begin{array}{l}\text { N75177.2 } \\
\text { E51149.8 }\end{array}$ & $\begin{array}{l}33.275499^{\circ} \mathrm{N} \\
81.678141^{\circ} \mathrm{N}\end{array}$ & $220.9-200.9 \mathrm{ft} \mathrm{msl}$ & $271 \mathrm{ft} \mathrm{msl}$ & 4" PVC & V & Water Table (IIB2) \\
\hline
\end{tabular}

SAMPLE DATE

07/10/98

$10 / 07 / 98$

FIELD DATA

Constituents

3Q98

Water elevation

Sp. conductance

Water temperature

Alkalinity as $\mathrm{CaCO}_{3}$

Turbidity

320

22.8

1

Volumes purged

Sampling code

Synchronous water level

\section{(10)}

4Q98

216.3

3.8

130

19.8

0

2.4

$215.4(12 / 18 / 98)$
Unit

ft ms!

$\mathrm{pH}$

${ }^{\circ} \mathrm{C} / \mathrm{cm}$

$\mathrm{mg} / \mathrm{L}$

NTU

well vol

ft ms]

ANALYTICAL DATA

Inorganic Constituemts

\begin{tabular}{|c|c|c|c|c|c|c|c|c|c|}
\hline Constituents & $\underline{3 Q 98}$ & Mod & Filt. & $\underline{\text { ST브 }}$ & $\underline{D F}$ & $\underline{\text { Lab }}$ & 4Q98 & Mod & Filt. \\
\hline Antimony, total recoverable & $<1.0$ & $\mathrm{U} / /$ & $<1.0$ & & 5 & GE & & & \\
\hline Arsenic, total recoverable & $<15$ & $\mathrm{U} / /$ & $<15.0$ & & 5 & GE & & & \\
\hline Barium, total recoverable & 110 & $/ 1$ & & & 5 & GE & & & \\
\hline Cadmium, total recoverable & 1.6 & $\mathrm{~J} / \mathrm{E} /$ & NDD & & 5 & GE & 0.79 & $\mathrm{~J} / \mathrm{I}$ & NDD \\
\hline Chromium, total recoverable & 6.9 & J/EV/ & NDD & & 5 & GE & & & \\
\hline Cobalt, total recoverable & 17 & 11 & & (a & 5 & GE & & & \\
\hline Copper, total recoverable & $<1.0$ & $\mathrm{U} / 1$ & $<1.0$ & & 5 & GE & & & \\
\hline Cyanide & $<10$ & $\mathrm{U} / /$ & $<10$ & & 1 & GE & & & \\
\hline Lead, total recoverable & 16 & $\|$ & & - & 5 & GE & 2.6 & $\|$ & \\
\hline Mercury, total recoverable & 3.2 & $/ 1$ & & $\mathbf{E}$ & 1 & GE & & & \\
\hline $\begin{array}{l}\text { Nickel, total recoverable } \\
\text { Nitrate as nitrogen }\end{array}$ & 14 & $\|$ & & & 5 & GE & & & \\
\hline Nitrate-nitrite as nitrogen & 30,000 & $16 /$ & & $\mathbf{\square}$ & 25 & GE & $\uparrow 1,000$ & /I & \\
\hline Selenium, total recoverable & $<25$ & $\mathrm{U} / /$ & $<25.0$ & & 5 & GE & & & \\
\hline Silver, total recoverable & $<5.0$ & $\mathrm{UJ} / \mathrm{C} /$ & $<5.0$ NDD & 5 & GE & & & & \\
\hline Thallium, total recoverable & $<13$ & $\mathrm{U} / /$ & $<12.5$ & & 5 & GE & & & \\
\hline Vanadium, total recoverable & $<10$ & $\mathrm{U} / /$ & $<10$ & & 5 & GE & & & \\
\hline Zinc, total recoverable & 83 & /l & & & 5 & GE & & & \\
\hline
\end{tabular}

Organic Constituents

\begin{tabular}{|c|c|c|c|c|c|}
\hline Constituents & $3 Q 98$ & Mod & Filt. & $\underline{\text { ST }} \underline{H}$ & DF \\
\hline Benzene & $<1.0$ & $\mathrm{UJ} / \mathrm{O} / 1$ & $<1.0$ & & 1 \\
\hline Bis(2-ethylhexyl) phthalate & $<10$ & $\mathrm{U} / /$ & $<10$ & & 1 \\
\hline Dichloromethane & $<2.5$ & UJNO8/1 & $<1.0$ & & 1 \\
\hline Phenols & $<5.0$ & $\mathrm{U} / /$ & $<5.0$ & & 1 \\
\hline Tetrachloroethylene & $<1.0$ & UJ/O/1 & $<1.0$ & & 1 \\
\hline Trichloroethylene & $<1.0$ & $\mathrm{UJ} / \mathrm{O} / 1$ & $<1.0$ & & 1 \\
\hline Trichlorofluoromethane & $<1.0$ & UJ/O/1 & $<1.0$ & & 1 \\
\hline
\end{tabular}

\section{otal recoverab}

Thallium, total recoverable

83

/l

Trichloroethylene

Trichlorofluoromethane

. 
WELL FSB107D (cont.)

Radioactive Constituents

\begin{tabular}{|c|c|c|c|c|c|c|c|c|c|c|c|c|c|}
\hline Constituents & 3098 & Mod & Filt. & $\underline{\mathrm{ST}} \underline{\mathrm{H}}$ & $\underline{\mathrm{DF}}$ & Lab & 4Q98 & Mod & Filt. & ST $\underline{H}$ & DF & Lab & Unit \\
\hline Americium-241 & $1.5 E+01$ & /I & & w & 1 & $\mathrm{GP}$ & & & & & & & $\mathrm{pCi} / \mathrm{L}$ \\
\hline Beta dose & 106.7378 & & & $\mathbf{a}$ & & & & & & & & & $\mathrm{pCi} / \mathrm{L}$ \\
\hline Cesium-137 & $<4.0 E-02$ & $\mathrm{UI} / /$ & $<4.1900$ & & 1 & GP & & & & & & & $\mathrm{pCi} / \mathrm{L}$ \\
\hline Cobalt-60 & $<6.1 E-01$ & $\mathrm{UI} / /$ & $<3.6900$ & & 1 & GP & & & & & & & $\mathrm{pCi} / \mathrm{L}$ \\
\hline Curium-242 & $<1.7 \mathrm{E}-01$ & $\mathrm{Ui} / /$ & $<0.4980$ & & 1 & GP & & & & & & & $\mathrm{pCi} / \mathrm{L}$ \\
\hline Curium-243/244 & $4.5 E+00$ & $/ /$ & & & 1 & GP & & & & & & & $\mathrm{pCil}$ \\
\hline Curium-245/246 & $<2.9 \mathrm{E}-01$ & $\mathrm{UI} / I$ & $<0.4350$ & & 1 & GP & & & & & & & $\mathrm{pCi} / \mathrm{L}$ \\
\hline Gross alpha & $3.0 \mathrm{E}+02$ & $\|$ & & $\mathbf{a}$ & 1 & GP & $1.8 \mathrm{E}+02$ & $\|$ & & $\mathbf{E}$ & 1 & GE & $\mathrm{pCill}$ \\
\hline Nonvolatile beta & $4.6 E+02$ & /l & & 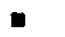 & 1 & GP & $1.8 E+02$ & $\|$ & & $\mathbf{\square}$ & 1 & GE & pCirl \\
\hline Plutonium-238 & $<0.0 \mathrm{E}+00$ & $\mathrm{UI} / /$ & $<0.0490$ & & 1 & GP & & & & & & & $\mathrm{pCi} / \mathrm{L}$ \\
\hline Plutonium-239/240 & $<-3.9 E-03$ & $\mathrm{UI} / /$ & $<0.0861$ & & 1 & GP & & & & & & & pCill \\
\hline Radium-226 & $1.4 E+01$ & 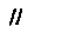 & & $\mathbf{\sigma}$ & 1 & GP & & & & & & & $\mathrm{pCill}$ \\
\hline Radium-228 & $2.1 E+00$ & $\|$ & & & $i$ & GP & & & & & & & pCi/L \\
\hline Strontium-90 & $1.7 E+02$ & $\|$ & & - & 1 & GP & & & & & & & $\mathrm{pCi} / \mathrm{L}$ \\
\hline Technetium-99 & $6.1 E+01$ & $/ 1$ & & a & 1 & GP & & & & & & & $\mathrm{pCi} / \mathrm{L}$ \\
\hline Thorium-228 & $<2.7 \mathrm{E}-01$ & vll/ & $<1.3000$ & & 1 & GP & & & & & & & pCill \\
\hline Thorium-230 & $<8.6 \mathrm{E}-02$ & UIJ/Cl & & & 1 & GP & & & & & & & PCill \\
\hline Sum of alphas & 4.7E+02 & & & घ & & & & & & & & & pCir \\
\hline Sum of betas & $3.1 E+02$ & & & घ & & & & & & & & & pCill \\
\hline Total radium & $1.6 E+01$ & & & a & & & & & & & & & $\mathrm{pCilL}$ \\
\hline Tritium & $2.9 E+02$ & $\|$ & & घ & 1 & GP & $6.0 \mathrm{E}+01$ & $\|$ & & $=$ & 1 & GE & $\mathrm{pCi} / \mathrm{mL}$ \\
\hline Uranium-233/234 & $1.9 E+02$ & $\|$ & & ם & 1 & GP & & & & & & & $\mathrm{pCi} / \mathrm{L}$ \\
\hline Uranium-235 & $2.1 E+01$ & $\|$ & & $\mathbf{z}$ & 1 & GP & & & & & & & $\mathrm{pCin}$ \\
\hline Uranium-238 & $2.4 E+02$ & $\|$ & & $\boldsymbol{\omega}$ & 1 & GP & & & & & & & pCir \\
\hline
\end{tabular}

Notes:

- exceeded holding time

E = exceeded groundwater protection or monitoring constituent standard (See Appendix A.) 


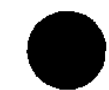

WELL FSB108D

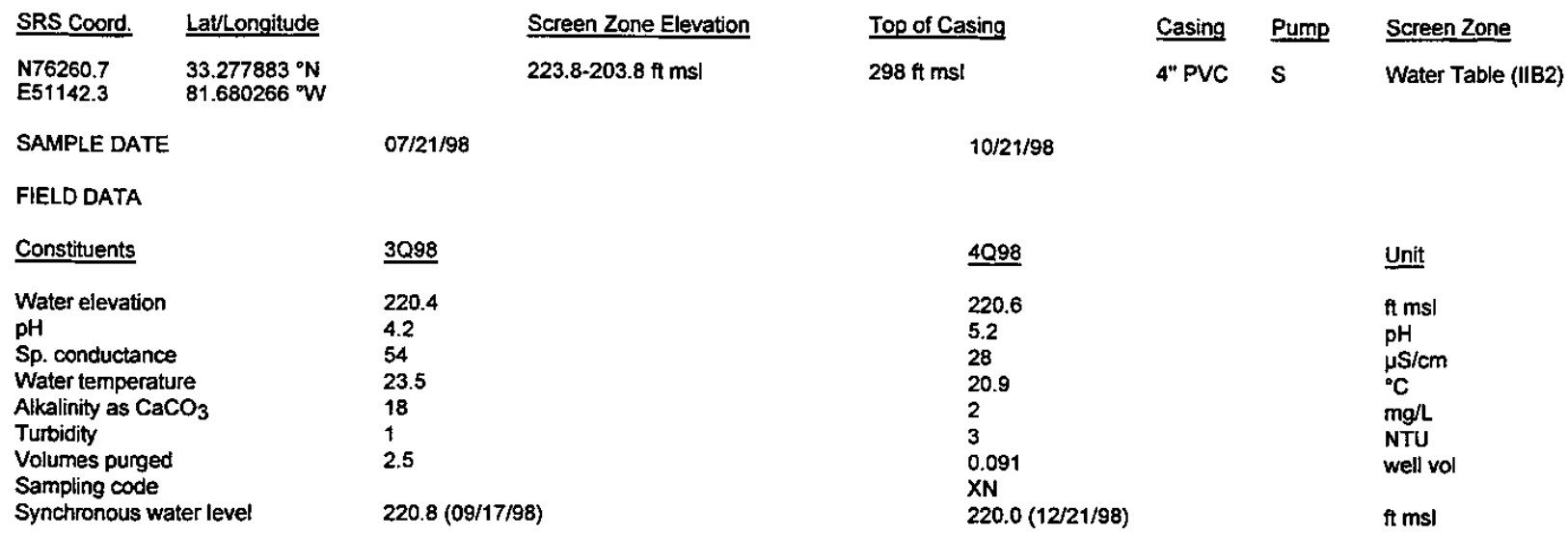

ANALYTICAL DATA

Inorganic Constituents

Constituents

Antimony, total recoverable Arsenic, total recoverable Barium, total recoverable Cadmium, total recoverable Chromium, total recoverable Cobalt, total recoverable Copper, total recoverable Cyanide

Lead total recoverable Mercury, total recoverable Nickel, total recoverable

Nitrate as nitrogen

Nitrate-nitrite as nitrogen

Selenium, total recoverable

Silver, total recoverable

Thallium, total recoverable

Vanadium, total recoverable

Zinc, total recoverable

Organic Constituents

$\stackrel{4 a}{\longrightarrow}$

Constituents
$<1.0$

3.3

1,400

NI

U/I

$<1.0$

1 GE $<1.0$

UII

1 GE 7.1

0.20

1 GE 1,100

$/ /$

II

3Q98

Filt.

$\underline{\text { ST }} \underline{\mathrm{H}}$

DF Lab 4098

Mod

Bis(2-ethylhexyl) phthalate

Dichloromethane

Phenols

Tetrachloroethylene

Trichloroethylene

Trichlorofluoromethane

Notes:

- = exceeded holding time

= exceeded groundwater protection or monitoring constituent standard (See Appendix A.) 
WELL FSB108D (cont.)

Radioactive Constituents

Constituents

Americium-241

Cesium-137

Cobalt -60

Curium-242

Curium-243/244

Curium-245/246

Gross alpha

lodine-129

Nonvolatile beta

Plutonium-238

Plutonium-239/240

Radium-226

Radium-228

Strontium-90

Technetium-99

Thorium-228

Thorium-230

Thorium-232

Sum of alphas

Sum of betas

Tritium

Uranium-233/234

Uranium-235

Uranium-238
3098

$1.3 \mathrm{E}+00 \quad / 1$

$<1.1 \mathrm{E}+00 \quad \mathrm{UI} / \mathrm{I}$

$<1.1300$

$1 \quad$ GP $<1.5 E+00$

U/I

1 GP $<2.0 \mathrm{E}+00$

U/I

<4.7E-01

$<1.0 \mathrm{E}+00$

1 GE PCi/l

1 GE pCill
1.4E+01 //
GP 1.1E+01 $/ /$

$1 \mathrm{GE} \quad \mathrm{pCi} / \mathrm{mL}$

Notes:

- = exceeded holding time

- exceeded groundwater protection or monitoring constituent standard (See Appendix A.) 


\section{WELL FSB109D}

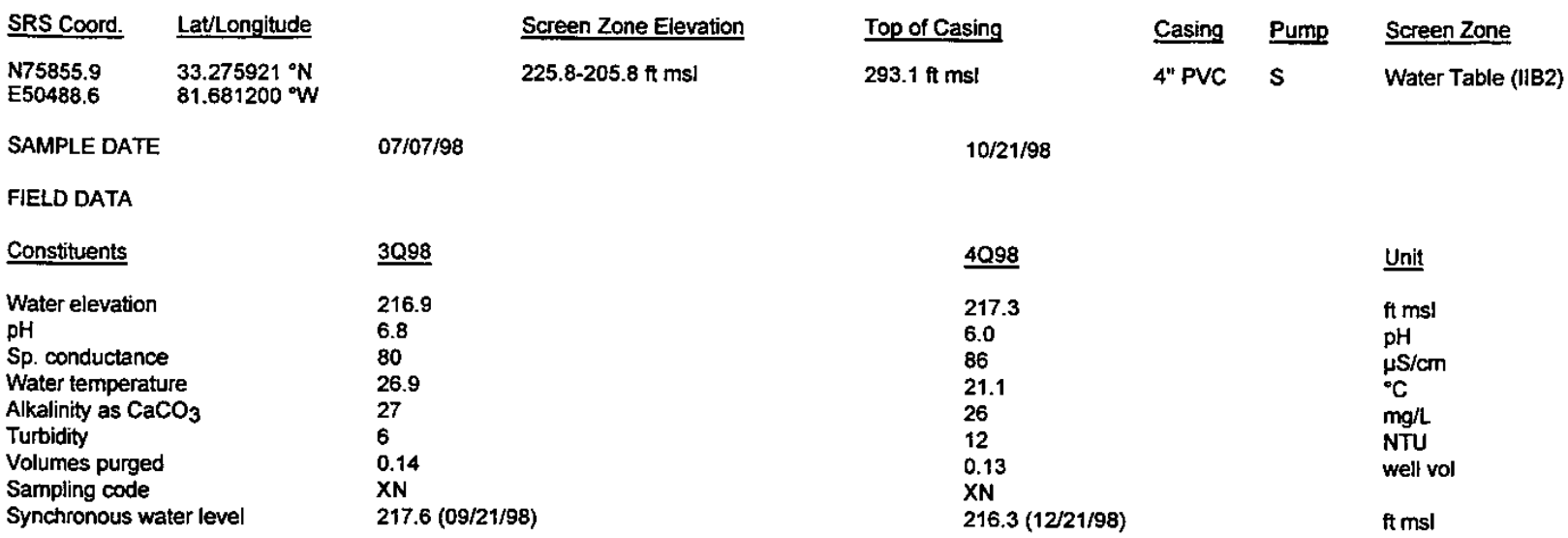

ANALYTICAL DATA

Inorganic Constituents

\begin{tabular}{|c|c|c|c|c|c|c|c|c|c|c|c|c|c|}
\hline Constituents & $\underline{3 Q 98}$ & $\underline{\text { Mod }}$ & Filt. & ST브 & $\underline{D F}$ & $\underline{\text { Lab }}$ & 4098 & Mod & Filt. & $\underline{\mathrm{ST}} \underline{\mathrm{H}}$ & $\underline{\text { DF }}$ & $\underline{L a b}$ & Unit \\
\hline Antimony, total recoverable & $<0.22$ & UNI & $<0.20$ & & 1 & GE & & & & & & & $\mu g / L$ \\
\hline Arsenic, total recoverable & 0.98 & $\mathrm{~J} / \mathrm{E} /$ & NDD & & 1 & $\overline{G E}$ & & & & & & & $\mu g / L$ \\
\hline Barium, total recoverable & 13 & $\|$ & & & 1 & GE & & & & & & & $\mu g / L$ \\
\hline Cadmium, total recoverable & $<1.0$ & $\mathrm{U} / /$ & $<1.0$ & & 1 & GE & $<1.0$ & $\mathrm{U} / /$ & $<1.0$ & & 1 & GE & $\mu g / L$ \\
\hline Chromium, total recoverable & 3.8 & $\|$ & & & 1 & GE & & & & & & & $\mu g / L$ \\
\hline Cobalt, total recoverable & 0.48 & $\|$ & & & 1 & GE & & & & & & & $\mu g / L$ \\
\hline Copper, total recoverable & 20 & $\|$ & & & 1 & $\mathbf{G E}$ & & & & & & & $\mu g / L$ \\
\hline Cyanide & $<10$ & $\mathrm{U} / /$ & $<10$ & & 1 & GE & & & & & & & $\mu g / L$ \\
\hline Lead, total recoverable & 19 & $N I$ & & $\mathbf{0}$ & 1 & GE & 8.7 & $\|$ & & & 1 & GE & $\mu g / L$ \\
\hline Mercury, total recoverable & $<0.20$ & $\mathrm{U} / /$ & $<0.20$ & & 1 & GE & & & & & & & $\mu g / L$ \\
\hline $\begin{array}{l}\text { Nickel, total recoverable } \\
\text { Nitrate as nitrogen }\end{array}$ & 5.0 & $/ /$ & & & 1 & GE & & & & & & & $\mu g / L$ \\
\hline Nitrate-nitrite as nitrogen & 640 & $N I$ & & & 1 & GE & 600 & $\|$ & & & 1 & GE & $\mu g / L$ \\
\hline Selenium, total recoverable & $<5.0$ & $\mathrm{U} / \mathrm{I}$ & $<5.0$ & & 1 & GE & & & & & & & $\mu g / L$ \\
\hline Silver, total recoverable & $<1.0$ & $\mathrm{U} / I$ & $<1.0$ & & 1 & GE & & & & & & & $\mu \mathrm{g} / \mathrm{L}$ \\
\hline Thallium, total recoverable & $<2.5$ & $\mathrm{U} / /$ & $<2.5$ & & 1 & GE & & & & & & & $\mu g / L$ \\
\hline Vanadium, total recoverable & $<2.0$ & $\mathrm{U} / /$ & $<2.0$ & & 1 & GE & & & & & & & $\mu g / L$ \\
\hline Zinc, total recoverable & 280 & $/ 1$ & & & 1 & GE & & & & & & & $\mu g / L$ \\
\hline \multicolumn{14}{|l|}{ Organic Constituents } \\
\hline Constituents & 3Q98 & Mod & Filt. & $\underline{\text { ST브 }}$ & $\underline{\mathrm{DF}}$ & $\underline{L a b}$ & $\underline{4 Q 98}$ & Mod & Filt. & $\underline{\text { ST }} \underline{\mathrm{H}}$ & $\underline{\text { DF }}$ & Lab & Unit \\
\hline Benzene & $<1.0$ & $\mathrm{U} / /$ & $<1.0$ & & 1 & GE & & & & & & & $\mu g / l$ \\
\hline Bis(2-ethylhexyl) phthalate & $<11$ & $\mathbf{U} / /$ & $<11$ & & 1 & GE & & & & & & & $\mu g / L$ \\
\hline Dichloromethane & $<1.0$ & $\mathrm{U} / /$ & $<1.0$ & & 1 & GE & & & & & & & $\mu \mathrm{g} / \mathrm{L}$ \\
\hline Phenols & $<5.0$ & $\mathrm{U} / /$ & $<5.0$ & & 1 & GE & & & & & & & $\mu g / L$ \\
\hline Tetrachloroethylene & $<1.0$ & $\mathbf{U} / /$ & $<1.0$ & & 1 & GE & & & & & & & $\mu g / L$ \\
\hline Trichloroethylene & $<1.0$ & $\mathbf{U} / /$ & $<1.0$ & & 1 & GE & & & & & & & $\mu g / L$. \\
\hline Trichlorofluoromethane & $<1.0$ & $\mathbf{U} / /$ & $<1.0$ & & 1 & GE & & & & & & & L \\
\hline
\end{tabular}

Notes:

- exceeded holding time

- exceeded groundwater protection or monitoring constituent standard (See Appendix A.) 


\section{WELL FSB109D (cont.)}

Radioactive Constituents

\begin{tabular}{|c|c|c|c|c|c|c|c|c|c|c|c|c|c|}
\hline Constituents & 3098 & Mod & Filt. & $\underline{\mathrm{ST}} \underline{\underline{ }}$ & $\underline{\text { DF }}$ & Lab & 4Q98 & Mod & Filt. & ST $\underline{\text { ㅂ }}$ & $\underline{\mathrm{DF}}$ & $\underline{L a b}$ & Unit \\
\hline Americium-241 & $<6.0 \mathrm{E}-02$ & $\mathrm{U} \mid \prime \prime$ & $<0.0522$ & & 1 & GP & & & & & & & pCill \\
\hline Cesium-137 & $<-4.6 E-01$ & $U I I I$ & $<3.6700$ & & 1 & GP & & & & & & & $\mathrm{pCill}$ \\
\hline Cobalt-60 & $1.0 E+01$ & $R / 4 /$ & $\operatorname{Rej}$ & & 1 & GP & & & & & & & pCill \\
\hline Curium-243/244 & $<-8.4 E-03$ & $\mathrm{UI} / \prime$ & $<0.1080$ & & 1 & GP & & & & & & & $\mathrm{pCi} / \mathrm{L}$ \\
\hline Curium-245/246 & $<0.0 \mathrm{E}+00$ & $\mathrm{UII} /$ & $<0.0522$ & & 1 & GP & & & & & & & $\mathrm{pCi} / \mathrm{L}$ \\
\hline Gross alpha & $1.3 E+00$ & $/ 1$ & & & 1 & GP & $4.7 \mathrm{E}+00$ & $\|$ & & & 1 & GE & $\mathrm{pCi} / \mathrm{L}$ \\
\hline lodine-129 & $<4.7 E-01$ & UIII & $<0.4840$ & & 1 & $\mathrm{GP}$ & & & & & & & pCill \\
\hline Plutonium-238 & $<1.0 E-07$ & UWI & $<0.2300$ & & 1 & GP & & & & & & & $\mathrm{pCi} / \mathrm{L}$ \\
\hline Plutonium-239/240 & $<8.3 E-03$ & $\mathrm{UI} / /$ & $<0.0995$ & & 1 & GP & & & & & & & $\mathrm{pCi} / \mathrm{L}$ \\
\hline Radium-226 & $<5.0$ E-01 & $\mathrm{U} / /$ & $<0.7650$ & & 1 & GP & & & & & & & $\mathrm{pCi} / \mathrm{L}$ \\
\hline Radium-228 & $<1.5 E-01$ & UIII & $<1.1500$ & & 1 & GP & & & & & & & $\mathrm{pCi} / \mathrm{L}$ \\
\hline Strontium-90 & $<-8.0 E-02$ & $\mathrm{U} \mathrm{y} / \mathrm{I}$ & $<1.4600$ & & 1 & GP & & & & & & & $\mathrm{pCi} / \mathrm{L}$ \\
\hline Technetium-99 & $<1.3 E+00$ & $\mathrm{Uy} /$ & $<21.0$ & & 1 & GP & & & & & & & $\mathrm{pCi} / \mathrm{L}$ \\
\hline Thorium-228 & $<2.4 \mathrm{E}-01$ & U: $/ /$ & $<0.4530$ & & 1 & GP & & & & & & & $\mathrm{pCi} / \mathrm{L}$ \\
\hline Thorium-230 & $<5.9 E-02$ & UII/ & $<0.2190$ & & 1 & GP & & & & & & & pCilL \\
\hline Thorium-232 & $<-3.5 \mathrm{E}-02$ & $\mathrm{U} 1 / /$ & $<0.2350$ & & 1 & GP & & & & & & & pCill \\
\hline Tritium & $1.3 E+01$ & /I & & & 1 & GP & 1.3E+01 & $\|$ & & & 1 & GE & \\
\hline Uranium-233/234 & $<2.9 E-02$ & $\mathrm{U} / \mathrm{I}$ & $<0.0862$ & & 1 & GP & & & & & & & $\mathrm{pCi} / \mathrm{L}$ \\
\hline Uranium-235 & $<1.6 \mathrm{E}-02$ & UII/ & $<0.0491$ & & 1 & GP & & & & & & & $\mathrm{pCi} / \mathrm{L}$ \\
\hline Uranium-238 & $<2.9 E-02$ & $\mathrm{U} \mid / /$ & $<0.0862$ & & 1 & GP & & & & & & & $\mathrm{pCi} / \mathrm{L}$ \\
\hline
\end{tabular}

\section{Notes:}




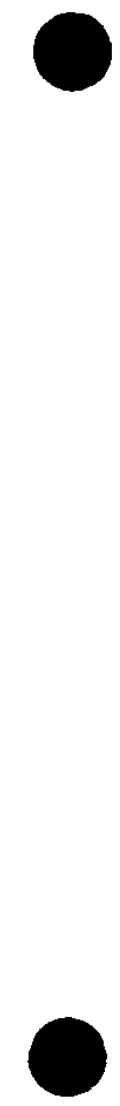

WELL FSB110C

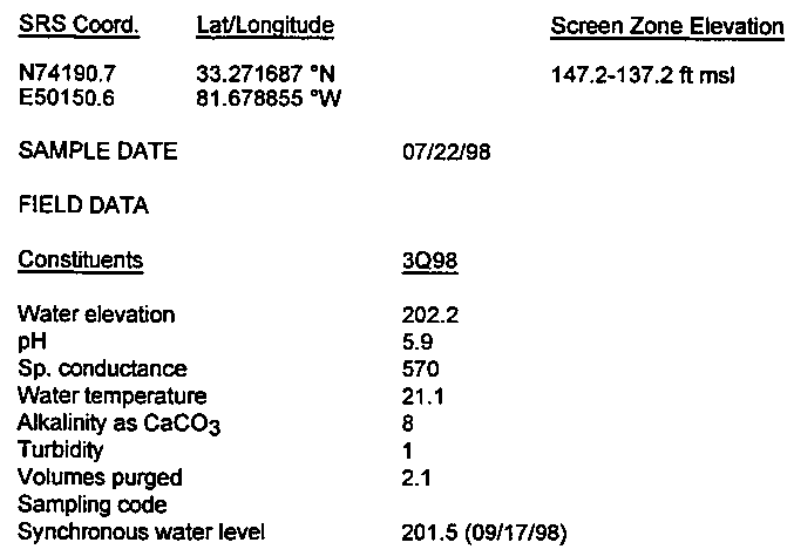

\begin{tabular}{|c|c|c|c|}
\hline Top of Casing & Casing & Pump & Screen Zone \\
\hline $234.5 \mathrm{ft} \mathrm{ms!}$ & 4" PVC & $\mathbf{s}$ & Barnwell $\left(\| B_{1}\right)$ \\
\hline $10 / 28 / 98$ & & & \\
\hline $4 Q 98$ & & & Unit \\
\hline $\begin{array}{l}200.8 \\
5.0 \\
700 \\
21.4 \\
5 \\
1 \\
2.3\end{array}$ & & & $\begin{array}{l}\text { ft msl } \\
\text { pH } \\
\mu \mathrm{S} / \mathrm{cm} \\
{ }^{\circ} \mathrm{C} \\
\mathrm{mg} / \mathrm{L} \\
\mathrm{NTU} \\
\text { well vol }\end{array}$ \\
\hline $200.9(12 / 18 / 98)$ & & & $\mathrm{ft} \mathrm{msl}$ \\
\hline
\end{tabular}

ANALYTICAL DATA

Inorganic Constituents

\begin{tabular}{|c|c|c|c|c|c|c|c|c|c|c|c|c|c|}
\hline Constituents & $\underline{3098}$ & Mod & Filt. & $\underline{\text { STI }} \underline{H}$ & $\underline{\mathrm{DF}}$ & $\underline{L a b}$ & $\underline{4 Q 98}$ & Mod & Filt. & $\underline{S T} \underline{H}$ & DF & $\underline{\text { Lab }}$ & $\underline{\text { Unit }}$ \\
\hline Antimony, total recoverable & $<0.20$ & UII & $<0.20$ & & 1 & GE & & & & & & & $\mu g / L$ \\
\hline Arsenic, total recoverable & $<3.0$ & $\mathrm{U} / /$ & $<3.0$ & & 1 & GE & & & & & & & $\mu g / L$ \\
\hline Barium, total recoverable & 130 & II & & & 1 & GE & & & & & & & $\mu \mathrm{g} / \mathrm{L}$ \\
\hline Cadmium, total recoverable & 1.6 & "I & & & 1 & $\overline{G E}$ & 2.3 & $\|$ & & & 1 & GE & $\mu g / L$ \\
\hline Chromium, total recoverable & $<3.0$ & $\mathrm{U} / /$ & $<3.0$ & & 1 & GE & & & & & & & $\mu g / L$ \\
\hline Cobalt, total recoverable & 8.7 & $N /$ & & - & 1 & WA & & & & & & & $\mu g / \mathrm{h}$ \\
\hline Copper, total recoverable & 15 & $16 /$ & & & 1 & GE & & & & & & & $\mu g / 2$ \\
\hline Cyanide & $<10$ & $\mathrm{U} / I$ & $<10$ & & 1 & GE & & & & & & & $\mu g / \mathrm{L}$ \\
\hline Lead, total recoverable & 2.3 & $" 1$ & & & 1 & GE & 1.3 & $\mathrm{~J} / / l$ & NDD & & 1 & $\mathrm{GE}$ & $\mu g / \mathrm{L}$ \\
\hline Mercury, total recoverable & $<0.20$ & UII & $<0.20$ & & 1 & GE &. .0 & 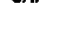 & Not & & & & $\mu g / L$ \\
\hline $\begin{array}{l}\text { Nickel, total recoverable } \\
\text { Nitrate as nitrogen }\end{array}$ & 20 & $\mathrm{~J} / \mathrm{E}$ & NDD & & 1 & WA & & & & & & & $\mu g / L$ \\
\hline Nitrate-nitrite as nitrogen & 63,000 & $N I$ & & - & 500 & WA & 85,000 & II & & $=$ & 50 & GE & \\
\hline Selenium, total recoverable & $<5.0$ & $\mathrm{u} / \mathrm{f}$ & $<5.0$ & & 1 & $\mathrm{GE}$ & & & & & & & Hg/L \\
\hline Silver, total recoverable & $<1.0$ & $\mathrm{U} / \mathrm{I}$ & $<1.0$ & & 1 & GE & & & & & & & $\mu g / L$ \\
\hline Thallium, total recoverable & $<0.22$ & UNI & $<2.5$ & & 1 & GE & & & & & & & $\mu g / L$ \\
\hline Vanadium, total recoverable & $<2.0$ & $\mathrm{U} / \mathrm{I}$ & $<2.0$ & & 1 & GE & & & & & & & $\mu g / L$ \\
\hline Zinc, total recoverable & 150 & "I & & & 1 & GE & & & & & & & $\mu \mathrm{g} / \mathrm{L}$ \\
\hline \multicolumn{14}{|l|}{ Organic Constituents } \\
\hline Constituents & $\underline{3098}$ & Mod & Filt. & $\underline{\text { ST }} \underline{H}$ & $\underline{\mathrm{DF}}$ & $\underline{\text { Lab }}$ & $\underline{4 Q 98}$ & Mod & Filt. & $\underline{\text { ST }} \underline{H}$ & DF & $\underline{\text { Lab }}$ & Unit \\
\hline Benzene & $<1.0$ & UJ/O/1 & $<1.0$ & & 1 & GE & & & & & & & \\
\hline Bis(2-ethylhexyl) phthalate & $<0.65$ & UNI & $<5.1$ & & 1 & WA & & & & & & & $\mu g / L$ \\
\hline Dichloromethane & $<1.0$ & $U \mathrm{~J} / \mathrm{O} / \mathrm{i}$ & $<1.0$ & & 1 & GE & & & & & & & $\mu g / L$ \\
\hline Phenols & $<5.0$ & & $<5.0$ & & 1 & GE & & & & & & & $\mu g / L$ \\
\hline Tetrachloroethylene & $<1.0$ & UJ/O/1 & $<1.0$ & & 1 & $\mathrm{GE}$ & & & & & & & $\mu g / L$ \\
\hline Trichloroethylene & $<1.0$ & UJ/O/1 & $<1.0$ & & 1 & $\mathrm{GE}$ & & & & & & & $\mu g / L$ \\
\hline Trichlorofluoromethane & $<1.0$ & UJ/O/1 & $<1.0$ & & 1 & GE & & & & & & & $g / \mathrm{L}$ \\
\hline
\end{tabular}

Notes:

- exceeded holding time

- = exceeded groundwater protection or monitoring constituent standard (See Appendix A.) 
WELL FSB110C (cont.)

Radioactive Constituents

\begin{tabular}{|c|c|c|c|c|c|c|c|c|c|c|c|c|c|}
\hline Constituents & 3098 & Mod & Filt. & $\underline{\text { ST브 }}$ & DF & $\underline{\text { Lab }}$ & 4Q98 & Mod & Filt. & $\underline{\mathrm{ST}} \underline{\mathrm{H}}$ & $\underline{\mathrm{DF}}$ & Lab & Unit \\
\hline Americium-241 & $<2.6 \mathrm{E}-02$ & $\mathrm{UU} / /$ & $<0.1040$ & & 1 & GP & & & & & & & pCill \\
\hline Beta dose & 25.1222 & & & $\mathbf{\square}$ & & & & & & & & & pCill \\
\hline Cesium-137 & $<3.3 E-01$ & $\mathrm{U} \mid / /$ & $<2.8000$ & & 1 & GP & & & & & & & pCill \\
\hline Cobalt- 60 & $<7.4 \mathrm{E}-01$ & $\mathrm{U} \mathbf{V} /$ & $<3.7700$ & & 1 & $\pi M$ & & & & & & & pCirl \\
\hline Curium-242 & $<0.0 \mathrm{E}+00$ & U1/l & $<0.6800$ & & 1 & $\mathrm{TM}$ & & & & & & & $\mathrm{pCi} /$ \\
\hline Curium-243/244 & $<-1.9 E-02$ & $\mathrm{U} 1 / /$ & $<0.1820$ & & 1 & GP & & & & & & & pCi/L \\
\hline Curium-245/246 & $<0.0 E+00$ & $\mathrm{UI} / /$ & $<0.0785$ & & 1 & GP & & & & & & & $\mathrm{pCi} / \mathrm{L}$ \\
\hline Gross alpha & $9.2 \mathrm{E}+00$ & $\|$ & & & 1 & $T M$ & $6.7 E+00$ & $1 /$ & & & 1 & GE & pCir \\
\hline lodine-129 & $2.5 \mathrm{E}+01$ & $\|$ & & & 1 & $\pi M$ & & & & & & & pCill \\
\hline Nonvolatile beta & 8.7E+01 & $\|$ & & $\mathbf{\square}$ & 1 & $T M$ & $8.1 E+01$ & $\|$ & & $\mathbf{E}$ & 1 & GE & $\mathrm{pCi} / \mathrm{L}$ \\
\hline Plutonium-238 & $<-4,7 E-03$ & $\mathrm{UI} / \mathrm{I}$ & $<0.0993$ & & 1 & GP & & & & & & & pCill \\
\hline Plutonium-239/240 & $<-9.5 E-03$ & $\mathrm{UI} / /$ & $<0.1620$ & & 1 & GP & & & & & & & $\mathrm{pCi} / \mathrm{L}$ \\
\hline Radium-226 & $2.6 E+00$ & $\|$ & & & 1 & $G P$ & & & & & & & $\mathrm{pCi} / \mathrm{L}$ \\
\hline Radium-228 & $9.7 E+00$ & $R / 4 /$ & Rej & & 1 & $T M$ & & & & & & & $\mathrm{pCi} / \mathrm{L}$ \\
\hline Strontium-90 & $5.4 E+00$ & $\mathrm{~J} / \mathrm{Cl}$ & NDD & & 1 & TM & & & & & & & pCill \\
\hline Technetium-99 & $1.1 E+02$ & $\|$ & & - & 1 & GP & & & & & & & $\mathrm{pCi} / \mathrm{L}$ \\
\hline Thorium-228 & $<5.0 \mathrm{E}-02$ & $\mathrm{UI} / /$ & $<0.5100$ & & 1 & MM & & & & & & & pCill \\
\hline Thorium-230 & $<5.5 \mathrm{E}-02$ & $\mathrm{UI} / /$ & $<0.1640$ & & 1 & GP & & & & & & & pCill \\
\hline Thorium-232 & $<-7.5 E-03$ & $\mathrm{UI} / /$ & $<0.1640$ & & 1 & GP & & & & & & & pCirl \\
\hline Sum of alphas & & & & & & & & & & & & & \\
\hline Sum of betas & $1.4 E+02$ & & & घ & & & & & & & & & pCi/l \\
\hline Total radium & $1.2 \mathrm{E}+01$ & & & - & & & & & & & & & pCirl \\
\hline Tritium & $2.0 \mathrm{E}+03$ & /I & & घ & 1 & $T M$ & $1.9 E+03$ & /I & & घ & 1 & GE & $\mathrm{pCi} / \mathrm{mL}$ \\
\hline Uranium-233/234 & $<1.8 \mathrm{E}-02$ & $\mathrm{UI} / /$ & $<0.2230$ & & 1 & GP & & & & & & & pCill \\
\hline Uranium-235 & $<5.4 \mathrm{E}-02$ & UWI & $<0.3770$ & & 1 & GP & & & & & & & pCil \\
\hline Uranium-238 & $<0.0 E+00$ & $\mathrm{UI} / /$ & $<0.2140$ & & 1 & GP & & & & & & & pCitl \\
\hline
\end{tabular}

Notes:

- exceeded holding time

- exceeded groundwater protection or monitoring constituent standard (See Appendix A.) 


\section{WELL FSB110D}

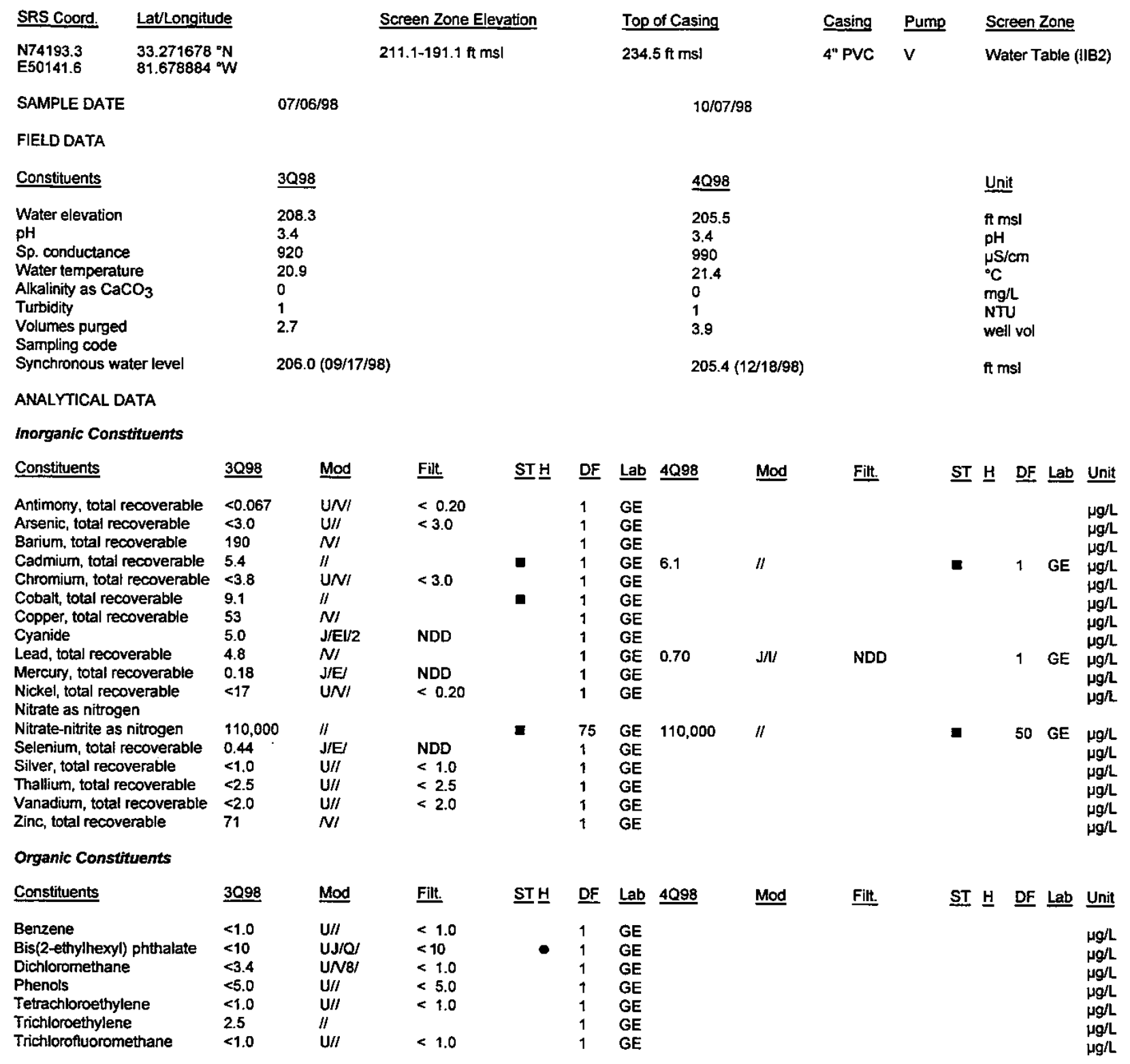

Notes:

- = exceeded holding time

- exceeded groundwater protection or monitoring constituent standard (See Appendix A.)

F-Area HWMF

C-123

Third and Fourth Quarter 1998 


\section{WELL FSB110D (cont.)}

Radloactive Constituents

\begin{tabular}{|c|c|c|c|c|c|c|c|c|}
\hline Constituents & 3098 & Mod & Filt. & ST브 & $\underline{\mathrm{DF}}$ & $\underline{L a b}$ & 4Q98 & Mod \\
\hline Americium-241 & $2.0 E+01$ & $\prime \prime$ & & घ & 1 & $\mathrm{GP}$ & & \\
\hline Beta dose & 565.0967 & & & $\omega$ & & & & \\
\hline Cesium-137 & $<1.5 \mathrm{E}+00$ & $\mathrm{UI} / /$ & $<4.1600$ & & 1 & GP & & \\
\hline Cobalt-60 & $<2.4 \mathrm{E}+00$ & $\mathrm{UV} / /$ & $<4.1900$ & & 1 & GP & & \\
\hline Curium-242 & $<3.1 E-02$ & $\mathrm{UI} / /$ & $<0.0926$ & & 1 & GP & & \\
\hline Curium-243/244 & $3.0 E+01$ & $16 /$ & & 른 & 1 & $G P$ & & \\
\hline Curium-245/246 & $3.6 E-01$ & $\|$ & & & 1 & GP & & \\
\hline Gross alpha & $5.9 E+02$ & $\mathrm{~J} / / 1 / 1$ & NDD & & 1 & GP & $6.7 E+02$ & $\|$ \\
\hline lodine-129 & $5.3 E+02$ & $\|$ & & - & 1 & GP & & \\
\hline Nonvolatile beta & $8.8 E+02$ & $\mathrm{~J} / \mathrm{IV} / 1$ & NDD & & 1 & GP & $1.1 E+03$ & $\|$ \\
\hline Plutonium-238 & $2.1 \mathrm{E}+00$ & $R / 4 /$ & Rej & & 1 & GP & & \\
\hline Plutonium-239/240 & 4.5E-01 & $\mathrm{J} / \mathrm{C} /$ & NDD & & 1 & GP & & \\
\hline Radium-226 & $1.3 E+01$ & $/ /$ & & घ & 1 & GP & & \\
\hline Radium-228 & $<-5.2 E-01$ & $\mathrm{UI} / /$ & $<1.3400$ & & 1 & GP & & \\
\hline Strontium-90 & $2.8 E+02$ & $\|$ & & - & 1 & GP & & \\
\hline Technetium-99 & $8.7 E+01$ & II & & घ & 1 & GP & & \\
\hline Thorium-228 & $<4.9 E-01$ & $\mathrm{UI} / I$ & $<0.5540$ & & 1 & GP & & \\
\hline Thorium-230 & $<1.3 E-01$ & $\mathrm{U} \mid / /$ & $<0.3090$ & & 1 & GP & & \\
\hline Thorium-232 & $<-1.7 \mathrm{E}-02$ & $\mathrm{UI} / /$ & $<0.2240$ & & 1 & $G P$ & & \\
\hline Sum of aiphas & $6.9 E+02$ & & & $\mathbf{n}$ & & & & \\
\hline Sum of betas & $9.0 E+02$ & & & ט & & & & \\
\hline Total radium & $1.3 E+01$ & & & $\mathbf{z}$ & & & & \\
\hline Tritium & $4.6 E+03$ & $/ 1$ & & $\boldsymbol{m}$ & 1 & GP & $4.2 E+03$ & $/ /$ \\
\hline Uranium-233/234 & $1.2 E+02$ & /I & & ש & 1 & $\mathrm{GP}$ & & \\
\hline Uranium-235 & 1.7E+01 & /I & & a & 1 & $\mathrm{GP}$ & & \\
\hline Uranium-238 & $5.0 \mathrm{E}+02$ & /I & & 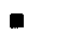 & 1 & GP & & \\
\hline
\end{tabular}

Filt. $\quad \underline{\text { ST }} \underline{H}$ DF Lab Unit

$\mathrm{pCin}$

pCi/L

$\mathrm{pCin}$

pCir

pCin

pCilL

- 1 Ge $\stackrel{\mathrm{pCil}}{\mathrm{pCil}}$

- pCill

- 1 Ge pCil

pCill.

pCill

pCill

pCill

pcin

pCill

pCill

pCir

pCirh

$\mathrm{PCi} / \mathrm{L}$

pCin

- 1 GE $\begin{aligned} & \mathrm{pCi} / \mathrm{p} \\ & \mathrm{pCi} / \mathrm{mL}\end{aligned}$

pCill

pCill.

pCill

Notes:

- = exceeded holding time

= exceeded groundwater protection or monitoring constituent standard (See Appendix A.) 


\section{WELL FSB111C}

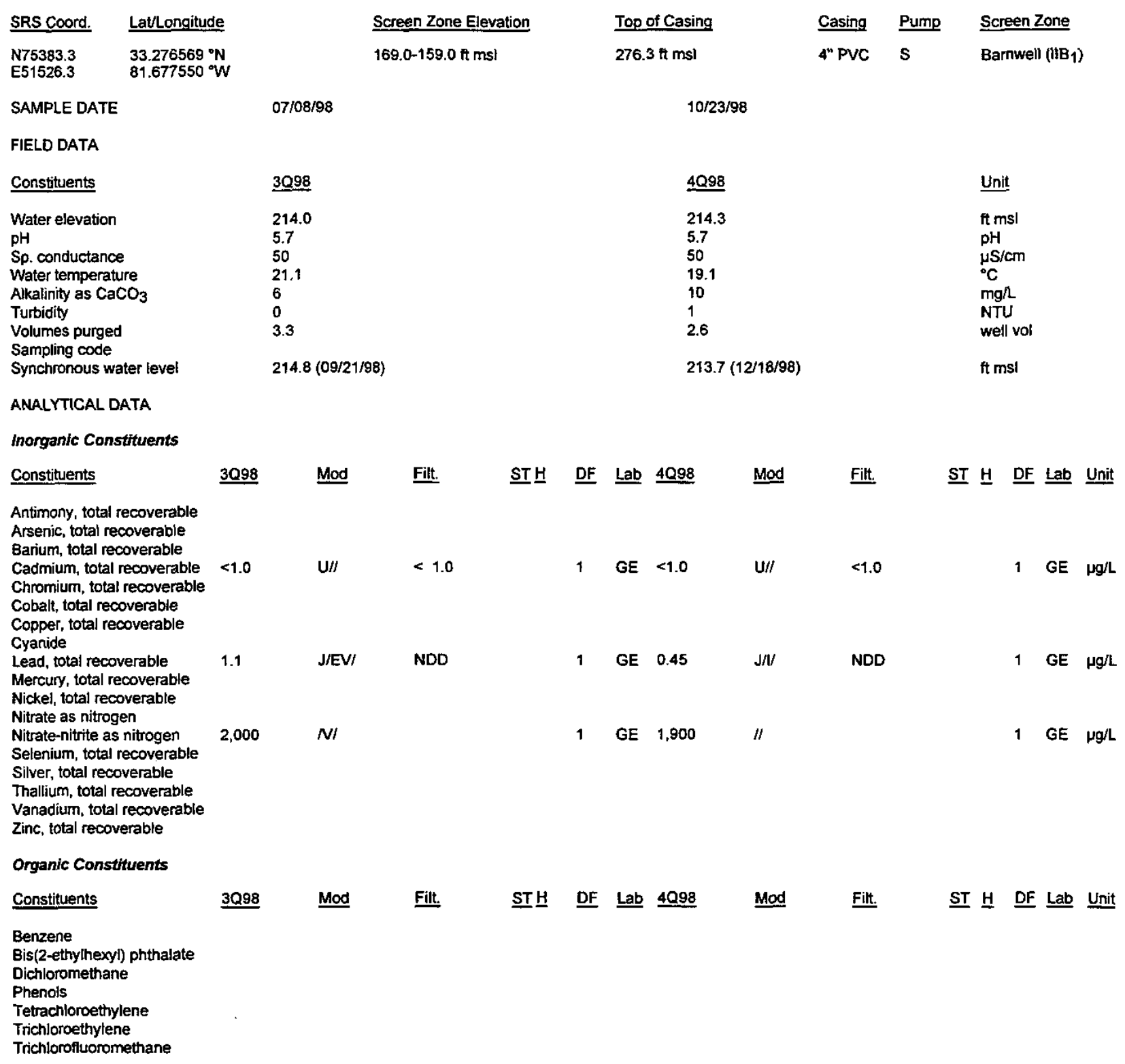

Notes:

- = exceeded holding time

= exceeded groundwater protection or monitoring constituent standard (See Appendix A.) 


\section{WELL FSB111C}

Radioactive Constituents

Constituents

Americium-241

Cesium-137

Cobalt-60

Curium-242

Curium-243/244

Curium-245/246

Gross alpha

lodine-129

Nonvolatile beta

Plutonium-238

Plutonium-239/240

Radium-226

Radium-228

Strontium-90

Technetium-99

Thorium-228

Thorium-230

Thorium-232

Sum of alphas

Sum of betas

Tritium

Uranium-233/234

Filt.

ST브 DF Lab 4098

Mod

Filt.

ST 브 DF Lab Unit

Uranium-235

Uranium-238

$<2.3 E-01 \quad$ UI $/ / \quad<0.5280$

$<2.9 E-01$

UI/I

$<1.1600$

1 GP

$<4.5 E-02$

$\mathrm{U} / I$

$\mathrm{U} / \mathrm{I}$

$<6,6 \mathrm{E}-01$

$<1.4 \mathrm{E}+00$

1 GE pCi/L

1 GE pCil

4.8E+00 $/ /$

1 GP $3.7 E+00 \quad / /$

$1 \mathrm{GE} \mathrm{pCi} / \mathrm{mL}$.

Notes:

- exceeded holding time

- exceeded grounowater protection or monitoring constituent standard (See Appendix A.) 
WELL FSB111D

\begin{tabular}{|c|c|}
\hline SRS Coord & Lat/Longitude \\
\hline $\begin{array}{l}\text { N75382.9 } \\
\text { E51515.9 }\end{array}$ & 81.677577 of \\
\hline
\end{tabular}

SAMPLE DATE

\section{Screen Zone Elevation}

221.7-201.7 ft ms

FIELD DATA

Constituents

Water elevation

pH

Sp. conductance

Water temperature

Alkalinity as $\mathrm{CaCO}_{3}$

Turbidity

Volumes purged

Sampling code

Synchronous water level
3098

218.2

5.1
38

21.9

0

1

4.9

$218.1(09 / 21 / 98)$
Top of Casing

$276.6 \mathrm{ft} \mathrm{ms}$
Casing Pump

4" PVC S
Screen Zone

Water Table (IIB2)
ANALYTICAL DATA

Inorganic Constituents
Constituents

Antimony, total recoverable

Arsenic, total recoverable

Barium, total recoverable

Chromium, total recoverable $<3.0$

Cobalt total recoverable $\quad 0.75$

Copper, total recoverable $\quad 54$

Cyanide

Lead, total recoverable $\quad 4.3$

Mercury, total recoverable $\quad 0.12$

Nickel, total recoverable $\quad<1.2$

Nitrate as nitrogen

Nitrate-nitrite as nitrogen $\quad 1,900$

Selenium, total recoverable $<5,0$

Silver, total recoverable $\quad<1.0$

Thallium, total recoverable $<2.5$

Vanadium, total recoverable $<2.0$

Zinc, total recoverable
Cadmium, total recoverable $<1.0$

Organic Constituents

$\begin{array}{ll}\text { Constituents } & \\ \text { Benzene } & <1.0 \\ \text { Bis(2-ethylhexyl) phthalate } & <10 \\ \text { Dichloromethane } & <3.7 \\ \text { Phenols } & <5 \\ \text { Tetrachloroethylene } & <1.0 \\ \text { Trichloroethylene } & <1.0 \\ \text { Trichlorofluoromethane } & \end{array}$

\begin{tabular}{|c|c|}
\hline Mod & Filt. \\
\hline $\mathrm{U} / /$ & $<0.20$ \\
\hline $\mathrm{U} / 1$ & $<3.0$ \\
\hline II & \\
\hline $\mathrm{U} / I$ & $<1.0$ \\
\hline $\mathrm{U} / /$ & $<3.0$ \\
\hline$N I$ & \\
\hline $\begin{array}{l}\mathrm{J} / \mathrm{E} / \\
\mathrm{N} /\end{array}$ & NDD \\
\hline $\begin{array}{l}\mathrm{J} / \mathrm{E} / \\
\mathrm{U} / \mathrm{N} /\end{array}$ & $\begin{array}{l}\text { NDD } \\
<0.20\end{array}$ \\
\hline$N /$ & \\
\hline$U / I$ & $<5.0$ \\
\hline $\mathrm{U} / /$ & $<1.0$ \\
\hline $\mathrm{U} / I$ & $<2.5$ \\
\hline $\mathrm{U} / /$ & $<2.0$ \\
\hline
\end{tabular}

ST브

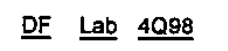

Mod

GE

1 GE

1 GE

1 GE

$<1.0$

$\mathrm{U} / /$

E

$11 / 1$

GE

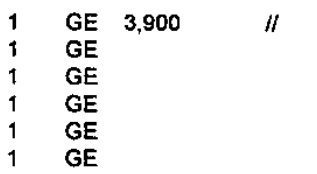

Unit

ft msl

$\mathrm{pH}$

$\mu \mathrm{S} / \mathrm{cm}$

${ }^{\circ} \mathrm{C}$

NTU

well vol

ft msl

$\begin{array}{ll}\text { Mod } & \text { Filt. } \\ \text { UJ/O/1 } & <1.0 \\ U / / & <10 \\ U J / O V / 1 & <1.0 \\ U / I & <5.0 \\ U J / O / 1 & <1.0 \\ U J / O / 1 & <1.0 \\ U J / O / 1 & <1.0\end{array}$

DF Lab 4098

$1 \mathrm{GE}$

1 GE

1 GE

$1 \mathrm{GE}$

1 GE

1 GE

Notes:

- = exceeded holding time

- = exceeded groundwater protection or monitoring constituent standard (See Appendix A.)

F-Area HWMF

C-127

Third and Fourth Quarter 1998 
WELL FSB111D (cont.)

Radloactive Constituents

\begin{tabular}{|c|c|c|c|c|c|c|c|c|c|c|c|c|c|}
\hline Constituents & $3 Q 998$ & Mod & Filt. & $\underline{\text { ST }} \underline{\underline{H}}$ & DF & Lab & $\underline{4098}$ & Mod & Filt. & $\underline{\text { ST }} \underline{\underline{H}}$ & $\underline{\mathrm{DF}}$ & $\underline{\text { Lab }}$ & Unit \\
\hline Americium-241 & $<3.7 E-02$ & $U 1 / I$ & $<0.1400$ & & 1 & GP & & & & & & & $p C i / L$ \\
\hline Beta dose & 0.4750 & & & & & & & & & & & & pCill \\
\hline Cesium-137 & $<-1,1 \mathrm{E}+00$ & $\mathrm{UI} / \mathrm{I}$ & $<3.9600$ & & 1 & GP & & & & & & & $\mathrm{pCi} / \mathrm{L}$ \\
\hline Cobalt-60 & $<1.2 E-01$ & $\mathrm{UI} / \mathrm{I}$ & $<3.9400$ & & 1 & GP & & & & & & & $\mathrm{pCi} / \mathrm{L}$ \\
\hline Curium-242 & $<0.0 \mathrm{E}+00$ & $\mathrm{UI} / /$ & $<0.0638$ & & 1 & $\mathrm{GP}$ & & & & & & & $\mathrm{pCi} / \mathrm{L}$ \\
\hline Curium-243/244 & $<-4.9 \mathrm{E}-03$ & UIII & $<0.1070$ & & 1 & GP & & & & & & & $\mathrm{pCi} / \mathrm{L}$ \\
\hline Curium-245/246 & $<-4.9 \mathrm{E}-03$ & $\mathrm{UI} / \mathrm{I}$ & $<0.1070$ & & 1 & $\mathrm{GP}$ & & & & & & & $\mathrm{pCi} / \mathrm{L}$ \\
\hline Gross alpha & $2.6 \mathrm{E}+00$ & $\|$ & & & 1 & GP & $2.5 E+00$ & /I & & & 1 & GE & pCill \\
\hline lodine-129 & $<5.0 \mathrm{E}-01$ & $\mathrm{U} \mid / /$ & $<1.2500$ & & 1 & GP & & & & & & & pCill \\
\hline Nonvolatile beta & $2.8 \mathrm{E}+00$ & $/ /$ & & & 1 & GP & $4.7 E+00$ & $/ 1$ & & & 1 & GE & $\mathrm{pCi} / \mathrm{L}$ \\
\hline Plutonium-238 & $<-5.7 \mathrm{E}-03$ & $\mathrm{U} \mid \mathrm{I} /$ & $<0.1820$ & & 1 & GP & & & & & & & $\mathrm{pCi} / \mathrm{L}$ \\
\hline Plutonium-239/240 & $<2.7 \mathrm{E}-02$ & $\mathrm{UIII}$ & $<0.1110$ & & 1 & GP & & & & & & & $\mathrm{pCi} / \mathrm{L}$ \\
\hline Radium-226 & $1.8 \mathrm{E}+\infty 0$ & /I & & & 1 & GP & & & & & & & $\mathrm{pCi} / \mathrm{L}$ \\
\hline Radium-228 & $<5.4 E-01$ & $\mathrm{U} 1 / 1 /$ & $<0.8290$ & & 1 & GP & & & & & & & $\mathrm{pCi} / \mathrm{L}$ \\
\hline Strontium-90 & $3.8 E+00$ & /I & & & 1 & $\mathrm{GP}$ & & & & & & & $\mathrm{pCil}$ \\
\hline Technetium-99 & $<9.8 E-01$ & $\mathrm{U} ! I I$ & $<20$ & & 1 & GP & & & & & & & pCill \\
\hline Thorium-228 & $<3.9 E-01$ & $\mathrm{U} I I I$ & $<0.5140$ & & 1 & GP & & & & & & & pCill \\
\hline Thorium-230 & $<0.0 E+00$ & $\mathrm{UII} /$ & $<0.2170$ & & 1 & GP & & & & & & & $\mathrm{pCi} / \mathrm{L}$ \\
\hline Thorium-232 & $<-1.7 E-02$ & $\mathrm{UI} / /$ & $<0.3820$ & & 1 & $G P$ & & & & & & & pCill \\
\hline \multicolumn{14}{|l|}{ Sum of alphas } \\
\hline $\begin{array}{l}\text { Sum of betas } \\
\text { Total radium }\end{array}$ & $\begin{array}{l}3.8 E+00 \\
1.8 E+00\end{array}$ & & & & & & & & & & & & $\begin{array}{l}\mathrm{pCi} / \mathrm{L} \\
\mathrm{pCi} / \mathrm{L}\end{array}$ \\
\hline Tritium & $2.2 \mathrm{E}+02$ & / & & च & 1 & GP & $2.8 E+02$ & $y$ & & $\boldsymbol{\square}$ & 1 & GE & $\mathrm{pCi} / \mathrm{mL}$ \\
\hline Uranium-233/234 & $<1.1 E-01$ & $\mathrm{UII} /$ & $<0.2430$ & & 1 & GP & & & & & & & oCill \\
\hline Uranium-235 & $<-3.8 \mathrm{E}-02$ & UIII & $<0.2020$ & & 1 & GP & & & & & & & $\mathrm{pCi} / \mathrm{L}$ \\
\hline Uranium-238 & $<6.4 \mathrm{E}-02$ & $\mathrm{U}: / /$ & $<0.2200$ & & $i$ & GP & & & & & & & $\mathrm{pCi} / \mathrm{L}$ \\
\hline
\end{tabular}

\section{Notes:}

- exceeded holding time

- exceeded groundwater protection or monitoring constituent standard (See Appendix A.) 


\section{WELL FSB112A}

$\begin{array}{lll}\text { SRS Coord. } & \text { Lat/Longitude } \\ \text { N74231.4 } & & 33.269588^{\circ} \mathrm{N} \\ \text { E48809.1 } & 81.682466^{\circ} \mathrm{W}\end{array}$

SAMPLE DATE

Screen Zone Elevation

$91.0-81.0 \mathrm{ft} \mathrm{ms!}$

FIELD DATA

Constituents

Water elevation

pH

Sp. conductance

Water temperature

Alkalinity as $\mathrm{CaCO}_{3}$

Turbidity

Volumes purged

Sampling code

Synchronous water leve

ANALYTICAL DATA

Inorganic Constituents

Constituents

Antimony, total recoverable $\quad<0.20$

Arsenic, total recoverable $\quad<3.0$

Barium, total recoverable $\quad 43$

Cadmium, total recoverable $<1.0$

Chromium, total recoverable 0.72

Cobalt, total recoverable $\quad 0.099$

Copper, total recoverable $\quad 1.2$

Cyanide

Mercury, total recoverable $\quad<0.20$

Nickel, total recoverable $\quad 0.92$

Nitrate as nitrogen

Nitrate-nitrite as nitrogen $\quad 1,500$

Selenium total recoverable 0.38

Silver, total recoverable $<1.0$

Thallium, total recoverable $<2.5$

Vanadium, total recoverable $<2.0$

Zinc, total recoverable

7.1

Organic Constituents

\begin{tabular}{ll} 
Constituents & 3098 \\
\hline Benzene & $<1.0$ \\
Bis(2-ethylhexyl) phthalate & $<10$ \\
Dichloromethane & $<2.8$ \\
Phenols & $<5.0$ \\
Tetrachloroethylene & $<1.0$ \\
Trichloroethylene & $<1.0$ \\
Trichlorofluoromethane & $<1.0$
\end{tabular}

30998

153.7

6.7

140

20.6

29

6.0

$153.8(09 / 18 / 98)$
Lead, total recoverable $\quad 0.15$
Top of Casing

$229.1 \mathrm{ft} \mathrm{ms}$

$10 / 29 / 98$
Casing Pump Screen Zone

4" PVC S $\quad$ U. Congaree (IIA)

\begin{tabular}{ll}
\hline 4098 & Unit \\
153.7 & $\mathrm{ftmsl}$ \\
6.5 & $\mathrm{pH}$ \\
160 & $\mathrm{HS} / \mathrm{cm}$ \\
19.8 & ${ }^{\circ} \mathrm{C}$ \\
53 & $\mathrm{mg} / \mathrm{L}$ \\
1 & $\mathrm{NTU}$ \\
2.2 & well vol \\
$153.5(12 / 21 / 98)$ & $\mathrm{ft} \mathrm{msl}$
\end{tabular}

\begin{tabular}{|c|c|c|c|c|c|c|c|c|c|c|c|}
\hline Mod & Filt. & ST $\underline{H}$ & DF & Lab & 4Q98 & Mod & Filt. & $\underline{\text { ST }} \underline{H}$ & DF & Lab & Unit \\
\hline $\mathrm{U} / /$ & $<0.20$ & & 1 & GE & & & & & & & $\mu g / L$ \\
\hline $\mathrm{U} / /$ & $<3.0$ & & 1 & GE & & & & & & & $\mu g / L$ \\
\hline$\|$ & & & 1 & GE & & & & & & & $\mu g /$ \\
\hline $\mathrm{U} / /$ & $<1.0$ & & 1 & GE & $<1.0$ & $\mathrm{u} / I$ & $<1.0$ & & 1 & GE & Lا \\
\hline $\mathrm{J} / \mathrm{E} /$ & NDD & & 1 & GE & & & & & & & $\mu g / L$ \\
\hline $\mathrm{J} / \mathrm{E} /$ & NDD & & 1 & GE & & & & & & & $\mu g / L$ \\
\hline$N /$ & & & 1 & GE & & & & & & & $\mu g / L$ \\
\hline $\mathrm{U} / /$ & $<10$ & & 1 & GE & & & & & & & $\mu g / \mathrm{L}$ \\
\hline $\mathrm{J} / \mathrm{E} /$ & NDD & & 1 & GE & $<2.0$ & $\mathrm{u} / I$ & $<2.0$ & & 1 & GE & $\mu g / L$ \\
\hline$U \mathrm{U} / / 1 / 1$ & $<0.20$ & & 1 & GE & & & & & & & $\mu \mathrm{g} / \mathrm{L}$ \\
\hline 11 & & & 1 & GE & & & & & & & $\mu g^{\prime} L$ \\
\hline$N /$ & & & 1 & $\mathrm{GE}$ & 3,500 & $\|$ & & & 2 & GE & $\mu g / L$ \\
\hline J/E & NDD & & 1 & GE & & & & & & & $\mu g /$ \\
\hline $\mathrm{U} / 1$ & $<1.0$ & & 1 & GE & & & & & & & $\mu g / L$ \\
\hline $\mathrm{U} / /$ & $<2.5$ & & 1 & GE & & & & & & & $\mu g / L$ \\
\hline $\mathrm{U} / /$ & $<2.0$ & & 1 & GE & & & & & & & $\mathrm{Hg} / \mathrm{L}$ \\
\hline$\|$ & & & 1 & GE & & & & & & & $\mu g / L$ \\
\hline
\end{tabular}

$\begin{array}{lllll}\text { Mod } & \text { Filt. } & \text { STㅂ } & \underline{\text { DF }} & \underline{\text { Lab }} \\ \text { UJ/O/1 } & <1.0 & & 1 & \text { GE } \\ U / / & <10 & & 1 & \text { GE } \\ U J / N O 8 / 1 & <1.0 & & 1 & \text { GE } \\ U / / & <5.0 & & 1 & \text { GE } \\ U J / O / 1 & <1.0 & 1 & \text { GE } \\ U J / O / 1 & <1.0 & 1 & \text { GE } \\ U J / O / 1 & <1.0 & 1 & \text { GE }\end{array}$

Mod

Filt.

ST 브 DF Lab Unit

$\mu g / 1$

$\mu g /$

$\mu g / L$

$\mu g / L$

$\mu g / L$

$\mathrm{Hg} / \mathrm{L}$

$\mu g / L$

Notes:

- exceeded holding time

= exceeded groundwater protection or monitoring constituent standard (See Appendix A.) 
WSRC-TR-99-00012

Unclassified

WELL FSB112A (cont.)

Radioactive Constituents

\begin{tabular}{|c|c|c|c|c|c|c|c|c|c|c|c|c|c|}
\hline Constituents & $\underline{3098}$ & Mod & Filt. & ST브 & $\underline{D F}$ & Lab & 4Q98 & Mod & Filt. & $\underline{\text { ST }} \underline{H}$ & DF & Lab & Unit \\
\hline Americium-241 & $<-1.0 \mathrm{E}-02$ & $\mathrm{U} ! / /$ & $<0.2030$ & & 1 & GP & & & & & & & $\mathrm{pCi} / \mathrm{L}$ \\
\hline Beta dose & 1.2000 & & & घ & & & & & & & & & pCill \\
\hline Cesium-137 & $<1.3 E+00$ & $u I / /$ & $<4.4000$ & & 1 & GP & & & & & & & $\mathrm{pCil}$ \\
\hline Cobalt- 60 & $<-1.0 E+\infty 0$ & $\mathrm{UIII}$ & $<2.7800$ & & 1 & GP & & & & & & & pCill \\
\hline Curium-242 & $<-2.0 \mathrm{E}-02$ & $U m$ & $<0.1880$ & & 1 & GP & & & & & & & $\mathrm{pCin}$ \\
\hline Curium-243/244 & $<-5.0 \mathrm{E}-02$ & $\mathrm{UI} / /$ & $<0.2590$ & & 1 & GP & & & & & & & $\mathrm{pCi} / \mathrm{L}$ \\
\hline Curium-245/246 & $<-6.0 \mathrm{E}-03$ & $U 1 / /$ & $<0.0751$ & & 1 & GP & & & & & & & $\mathrm{pCi} / \mathrm{L}$ \\
\hline Gross alpha & 1. $1 E+00$ & $\|$ & & & 1 & GP & $2.2 E+00$ & /1 & & & 1 & GE & $\mathrm{pCi} / \mathrm{L}$ \\
\hline lodine-129 & $<4.0 \mathrm{E}-01$ & $\mathrm{U} \mid / /$ & $<0.5120$ & & 1 & GP & & & & & & & $\mathrm{pCI} / \mathrm{L}$ \\
\hline Nonvolatile beta & $8.9 E+00$ & "I & & & 1 & GP & $1.8 E+01$ & /I & & & 1 & $\mathrm{TM}$ & $\mathrm{pCi} / \mathrm{L}$ \\
\hline Plutonium-238 & $<9.3 E-03$ & $\mathrm{UIII}$ & $<0.2640$ & & 1 & GP & & & & & & & $\mathrm{pCi} / \mathrm{L}$ \\
\hline Plutonium-239/240 & $<1.4 \mathrm{E}-02$ & $\mathrm{UI} / / \mathrm{I}$ & $<0.0939$ & & 1 & GP & & & & & & & pCill \\
\hline Radium-226 & $1.5 \mathrm{E}+00$ & "I & & & 1 & GP & & & & & & & $\mathrm{pCi} / \mathrm{L}$ \\
\hline Radium-228 & $<1.3 \mathrm{E}-01$ & $\mathrm{U} \mathrm{I} / \mathrm{I}$ & $<0.8800$ & & 1 & GP & & & & & & & $\mathrm{pCin}$ \\
\hline Strontium-90 & $9.6 E+00$ & /I & & - & 1 & GP & & & & & & & $\mathrm{pCi} / \mathrm{L}$ \\
\hline Technetium-99 & $<4.6 \mathrm{E}+\infty 0$ & UIIII & $<22.0$ & & 1 & GP & & & & & & & $\mathrm{pCi} / \mathrm{L}$ \\
\hline Thorium-228 & $<-2.0 \mathrm{E}-02$ & $\mathrm{UI} / /$ & $<0.3870$ & & 1 & GP & & & & & & & $\mathrm{pCi} / \mathrm{L}$ \\
\hline Thorium-230 & $<1.8 \mathrm{E}-01$ & $\mathrm{UI} / /$ & $<0.1320$ & & 1 & GP & & & & & & & $\mathrm{pCill}$ \\
\hline $\begin{array}{l}\text { Thorium-232 } \\
\text { Sum of aiphas }\end{array}$ & $<0.0 E+00$ & UiII & $<0.9320$ & & 1 & GP & & & & & & & $\mathrm{pCin}$ \\
\hline $\begin{array}{l}\text { Sum of betas } \\
\text { Total radium }\end{array}$ & $\begin{array}{l}9.6 E+00 \\
1.5 E+00\end{array}$ & & & & & & & & & & & & $\begin{array}{l}\mathrm{pCilL} \\
\mathrm{pCi} / \mathrm{L}\end{array}$ \\
\hline Tritium & $3.8 \mathrm{E}+01$ & "I & & m & 1 & GP & $1.0 E+02$ & "I & & - & 1 & GE & $\mathrm{pCi} / \mathrm{mL}$ \\
\hline Uranium-233/234 & $<1.4 E-01$ & $\mathrm{U} I / /$ & $<0.1570$ & & 1 & $G P$ & & & & & & & $\mathrm{pCin}$ \\
\hline Uranium-235 & $<6.1 \mathrm{E}-02$ & $\mathrm{U} U / /$ & $<0.1080$ & & 1 & GP & & & & & & & $\mathrm{pCi} / \mathrm{L}$ \\
\hline Uranium-238 & $<3.5 \mathrm{E}-02$ & $\mathrm{UI} / /$ & $<0.0521$ & & 1 & GP & & & & & & & $\mathrm{pCi} / \mathrm{L}$ \\
\hline
\end{tabular}

Notes:

- exceeded holding time

- exceeded groundwater protection or monitoring constituent standard (See Appendix A.) 
WELL FSB112C

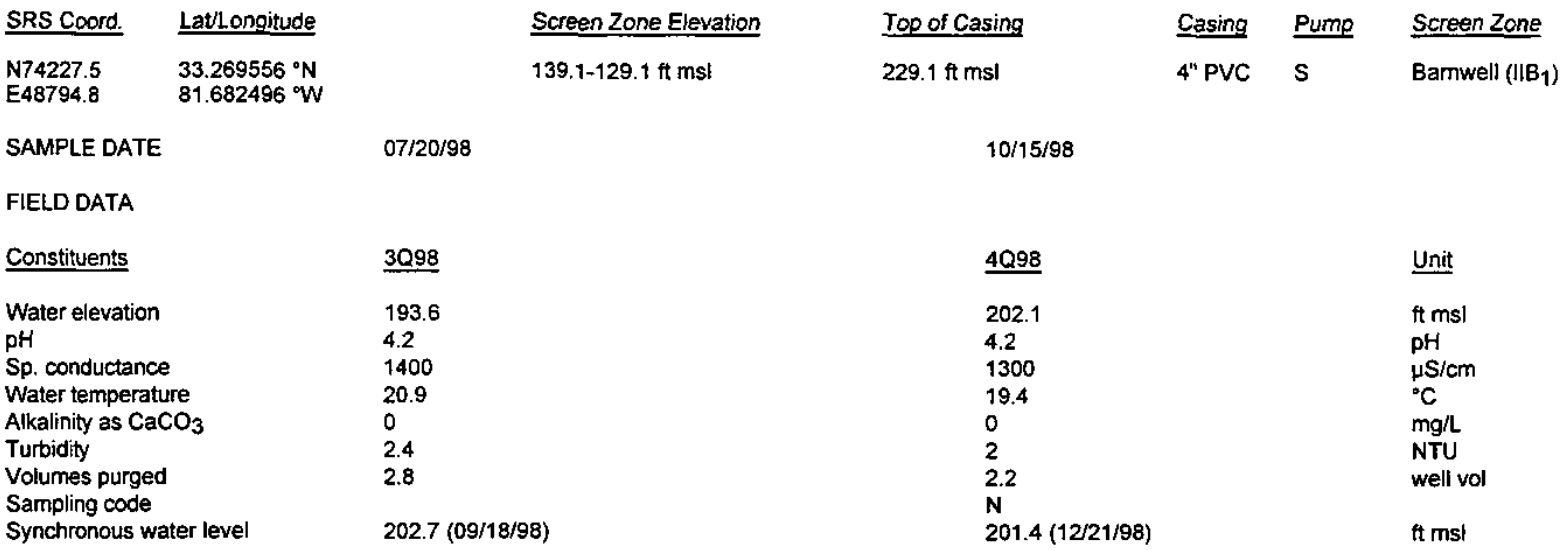

ANALYTICAL DATA

Inorganic Constituents

\begin{tabular}{|c|c|c|c|c|c|c|c|c|c|c|c|c|c|}
\hline Constituents & 3098 & Mod & Filt. & ST브 & $\underline{\text { DF }}$ & $\underline{\text { Lab }}$ & 4Q98 & Mod & Filt. & $\underline{\mathrm{ST}} \underline{\mathrm{H}}$ & DF & $\underline{\text { Lab }}$ & Unit \\
\hline Antimony, total recoverable & $<0.20$ & $\mathrm{U} / /$ & $<0.20$ & & 1 & GE & & & & & & & $\mu g / L$ \\
\hline Arsenic, total recoverable & $<150$ & $\mathrm{U} / /$ & $<150$ & & 50 & GE & & & & & & & $\mu g / 1$ \\
\hline Barium, total recoverable & 720 & $/ 1$ & & & 1 & $\overline{G E}$ & & & & & & & $\mu g / L$ \\
\hline Cadmium, total recoverable & 36 & 11 & & च & 1 & GE & 36 & "I & & e & 1 & GE & $\mu \mathrm{g} / \mathrm{L}$ \\
\hline Chromium, total recoverable & $<150$ & $\mathrm{U} / /$ & $<150$ & & 50 & GE & & & & & & & $\mu \mathrm{g} / \mathrm{L}$ \\
\hline Cobalt, total recoverable & 190 & $\|$ & & $\mathbf{0}$ & 50 & GE & & & & & & & $\mu g^{\prime}$ \\
\hline Copper, total recoverable & 27 & $\|$ & & & 1 & $\overline{G E}$ & & & & & & & $\mu g / \mathrm{L}$ \\
\hline Cyanide & $<10$ & $\mathrm{U} / /$ & $<10$ & & 1 & GE & & & & & & & $\mu g / L$ \\
\hline Lead, total recoverable & 0.86 & $J / E J$ & NDO & & 1 & $G E$ & 1.4 & Jill & NOD & & 1 & GE & $\mu g / 1$ \\
\hline Mercury, total recoverable & 0.38 & $/ 1$ & & & 1 & $\mathrm{GE}$ & & & & & & & $\mu g / L$ \\
\hline $\begin{array}{l}\text { Nickel, total recoverable } \\
\text { Nitrate as nitrogen }\end{array}$ & 93 & $\|$ & & & 1 & GE & & & & & & & $\mu g / L$ \\
\hline Nitrate-nitrite as nitrogen & 220,000 & $\mathrm{~J} / \mathrm{IV} / 1$ & NDD & & 100 & GE & 230,000 & $/ 1$ & & a & 100 & GE & $\mu g / L$ \\
\hline Selenium, total recoverable & $<250$ & $\mathrm{U} / /$ & $<250$ & & 50 & GE & & & & & & & $\mu g / L$ \\
\hline Silver, total recoverable & $<1.0$ & $\mathrm{U} / /$ & $<1.0$ & & 1 & GE & & & & & & & $\mu g / L$ \\
\hline Thallium, total recoverable & $<0.068$ & $\mathrm{UN} /$ & $<2.5$ & & 1 & GE & & & & & & & $\mu g / L$ \\
\hline Vanadium, total recoverabie & $<100$ & $\mathrm{U} / 1$ & $<100.0000$ & & 50 & $\mathrm{GE}$ & & & & & & & $\mu g / L$ \\
\hline Zinc, total recoverable & 230 & /I & & & $\uparrow$ & GE & & & & & & & $\mu g /$ \\
\hline \multicolumn{14}{|l|}{ Organic Constituents } \\
\hline Constituents & 3098 & Mod & Filt. & ST브 & $\underline{\mathrm{DF}}$ & Lab & 4Q98 & Mod & Filt. & $\underline{\mathrm{ST}} \underline{\mathrm{H}}$ & $\underline{D F}$ & $\underline{L a b}$ & Unit \\
\hline Benzene & $<1.0$ & $\mathrm{u} / /$ & $<1.0$ & & 1 & $G E$ & & & & & & & $\mu g / L$ \\
\hline Bis(2-ethylhexyl) phthalate & $<10$ & $\mathrm{UJ} / \mathrm{Q} /$ & $<10$ & $\bullet$ & 1 & GE & & & & & & & $\mu g / L$ \\
\hline Dichloromethane & $<1.9$ & $\mathrm{U} / 8 /$ & $<1.0$ & & 1 & GE & & & & & & & $\mu g / L$ \\
\hline Phenols & $<5.0$ & $\mathrm{U} / /$ & $<5.0$ & & 1 & GE & & & & & & & $\mu g / L$ \\
\hline Tetrachloroethylene & $<1.0$ & $\mathrm{U} / /$ & $<1.0$ & & 1 & GE & & & & & & & $\mu g / L$ \\
\hline Trichloroethylene & $<1.0$ & $\mathrm{U} / /$ & $<1.0$ & & 1 & GE & & & & & & & $\mu g / L$ \\
\hline Trichlorofluoromethane & $<1.0$ & $\mathrm{U} / /$ & $<1.0$ & & 1 & GE & & & & & & & $\mu g / L$ \\
\hline
\end{tabular}


WELL FSB112C (cont.)

Radioactive Constituents

\begin{tabular}{|c|c|c|c|c|c|c|c|c|c|c|c|c|c|}
\hline Constituents & $\underline{3098}$ & Mod & Filt. & ST브 & $\underline{\mathrm{DF}}$ & Lab & 4Q98 & Mod & Filt. & $\underline{\text { ST }} \underline{H}$ & DF & Lab & $\underline{\text { Unit }}$ \\
\hline Americium-241 & $<1.5 E-01$ & $\mathrm{UI} / /$ & $<0.1720$ & & 1 & GP & & & & & & & $\mathrm{pCi} / \mathrm{L}$ \\
\hline Beta dose & 190.1416 & & & 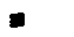 & & & & & & & & & $\mathrm{pCi} / \mathrm{L}$ \\
\hline Cesium-137 & $<1.6 \mathrm{E}+00$ & $\mathrm{UH} / \mathrm{I}$ & $<3.3300$ & & 1 & GP & & & & & & & $\mathrm{pCill}$ \\
\hline Cobalt-60 & $3.6 \mathrm{E}+00$ & $/ /$ & & & 1 & GP & & & & & & & pCill \\
\hline Curium-242 & $<0.0 \mathrm{E}+00$ & UII/ & $<0.1850$ & & 1 & GP & & & & & & & $\mathrm{pCi} / \mathrm{L}$ \\
\hline Curium-243/244 & $<-1.4 \mathrm{E}-02$ & $\mathrm{U} / /$ & $<0.3030$ & & 1 & GP & & & & & & & $\mathrm{pCi} / \mathrm{L}$ \\
\hline Curium-245/246 & $<0.0 E+00$ & $\mathrm{UL} / /$ & $<0.1720$ & & 1 & GP & & & & & & & pCill \\
\hline Gross alpha & $1.6 \mathrm{E}+02$ & $\|$ & & 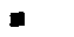 & 1 & GP & $1.6 E+02$ & 11 & & - & 1 & GE & $\mathrm{pCi} / \mathrm{L}$ \\
\hline Nonvolatile beta & $1.8 \mathrm{E}+03$ & $\|$ & & ט & 1 & GP & $1.9 E+03$ & $\|$ & & a & 1 & GE & $\mathrm{pCi} / \mathrm{L}$ \\
\hline Plutonium-238 & $<0.0 \mathrm{E}+00$ & $\mathrm{UI} / /$ & $<0.1880$ & & 1 & GP & & & & & & & pCill \\
\hline Plutonium-239/240 & $<6.3 E-02$ & $\mathrm{UIII}$ & $<0.1880$ & & 1 & GP & & & & & & & pCill \\
\hline Radium-226 & $1.3 E+02$ & $/ /$ & & $\boldsymbol{n}$ & 1 & GP & & & & & & & pCill \\
\hline Radium-228 & $3.0 E+01$ & $\|$ & & - & 1 & GP & & & & & & & pcill \\
\hline Strontium-90 & $5.1 \mathrm{E}+02$ & $\|$ & & $\mathbf{n}$ & 1 & GP & & & & & & & pCill \\
\hline Technetium-99 & $3.2 E+02$ & $/ 1$ & & 口 & 1 & GP & & & & & & & $\mathrm{pCil}$ \\
\hline Thorium-228 & $1.1 E+00$ & R/4/ & Rej & & 1 & GP & & & & & & & $\mathrm{pCi} / \mathrm{L}$ \\
\hline Thorium-230 & $<7.9 \mathrm{E}-02$ & $\mathrm{UI} / 1$ & $<0.2360$ & & 1 & GP & & & & & & & $\mathrm{pCi} / \mathrm{L}$ \\
\hline Sum of alphas & $7.9 \mathrm{E}+00$ & & & & & & & & & & & & pCil \\
\hline Sum of betas & $9.8 \mathrm{E}+02$ & & & 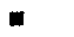 & & & & & & & & & $\mathrm{pCin}$ \\
\hline Total radium & $1.6 \mathrm{E}+02$ & & & - & & & & & & & & & pCill \\
\hline Tritium & $6.4 E+03$ & /1 & & च & 1 & GP & $7.3 E+03$ & /I & & घ & 1 & GE & $\mathrm{pCi} / \mathrm{ml}$ \\
\hline Uranium-233/234 & $4.1 E+00$ & $/ /$ & & & 1 & GP & & & & & & & pCill \\
\hline Uranium-235 & $3.2 \mathrm{E}-01$ & $\|$ & & & 1 & GP & & & & & & & $\mathrm{pCi} / \mathrm{L}$ \\
\hline Uranium-238 & $3.5 E+00$ & $\|$ & & & 1 & GP & & & & & & & pCilL \\
\hline
\end{tabular}

Notes:

- = exceeded holding time

= exceeded groundwater protection or monitoring constituent standard (See Appendix A.) 


\section{WELL FSB112D}

$\begin{array}{ll}\text { SRS Coord. } & \text { Lat/Longitude } \\ \text { N74223.7 } & 33.269523^{\circ} \mathrm{N} \\ \text { E48780.0 } & 81.682527^{\circ} \mathrm{W}\end{array}$

SAMPLE DATE

FIELD DATA

Constituents

Water elevation

$\mathrm{pH}$

Sp. conductance

Water temperature

Alkalinity as $\mathrm{CaCO}_{3}$

Turbidity

Volumes purged

Sampling code

Synchronous water level

ANALYTICAL DATA

Inorganic Constituents

Constituents

Antimony total recoverable $<0.20$

Arsenic, total recoverable $<15$

Barium, total recoverable 310

Cadmium, total recoverable 3.2

Chromium, total recoverable $\mathbf{5 . 5}$

Cobalt, total recoverable $\quad 9.5$

Copper, total recoverable 50

Cyanide

Lead, total recoverable

Mercury, total recoverable $\quad 0.039$

Nickel, total recoverable

Nitrate as nitrogen

Nitrate-nitrite as nitrogen $\quad 25,000$

Selenium, total recoverable 7.1

Silver, total recoverable $<1$.

Thallium, total recoverable $<2.5$

Vanadium, total recoverable $<10$

Zinc, total recoverable

55

Onganic Constituents

Constituents

Benzene

3Q98

Bis(2-ethylhexyt) phthalate

Dichloromethane

Phenols

Tetrachloroethylene

Trichloroethylene

Trichlorofluoromethane
Screen Zone Elevation

$208.9-188.9 \mathrm{ft} \mathrm{ms}$

$07 / 20 / 98$

3Q98

207.9

4

240

22.3

0

3.2

$207.2(09 / 18 / 98)$
Top of Casing

$229.6 \mathrm{ft} \mathrm{msl}$

$10 / 14 / 98$

4098
206.6
3.7
180
18.3
0
1
4.5

$205.8(12 / 21 / 98)$
Casing Pump Screen Zone

4" PVC S Water Table (IIB2) 
WELL FSB112D (cont.)

Radioactive Constituents

\begin{tabular}{|c|c|c|c|c|c|c|c|c|c|c|c|c|c|}
\hline Constituents & $\underline{3098}$ & Mod & Filt. & $\underline{\text { ST }} \underline{H}$ & $\underline{D F}$ & Lab & $\underline{4 Q 98}$ & $\underline{\text { Mod }}$ & Filt. & $\underline{\mathrm{ST}} \underline{\mathrm{H}}$ & $\underline{\mathrm{DF}}$ & $\underline{\text { Lab }}$ & $\underline{\text { Unit }}$ \\
\hline Americium-241 & $<7.9 E-02$ & $\mathrm{U}: / /$ & $<0.2930$ & & 1 & GP & & & & & & & $\mathrm{pCV} / \mathrm{L}$ \\
\hline Beta dose & 46.7000 & & & a & & & & & & & & & pCir. \\
\hline Cesium-137 & $<1.0 E+00$ & $\mathrm{UI} / /$ & $<3.3800$ & & 1 & GP & & & & & & & $\mathrm{pCilL}$ \\
\hline Cobalt-60 & $<8.8 \mathrm{E}-02$ & $\mathrm{UI} / \mathrm{I}$ & $<3.5100$ & & 1 & GP & & & & & & & $\mathrm{pCilL}$ \\
\hline Curium-242 & $<1.1 \mathrm{E}-01$ & $\mathrm{UI} / \mathrm{I}$ & $<0.3160$ & & 1 & GP & & & & & & & $\mathrm{pCi} / \mathrm{L}$ \\
\hline Curium-243/244 & $<5.1 \mathrm{E}-02$ & $U: / /$ & $<0.6090$ & & 1 & GP & & & & & & & pCill \\
\hline Curium-245/246 & $<0.0 E+00$ & $\mathrm{UI} / /$ & $<0.2930$ & & 1 & GP & & & & & & & $\mathrm{pCill}$ \\
\hline Gross alpha & $5.0 \mathrm{E}+01$ & "I & & - & 1 & GP & $4.6 \mathrm{E}+01$ & $" 1$ & & = & 1 & GE & pCil \\
\hline lodine-129 & $1.2 \mathrm{E}+01$ & "I & & & 1 & GP & & & & & & & $\mathrm{pCill}$ \\
\hline Nonvolatile beta & $7.1 \mathrm{E}+02$ & $\|$ & & m & 1 & GP & $5.2 E+02$ & $\|$ & & a & 1 & $\mathrm{GE}$ & pCill \\
\hline Plutonium-238 & $<1.2 \mathrm{E}-01$ & $\mathrm{U} \mid / /$ & $<0.1810$ & & 1 & GP & & & & & & & $\mathrm{pCin}$ \\
\hline Plutonium-239/240 & $<1.1 \mathrm{E}-01$ & $\mathrm{U} 1 / /$ & $<0.3180$ & & 1 & GP & & & & & & & $\mathrm{pCilL}$ \\
\hline Radium-226 & $3.3 E+01$ & "I & & - & 1 & GP & & & & & & & pCill \\
\hline Radium-228 & 1.1E+01 & "I & & - & 1 & GP & & & & & & & $\mathrm{pCi} / \mathrm{L}$ \\
\hline Strontium-90 & $2.6 E+02$ & 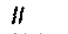 & & 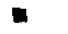 & 1 & GP & & & & & & & $\mathrm{pCi} / \mathrm{L}$ \\
\hline Technetium-99 & $<8.0 E+00$ & $U I / I$ & $<21.7000$ & & 1 & GP & & & & & & & pCill \\
\hline Thorium-228 & $6.4 \mathrm{E}-01$ & $R / 4 /$ & & & 1 & GP & & & & & & & $\mathrm{pCilL}$ \\
\hline Thorium-230 & $<7,7 \mathrm{E}-02$ & $\mathrm{U} \mid / /$ & $<0.2320$ & & 1 & GP & & & & & & & $\mathrm{pCi} / \mathrm{L}$ \\
\hline Thorium-232 & $<0.0 \mathrm{E}+00$ & $\mathrm{U}: / /$ & $<0.2320$ & & 1 & GP & & & & & & & $\mathrm{pCilL}$ \\
\hline Sum of alphas & $8.9 E+\infty 0$ & & & & & & & & & & & & PCilL \\
\hline Sum of betas & $2.8 \mathrm{E}+02$ & & & - & & & & & & & & & $\mathrm{pCil}$ \\
\hline Total radium & $4.4 E+01$ & & & - & & & & & & & & & $\mathrm{pCilh}$ \\
\hline Tritium & $6.0 \mathrm{E}+02$ & 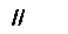 & & m & 1 & GP & $3.7 \mathrm{E}+02$ & $/ 1$ & & = & 1 & GE & $\mathrm{pCi} / \mathrm{mL}$ \\
\hline Uranium-233/234 & $5.5 E+00$ & $\|$ & & & 1 & GP & & & & & & & $\mathrm{pCi} / \mathrm{L}$ \\
\hline Uranium-235 & $<1.8 E-01$ & Uil/ & $<0.1800$ & & 1 & GP & & & & & & & $\mathrm{pCi} / \mathrm{L}$ \\
\hline Uranium-238 & $3.5 E+00$ & " & & & 1 & GP & & & & & & & $\mathrm{pCil}$ \\
\hline
\end{tabular}

Notes:

$-=$ exceeded holding time

- = exceeded groundwater protection or monitoring constituent standard (See Appendix A.) 


\section{WELL FSB113A}

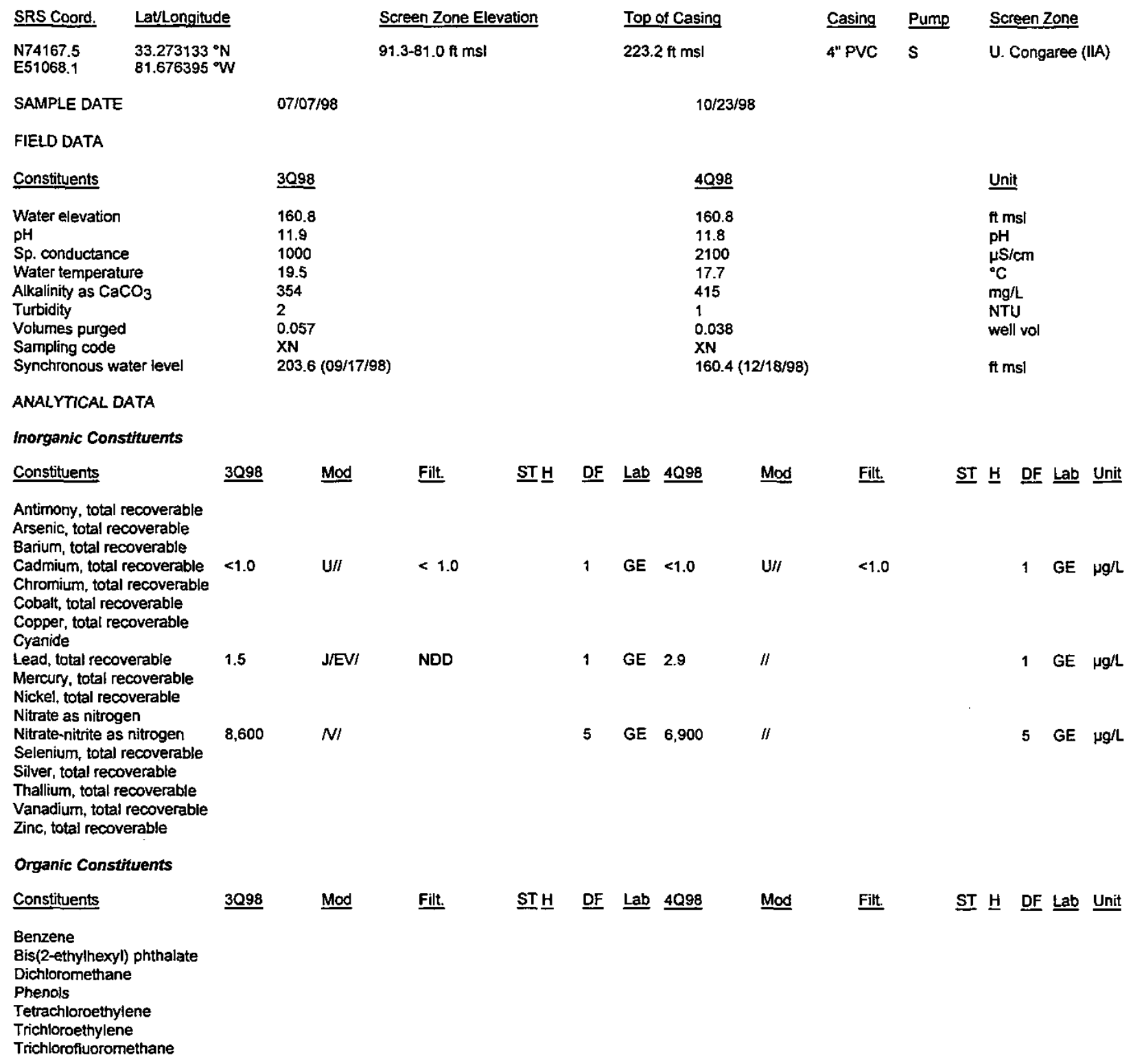

Notes:

- exceeded holding time

a = exceeded grounowater protection or monitoring constituent standard (See Appendix A.) 
WELL FSB113A (cont.)

Radioactive Consthuents

Constituents

$\underline{3 Q 98}$

Mod

Filt.

STH DF Lab $\underline{4098}$

Mod

Filt.

$\underline{\text { ST }} \underline{\mathrm{H}} \underline{\mathrm{DF}}$ Lab Unit

Americium-241

Cesium-137

Cobalt-60

Curium-242

Curium-243/244

Curium-245/246

Gross alpha

lodine-129

Nonvolatile beta

Plutonium-238

Plutonium-239/240

Radium-226

Radium-228

Strontium-90

Technetium-99

Thorium-228

Thorium-230

Thorium-232

Sum of alphas

Sum of betas

Tritium

Uranium-233/234

$3.4 \mathrm{E}+00$

1 GP $4.3 E+00 \quad / /$

7.2E+00 /

1 GP 4.4E+00 //

1 GE pCill

Uranium-235

Uranium-238

Notes:

- = exceeded holding time

E = exceeded groundwater protection or monitoring constituent standard (See Appendix A.) 
WELL FSB113C

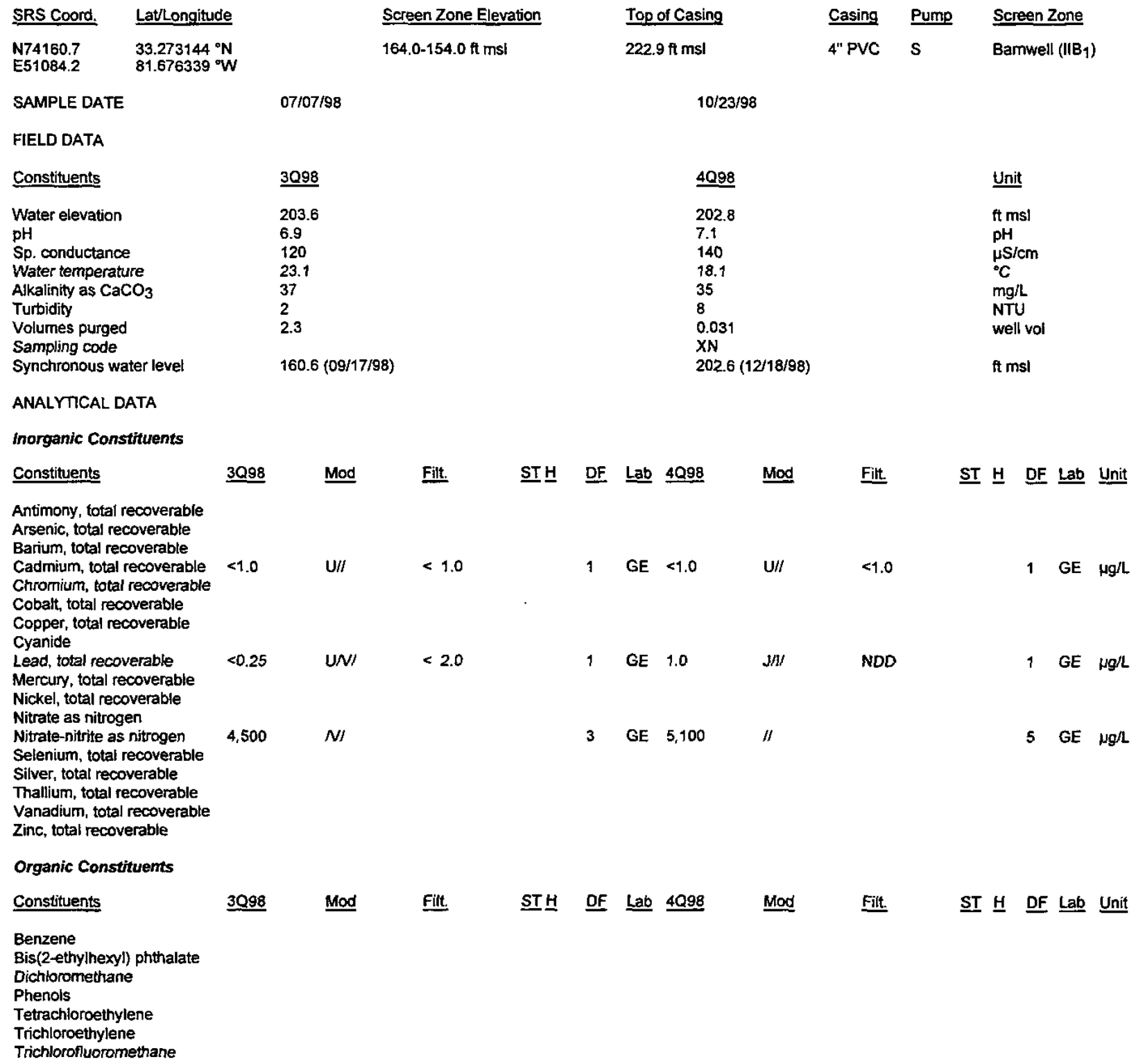

Notes:

- exceeded holding time

- exceeded groundwater protection or monitoring constituent standard (See Appendix A.) 


\section{WELL FSB113C (cont.)}

Radioactive Constituents

Constituents

Americium-241

Cesium-137

Cobalt-60

Curium-242

Curium-243/244

Curium-245/246

Gross alpha

lodine-129

Nonvolatile beta

Plutonium-238

Plutonium-239/240

Radium-226

Radium-228

Strontium-90

Technetium-99

Thorium-228

Thorium-230

Thorium-232

Sum of alphas

Sum of betas

Tritium

Uranium-233/234

Uranium-235

Uranium-238
3098

$\underline{\operatorname{Mod}}$

Filt.

ST브 DF LLab 4098

Mod

Filt.

$\underline{\text { ST }} \underline{\text { ㅍ }}$ Lab Unit

$3.0 \mathrm{E}+00$

$1.4 \mathrm{E}+00 \quad / /$

1 GP $<6.3 E-01$

$\mathrm{U} / 1$

1 GP $<1.4 E+00 \quad U / 1$

$<1.4 \mathrm{E}+00$

$<1.4 \mathrm{E}+00$

$1 \mathrm{GE} \rho \mathrm{Cj} / \mathrm{L}$

$1 \mathrm{GE} \mathrm{pCi} / \mathrm{L}$

- exceeded holding time

- exceeded groundwater protection or monitoring constituent standard (See Appendix A.) 


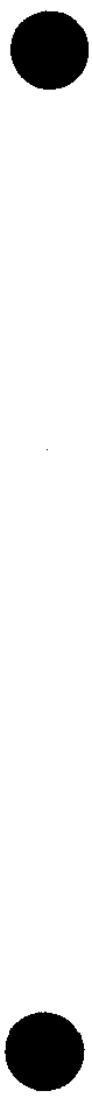

\section{WELL FSB113D}

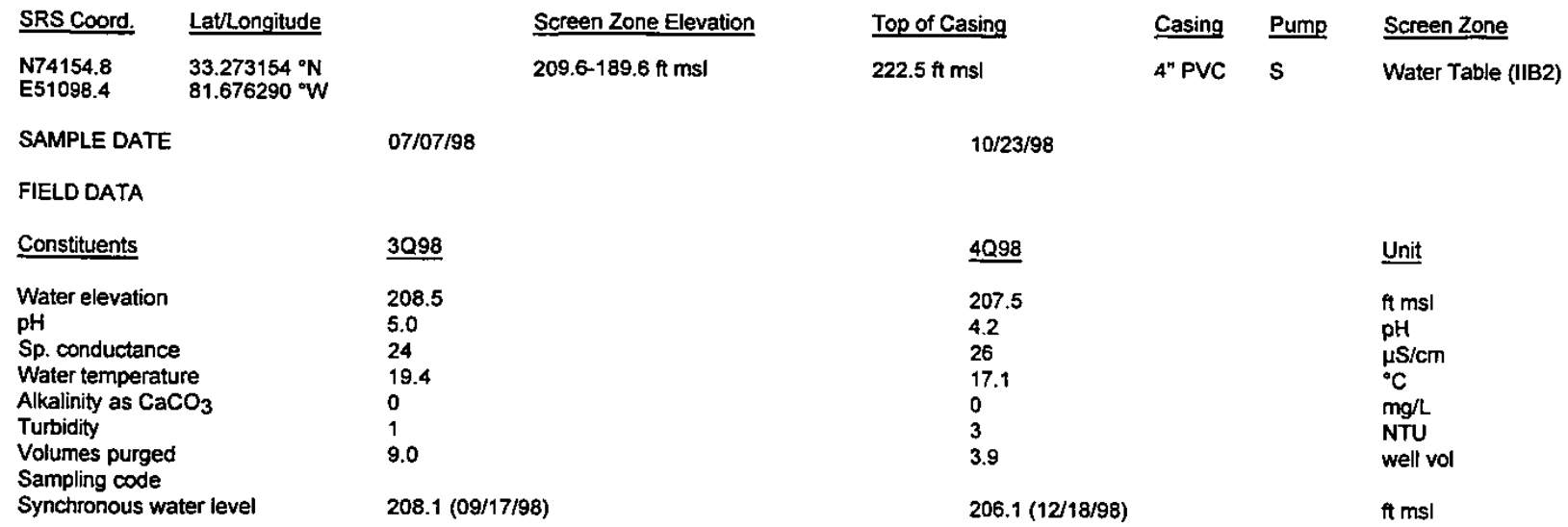

\section{ANALYTICAL DATA}

Inorganic Consthuemts

Constituents

Antimony, total recoverable

Arsenic, total recoverable

Barium, total recoverable

Cadmium, total recoverable

Chromium, total recoverable

$<1.0$

Cobalt, total recoverable

Copper, total recoverable

Cyanide

Lead, total recoverable

Mercury, total recoverable

Nickel, total recoverable

Nitrate as nitrogen

Nitrate-nitrite as nitrogen

Selenium, total recoverable

Silver, total recoverable

Thallium, total recoverable

Vanadium, total recoverable

Zinc, total recoverable

Organic Constituents

Constituents

Benzene

Bis(2-ethylhexyl) phthalate

Dichloromethane

Phenols

Tetrachloroethylene

Trichloroethylene

Trichlorofluoromethane
3098

0.66

J/EV $/$

NDD

1 GE $\quad 0.88$

$\mathrm{J} / \mathrm{I}$

NI

1 GE 660

/I

Mod

Mod Filt.

STH
Mod

$\mathrm{U} / 1$

Notes:

- exceeded holding time

- = exceeded groundwater protection or monitoring constituent standard (See Appendix A.) 
WSRC-TR-99-00012

Unclassified

WELL FSB113D (cont.)

Radioactive Constituents

Constituents

Americium-241

Cesium-137

Cobalt-60

Curium-242

Curium-243/244

Curium-245/246

Gross alpha

lodine-129

Nonvolatile beta

Plutonium-238

Plutonium-239/240

Radium-226

Radium-228

Strontium-90

Technetium-99

Thorium-228

Thorium-230

Thorium-232

Sum of alphas

Sum of betas

Tritium

Uranium-233/234

Uranium-235

Uranium-238
309

$<8.9 E-01 \quad$ UII $\quad<0.9610$

$<-1.9 \mathrm{E}-01 \quad$ UI $/ 1$

$<1.9500$

$1 \quad G P<6.1 E-01$

$\mathrm{U} / 1$

1 GP $<7,5 E-01$

$\mathrm{U} / /$

$<7.3 E-01$

$<1.4 \mathrm{E}+00$

1 GE pCill

1 GE pCill

$6.8 \mathrm{E}+00 \quad / 1$

1 GP $6.3 \mathrm{E}+00 \quad / /$

$1 \mathrm{GE} \quad \mathrm{pCi} / \mathrm{mL}$

Notes:

- = exceeded holding time

- =xceeded groundwater protection or monitoring constituent standard (See Appendix A.)

F-Area HWMF 


\section{WELL FSB114A}

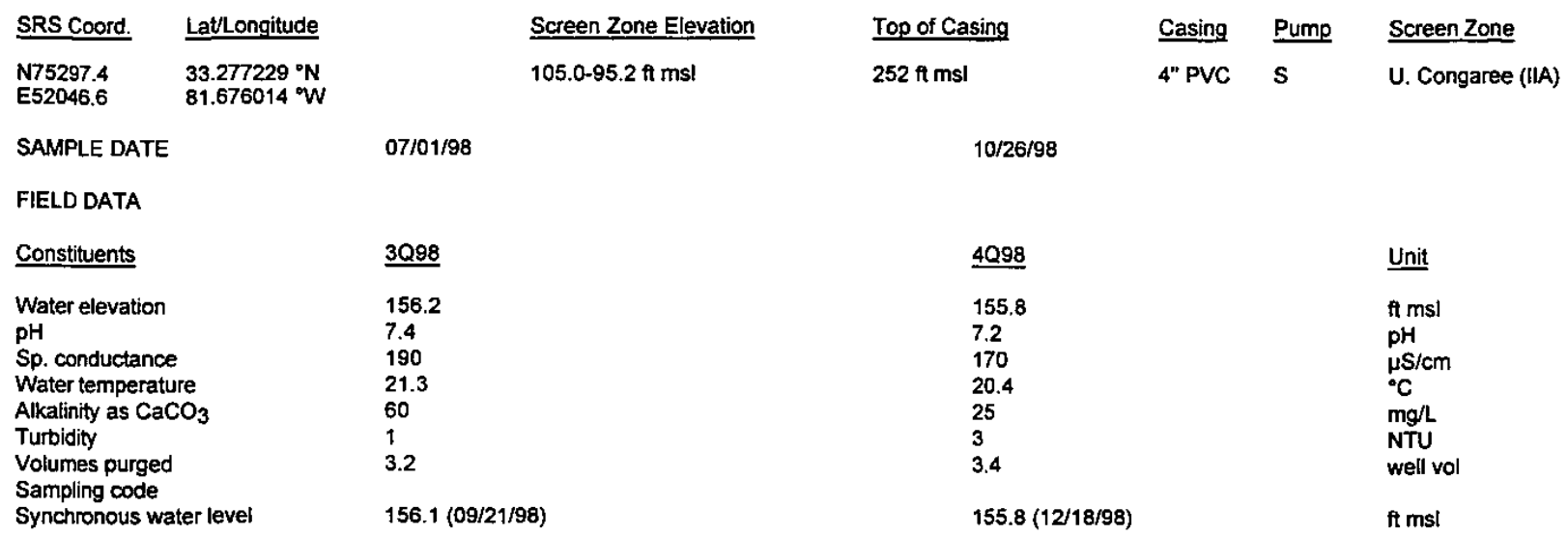

\section{ANALYTICAL DATA}

Inorganic Constituents

Constituents

Antimony, total recoverable

Arsenic, total recoverable

Barium, total recoverable

Cadmium, total recoverable

Chromium, total recoverable

Cobalt, total recoverable

Copper, total recoverable

Cyanide

Lead, total recoverable

Mercury, total recoverable

Nickel, total recoverable

Nitrate as nitrogen

Nitrate-nitrite as nitrogen

Selenium, total recoverable

Silver, total recoverable

Thallium, total recoverable

Vanadium, total recoverable

Zinc, total recoverable

Organic Constituents

Constituents

Benzene

Bis(2-ethylhexyl) phthalate

Dichloromethane

Phenois

Tetrachloroethylene

Trichloroethylene

Trichlorofluoromethane
$\mathrm{J} / \mathrm{E}$

NDD

$<1.0$

$1 \quad \mathrm{GE}<1.0$

UII

1 GE 2,000

/I

Mod

Filt.

ST $\underline{\text { Lab }}$
$\mathrm{J} / \mathrm{I} /$

Mod

.

GE $\mu g / /$

1 GE $\mu g / L$

Mod
Filt.

ST 브 DF Lab Unit

Notes:

- exceeded holding time

a = exceeded groundwater protection or monitoring constituent standard (See Appendix A.) 


\section{WELL FSB114A (cont.)}

Radloactive Constituents

Constituents

Americium-241

Cesium-137

Cobalt-60

Curium-242

Curium-243/244

Curium-245/246

Gross alpha

lodine-129

Nonvolatile beta

Plutonium-238

Plutonium-239/240

Radium-226

Radium-228

Strontium-90

Technetium-99

Thorium-228

Thorium-230

Thorium-232

Sum of alphas

Sum of betas

Tritium

Uranium-233/234

Uranium-235

Uranium-238
3098

Mod

Filt.

STI H DF Lab 4 QQ98

7.9E-01

$\mathrm{J} / \mathrm{C} /$

NDD

1.7E+00 //

1 GP $<6.7 \mathrm{E}-01 \quad \mathrm{U} / \prime$

1 GP $<6.6 \mathrm{E}-01 \quad \mathrm{U} / 1$

$<6.2 \mathrm{E}-01$

$<1.1 E+00$

1 GE pCi/l

$1 \mathrm{GE} \mathrm{pCi} / \mathrm{L}$

8.5E-01 $/ /$

1 GP $<4.7 E-01 \quad U / I$

$<6.3 E-01$

1 GE $p C i / m L$

Notes:

- exceeded holding time

- = exceeded groundwater protection or monitoring constituent standard (See Appendix A) 
WELL FSB114C

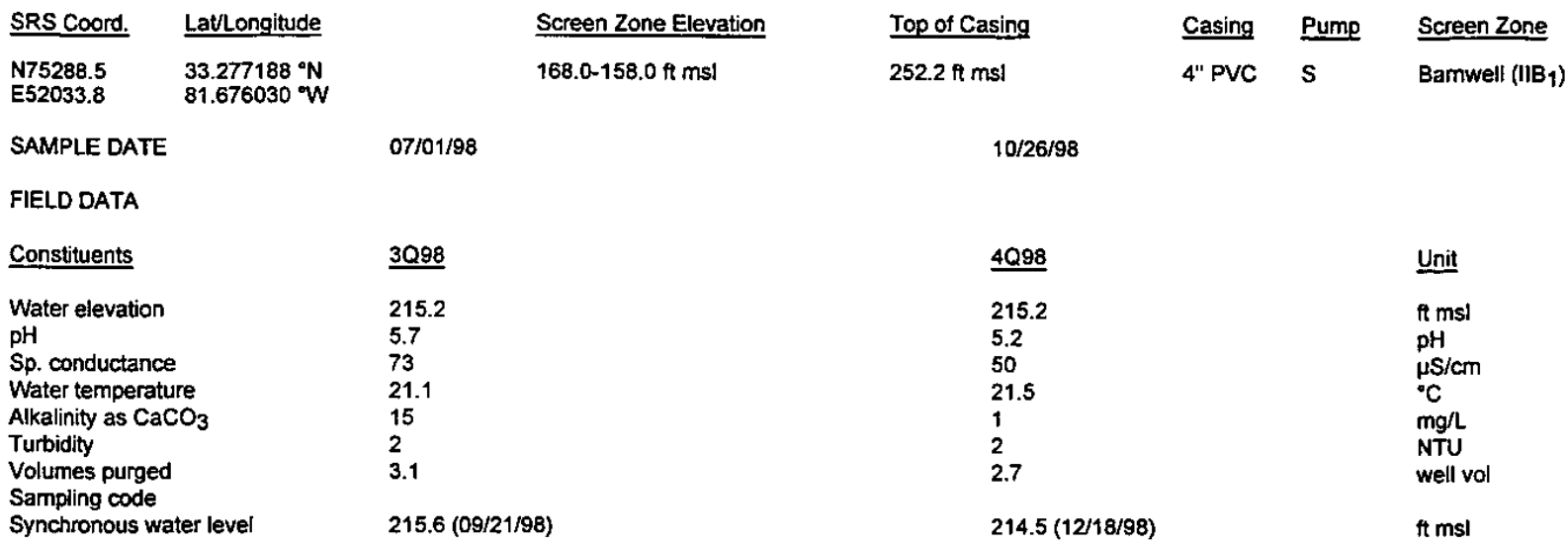

\section{ANALYTICAL DATA}

Inorganic Constituents

Constituents

Antimony, total recoverable

Arsenic, total recoverable

Barium, total recoverable

Cadmium, total recoverable

Chromium, total recoverable

Cobalt, total recoverable

Copper, total recoverable

Cyanide

Lead, total recoverable

Mercury, total recoverable

Nickel, total recoverable

Nitrate as nitrogen

Nitrate-nitrite as nitrogen

Selenium, total recoverable

Silver, total recoverable

Thallium, total recoverable

Vanadium, total recoverable

Zinc, total recoverable

Organic Constituents

Constituents

$\underline{\operatorname{Mod}}$

Filt.

ST브

DF Lab 4 Q998

Mod

Filt.

$\underline{\text { ST }}$ H DF Lab Unit

$<1.0 \quad U /$

$<1.0$

1 GE $<1.0$

$\mathrm{U} / 1$

$<1.0$

1 GE $\mu g / L$

2.5

/I

$1 \quad$ GE $\quad 2.2$

/

1 GE 2,100

1 GE $\mu g / L$

$2,100 \quad$ II

3Q998

Mod

Filt.

ST브

DF L

4Q98

Mod

Filt.

ST $\underline{H}$ DF Lab Unit

Benzene

Bis(2ethylhexyl) phthalate

Dichloromethane

Phenols

Tetrachloroethylene

Trichtoroethylene

Trichlorofiuoromethane

Notes:

- = exceeded holding time

= exceeded groundwater protection or monitoring constituent standard (See Appendix A.) 
WELL FSB114C (cont.)

Radioactive Constituents

Constituents

Americium-241

Cesium-137

Cobalt-60

Curium-242

Curium-243/244

Curium-245/246

Gross atpha

lodine-129

Nonvolatile beta

Plutonium-238

Plutonium-239/240

Radium-226

Radium-228

Strontium-90

Technetium-99

Thorium-228

Thorium-230

Thorium-232

Sum of alphas

Sum of betas

Tritium

Uranium-233/234

Uranium-235

Uranium-238
3098

Mod

$1.2 \mathrm{E}+00 \quad \mathrm{~J} / \mathrm{C} /$

NDD

$<9.4 \mathrm{E}-01 \quad$ UIII

$<1.1700$

$1 \quad \mathrm{GP}<5.5 \mathrm{E}-02$

$\mathrm{U} / 1$

1 GP $<1,1 E+00$

$<5.7 \mathrm{E}-01$

$<1.2 \mathrm{E}+00$

1 GE $\mathrm{pCin}$

1 GE $\mathrm{pCi} / \mathrm{L}$

$3.3 E+00 \quad / /$

1 GP $3.3 E+00 \quad / 1$

1 GE pCi/mL

Notes:

= exceeded holding time

= exceeded groundwater protection or monitoring constituent standard (See Appendix A.) 
WELL FSB114D

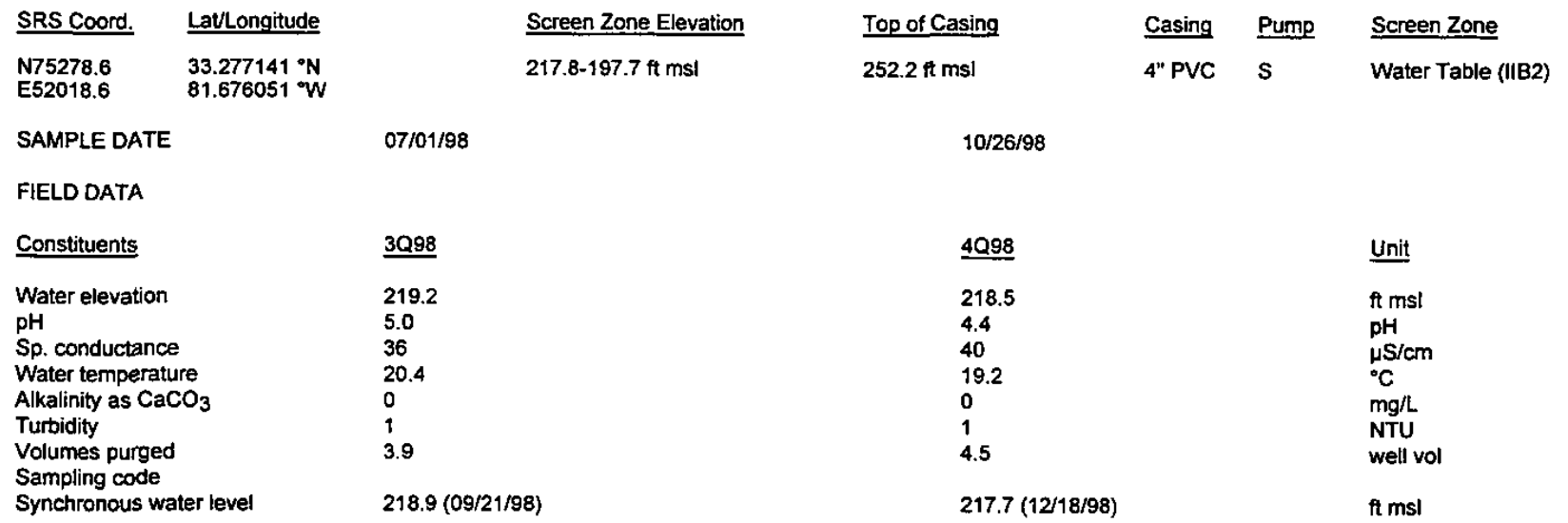

ANALYTICAL DATA

Inorganic Constituents

Constituents

Antimony, total recoverable

Arsenic, total recoverable

Barium, total recoverable

Cadmium, total recoverable

Chromium, total recoverable

Cobalt, total recoverable

Copper, total recoverable

Cyanide

Lead, total recoverable

Mercury, total recoverable

Nickel, total recoverable

Nitrate as nitrogen

Nitrate-nitrite as nitrogen

Selenium, total recoverable

Silver, total recoverable

Thallium, total recoverable

Vanadium, total recoverable

Zinc, total recoverable

Organic Constituents

Constituents

3Q9

$<1.0$

6.9

1,900
$\mathrm{U} / \prime$

II

1 GE 8.2

1 GE 2,000

II

Mod

Filt.

ST브

DF Lab 4 QQ98

Mod

Filt.

ST H $\underline{\text { DF Lab Unit }}$

Bis(2-ethylhexyl) phthalate

Dichloromethane

Phenols

Tetrachloroethylene

Trichloroethylene

Trichlorofluoromethane

Notes:

- exceeded holding tirne

- exceeded groundwater protection or monitoring constituent standard (See Appendix A.) 


\section{WELL FSB114D (cont.)}

\section{Radioactre Constituents}

Constituents

3098

Mod

Filt.

ST브 DF Lab $\underline{4 Q 98}$

Mod

Filt.

ST 브 DF Lab Unit

Americium-241

Cesium-137

Cobalt-60

Curium-242

Curium-243/244

Curium-245/246

Gross alpha

lodine-129

Nonvolatile beta

Plutonium-238

Plutonium-239/240

Radium-226

Radium-228

Strontium-90

Technetium-99

Thorium-228

Thorium-230

Thorium-232

Sum of alphas

Sum of betas

Tritium

Uranium-233/234

Uranium-235

Uranium-238

$3.0 \mathrm{E}+00 \quad \mathrm{~J} / \mathrm{C} / \quad$ NDD

$2.6 \mathrm{E}+00 \quad / /$

$5.8 E+00 \quad / /$
1 GP $<1.4 E+00 \quad U / \prime$

1 GP $<1.4 E+00 \quad \mathrm{U} / \prime$

1 GP $6.0 E+00 \quad / /$
1 GE pCill

1 GE PCill

1 GE $\mathrm{pCi} / \mathrm{mL}$

Notes: 
WELL FSB115C

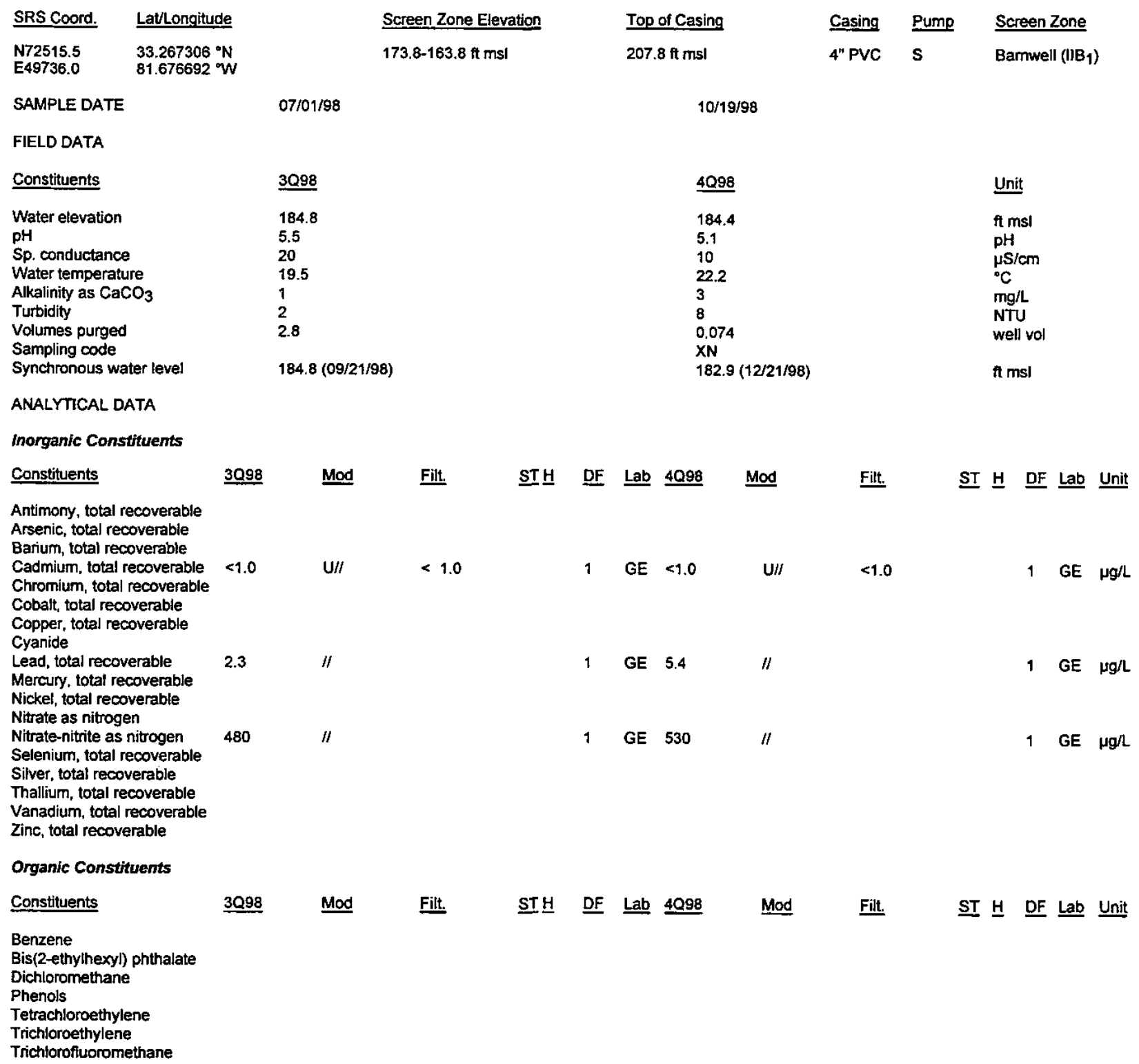

Notes:

- exceeded holding time

a = exceeded groundwater protection or monitoring constituent standard (See Appendix A.) 
WELL FSB115C (cont.)

Radfoactive Constituents

Constituents

3098

Mod

Filt.

ST

DF Lab 4098

Mod

Filt.

ST 브 DF Lab Unit

Americium-241

Cesium-137

Cobalt-60

Curium-242

Curium-243/244

Curium-245/246

Gross alpha

todine-129

Nonvolatile beta

Plutonium-238

Plutonium-239/240

Radium-226

Radium-228

Strontium-90

Technetium-99

Thorium-228

Thorium-230

Thorium-232

Sum of alphas

Sum of betas

Tritium

$<1.9 E-01 \quad \mathrm{UI} / / \quad<0.5680$

1 GP $<3.5 \mathrm{E}-01$

$\mathrm{U} / 1$

$1 \mathrm{GP}$

$<1.3 E+00$

U/I

$<4.5 E-01$

1 GE $\mathrm{pCil}$

$<7.0 \mathrm{E}-01 \quad \mathrm{UI} / !$

$<1.1600$

$<1.1 E+00$

1 GE pCill

Uranium-233/234

Uranium-235

Uranium-238

7.7E+00 $/ I$

1 GP $8.5 \mathrm{E}+00 \quad / /$

1 GE $\mathrm{pCi} / \mathrm{ml}$

Notes:

- = exceeded holding time

- = exceeded groundwater protection or monitoring constituent standard (See Appendix A.) 
WSRC-TR-99-00012

Unclassified

\section{WELL FSB115D}

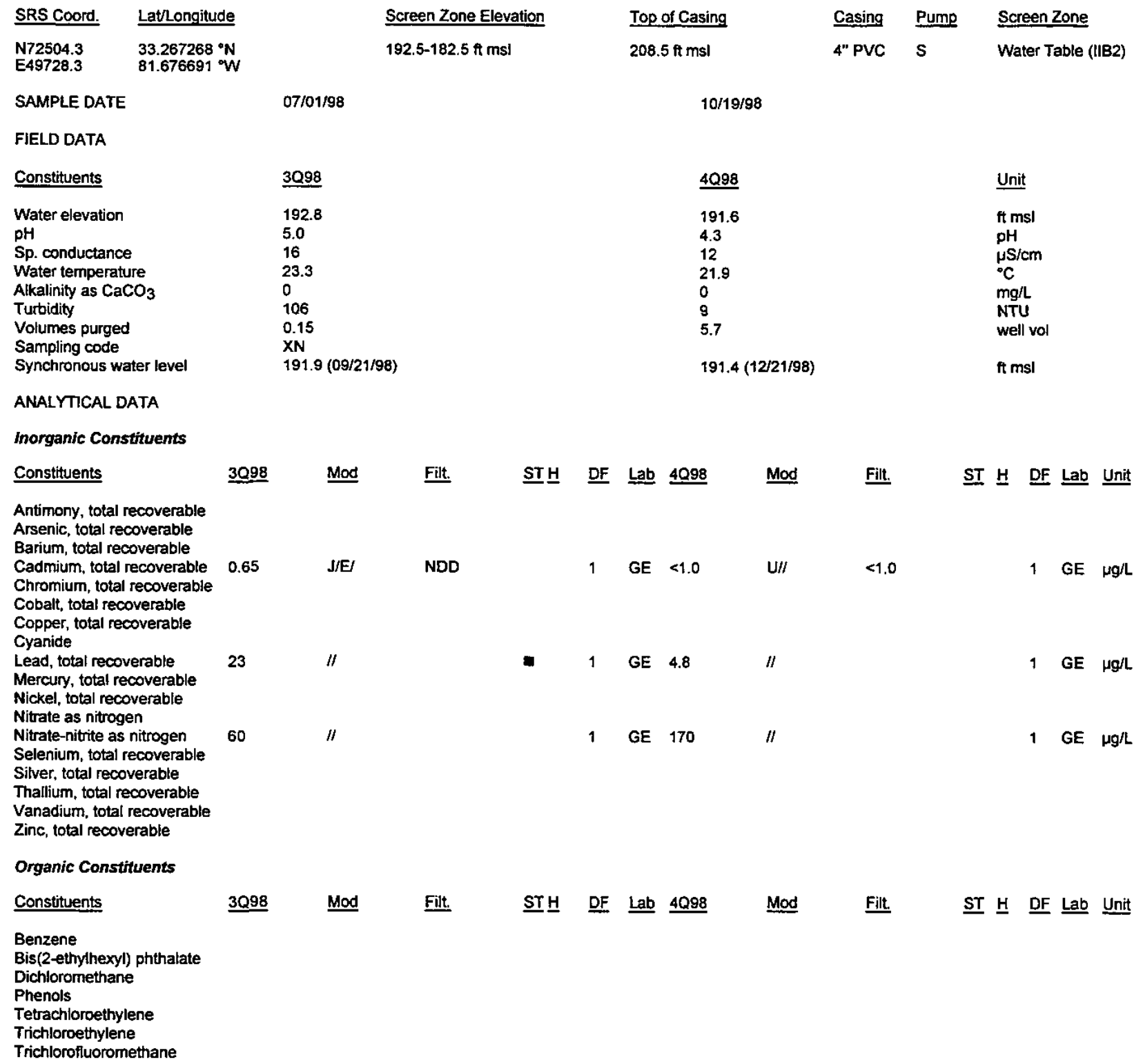

Notes:

- = exceeded holding time

- exceeded groundwater protection or monitoring constituent standard (See Appendix A.) 


\section{WELL FSB115D (cont.)}

Radioactive Constituents

Constituents

3098

Mod

Filt.

ST브

DF Lab $\underline{4 Q 98}$

Mod

Filt.

ST 브 DF Lab Unit

Americium-241

Cesium-137

Cobalt-60

Curium-242

Curium-243/244

Curium-245/246

Gross alpha

lodine-129

Nonvolatile beta

Plutonium-238

Plutonium-239/240

Radium-226

Radium-228

Strontium-90

Technetium-99

Thorium-228

Thorium-230

Thorium-232

Sum of alphas

Sum of betas

Tritium

Uranium-233/234

$7.2 E+00 \quad \mathrm{~J} / \mathrm{C} /$

NDD

$5.7 \mathrm{E}+00$

1 GP $<7.8 E-01 \quad U / I$

$<5.4 E-01$

1 GP $<1.3 E+00 \quad U / I$

$<1.2 E+00$

1 GE pCi/L

Uranium-235

$7.3 \mathrm{E}+00 \quad / 1$

1 GP $9.0 \mathrm{E}+00 \quad / /$

1 GE $\mathrm{pCV} / \mathrm{mL}$

Notes:

$\bullet=$ exceeded holding time

- exceeded groundwater protection or monitoring constituent standard (See Appendix A.)
$1 \mathrm{GE} \mathrm{PCV} / \mathrm{L}$ 


\section{WELL FSB116C}

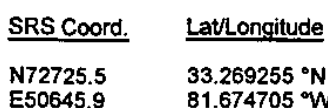

SAMPLE DATE

FIELD DATA

Constituents

Water elevation

pH

Sp. conductance

Water temperature

Alkalinity as $\mathrm{CaCO}_{3}$

Turbidity

Volumes purged

Sampling code

Synchronous water level

ANALYTICAL DATA

Inorganic Constituents

Constituents

Antimony, total recoverable

Arsenic, total recoverable

Barium, total recoverable

Cadmium, total recoverable

Chromium, total recoverable

3Q98

190.5

5.1

20

19.5

1

4.1

$177.3(09 / 21 / 98)$
Screen Zone Elevation

$170.5-160.5 \mathrm{ft} \mathrm{ms}$

Top of Casing

$202.5 \mathrm{ft} \mathrm{msl}$

$10 / 21 / 98$

4Q98

190.0

4.3

10

19.2

0

1
3.7

$\uparrow 89.8(12 / 21 / 98)$

\section{Casing Pump Screen Zone}

4" PVC S Barnwell (IIB

Cobalt, total recoverable

Copper, total recoverable

Cyanide

Lead, total recoverable

Mercury, total recoverable

Nickel, total recoverable

Nitrate as nitrogen

Nitrate-nitrite as nitrogen

Selenium, total recoverable

Silver, total recoverable

Thallium, total recoverable

Vanadium, total recoverable

Zinc, total recoverable

Organic Constituents

Constituents

$<1,0$

1 GE $<1.0$

$\mathrm{U} / 1$

$<1.0$

1 GE $\mu g / L$

Benzene

Bis(2-ethylhexyl) phthalate

Dichloromethane

Phenols

Tetrachloroethylene

Trichloroethylene

Trichlorofluoromethane

$J / E J$

NDD

$\mathrm{J} / \mathrm{I} /$

1 GE 850

/l

NDD

1 GE $\mu g / L$

$1 \mathrm{GE} \mu g / l$

Mo
Filt.

\section{브}

DF 느

4Q98

ST $\underline{H}$ DF Lab Unit

$\mathrm{pH}$

$\mu \mathrm{S} / \mathrm{cm}$

${ }^{\circ} \mathrm{C}$

NTU

well vol

ft $\mathrm{ms}$

Notes:

- exceeded holding time

- exceeded groundwater protection or monitoring constituent standard (See Appendix A.)

F-Area HWMF 


\section{WELL FSB116C (cont.)}

Radioactive Constituents

Constituents

Americium-241

Cesium-137

Cobalt-60

Curium-242

Curium-243/244

Curium-245/246

Gross alpha

lodine-129

Nonvolatile beta

Plutonium-238

Plutonium-239/240

Radium-226

Radium-228

Strontium-90

Technetium-99

Thorium-228

Thorium-230

Thorium-232

Sum of alphas

Sum of betas

Tritium

Uranium-233/234

Uranium-235

Uranium-238
$<-7.0 \mathrm{E}-02 \quad \mathrm{U} \mathrm{I} / \mathrm{I}$

$<0.6010$

$<1.9 \mathrm{E}-0$

UII

$<1.2600$

$1 \quad \mathrm{GP}<4.5 \mathrm{E}-01 \quad \mathrm{U} / 1$

1 GP $<2.5 E-01 \quad U I I$

1 GP 1.2E+01 //
$<6.7 E-01$

$<1.3 E+00$

Filt. $\quad \underline{\text { ST }} \underline{\mathrm{H}}$ DF Lab Unit

1 GE PCill

1 GE PCIL

1 GE $\mathrm{pCi} / \mathrm{mL}$

Notes:

= exceeded holding time

- = exceeded groundwater protection or monitoring constituent standard (See Appendix A.) 
WELL FSB116D

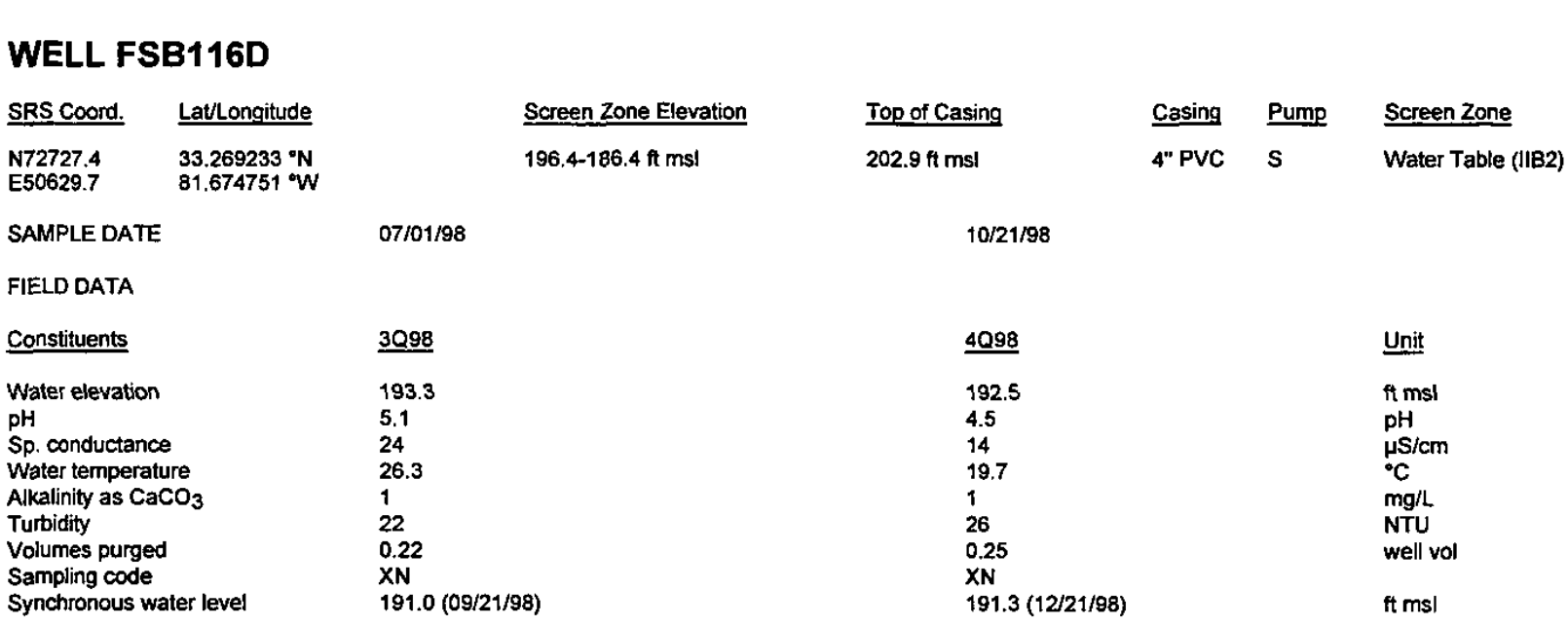

ANALYICAL DATA

Inonganic Constituents

Constituents

Antimony, total recoverable

Arsenic, total recoverable Barium, total recoverable

Cadmium, total recoverable

Chromium, total recoverable

Cobalt, total recoverable

Copper, total recoverable

Cyanide

Lead, total recoverable

Mercury, total recoverable

Nickel, total recoverable

Nitrate as nitrogen

Nitrate-nitrite as nitrogen

Selenium, total recoverable

Silver, total recoverable

Thallium, total recoverable

Vanadium, total recoverable

Zinc, total recoverable

Organic Constrituents

Constituents

Benzene

Bis(2-ethylhexyl) phthalate

Dichloromethane

Phenols

Tetrachloroethylene

Trichloroethylene

Trichlorofluoromethane

$\underline{3098}$

38
Mod

Filft

$J / E J$

NDD

$\mathrm{J} / \mathrm{l} /$

/I

1 GE 29

1 GE $170 \quad$ /l

$\underline{\text { Mod }}$

Filt.

șr브

(n)

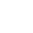 \\ Unclassified}




\section{WELL FSB116D (cont.)}

Radioactive Consthtuents

\section{Constituents}

Americium-241

Cesium-137

Cobalt-60

Curium-242

Curium-243/244

Curium-245/246

Gross alpha

lodine-129

Nonvolatile beta

Plutonium-238

Plutonium-239/240

Radium-226

Radium-228

Strontium-90

Technetium-99

Thorium-228

Thorium-230

Thorium-232

Sum of alphas

Sum of betas

Tritium

Uranium-233/234

Uranium-235

Uranium-238
3Q98

Mod

Filt.

ST보

DF Lab $4 Q 98$

Mod

Filt.

ST $\underline{\text { H }}$ DF Lab Unit
$<1.3 E+00 \quad$ UIII $<1.3900$

$3.6 \mathrm{E}+00 \quad / /$

$8.7 E+00 \quad / /$
1 GP $3.4 E+00$

1 GP 5.1E+01 /

1 GP 1.1E+01 /l
1 GE PCill

- 1 GE pCill
1 GE $\mathrm{pCi} / \mathrm{mL}$

Notes:

- = exceeded holding time

- = exceeded groundwater protection or monitoring constituent standard (See Appendix A.) 
WELL FSB117D

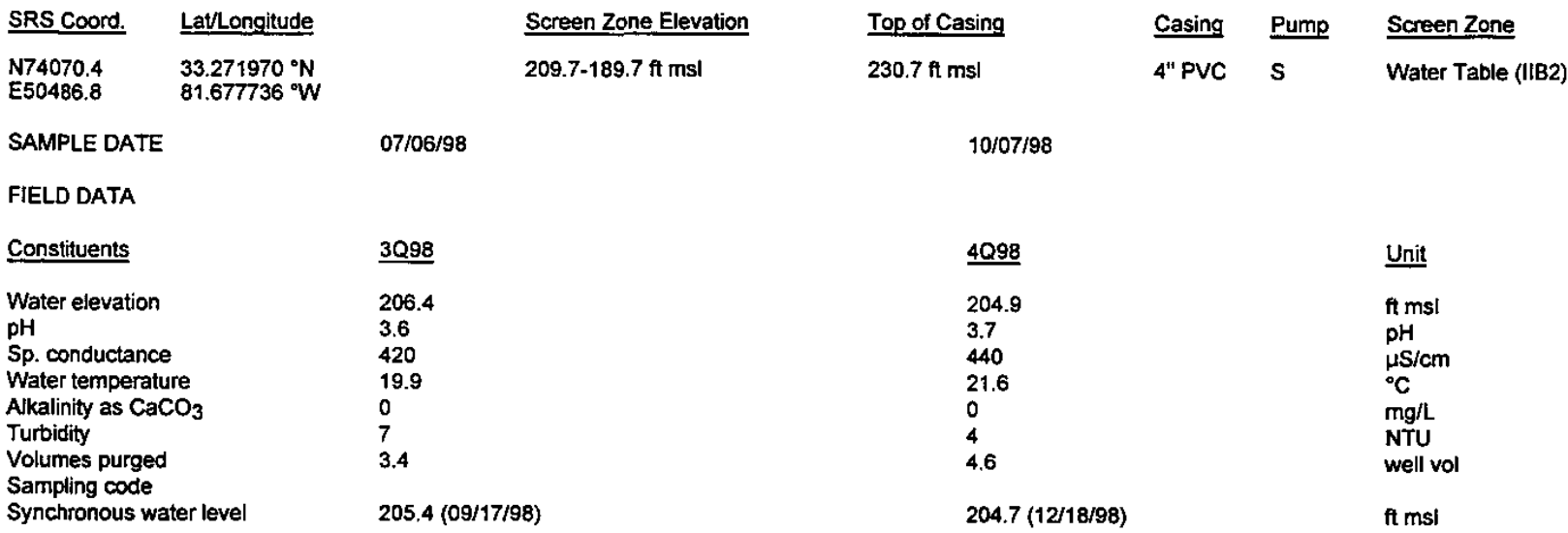

ANALYTICAL DATA

Inorganic Constituents

Constituents$$
3
$$

Antimony, total recoverable

Arsenic, total recoverable

Barium, total recoverable

Cadmium, total recoverable

Chromium, total recoverable

Cobalt, total recoverable

Copper, total recoverable

Cyanide

Lead, total recoverable

Mercury, total recoverable

Nickel, total recoverable

Nitrate as nitrogen

Nitrate-nitrite as nitrogen

Selenium, total recoverable

Silver, total recoverable

Thallium, total recoverable

Vanadium, total recoverable

Zinc, total recoverable

Organic Constituents

Constituents

$3 Q 98$

$3.7 \quad 1$

Mod Filt.

ST브 DF Lab

Mod

Filt.

ST 브 DF Lab Unit

3.8

II

$1 \quad$ GE $\quad 4.4$

II

1 GE $\mu g /$.

$42,000 \quad N /$

1 GE 1.3

$\mathrm{J} / \mathrm{l}$

NDD

1 GE $\mu g / L$

Benzene

Bis(2-ethylhexyl) phthalate

Dichloromethane

Phenols

Tetrachloroethylene

Trichloroethyiene

Trichlorofluoromethane

Notes:

- = exceeded holding time

= exceeded groundwater protection or monitoring constituent standard (See Appendix A.) 


\section{WELL FSB117D (cont.)}

Radioactive Constituents

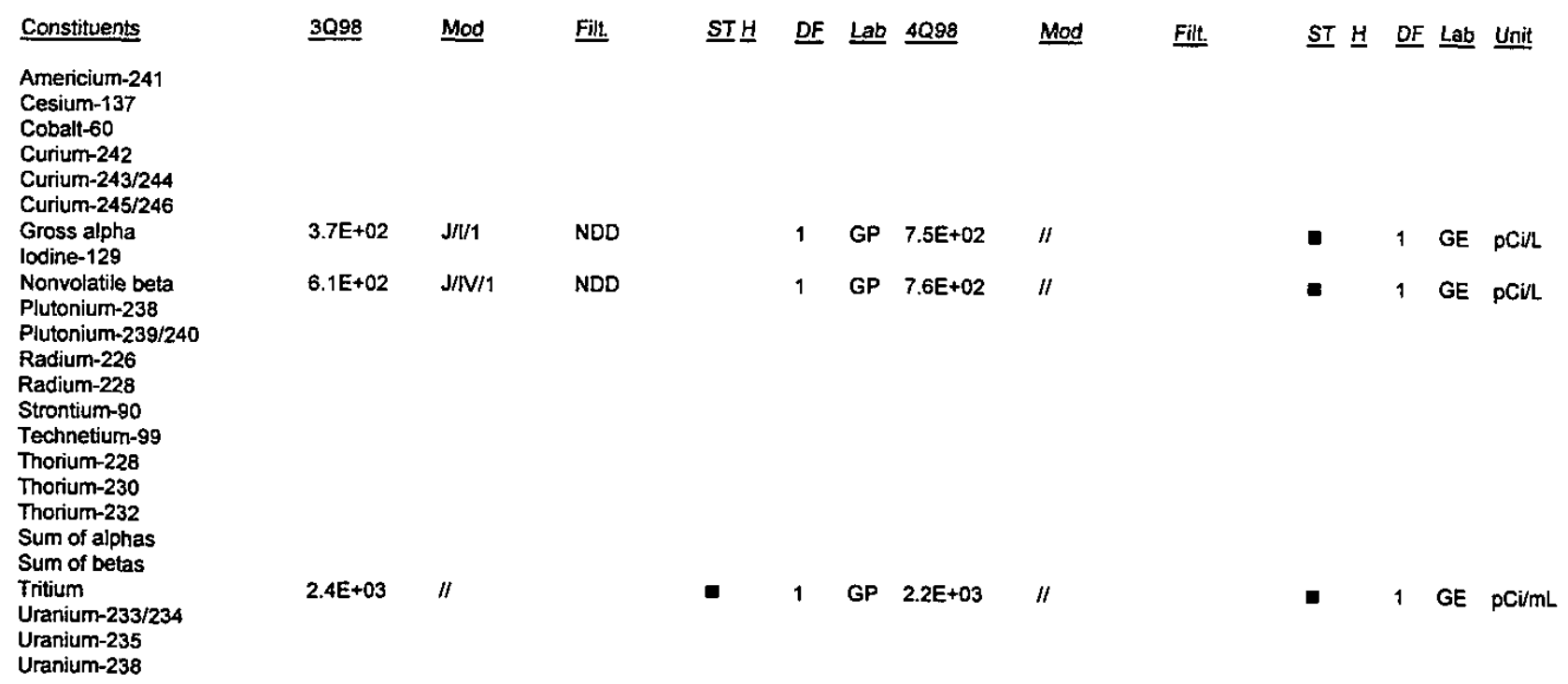

Notes:

- = exceeded holding time

- = exceeded groundwater protection or monitoring constituent standard (See Appendix A.) 
WELL FSB118D

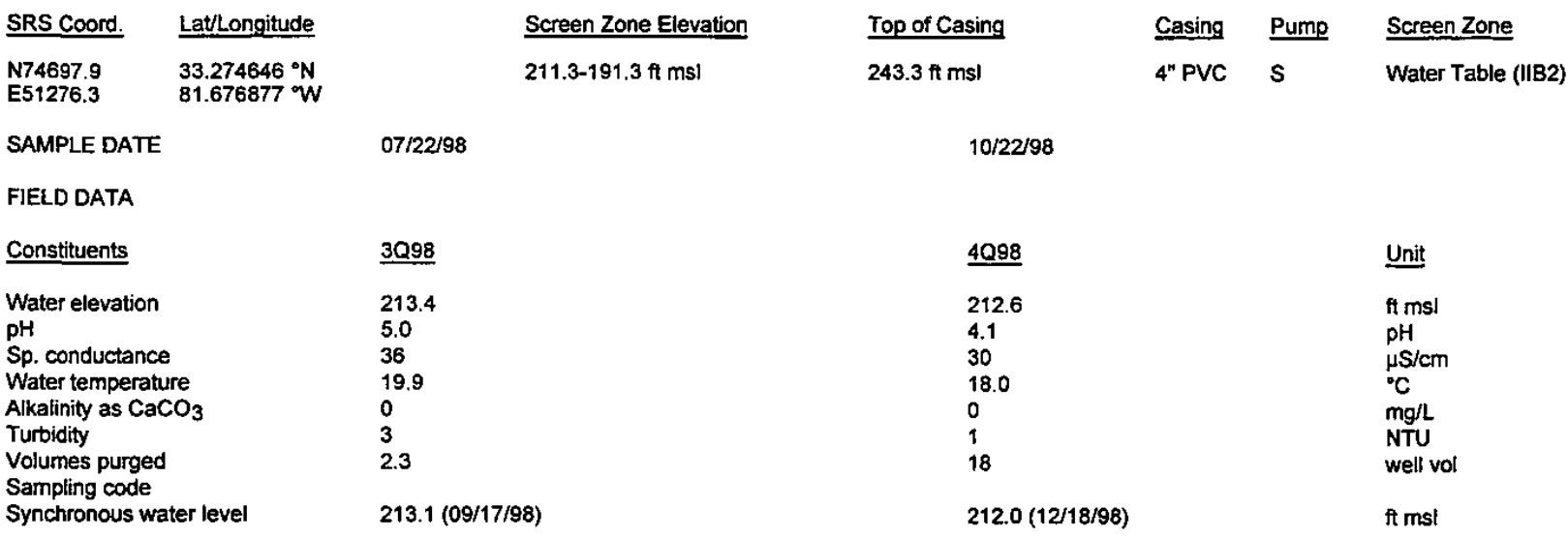

ANALYTICAL DATA

Inorganic Constituents

\begin{tabular}{|c|c|c|c|c|c|c|c|c|c|c|c|c|c|}
\hline Constituents & 3098 & Mod & Filt. & ST브 & $\underline{\text { DF }}$ & Lab & 4Q98 & Mod & Filt. & $\underline{\mathrm{ST}} \underline{\mathrm{H}}$ & $\underline{D F}$ & $\underline{\mathrm{Lab}}$ & Unit \\
\hline Antimony, total recoverable & $<0.21$ & $\mathrm{UN} /$ & $<0.20$ & & 1 & GE & & & & & & & $\mu g /$ \\
\hline Arsenic, total recoverable & $<3.0$ & $\mathrm{U} / /$ & $<3.0$ & & 1 & GE & & & & & & & $\mu g /$ \\
\hline Barium, total recoverable & 9.3 & $1 /$ & & & 1 & GE & & & & & & & $\mu g /$ \\
\hline Cadmium, total recoverable & $<1.0$ & $\mathrm{U} / /$ & $<1.0$ & & 1 & GE & $<1.0$ & $\mathrm{U} / /$ & $<1.0$ & & 1 & GE & $\mu g / L$ \\
\hline Chromium, total recoverable & 8.6 & $/ /$ & & & 1 & GE & & & & & & & $\mu g /$ \\
\hline Cobalt, total recoverable & 0.74 & $/ /$ & & & 1 & GE & & & & & & & $\mu g / L$ \\
\hline Copper, total recoverable & 50 & $/ /$ & & & 1 & GE & & & & & & & $\mu g / L$ \\
\hline Cyanide & $<10$ & $\mathrm{U} / /$ & $<10$ & & 1 & GE & & & & & & & $\mu g / L$ \\
\hline Lead, total recoverable & 5.3 & $/ /$ & & & 1 & GE & 2.3 & /I & & & 1 & GE & $\mu g / L$ \\
\hline Mercury, total recoverable & $<0.20$ & $\mathrm{U} / /$ & $<0.20$ & & 1 & GE & & & & & & & $\mu g / L$ \\
\hline $\begin{array}{l}\text { Nickel, total recoverable } \\
\text { Nitrate as nitrogen }\end{array}$ & 9.6 & $\|$ & & & 1 & GE & & & & & & & $\mu g / L$ \\
\hline Nitrate-nitrite as nitrogen & 900 & $N \prime$ & & & 1 & GE & 980 & $\|$ & & & 1 & GE & $\mu g / L$ \\
\hline Selenium, total recoverable & $<5.0$ & $\mathrm{U} / \mathrm{I}$ & $<5.0$ & & 1 & GE & & & & & & & $\mu g / L$ \\
\hline Silver, total recoverable & $<1.0$ & $\mathrm{U} / l$ & $<1.0$ & & 1 & GE & & & & & & & $\mu$ gl. \\
\hline Thallium, total recoverable & $<0.048$ & $\mathrm{UNI}$ & $<2.5$ & & 1 & GE & & & & & & & jgl \\
\hline Vanadium, total recoverable & $<2.0$ & $\mathrm{U} / /$ & $<2.0$ & & 1 & GE & & & & & & & $\mu g / L$ \\
\hline Zinc, total recoverable & 31 & $\|$ & & & 1 & GE & & & & & & & $\mu g / L$ \\
\hline \multicolumn{14}{|l|}{ Organic Constituents } \\
\hline Constituents & $\underline{3 Q 98}$ & Mod & Filt. & $\underline{\text { ST }} \underline{\underline{H}}$ & $\underline{\text { DF }}$ & $\underline{\text { Lab }}$ & 4Q98 & Mod & Filt. & $\underline{\text { ST }} \underline{H}$ & DF & Lab & $\underline{\text { Unit }}$ \\
\hline Benzene & $<1.0$ & $\mathrm{UJ} / \mathrm{O} / 1$ & $<1.0$ & & 1 & GE & & & & & & & $\mu g / L$ \\
\hline Bis(2-ethylhexyl) phthalate & $<9.9$ & $\mathrm{U} / /$ & $<9.9$ & & 1 & GE & & & & & & & $\mu g / L$ \\
\hline Dichloromethane & $<1.0$ & $\mathrm{U} J / O / 1$ & $<1.0$ & & 1 & GE & & & & & & & $\mu g /$ \\
\hline Phenols & $<5.0$ & $\mathrm{U} / /$ & $<5.0$ & & 1 & GE & & & & & & & $\mu g / L$ \\
\hline Tetrachloroethylene & $<1.0$ & $\mathrm{UJ} / \mathrm{O} / 1$ & $<1.0$ & & 1 & GE & & & & & & & $\mu g / 1$ \\
\hline Trichloroethylene & $<1.0$ & $\mathrm{UJ} / \mathrm{O} / 1$ & $<1.0$ & & 1 & GE & & & & & & & $\mu g /$ \\
\hline Trichlorofluoromethane & $<1.0$ & UJ/O/1 & $<1.0$ & & 1 & GE & & & & & & & \\
\hline
\end{tabular}

Notes:

- = exceeded holding time

- exceeded groundwater protection or monitoring constituent standard (See Appendix A.) 
WELL FSB118D (cont.)

Radloactive Constituents

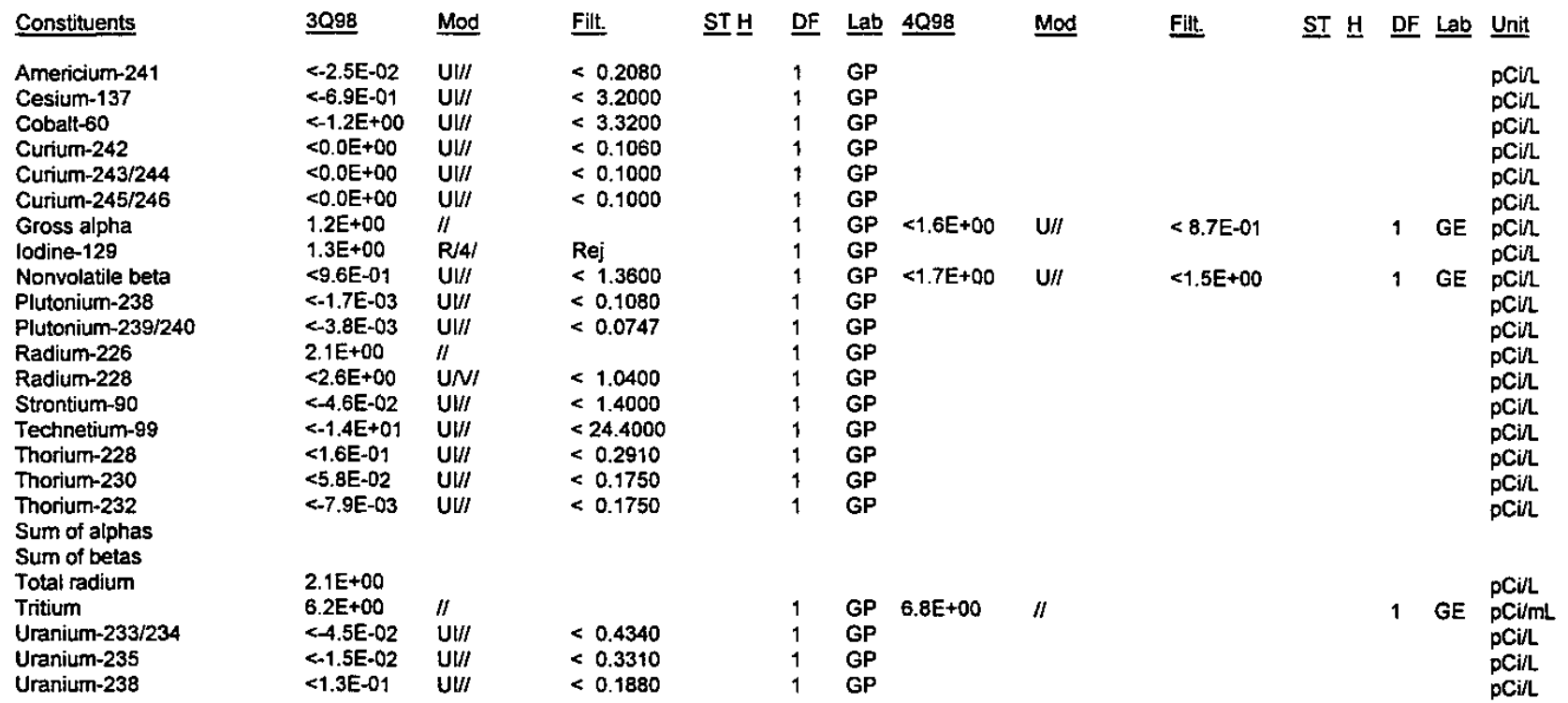

Notes:

- exceeded holding time

- = exceeded groundwater protection or monitoring constituent standard (See Appendix A.) 
WELL FSB119D

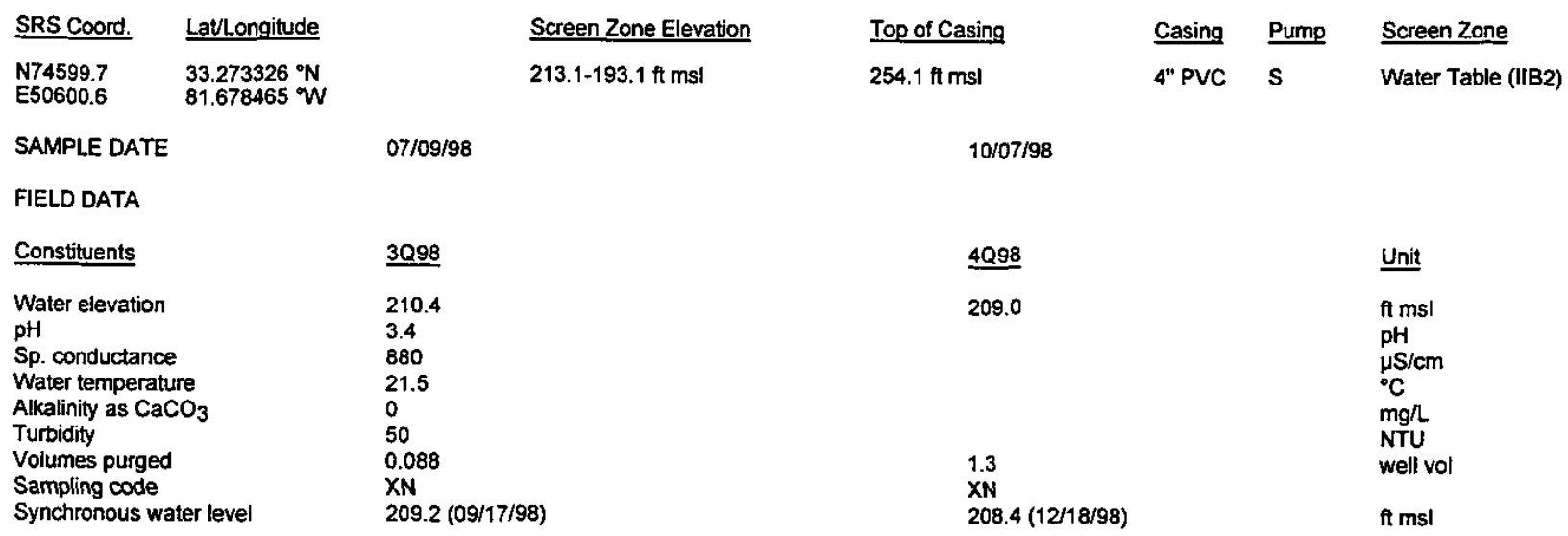

ANALYTICAL DATA

Inorganic Constituents

Constituents

Arsenic, total recoverabie

Barium, total recoverable

Cadmium, total recoverable

Chromium, total recoverable

6.8

Cobalt, total recoverable

Copper, total recoverable

Cyanide

Lead, total recoverable

4.4

1 GE 5.1

Mercury, total recoverabise

Nickel, total recoverable

Nitrate as nitrogen

Nitrate-nitrite as nitrogen

Selenium, total recoverable

Silver, total recoverable

Thallium, total recoverable

Vanadium, total recoverable

Zinc, total recoverable

Organic Constituents

Constituents

Benzene

Bis(2-ethylhexyl) phthalate

Dichloromethane

Phenols

Tetrachloroethylene

Trichloroethylene

Trichlorofluroromethane

Notes:

- = exceeded holding time

- = exceeded groundwater protection or monitoring constituent standard (See Appendix A.)

F-Area HWMF 


\section{WELL FSB119D (cont.)}

Radioactive Constituents

Constituents

$\underline{3 Q 98}$

\section{Mod}

Filt.

ST브 DF Lab $\underline{4 Q 98}$

Mod

Filt.

ST $\underline{H}$ DF Lab Unit

Americium-241

Cesium-137

Cobalt-60

Curium-242

Curium-243/244

Curium-245/246

Gross alpha

lodine-129

Nonvolatile beta

Plutonium-238

Plutonium-239/240

Radium-226

Radium-228

Strontium-90

Technetium-99

Thorium-228

Thorium-230

Thorium-232

Sum of alphas

Sum of betas

Tritium

Uranium-233/234

$1.2 \mathrm{E}+03 \quad / 1$

$1.1 \mathrm{E}+03 \quad / /$

- 1 GP $1.1 \mathrm{E}+03 \quad / 1$

- 1 GP $1.2 \mathrm{E}+03 \quad / /$

- 1 GE pCir

Uranium-235

Uranium-238

Notes:

- = exceeded holding time

= exceeded groundwater protection or monitoring constituent standard (See Appendix A.) 


\section{WELL FSB120A}

\begin{tabular}{|c|c|c|c|}
\hline SRS Coord. & Lat/Longitude & & Screen Zone Elevation \\
\hline $\begin{array}{l}N 75538.9 \\
E 49175.7\end{array}$ & $\begin{array}{l}33.273077^{\circ} \mathrm{N} \\
81.684041^{\circ} \mathrm{W}\end{array}$ & & $109.0-99.0 \mathrm{ft} \mathrm{msl}$ \\
\hline \multicolumn{2}{|c|}{ SAMPLE DATE } & $07 / 09 / 98$ & \\
\hline \multicolumn{4}{|l|}{ FIELD DATA } \\
\hline \multicolumn{2}{|l|}{ Constituents } & 3098 & \\
\hline \multicolumn{2}{|c|}{ Water elevation } & 175.8 & \\
\hline \multicolumn{2}{|c|}{$\begin{array}{l}\text { Water elevation } \\
\text { pH }\end{array}$} & 11.8 & \\
\hline \multirow{2}{*}{\multicolumn{2}{|c|}{$\begin{array}{l}\text { Sp. conductance } \\
\text { Water temperature }\end{array}$}} & 2000 & \\
\hline & & 25.8 & \\
\hline \multicolumn{2}{|c|}{ Alkalinity as $\mathrm{CaCO}_{3}$} & 524 & \\
\hline \multicolumn{2}{|c|}{ Turbidity } & 2 & \\
\hline \multicolumn{2}{|c|}{ Volumes purged } & 0.020 & \\
\hline \multicolumn{2}{|c|}{ Sampling code } & $\mathrm{XN}$ & \\
\hline \multicolumn{2}{|c|}{ Synchronous water level } & $177.5(0$ & 98) \\
\hline
\end{tabular}

ANALYTICAL DATA

inorganic Constituents

\begin{tabular}{|c|c|c|c|c|c|c|c|c|c|c|c|c|c|c|}
\hline Constituents & $\underline{3098}$ & Mod & Filt. & $\underline{\text { ST }} \underline{H}$ & $\underline{\text { DF }}$ & $\underline{\text { Lab }}$ & 4Q98 & Mod & Filt. & & $\underline{\text { ST }} \underline{H}$ & $\underline{\mathrm{DF}}$ & $\underline{\text { Lab }}$ & $\underline{\text { Unit }}$ \\
\hline Antimony, total recoverable & $<0.20$ & $\mathrm{U} / /$ & $<0.20$ & & 1 & GE & & & & & & & & $\mu g / L$ \\
\hline Arsenic, total recoverable & $<3.0$ & $\mathrm{U} / I$ & $<3.0$ & & 1 & GE & & & & & & & & $\mu g / L$ \\
\hline Barium, total recoverable & 130 & $/ /$ & & & 1 & $\overline{\mathrm{GE}}$ & & & & & & & & $\mathrm{\mu g} / \mathrm{L}$ \\
\hline Cadmium, total recoverable & $<1.0$ & $\mathrm{u} / /$ & $<1.0$ & & 1 & GE & 1.4 & $/ /$ & & & & 1 & GE & $\mu g / L$ \\
\hline Chromium, total recoverable & $<3.7$ & UNI & $<3.0$ & & 1 & GE & & & & & & & & $\mu g / L$ \\
\hline Cobalt, total recoverable & 0.63 & $\|$ & & & 1 & GE & & & & & & & & $\mu g / L$ \\
\hline Copper, total recoverable & 1.2 & $\|$ & & & 1 & GE & & & & & & & & $\mu g / L$ \\
\hline Cyanide & $<10$ & $\mathrm{U} / /$ & $<10$ & & 1 & GE & & & & & & & & $\mu g / L$ \\
\hline Lead, total recoverable & 4.6 & $/ /$ & & & 1 & $\mathrm{GE}$ & 2.2 & $\|$ & & & & 1 & GE & $\mu g / L$ \\
\hline Mercury, total recoverable & $<0.20$ & $\mathrm{U} / /$ & $<0.20$ & & 1 & GE & & & & & & & & $\mu g / L$ \\
\hline $\begin{array}{l}\text { Nickel, total recoverable } \\
\text { Nitrate as nitrogen }\end{array}$ & 3.7 & $N \prime$ & & & 1 & GE & & & & & & & & $\mu g / L$ \\
\hline Nitrate-nitrite as nitrogen & 18,000 & $N I$ & & - & 10 & GE & 20,000 & $\|$ & & & $\mathbf{a}$ & 25 & GE & $\mu g / 1$ \\
\hline Selenium, total recoverable & 1.5 & $\mathrm{~J} / \mathrm{E} /$ & NDD & & 1 & $\overline{G E}$ & & & & & & & & $\mu g / h$ \\
\hline Silver, total recoverable & $<0.18$ & UNI & $<1.0$ & & 1 & $\overline{G E}$ & & & & & & & & $\mu g / L$ \\
\hline Thallium, total recoverable & $<2.5$ & $\mathrm{U} / /$ & $<2.5$ & & 1 & GE & & & & & & & & $\mu g / L$ \\
\hline Vanadium, total recoverable & $<2.0$ & $\mathrm{U} / /$ & $<2.0$ & & 1 & GE & & & & & & & & $\mu g / L$ \\
\hline Zinc, total recoverable & 7.4 & $\|$ & & & 1 & GE & & & & & & & & $\mu g /$ \\
\hline \multicolumn{15}{|l|}{ Organic Constituents } \\
\hline Constituents & 3098 & Mod & Filt. & ST브 & DF & $\underline{\mathrm{Lab}}$ & 4Q98 & Mod & Filt. & • & $\underline{\text { ST }} \underline{H}$ & DF & Lab & Unit \\
\hline Benzene & $<1.0$ & $\mathrm{u} / /$ & $<1.0$ & & 1 & GE & & & & & & & & $\mu g / L$ \\
\hline Bis(2-ethylhexyl) phthalate & $<10$ & $\mathrm{U} / /$ & $<10$ & & 1 & GE & & & & & & & & $\mu g / 2$ \\
\hline Dichloromethane & $<1.0$ & $\mathrm{U} / /$ & $<1.0$ & & 1 & GE & & & & & & & & $\mu g / 2$ \\
\hline Phenols & $<5.0$ & $\mathrm{U} / \mathrm{I}$ & $<5.0$ & & 1 & GE & & & & & & & & $\mu g / L$ \\
\hline Tetrachloroethylene & $<1.0$ & $\mathrm{U} / /$ & $<1.0$ & & 1 & GE & & & & & & & & $\mu g / L$ \\
\hline Trichloroethylene & 1.7 & $/ /$ & & & 1 & $\overline{G E}$ & & & & & & & & $\mu g / 2$ \\
\hline Trichlorofluoromethane & $<1.0$ & $\mathrm{U} / /$ & $<1.0$ & & 1 & $\overline{G E}$ & & & & & & & & \\
\hline
\end{tabular}

Notes:

= exceeded holding time

E = exceeded groundwater protection or monitoring constituent standard (See Appendix A.)

$\begin{array}{llll}\text { Top of Casing } & \text { Casing } & \text { Pump } & \text { Screen Zone } \\ 280.1 \mathrm{ft} \mathrm{msl} & \text { 4" PVC } & \text { S } & \text { U. Congaree (IIA) }\end{array}$

$10 / 26 / 98$

4098
176.4
11.3
1400
21.9
253
3
0.020
$X N$
$173.3(12 / 21 / 98)$

$\underline{\text { Unit }}$

ft msl

$\mathrm{pH}$

$\mu \mathrm{S} / \mathrm{cm}$

$\mathrm{mglt}$

NTU

well vol

ft msl $\mu g / L$

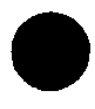


WELL FSB120A (cont.)

Radioactive Constituents

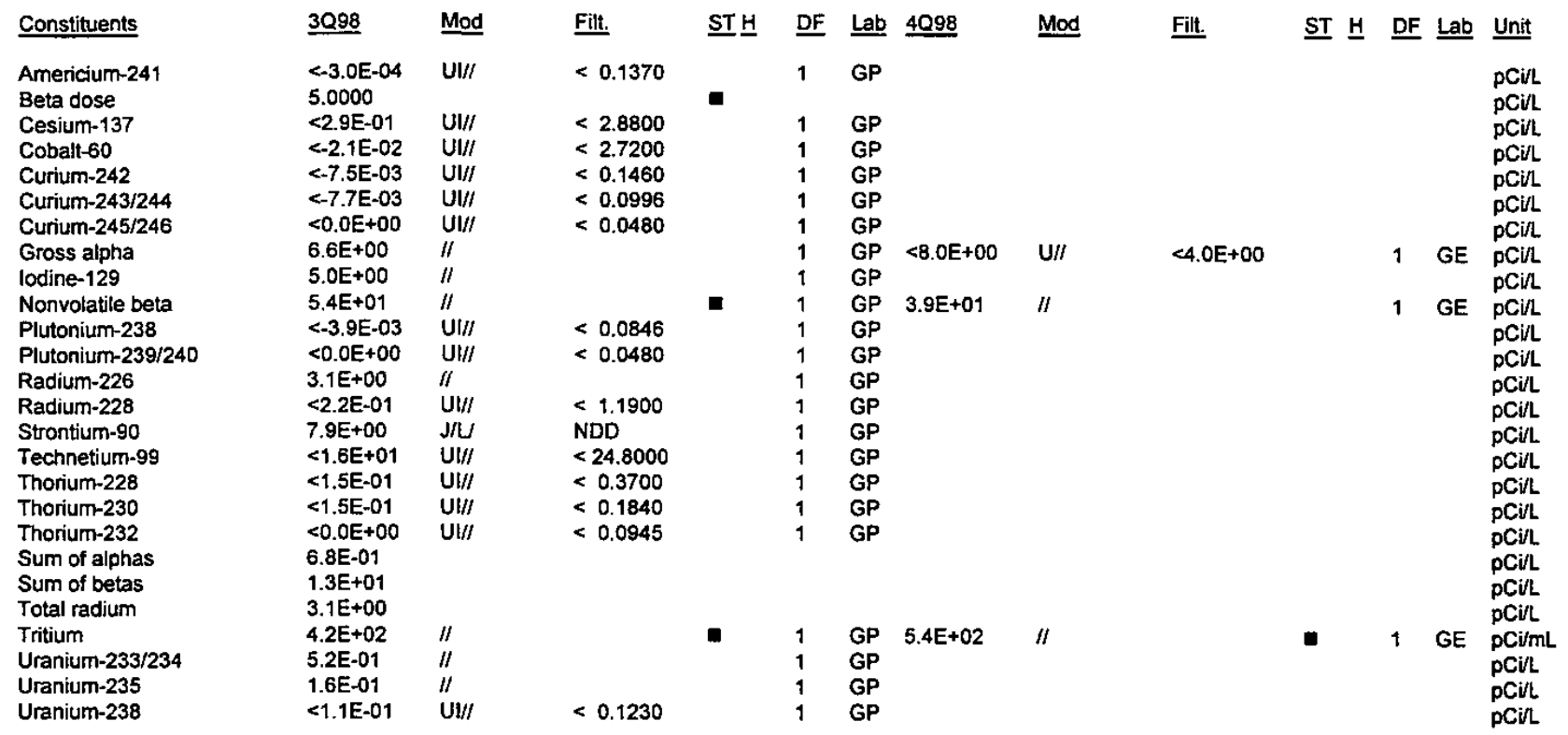

Notes: 


\section{WELL FSB120C}

\begin{tabular}{|c|c|}
\hline SRS Coord. & Lat/Longitude \\
\hline $\begin{array}{l}N 75549.8 \\
\text { E49171.1 }\end{array}$ & 81.684074 \\
\hline
\end{tabular}

SAMPLE DATE

FIELD DATA

Constituents

Water elevation

$\mathrm{pH}$

Sp. conductance

Water temperature

Alkalinity as $\mathrm{CaCO}_{3}$

Turbidity

Volumes purged

Sampling code

Synchronous water level

ANALYTICAL DATA

\section{Screen Zone Elevation}

160.7-150.7 ft ms

$07 / 21 / 98$

3Q98

208.5

6.3

260

21.7

1
7.3

$207.5(09 / 21 / 98)$
Top of Casing

$279.7 \mathrm{ft} \mathrm{msl}$

$10 / 26 / 98$

4Q98

207.

6.1

250

3.5

$206.5(12 / 21 / 98)$

Casing Pump Screen Zone

4" PVC S
Bamwell (IIB 1 )

Inorganic Constituents

Constituents

Antimony, total recoverable $<0.20$

Arsenic, total recoverable $\quad<3.0$

Barium, total recoverable 81

Cadmium, total recoverable 0.45

Chromium, total recoverable 0.99

Cobalt, total recoverable

Copper, total recoverable

Cyanide

Lead, total recoverable

Mercury, total recoverable $\quad<0.20$

Nickel, total recoverable

Nitrate as nitrogen

Nitrate-nitrite as nitrogen $\quad 30,000$

Selenium, total recoverable $<5.0$

Silver, total recoverable $\quad<1.0$

Thallium, total recoverable $\quad 0.053$

Vanadium, total recoverable $<20$

Zinc, total recoverable

$<2.0$
18

Organic Constituemts

\begin{tabular}{ll} 
Constituents & 3098 \\
\hline & $<1.0$ \\
Benzene & $<10$ \\
Bis(2-ethylhexyl) phthalate & $<2.1$ \\
Dichloromethane & $<5.0$ \\
Phenols & $<1.0$ \\
Tetrachloroethylene & $<1.4$ \\
Trichloroethylene & $<1.0$ \\
Trichlorofluoromethane &
\end{tabular}

\begin{tabular}{|c|c|}
\hline Mod & Filt. \\
\hline $\mathrm{u} / /$ & $<0.2$ \\
\hline $\mathrm{U} / /$ & $<3.0$ \\
\hline lI & \\
\hline $\mathrm{J} / \mathrm{E}$ & NDD \\
\hline $\mathrm{J} / \mathrm{E} /$ & NDD \\
\hline$\|$ & \\
\hline 11 & \\
\hline $\mathrm{J} / \mathrm{E} /$ & NDD \\
\hline $\mathrm{U} / /$ & $<2.0$ \\
\hline$U / I$ & $<0.2$ \\
\hline$/ 1$ & \\
\hline$N I$ & \\
\hline $\mathrm{U} / /$ & $<5.0$ \\
\hline $\mathrm{U} / l$ & $<1.0$ \\
\hline J/E & NDD \\
\hline $\mathrm{U} / /$ & $<2.0$ \\
\hline
\end{tabular}

$\begin{array}{ll}\text { Mod } & \text { Filt. } \\ \text { UJ/O/1 } & <1.0 \\ U / / & <10 \\ U J / N O 8 / 1 & <1.0 \\ U / I & <5.0 \\ U J / O / 1 & <1.0 \\ U J / O / 1 & <1.0 \\ U J / O / 1 & <1.0\end{array}$

ST브

-

$\begin{array}{ll}1 & G E \\ 1 & G E \\ 1 & G E \\ 1 & G E \\ 1 & G E \\ 1 & G E \\ 1 & G E \\ 1 & G E \\ 1 & G E \\ 1 & G E \\ 1 & G E \\ & \\ 25 & G E \\ 1 & G E \\ 1 & G E \\ 1 & G E \\ 1 & G E \\ 1 & G E\end{array}$

ST브
0.39

$<2.0$

28,000

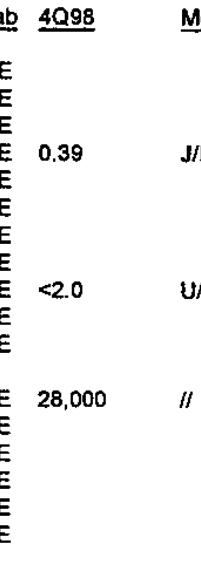

Lab
GE
GE
GE
GE
GE
GE
GE
Mod Filt.

J/N

U/I

//

$\operatorname{Mod}$

Filt.

$$
\begin{aligned}
& \text { Unit } \\
& \text { ft msl } \\
& \text { pH } \\
& \mu \mathrm{s} / \mathrm{cm} \\
& { }^{\circ} \mathrm{C} \\
& \mathrm{mg} / \mathrm{L} \\
& \mathrm{NTU} \\
& \text { well vol } \\
& \mathrm{ft} \mathrm{ms}
\end{aligned}
$$


WELL FSB120C (cont.)

Radioactive Constituents

\begin{tabular}{|c|c|c|c|c|c|c|c|c|c|c|c|c|c|}
\hline Constituents & 3098 & Mod & Filt. & $\underline{\text { ST }} \underline{\underline{H}}$ & DF & $\underline{L a b}$ & $4 Q 98$ & Mod & Filt. & $\underline{\mathrm{ST}} \underline{\mathrm{H}}$ & DF & $\underline{L a b}$ & Unit \\
\hline $\begin{array}{l}\text { Americium-241 } \\
\text { Beta dose }\end{array}$ & $\begin{array}{l}1.4 \mathrm{E}-01 \\
0.9392\end{array}$ & $\|$ & & & 1 & $G P$ & & & & & & & pCill \\
\hline $\begin{array}{l}\text { Beta cose } \\
\text { Cesium-137 }\end{array}$ & $<1.7 E+00$ & $\mathrm{UI} / /$ & $<2.9300$ & & 1 & GP & & & & & & & $\mathrm{OCi}$ \\
\hline Cutium-242 & $<0.0 E+00$ & UVI & $<0.0849$ & & 1 & GP & & & & & & & $\mathrm{pCi} / \mathrm{L}$ \\
\hline Curiurn-243/244 & $2.9 \mathrm{E}-01$ & $\|$ & & & 1 & GP & & & & & & & $\mathrm{pCi} / \mathrm{L}$ \\
\hline Curium-245/246 & $<0.0 \mathrm{E}+00$ & $\mathrm{UI} / \mathrm{I}$ & $<0.0745$ & & 1 & GP & & & & & & & pCirt \\
\hline Gross alpha & $1.0 \mathrm{E}+01$ & $\| l$ & & & 1 & GP & $5.3 E+00$ & $\|$ & & & 1 & GE & $\mathrm{pCil}$ \\
\hline Nonvolatile beta & $2.7 E+01$ & $/ /$ & & & 1 & GP & $3.3 E+01$ & $/ 1$ & & & 1 & GE & $\mathrm{pCin}$ \\
\hline Plutonium-238 & $<-2,1 E-02$ & $\mathrm{UU} / /$ & $<0.1270$ & & 1 & GP & & & & & & & $\mathrm{pCil}$ \\
\hline Plutonium-239/240 & $<-7.1 \mathrm{E}-03$ & $\mathrm{UI} / I$ & $<0.0917$ & & 1 & GP & & & & & & & $\mathrm{pCil}$ \\
\hline Radium-226 & $5.0 \mathrm{E}+00$ & II & & $\mathbf{0}$ & 1 & GP & & & & & & & pCill \\
\hline Radium-228 & $1.6 E+00$ & 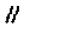 & & & 1 & GP & & & & & & & pCin \\
\hline Strontium-90 & $4.5 E+00$ & /I & & & 1 & GP & & & & & & & pCill \\
\hline Technetium-99 & $5.1 E+01$ & /l & & $\mathbf{n}$ & 1 & GP & & & & & & & pCil \\
\hline Thorium-228 & 1.7E-01 & $\|$ & & & 1 & $G P$ & & & & & & & pCill \\
\hline Thorium-230 & $<3.4 E-02$ & UII/ & $<0.1400$ & & 1 & GP & & & & & & & pCill \\
\hline Sum of alphas & $6.1 E-01$ & & & & & & & & & & & & pCill \\
\hline Sum of betas & $5.7 E+01$ & & & $\mathbf{0}$ & & & & & & & & & pCirl \\
\hline Total radium & $6.6 \mathrm{E}+00$ & & & $\bar{\square}$ & & & & & & & & & $\mathrm{pCil}$ \\
\hline Tritium & $7.2 E+02$ & $\|$ & & $\boldsymbol{\square}$ & 1 & GP & $6.8 \mathrm{E}+02$ & $/ 1$ & & ש & 1 & GE & pCi/mL \\
\hline Uranium-233/234 & $<3.2 \mathrm{E}-02$ & $\mathrm{U} U /$ & $<0.2340$ & & 1 & GP & & & & & & & pCill \\
\hline Uranium-235 & $<5.5 \mathrm{E}-02$ & $\mathrm{U} 1 / /$ & $<0.1640$ & & 1 & GP & & & & & & & $\mathrm{pCil}$ \\
\hline Uranium-238 & $<8.6 \mathrm{E}-02$ & $\mathrm{U}: / /$ & $<0.1640$ & & 1 & GP & & & & & & & pCill \\
\hline
\end{tabular}

Notes:

- = exceeded holding time

E = exceeded groundwater protection or monitoring constituent standard (See Appendix A.) 
WELL FSB120D

$\begin{array}{ll}\text { SRS Coord. } & \text { Lat/Longitude } \\ \text { N75568.7 } & 33.273124^{\circ} \mathrm{N} \\ \text { E49163.7 } & 81.684130^{\circ} \mathrm{W}\end{array}$

SAMPLE DATE

FIELD DATA

Constituents

Water elevation

$\mathrm{pH}$

Sp. conductance

Water temperature

Alkalinity as $\mathrm{CaCO}_{3}$

Turbidity

Volumes purged

Sampling code

Synchronous water leve

212.5

7.0

90

22.7

16

$\mathrm{XN}$

$212.0(09 / 21 / 98)$

Screen Zone Elevation

216.5-196.5 ft ms

ANALYTICAL DATA

Inonganic Constituents

Constituents

Antimony, total recoverable $<0.20$

Arsenic, total recoverable $\quad<3.0$

Barium, total recoverable $\quad 47$

Cadmium, total recoverable $<1.0$

Chromium, total recoverable 3.3

Cobalt, total recoverable $\quad 0.051$

Copper, total recoverable $\quad 5.9$

Cyanide

Lead, total recoverable $\quad 5.2$

Mercury, total recoverable $\quad<0.20$

Nickel, total recove

Nitrate as nitrogen

Nitrate-nitrite as nitrogen $\quad 240$

Selenium, total recoverable $<5.0$

Silver, total recoverable $<1.0$

Thallium, total recoverable $\quad<2.5$

Vanadium, total recoverable $<2.0$

Zinc, total recoverable

\begin{tabular}{|c|c|}
\hline Mod & Filt. \\
\hline $\begin{array}{l}\mathrm{U} N / \\
\mathrm{U} / I\end{array}$ & $\begin{array}{l}<0.20 \\
<3.0\end{array}$ \\
\hline II & \\
\hline $\begin{array}{l}\mathrm{U} / / \\
/ I\end{array}$ & $<1.0$ \\
\hline $\begin{array}{l}\mathrm{J} / \mathrm{E} / \\
/ /\end{array}$ & NDD \\
\hline $\begin{array}{l}\mathrm{U} / / \\
\| /\end{array}$ & $<10$ \\
\hline $\begin{array}{l}\mathrm{w} / / \\
/ /\end{array}$ & $<0.20$ \\
\hline$N \prime$ & \\
\hline $\mathrm{U} / /$ & $<5.0$ \\
\hline $\mathrm{U} / /$ & $<1.0$ \\
\hline $\mathrm{U} / /$ & $<2.5$ \\
\hline $\begin{array}{l}\mathrm{U} / / \\
/ /\end{array}$ & $<2.0$ \\
\hline
\end{tabular}

$<1.0$

$<2.5$

Organic Consttuents

Benzene

Bis(2-ethylhexyl) phthalate

Dichloromethane

Phenols

Tetrachloroethylene $<1.0$

Irichloroethylene

Trichlorofluoromethane $\underline{3098}$

Mod

$\mathrm{UJ} / \mathrm{O} / 1$

UII

$\mathrm{UJ} / \mathrm{O} / 1$

UII

UJ/O/1

UJ/O/1

$\mathrm{UJ} / \mathrm{O} / 1$
Filt.

$<1.0$

$<11$

$<5.0$

$<1.0$

$<1.0$
$<1.0$
$<1.0$

$\begin{array}{ll}\text { DF } & \text { Lab } \\ 1 & \text { GE } \\ 1 & \text { GE } \\ 1 & \text { GE } \\ 1 & \text { GE } \\ 1 & \text { GE } \\ 1 & \text { GE } \\ 1 & \text { GE }\end{array}$

GE
GE
GE
GE
GE
GE
GE
4Q98

211.6

6.2

70

30

7

0.10
$X N$

$211.0(12 / 21 / 98)$
Casing Pump

4" PVC S
Screen Zone

Water Table (IIB2)

0/26/98

Unit

ft ms!

$\mathrm{pH}$

$\mu \mathrm{S} / \mathrm{cm}$

$\mathrm{mg} / \mathrm{L}$

NTU

ft msl

ST브

DF Lab 4Q998

GE

GE

GE

GE

GE

GE

GE

1 GE

1 GE

GE

$3.2 \quad 11$

110

$<1.0 \quad \mathrm{U} / / \quad<1.0$
Mod

"l

Mod
Filt.

ST브

Filt.

ST 브 DF Lab Unit

$\mu g / L$
$\mu g / L$
$\mu g / L$
$\mu g^{\prime} L$
$\mu g / L$
$\mu g^{\prime} L$

$\mu g /$.

Notes:

- exceeded holding time

- = exceeded groundwater protection or monitoring constituent standard (See Appendix A.)

F-Area HWMF

C-165

Third and Fourth Quarter 1998 
WELL FSB120D (cont.)

Radioactive Constituents

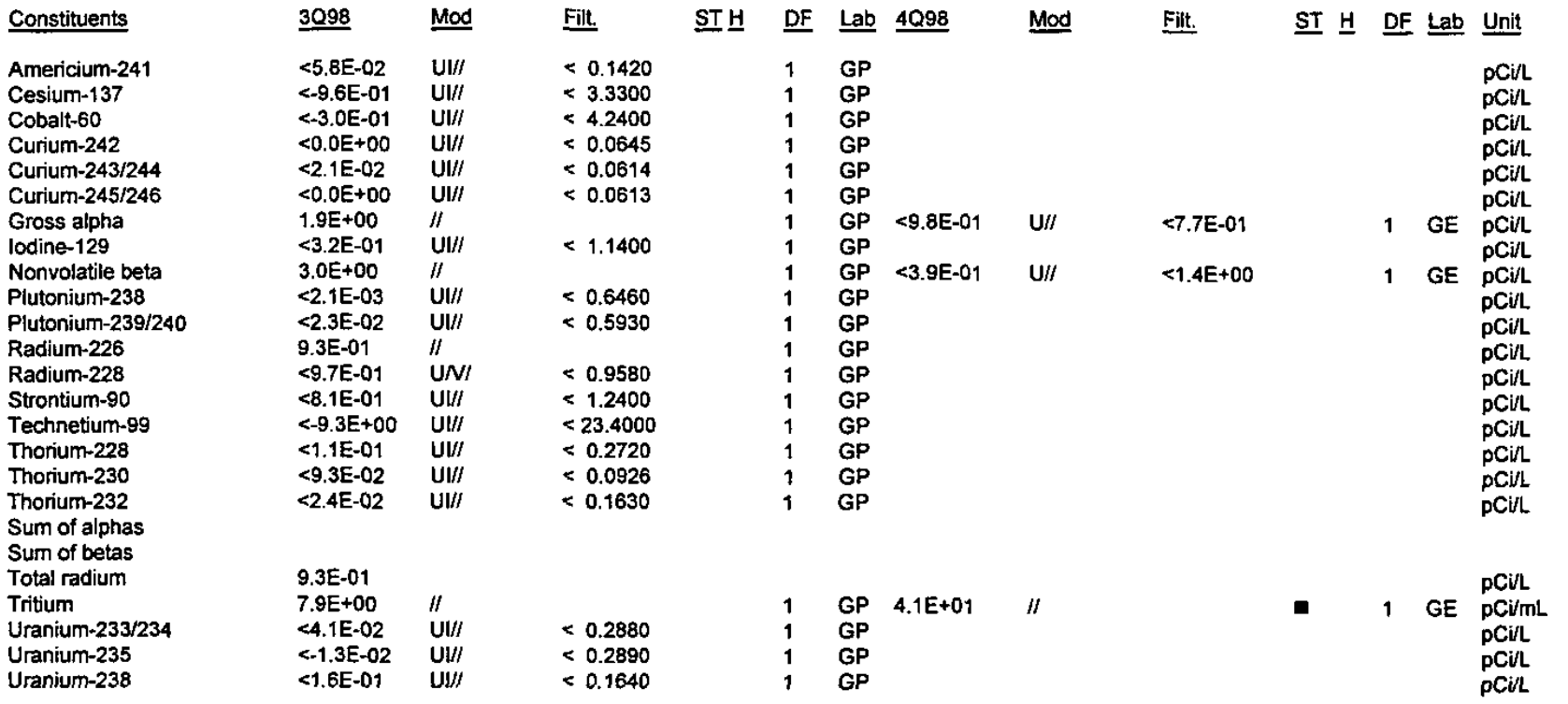

\section{Notes:}

- exceeded holding time

- exceeded groundwater protection or monitoring constituent standard (See Appendix A.) 
WSRC-TR-99-00012

Unclassified

\section{WELL FSB121C}

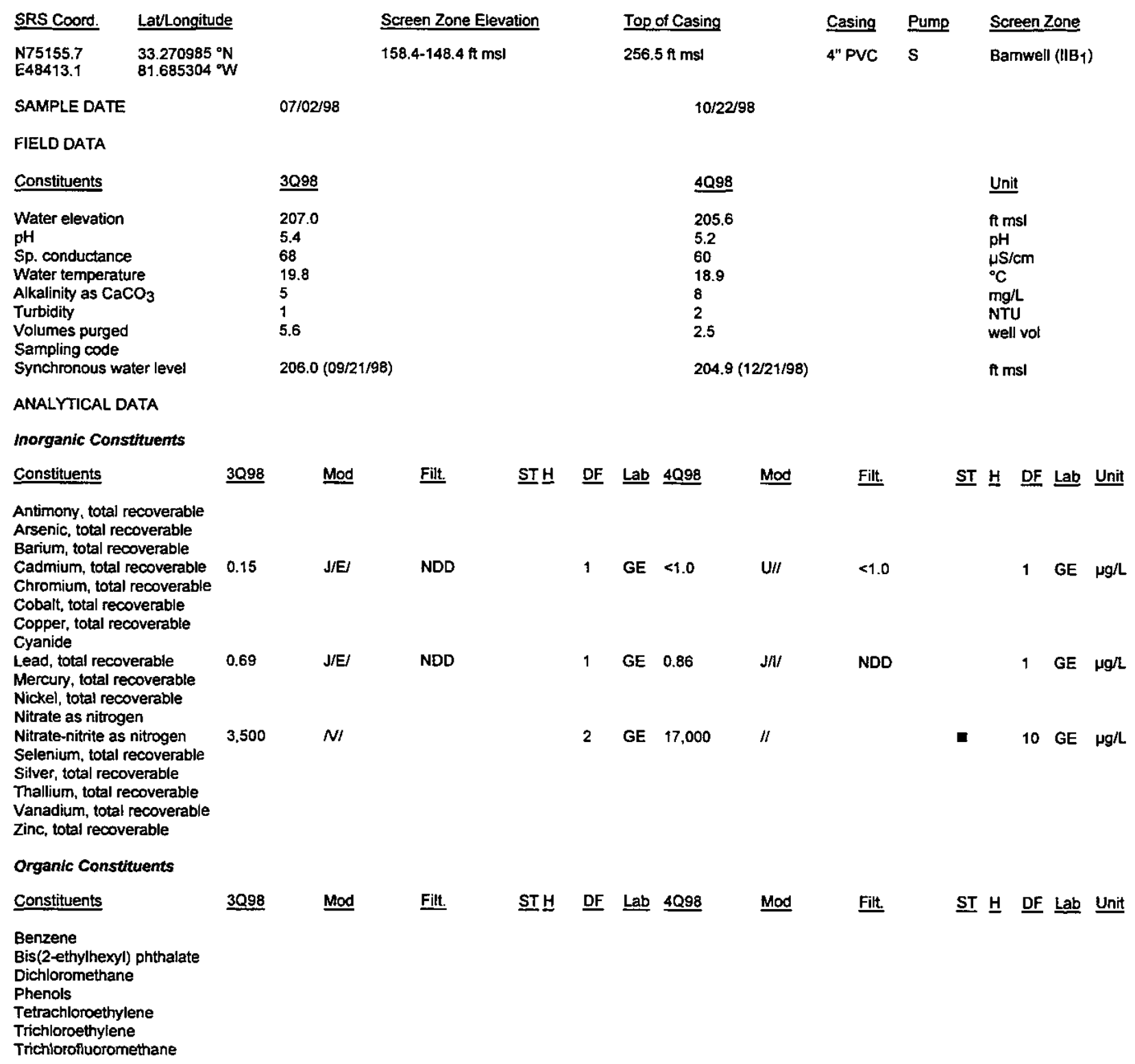

Notes:

- = exceeded holding time

= exceeded groundwates protection or monitoring constituent standard (See Appendix A.)

F-Area HWMF 


\section{WELL FSB121C (cont.)}

Radioactive Constituents

Constituents

Americium-241

Cesium-137

Cobalt-60

Curium-242

Curium-243/244

Curium-245/246

Gross alpha

lodine-129

Nonvolatile beta

Plutonium-238

Plutonium-239/240

Radium-226

Radium-228

Strontium-90

Technetium-99

Thorium-228

Thorium-230

Thorium-232

Sum of alphas

Sum of betas

Tritium

Uranium-233/234

Uranium-235

Uranium-238 $\underline{3098}$

Mod

Filt.

ST브 DF Lab

Mod

Filt.

ST $\underline{H}$ DF Lab Unit

$1.4 \mathrm{E}+00 \quad / /$

$2.8 \mathrm{E}+00 \quad / /$

$1 \quad \mathrm{GP}<1.8 \mathrm{E}+00 \quad \mathrm{U} / /$

$<9.5 E-01$

1 GP $<2.6 \mathrm{E}+00 \quad \mathrm{U} / /$

$<1.6 \mathrm{E}+00$

1 GE $p \mathrm{Ci} / \mathrm{L}$

1 GE pCill

$<2.9 \mathrm{E}-01 \quad \mathrm{UI} / \prime \quad<5.8 \mathrm{E}-01 \quad 1 \quad \mathrm{GP} \quad 1.3 \mathrm{E}+02 \quad \|$

- $\quad 1$ GE pCimL

Notes:

- exceeded holding time

E = exceeded groundwater protection or monitoring constituent standard (See Appendix A.) 


\section{WELL FSB121DR}

$\begin{array}{ll}\text { SRS Coord. } & \text { Lat/Longitude } \\ \text { N75151.9 } & 33.271004^{\circ} \mathrm{N} \\ \text { E48429.7 } & 81.685253^{\circ} \mathrm{W}\end{array}$

SAMPLE DATE

FIELD DATA

Constituents

Water elevation

pH

Sp. conductance

Water temperature

Alkalinity as $\mathrm{CaCO}_{3}$

Turbidity

Volumes purged

Sampling code

Synchronous water level

ANALYTICAL DATA

Inorganic Constituents

Constituents

Antimony, total recoverable

Arsenic, total recoverable

Barium, total recoverable

Cadmium, total recoverable

Chromium, total recoverable

0.37

3098

210.1

5.0

34

18.8

2

3

0.081

$209.3(09 / 21 / 98)$

3098

Mod
Screen Zone Elevation

211.3-191.3 ft ms

Top of Casing

$255.5 \mathrm{ft} \mathrm{msl}$

$10 / 22 / 98$

$\underline{4098}$

208.9

5.3

50

17.5

9

4

$208.3(12 / 21 / 98)$
Casing Pump Screen Zone

4" PVC S
Water Table (IIB2)

$\underline{\text { Unit }}$

ft mst

pH

$\mu \mathrm{S} / \mathrm{cm}$

${ }^{\circ} \mathrm{C}$

$\mathrm{mg} / \mathrm{L}$.

NTU

well vol

ft ms

Cobalt, total recoverable

Copper, total recoverable

Cyanide

Lead, total recoverable

Mercury, total recoverable

Nickel, total recoverable

Nitrate as nitrogen

Nitrate-nitrite as nitrogen

Selenium, total recoverable

Silver, total recoverable

Thallium, total recoverable

Vanadium, total recoverable

Zinc, total recoverable

Organic Constituents

Constituents

Filt.

ST H DF Lab $\underline{4098}$

Mod

Filt.

ST 브 DF Lab Unit

Benzene

Bis(2-ethylhexyl) phthalate

Dichloromethane

Phenols

Tetrachloroethylene

Trichloroethylene

Trichlorofluoromethane

38

NDD

$1 \mathrm{GE} \mu g / L$

$3,000 \quad \quad N \prime$

1

GE $\quad 0.24$

$\mathrm{J} / / /$

$\begin{array}{llll}\text { - } & 1 & \text { GE } & 11\end{array}$

II

2 GE 1,200

II

Mod

Filt.

ST $\underline{H}$ DF Lab Unit
1 GE $\mu g /$

1 GE $\mu g / L$

Notes:

- exceeded holding time

- = exceeded groundwater protection or monitoring constituent standard (See Appendix A.)

F-Area HWMF

C-169

Third and Fourth Quarter 1998 


\section{WELL FSB121DR (cont.)}

Radioactive Constituents

\section{Constituents}

Americium-241

Cesium-137

Cobalt-60

Curium-242

Curium-243/244

Curium-245/246

Gross alpha

lodine-129

Nonvolatile beta

Plutonium-238

Plutonium-239/240

Radium-226

Radium-228

Strontium-90

Technetium-99

Thorium-228

Thorium-230

Thorium-232

Sum of alphas

Sum of betas

Tritium

Uranium-233/234

Uranium-235

Uranium-238
30.98

$7.6 \mathrm{E}+00 \quad / /$

4.1E+01 //

$5.6 \mathrm{E}+01 \quad \|$
ST브 DF Lab $4098 \quad \underline{M o d}$

1 GP $9.0 E+00$

/I

1 GP $3.4 \mathrm{E}+01$ //

- 1 GP $1.5 \mathrm{E}+01 \quad / /$
1 GE PCi/L

1 GE PCin

1 GE PCi/mL

Notes:

- = exceeded holding time

= exceeded groundwater protection or monitoring constituent standard (See Appendix A.) 


\section{WELLL FSB122C}

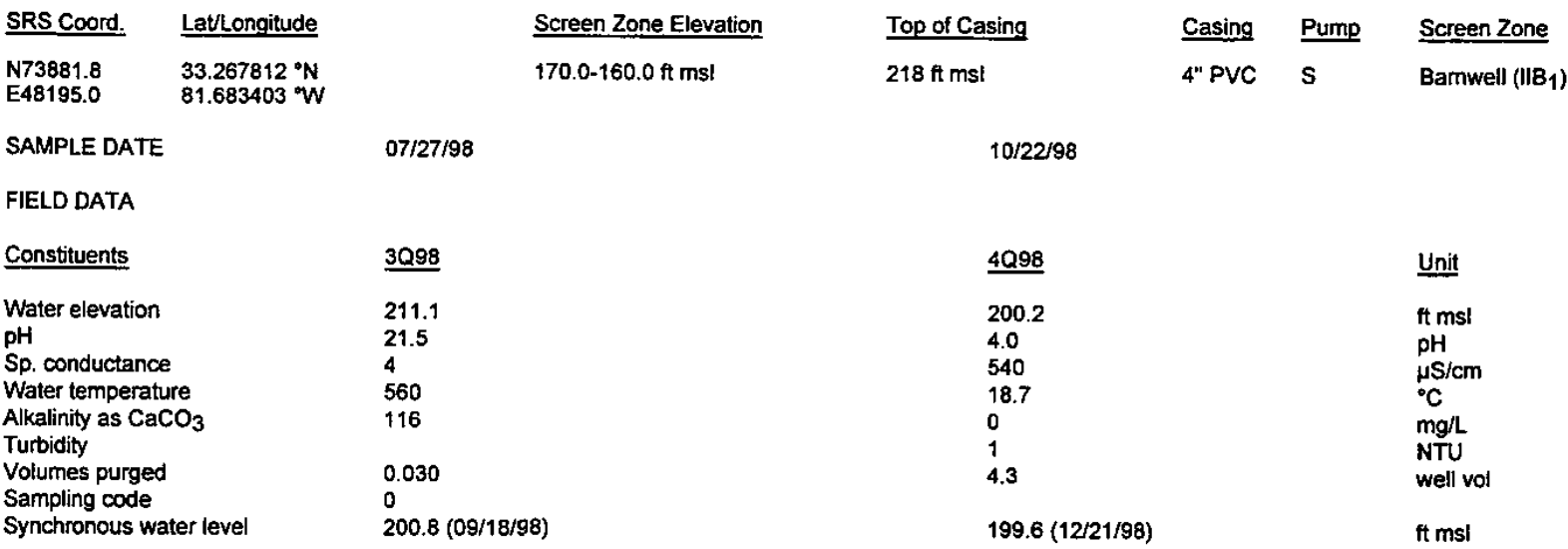

\section{ANALYTICAL DATA}

Inorganic Constituents

Constituents

Antimony, total recoverable

Arsenic, total recoverable

Barium, total recoverable

Cadmium, total recoverable

Chromium, total recoverable

Cobalt, total recoverable

Copper, total recoverable

Cyanide

Lead, total recoverable

Mercury, total recoverable

Nickel, total recoverable

Nitrate as nitrogen

Nitrate-nitrite as nitrogen

Selenium, total recoverable

Silver, total recoverable

Thallium, total recoverable

Vanadium, total recoverable

Zinc, total recoverable

Organic Constituents

\section{Constituents}

1.1

$\mathrm{J} / \mathrm{E}$

Mod

Filt.

ST브

DF Lab 4Q98

Mod

$66,000 \quad N \prime$

Benzene

Bis(2-ethylhexyl) phthalate

Dichloromethane

Phenols

Tetrachloroethylene

Trichloroethylene

Trichlorofluoromethane

Notes:

- exceeded holding time

E = exceeded groundwater protection or monitoring constituent standard (See Appendix A.)

F-Area HWMF

C.171

Third and Fourth Quarter 1998 
WSRC-TR-99-00012

Unclassified

WELL FSB122C (cont.)

Radioactive Constituents

Constituents

Americium-241

Cesium-137

Cobalt-60

Curium-242

Curium-243/244

Curium-245/246

Gross alpha

lodine-129

Nonvolatile beta

Plutonium-238

Plutonium-239/240

Radium-226

Radium-228

Strontium-90

Technetium-99

Thorium-228

Thorium-230

Thorium-232

Sum of alphas

Sum of betas

Tritium

Uranium-233/234

Uranium-235

Uranium-238 $\underline{3098}$

Mod

Filt.

ST브 DF Lab $4 Q 98$

Mod

Filt.

ST $\underline{\text { H }} \underline{\mathrm{DF}} \underline{\mathrm{Lab}}$ Unit

$1.9 \mathrm{E}+01 \quad / /$

$6.6 \mathrm{E}+01 \quad / /$

$1.6 \mathrm{E}+03 \quad / /$

-

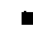

$\mathrm{GP}<7.6 \mathrm{E}+00 \quad \mathrm{U} / 1$

$<7.1 \mathrm{E}+00$

$1 \mathrm{GE} \quad \mathrm{pCi} / \mathrm{L}$

$+$

GP

$\mathrm{J} / \mathrm{K} / \mathrm{I}$

NDD

1 GE pCi/L

1 GP $1.7 \mathrm{E}+03 \quad /$

- 1 GE pCi/mL

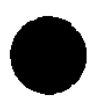

Notes:

- = exceeded holding time

a = exceeded groundwater protection or monitoring constituent standard (See Appendix A.) 
WELL FSB122D

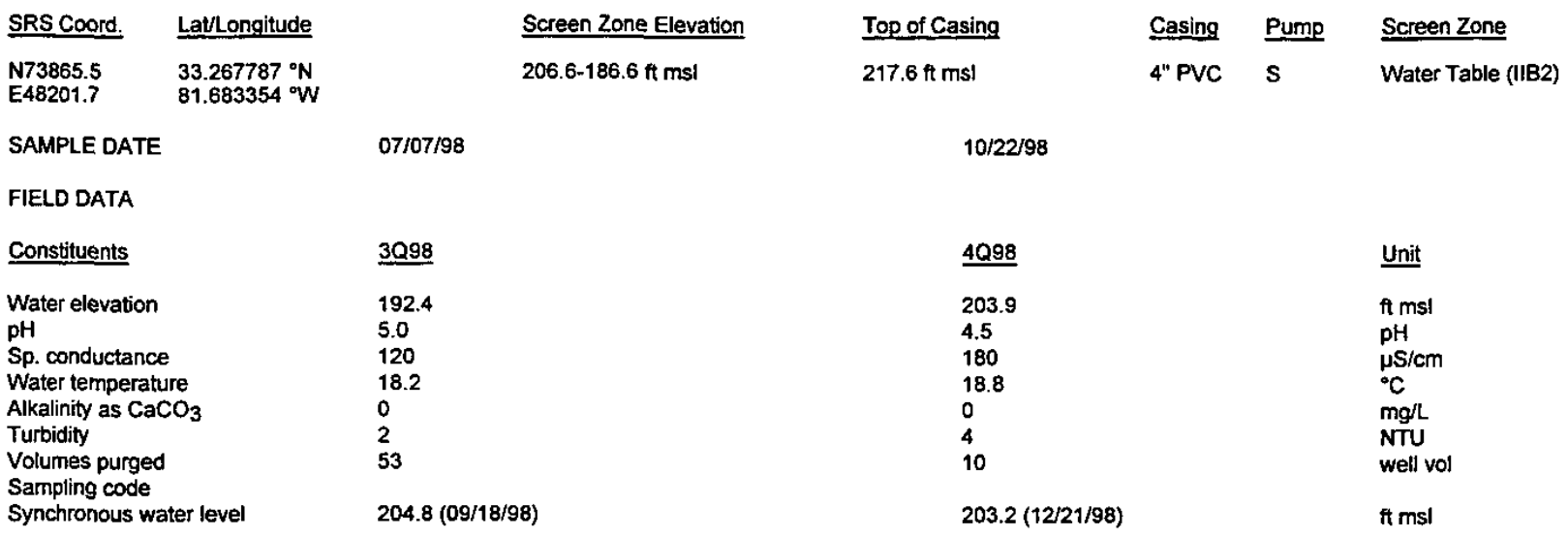

\section{ANALYTICAL DATA}

Inorganic Constituents

\begin{tabular}{|c|c|c|c|c|c|c|c|c|c|c|c|c|c|}
\hline Constituents & 3098 & Mod & Filt. & ST브 & DF & $\underline{L a b}$ & 4Q98 & Mod & Filt. & $\underline{\mathrm{ST}} \underline{\mathrm{H}}$ & $\underline{\text { DF }}$ & $\underline{\text { Lab }}$ & $\underline{\text { Unit }}$ \\
\hline Antimony, total recoverable & $<0.20$ & $U / I$ & $<0.20$ & & 1 & GE & & & & & & & $\mu g /$ \\
\hline Arsenic, total recoverable & $<3.0$ & $\mathrm{U} / I$ & $<3.0$ & & 1 & GE & & & & & & & $\mu g /$. \\
\hline Barium, total recoverable & 60 & $/ /$ & & & 1 & GE & & & & & & & $\mu g / L$ \\
\hline Cadmium, total recoverable & $<1.0$ & $\mathrm{U} / I$ & $<1.0$ & & 1 & GE & $<1.0$ & $\mathrm{U} / /$ & $<1.0$ & & 1 & GE & $\mu g / L$ \\
\hline Chromium, total recoverable & 0.68 & $\mathrm{~J} / \mathrm{E} /$ & NDD & & 1 & $\overline{G E}$ & & & & & & & $\mu g / L$ \\
\hline Cobalt, total recoverable & 1.8 & 11 & & & 1 & GE & & & & & & & $\mu g / L$ \\
\hline Copper, total recoverable & 6.3 & $\| 1$ & & & 1 & GE & & & & & & & $\mu g / L$ \\
\hline Cyanide & $<10$ & $\mathrm{U} / \prime$ & $<10$ & & 1 & GE & & & & & & & $\mu g h$. \\
\hline Lead, total recoverable & 3.0 & $N I$ & & & 1 & GE & 2.6 & $\|$ & & & 1 & GE & $\mu \mathrm{g} / \mathrm{L}$ \\
\hline Mercury, total recoverable & $<0.20$ & $U / I$ & $<0.20$ & & 1 & GE & & & & & & & $\mu g / L$ \\
\hline $\begin{array}{l}\text { Nickel, total recoverable } \\
\text { Nitrate as nitrogen }\end{array}$ & 2.9 & $\|$ & & & 1 & GE & & & & & & & $\mu g / L$ \\
\hline Nitrate-nitrite as nitrogen & 13,000 & $N I$ & & $\mathbf{e}$ & 25 & GE & 3,000 & $\|$ & & & 3 & GE & $\mu g / L$ \\
\hline Selenium, total recoverable & $<5.0$ & $\mathrm{U} / /$ & $<5.0$ & & 1 & GE & & & & & & & $\mu g$. \\
\hline Silver, total recoverable & $<1.0$ & $\mathrm{U} / /$ & $<1.0$ & & 1 & GE & & & & & & & $\mu g / L$ \\
\hline Thallium, total recoverable & $<2.5$ & $\mathrm{U} / l$ & $<2.5$ & & 1 & GE & & & & & & & $\mu \mathrm{g} / \mathrm{L}$ \\
\hline Vanadium, total recoverable & $<2.0$ & U/I & $<2.0$ & & 1 & GE & & & & & & & $\mu g / L$ \\
\hline Zinc, total recoverable & 34 & $\|$ & & & 1 & GE & & & & & & & $\mu g / L$ \\
\hline \multicolumn{14}{|l|}{ Organic Constituents } \\
\hline Constituents & $\underline{3098}$ & Mod & Filt. & $\underline{\text { ST브 }}$ & DF & $\underline{\text { Lab }}$ & 4Q98 & Mod & Filt. & $\underline{\text { ST }} \underline{\text { H }}$ & $\underline{\text { DF }}$ & $\underline{\text { Lab }}$ & Unit \\
\hline Benzene & $<1.0$ & $U / I$ & $<1.0$ & & 1 & GE & & & & & & & $\mu g / L$ \\
\hline Bis(2-ethylhexyl) phthalate & $<10$ & UII & $<10$ & & 1 & GE & & & & & & & $\mu g / L$ \\
\hline Dichloromethane & $<1.0$ & $\mathrm{U} / /$ & $<1.0$ & & 1 & GE & & & & & & & $\mu \mathrm{g} / \mathrm{L}$ \\
\hline Phenols & $<5.0$ & $\mathrm{U} / I$ & $<5.0$ & & 1 & GE & & & & & & & $\mu g / L$ \\
\hline Tetrachloroethylene & $<1.0$ & $\mathbf{U} / /$ & $<1.0$ & & 1 & GE & & & & & & & $\mu g / L$ \\
\hline Trichloroethylene & $<1,0$ & $\mathrm{U} / 1$ & $<1.0$ & & 1 & GE & & & & & & & $\mu \mathrm{g} / \mathrm{L}$ \\
\hline Trichlorofluoromethane & $<1.0$ & $\mathrm{U} / 1$ & $<1.0$ & & 1 & GE & & & & & & & $g / L$ \\
\hline
\end{tabular}

Notes:

- = exceeded holding time

a = exceeded groundwater protection or monitoring constituent standard (See Appendix A.)

F-Area HWMF 


\section{WELL FSB122D (cont.)}

Radioactive Constituents

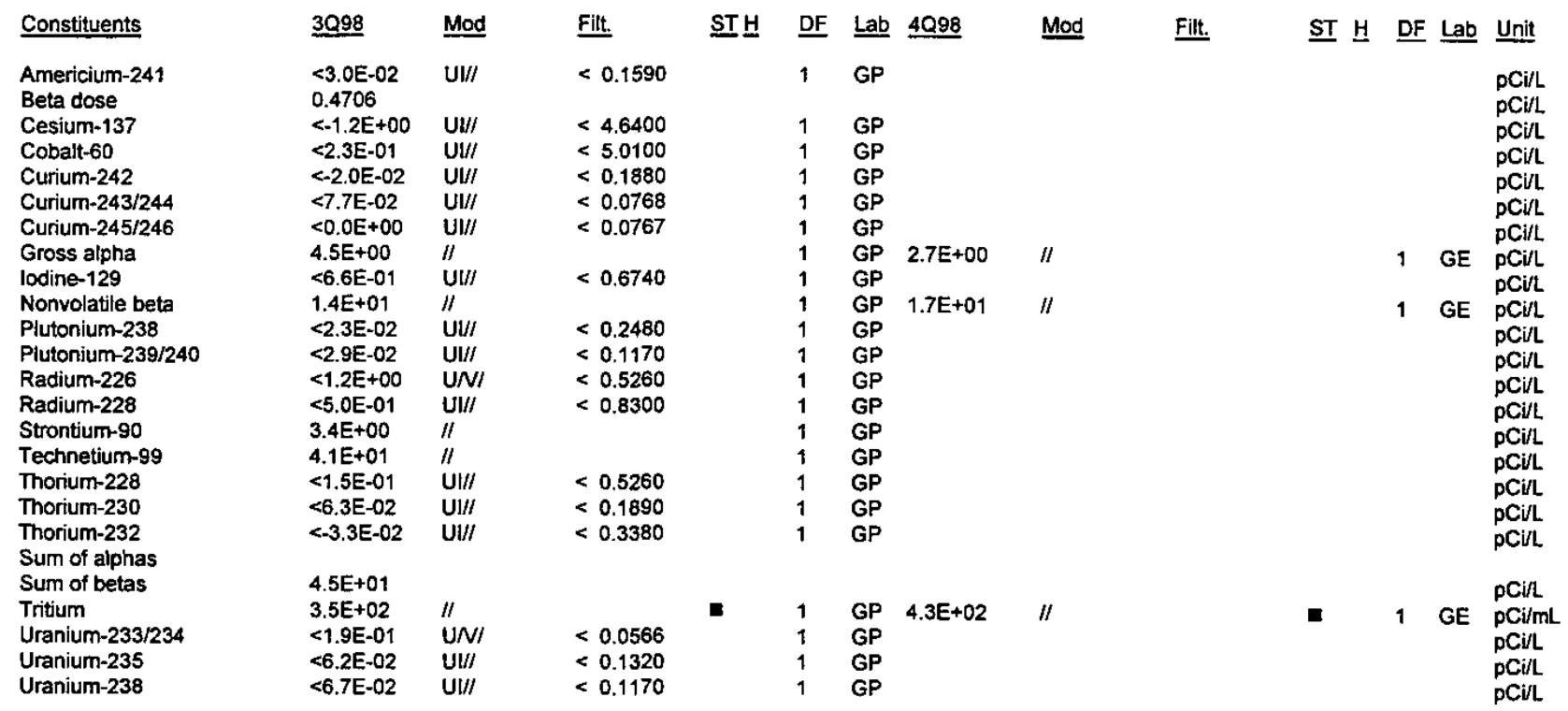

Notes:

- = exceeded holding time

- exceeded groundwater protection or monitoring constituent standard (See Appendix A.) 
WELL FSB123C

$\begin{array}{ll}\text { SRS Coord. } & \text { Latlongitude } \\ \text { N74566.7 } & 33.275129^{\circ} \mathrm{N} \\ E^{51750.5} & 81.675374^{\circ} \mathrm{W}\end{array}$

SAMPLE DATE

FIELD DATA

Constituents

Water elevation

$\mathrm{pH}$

Sp. conductance

Water temperature

Alkalinity as $\mathrm{CaCO}_{3}$

Turbidity

Volumes purged

Sampling code

Synchronous water level

ANALYTICAL DATA

\section{Inorganic Constituents}

\section{Constituents}

Antimony, total recoverable

Arsenic, total recoverable

Barium, total recoverable

Cadmium, total recoverable

Chromium, total recoverable

Cobalt, total recoverable

Copper, total recoverable

Cyanide

Lead, total recoverable

Mercury, total recoverable

Nickel, total recoverable

Nitrate as nitrogen

Nitrate-nitrite as nitrogen

Selenium, total recoverable

Silver, total recoverable

Thallium, total recoverable

Vanadium, total recoverable

Zinc, total recoverable

Organic Constituents

Constituents

Screen Zone Elevation

$165.3-155.3 \mathrm{ft} \mathrm{ms}$

07/07/98

$\underline{3098}$

211.7

60

21.2

1
4.7

$211.8(09 / 17 / 98)$
Top of Casing

$238.1 \mathrm{ft} \mathrm{ms}$

$10 / 21 / 98$

$\underline{4098}$

211.5

5.4

48

19.1

7

3.6

$210.8(12 / 18 / 98)$

$\begin{array}{lll}\text { Casing } & \text { Pump } & \text { Screen Zone } \\ 4^{n} \text { PVC } & S & \left.\text { Bamwell (IIB } B_{1}\right)\end{array}$

Bamwell $\left(1 \mathrm{~B}_{1}\right)$

Benzene

Bis(2-ethylhexyl) phthalate

Dichloromethane

Phenols

Tetrachloroethylene

Trichloroethylene

Trichlorofluoromethane

UN

ull

Mod

Filt.

$<1.0$

3,000

NI

$\underline{3 Q 9}$

$<1.0 \quad u$

$<2.0$

1 GE $<2.0$

U/I

3 GE 3,000

/

Mod

Filt.
Unit

ft msl

$\mathrm{pH}$

$\mu \mathrm{S} / \mathrm{cm}$

${ }^{\circ} \mathrm{C}$

$\mathrm{mg} / \mathrm{L}$

NTU

well vol

$\mathrm{ft} \mathrm{msl}$

Notes:

- = exceeded holding time

- exceeded groundwater protection or monitoring constituent standard (See Appendix A.) 


\section{WELL FSB123C (cont.)}

Radioactive Constituents

Constituents

Mod

Filt.

ST브 므 느 4Qb98

Mod

Filt.

ST $\underline{H}$ DF Lab Unit

Americium-241

Cesium-137

Cobalt-60

Curium-242

Curium-243/244

Curium-245/246

Gross alpha

lodine-129

Nonvolatile beta

Plutonium-238

Plutonium-239/240

Radium-226

Radium-228

Strontium-90

Technetium-99

Thorium-228

Thorium-230

Thorium-232

Sum of alphas

Sum of betas

Tritium

Uranium-233/234

Uranium-235

$8.2 \mathrm{E}+00 \quad / /$

$<9.5 E-01 \quad U U /$

$<1.7900$

$1 \quad$ GP $<1.8 E-01$

U/I

$1 \quad G P$

$<1.3 E+00$

$\mathrm{U} / 1$

$<7.4 E-01$

$<1,6 \mathrm{E}+00$

1 GE pCill

$1 \mathrm{GE}$ pCi凡

Uranium-238

Notes:

- = exceeded holding time

- = exceeded grounowater protection or monitoring constituent standard (See Appendix A.) 
WSRC-TR-99-00012

Unclassified

WELL FSB123D

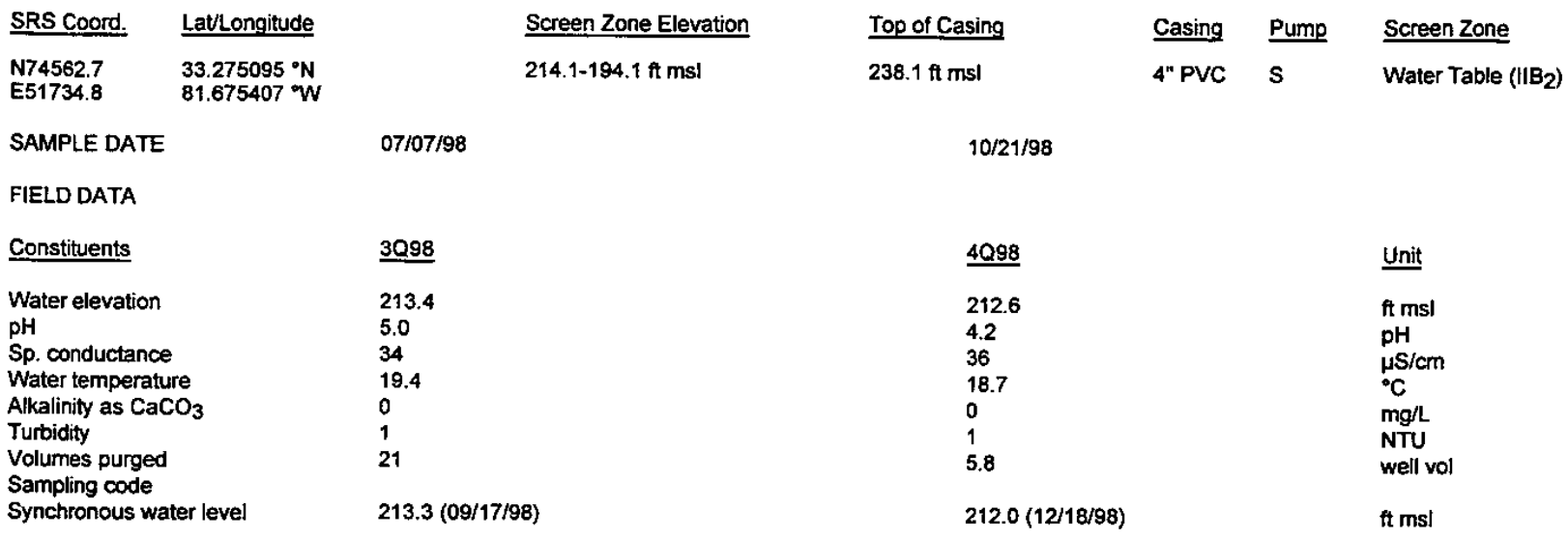

ANALYTICAL DATA

Inorganic Constrituents

Constituents

Antimony, total recoverable

Arsenic, total recoverable

Barium, total recoverable

Cadmium, total recoverable

Chromium, total recoverable

$<1.0$

Cobalt, total recoverable

Copper, total recoverable

Cyanide

Lead, total recoverable

Mercury, total recoverable

Nickel, total recoverable

Nitrate as nitrogen

Nitrate-nitrite as nitrogen

Selenium, total recoverable

Silver, total recoverable

Thallium, total recoverable

Vanadium, total recoverable

Zinc, total recoverable

Organic Constituents

Constituents

Benzene

Bis(2-ethylhexyl) phthalate

Dichloromethane

Phenols

Tetrachloroethylene

Trichloroethylene

Trichlorofluoromethane

1.2

1,600
Filt.

ST브

DF Lab 4 Q998

Mod

Filt.

$\underline{\text { ST }} \underline{\mathrm{H}}$ OF Lab Unit

J/EV $/$

NDD

$1 \quad$ GE $\quad 1.5$

$\mathrm{J} / \mathrm{l}$

1 GE 2,200 $\quad$ /l

NI

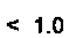

1 GE $<1.0$

U/I

$<1.0$

$1 \mathrm{GE} \mu g / \mathrm{L}$

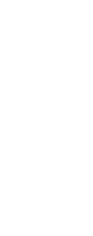

Mod

Filt.

ST브

DF Lab 4098

Mod

Filt.

ST $\underline{H}$ DF Lab Unit

2 GE $\mu g / L$

Notes:

- = exceeded holding time

- = exceeded groundwater protection or monitoring constituent standard (See Appendix A.) 


\section{WELL FSB123D (cont.)}

Radioactive Constituents

Constituents

Americium-241

Cesium-137

Cobalt-60

Curium-242

Curium-243/244

Curium-245/246

Gross alpha

lodine-129

Nonvolatile beta

Plutonium-238

Plutonium-239/240

Radium-226

Radium-228

Strontium-90

Technetium-99

Thorium-228

Thorium-230

Thorium-232

Sum of aiphas

Sum of betas

Tritium

Uranium-233/234

Uranium-235

Uranium-238
3098

Mod

Filt.

ST

DF Lab 4 Q998

Mod

Filt.

ST 브 Df Lab Unit

$2.1 E+00 \quad / /$

$<1.0 \mathrm{E}+00 \quad \mathrm{UI} / /$

$<1.7200$

$1 \quad \mathrm{GP}<1.6 \mathrm{E}+\infty \quad \mathrm{U} / 1$

$1 \quad G P<1.9 E+00 \quad U / /$

$<7.2 E-01$

$<1.3 E+00$

1 GE pCill

1 GE pCill

Notes:

- exceeded holding time

- = exceeded groundwater protection or monitoring constituent standard (See Appendix A.) 
WELL FSL 1D

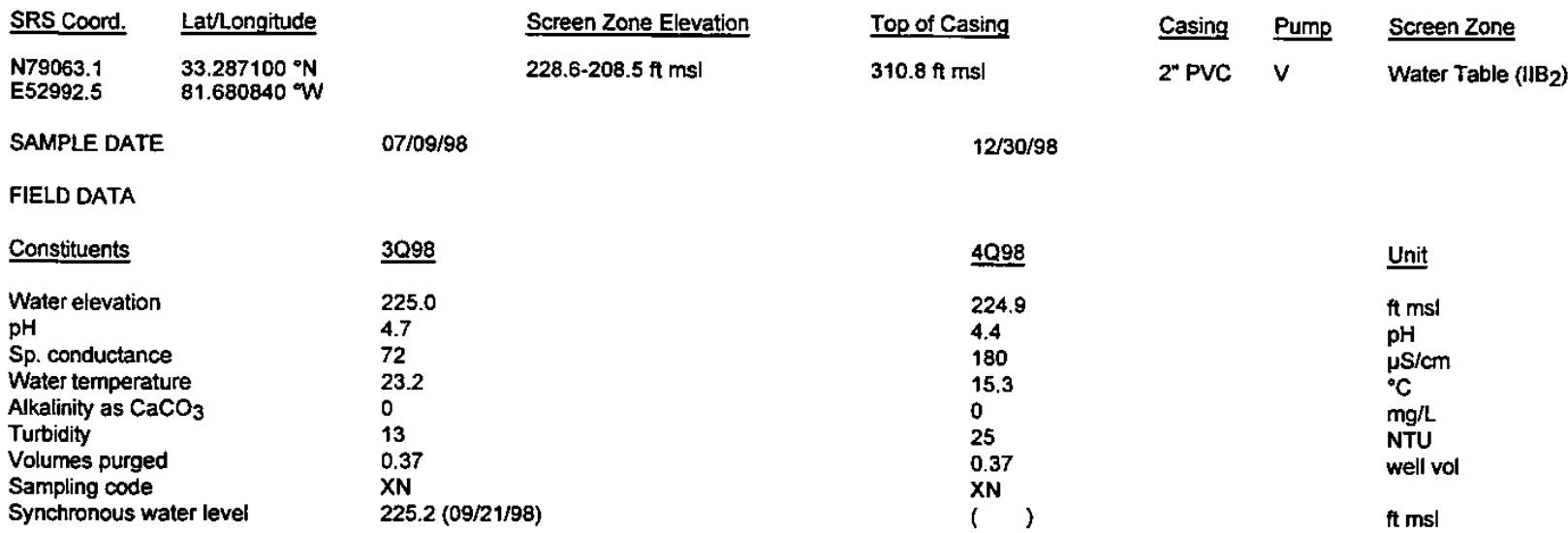

ANALYTICAL DATA

Inorganic Constituents

STH DF Lab $\underline{4 Q 98} \quad$ Mod

Filt.

$\underline{\text { ST }}$ H DF Lab Unit

Antimony, total recoverable

Arsenic, total recoverable

Barium, total recoverable

Cadmium, total recoverable

$\mathrm{J} / \mathrm{E}$

NDD

1 GE $\begin{array}{lll}1.4 & \end{array}$

1 GE $\mu g / L$

Chromium, total recoverable

Cobalt, total recoverable

Copper, total recoverable

Cyanide

Lead, total recoverable

Mercury, total recoverable

Nickel, total recoverable

Nitrate as nitrogen

Nitrate-nitrite as nitrogen

Selenium, total recoverable

Silver, total recoverable

Thallium, total recoverable

Vanadium, total recoverable

Zinc, total recoverable

Organic Constituents

Constituents

309

Mod

Filt.

ST브

DF Lab $\underline{4 Q 98}$

Mod

1.0

/l

Bis(2-ethylhexyl) phthalate

Dichloromethane

Phenols

Tetrachloroethylene

Trichloroethylene

Trichlorofluoromethane

1.0
2.8

Notes:

- = exceeded holding time

E = exceeded groundwater protection or monitoring constituent standard (See Appendix A.) 


\section{WELL FSL 1D (cont.)}

Radioactive Constituents

\section{Constituents}

3098

Mod

Filt.

ST브 DF Lab $\underline{4098}$

Mod

Filt.

ST 브 DF Lab Unit

Americium-241

Cesium-137

Cobalt-60

Curium-242

Curium-243/244

Curium-245/246

Gross alpha

lodine-129

Nonvolatile beta

Plutonium-238

Plutonium-239/240

Radium-226

Radium-228

Strontium-90

Technetium-99

Thorium-228

Thorium-230

Thorium-232

Sum of alphas

Sum of betas

Tritium

Uranium-233/234

8.9E+00

8.7E+00 $/ 1$

$\begin{array}{llll}1 & G P & 8.6 E+00 & / / \\ 1 & G P & 1.2 \mathrm{E}+01 & / /\end{array}$

1 GP $p C i / L$

Uranium-235

Uranium-238

Notes:

= exceeded holding time

- = exceeded groundwater protection or monitoring constituent standard (See Appendix A.) 
WELL FSL 2D

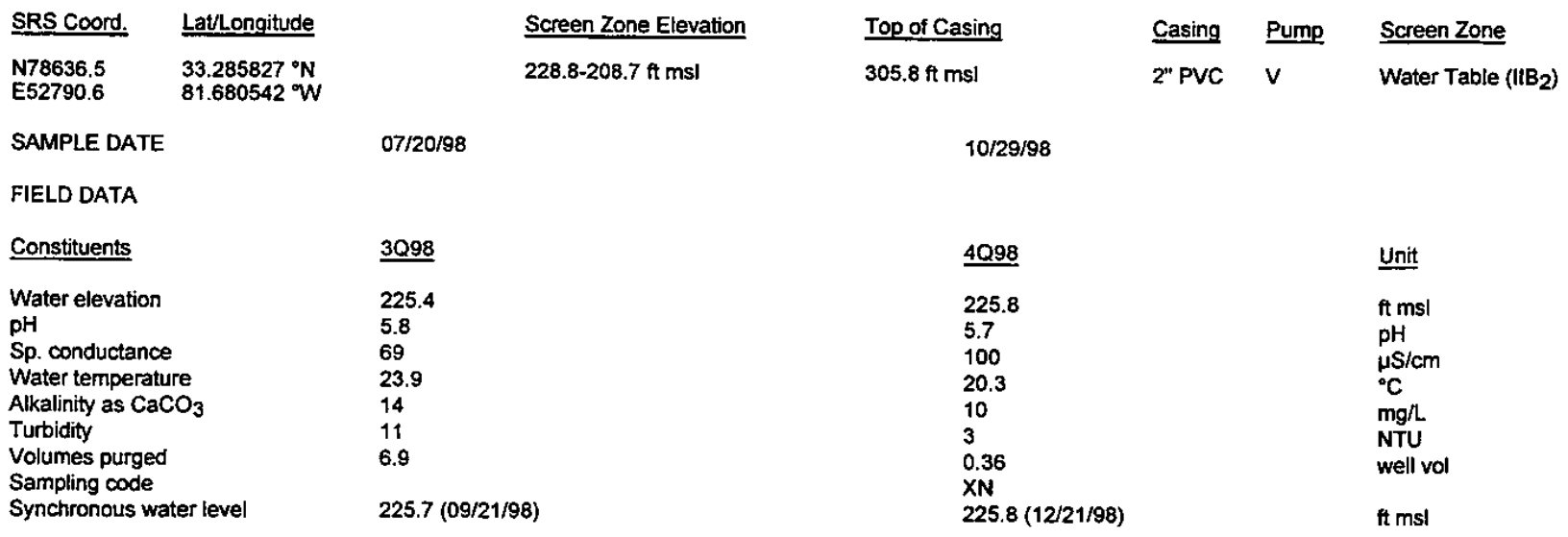

ANALYTICAL DATA

Inonganic Constituents

Constituents

Antimony, total recoverable

Arsenic, total recoverable

Barium, total recoverable

Cadmium, total recoverable

Chromium, total recoverable

Cobalt, total recoverable

Copper, total recoverable

Cyanide

Lead, total recoverable

Mercury, total recoverable

Nickel, total recoverable

Nitrate as nitrogen

Nitrate-nitrite as nitrogen

Selenium, total recoverable

Silver, total recoverable

Thallium, total recoverable

Vanadium, total recoverable

Zinc, total recoverable

Onganic Constituents

Constituents

3Q98

0.14

4.5

1,400
$N$

$\mathrm{J} / \mathrm{E} /$

NDD

II

Benzene

Bis(2-ethylhexyl) phthaiate

Dichloromethane

Phenols

Tetrachloroethylene

Trichloroethylene

Trichlorofluoromethane

\section{Mod Filt}

Filt.

Mod $\underline{\text { ST }} \underline{\underline{H}}$

HF Lab

1 GE $<1.0$

U/I

$<$

1 GE $\mu g / L$

$1 \quad G E \quad 2.6$

0.20

"I

1 GE $1,100 \quad$ /I

1 GE $\mu g / L$

1 GE $\mu g / L$

ST브

Notes:

- = exceeded holding time

= exceeded groundwater protection or monitoring constituent standard (See Appendix A.) 
WELL FSL 2D

Radioactive Constituents

Constituents

Americium-241

Cesium-137

Cobalt-60

Curium-242

Curium-243/244

Curium-245/246

Gross alpha

lodine-129

Nonvolatile beta

Plutonium-238

Plutonium-239/240

Radium-226

Radium-228

Strontium-90

Technetium-99

Thorium-228

Thorium-230

Thorium-232

Sum of alphas

Sum of betas

Tritium

Uranium-233/234

Uranium-235

Uranium-238
$3 \mathrm{Q}$

$1.7 E+00$

$1.7 \mathrm{E}+00 \quad / /$

$8.9 E+00 \quad /$
1 GE $\mathrm{pCin}$

1 GP $<1.7 E+00 \quad \mathrm{U} / 1$

1 GP $4.4 \mathrm{E}+00 \quad /$

1 GE $\mathrm{pCi}$

1 GE $\mathrm{pCi} / \mathrm{mL}$

Notes:

- exceeded holding time

- = exceeded groundwater protection or monitoring constituent standard (See Appendix A.) 
WSRC-TR-99-00012

Unclassified

WELL FSL 3D

$\begin{array}{ll}\text { SRS Coord. } & \text { Latlongitude } \\ \text { N77765.2 } & 33.283369{ }^{\circ} \mathrm{N} \\ \text { E52465.2 } & 81.679706{ }^{\circ} \mathrm{W}\end{array}$

SAMPLE DATE

FIELD DATA

Constituents

Water elevation

$\mathrm{pH}$

Sp. conductance

Water temperature

Alkalinity as $\mathrm{CaCO}_{3}$

Turbidity

Volumes purged

Sampling code

Synchronous water level

ANALYTICAL DATA

Inorganic Constituents

Constituents

Antimony, total recoverable

Arsenic, total recoverable

Barium, total recoverable

Cadmium, total recoverable

Chromium, total recoverable

$<1.0 \quad U$

Screen Zone Elevation

$226.0-205.9 \mathrm{ft} \mathrm{ms}$

Top of Casing

$302 \mathrm{ft}$ msl

Casing Pump

2" PVC V

Screen Zone

Water Table (IIB 2 )

$10 / 28 / 98$

3Q998

223.0

6.5

140

25.7

2.5

$223.6(09 / 21 / 98)$ $\underline{4 Q 98}$

223.8

6.9

120

5.8

$223.8(12 / 21 / 98)$
Unit

fi ms

$\mathrm{pH}$

$\mu \mathrm{S} / \mathrm{cm}$

${ }^{\circ} \mathrm{C}$

$\mathrm{mg} / \mathrm{L}$

NTU

well vol

ft msl
3Q98

Mod

Fitt.

ST브

DF Lab 4Q98

Mod

Filt.

ST $\underline{H}$ DF Lab Unit

Cobalt, total recoverable

Copper, total recoverable

Cyanide

Lead, total recoverable

2.0

Mercury, total recoverable

Nickel, total recoverable

Nitrate as nitrogen

Nitrate-nitrite as nitrogen

Selenium, total recoverable

Silver, total recoverable

Thallium, total recoverable

Vanadium, total recoverable

Zinc, total recoverable

Organic Constituents

Constituents

3Q98

Filt.

ST보

DF Lab 4Q98

Mod

$1.0 \quad 11$

Filt.

Benzene

Bis(2-ethylhexyl) phthalate

Dichloromethane

Phenols

Tetrachloroethylene

Trichloroethylene

Trichiorofluoromethane

$1.0 \quad / 1$

1.5

1 GE $\mu g /$ L

1 GE $\mu g / L$

Notes:

= exceeded holding time

v = exceeded groundwater protection or monitoring constituent standard (See Appendix A.)

F-Area HWMF

C-183

Third and Fourth Quarter 1998 
WELL FSL 3D

Radioactive Constituents

Constituents

3098

Mod

Filt.

STI브 DF ㄴab 4 QQ98

Mod

Filt.

$\underline{\text { ST }} \underline{H}$ DF Lab Unit

Americium-241

Cesium-137

Cobalt-60

Curium-242

Curium-243/244

Curium-245/246

Gross alpha

lodine-129

Nonvolatile beta

Plutonium-238

Plutonium-239/240

Radium-226

Radium-228

Strontium-90

Technetium-99

Thorium-228

Thoium-230

Thorium-232

Sum of alphas

Sum of betas

Tritium

Uranium-233/234

Uranium-235

Uranium-238

$1.5 \mathrm{E}+00$

3.1E+00 /

3.6E+01 /

1 GP $<9.6 \mathrm{E}-01 \quad \mathrm{U} / /$

1 GP 2.7E+00 //

1 GE pCill

1 GE pCill.

- 1 GP $3.3 \mathrm{E}+01 \quad / 1$

- 1 GE $\mathrm{pCi} / \mathrm{mL}$

Notes:

- = exceeded holding time

$\square$ = exceeded groundwater protection or monitoring constituent standard (See Appendix A.) 
WELL FSL 4D

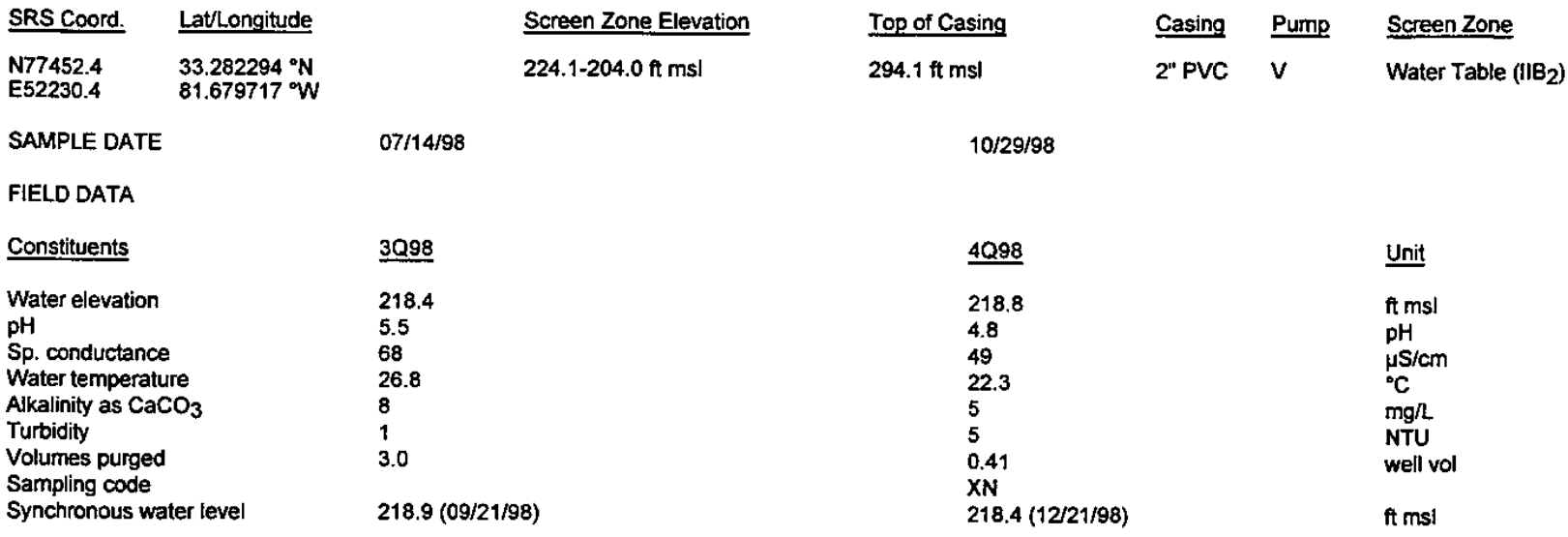

ANALYTICAL DATA

Inorganic Constituents

Constituents

Antimony, total recoverable

Arsenic, total recoverable

Barium, total recoverable

Cadmium, total recoverable

Chromium, total recoverable

Cobatt, total recoverable

Copper, total recoverable

Cyanide

Lead, total recoverable

Mercury, total recoverable

Nickel, total recoverable

Nitrate as nitrogen

Nitrate-nitrite as nitrogen

Selenium, total recoverable

Silver, total recoverable

Thallium, total recoverable

Vanadium, total recoverable

Zinc, total recoverable

Organic Constituents

Constituents

Benzene

Bis(2-ethylhexyl) phthalate

Dichloromethane

Phenols

Tetrachloroethylene

Trichloroethylene

Trichlorofluoromethane

0.17

2,000

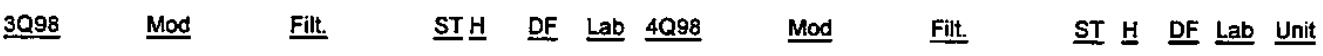

$\mathrm{J} / \mathrm{E} /$

NDD

1 GE $<1.0$

$\mathrm{U} / /$

$43 \quad 1$

/

-

GE 40

40
0.20

$/ 1$

1 GE $2,200 \quad / 1$

- 1 GE $\mu g /$ L

1 GE $\mu g / L$

Mod

Filt.

ST브

Mod

Filt.

$\underline{\text { ST }} \underline{H}$ DF Lab Unit

Notes:

- = exceeded holding time

= exceeded groundwater protection or monitoring constituent standard (See Appendix A.) 
WELL FSL 4D (cont.)

Radioactive Constituents

Constituents

ST브

DF Lab 4 QQ98

Mod

Filt.

ST H DF Lab Unit

Americium-241

Cesium-137

Cobalt-60

Curium-242

Curium-243/244

Curium-245/246

Gross alpha

lodine-129

Nonvolatile beta

Plutonium-238

Plutonium-239/240

Radium-226

Radium-228

Strontium-90

Technetium-99

Thorium-228

Thorium-230

Thorium-232

Sum of alphas

Sum of betas

Tritium

Uranium-233/234

$<2.5 \mathrm{E}-01 \quad$ UII $\quad<0.7310$

1 GP 1.7E+00 $/ /$

2.9E+00 $/ /$

1 GP $5.0 \mathrm{E}+00 \quad / /$

1 GE pCill

Uranium-235

Uranium-238

= exceeded groundwater protection or monitoring constituent standard (See Appendix A.) 
WELL FSL 5D

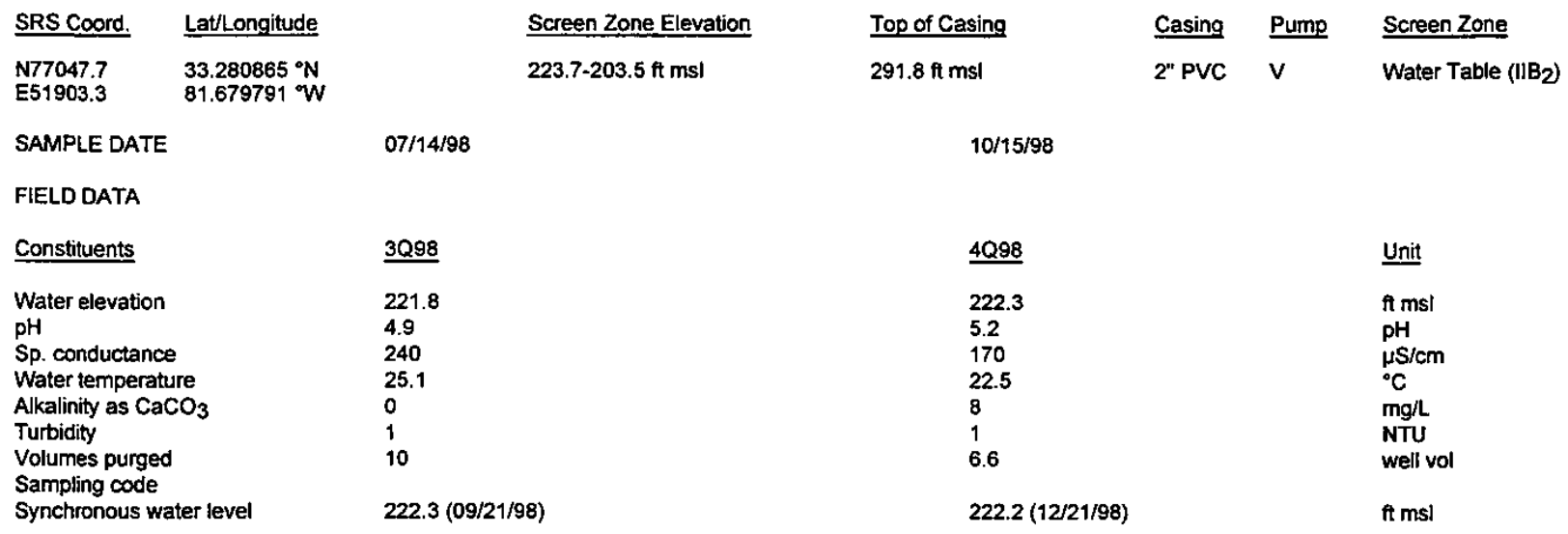

ANALYTICAL DATA

Inorganic Constituents

Constituents

Antimony, total recoverable

Assenic, total recoverable

Barium, total recoverable

Cadmium, total recoverable

Chromium, total recoverable

Cobalt, total recoverable

Copper, total recoverable

Cyanide

Lead, total recoverable

Mercury, total recoverable

Nickel, total recoverable

Nitrate as nitrogen

Nitrate-nitrite as nitrogen

Selenium, total recoverable

Silver, total recoverable

Thallium, total recoverable

Vanadium, total recoverable

Zinc, total recoverable

Organic Constituents

Constituents

3098

Filt.

ST

DF Lab 4 Q998

Mod

NDD

$1 \quad$ GE $\quad 0.21$

$\mathrm{J} / \mathrm{TV} /$

$2.2 \quad 1 /$

23,000

GE 0.75

$$
0.2
$$

$\mathrm{J} / \mathrm{V} /$

- 15 GE 19,000

Benzene

Bis(2-ethylhexyl) phthalate

Dichloromethane

Phenols

Tetrachloroethylene

Trichloroethylene

Trichlorofluoromethane

Notes:

- = exceeded holding time

= exceeded groundwater protection or monitoring constituent standard (See Appendix A.)

F-Area HWMF

C-187

Third and Fourth Quarter 1998 


\section{WELL FSL $5 D$ (cont.)}

Radioactive Constituents

Constituents

Americium-241

Cesium-137

Cobalt-60

Curium-242

Curium-243/244

Curium-245/246

Gross alpha

lodine-129

Nonvolatile beta

Plutonium-238

Plutonium-239/240

Radium-226

Radium-228

Strontium-90

Technetium-99

Thorium-228

Thorium-230

Thorium-232

Sum of alphas

Sum of betas

Tritium

Uranium-233/234

Uranium-235

Uranium-238
Mod

Filt.

ST브 DF ㄴab $\underline{4098}$

Mod

Filt.

ST 브 DF Lab Unit

$1.3 E+01 \quad /$

$3.4 E+02 \quad / 1$

$3.3 \mathrm{E}+02 \quad / /$

$\begin{array}{lll}1 & \mathrm{GE} & \mathrm{pCi} \\ -\quad 1 & \mathrm{GE} & \mathrm{pCi} / \mathrm{L}\end{array}$

- 1 GP $1.9 \mathrm{E}+02 \quad$ II

1 GP $1.8 \mathrm{E}+02 \quad /$

- $1 \mathrm{GE} \quad \mathrm{pCi} / \mathrm{mL}$

Notes:

- = exceeded holding time

a = exceeded groundwater protection or monitoring constituent standard (See Appendix A.) 
WELL FSL 60

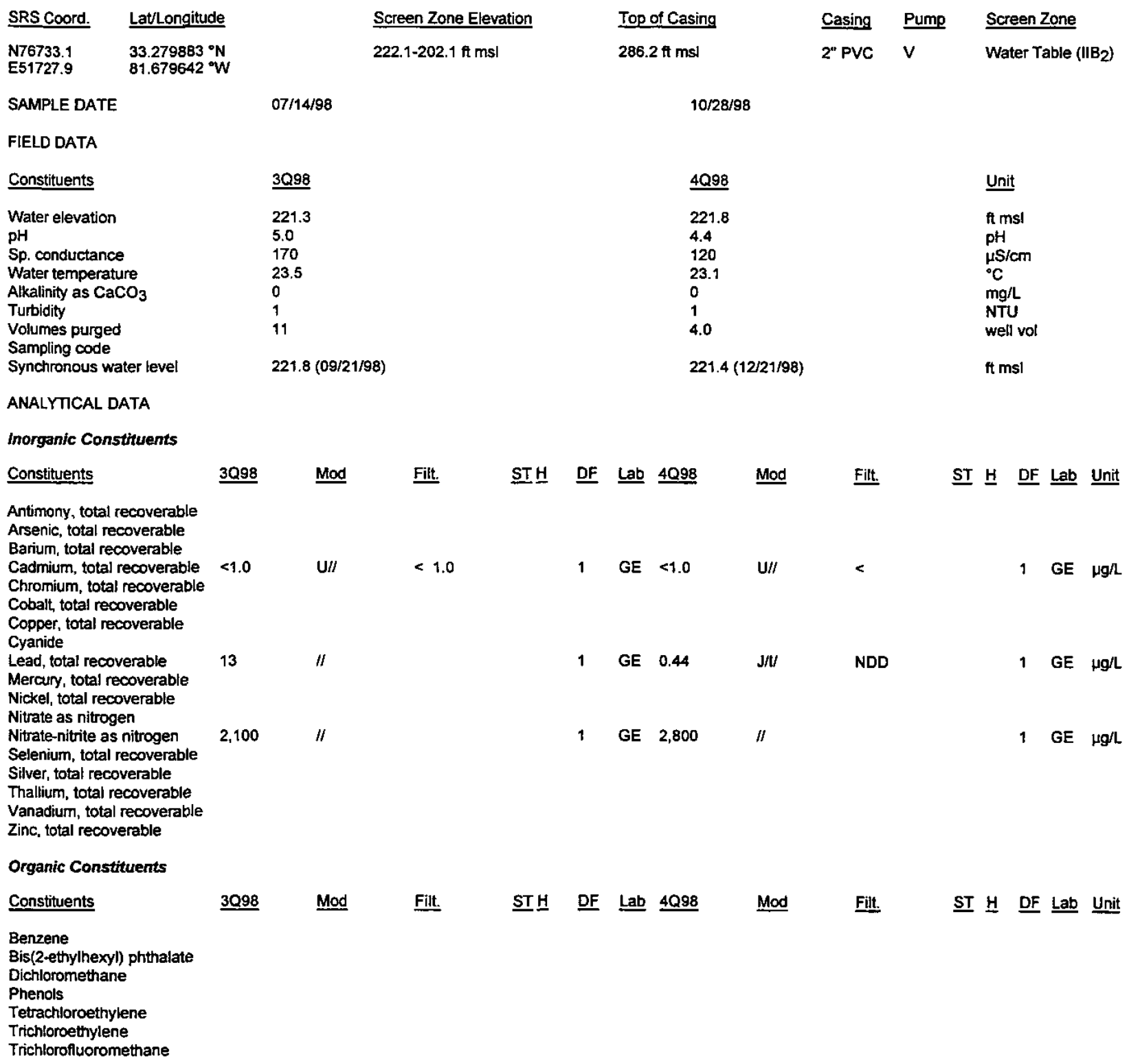

Notes:

- = exceeded holding time

- = exceeded groundwater protection or monitoring constituent standard (See Appendix A.) 
WELL FSL 6D (cont.)

Radioactive Constituents

Constituents

Americium-241

Cesium-137

Cobalt-60

Curium-242

Curium-243/244

Curium-245/246

Gross alpha

lodine-129

Nonvolatile beta

Plutonium-238

Plutonium-239/240

Radium-226

Radium-228

Strontium-90

Technetium-99

Thorium-228

Thorium-230

Thorium-232

Sum of alphas

Sum of betas

Tritium

Uranium-233/234

Uranium-235

Uranium-238

\section{Notes:}

= exceeded holding time

- = exceeded groundwater protection or monitoring constituent standard (See Appendix A.)

F-Area HWMF

C-190

2.1E+00 / $\underline{3098} \quad$ Mod $\quad \underline{\text { Filt. }} \quad \underline{\text { STH }}$ DF Lab $\underline{4 Q 98} \quad \underline{\text { Mod }} \quad \underline{\text { Filt. }} \underline{\text { ST }} \underline{H}$ DF Lab Unit

$9.6 \mathrm{E}+00 \quad / 1$

$1 \mathrm{GP}$

$<1.5 \mathrm{E}+00 \quad \mathrm{U} / \mathrm{I}$

$<$

1 GE PCiLL

1 GP $6.6 \mathrm{E}+00 \quad / /$

1 GE $\mathrm{pCill}$

7.5E+01

1

GP

6.1E+01

"l

-

1 GE pCi/mL 
WELL FSL 7D

\begin{tabular}{|c|c|c|c|c|c|c|}
\hline SRS Coord. & Lat/Longitude & Screen Zone Elevation & Top of Casing & Casing & Pump & Screen Zone \\
\hline $\begin{array}{l}\text { N76327.8 } \\
\text { E51485.6 }\end{array}$ & $\begin{array}{l}33.278592^{\circ} \mathrm{N} \\
81.679492^{\circ} \mathrm{W}\end{array}$ & $219.6-199.5 \mathrm{ft} \mathrm{msl}$ & $287.6 \mathrm{ft} \mathrm{msl}$ & 2" PVC & v & Water Table $\left(\mathrm{IIB}_{2}\right)$ \\
\hline \multicolumn{2}{|c|}{ SAMPLE DATE } & $07 / 43 / 98$ & \multicolumn{2}{|l|}{$10 / 28 / 98$} & & \\
\hline \multicolumn{7}{|l|}{ FIELD DATA } \\
\hline \multicolumn{2}{|l|}{ Constituents } & 3QQ98 & 4Q98 & & & Unit \\
\hline \multicolumn{2}{|c|}{ Water elevation } & 216.8 & 220.8 & & & $\mathrm{ft} \mathrm{ms} !$ \\
\hline & 4.0 & 3.9 & & & $\mathrm{pH}$ \\
\hline & 370 & 398 & & & $\mu \mathrm{S} / \mathrm{cm}$ \\
\hline \multirow{2}{*}{\multicolumn{2}{|c|}{$\begin{array}{l}\text { Water temperature } \\
\text { Alkalinity as } \mathrm{CaCO}_{3}\end{array}$}} & 21.8 & 22.3 & & & ${ }^{\circ} \mathrm{C}$ \\
\hline & & 0 & 0 & & & mgll \\
\hline \multicolumn{2}{|c|}{ Turbidity } & 7 & 1 & & & NTU \\
\hline \multirow{2}{*}{\multicolumn{2}{|c|}{ Volumes purged }} & 0.0 & 3.7 & & & well vol \\
\hline \multirow{2}{*}{\multicolumn{2}{|c|}{$\begin{array}{l}\text { Sampling code } \\
\text { Synchronous water level }\end{array}$}} & \multirow{2}{*}{$\begin{array}{l}X N \\
220.9(09 / 17 / 98)\end{array}$} & & & & \\
\hline & & & $220.1(12 / 18 / 98)$ & & & $\mathrm{ft} \mathrm{msl}$ \\
\hline
\end{tabular}

ANALYTICAL DATA

Inorganic Constituents

Constituents

Antimony, total recoverable

Arsenic, total recoverable Barium, total recoverable

Cadmium, total recoverable

Chromium, total recoverable

Cobalt, total recoverable

Copper, total recoverable

Cyanide

Lead, total recoverable

Mercury, total recoverable

Nickel, total recoverable

Nitrate as nitrogen

Nitrate-nitrite as nitrogen

Selenium, total recoverable

Silver, total recoverable

Thallium, total recoverable

Vanadium, total recoverable

Zinc, total recoverable

Organic Constituents

Constituents

Benzene

Bis(2-ethylhexyl) phthalate

Dichloromethane

Phenols

Tetrachloroethylene

Trichloroethylene

Trichlorofluoromethane
1.7

J/EV

NDD

$1 \quad$ GE $\quad 0.44$

$\mathrm{J} / \mathrm{I}$

44,000

/I

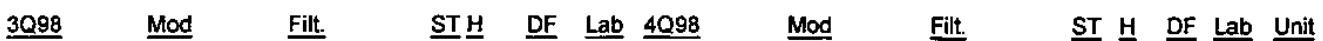

- 25 GE $46,000 \quad / /$

25 GE $\mu g / L$

Mod Filt

$\underline{\text { ST }}$

Notes:

- = exceeded holding time

a exceeded groundwater protection or monitoring constituent standard (See Appendix A.) 
WELL FSL 7D (cont.)

Radioactive Constituents

Constituents

Americium-241

Cesium-137

Cobalt-60

Curium-242

Curium-243/244

Curium-245/246

Gross alpha

lodine-129

Nonvolatile beta

Plutonium-238

Plutonium-239/240

Radium-226

Radium-228

Strontium-90

Technetiurn-99

Thorium-228

Thorium-230

Thorium-232

Sum of alphas

Sum of betas

Tritium

Uranium-233/234

Uranium-235

Uranium-238
3Q98

Mod

5.7E+D1

$3.4 \mathrm{E}+02$

8.9E+01 $/ /$

1 GP $4.6 \mathrm{E}+01 \quad / /$

- 1 GP $7.3 \mathrm{E}+02$ /I

1 GP $1.5 \mathrm{E}+02 \quad / /$
- 1 GE pCill

- 1 GE pCill

Notes:

- = exceeded holding time

E = exceeded groundwater protection or monitoring constituent standard (See Appendix A.) 


\section{WELL FSL 8D} $\begin{array}{ll}\text { SRS Coord. } & \text { LatlLongitude } \\ \text { N76054.7 } & 33.278033^{\circ} \mathrm{N} \\ \text { E51513.5 } & \mathbf{8 1 . 6 7 8 8 8 8} \mathrm{W}\end{array}$

SAMPLE DATE

FIELD DATA

Constituents

Water elevation

pH

Sp. conductance

Water temperature

Alkalinity as $\mathrm{CaCO}_{3}$

Turbidity

Volumes purged

Sampling code

Synchronous water level

ANALYTICAL DATA

$\begin{array}{lllll}\text { Screen Zone Elevation } & \text { Top of Casing } & \text { Casing } & \text { Pump } & \text { Screen Zone } \\ 222.8-202.7 \mathrm{ft} \mathrm{msl} & 290.8 \mathrm{ft} \mathrm{msl} & 2^{\prime \prime} \text { PVC } & \text { V } & \text { Water Table (IIB } 2)\end{array}$

$10 / 28 / 98$

$219.3(12 / 18 / 98)$
4Q98

220.0

4.4

92

22.0

0

3.9

Unit

ft ms:

pH

$\mu \mathrm{S} / \mathrm{cm}$

${ }^{\circ} \mathrm{C}$

$\mathrm{mg} / \mathrm{L}$

NTU

well vol

$220.2(09 / 17 / 98)$

ft msl

Inorganic Constituents

Constituents

Antimony, total recoverable

Arsenic, total recoverable

Barium, total recoverable

Cadmium, total recoverable

Chromium, total recoverable

Cobalt, total recoverabie

Copper, total recoverable

Cyanide

Lead, total recoverable

Mercury, total recoverable

Nickel, total recoverable

Nitrate as nitrogen

Nitrate-nitrite as nitrogen

Selenium, total recoverable

Silver, total recoverable

Thallium, total recoverable

Vanadium, total recoverable

Zinc, total recoverable

Organic Constfuents

\section{Constituents}

3Q98

Benzene

Bis(2-ethylhexyl) phthalate

Dichloromethane

Phenols

Tetrachloroethylene

Trichloroethylene

Trichlorofluoromethane

$\underline{3098}$

$<1.0$
Mod

Filt.

SITH

DF Lab 4 Q98

Mod

Filt.

ST $\underline{H} \quad \underline{\text { DF Lab Unit }}$

UII

$<1.0$

1 GE $<1.0$

$\mathrm{U} / /$

$<$

1 GE $\mu g / L$

$15 \quad$ II

/I

5,800

\section{$1 \quad$ GE $\quad 2.4$}

$$
\begin{aligned}
& 2.4 \\
& 0.25
\end{aligned}
$$

3 GE 7,300

$\|$

$\begin{array}{lll}1 & \text { GE } & \mu g / L \\ 1 & \text { GE } & \mu g / L\end{array}$

5 GE $\mu g / L$

Notes:

- exceeded holding time

= exceeded groundwater protection or monitoring constituent standard (See Appendix A.)

F-Area HWMF

C-193

Third and Fourth Quarter 1998 
WELL FSL 8D (cont.)

Radioactive Constituents

Constituents

Americium-241

Cesium-137

Cobalt-60

Curium-242

Curium-243/244

Curium-245/246

Gross alpha

lodine-129

Nonvolatile beta

Plutonium-238

Plutonium-239/240

Radium-226

Radium-228

Strontium-90

Technetium-99

Thorium-228

Thorium-230

Thorium.232

Sum of alphas

Sum of betas

Tritium

Uranium-233/234

Uranium-235

Uranium-238
4.4E+00

4.3E+00 $/$

$1 \quad$ GP $<2.4 E+00 \quad$ U $/ l$

1 GP $8.1 \mathrm{E}+00$ //

1 GE pCill

1 GE pCill
1 GP 2.7E+01 //
Filt.

ST $\underline{H}$ DE Lab Unit

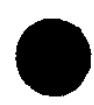

$1.8 \mathrm{E}+01 \quad / /$

-

GE $\mathrm{pCi} / \mathrm{mL}$

Notes:

- = exceeded holding time

- exceeded groundwater protection or monitoring constituent standard (See Appendix A.) 
WSRC-TR-99-00012

Unclassified

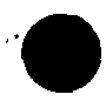

WELL FSL 9D

\begin{tabular}{|c|c|}
\hline SRS coord. & Lat/Longitude \\
\hline $\begin{array}{l}N 75768.4 \\
E^{51543.9}\end{array}$ & $\begin{array}{l}33.277450^{\circ} \\
81.678252^{\circ}\end{array}$ \\
\hline
\end{tabular}

SAMPLE DATE

$07 / 14 / 98$

\section{Screen Zone Elevation}

221.5-201.4 ft ms!

Top of Casing

$285.9 \mathrm{ft} \mathrm{ms!}$

$10 / 28 / 98$

FIELD DATA

Constituents

Water elevation

pH

Sp. conductance

Water temperature

Alkalinity as $\mathrm{CaCO}_{3}$

Turbidity

Volumes purged

Sampling code

Synchronous water level

ANALYTICAL DATA

Inorganic Constituemts

Constituents

$\underline{3098}$

3Q98

219.1

4.1

160

21.5

0

12

$219.2(09 / 17 / 98)$

Filt.

ST브

DF Lab $\underline{4 Q 98}$
4098

219.0

220

22.

0

4.5

$218.1(12 / 18 / 98)$
Screen Zone

Water Table (IIB2)

Antimony, total recoverable

Arsenic, total recoverable

Barium, total recoverable

Cadmium, total recoverable

Chromium, total recoverable

Cobalt, total recoverable

Copper, total recoverable

Cyanide

Lead, total recoverable

Mercury, total recoverable

$0.87 \quad \mathrm{~J} / \mathrm{E} / \quad$ NDD

Mod

Filt.

ST 브 DF Lab Unit

0.35

$\mathrm{J} / \mathbf{E}^{\prime}$

NDD

$1 \quad$ GE $\quad 0.44$

$\mathrm{J} / /$

NDD

1 GE $\mu g / L$

Nickel, total recoverable

Nitrate as nitrogen

Nitrate-nitrite as nitrogen

Selenium, total recoverable

Silver, total recoverable

Thallium, total recoverable

Vanadium, total recoverable

Zinc, total recoverable

Organic Constituents

Constituents

NDD

10 GE $21,000 \quad / /$

Benzene

Bis(2-ethylhexyl) phthalate

Dichtoromethane

Phenols

Tetrachloroethylene

Trichloroethylene

Trichlorofluoromethane

Notes:

- exceeded holding time

w = exceeded groundwater protection or monitoring constituent standard (See Appendix A.)

F-Area HWMF 


\section{WELL FSL 9D (cont.)}

\section{Radioactive Constituents}

Constituents

3098

Mod

Filt.

ST브 DF Lab $\underline{4 Q 98}$

Mod

Filt.

ST $\underline{\text { DF Lab Unit }}$

Americium-241

Cesium-137

Cobalt-60

Curium-242

Curium-243/244

Curium-245/246

Gross alpha

lodine-129

Nonvolatile beta

Plutonium-238

Plutonium-239/240

Radium-226

Radium-228

Strontium-90

Technetium-99

Thorium-228

Thorium-230

Thorium-232

Sum of alphas

Sum of betas

Tritium

7.1E+01

$\begin{array}{lllll}-1 & \mathrm{GP} & 1.1 E+02 & / /\end{array}$

$1.1 \mathrm{E}+02$

- 1 GP $1.9 \mathrm{E}+02$ //

233/234

Uranium-235

$3.8 E+02 \quad / /$

1 GP $6.1 E+02 \quad /$

- 1 GE pCir

- 1 GE pCill

Uranium-238

Notes:

- = exceeded holding time

- = exceeded groundwater protection or monitoring constituent standard (See Appendix A.) 
WELL HSB 85A

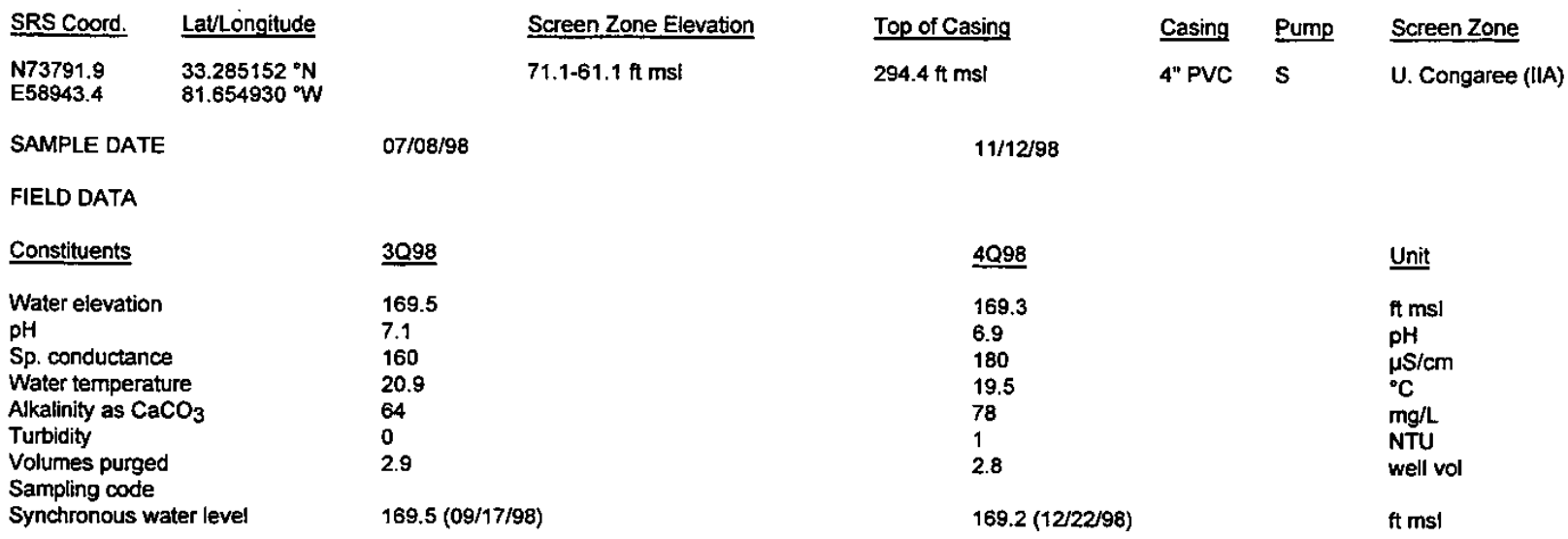

ANALYTICAL DATA

Inorganic Constituerts

\begin{tabular}{|c|c|c|c|c|c|c|c|c|c|c|c|c|c|}
\hline Constituents & $\underline{3098}$ & $\underline{\text { Mod }}$ & Filt. & ST브 & $\underline{\text { DF }}$ & $\underline{\text { Lab }}$ & $\underline{4 Q 98}$ & Mod & Filt. & $\underline{\mathrm{ST}} \underline{\mathrm{H}}$ & $\underline{\text { DF }}$ & $\underline{\underline{L a b}}$ & $\underline{\text { Unit }}$ \\
\hline Antimony, total recoverable & $<1.0$ & $\mathrm{U} / /$ & $<1.0$ & & 5 & GE & $<27$ & $\mathrm{U} / /$ & $<$ & & 1 & WA & $\mu g / L$ \\
\hline Arsenic, total recoverable & & & & & & & $<40$ & $\mathrm{U} / 1$ & $<$ & & 1 & WA & $\mu g / L$ \\
\hline Barium, total recoverable & & & & & & & 29 & /I & & & 1 & WA & $\mu g / L$ \\
\hline Cadmium, total recoverable & $<1.0$ & $\mathbf{U} / \prime$ & $<1.0$ & & 1 & GE & $<1.0$ & $\mathrm{U} / /$ & $<$ & & 1 & GE & $\mu g / L$ \\
\hline Chromium, total recoverable & & & & & & & $<7.0$ & $\mathrm{U} / \prime$ & $<$ & & 1 & WA & $\mu g / L$ \\
\hline Cobalt, total recoverable & 0.64 & $\mathrm{~J} / \mathrm{E} /$ & NDD & & 5 & GE & & & & & & & $\mu g / L$ \\
\hline $\begin{array}{l}\text { Copper, total recoverable } \\
\text { Cyanide }\end{array}$ & 5.8 & $N I$ & & & 5 & GE & 3.3 & $\mathrm{~J} / \mathrm{I} /$ & NDD & & 1 & WA & $\mu g / L$ \\
\hline Lead, total recoverable & 4.0 & J/EV/ & NDD & & 5 & GE & 0.81 & $\mathrm{~J} / \mathrm{I} /$ & NDD & & 1 & GE & $\mu g / L$ \\
\hline Mercury, total recoverable & $<0.20$ & $\mathrm{U} / /$ & $<0.20$ & & 1 & GE & 1.2 & $/ /$ & & & 1 & GE & $\mu g / L$ \\
\hline $\begin{array}{l}\text { Nickel, total recoverable } \\
\text { Nitrate as nitrogen }\end{array}$ & $<2.1$ & UNI & $<1.0$ & & 5 & GE & $<26$ & $\mathrm{U} / /$ & $<$ & & 1 & WA & $\mu g^{\prime} L$ \\
\hline Nitrate-nitrite as nitrogen & 22 & $\|$ & & & 1 & WA & $<20$ & UNI & $<$ & & 1 & GE & $\mu g / L$ \\
\hline Selenium, total recoverable & & & & & & & $<66$ & $\mathrm{U} / /$ & $<$ & & 1 & WA & $\mu g / L$ \\
\hline Silver, total recoverable & & & & & & & $<5.0$ & $\mathrm{U} / /$ & $<$ & & 1 & WA & $\mu g h$ L. \\
\hline Thallium, total recoverable & $<13$ & $U / I$ & $<12.5$ & & 5 & GE & & & & & & & $\mu g / L$ \\
\hline Vanadium, total recoverable & 13 & $N I$ & & - & 5 & $\mathrm{GE}$ & & & & & & & $\mu g / 2$ \\
\hline Zinc, total recoverable & 12 & J/EV $/$ & NDD & & 5 & GE & $<53$ & $\mathrm{U} / /$ & $<$ & & 1 & WA & $\mu g / L$ \\
\hline \multicolumn{14}{|l|}{ Organic Constituents } \\
\hline Constituents & $\underline{3 Q 98}$ & Mod & Filt. & ST브 & $\underline{\mathrm{DF}}$ & $\underline{\text { Lab }}$ & 4Q98 & Mod & Filt. & ST 브 & $\underline{\mathrm{DF}}$ & Lab & Unit \\
\hline $\begin{array}{l}\text { Benzene } \\
\text { Bis(2-ethylhexyl) phthalate }\end{array}$ & & & & & & & $<5.0$ & $\mathrm{U} / \mathrm{I}$ & $<$ & & 1 & WA & $\mu g / L$ \\
\hline Dichloromethane & & & & & & & $<5.0$ & $\mathrm{U} / 1$ & $<$ & & 1 & WA & $\mu g / L$ \\
\hline Phenols & & & & & & & $<37$ & $\mathrm{U} / 1$ & $<$ & & 1 & WA & $\mu g / L$ \\
\hline Tetrachioroethylene & & & & & & & $<5.0$ & U/I & $<$ & & 1 & WA & $\mu g / L$ \\
\hline Trichloroethylene & & & & & & & $<5.0$ & $\mathrm{U} / 1$ & $<$ & & 1 & WA & $\mu g / L$ \\
\hline Trichlorofluoromethane & & & & & & & $<5.0$ & $\mathrm{U} / /$ & $<$ & & 1 & WA & \\
\hline
\end{tabular}

Notes:

- =xceeded holding time

- exceeded groundwater protection or monitoring constituent standard (See Appendix A.) 
WSRC-TR-99-00012

Unclassified

WELL HSB 85A (cont.)

Radioactive Constituents

Constituents

Americium-241

Cesium-137

Cobalt-60

Curium-242

Curium-243/244

Curium-245/246

Gross alpha

lodine-129

Nonvolatile beta

Plutonium-238

Plutonium-239/240

Radium, total alpha-emitting

Radium-226

Radium-228

Strontium-90

Technetium-99

Thorium-228

Thorium-230

Thorium-232

Sum of alphas

Sum of betas

Tritium

Uranium-233/234

Uraniurr-235

Uranium-238
$<1.5 \mathrm{E}-01$

บ/I

$1.9 \mathrm{E}+00 \quad \mathrm{~J} / \mathrm{l}$

$\mathrm{J} / \mathrm{I} /$

<-1.2E-01 U\} $/ /$

$<1.3 E+00$

$1 \mathrm{TM}<\begin{aligned} & 1.9 \mathrm{E}+00 \\ & <3.4 \mathrm{E}-02\end{aligned}$

U/I

$<6.4 \mathrm{E}-01$

NDD

$<1.1 E+00$

$<8.8 \mathrm{E}-01$

NDD
Notes:

- = exceeded holding time

= exceeded groundwater protection or monitoring constituent standard (See Appendix A.)

F-Area HWMF

C-198
$1 \mathrm{TM} \mathrm{PCi} / \mathrm{L}$

1 GE PCill

$1 \mathrm{TM} p \dot{\mathrm{Ci} / L}$

1 TM pCill

1 GE $\mathrm{PCH}$

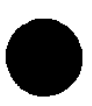

Third and Fourth Quarter 1998 


\begin{tabular}{|c|c|c|c|c|c|c|c|c|}
\hline UIC Permitted Constituents & & Reg & Jul-98 & Aug-98 & Sep-98 & Oct-98 & Nov-98 & Dec-98 \\
\hline Constituent & Unit & Limit & Result & Result & Result & Result & Result & Result \\
\hline \multicolumn{9}{|l|}{ Section I, INORGANICS } \\
\hline Arsenic & $\mu \mathrm{g} / \mathrm{L}$ & 50 & $<3$ & $<3$ & $<3$ & $<3$ & $<3$ & $<3$ \\
\hline Barium & $\mu g / L$ & 2000 & 15.70 & 13.90 & 9.49 & 16.30 & $<0.2$ & 25.30 \\
\hline Cadmium & $\mu g / L$ & 5 & $<1$ & $<1$ & $<1$ & $<1$ & $<1$ & $<1$ \\
\hline Chromium & $\mu \mathrm{g} / \mathrm{L}$ & 100 & $<3$ & $<3$ & $<3$ & $<3$ & $<3$ & $<3$ \\
\hline Lead & ! $\mu g / L$ & 150 & $<2$ & $<2$ & $<2$ & $<2$ & $<2$ & $<2$ \\
\hline Mercury & $\mu g / L$ & !2 & $<0.2$ & $<0.2$ & $<0.2$ & $<0,2$ & $<0.2$ & $<0.2$ \\
\hline Selenium & $\mu g / L$ & 50 & $<5$ & $<5$ & $<5$ & $<5$ & $<5$ & $<5$ \\
\hline Silver & $\mu g / L$ & 50 & $<1$ & $<1$ & $<1$ & $<1$ & $<1$ & $<1$ \\
\hline \multicolumn{9}{|l|}{ Section II, ORGANICS } \\
\hline Antimony & $\mu \mathrm{g} / \mathrm{L}$ & 6 & $<0.2$ & $<0.2$ & $<0.2$ & 0.23 & $<0.2$ & $<0.2$ \\
\hline Cobalt & $\mu g / L$ & 140 & 7.46 & 2.37 & $1 . \overline{81}$ & 1.33 & $<0.2$ & 3.53 \\
\hline Copper & $\mid \mu g / L$ & 11300 & 1.85 & 2.10 & 5.31 & 2.85 & $<0.2$ & 4.87 \\
\hline Cyanide & $\mu g / L$ & 200000 & $<10$ & $<10$ & $<10$ & $<1 \overline{0}$ & $<10$ & $<10$ \\
\hline Benzene & $\mu g / L$ & 5 & $<1$ & $<1$ & $<1$ & $<1$ & $<1$ & $<1$ \\
\hline BEHP & $\mu g / L$ & 140 & $<10.3$ & $<9.9$ & $<10.8$ & $<10$ & $<10$ & $<10.4$ \\
\hline Methylene Chloride & $\mu g / L$ & 15 & $<1$ & $<1$ & $<5$ & $<5$ & $<5$ & $<5$ \\
\hline Nickel & ! $\mathrm{g} / \mathrm{L}$ & 100 & 3.84 & 0.99 & 0.94 & $<0.2$ & $<0.2$ & 1.43 \\
\hline Phenol & $\operatorname{l\mu g} / \mathrm{L}$ & 110 & $<5$ & $<5$ & $<5$ & $<5$ & $<5$ & $<5$ \\
\hline Tetrachloroethylene & l $\mu g / L$ & 5 & $<1$ & $<1$ & $<1$ & $<1$ & $<1$ & $<1$ \\
\hline Thallium & $\mu g / L$ & 2 & $<2.5$ & $<2.5$ & $<2.5$ & $<2.5$ & $<2.5$ & $<2.5$ \\
\hline Tin & $\mid \mu g / L$ & $! 50$ & NA & NA & NA & NA & $\overline{\mathrm{NA}}$ & NA \\
\hline Trichloroethylene & $\mu g / L$ & $i 5$ & $<1$ & $<1$ & $<1$ & $<1$ & $<1$ & $<1$ \\
\hline Trichlorofluoromethane & $\mu g / L$ & 100 & $<1$ & $<1$ & $<5$ & $<5$ & $<5$ & $<5$ \\
\hline Vanadium & i $\mathrm{g} / \mathrm{L}$ & 149 & $<2$ & $<2$ & $<2$ & $<2$ & $<2$ & $<2$ \\
\hline Zinc & $\mu g / L$ & 5000 & $<5$ & $<5$ & $<5$ & $<5$ & $<5$ & $<5$ \\
\hline \multirow{2}{*}{\multicolumn{9}{|c|}{ Section III, RADIONUCLIDES }} \\
\hline & & & & & & & & \\
\hline Gross Alpha & $\frac{\mid \mathrm{pCi} / \mathrm{L}}{\mathrm{pCi} / \mathrm{L}}$ & 150 & 9.10 & $\begin{array}{l}4.23 \\
9.36\end{array}$ & $\frac{0.22}{1620}$ & $<12.13$ & 18.60 & $<16.15$ \\
\hline Gross Beta & & $\frac{100}{15}$ & 285 & 169 & 76.20 & 40.40 & 41.60 & $<16.29$ \\
\hline Total Radium $(226+228)$ & pCi/L. & 10 & $\mathbf{2 . 8 5}$ & 1.69 & 0.84 & BDL & BDL & BDL \\
\hline \multicolumn{9}{|l|}{ Section IV, RADIONUCLIDES } \\
\hline Americium-241 & $\mathrm{pCi} / \mathrm{L}$ & SOA & 0.55 & $<0.668$ & $<0.569$ & $<0.46$ & 3.54 & $<1$ \\
\hline Cesium-137 & $\mathrm{pCi} / \mathrm{L}$ & SOB & $<4.05$ & $<3.78$ & $<3.97$ & $<7 . \overline{51}$ & $<7.62$ & $<7.67$ \\
\hline Curium-242 & $\mathrm{IpCi} / \mathrm{L}$ & SOA & $<0.3$ & $<0.792$ & $<0.517$ & $<0.1874$ & $<0.287$ & $<0.198$ \\
\hline Curium-243/244 & $\mathrm{pCi} / \mathrm{L}$ & SOA & $<0.339$ & $\mathbf{R}$ & $<1$ & $<0.429$ & $<0.865$ & $<0.832$ \\
\hline Curium-246 & IpCilL & SOA & $<0.0994$ & $<0.516$ & $<0.127$ & $<0.2092$ & $<0.3343$ & $<0.191$ \\
\hline Carbon-14 & IpCi/L & SOB & $<16.6$ & $<16.8$ & 48.80 & 60.10 & $<20.35$ & 25.40 \\
\hline Cobalt-60 & $\mathrm{pCi} / \mathrm{L}$ & ISOB & $<3.59$ & $<2.47$ & $<4.07$ & $<7.17$ & $<7.36$ & $<8.27$ \\
\hline lodine-129 & IpCill & SOB & 46.60 & 64.30 & 58.20 & 22.80 & 97.50 & 32.40 \\
\hline Plutonium-238 & pCi/L & SOA & $<0.305$ & $\mathbf{R}$ & $\mathbf{R}$ & $<0.1508$ & $<0.456$ & $<0.626$ \\
\hline Plutonium-239/240 & $\rho \mathrm{Ci} / \mathrm{L}$ & ISOA & $<0.199$ & $\bar{R}$ & $<0.526$ & $<0.1491$ & $<0.1882$ & $<0 . \overline{441}$ \\
\hline Nickel-63 & $\mathrm{pCi} / \mathrm{L}$ & SOB & NA & NA & NA & NA & NA & NA \\
\hline Radium-226 & $\mathrm{pCi} / \mathrm{L}$ & SOR & 1.14 & 1.69 & 0.84 & $<1.609$ & $<1.946$ & $<1.412$ \\
\hline Radium-228 & $\overline{\mathrm{pCi} / L}$ & SOR & 1.71 & $<1.81$ & $<2.11$ & $<4.024$ & $<4 . \overline{97}$ & $<2.398$ \\
\hline Strontium-90 & $\mathrm{pCi} / \mathrm{L}$ & SOB & 14.90 & 9.59 & 9.30 & $<5.07$ & $<5.73$ & 5.09 \\
\hline Technetium-99 & $\mathrm{pCi} / \mathrm{L}$ & SOB & 11.30 & 25.60 & 25.40 & $R$ & $<42.1$ & 27.60 \\
\hline Thorium-228 & $\mathrm{pCi} / \mathrm{L}$ & SOA & $<0.212$ & $<1.12$ & 0.25 & $<0.511$ & $<0.503$ & $<0.661$ \\
\hline Thorium-230 & $\mathrm{pCi} / \mathrm{L}$ & SOA & $\mathbf{R}$ & $<0.43$ & $<0.087$ & $<0.355$ & $<0.377$ & $<0.518$ \\
\hline Thorium-232 & $\mathrm{pCi} / \mathrm{L}$ & SOA & $<0.254$ & $<0.365$ & $<0.03$ & $<0.2482$ & $<0.1838$ & $<0.0696$ \\
\hline Uranium-233/234 & $\mathrm{pCi} / \mathrm{L}$ & ISOA & 2.77 & 2.80 & 2.69 & 2.57 & 13.30 & 3.86 \\
\hline Uranium-234 & $\mathrm{pCi} / \mathrm{L}$ & SOA & NA & NA & NA & NA & NA & NA \\
\hline Uranium-235 & $\mathrm{PCi} / \mathrm{L}$ & SOA & $<0.0851$ & 0.70 & 0.49 & $<0.479$ & 0.41 & $<0.842$ \\
\hline Uranium-238 & $\mathrm{pCi} / \mathrm{L}$ & SOA & 5.15 & 4.17 & 3.99 & 4.87 & 6.83 & 6.70 \\
\hline Sum of Alphas & & 1 & 9.61 & 9.36 & 8.26 & 7.44 & 24.08 & 10.56 \\
\hline Sum of Betas & & 1 & 74.51 & 99.49 & 92.90 & 82.90 & 97.50 & 90.49 \\
\hline
\end{tabular}




\begin{tabular}{|c|c|c|c|c|c|c|c|c|c|}
\hline UIC Permitted Constituents & & TReg & Raw & Rad & & $i$ & July & Exceedence & \\
\hline Constituent & Unit & Limit & Result & Acc & Qual. & $\mathrm{QL}$ & Result & $\mathbf{Y} / \mathbf{N}$ & Notes \\
\hline Section I, INORGANICS & & - & & & & & & & $\begin{array}{l}\text { Samples collected } \\
7 / 30 / 98\end{array}$ \\
\hline Arsenic & $\mu g / L$ & 50 & 3 & & $\mathrm{U}$ & 3 & $<3$ & $\mathrm{~N}$ & \\
\hline Barium & $\mu g / L$ & 2000 & 15.7 & & & 0.2 & 15.70 & $\mathrm{~N}$ & \\
\hline Cadmium & $\mu \mathrm{g} / \mathrm{L}$ & 15 & 1 & & $\mathrm{U}$ & 1 & $<1$ & $\mathrm{~N}$ & \\
\hline Chromium & $\mu g / L$ & 100 & 3 & & $\mathrm{U}$ & 3 & $<3$ & $\mathrm{~N}$ & \\
\hline Lead & $\mu g / L$ & 50 & 2 & & $\bar{U}$ & 2 & $<2$ & N & \\
\hline Mercury & $\mu g / L$ & 2 & 0.2 & & $\bar{U}$ & 0.2 & $<0.2$ & $\mathrm{~N}$ & \\
\hline Selenium & $1 \mu \mathrm{g} / \mathrm{L}$ & 50 & 5 & & $U$ & 5 & $<5$ & $N$ & \\
\hline Silver & $\mu g / L$ & 50 & 1 & & $\mathrm{U}$ & 1 & $<1$ & $\mathbf{N}$ & \\
\hline Section II, ORGANICS & & & & & & & & . & \\
\hline Antimony & $\mu \mathrm{g} / \mathrm{L}$ & 76 & 0.2 & & U & 0.2 & $<0.2$ & $\bar{N}$ & \\
\hline Cobalt & $\mu g / L$ & 140 & 7.46 & 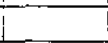 & & 0.2 & 7.46 & $\mathrm{~N}$ & \\
\hline Copper & $\mu g / L$ & 1300 & 1.85 & & & 0.2 & 1.85 & $\mathbf{N}$ & \\
\hline Cyanide & $\mu g / L$ & 200000 & 10 & & $\bar{U}$ & 10 & $<10$ & $\bar{N}$ & \\
\hline Benzene & $\mu \mathrm{g} / \mathrm{L}$ & 15 & 1 & & $\bar{U}$ & 1 & $<1$ & $\mathrm{~N}$ & \\
\hline $\mathrm{BEHP}$ & $\mu g / L$ & 140 & 10.3 & & $\mathrm{U}$ & 10.3 & $<10.3$ & $\mathrm{~N}$ & \\
\hline Methylene Chloride & $\operatorname{lng} / \mathrm{L}$ & 15 & 1 & & $\mathrm{U}$ & 1 & $<1$ & $N$ & \\
\hline Nickel & $\operatorname{l\mu g} / L$ & 100 & 3.84 & & & 0.2 & 3.84 & $\mathrm{~N}$ & \\
\hline Phenol & $\mu g / L$ & 10 & 5 & & $\mathrm{U}$ & 5 & $<5$ & $\mathbf{N}$ & \\
\hline Tetrachloroethylene & $\mu \mathrm{g} / \mathrm{L}$ & 5 & 1 & & $\mathrm{U}$ & 1 & $<1$ & $\mathrm{~N}$ & \\
\hline Thallium & $\mu g / L$ & 2 & 0.337 & & $\mathrm{~J}$ & 2.5 & $<2.5$ & & DL too high \\
\hline Trichloroethylene & $\mathrm{i \mu g} / \mathrm{L}$ & $! 5$ & 1 & & $\bar{U}$ & 1 & $<1$ & $N$ & \\
\hline Trichlorofluoromethane & $\operatorname{l\mu g} / \mathrm{L}$ & 100 & 1 & & $\mathrm{U}$ & 1 & $<1$ & $N$ & \\
\hline Vanadium & $\mu \mathrm{g} / \mathrm{L}$ & 49 & 2 & & $\mathrm{U}$ & 2 & $<2$ & $\mathbf{N}$ & \\
\hline Zinc & $\mu \mathrm{g} / \mathrm{L}$ & .5000 & 3.19 & & $J$ & 5 & $<5$ & $N$ & \\
\hline Section III, RADIONUCLIDES & & & & & & & & & \\
\hline Gross Alpha & $\mathrm{pCi} / \mathrm{L}$ & 15 & 3.1 & $0 . \overline{9} 99^{-1}$ & & 1.06 & 3.10 & $\mathrm{~N}$ & \\
\hline Gross Beta & $\mathrm{pCi} / \mathrm{L}$ & 50 & 9.73 & 1.06 & & 1.18 & 9.73 & $\mathbf{N}$ & \\
\hline Total Radium $(226+228)$ & $\mathrm{ipCi/L}$ & 15 & & & & & 2.85 & $\mathbf{N}$ & \\
\hline Section IV, RADIONUCLIDES & & & & & & & & & \\
\hline Americium-241 & $\mathrm{pCi} / \mathrm{L}$ & SOA & 0.547 & 0.29 & & 0.251 & 0.55 & $\mathrm{~N}$ & \\
\hline Cesium-137 & $\mathrm{pCi} / \mathrm{L}$ & SOB & 3.26 & 3.53 & UI & 4.05 & $<4.05$ & $\mathrm{~N}$ & \\
\hline Curium-242 & $1 \mathrm{pCi} / \mathrm{L}$ & 'SOA & 0.0196 & 0.107 & Ul & 0.3 & $<0.3$ & $\mathrm{~N}$ & \\
\hline Curium-243/244 & $\mathrm{ipCi} / \mathrm{L}$ & SOA & 0.285 & 0.229 & UI & 0.339 & $<0.339$ & $\mathrm{~N}$ & \\
\hline Curium-246 & $\mathrm{pCi} / \mathrm{L}$ & SOA & 0.0331 & 0.0665 & UI & 0.0994 & $<0.0994$ & $\mathrm{~N}$ & \\
\hline Carbon-14 & $\mathrm{pCi} / \mathrm{L}$ & $\mathrm{SOB}$ & -3.96 & 9.49 & UI & 16.6 & $<16.6$ & $\mathrm{~N}$ & \\
\hline Cobalt-60 & $\mathrm{pCi} / \mathrm{L}$ & ISOB & -0.972 & 2.09 & UI & 3.59 & $<3.59$ & $\mathrm{~N}$ & \\
\hline lodine-129 & ipCi/L & ISOB & 46.6 & 5.92 & & 0.769 & 46.60 & $N$ & \\
\hline Plutonium-238 & |pCill & SOA & -0.0452 & 0.0411 & UI & 0.305 & $<0.305$ & $\mathrm{~N}$ & \\
\hline Plutonium-239/240 & $p C i / 2$ & SOA & -0.009 & 0.0181 & UI & 0.199 & $<0.199$ & $\mathrm{~N}$ & \\
\hline Radium-226 & $\mathrm{pCi} / \mathrm{L}$ & SOR & 1.14 & 0.618 & & 0.237 & 1.14 & $N$ & \\
\hline Radium-228 & $\mathrm{pCi} / \mathrm{L}$ & SOR & 1.71 & 0.506 & & 0.867 & 1.71 & $\mathbf{N}$ & \\
\hline Strontium -90 & $\mathrm{pCi} / \mathrm{L}$ & ISOB & 14.9 & 2.42 & & 2.37 & 14.90 & $Y$ & \\
\hline Technetium-99 & $\mathrm{pCi} / \mathrm{L}$ & SOB & 11.3 & 3.77 & & 7.64 & 11.30 & $\bar{N}$ & \\
\hline Thorium-228 & $i p C i / L$ & SOA & 0.12 & 0.14 & UI & 0.212 & $<0.212$ & $\mathrm{~N}$ & \\
\hline Thorium-230 & $\mathrm{pCi} / \mathrm{L}$ & SOA & 0.322 & 0.235 & $\mathbf{R}$ & 0.289 & $\mathbf{R}$ & $\mathrm{N}$ & Rejected Analysis \\
\hline Thorium-232 & ipCi/l & SOA & -0.0323 & 0.0329 & UIJ & 0.254 & $<0.254$ & $\mathbf{N}$ & \\
\hline Uranium-233/234 & $1 \mathrm{pCi} / \mathrm{L}$ & SOA & 2.77 & 0.641 & & 0.229 & 2.77 & $\mathrm{~N}$ & \\
\hline Uranium-235 & $\mathrm{pC \textrm {i } / \mathrm { L }}$ & SOA & 0.113 & 0.114 & UI & 0.0851 & $<0.0851$ & $\mathrm{~N}$ & \\
\hline Uranium-238 & $\mathrm{pCi} / \mathrm{L}$ & SOA & 5.15 & 0.954 & & 0.196 & 5.15 & $\mathrm{~N}$ & \\
\hline Sum of Alphas & 5 & & & & & & 9.61 & $\mathrm{~N}$ & \\
\hline Sum of Betas & 1 & & & & & & 74.51 & $\bar{Y}$ & \\
\hline
\end{tabular}




\begin{tabular}{|c|c|c|c|c|c|c|c|c|c|}
\hline UIC Permitted Constituents & & Reg & Raw & Rad & & & August & Exceedence & \\
\hline Constituent & Unit & Limit & Result & Acc & Qual. & $\overline{Q L}$ & Result & $Y / N$ & Notes \\
\hline Section I, INORGANICS & & & & & & & & & $\begin{array}{l}\text { Samples collected } \\
8 / 13 / 98\end{array}$ \\
\hline Arsenic & $\mu g / L$ & 50 & 3 & & $U$ & 3 & $<3$ & $\mathrm{~N}$ & \\
\hline Barium & $\mu g / L$ & 2000 & 13.9 & & & 0.2 & 13.90 & $N$ & \\
\hline Cadmium & $\mu g / \mathrm{L}$ & 15 & 1 & & $\mathrm{U}$ & 1 & $<1$ & $\mathbf{N}$ & \\
\hline Chromium & $\mu g / L$ & $\overline{100}$ & 3 & & U & 3 & $<3$ & $\mathbf{N}$ & \\
\hline Lead & $\mu \mathrm{g} / \mathrm{L}$ & 50 & 2 & & $U$ & 2 & $<2$ & $\mathbf{N}$ & \\
\hline Mercury & $\mu g / L$ & 12 & 0.094 & & $\mathrm{~J}$ & 0.2 & $<0.2$ & $\mathrm{~N}$ & \\
\hline Selenium & $\mu g / L$ & 50 & 5 & & $\mathrm{U}$ & 5 & $<5$ & $N$ & \\
\hline Silver & $\mu \mathrm{g} / \mathrm{L}$ & 50 & 0.57 & & $U$ & 1 & $<1$ & $\mathrm{~N}$ & \\
\hline Section II, ORGANICS & & & & & & & 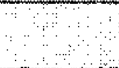 & 4 & 6 \\
\hline Antimony & $\mu g / L$ & 6 & 0.186 & & U & 0.2 & $<0.2$ & $\mathrm{~N}$ & \\
\hline Cobalt & $\mu \mathrm{\mu g} / \mathrm{L}$ & 140 & 2.37 & & & 0.2 & 2.37 & $\mathbf{N}$ & \\
\hline Copper & $\mu g / L$ & 1300 & 2.1 & & & 0.2 & 2.10 & $\mathbf{N}$ & \\
\hline Cyanide & $\mu g / L$ & 200000 & 10 & & $\mathrm{U}$ & 10 & $<40$ & $\mathbf{N}$ & \\
\hline Benzene & $\mu g / \mathrm{L}$ & 15 & 1 & & $U$ & 1 & $<1$ & $N$ & \\
\hline BEHP & $\mu g / L$ & 140 & 9.9 & & $U$ & 9.9 & $<9.9$ & $\mathrm{~N}$ & \\
\hline Methylene Chloride & $\mu g / L$ & 15 & 1 & & $\mathrm{U}$ & 1 & $<1$ & $\mathrm{~N}$ & \\
\hline Nickel & $\mu g / L$ & 1100 & 0.989 & & & 0.2 & 0.99 & $N$ & \\
\hline Phenol & $\mu g / L$ & 10 & 5 & & $\mathrm{U}$ & 5 & $<5$ & $N$ & \\
\hline Tetrachloroethylene & $\mu g / L$ & 5 & 1 & & $U$ & 1 & $<1$ & $N$ & \\
\hline Thallium & $\mu g / L$ & 2 & 0.655 & & U & 2.5 & $<2.5$ & & DL too high \\
\hline Trichloroethylene & $\mu \mathrm{g} / \mathrm{L}$ & 15 & 1 & & $\mathrm{U}$ & 1 & $<1$ & $\bar{N}$ & \\
\hline Trichlorofluoromethane & $\mu g / L$ & 100 & 1 & & $\mathrm{U}$ & 1 & $<1$ & $\mathrm{~N}$ & \\
\hline Vanadium & $\mu g / L$ & 49 & 2 & & $\bar{U}$ & 2 & $<2$ & $\bar{N}$ & \\
\hline Zinc & $\mu \mathrm{g} / \mathrm{L}$ & 5000 & 5 & & $u$ & 5 & $<5$ & $\mathrm{~N}$ & \\
\hline Section III, RADIONUCLIDES & & & & & & & & $:$ & \\
\hline Gross Alpha & $1 \mathrm{CCi} / \mathrm{L}$ & 15 & 4.23 & 1.12 & & 0.97 & 4.23 & $\mathbf{N}$ & \\
\hline Gross Beta & $\mathrm{pCi} / \mathrm{L}$ & 50 & 9.36 & 1 & & 1.06 & 9.36 & $\mathbf{N}$ & \\
\hline Total Radium $(226+228)$ & $\mathrm{pCi} / \mathrm{L}$ & 5 & & & & & 1.69 & $\mathrm{~N}$ & \\
\hline Section IV, RADIONUCLIDES & & & & & & & & & \\
\hline Americium-241 & $\overline{p C i} / L$ & $\widehat{S O A}$ & 0.419 & 0.435 & UI & 0.668 & $<0.668$ & $\mathrm{~N}$ & \\
\hline Cesium-137 & $\mathrm{pCi} / \mathrm{L}$ & ISOB & 0.0574 & 2.08 & UI & 3.78 & $<3.78$ & $\mathrm{~N}$ & \\
\hline Curium-242 & $\mathrm{IpCi} / \mathrm{L}$ & SOA & 0.59 & 0.566 & UI & 0.792 & $<0.792$ & $\mathbf{N}$ & \\
\hline Curium-243/244 & $\mathrm{pCi} / \mathrm{L}$ & SOA & 1.56 & 0.92 & $\mathbf{R}$ & 1.11 & $\mathbf{R}$ & $\hat{N}$ & Rejected Analysis \\
\hline Curium-246 & $\mathrm{pCi} / \mathrm{L}$ & ISOA & 0.289 & 0.361 & UI & 0.516 & $<0.516$ & $\mathbf{N}$ & \\
\hline Carbon-14 & $\mathrm{pCi} / \mathrm{L}$ & $\mathrm{SOB}$ & 14.5 & 10.2 & UI & 16.8 & $<16.8$ & $\mathbf{N}$ & \\
\hline Cobalt-60 & $\mathrm{pCi} / \mathrm{L}$ & SOB & -1.78 & 1.61 & UI & 2.47 & $<2.47$ & $N$ & \\
\hline lodine-129 & $\mathrm{pCi} / \mathrm{L}$ & SOB & 64.3 & 8.61 & & 1.62 & 64.30 & $\mathrm{~N}$ & \\
\hline Plutonium-238 & $\mathrm{ipCi} / \mathrm{L}$ & SOA & 1.75 & 0.381 & $R$ & 0.044 & $\mathbf{R}$ & $\mathrm{N}$ & Rejected Analysis \\
\hline Plutonium-239/240 & $\mathrm{pCi} / \mathrm{L}$ & SOA & 0.425 & 0.17 & $\mathrm{R}$ & 0.112 & $\mathbf{R}$ & $\mathrm{N}$ & Rejected Analysis \\
\hline Radium-226 & $\mid \mathrm{pCi} / \mathrm{L}$ & ISOR & 1.69 & 0.67 & & 0.562 & 1.69 & $\mathrm{~N}$ & \\
\hline Radium-228 & $\mathrm{pCi} / \mathrm{L}$ & SOR & -0.0164 & 0.791 & UI & 1.81 & $<1.81$ & $\mathbf{N}$ & \\
\hline Strontium-90 & $\mathrm{pCi} / \mathrm{L}$ & SOB & 9.59 & 2.07 & & 2.5 & 9.59 & $Y$ & \\
\hline Technetium-99 & $\mathrm{pCi} / \mathrm{L}$ & $\mathrm{SOB}$ & 25.6 & 4.78 & & 7.18 & 25.60 & $N$ & \\
\hline Thorium-228 & $i p C i / L$ & ISOA & 0.0929 & 0.464 & UI & 1.12 & $<1.12$ & $\mathrm{~N}$ & \\
\hline Thorium-230 & $\mathrm{pCi} / \mathrm{L}$ & SOA & 0.174 & 0.246 & U! & $0 . \overline{43}$ & $<0.43$ & $\mathrm{~N}$ & Rejected Analysis \\
\hline Thorium-232 & $\mathrm{pCi} / \mathrm{L}$ & SOA & 0.122 & 0.2 & UI & 0.365 & $<0.365$ & $\mathbf{N}$ & \\
\hline Uranium-233/234 & $\mathrm{pCi} / \mathrm{L}$ & SOA & 2.8 & 1.24 & & 0.595 & 2.80 & $\mathrm{~N}$ & \\
\hline Uranium-235 & $\mathrm{pCi} / \mathrm{L}$ & SOA & 0.7 & 0.613 & & 0.691 & 0.70 & $\mathbf{N}$ & \\
\hline Uranium-238 & $\mathrm{pCi} / \mathrm{L}$ & SOA & 4.17 & 1.54 & & 0.368 & 4.17 & $N$ & \\
\hline Sum of Alphas & & & & & & & 9.36 & $\mathbf{N}$ & \\
\hline Sum of Betas & & & & & & & 99.49 & $\mathrm{Y}$ & \\
\hline
\end{tabular}




\begin{tabular}{|c|c|c|c|c|c|c|c|c|c|}
\hline UIC Permitted Constituents & & Reg & Raw & Rad & & $i$ & September & Exceedence & \\
\hline Constituent & Unit & Limit & Result & $\overline{A c c}$ & Qual. & $\mathbf{Q L}$ & Result & $\mathbf{Y} / \mathbf{N}$ & Notes \\
\hline Section I, INORGANICS & & $\therefore$ & & & & $\therefore$ & 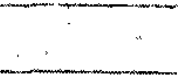 & ? & $\begin{array}{l}\text { Samples collected } \\
9 / 29 / 98\end{array}$ \\
\hline Arsenic & $\mu \mathrm{g} / \mathrm{L}$ & 50 & 3 & & $\mathrm{U}$ & 3 & $<3$ & $\mathrm{~N}$ & \\
\hline Barium & $\mu g / L$ & 2000 & 9.49 & & & 0.2 & 9.49 & $\mathrm{~N}$ & \\
\hline Cadmium & $\mu g / L$ & 5 & 1 & & U & 1 & $<1$ & $\bar{N}$ & \\
\hline Chromium & $\mu g / L$ & 100 & 9.03 & & $\mathrm{U}$ & 3 & $<3$ & $N$ & \\
\hline Lead & $\mu \mathrm{g} / \mathrm{L}$ & 50 & 2 & & $\mathrm{U}$ & 2 & $<2$ & $\mathrm{~N}$ & \\
\hline Mercury & $\mu g / L$ & 12 & 0.059 & & $\mathrm{~J}$ & 10.2 & $<0.2$ & $N$ & \\
\hline Selenium & $\mu \mathrm{g} / \mathrm{L}$ & 50 & 1.61 & & $\mathrm{U}$ & 5 & $<5$ & $N$ & \\
\hline Silver & $\mu g / L$ & 50 & 1 & 7 & $\mathrm{U}$ & 1 & $<1$ & $\mathrm{~N}$ & \\
\hline Section II, ORGANICS & & & . & & & & & & , \\
\hline Antimony & $\mu \mathrm{g} / \mathrm{L}$ & 6 & 0.2 & & $\mathrm{U}$ & 0.2 & $<0.2$ & $\mathrm{~N}$ & \\
\hline Cobalt & $\mu \mathrm{g} / \mathrm{L}$ & 140 & 1.81 & & & 0.2 & 1.81 & $\mathrm{~N}$ & \\
\hline Copper & $\mu \mathrm{g} / \mathrm{L}$ & 1300 & 5.31 & & & 0.2 & 5.31 & $N$ & \\
\hline Cyanide & $\mu g / L$ & 200000 & 10 & & $\bar{U}$ & 10 & $<10$ & $N$ & \\
\hline Benzene & $\mu g / L$ & 5 & 1 & & $U$ & 1 & $<1$ & $N$ & \\
\hline BEHP & $\mu \mathrm{H} / \mathrm{L}$ & 140 & 10.8 & & $\mathrm{U}$ & 10.8 & $<10.8$ & $N$ & \\
\hline Methylene Chloride & $\mu \mathrm{g} / \mathrm{L}$ & 5 & 5.3 & 1 & $\mathrm{U}$ & 5 & $<5$ & $N$ & \\
\hline Nickel & $\mu g / L$ & 100 & 0.941 & & & 0.2 & 0.94 & $\bar{N}$ & \\
\hline Phenol & $\mu \mathrm{g} / \mathrm{L}$ & 10 & 5 & 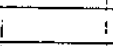 & $\mathrm{U}$ & 5 & $<5$ & $N$ & \\
\hline Tetrachloroethylene & $\mu g / L$ & 15 & 1 & & $\mathrm{U}$ & 1 & $<1$ & $\mathrm{~N}$ & \\
\hline Thallium & $\mu g / L$ & 12 & 0.341 & & $\mathrm{~J}$ & 2.5 & $<2.5$ & & DL too high \\
\hline Trichloroethylene & $\mu \mathrm{g} / \mathrm{L}$ & 5 & 0.841 & & $\mathrm{~J}$ & 1 & $<1$ & $\mathbf{N}$ & \\
\hline Trichlorofluoromethane & $\mu g / L$ & 100 & 5 & & $\mathrm{U}$ & 5 & $<5$ & $\mathrm{~N}$ & \\
\hline Vanadium & $\mu g / L$ & 149 & 2 & & $\mathrm{U}$ & 2 & $<2$ & $N$ & \\
\hline Zinc & $\mu \mathrm{g} / \mathrm{L}$ & 5000 & 5 & & $\underline{U}$ & 5 & $<5$ & $N$ & \\
\hline Section III, RADIONUCLIDES & & & & & & & & & \\
\hline Gross Alpha & $\mathrm{pCi} / \mathrm{L}$ & \15 & 6.22 & 2.08 & & 1.77 & 6.22 & $N$ & \\
\hline Gross Beta & $\mathrm{pCi} / \mathrm{L}$ & 50 & 16.2 & 1.89 & & 1.76 & 16.20 & $\mathrm{~N}$ & \\
\hline Total Radium $(226+228)$ & $\mathrm{pCi} / \mathrm{L}$ & 5 & & & & & 0.84 & $\mathbf{N}$ & \\
\hline Section IV, RADIONUCLIDES & & & & & & & & & \\
\hline Americium-241 & $i p C i / L$ & SOA & 0.468 & 0.354 & UI & 0.569 & $<0.569$ & $\mathrm{~N}$ & \\
\hline Cesium-137 & $\mathrm{pCi} / \mathrm{L}$ & ISOB & -0.133 & 2.25 & UI & 3.97 & $<3.97$ & $\mathbf{N}$ & \\
\hline Curium-242 & $\mathrm{pCi} / \mathrm{L}$ & SOA & 0.109 & 0.246 & UI & 0.517 & $<0.517$ & $N$ & \\
\hline Curium-243/244 & $\mid \mathrm{pCi} / \mathrm{L}$ & SOA & 0.351 & 0.523 & UI & 1 & $<1$ & $\mathbf{N}$ & Rejected Analysis \\
\hline Curium-246 & $\mathrm{pCi} / \mathrm{L}$ & SOA & 0 & 0 & UI & 0.127 & $<0.127$ & $\mathrm{~N}$ & \\
\hline Carbon-14 & $\mathrm{pCi} / \mathrm{L}$ & $\mathrm{SOB}$ & 48.8 & 6.53 & & 8.48 & 48.80 & N & \\
\hline Cobalt-60 & $\mathrm{pCi} / \mathrm{L}$ & SOB & 0.104 & 2.2 & Ui & 4.07 & $<4.07$ & N & \\
\hline lodine-129 & $\mathrm{pCi} / \mathrm{L}$ & iSOB & 58.2 & 8.09 & & 2.24 & 58.20 & $\bar{Y}$ & \\
\hline Plutonium-238 & $\mathrm{pCi} / \mathrm{L}$ & SOA & 0.897 & 0.619 & $\mathbf{R}$ & 0.299 & $\mathbf{R}$ & N & Rejected Analysis \\
\hline Plutonium-239/240 & $\mathrm{pCi} / \mathrm{L}$ & SOA & -0.0239 & 0.048 & U! & 0.526 & $<0.526$ & $N$ & Rejected Analysis \\
\hline Radium-226 & $\mathrm{pCi} / \mathrm{L}$ & SOR & 0.839 & 0.57 & & 0.618 & 0.84 & $N$ & \\
\hline Radium-228 & $\mathrm{pCi} / \mathrm{L}$ & ISOR & -1.7 & 0.821 & UI & 2.11 & $<2.11$ & $\mathrm{~N}$ & \\
\hline Strontium-90 & $\mathrm{pCi} / \mathrm{L}$ & SOB & 9.3 & 1.31 & & 1.42 & 9.30 & Y & \\
\hline Technetium-99 & pCill & SOB & $25.4 i$ & 4.35 & & 8.17 & 25.40 & $\mathrm{~N}$ & \\
\hline Thorium-228 & $\mathrm{pCi} / \mathrm{L}$ & ISOA & 0.253 & 0.124 & & 0.147 & 0.25 & $\mathrm{~N}$ & \\
\hline Thorium-230 & $\mathrm{pCi} / \mathrm{L}$ & SOA & 0.0449 & 0.0525 & UI & 0.087 & $<0.087$ & $\mathbf{N}$ & Rejected Analysis \\
\hline Thorium-232 & $\mathrm{pCi} / \mathrm{L}$ & SOA & 0.01 & 0.0201 & UI & 0.03 & $<0.03$ & $\mathrm{~N}$ & \\
\hline Uranium-233/234 & $\mathrm{pCi} / \mathrm{L}$ & SOA & 2.69 & 0.781 & & 0.593 & 2.69 & $\mathbf{N}$ & \\
\hline Uranium-235 & $\mathrm{pCi} / \mathrm{L}$ & SOA & 0.486 & 0.317 & & 0.339 & 0.49 & $\mathbf{N}$ & \\
\hline Uranium-238 & $\mathrm{PCi} / \mathrm{L}$ & SOA & 3.99 & 0.938 & & 0.278 & 3.99 & $\mathrm{~N}$ & \\
\hline Sum of Alphas & & & & & & & 8.26 & $N$ & \\
\hline Sum of Betas & & & & & & & 92.90 & $Y$ & \\
\hline
\end{tabular}




\begin{tabular}{|c|c|c|c|c|c|c|c|c|c|}
\hline UIC Permitted Constituents & & $\operatorname{Reg}$ & Raw & Rad & & I & October & Exceedence & \\
\hline Constituent & Unit & Limit & Result & Acc & Qual. & $\mathbf{Q L}$ & Result & $\mathbf{Y} / \mathbf{N}$ & Notes \\
\hline Section I, INORGANICS & & 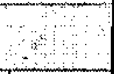 & & & & & & & $\begin{array}{l}\text { Samples collected } \\
10 / 19 / 98\end{array}$ \\
\hline Arsenic & $\mu \mathrm{g} / \mathrm{L}$ & 50 & 3 & & $\mathrm{U}$ & 3 & $<3$ & $\mathrm{~N}$ & \\
\hline Barium & $\mu \mathrm{g} / \mathrm{L}$ & 2000 & 16.3 & & & 0.2 & 16.30 & $\mathbf{N}$ & \\
\hline Cadmium & $\mu \mathrm{g} / \mathrm{L}$ & 5 & 1 & & $\mathrm{U}$ & 1 & $<1$ & $\mathrm{~N}$ & \\
\hline Chromium & $\mu \mathrm{g} / \mathrm{L}$ & 100 & 3 & & $\mathrm{U}$ & 3 & $<3$ & $N$ & \\
\hline Lead & $\mu \mathrm{g} / \mathrm{L}$ & 50 & 2 & 7 & $\mathrm{U}$ & 2 & $<2$ & $\mathbf{N}$ & \\
\hline Mercury & $\mu \mathrm{g} / \mathrm{L}$ & 2 & 0.2 & & $\mathrm{U}$ & 0.2 & $<0.2$ & $\mathbf{N}$ & \\
\hline Selenium & $\mu \mathrm{g} / \mathrm{L}$ & 50 & 5 & & U & 5 & $<5$ & $\mathbf{N}$ & \\
\hline Silver & $\mu \mathrm{g} / \mathrm{L}$ & 50 & 1 & 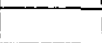 & $\mathrm{U}$ & 1 & $<1$ & $\bar{N}$ & \\
\hline Section II, ORGANICS & & & & 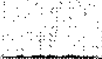 & & 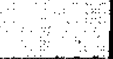 & 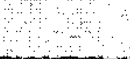 & 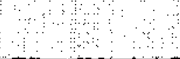 & \\
\hline Antimony & $\mu \mathrm{g} / \mathrm{L}$ & 76 & 0.226 & & & 0.2 & 0.23 & N & \\
\hline Cobalt & $\mu \mathrm{g} / \mathrm{L}$ & 1140 & 1.33 & & & 0.2 & 1.33 & $\mathrm{~N}$ & \\
\hline Copper & $\mathrm{i \mu g} / \mathrm{L}$ & 1300 & 2.85 & & & 0.2 & 2.85 & $\mathbf{N}$ & \\
\hline Cyanide & $\mu \mathrm{g} / \mathrm{L}$ & 200000 & 10 & & $U$ & 10 & $<10$ & $\mathbf{N}$ & \\
\hline Benzene & $\mu \mathrm{g} / \mathrm{L}$ & 15 & 1 & & U & 1 & $<1$ & $\mathbf{N}$ & \\
\hline BEHP & $\mid \mu g / L$ & 140 & 10 & & $\mathbf{U}$ & 10 & $<10$ & $\mathbf{N}$ & \\
\hline Methylene Chloride & $\mathrm{i} \mu \mathrm{g} / \mathrm{L}$ & 5 & 5 & & U & 5 & $<5$ & $\mathbf{N}$ & \\
\hline Nickel & $\mu \mathrm{g} / \mathrm{L}$ & 100 & 0.2 & & U & 0.2 & $<0.2$ & $\mathrm{~N}$ & \\
\hline Phenol & $\mu \mathrm{g} / \mathrm{L}$ & 10 & 5 & & $\bar{U}$ & 5 & $<5$ & $\mathrm{~N}$ & \\
\hline Tetrachloroethylene & $\mu \mathrm{g} / \mathrm{L}$ & 5 & 1 & & $\mathrm{U}$ & 1 & $<1$ & $\mathbf{N}$ & \\
\hline Thallium & $\mu \mathrm{g} / \mathrm{L}$ & 2 & 0.369 & & J & 2.5 & $<2.5$ & & DL too high \\
\hline Trichloroethylene & $\mu \mathrm{g} / \mathrm{L}$ & 5 & 0.912 & & $\mathrm{~J}$ & 1 & $<1$ & $N$ & \\
\hline Trichlorofluoromethane & $\mu \mathrm{g} / \mathrm{L}$ & 100 & 5 & & u & 5 & $<5$ & $\mathrm{~N}$ & \\
\hline Vanadium & $\mu g / L$ & 49 & 2 & & $\mathrm{U}$ & 2 & $<2$ & $N$ & \\
\hline Zinc & $\mu \mathrm{g} / \mathrm{L}$ & 5000 & 5 & & U & 5 & $<5$ & $\mathrm{~N}$ & \\
\hline Section III, RADIONUCLIDES & & & & & & & & & \\
\hline Gross Alpha & $\mathrm{pCi} / \mathrm{L}$ & 115 & 9.94 & 3.7 & $\mathrm{~J}$ & 12.13 & $<12.13$ & $\bar{N}$ & \\
\hline Gross Beta & $\mid p \mathrm{Ci} / \mathrm{L}$ & 50 & 40.4 & 5.98 & & 20.11 & 40.40 & $\mathbf{N}$ & \\
\hline Total Radium $(226+228)$ & $\mathrm{PCi} / \mathrm{L}$ & 5 & & & & & BDL & $\mathbf{N}$ & \\
\hline Section IV, RADIONUCLIDES & & & & & & & & & \\
\hline Americium-241 & $\mathrm{pCi} / \mathrm{L}$ & SOA & 0.168 & 0.146 & JI & 0.46 & $<0.46$ & $\mathrm{~N}$ & \\
\hline Cesium-137 & $\mathrm{pCi} / \mathrm{L}$ & SOB & -0.802 & 2.01 & $\mathrm{U}$ & 7.51 & $<7.51$ & $\mathrm{~N}$ & \\
\hline Curium-242 & $\mathrm{pCi} / \mathrm{L}$ & ISOA & -0.0075 & 0.0107 & $U$ & 0.1874 & $<0.1874$ & $\mathrm{~N}$ & \\
\hline Curium-243/244 & $\mathrm{pCi} / \mathrm{L}$ & SOA & 0.0645 & 0.106 & $\mathrm{U}$ & 0.429 & $<0.429$ & $\mathrm{~N}$ & \\
\hline Curium-246 & $\mathrm{pCi} / \mathrm{L}$ & SOA & 0.0298 & 0.0599 & U & 0.2092 & $<0.2092$ & $\mathrm{~N}$ & \\
\hline Carbon-14 & $1 \mathrm{pCi} / \mathrm{L}$ & ISOB & 60.1 & 10.5 & & 35.9 & 60.10 & $\bar{Y}$ & \\
\hline Cobalt-60 & $1 \mathrm{pCi} / \mathrm{L}$ & SOB & -0.0057 & 1.83 & $\mathrm{U}$ & 7.17 & $<7.17$ & $\mathrm{~N}$ & \\
\hline lodine-129 & $\mathrm{pCi} / \mathrm{L}$ & SOB & 22.8 & 3.46 & & 8.23 & 22.80 & $\mathrm{~N}$ & \\
\hline Plutonium-238 & $\mid \mathrm{pCi} / \mathrm{L}$ & SOA & -0.0152 & 0.0154 & $\mathrm{U}$ & 0.1508 & $<0.1508$ & $\mathrm{~N}$ & \\
\hline Plutonium-239/240 & $\mathrm{pCi} / \mathrm{L}$ & SOA & 0.0121 & 0.0327 & $U$ & 0.1491 & $<0.1491$ & $\mathbf{N}$ & \\
\hline Radium-226 & $\mathrm{pCi} / \mathrm{L}$ & ISOR & 0.751 & 0.493 & JI & 1.609 & $<1.609$ & $\overline{\mathbf{N}}$ & \\
\hline Radium-228 & $\mathrm{pCi} / \mathrm{L}$ & ISOR & 0.915 & 0.784 & $\mathrm{U}$ & 4.024 & $<4.024$ & $\mathrm{~N}$ & \\
\hline Strontium-90 & $\mathrm{IpCi/L}$ & ISOB & 2.51 & 1.29 & Ji & 5.07 & $<5.07$ & $\mathrm{~N}$ & \\
\hline Technetium-99 & $\mathrm{IpC \textrm {i } / \mathrm { L }}$ & ISOB & 25.2 & 5.49 & $R$ & 19.98 & $\mathbf{R}$ & $\mathrm{N}$ & Rejected Analysis \\
\hline Thorium-228 & $\mathrm{pCi} / \mathrm{L}$ & SOA & 0.0139 & 0.105 & $\mathrm{U}$ & 0.511 & $<0.511$ & $\mathbf{N}$ & \\
\hline Thorium-230 & pCi/L & SOA & 0.0791 & 0.102 & U & 0.355 & $<0.355$ & $\mathrm{~N}$ & \\
\hline Thorium-232 & $\mathrm{pCi} / \mathrm{L}$ & SOA & 0.0252 & 0.0581 & U & 0.2482 & $<0.2482$ & $\mathbf{N}$ & \\
\hline Uranium-233/234 & $\mathrm{pCi} / \mathrm{L}$ & ISOA & 2.57 & 0.659 & & 1.454 & 2.57 & $\mathrm{~N}$ & \\
\hline Uranium-235 & $\mathrm{pCi} / \mathrm{L}$ & !SOA & 0.233 & 0.171 & $\mathrm{Jl}$ & 0.479 & $<0.479$ & $N$ & \\
\hline Uranium-238 & $\mathrm{pCi} / \mathrm{L}$ & SOA & 4.87 & 1.02 & & 2.1286 & 4.87 & $N$ & \\
\hline Sum of Alphas & & 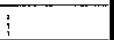 & & & & & 7.44 & $\mathbf{N}$ & \\
\hline Sum of Betas & $:$ & & & 7 & & & 82.90 & $\bar{Y}$ & \\
\hline
\end{tabular}




\begin{tabular}{|c|c|c|c|c|c|c|c|c|c|}
\hline \multirow{2}{*}{\begin{tabular}{|l|} 
UIC Permitted Constituents \\
Constituent \\
\end{tabular}} & & $\operatorname{Reg}$ & Raw & Rad & & & November & Exceedence & \multirow{3}{*}{\begin{tabular}{|l|} 
Notes \\
Samples collected \\
$11 / 17 / 98$
\end{tabular}} \\
\hline & Unit & Limit & Resuit & Acc & Qual. & $\mathbf{Q L}$ & Result & Y/N & \\
\hline Section I, INORGANICS & & \multicolumn{4}{|c|}{ (2) } & $\bullet$ & $\therefore$ & \\
\hline Arsenic & $\mu g / L$ & 50 & 3 & & $\mathrm{U}$ & 3 & $<3$ & $N$ & \\
\hline Barium & $\mu g / L$ & 2000 & 0.2 & i & $\mathrm{U}$ & 0.2 & $<0.2$ & $\bar{N}$ & \\
\hline Cadmium & $! \mathrm{gg} / \mathrm{L}$ & 5 & 1 & & $\mathrm{U}$ & 1 & $<1$ & $\bar{N}$ & \\
\hline Chromium & $\mu g / L$ & 100 & 2.46 & 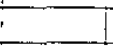 & $U$ & 3 & $<3$ & $\mathrm{~N}$ & \\
\hline Lead & $\mu g / L$ & 150 & 2 & & 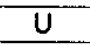 & 2 & $<2$ & $\mathrm{~N}$ & \\
\hline Mercury & $\mu \mathrm{\mu g} / \mathrm{L}$ & 12 & 0.0665 & & $\mathrm{~J}$ & 0.2 & $<0.2$ & $\mathrm{~N}$ & \\
\hline Selenium & $\mathrm{\mu g} / \mathrm{L}$ & 50 & 5 & 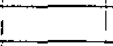 & $\mathrm{U}$ & 5 & $<5$ & $\mathrm{~N}$ & \\
\hline Silver & $\mu g / L$ & 50 & 1 & & $U$ & 1 & $<1$ & $\mathbf{N}$ & \\
\hline \multicolumn{3}{|l|}{ Section II, ORGANICS } & & & & & & & \\
\hline Antimony & $\mu g / L$ & 6 & 0.2 & & $\mathrm{U}$ & 0.2 & $<0.2$ & $\mathrm{~N}$ & \\
\hline Cobalt & $\mu g / L$ & 140 & 0.031 & & $\mathrm{U}$ & 0.2 & $<0 . \overline{2}$ & $\mathrm{~N}$ & \\
\hline Copper & $\mu g / L$ & 1300 & 0.2 & & $\mathrm{U}$ & 0.2 & $<0.2$ & $\mathbf{N}$ & \\
\hline Cyanide & $\mu \mathrm{g} / \mathrm{L}$ & 200000 & 10 & & $\mathrm{U}$ & 10 & $<10$ & $N$ & \\
\hline Benzene & $\mu \mathrm{g} / \mathrm{L}$ & 15 & 1 & & UJ & 1 & $<1$ & $N$ & \\
\hline BEHP & $\mid \mu g / L$ & 140 & 10 & & $\mathrm{U}$ & 10 & $<10$ & $\mathrm{~N}$ & \\
\hline Methylene Chloride & $\mu g / L$ & 5 & 5 & & UJ & 5 & $<5$ & $\mathbf{N}$ & \\
\hline Nickel & $\mu g / L$ & 100 & 0.2 & & $U$ & 0.2 & $<0.2$ & $\mathbf{N}$ & \\
\hline Phenol & $\mu g / L$ & 10 & 5 & & $\mathrm{U}$ & 5 & $<5$ & $\mathrm{~N}$ & \\
\hline Tetrachloroethylene & $\mu g / L$ & 5 & 1 & & UJ & 1 & $<1$ & $\mathrm{~N}$ & \\
\hline Thallium & $\mu g / L$ & 2 & 0.092 & & $\mathrm{U}$ & 2.5 & $<2.5$ & & DL too high \\
\hline Trichloroethylene & $\mu \mathrm{g} / \mathrm{L}$ & 15 & 0.93 & & $J$ & 1 & $<1$ & $\mathrm{~N}$ & \\
\hline Trichlorofluoromethane & $\mu \mathrm{g} / \mathrm{L}$ & 100 & 5 & & UJ & 5 & $<5$ & $\mathrm{~N}$ & \\
\hline Vanadium & $\mu g / L$ & 149 & 2 & & U & 2 & $<2$ & $\mathrm{~N}$ & \\
\hline Zinc & $\mu \mathrm{g} / \mathrm{L}$ & 5000 & 5 & & U & 5 & $<5$ & $N$ & \\
\hline \multicolumn{3}{|l|}{ Section III, RADIONUCLIDES } & & & & & & & \\
\hline Gross Alpha & $\mathrm{pCi} / \mathrm{L}$ & 15 & 18.6 & 4.72 & & 12.73 & 18.60 & $\bar{Y}$ & \\
\hline Gross Beta & $\mathrm{pCi} / \mathrm{L}$ & 50 & 41.6 & 4.15 & & 11.83 & 41.60 & $\mathrm{~N}$ & \\
\hline Total Radium $(226+228)$ & $\mathrm{pCi} / \mathrm{L}$ & 15 & & & & & $\mathrm{BDL}$ & $N$ & \\
\hline \multicolumn{3}{|l|}{ Section IV, RADIONUCLIDES } & & & & & & & \\
\hline Americium-241 & pCin & SOA & 3.54 & 0.869 & J & 1.904 & 3.54 & $\mathrm{~N}$ & \\
\hline Cesium-137 & $\mathrm{pCi} / \mathrm{t}$ & SOB & -0.409 & 2.03 & $\mathrm{U}$ & 7.62 & $<7.62$ & $\mathrm{~N}$ & \\
\hline Curium-242 & $\mathrm{pCi} / \mathrm{L}$ & SOA & -0.0424 & 0.0385 & U & 0.287 & $<0.287$ & $\mathrm{~N}$ & \\
\hline Curium-243/244 & $\mathrm{pCi} / \mathrm{L}$ & SOA & 0.78 & 0.298 & $\mathrm{JI}$ & 0.865 & $<0.865$ & $\mathrm{~N}$ & \\
\hline Curium-246 & IpCi/h & SOA & 0.208 & 0.135 & Jl & 0.3343 & $<0.3343$ & $\mathrm{~N}$ & \\
\hline Carbon-14 & $\mathrm{pCi} / \mathrm{L}$ & ISOB & 12.6 & 5.66 & J & 20.35 & $<20.35$ & $\mathrm{~N}$ & \\
\hline Cobalt-60 & $\mathrm{PCi} / \mathrm{L}$ & SOB & 0.032 & 1.9 & U & 7.36 & $<7.36$ & $N$ & \\
\hline lodine-129 & $\mathrm{pCi} / \mathrm{L}$ & SOB & 97.5 & 12.5 & & 26.84 & 97.50 & $\bar{Y}$ & \\
\hline Plutonium-238 & $\mathrm{pCCi/L}$ & SOA & 0.345 & 0.156 & $\mathrm{U}$ & 0.456 & $<0.456$ & $\mathrm{~N}$ & \\
\hline Plutonium-239/240 & ipcin & SOA & 0.0128 & 0.0411 & $\mathrm{U}$ & 0.1882 & $<0.1882$ & $\mathrm{~N}$ & \\
\hline Radium-226 & $\mathrm{pCi} / \mathrm{L}$ & SOR & 1.9 & 0.697 & U & 1.946 & $<1.946$ & $\mathrm{~N}$ & \\
\hline Radium-228 & $\mid \mathrm{pCi} / \mathrm{L}$ & SOR & -0.795 & 1.16 & $U$ & 4.97 & $<4.97$ & $\mathrm{~N}$ & \\
\hline Strontium-90 & $\mathrm{pCi} / \mathrm{L}$ & $\mathrm{SOB}$ & 12 & 1.77 & $\mathrm{~J}$ & 5.73 & $<5.73$ & $N$ & \\
\hline Technetium-99 & $! \mathrm{pCi} / \mathrm{L}$ & SOB & 28.7 & 10.6 & $\mathrm{~J}$ & 42.1 & $<42.1$ & $\mathrm{~N}$ & \\
\hline Thorium-228 & $\mathrm{pCi} / \mathrm{L}$ & ISOA & 0.0343 & 0.115 & $\mathrm{U}$ & 0.503 & $<0.503$ & $\mathrm{~N}$ & \\
\hline Thorium-230 & $\mathrm{pCi} / \mathrm{L}$ & SOA & 0.211 & 0.137 & $\mathrm{~J}$ & 0.377 & $<0.377$ & $\mathrm{~N}$ & \\
\hline Thorium-232 & $\mathrm{pCi} / \mathrm{L}$ & SOA & 0.0149 & 0.04041 & $U$ & 0.1838 & $<0.1838$ & $N$ & \\
\hline Uranium-233/234 & pCill & SOA & 13.3 & 3.57 & & 8.29 & 13.30 & $\mathrm{~N}$ & \\
\hline Uranium-235 & $\mathrm{pCi} / \mathrm{L}$ & ISOA & 0.412 & 0.111 & & 0.2416 & 0.41 & $\mathrm{~N}$ & \\
\hline Uranium-238 & $\mathrm{pCi} / \mathrm{L}$ & SOA & 6.83 & 0.781 & & 1.618 & 6.83 & $\mathrm{~N}$ & \\
\hline Sum of Alphas & 1 & & & & 1 & 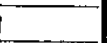 & 24.08 & $\bar{Y}$ & \\
\hline Sum of Betas & & & & & & & 97.50 & $\bar{Y}$ & \\
\hline
\end{tabular}




\begin{tabular}{|c|c|c|c|c|c|c|c|c|c|}
\hline \multicolumn{2}{|c|}{ UIC Permitted Constituents } & \multirow{2}{*}{\begin{tabular}{|l|} 
Reg \\
Limit \\
\end{tabular}} & \multirow{2}{*}{\multicolumn{2}{|c|}{\begin{tabular}{|c|c|} 
Raw & Rad \\
Result & Acc \\
\end{tabular}}} & \multirow[b]{2}{*}{ Qual. } & \multirow[b]{2}{*}{$\mathbf{Q L}$} & \multirow{2}{*}{$\begin{array}{c}\text { December } \\
\text { Result } \\
\end{array}$} & \multirow{2}{*}{$\frac{\text { Exceedence }}{\mathrm{Y} / \mathrm{N}}$} & \multirow[b]{2}{*}{ Notes } \\
\hline Constituent & Unit & & & & & & & & \\
\hline Section I, INORGANICS & & & & & & 8 & & 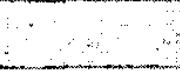 & $\begin{array}{l}\text { Samples collected } \\
12 / 21 / 98\end{array}$ \\
\hline Arsenic & $\mu \mathrm{g} / \mathrm{L}$ & 50 & 3 & & $\mathrm{U}$ & 3 & $<3$ & $\mathrm{~N}$ & \\
\hline Barium & $\mu g / \mathrm{L}$ & 2000 & 25.3 & & & 0.2 & 25.30 & $\mathrm{~N}$ & \\
\hline Cadmium & $\mu g / L$ & 5 & 1 & & U & 1 & $<1$ & $\mathbf{N}$ & \\
\hline Chromium & $\mu g / L$ & 100 & 3 & & $\mathrm{U}$ & 3 & $<3$ & $\mathbf{N}$ & \\
\hline Lead & $\mu g / L$ & 150 & 1.04 & & Jl & 2 & $<2$ & $\mathrm{~N}$ & \\
\hline Mercury & $\mu g / L$ & 2 & 0.111 & & JI & 0.2 & $<0.2$ & $\mathrm{~N}$ & \\
\hline Selenium & $\mu \mathrm{g} / \mathrm{L}$ & 50 & 5 & & $\mathrm{U}$ & 5 & $<5$ & $\mathrm{~N}$ & \\
\hline Silver & $\mu \mathrm{g} / \mathrm{L}$ & 50 & 1 & & $\mathrm{U}$ & 1 & $<1$ & $N$ & \\
\hline \multicolumn{10}{|l|}{ Section II, ORGANICS } \\
\hline Antimony & $\mu \mathrm{g} / \mathrm{L}$ & 6 & 0.2 & & $\mathrm{U}$ & 0.2 & $<0.2$ & $\mathrm{~N}$ & \\
\hline Cobalt & $\mu \mathrm{g} / \mathrm{L}$ & 140 & 3.53 & & & 0.2 & 3.53 & $N$ & \\
\hline Copper & $\mu g / L$ & 1300 & 4.87 & & & 0.2 & 4.87 & $\mathrm{~N}$ & \\
\hline Cyanide & $\mu g / \mathrm{L}$ & 200000 & 10 & & $\bar{U}$ & 10 & $<10$ & $\mathbf{N}$ & \\
\hline Benzene & $\operatorname{lng} / \mathrm{L}$ & 5 & 1 & & $\mathrm{U}$ & 1 & $<1$ & $N$ & \\
\hline $\mathrm{BEHP}$ & $\mu g / L$ & 140 & 10.4 & & $\mathrm{UJ}$ & 10.4 & $<10.4$ & $\mathbf{N}$ & \\
\hline Methylene Chloride & $\mu g / L$ & 5 & 5 & & $U$ & 5 & $<5$ & $N$ & \\
\hline Nickel & $\mu \mathrm{g} / \mathrm{L}$ & 100 & 1.43 & & & 0.2 & 1.43 & $\mathrm{~N}$ & \\
\hline Phenol & i $\mu g / L$ & 10 & 5 & & $\bar{U}$ & 5 & $<5$ & $N$ & \\
\hline Tetrachloroethylene & $\mu g / L$ & 5 & 1 & & $\mathrm{U}$ & 1 & $<1$ & $\mathbf{N}$ & \\
\hline Thallium & $\mu g / L$ & 2 & 0.244 & & $\bar{U}$ & 2.5 & $<2.5$ & & DL too high \\
\hline Trichloroethylene & $\mu \mathrm{g} / \mathrm{L}$ & 5 & 1 & & $\bar{U}$ & 1 & $<1$ & $\mathbf{N}$ & \\
\hline Trichlorofluoromethane & $\mu \mathrm{g} / \mathrm{L}$ & 100 & 5 & & $\bar{U}$ & 5 & $<5$ & $\mathbf{N}$ & \\
\hline Vanadium & $\mu g / L$ & 49 & 2 & & $\mathrm{U}$ & 2 & $<2$ & $\mathbf{N}$ & \\
\hline Zinc & $\mu \mathrm{g} / \mathrm{L}$ & 5000 & 5 & & U & 5 & $<5$ & $\mathbf{N}$ & \\
\hline \multicolumn{10}{|c|}{ Section III, RADIONUCLIDES } \\
\hline Gross Alpha & $\mathrm{pCi} / \mathrm{L}$ & 15 & 9.56 & 5.02 & ل & 16.15 & $<16.15$ & $\mathrm{~N}$ & $\begin{array}{l}\text { SsEQL too high, } \\
\text { result OK }\end{array}$ \\
\hline Gross Beta & $\mathrm{pCi} / \mathrm{L}$ & 50 & 15.5 & 4.66 & $\mathrm{JI}$ & 16.29 & $<16.29$ & $\mathrm{~N}$ & \\
\hline Total Radium $(226+228)$ & $\mathrm{pCi} / \mathrm{L}$ & 5 & & & & & BDL & $\mathbf{N}$ & \\
\hline \multicolumn{10}{|c|}{ Section IV, RADIONUCLIDES } \\
\hline Americium-241 & $\mathrm{pCi} / \mathrm{L}$ & ISOA & 0.255 & 277 & $\mathrm{U}$ & 1 & $<1$ & $\mathrm{~N}$ & \\
\hline Cesium-137 & $\mathrm{pCi} / \mathrm{L}$ & SOB & 1.33 & 2.41 & U & 7.67 & $<7.67$ & $N$ & \\
\hline Curium-242 & $\mathrm{pCi} / \mathrm{L}$ & SOA & 0 & 0 & $\mathrm{U}$ & 0.198 & $<0.198$ & $\mathrm{~N}$ & \\
\hline Curium-243/244 & $\mathrm{pCi} / \mathrm{L}$ & SOA & 0.383 & 0.32 & JI & 0.832 & $<0.832$ & $\mathrm{~N}$ & \\
\hline Curium-246 & $\mathrm{pCi} / \mathrm{L}$ & SOA & 0 & 0 & $U$ & 0.191 & $<0.191$ & $\mathbf{N}$ & \\
\hline Carbon-14 & $\mathrm{pCi} / \mathrm{L}$ & SOB & 25.4 & 5.43 & & 18.27 & 25.40 & $\mathbf{N}$ & \\
\hline Cobalt-60 & $\mathrm{pCi} / \mathrm{L}$ & SOB & 0.762 & 2.18 & $\mathrm{U}$ & 8.27 & $<8.27$ & $\mathbf{N}$ & \\
\hline lodine-129 & $\mathrm{pCi} / \mathrm{L}$ & SOB & 32.4 & 5.1 & & 12.24 & 32.40 & $\mathbf{N}$ & \\
\hline Plutonium-238 & $\mathrm{pCi} / \mathrm{L}$ & SOA & 0.0156 & 0.12 & U & 0.626 & $<0.626$ & $\mathbf{N}$ & \\
\hline Plutonium-239/240 & ipCi/L & ISOA & 0.0704 & 0.115 & $u$ & 0.441 & $<0.441$ & $N$ & \\
\hline Radium-226 & $\mathrm{pCi} / \mathrm{L}$ & SOR & 1.2 & 0.513 & JI & 1.412 & $<1.412$ & $N$ & \\
\hline Radium-228 & $\mathrm{pCi} / \mathrm{L}$ & SOR & -2.23 & 0.619 & $\mathrm{U}$ & 2.398 & $<2.398$ & $N$ & \\
\hline Strontium-90 & $\mathrm{pCi} / \mathrm{L}$ & $\mathrm{SOB}$ & 5.09 & 0.924 & & 3.158 & 5.09 & $\mathrm{~N}$ & \\
\hline Technetium-99 & $\mathrm{pCi} / \mathrm{L}$ & SOB & 27.6 & 6.75 & & 25.6 & 27.60 & $\mathrm{~N}$ & \\
\hline Thorium-228 & $1 \mathrm{pCi} / \mathrm{L}$ & SOA & -0.107 & $0 . \overline{136}$ & $U$ & 0.661 & $<0.661$ & $\mathbf{N}$ & \\
\hline Thorium-230 & $\mathrm{pCi} / \mathrm{L}$ & ISOA & 0.255 & $0 . \overline{17}$ & $U$ & 0.518 & $<0.518$ & $\mathrm{~N}$ & \\
\hline Thorium-232 & pCill & SOA & 0 & 0 & $U$ & 0.0696 & $<0.0696$ & $N$ & \\
\hline Uranium-233/234 & $1 \mathrm{pCi} / \mathrm{L}$ & SOA & 3.86 & 0.844 & & 2.013 & 3.86 & $N$ & \\
\hline Uranium-235 & $\mathrm{pCi} / \mathrm{L}$ & ISOA & 0.639 & 0.306 & $\mathrm{JI}$ & 0.842 & $<0.842$ & $\mathrm{~N}$ & \\
\hline Uranium-238 & $\mathrm{pCi} / \mathrm{L}$ & ISOA & 6.7 & 1.21 & & 2.519 & 6.70 & $\mathbf{N}$ & \\
\hline Sum of Alphas & & & & & & & 10.56 & $\mathrm{~N}$ & \\
\hline Sum of Betas & & $\vdots$ & & & & & 90.49 & $\bar{Y}$ & \\
\hline
\end{tabular}


WSRC-TR-99-00012

Unclassified

\section{Appendix D}

Time Series Plots 
THIS PAGE LEFT BLANK INTENTIONALLY. 

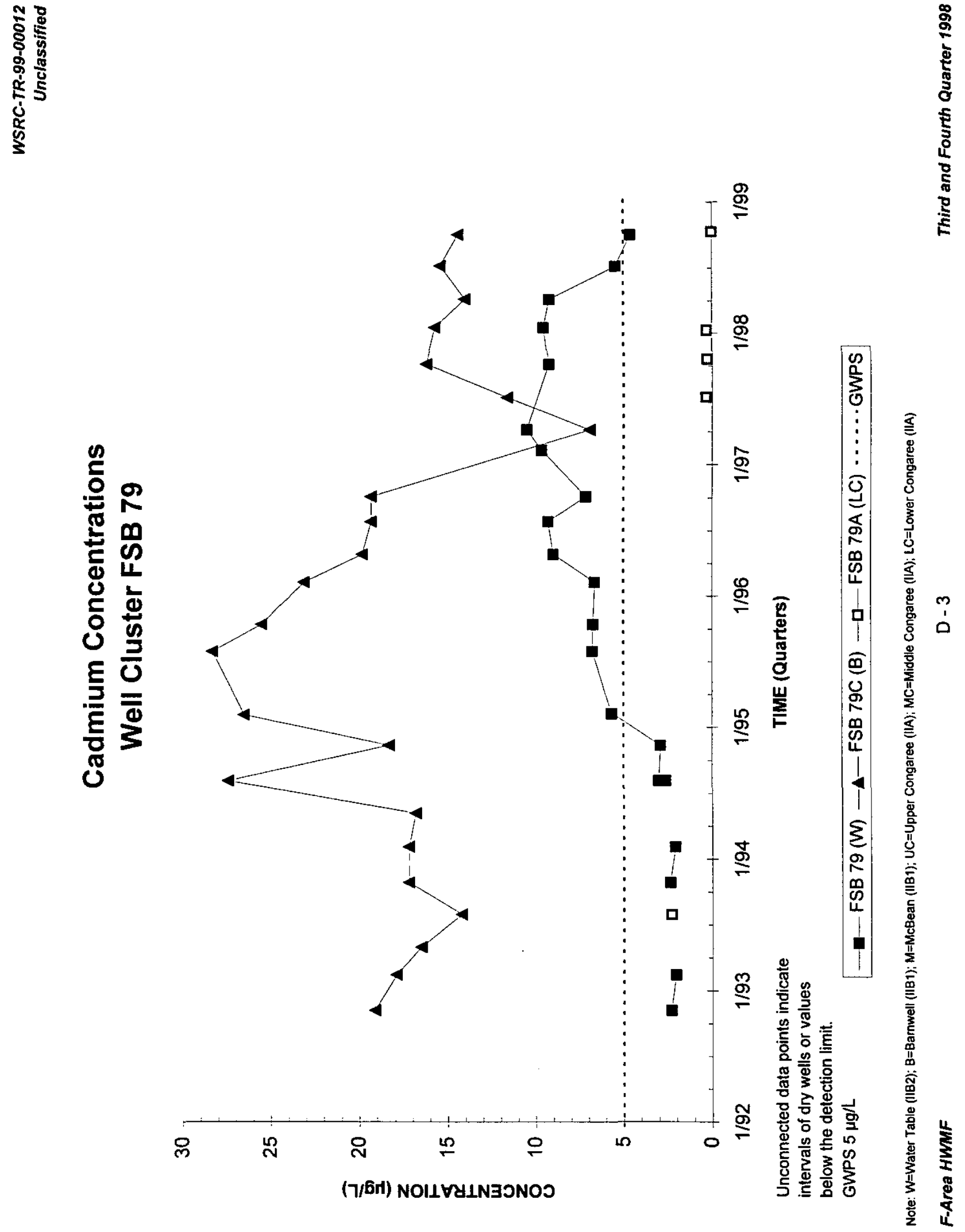


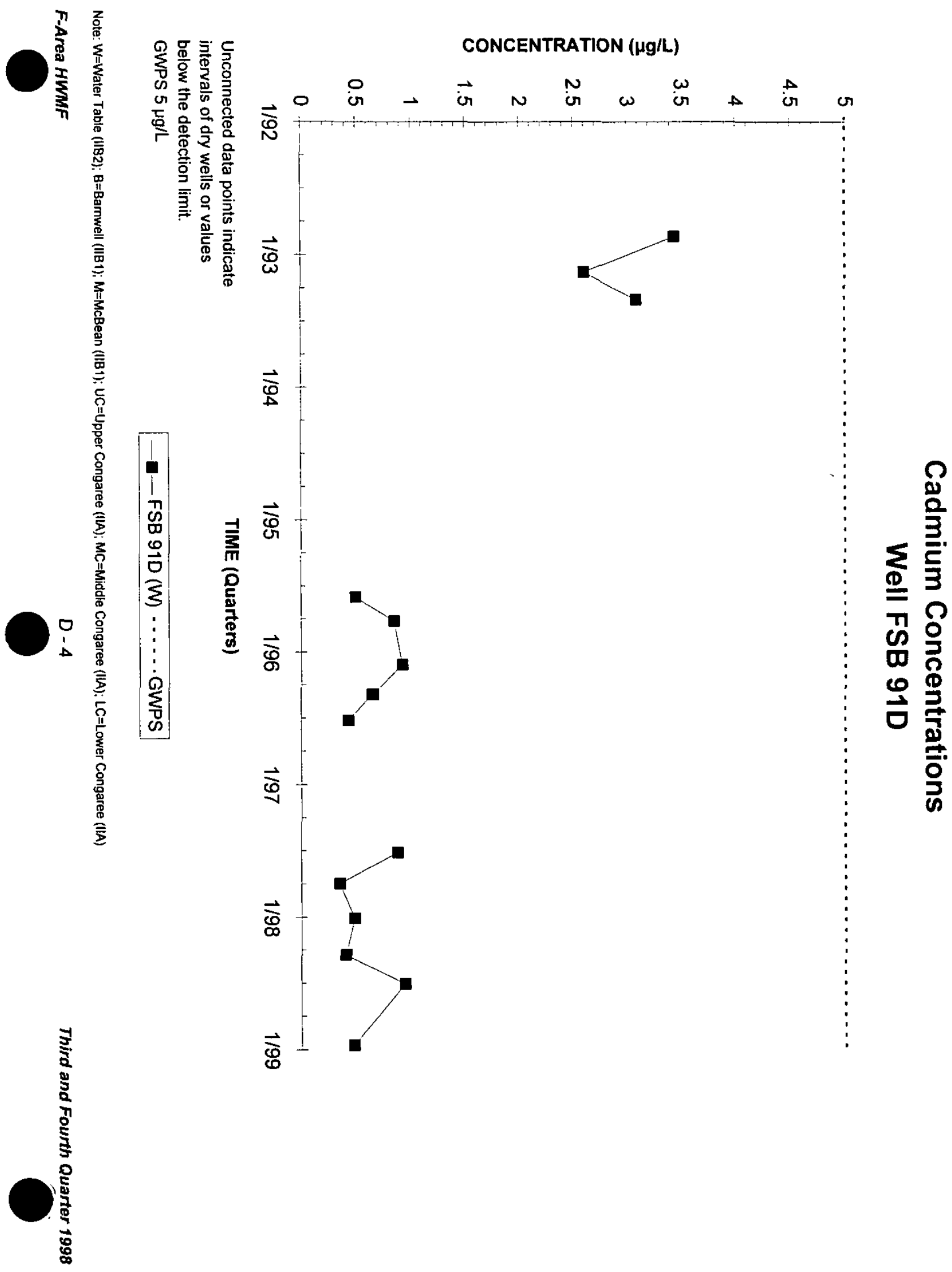



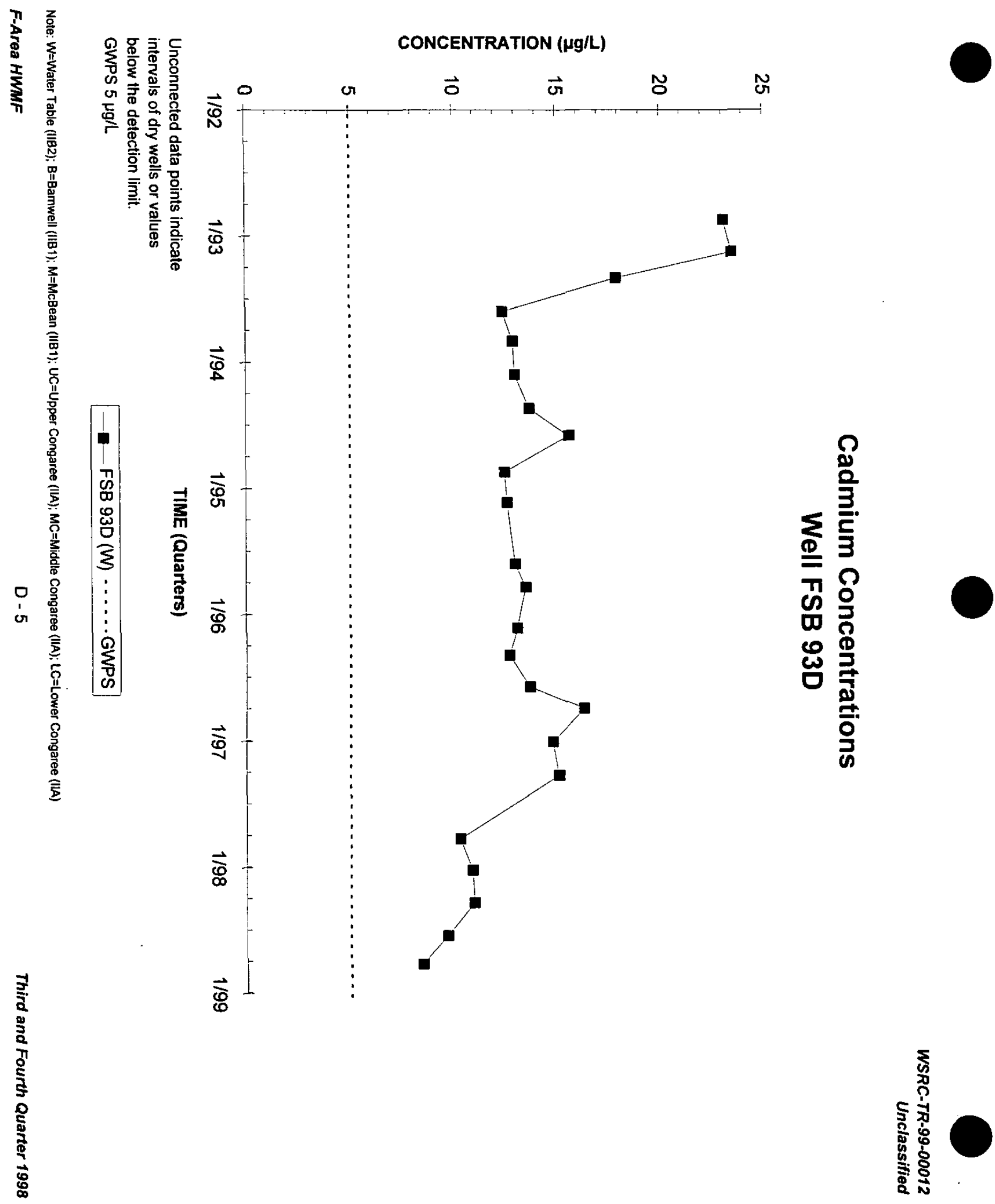


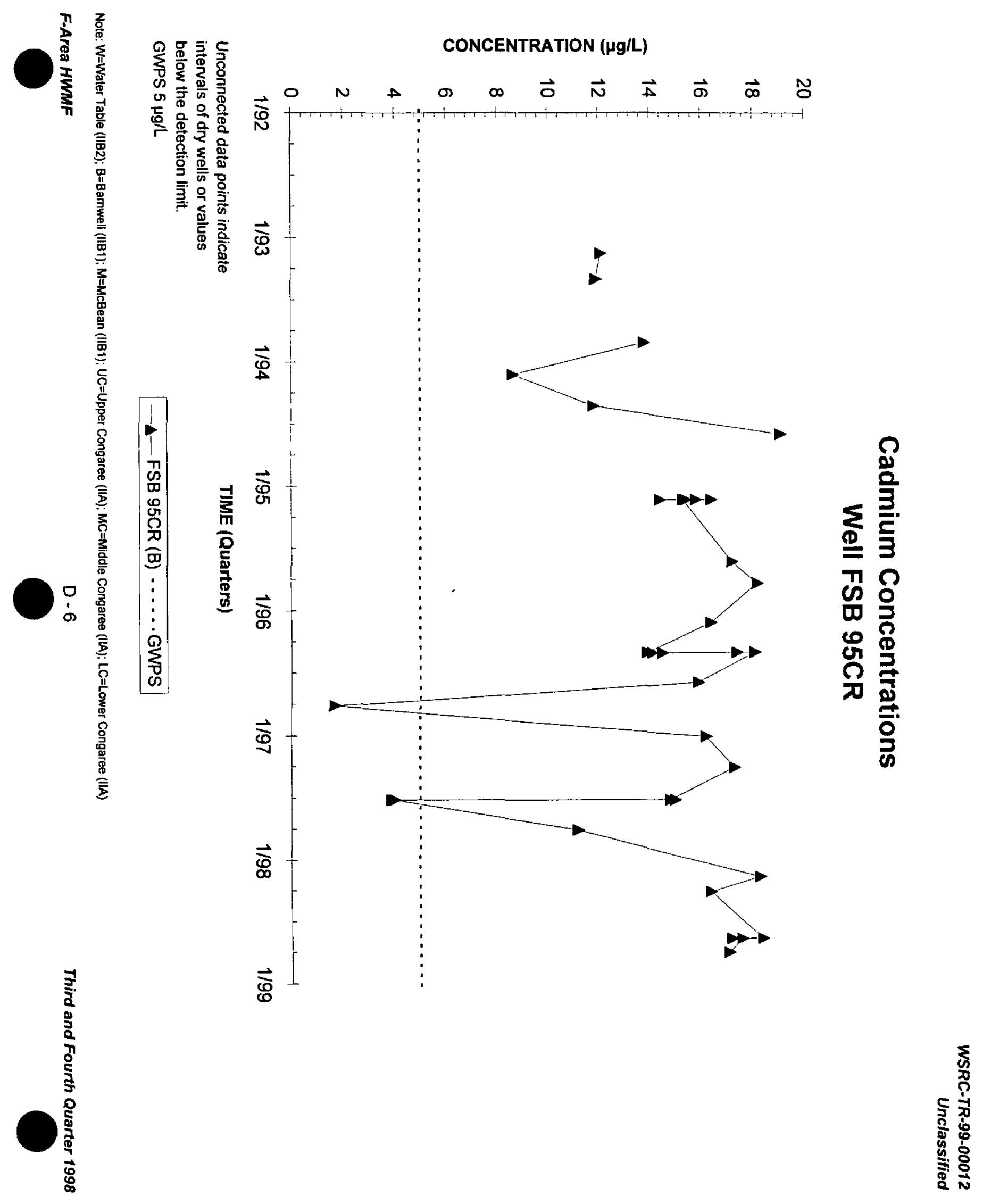



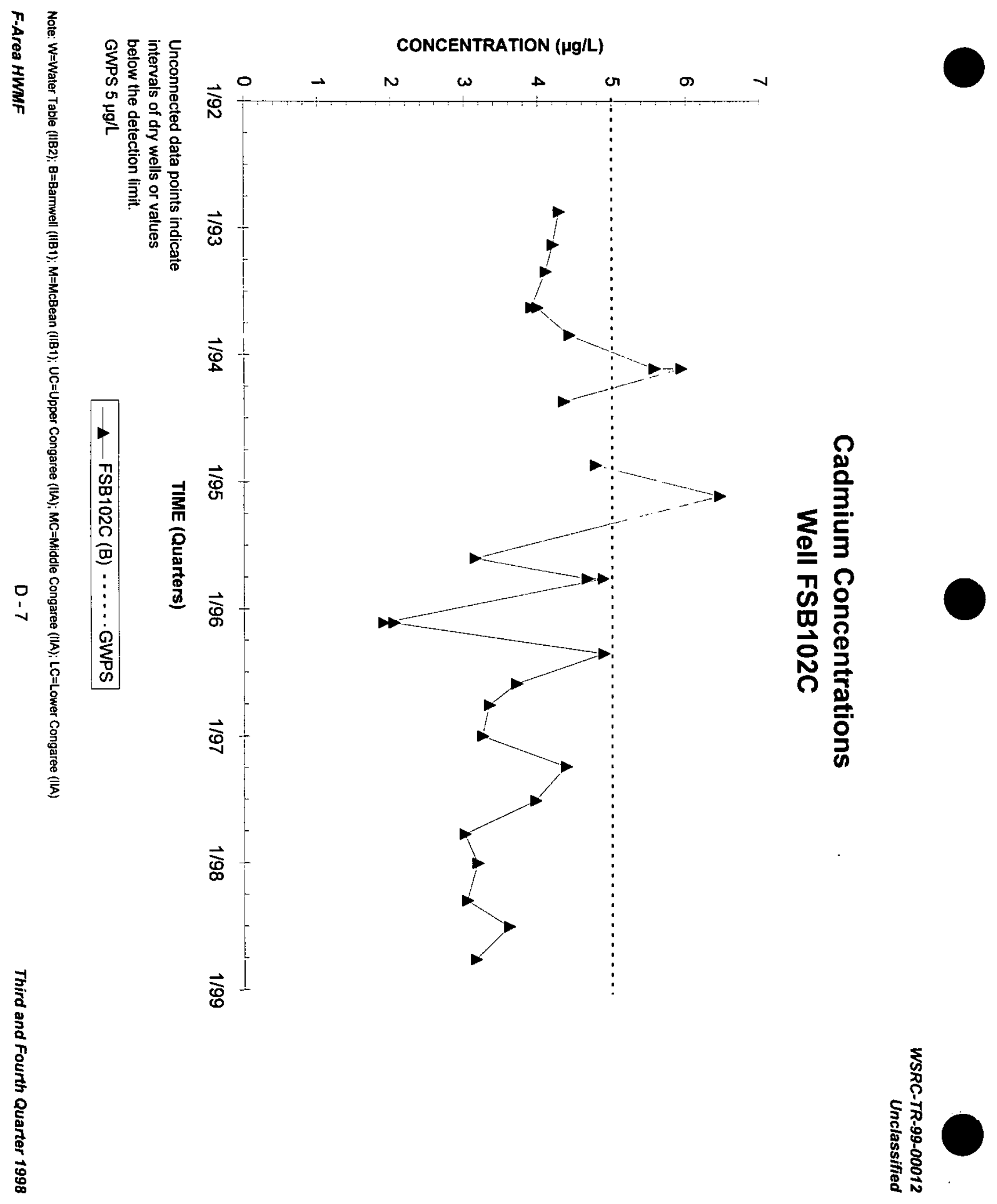

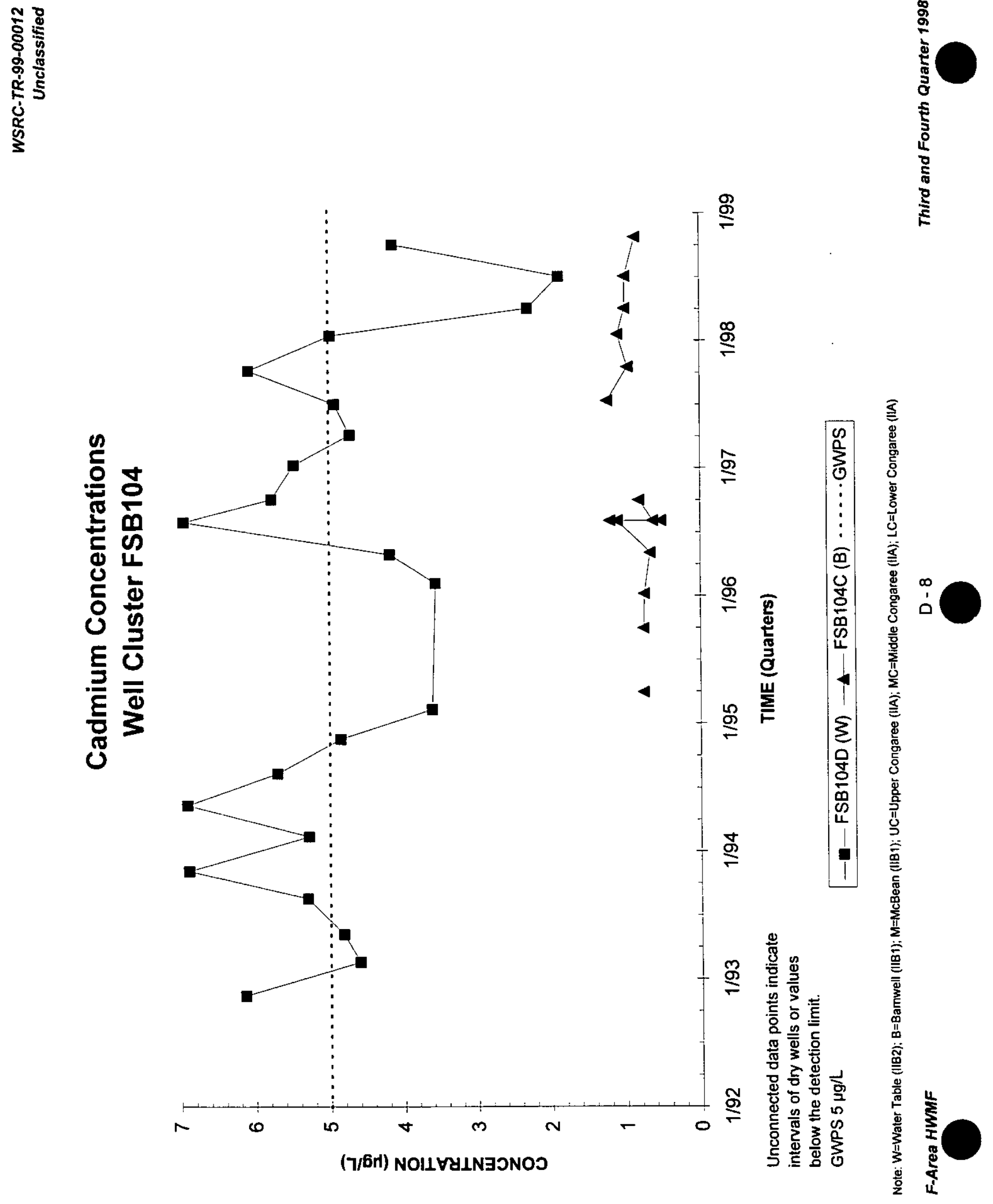

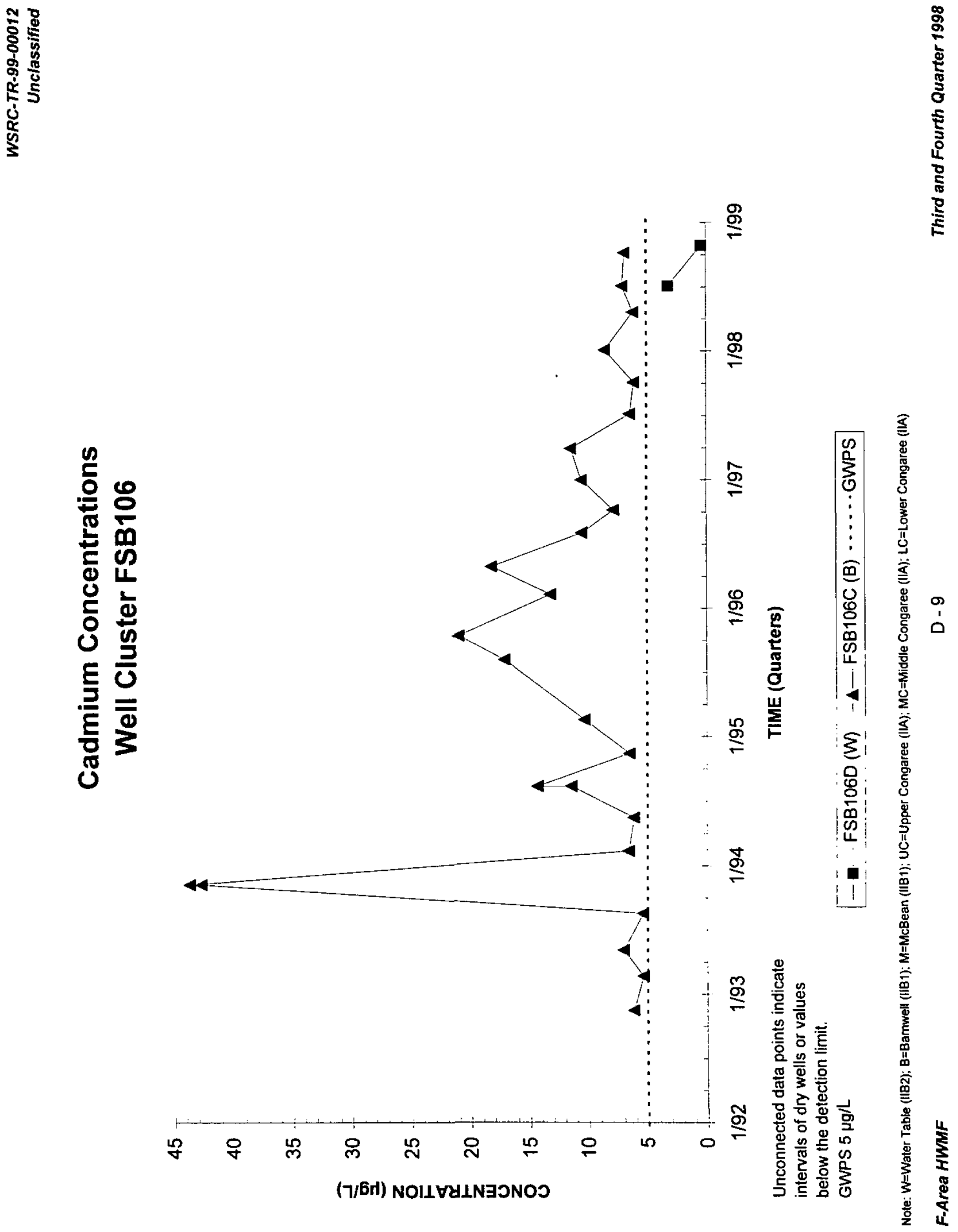

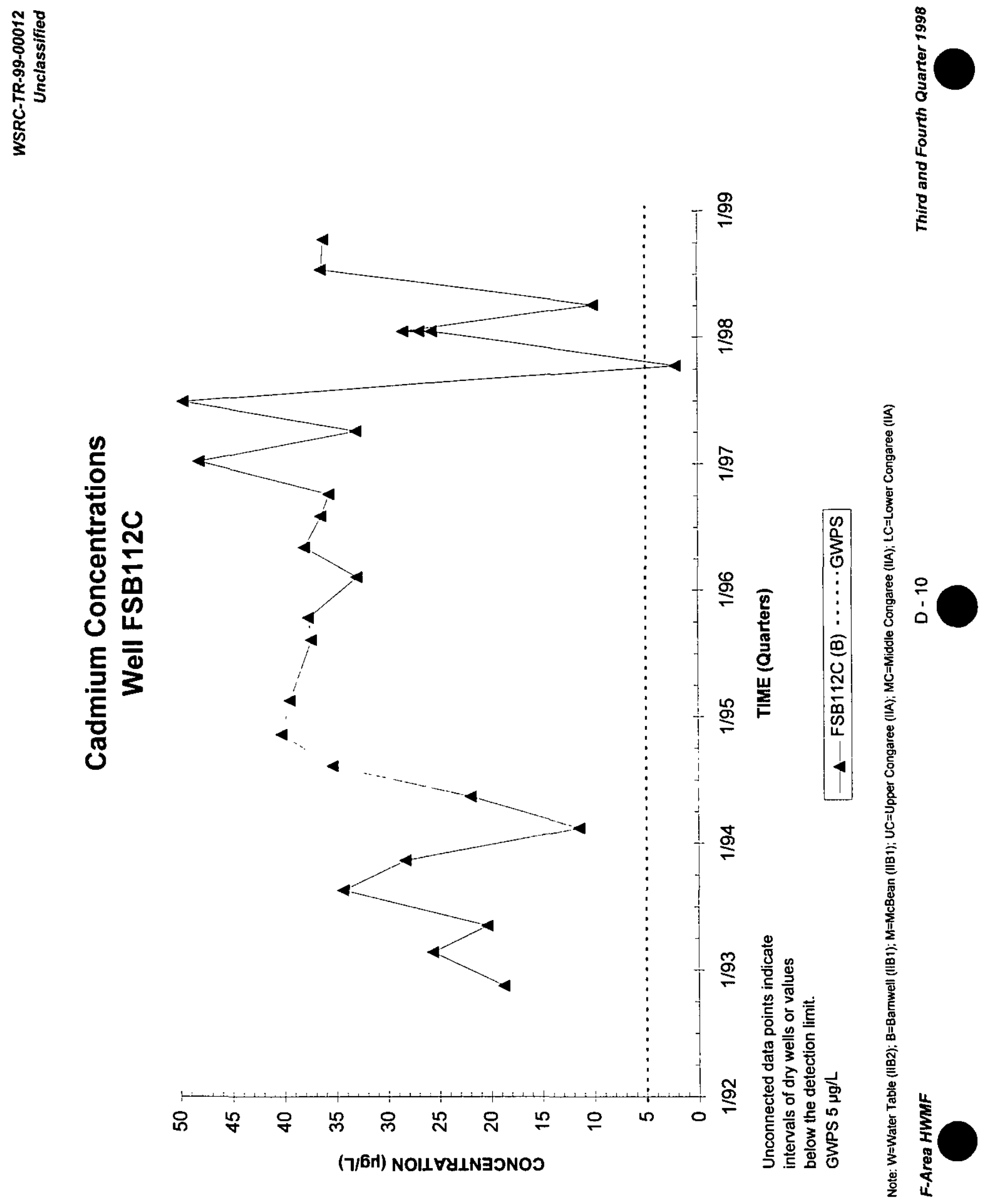

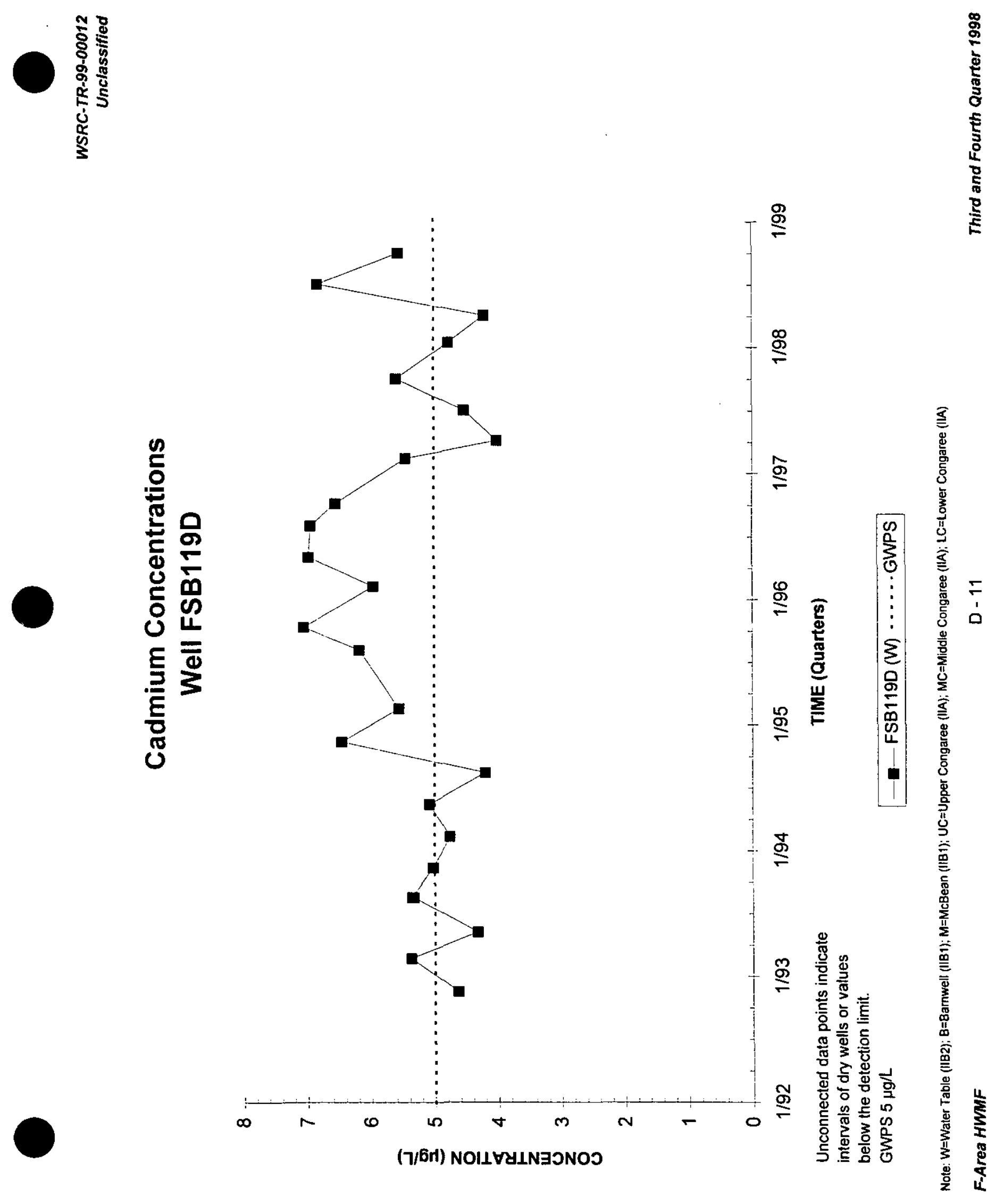


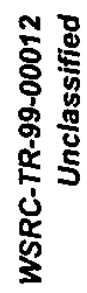
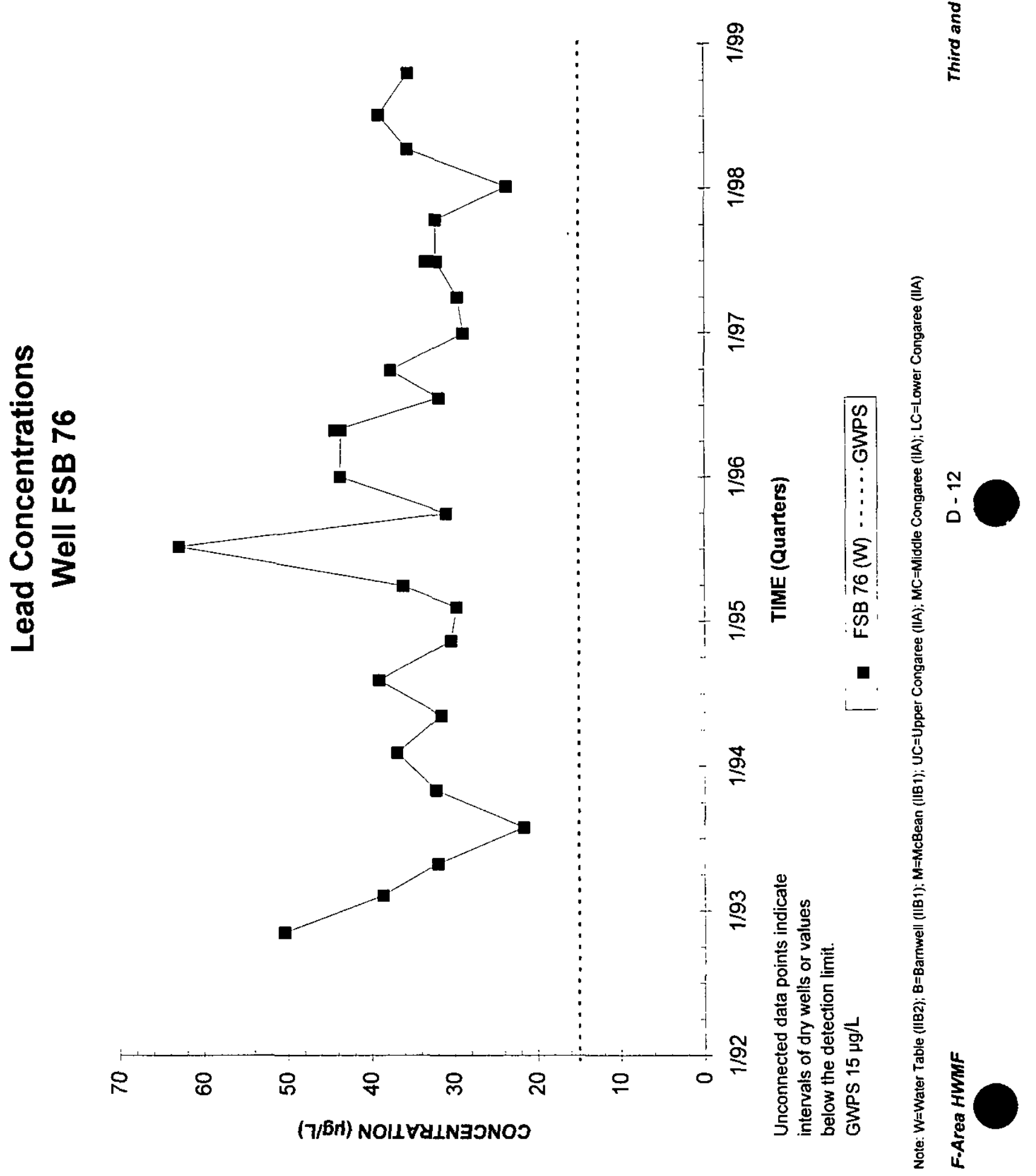

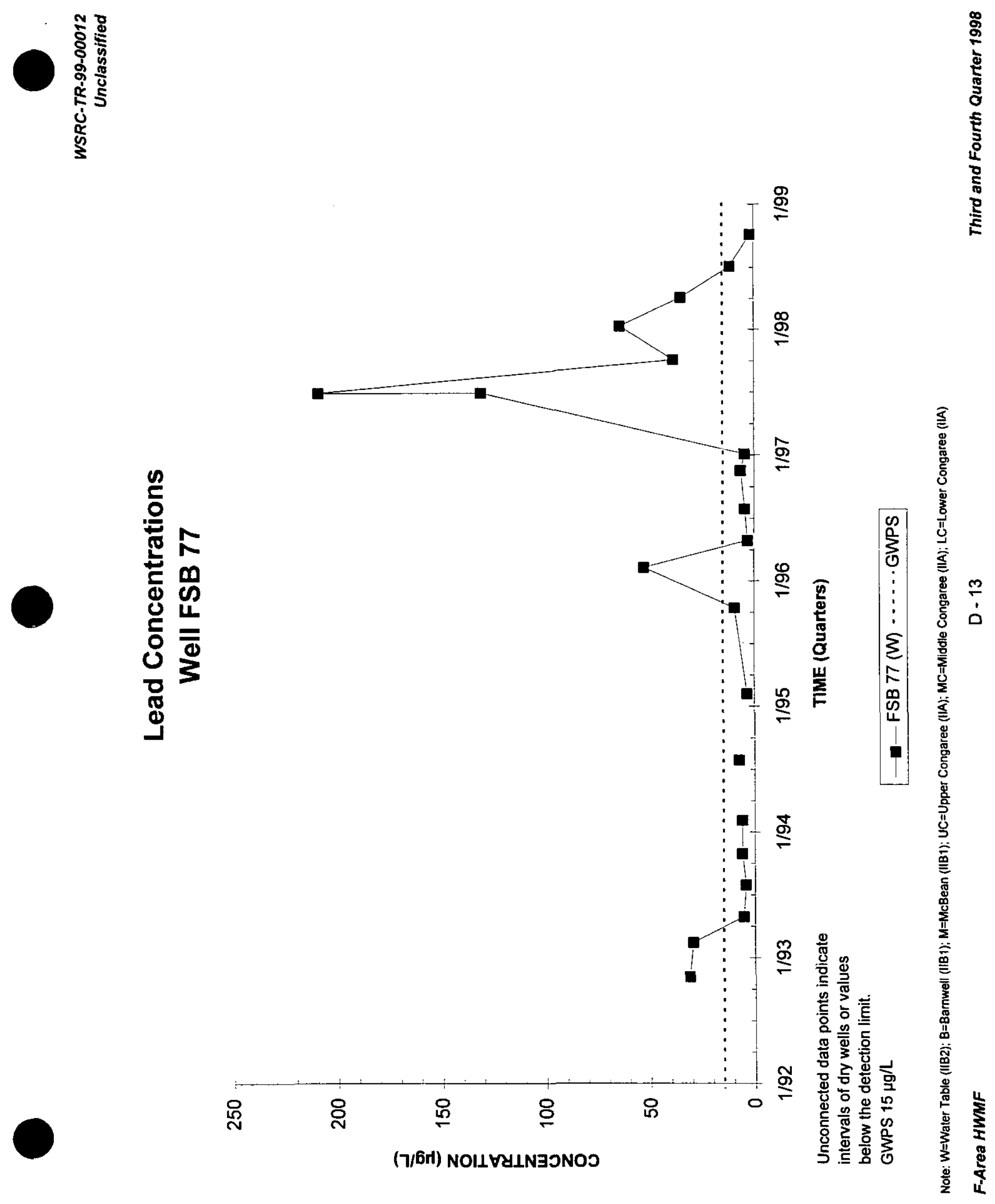

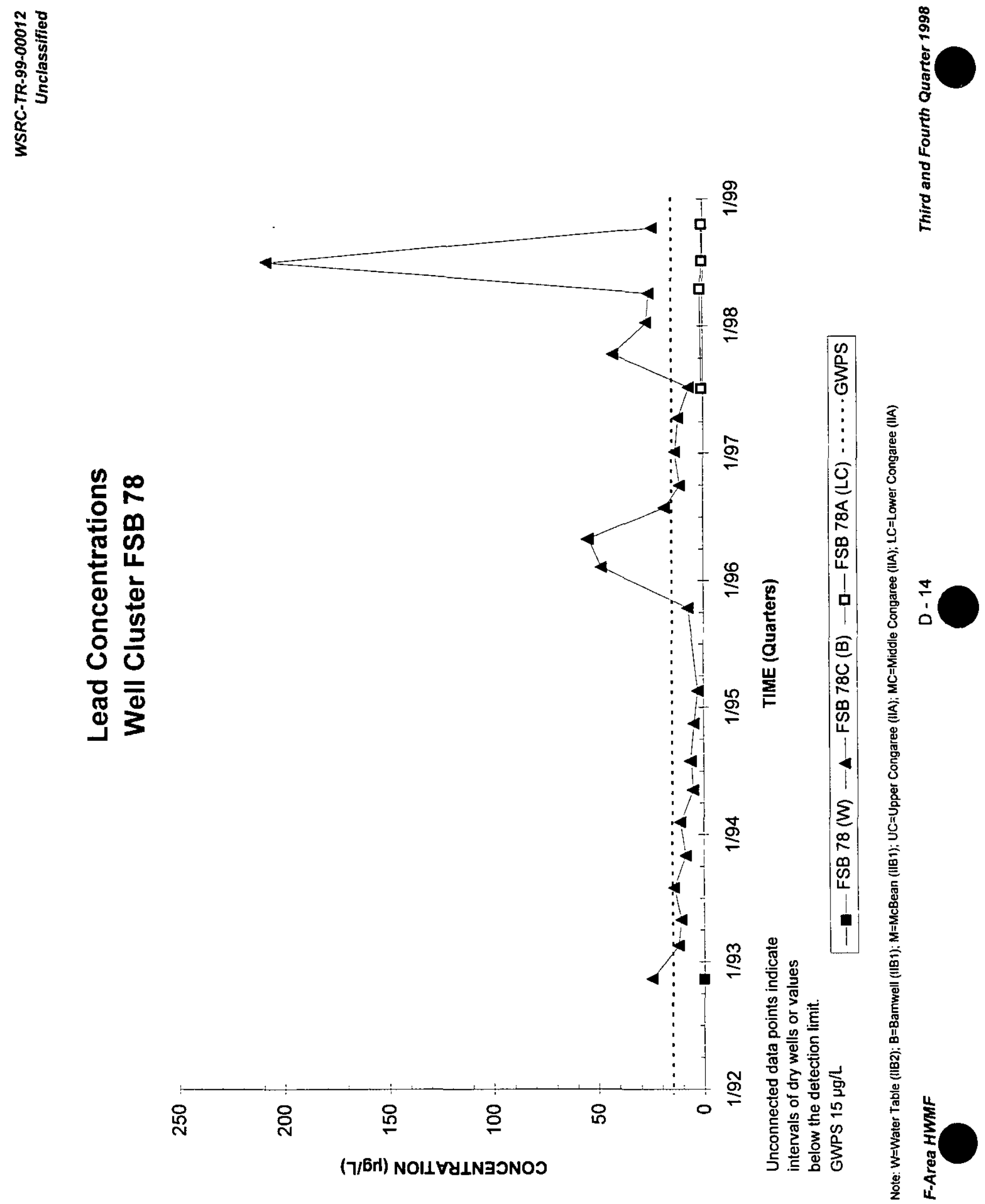

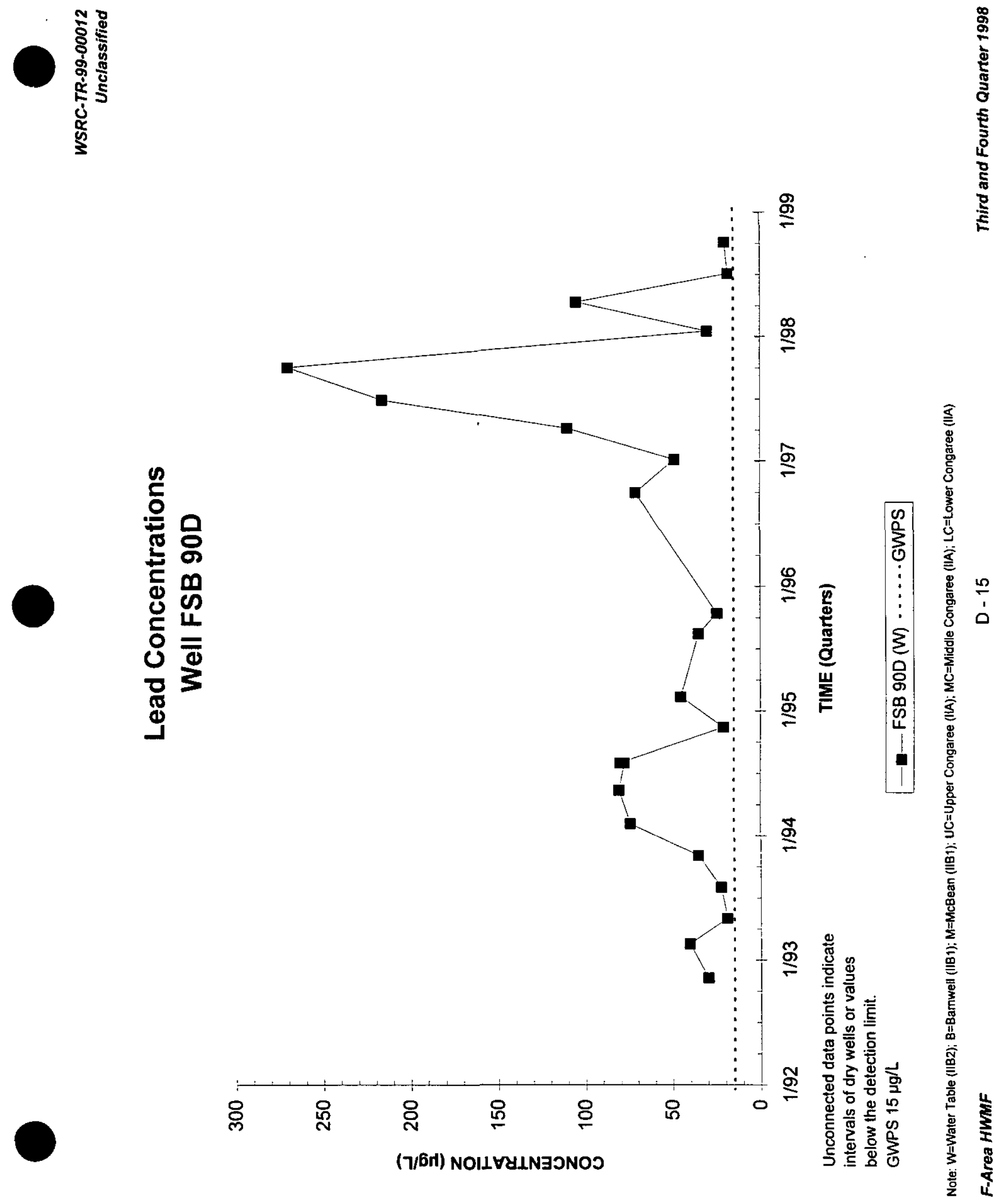

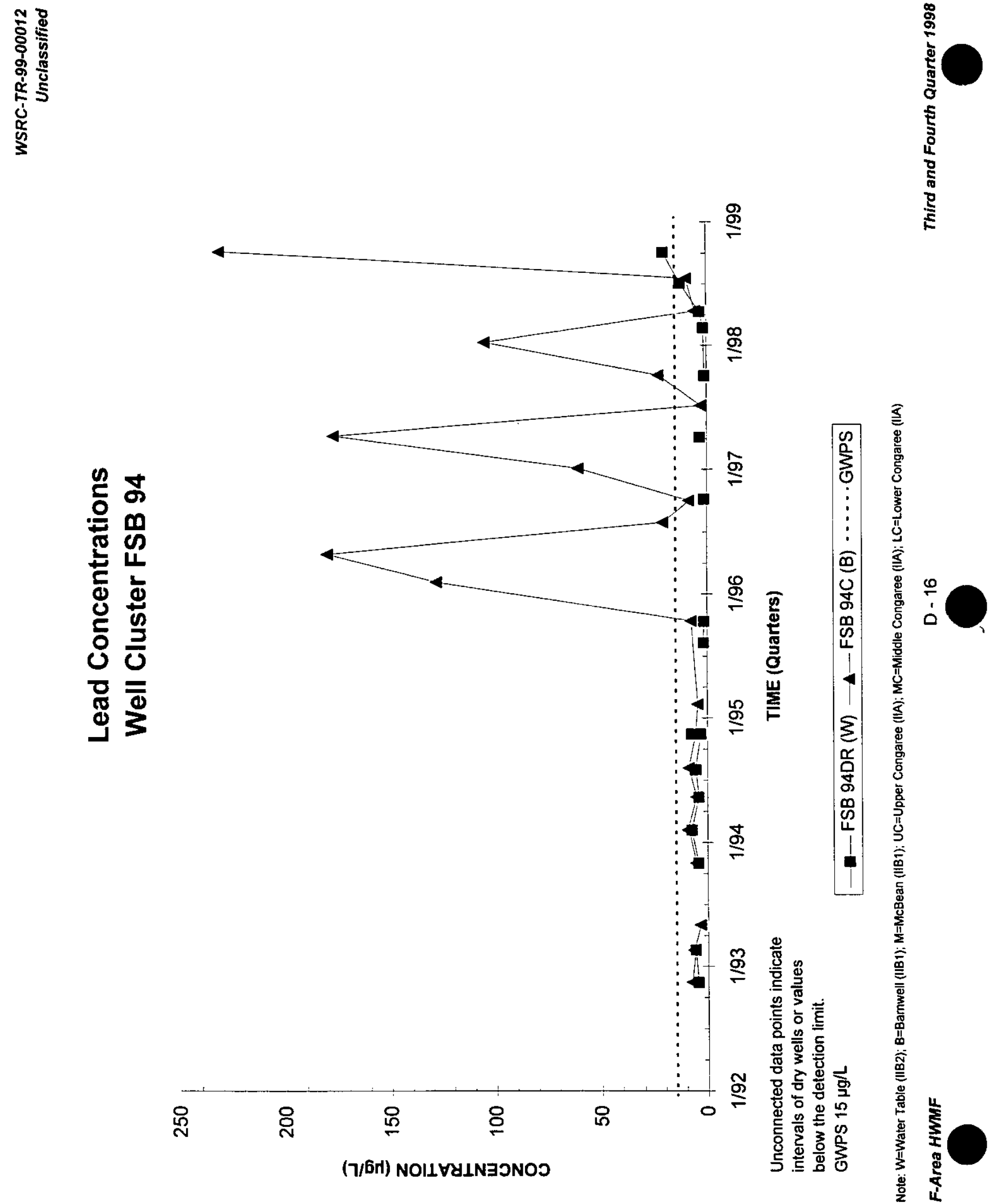

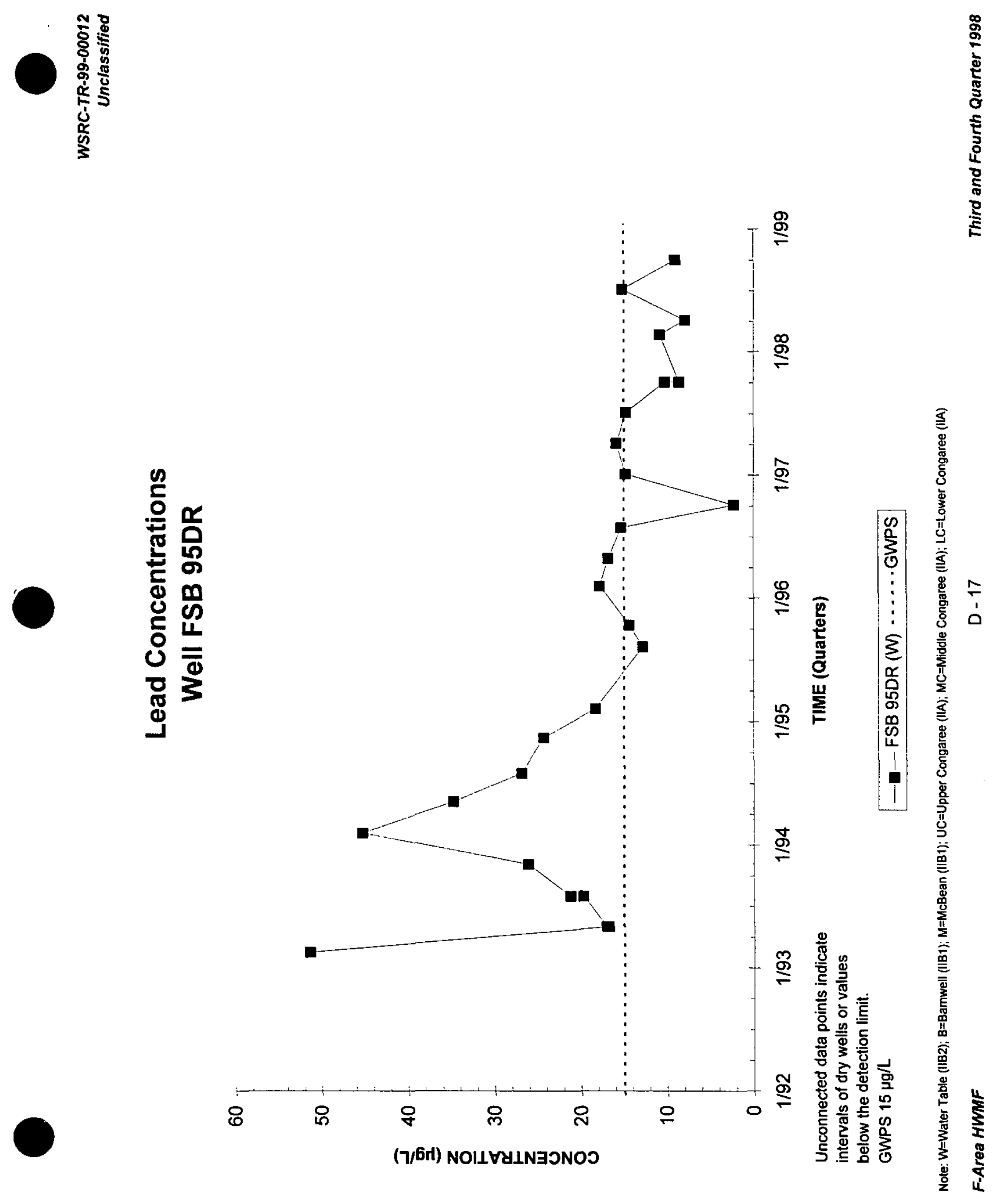


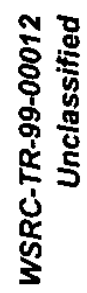

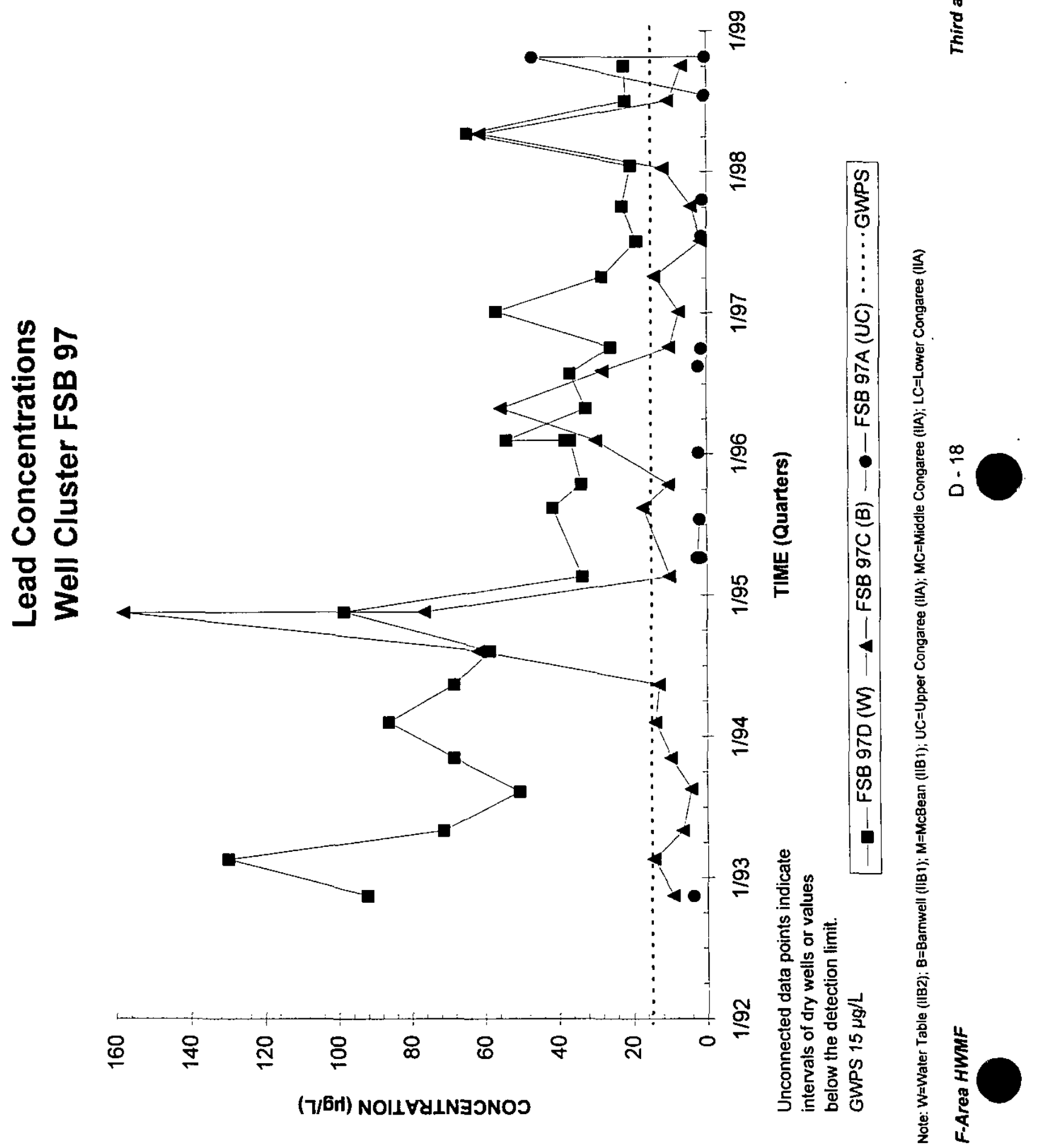



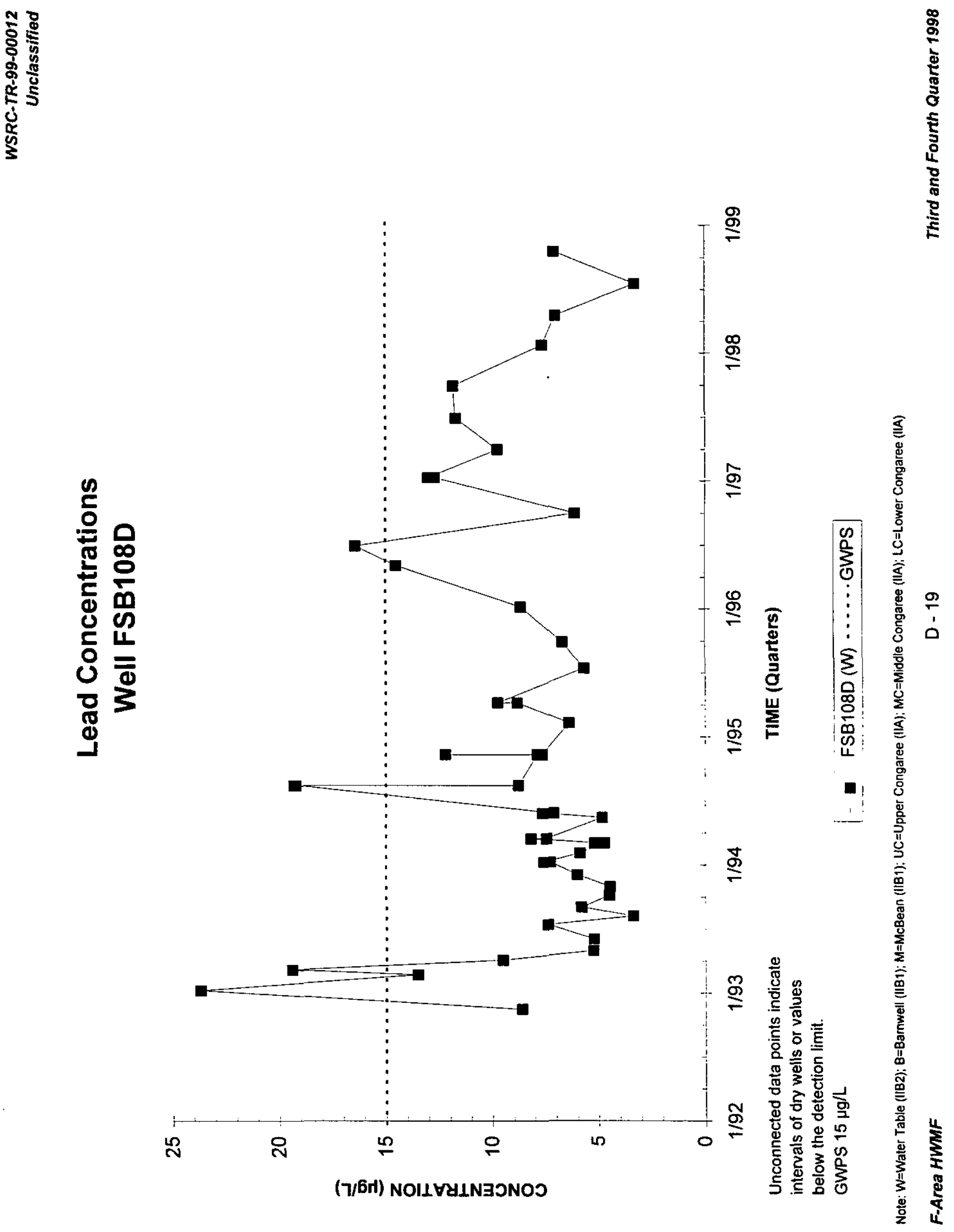

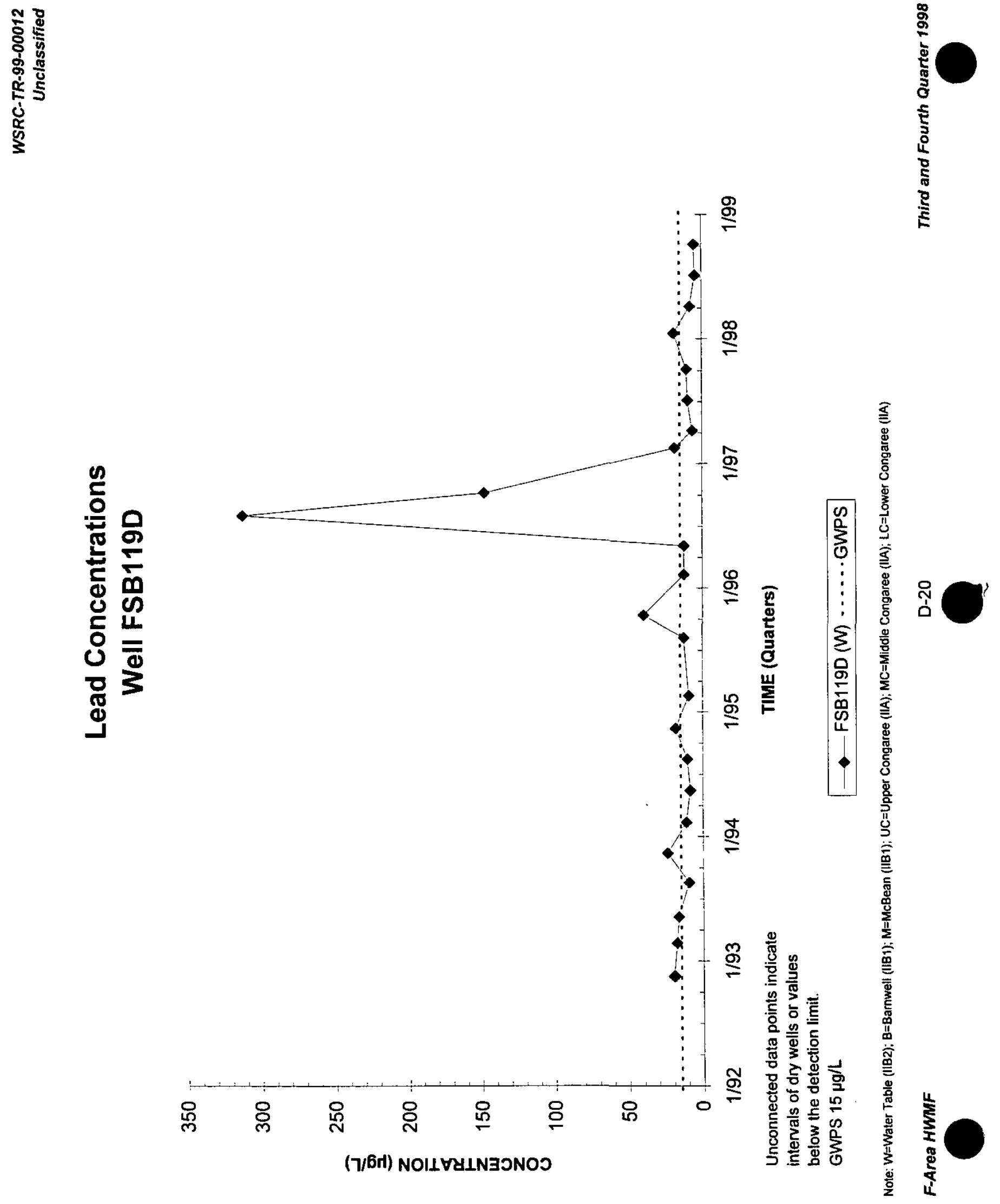

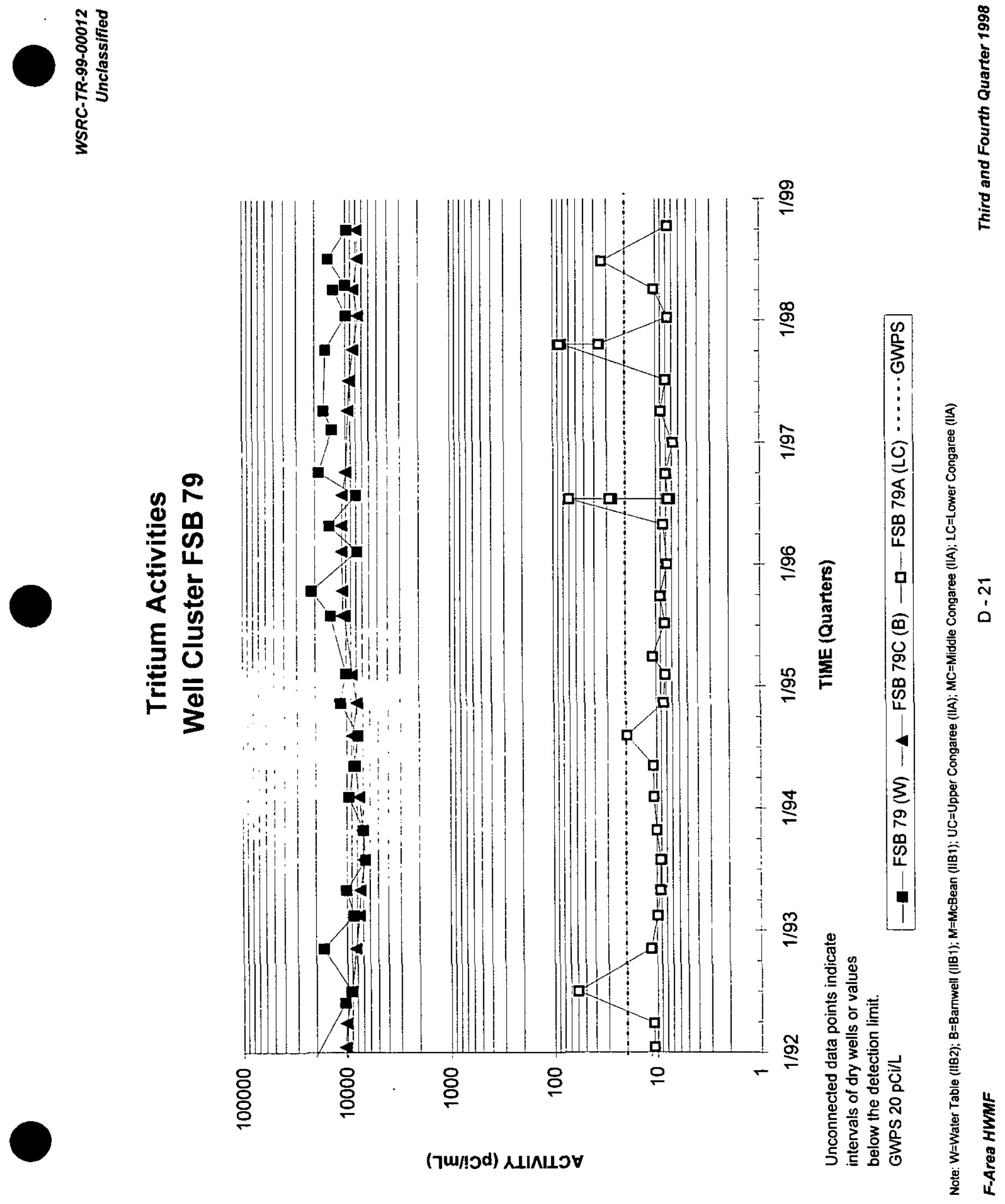


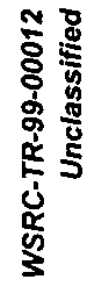

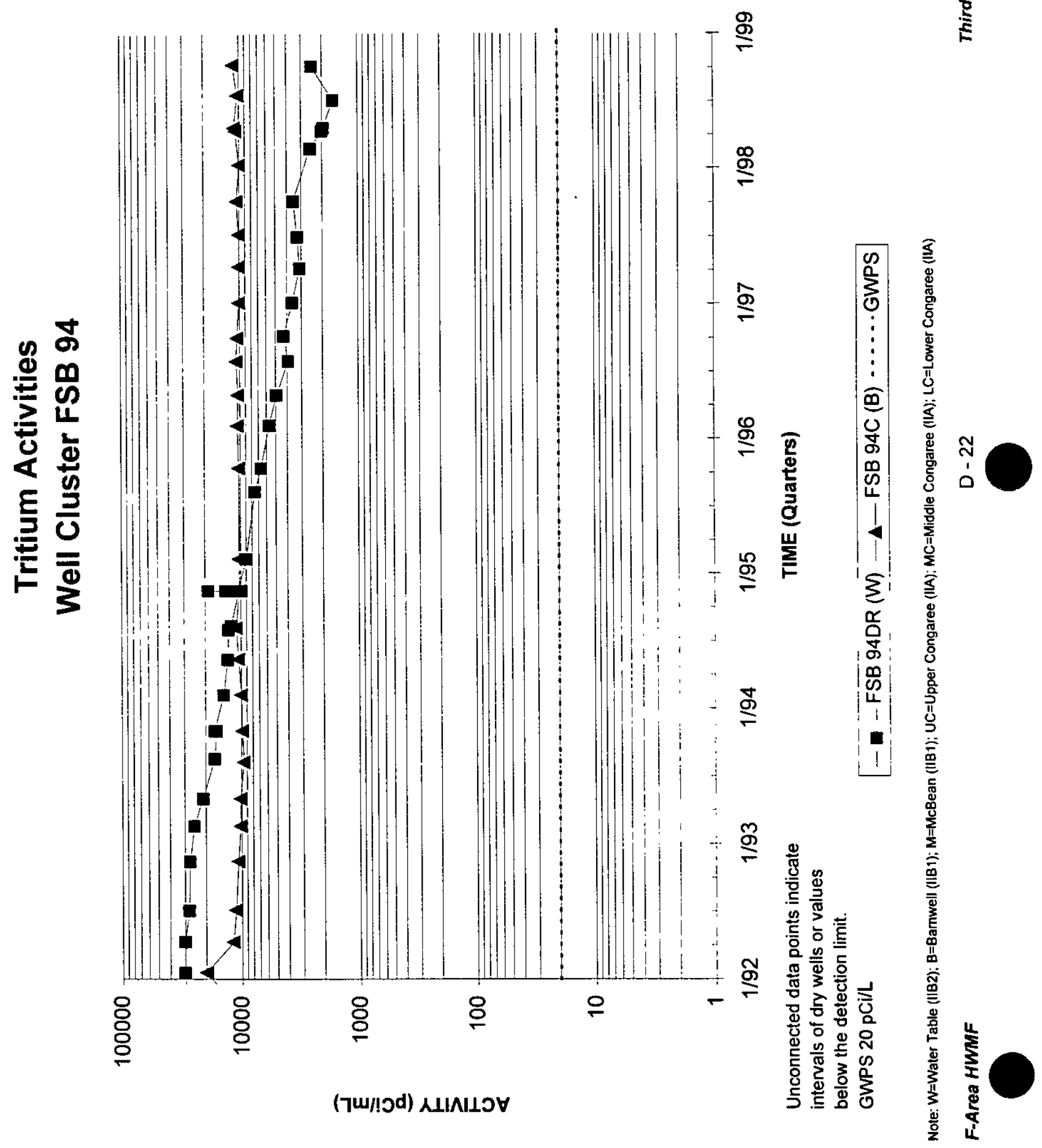



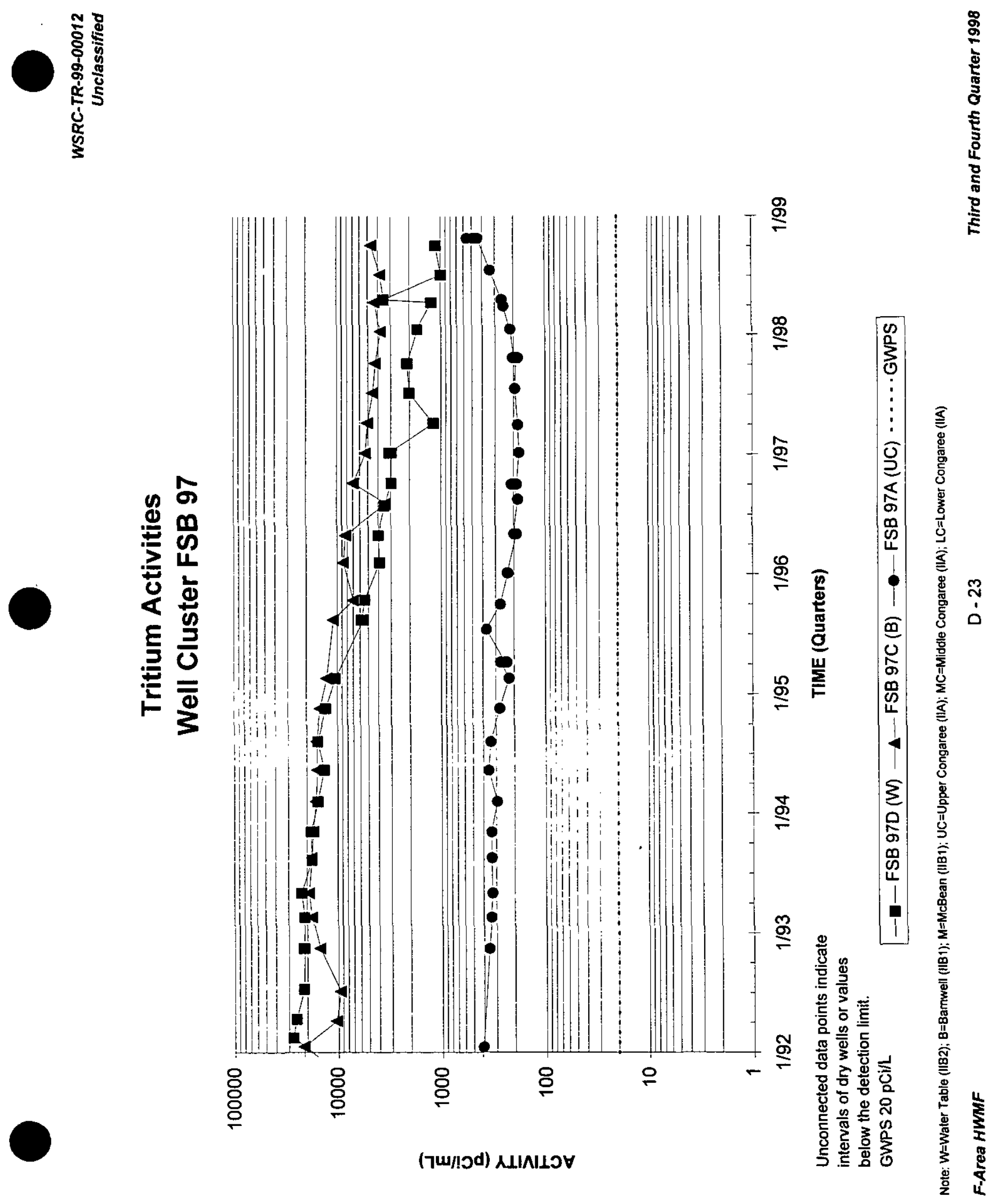

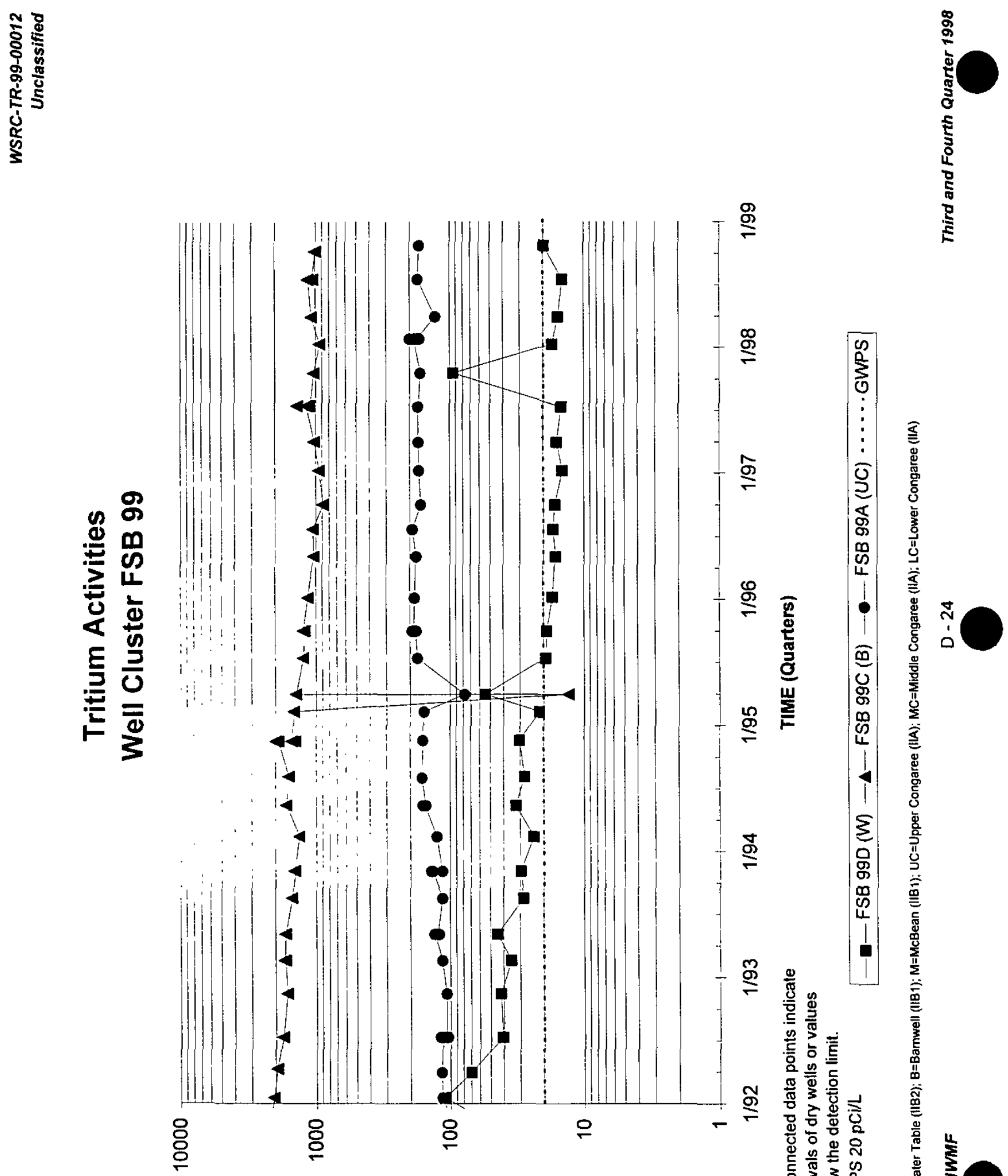

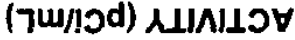

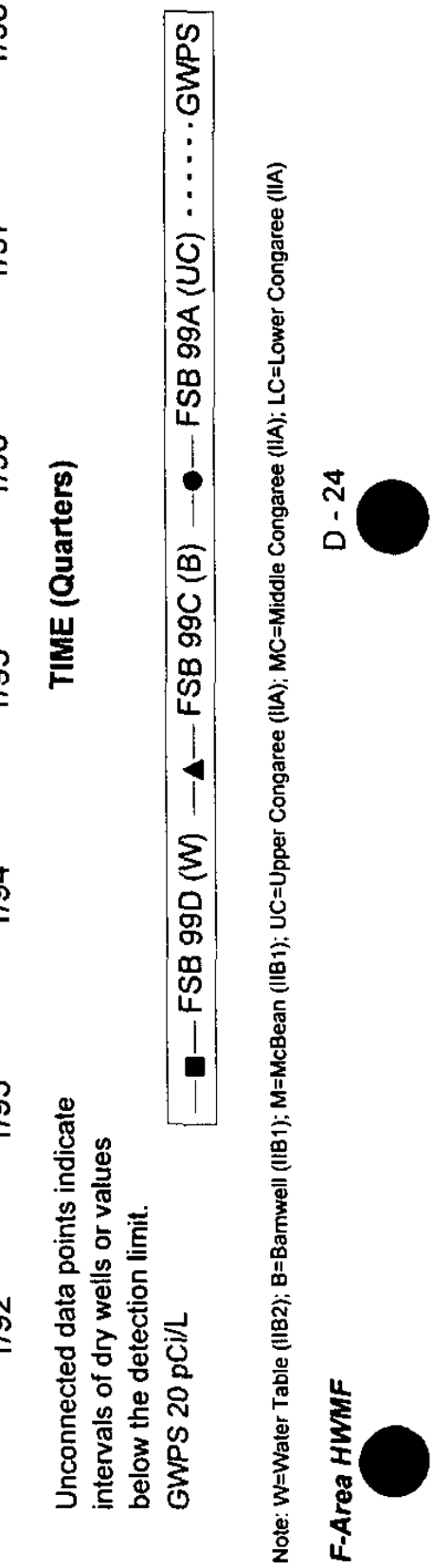



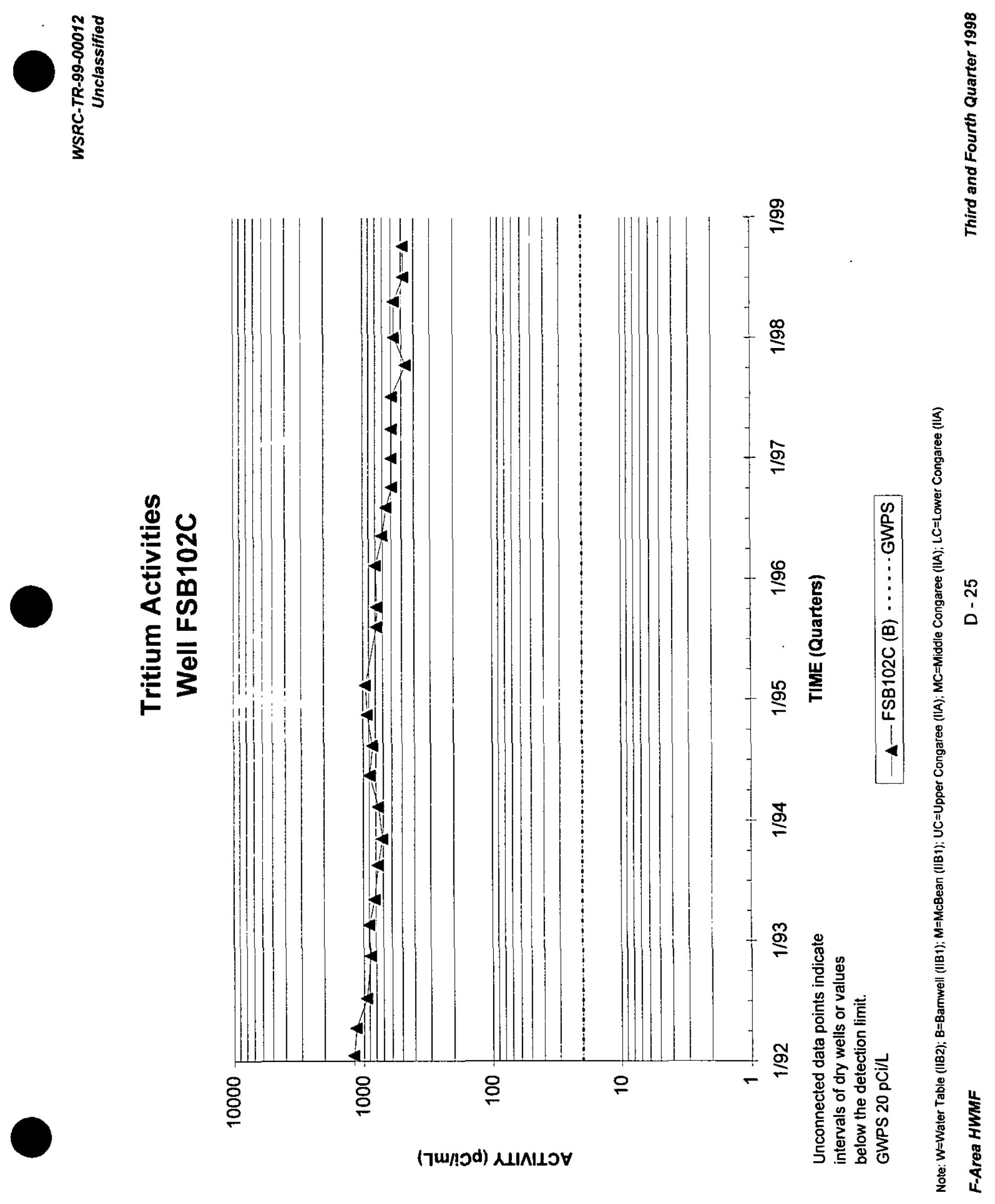

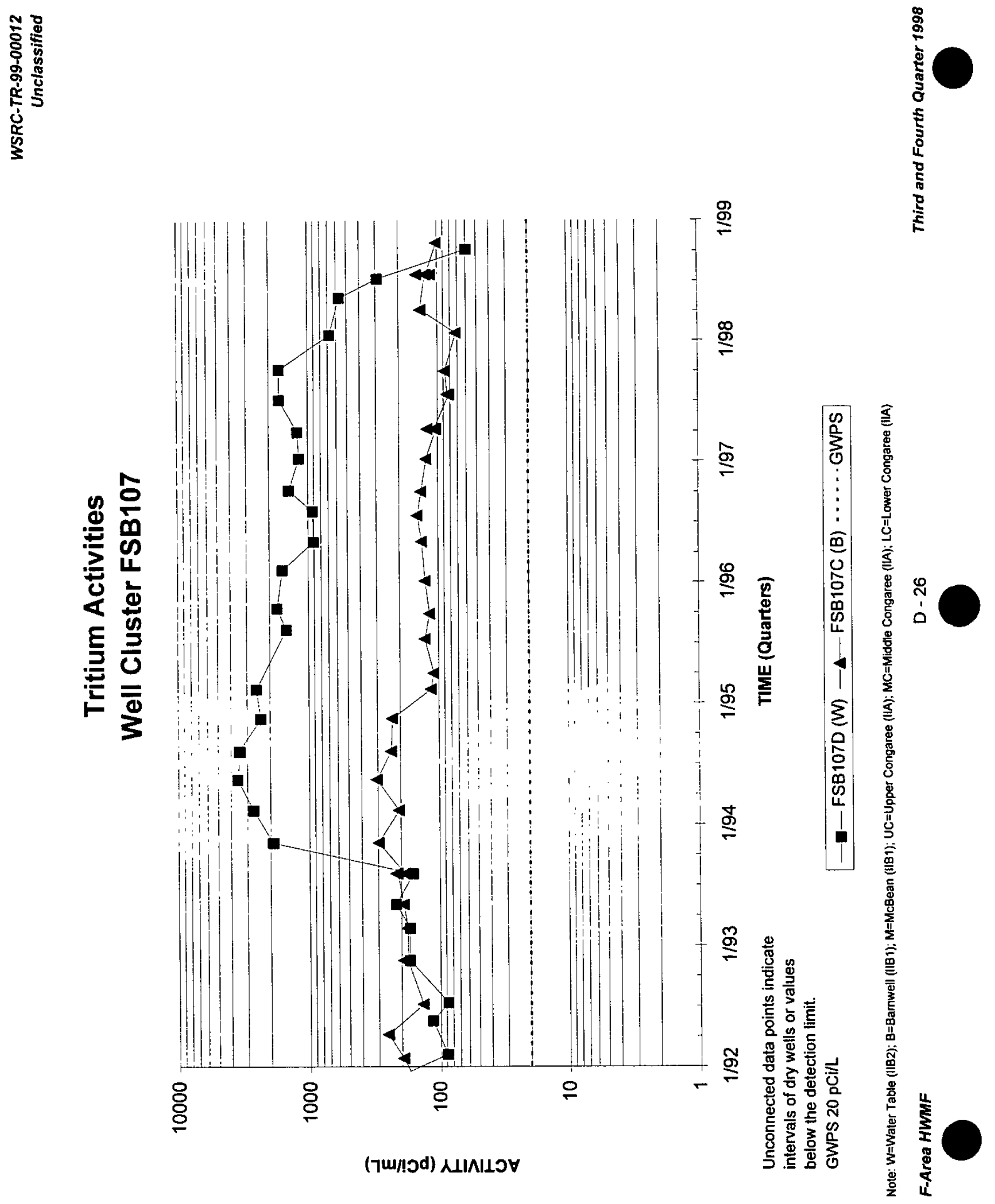

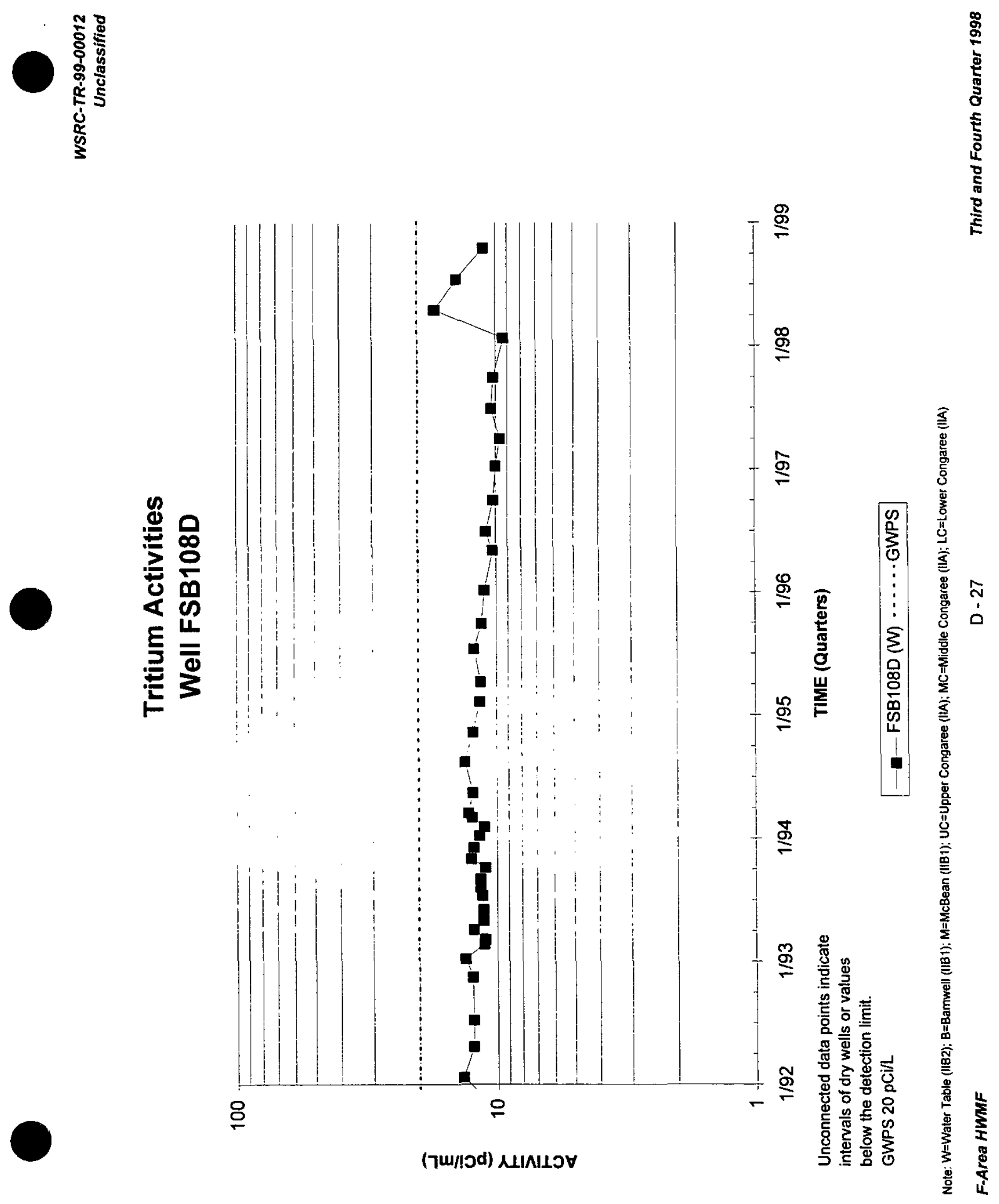


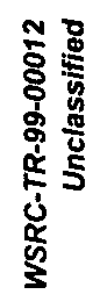

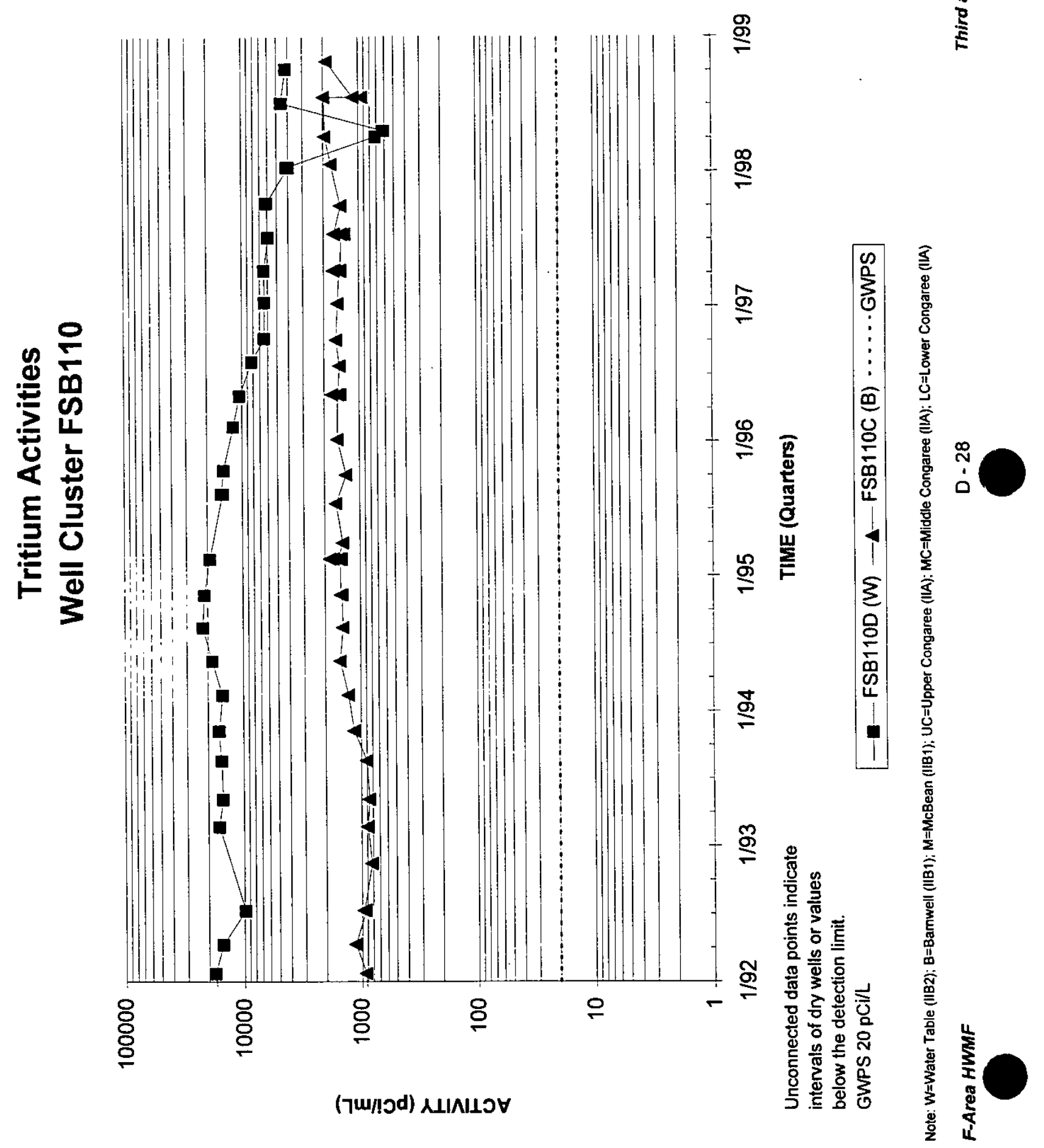



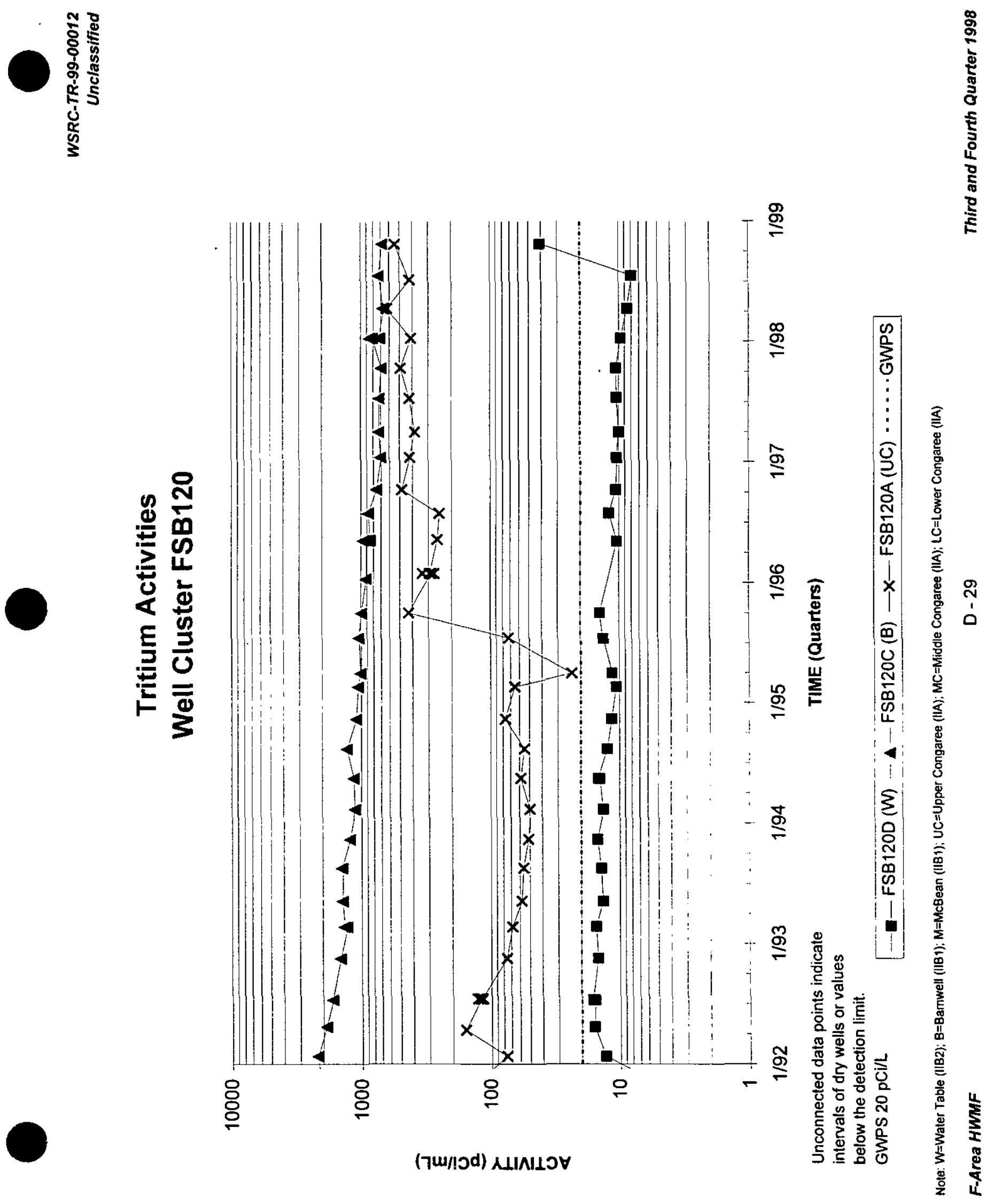


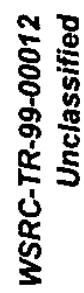
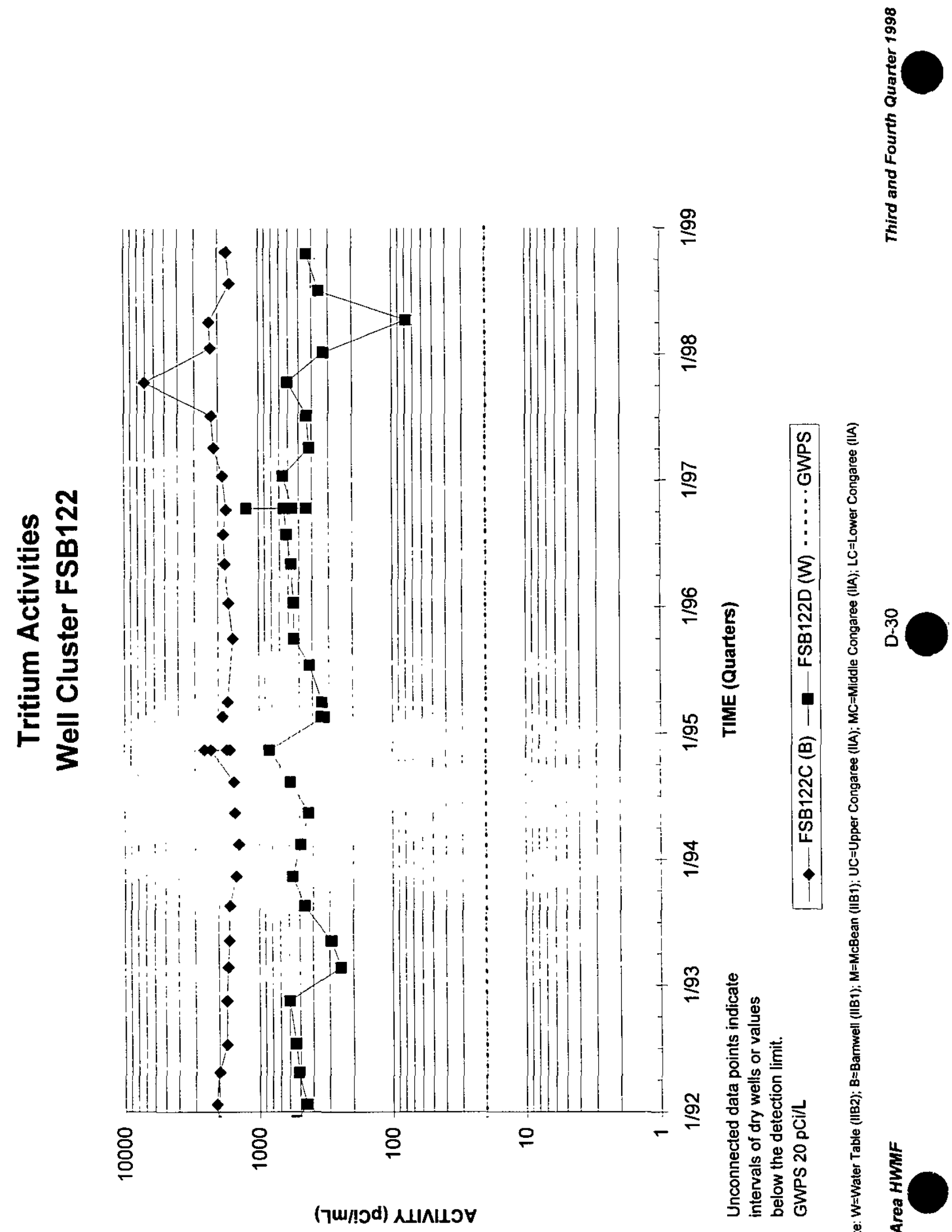


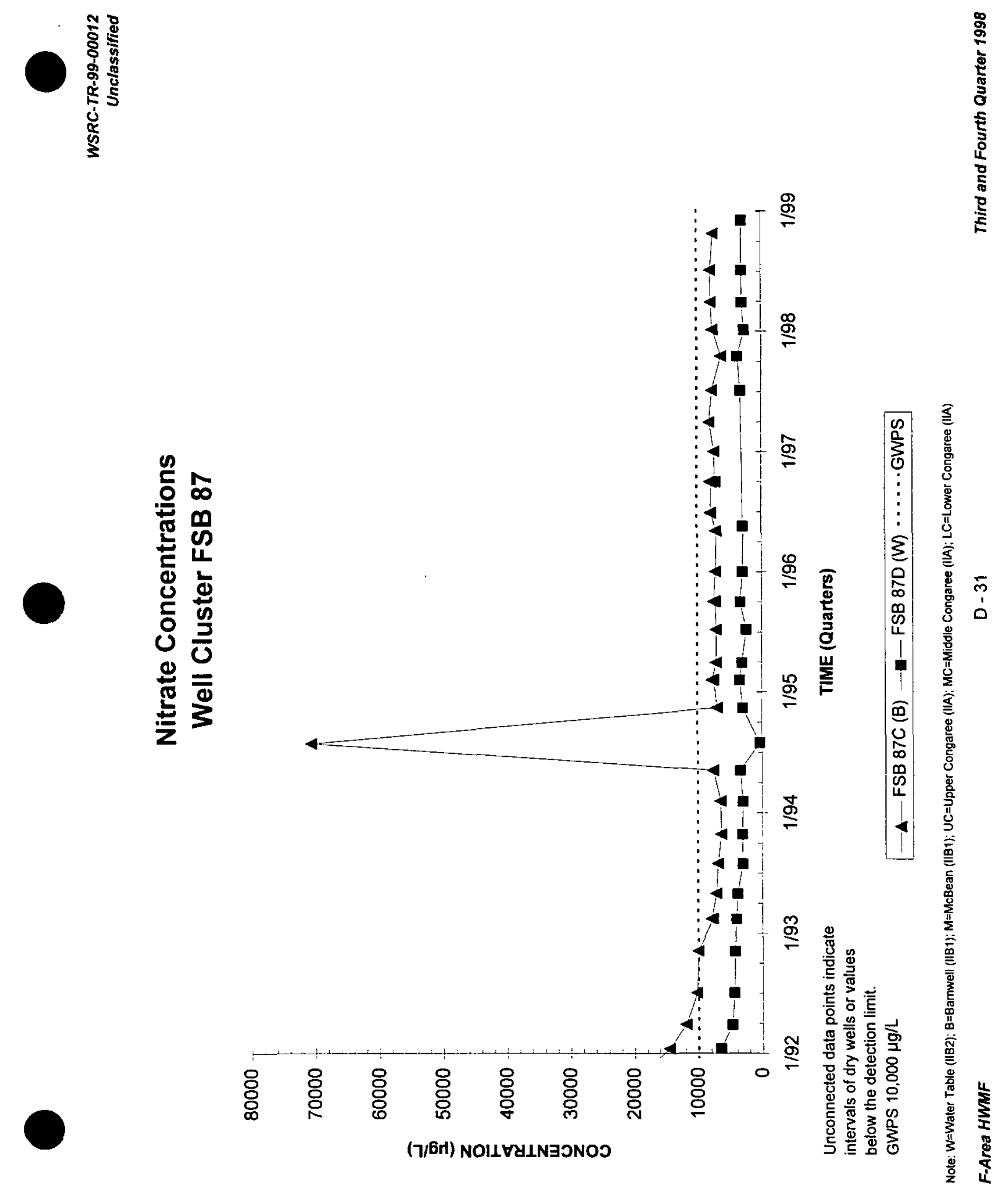



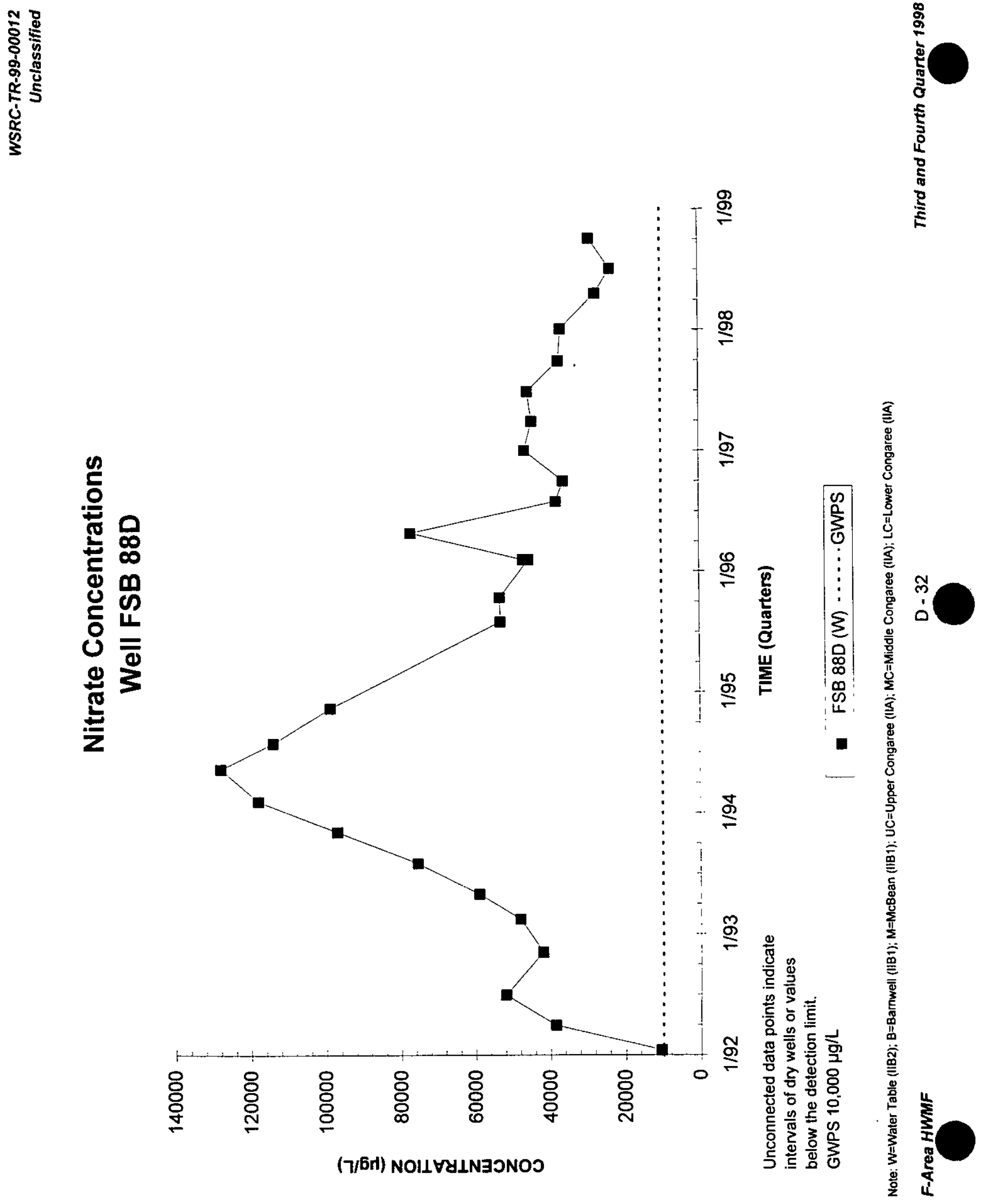


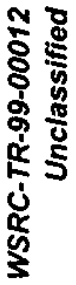
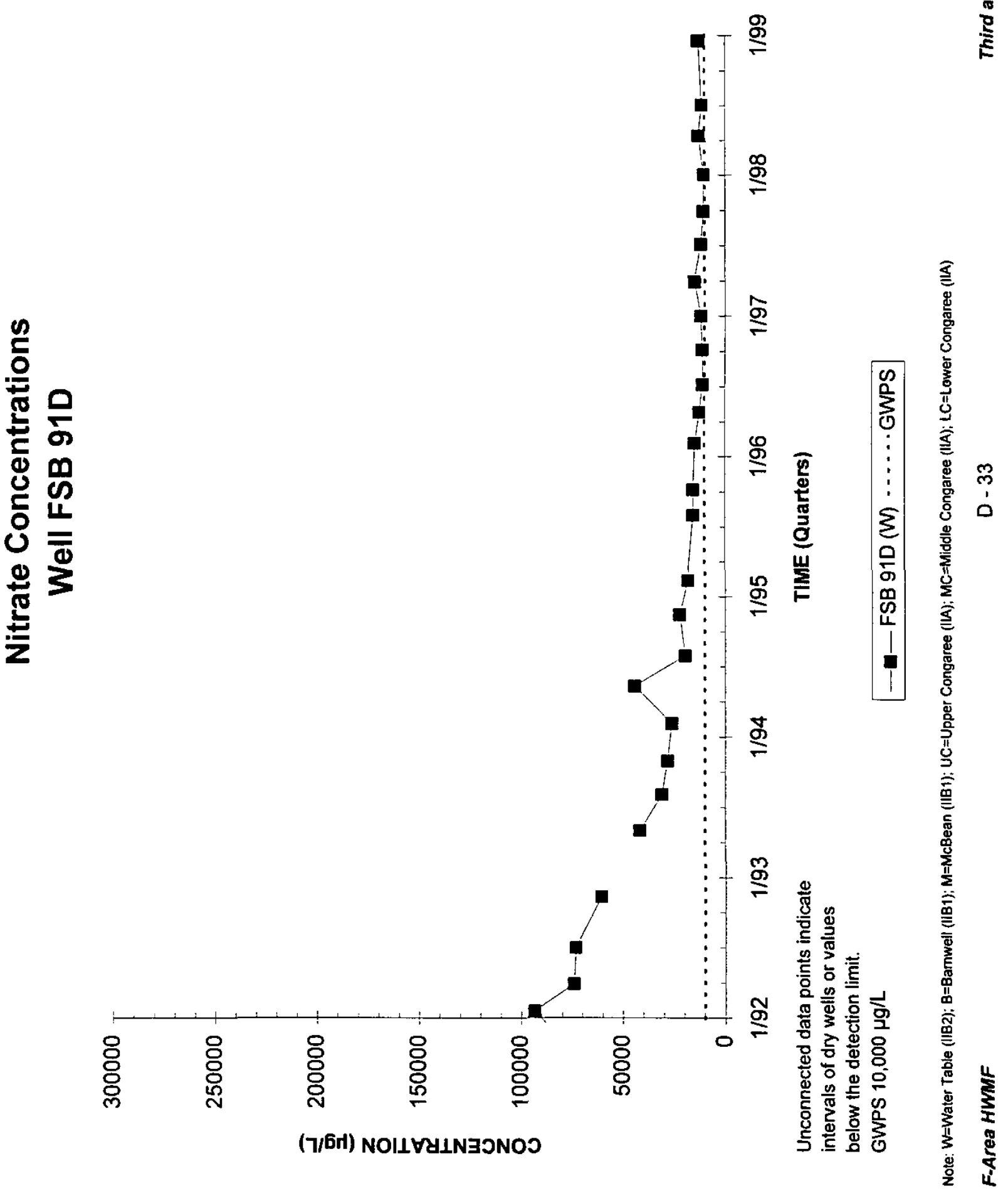


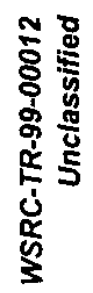

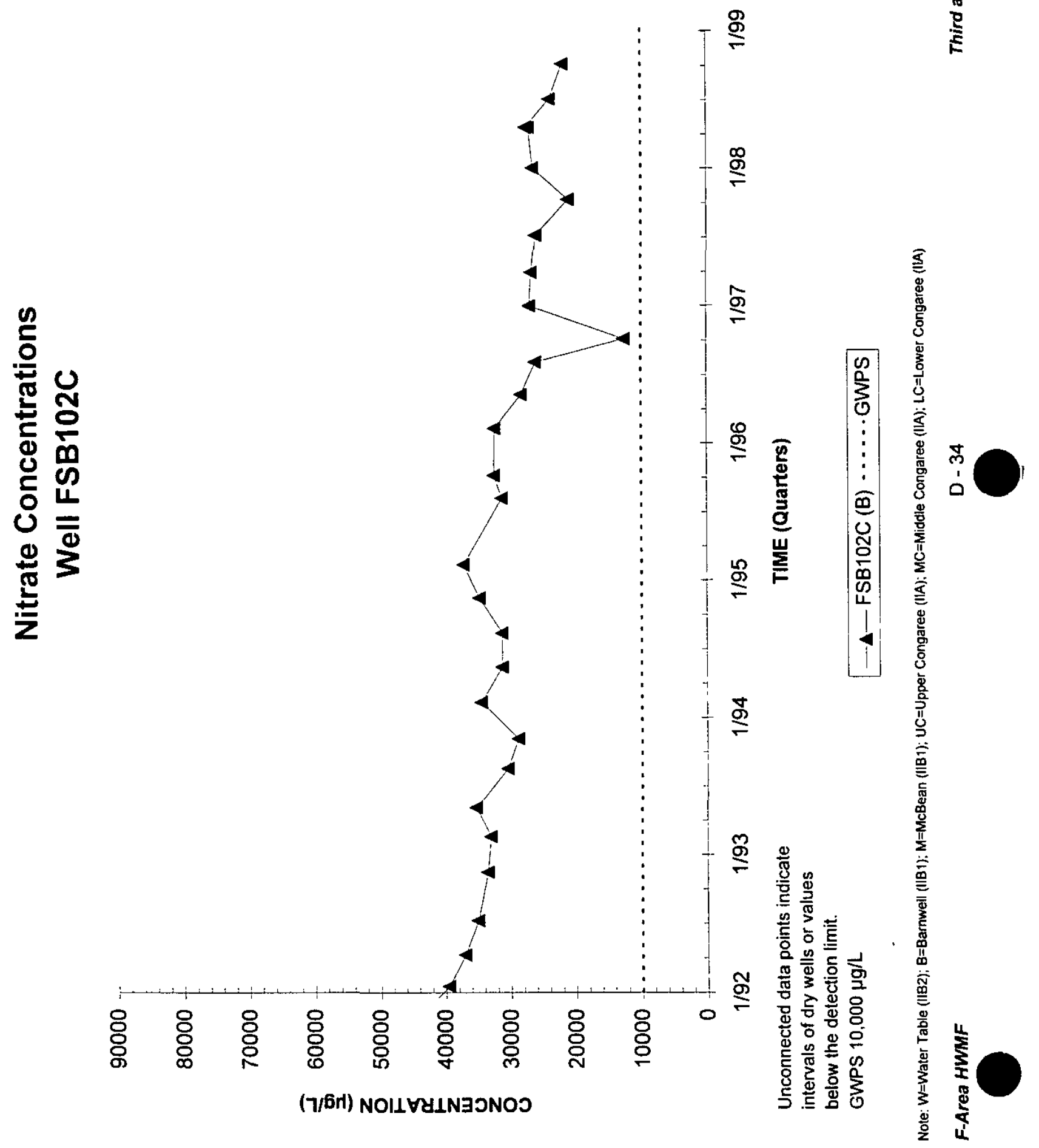




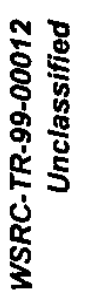

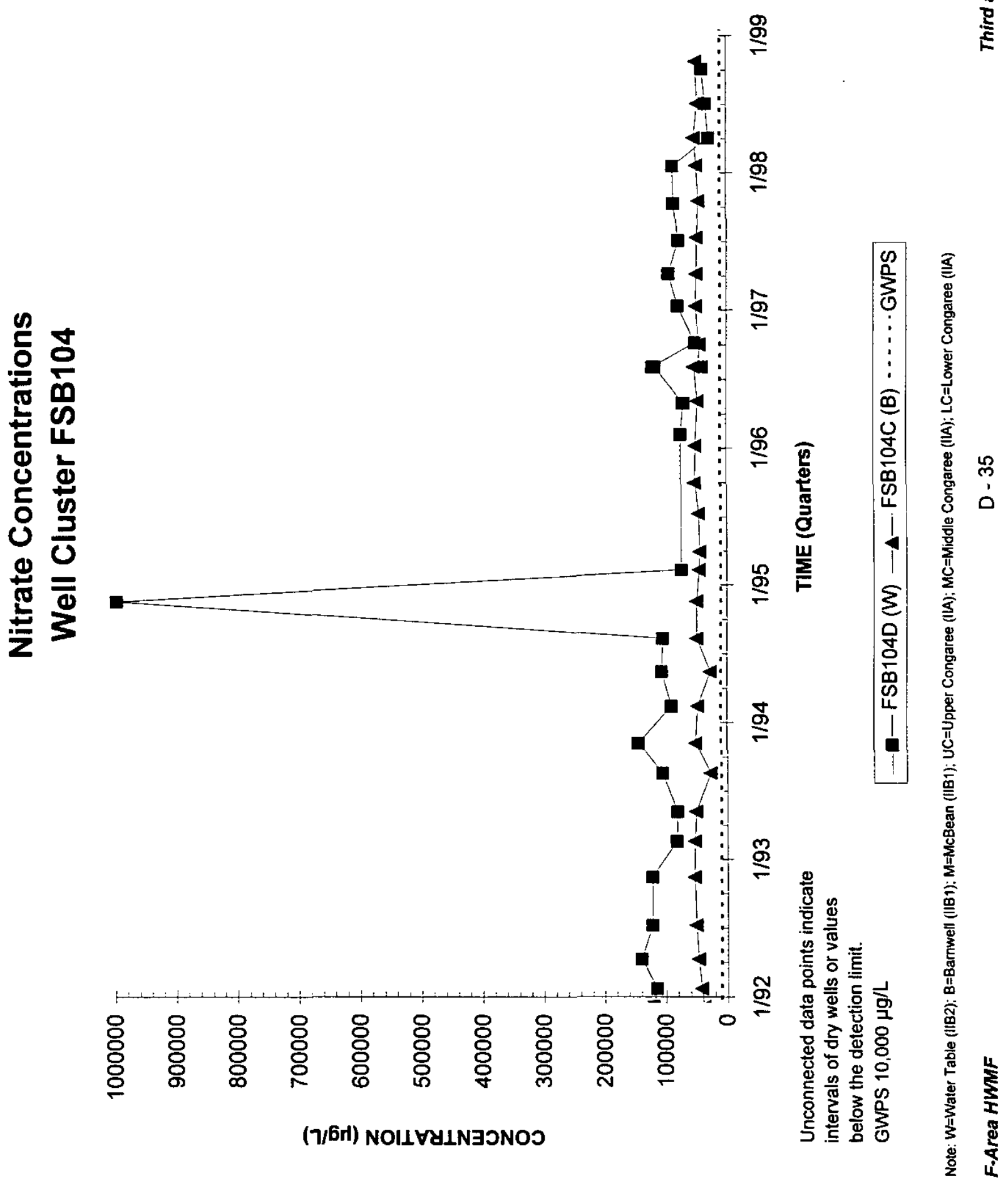



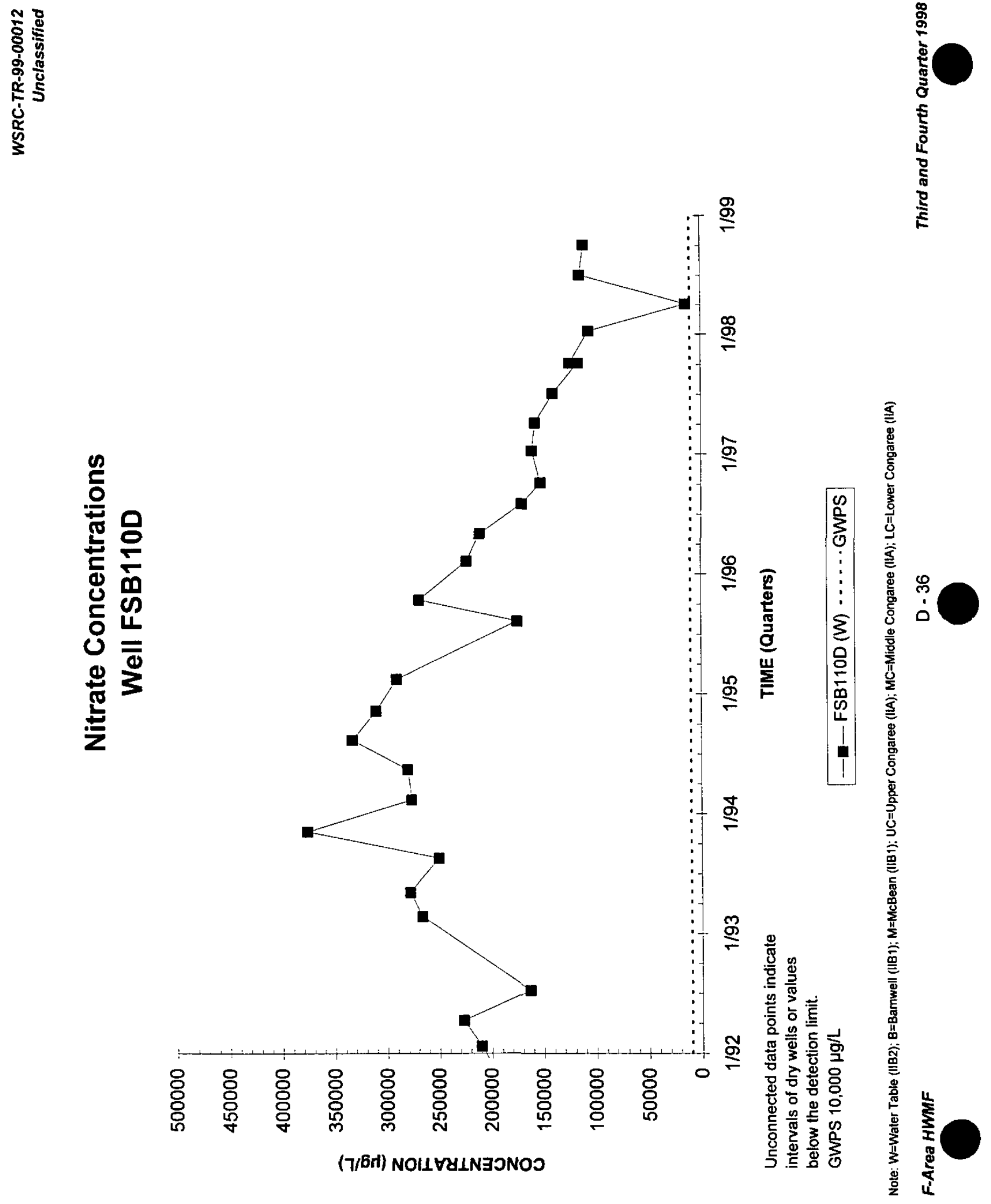

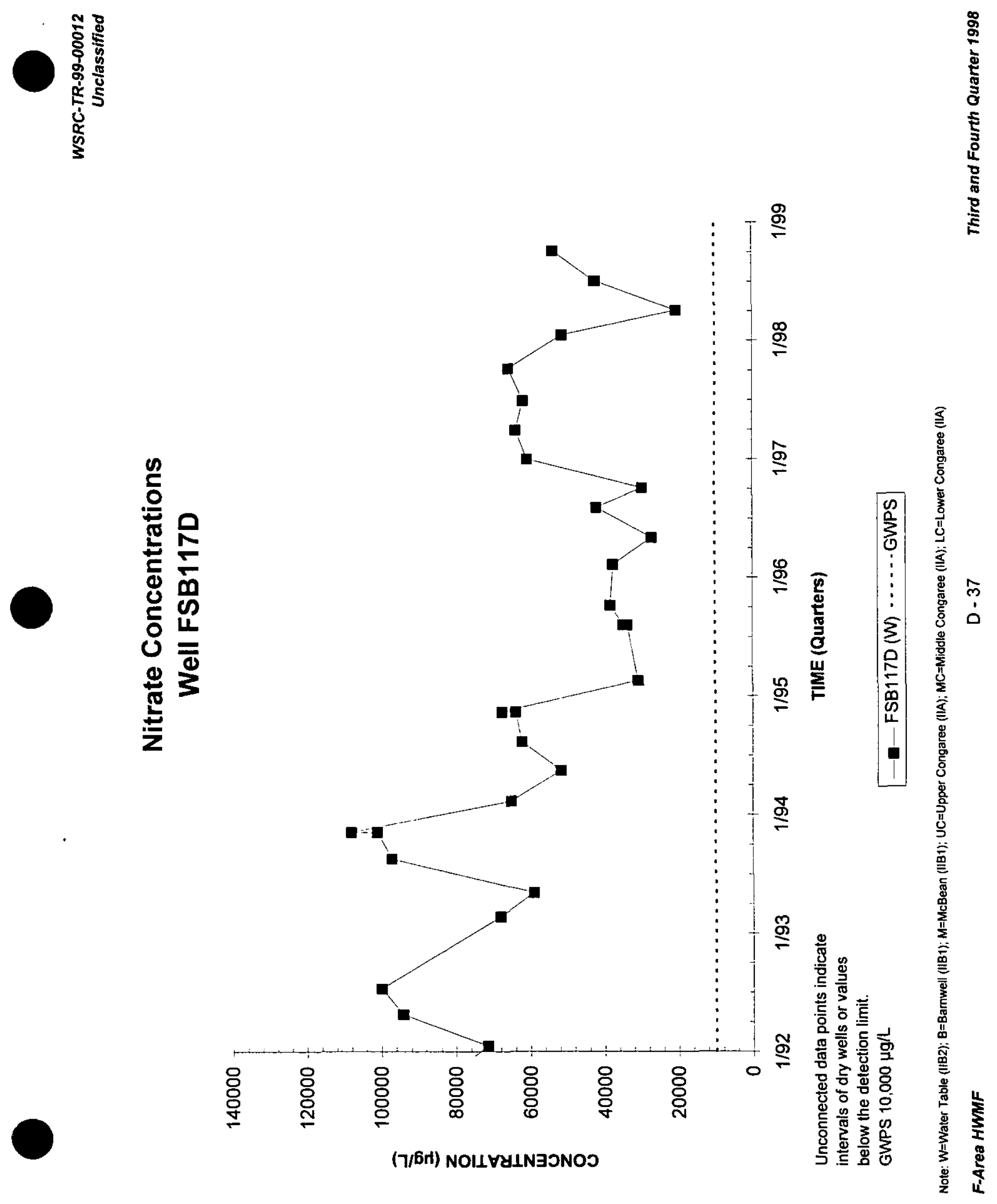

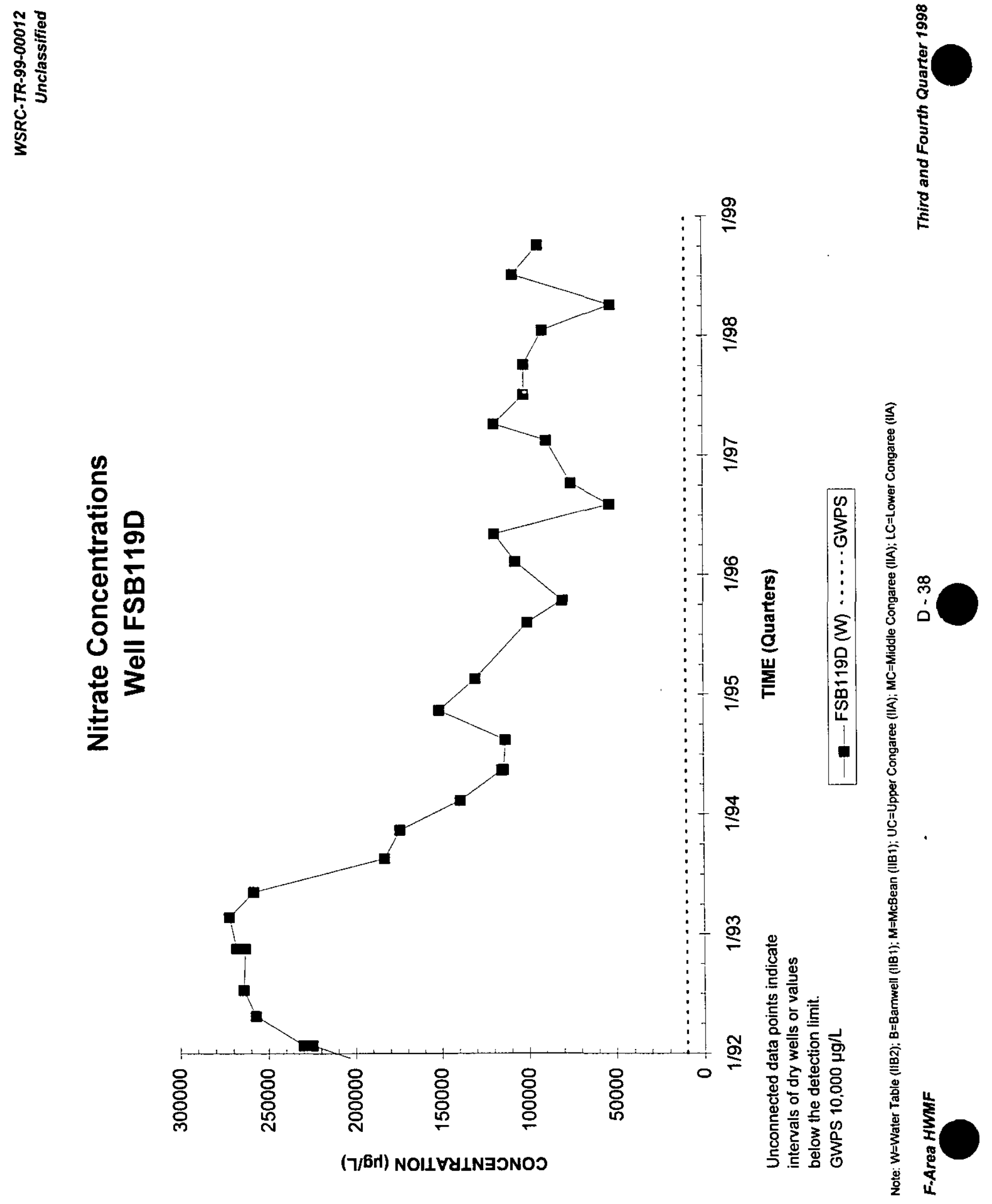

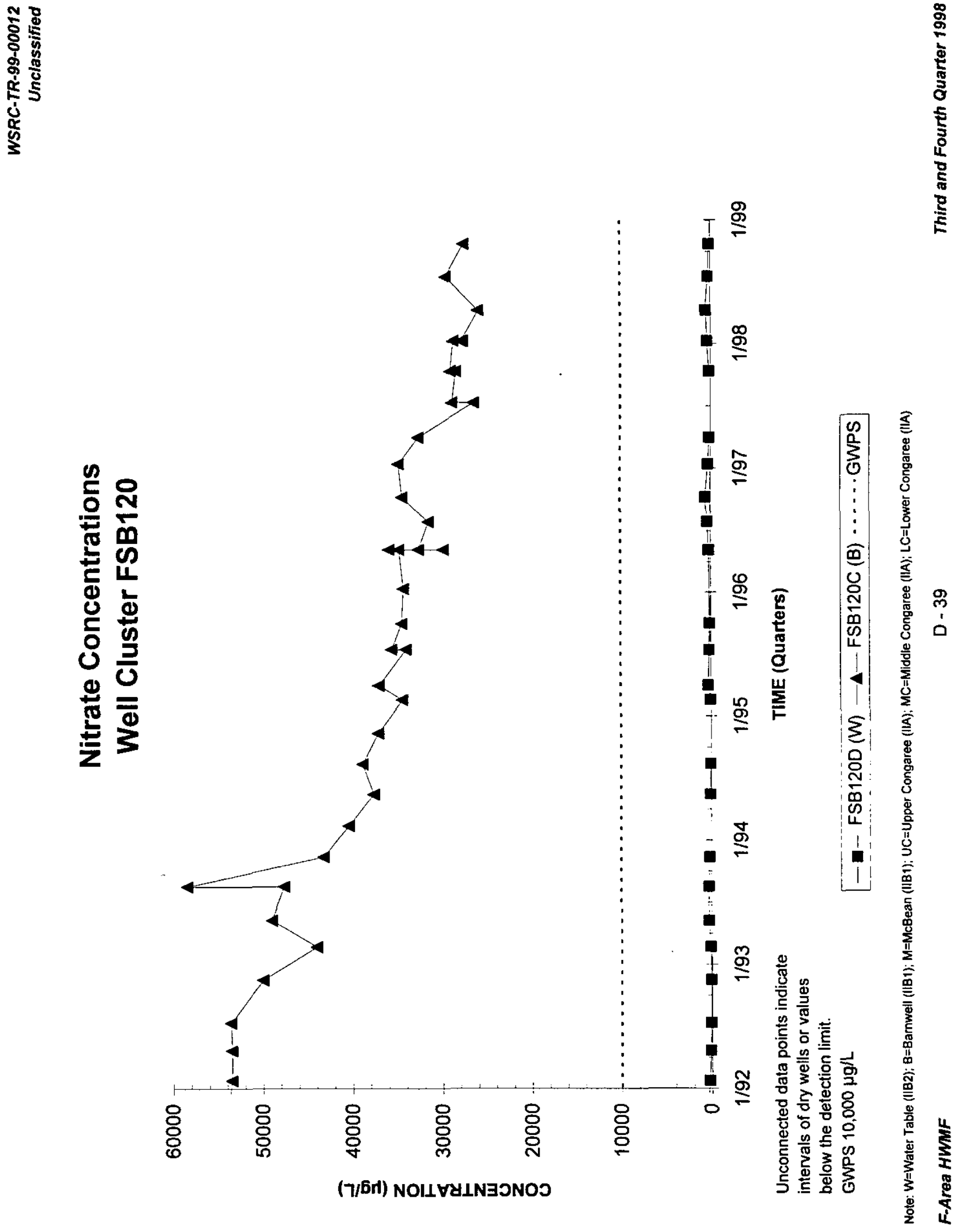


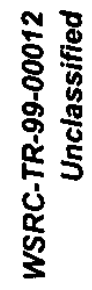

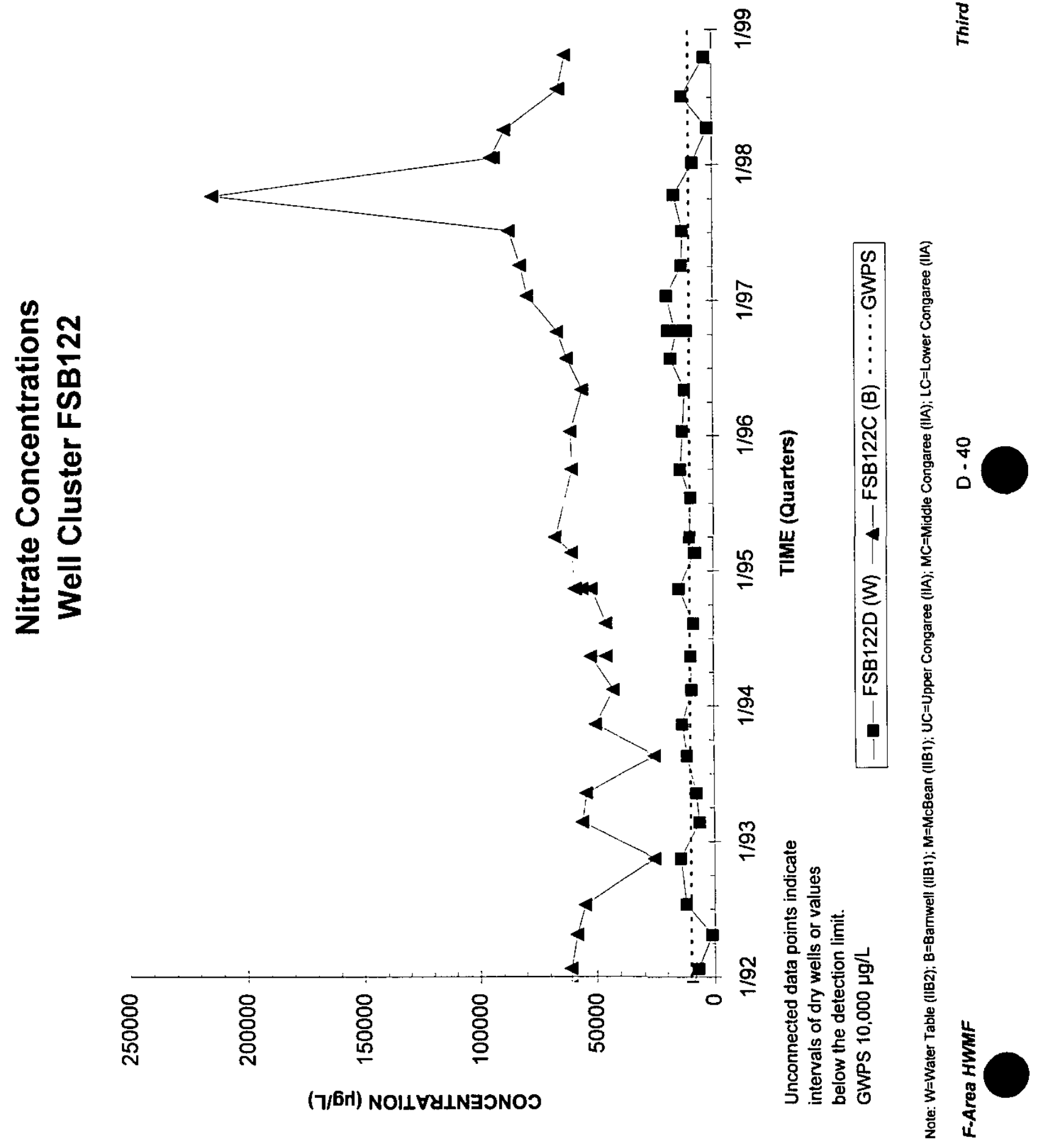




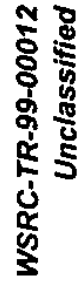

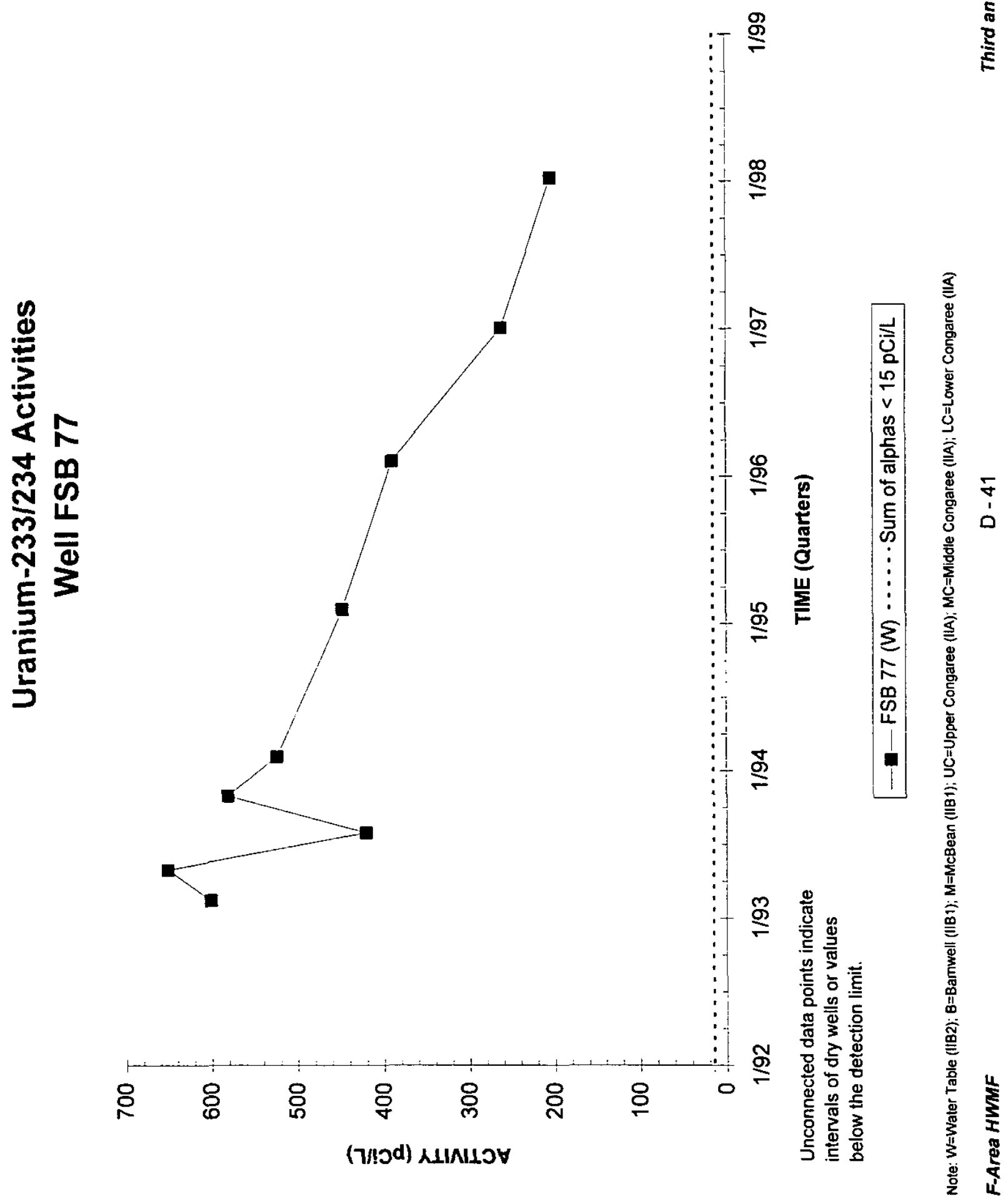



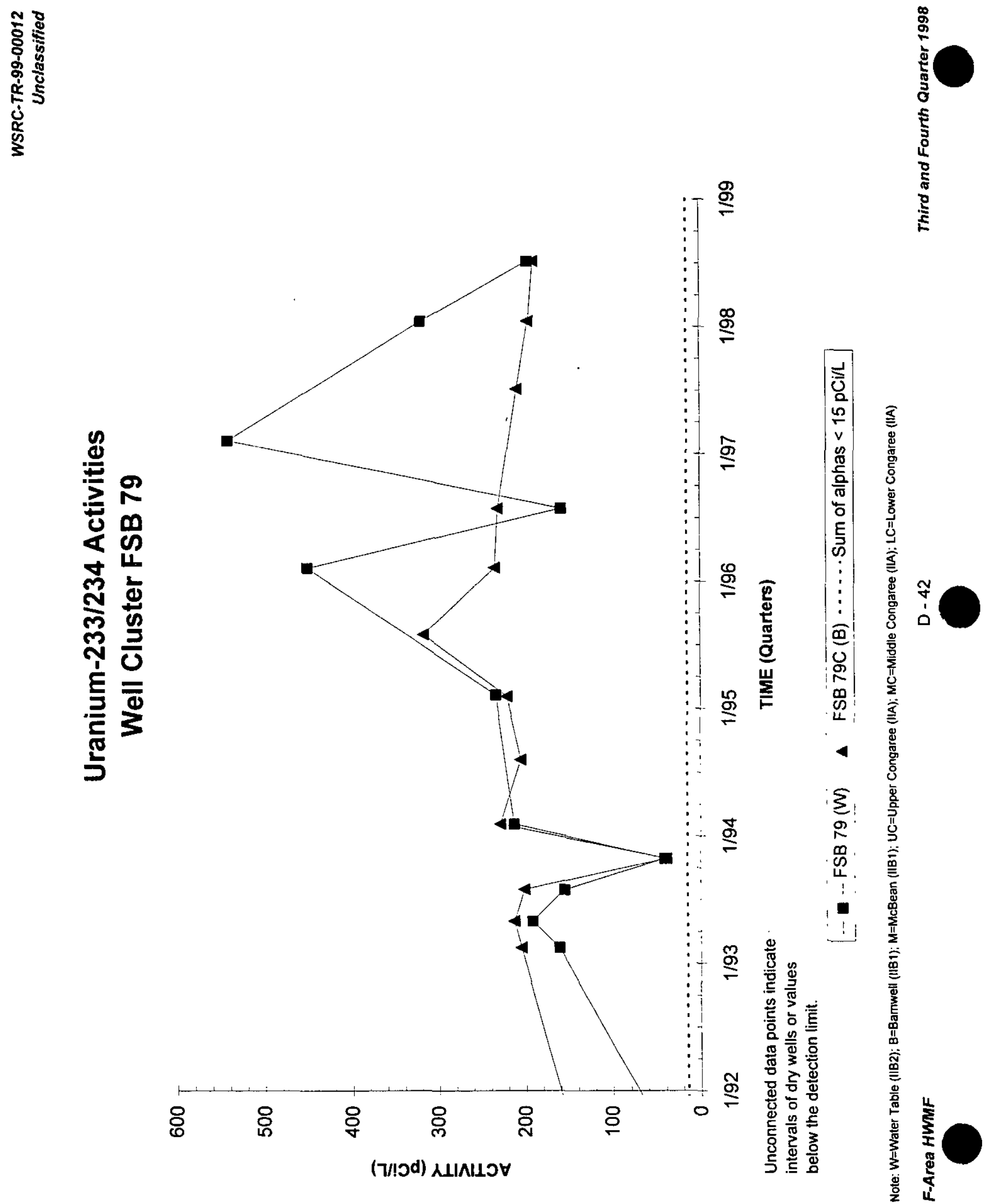


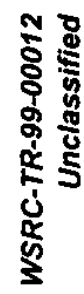

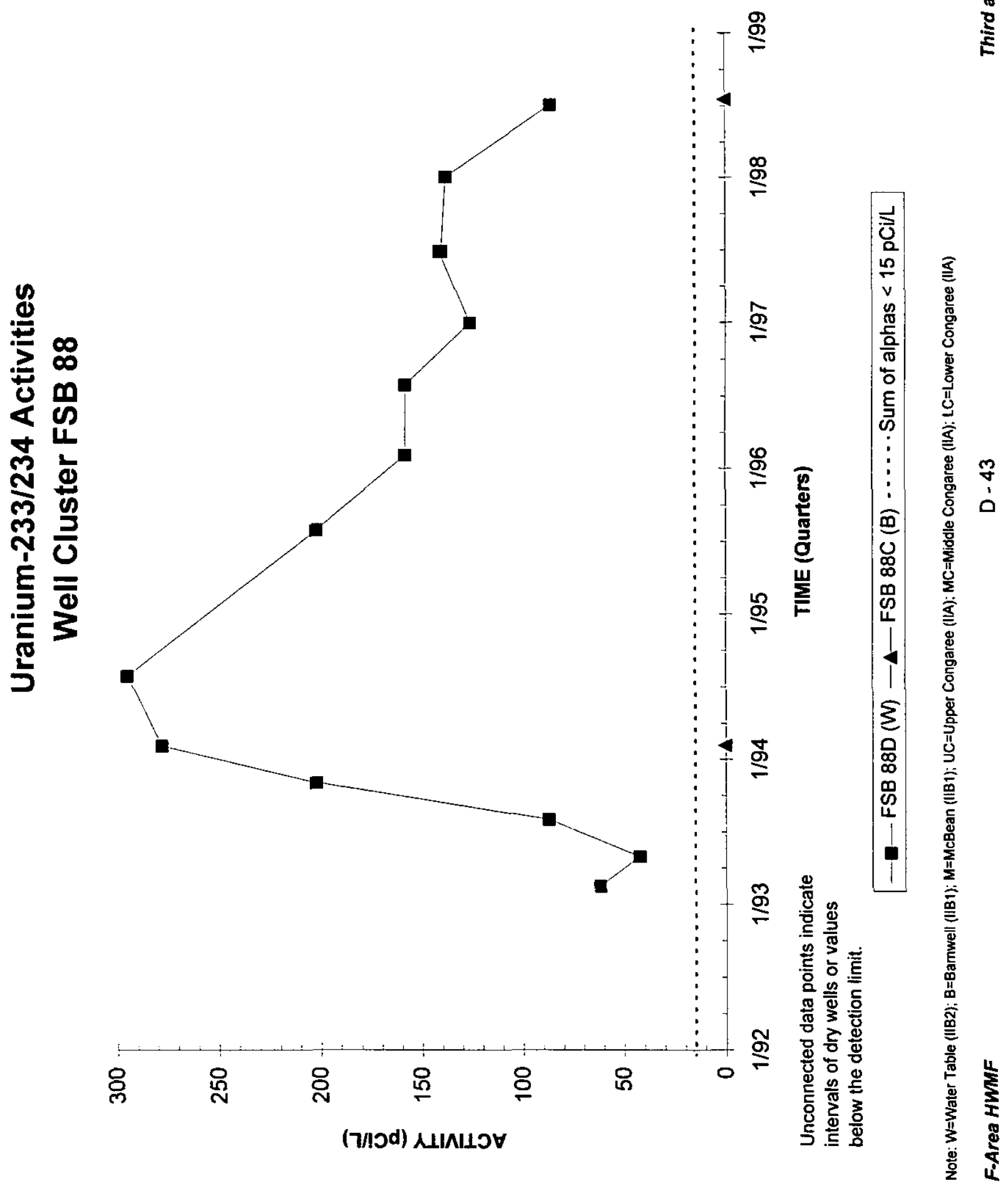




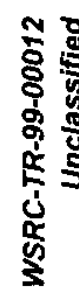
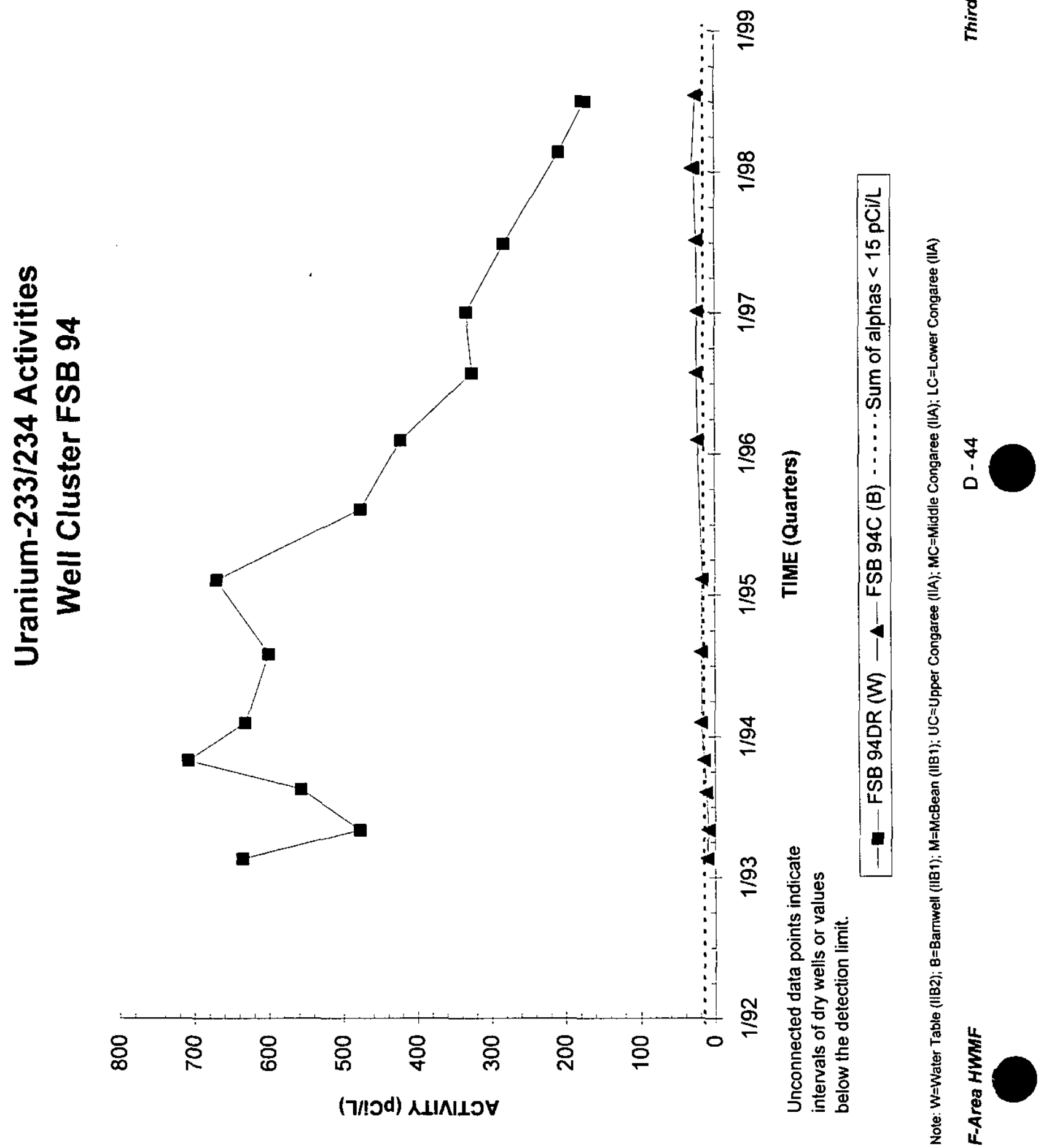

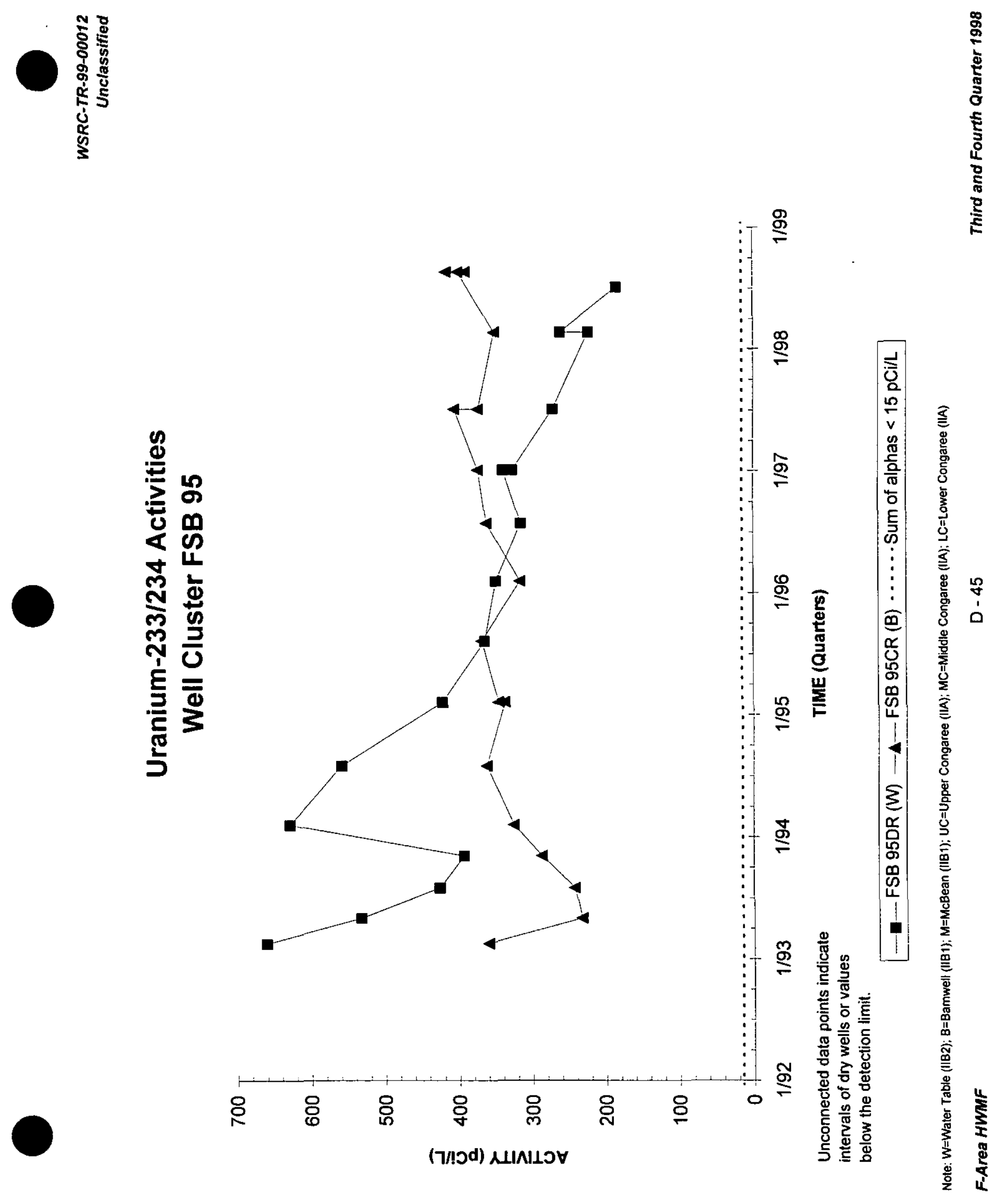


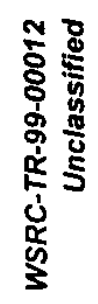

:
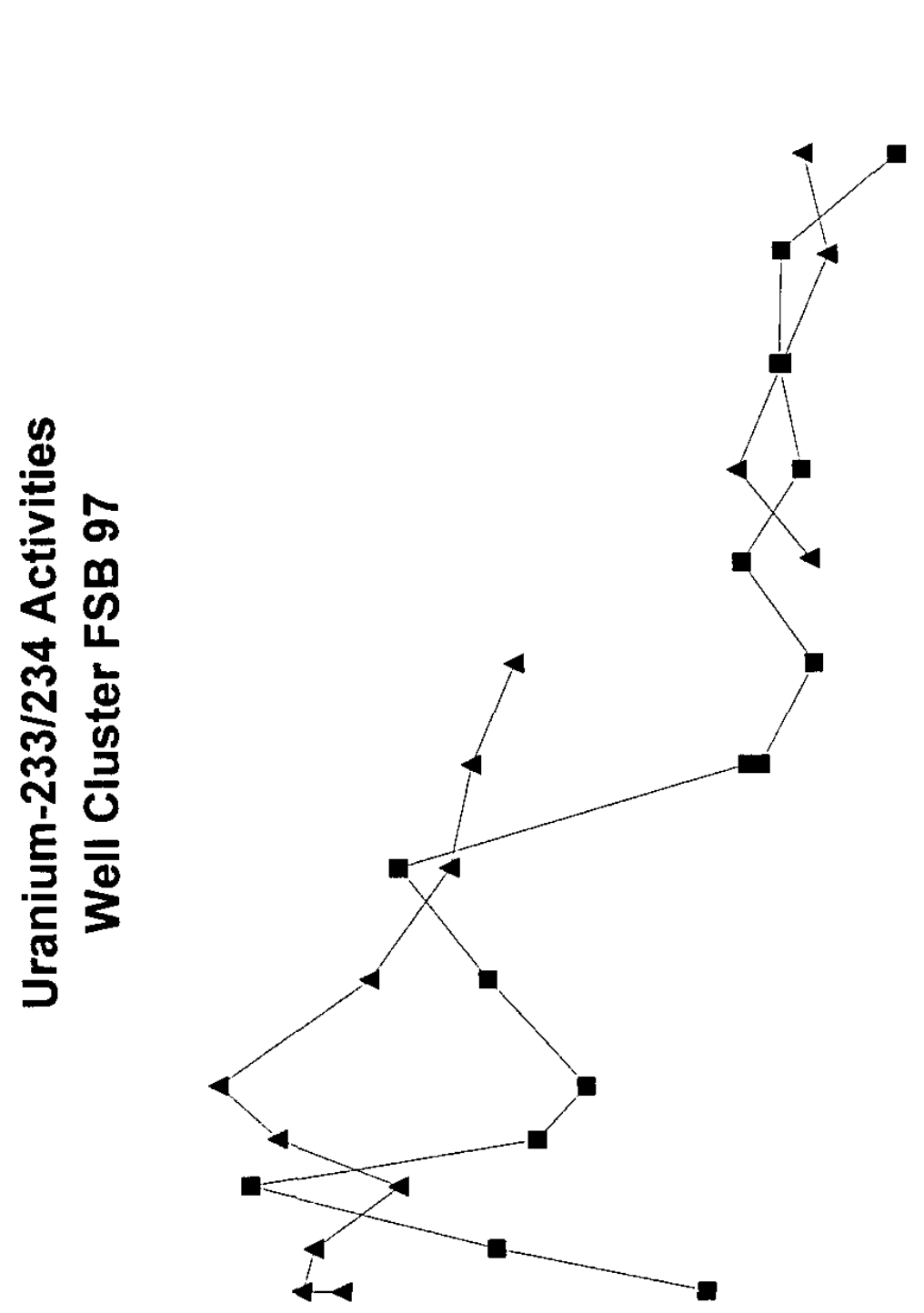

I

$\stackrel{9}{\stackrel{9}{7}}$

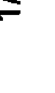

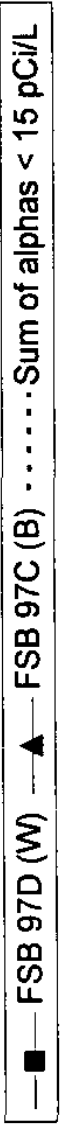

ฒำ

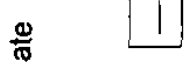

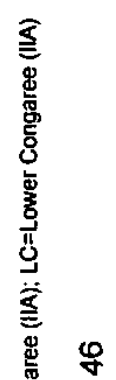

á

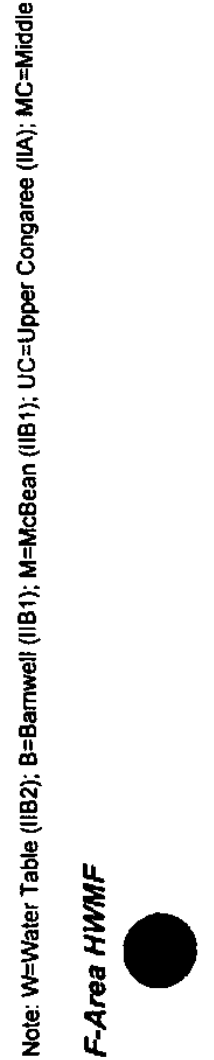



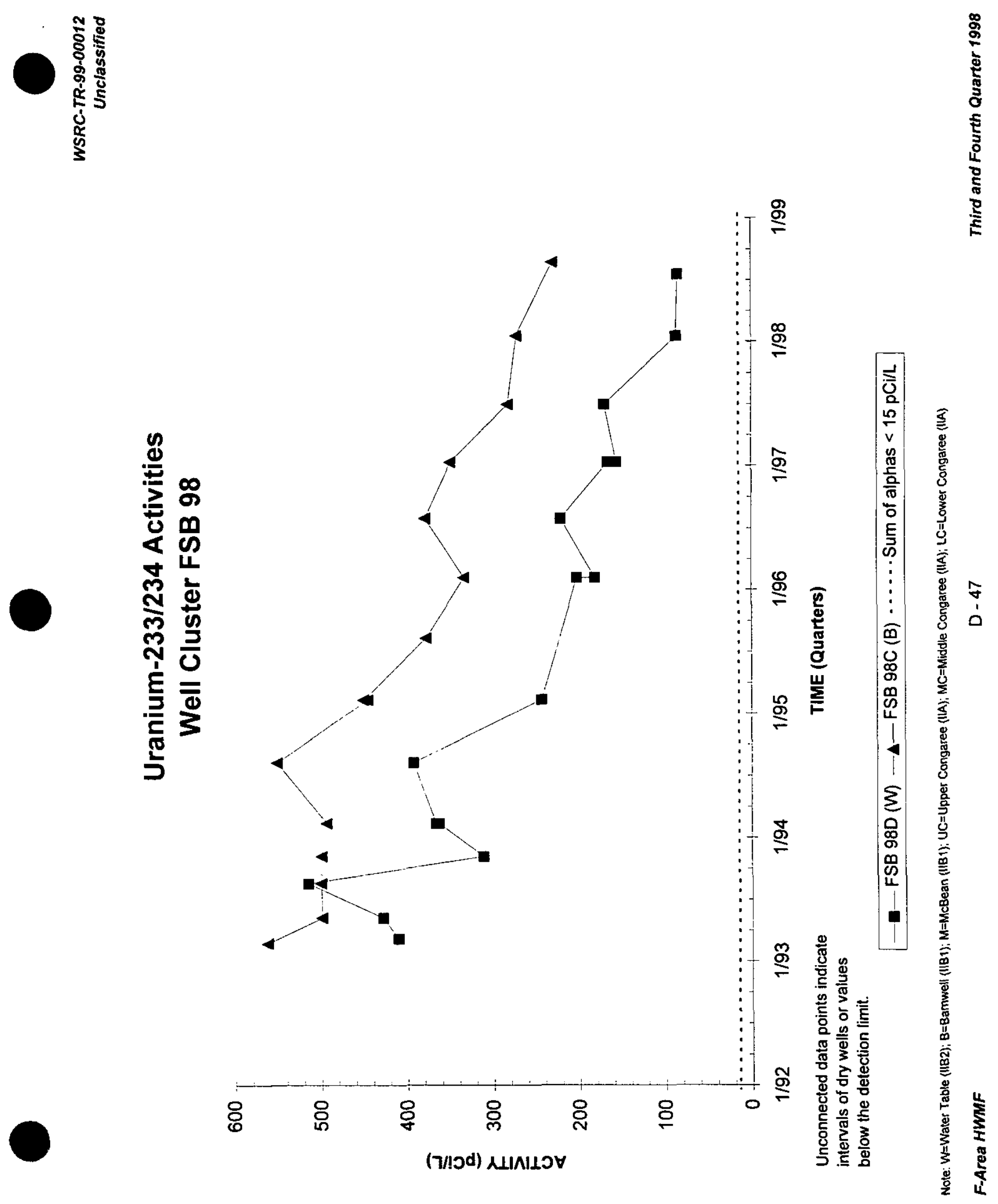

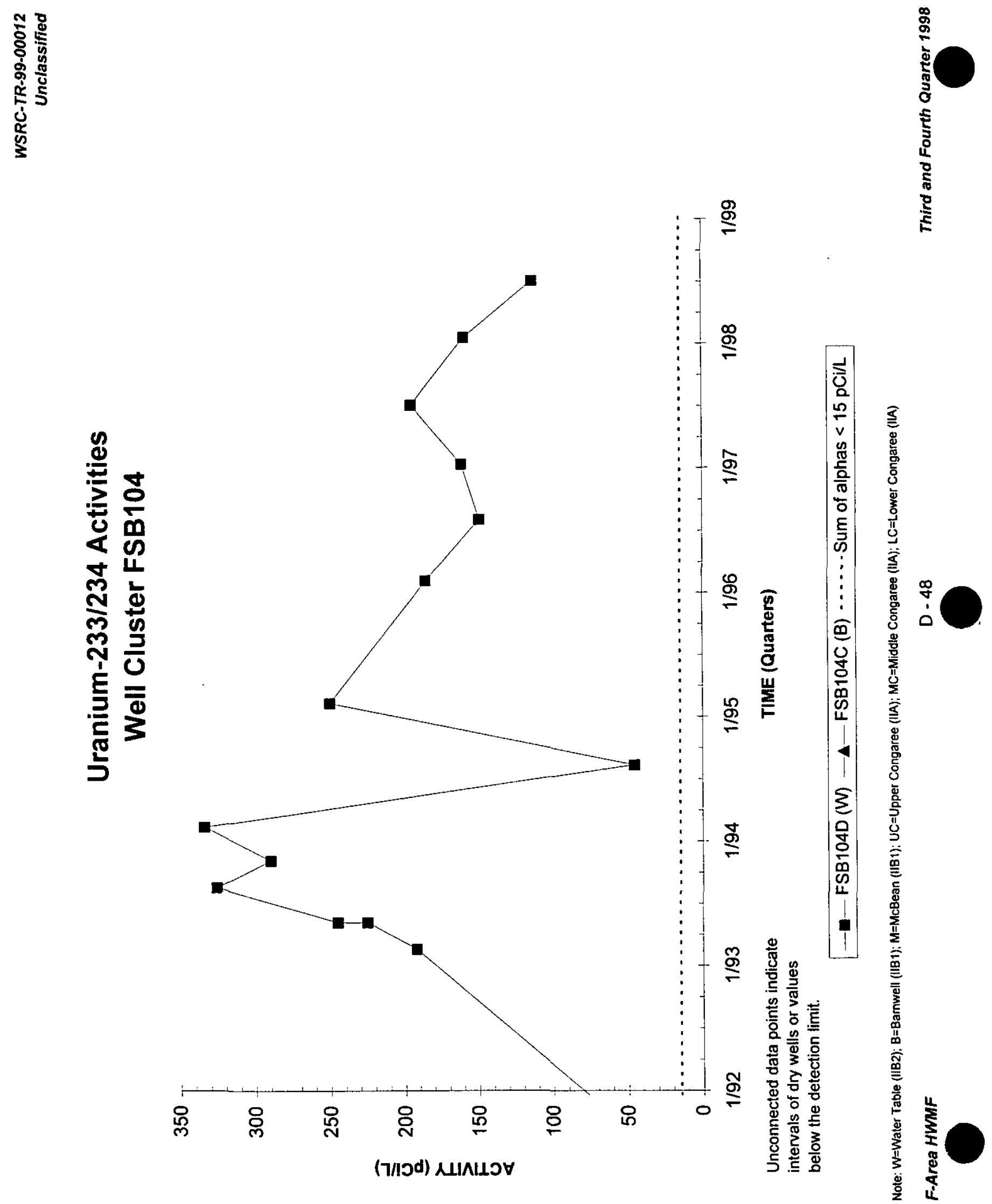

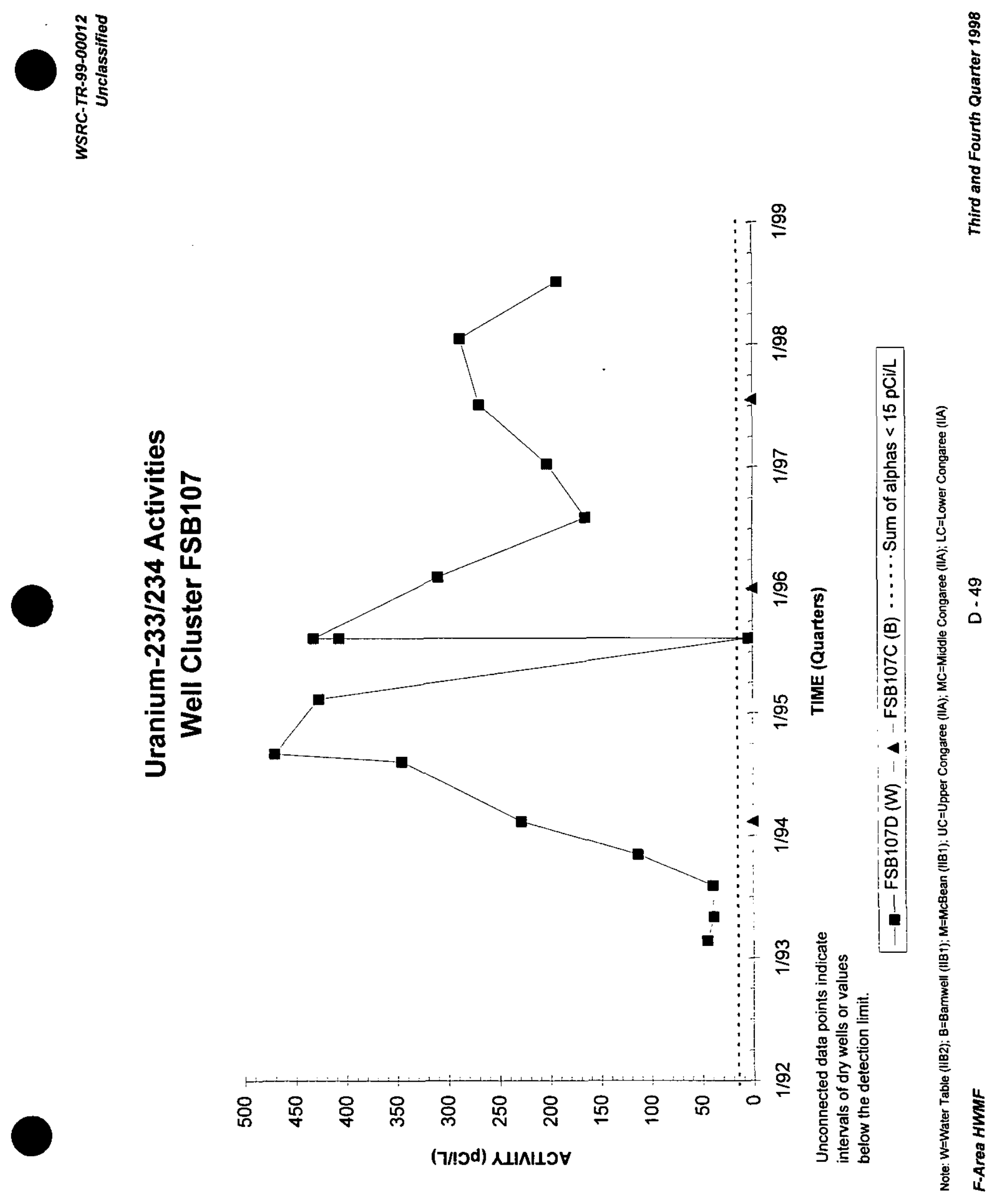


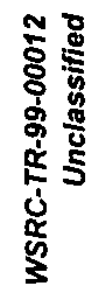

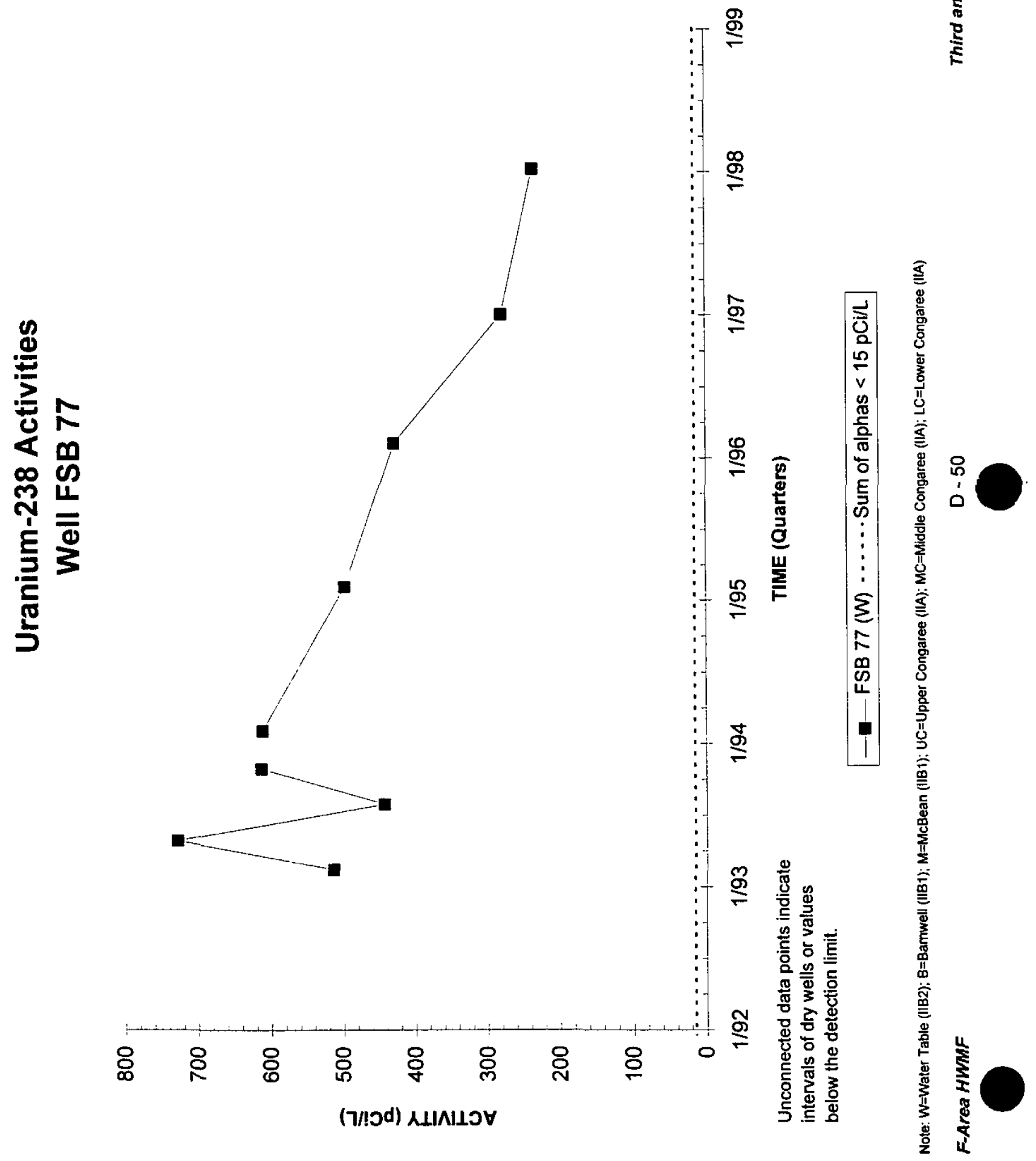




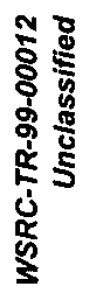

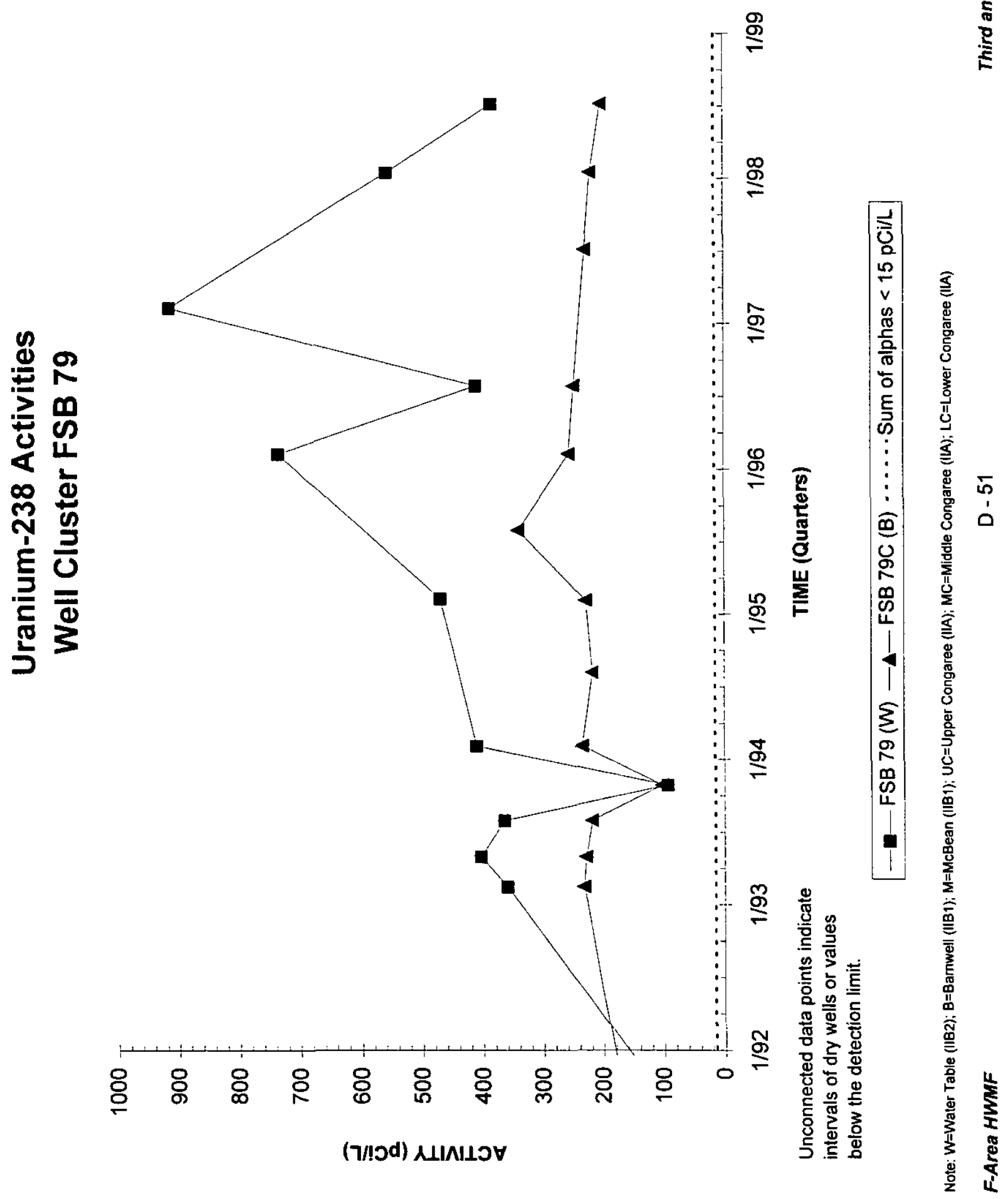



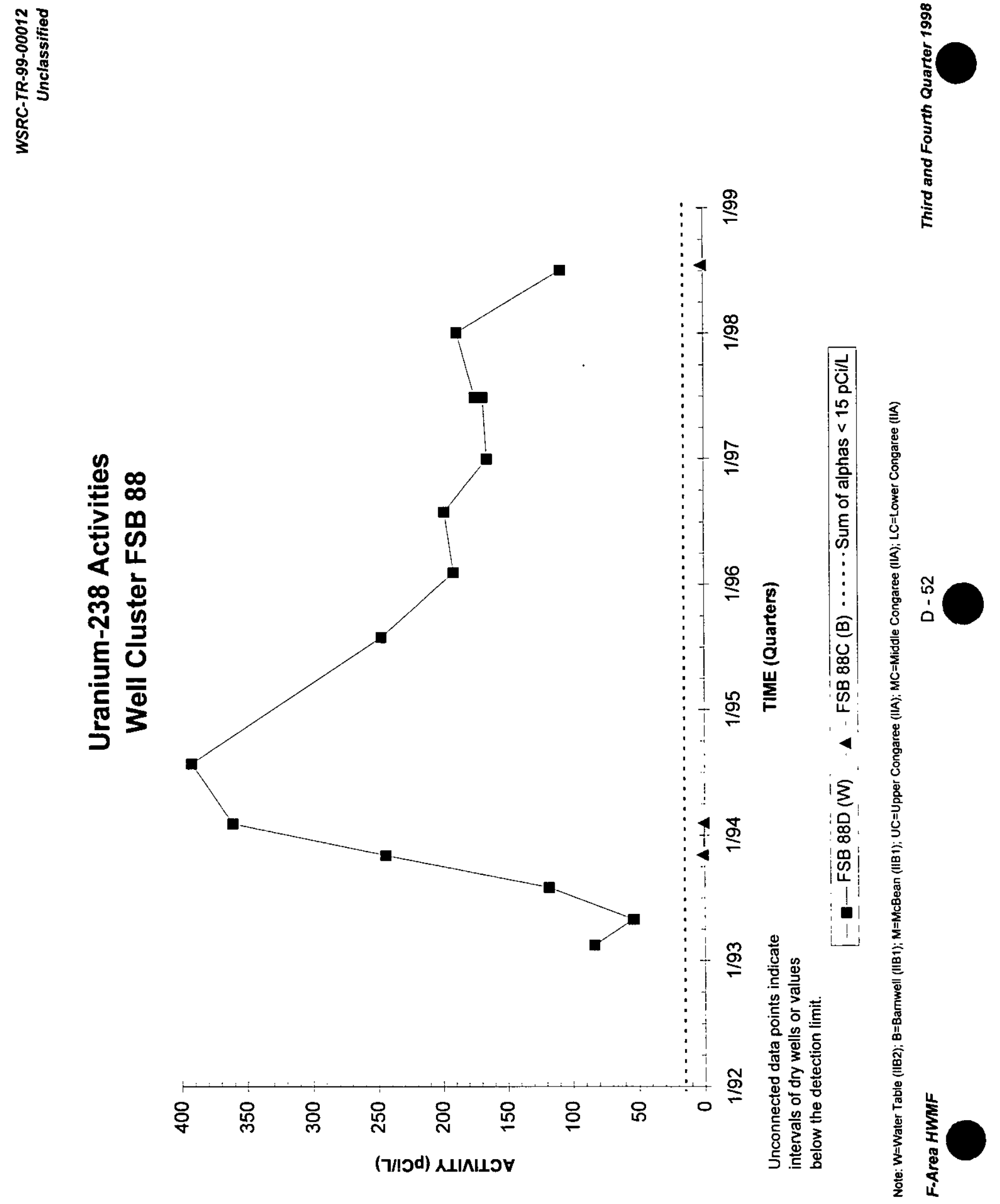


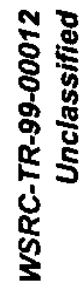

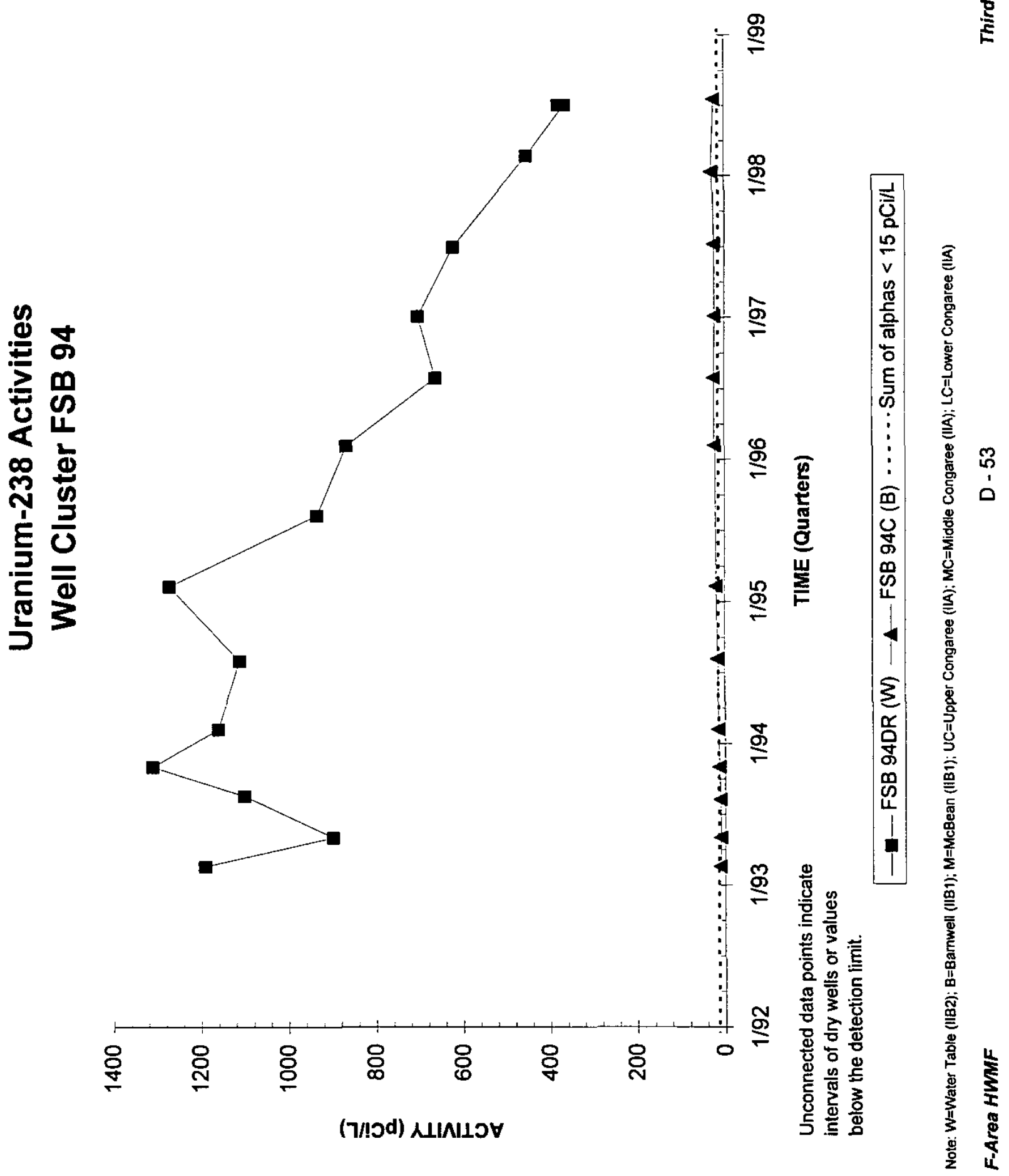



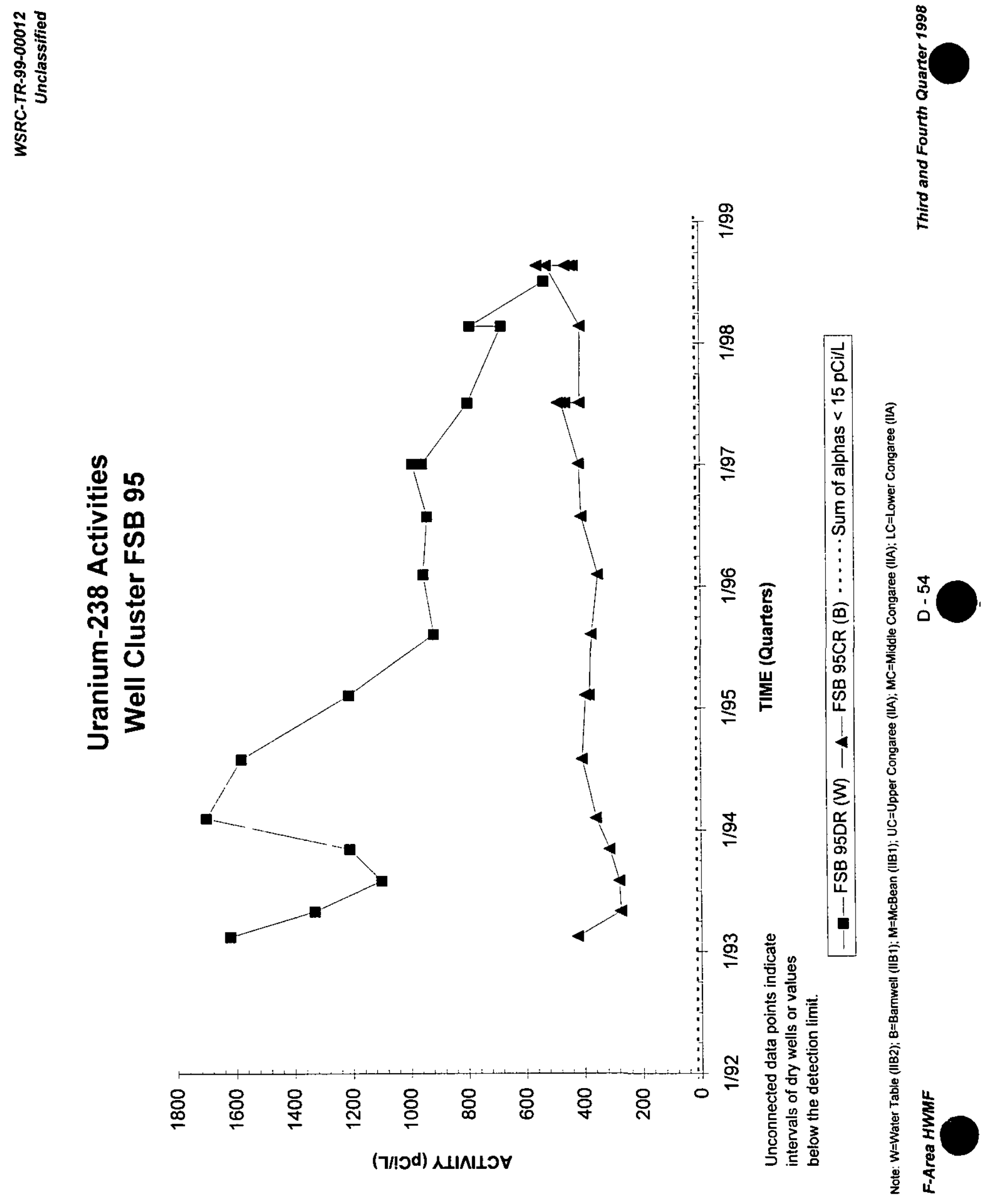

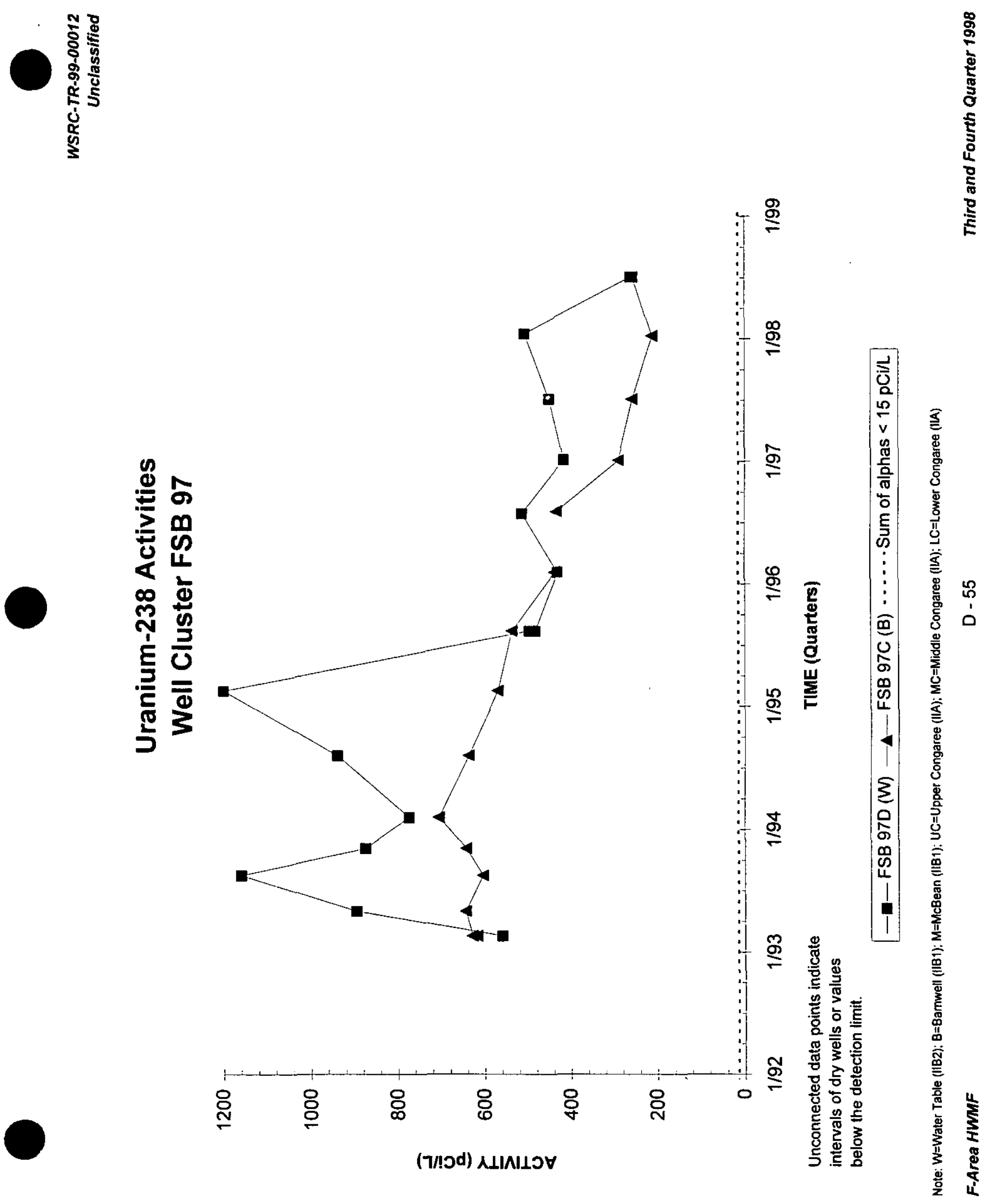

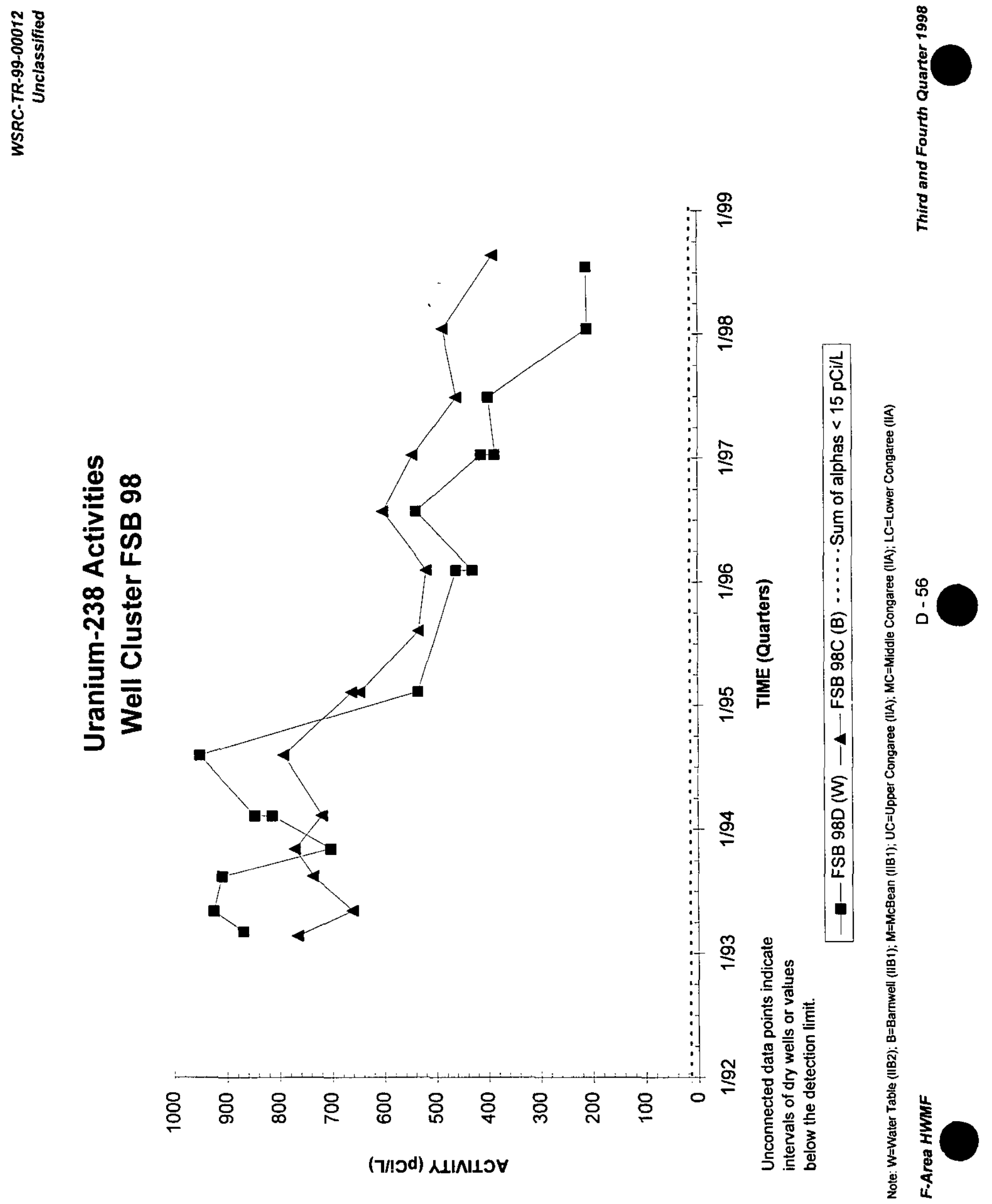

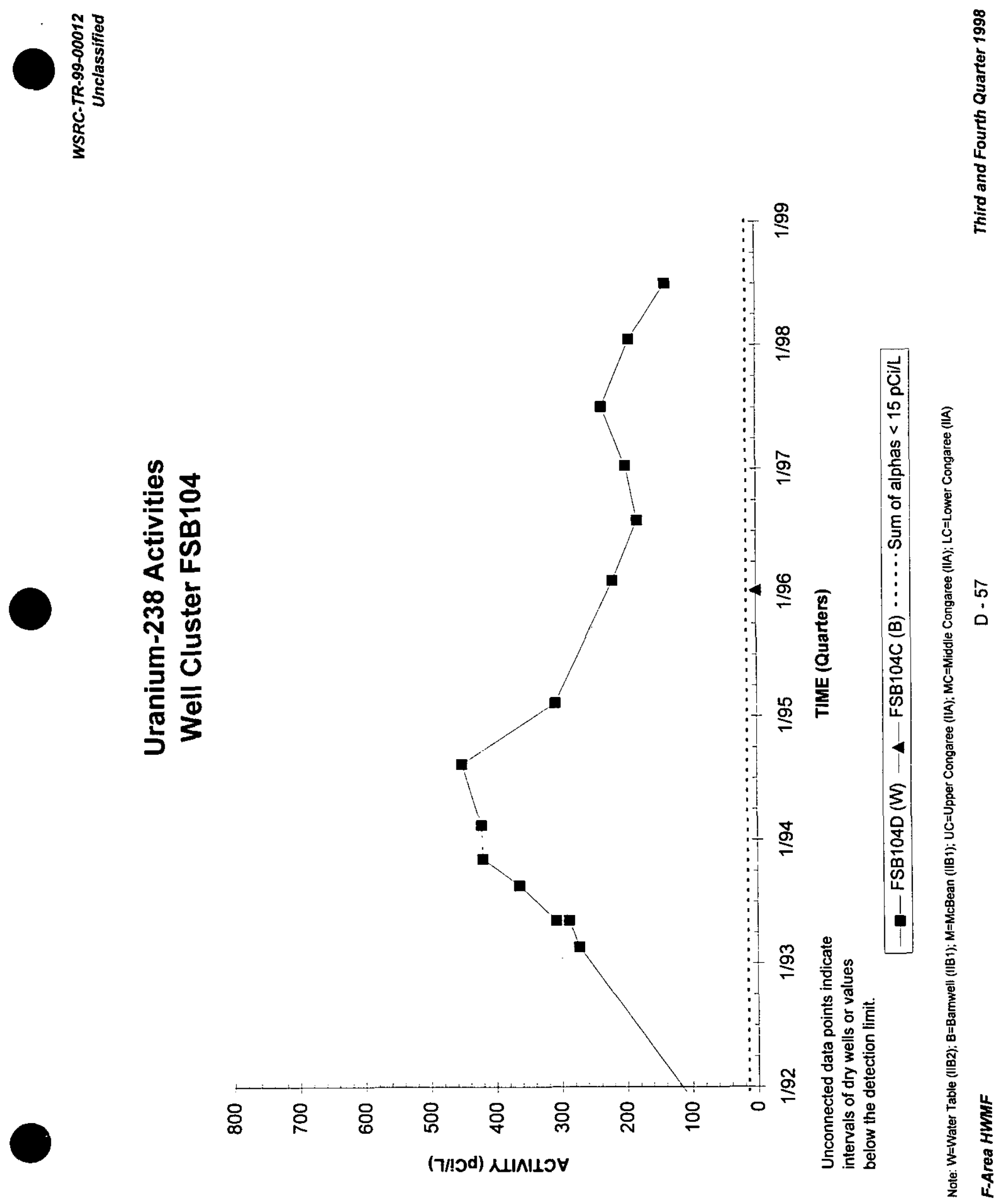


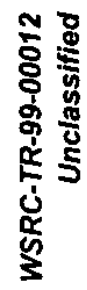

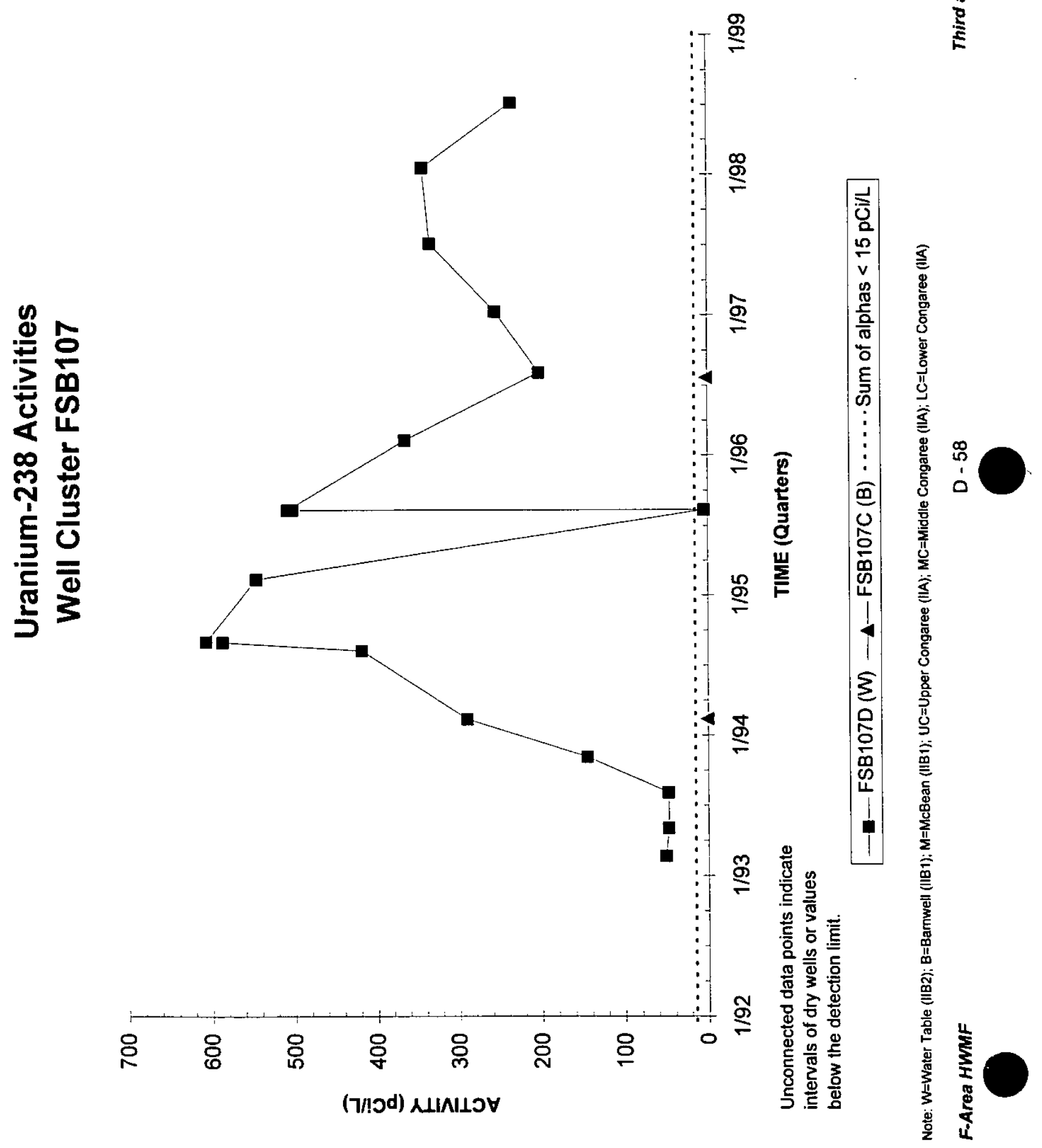



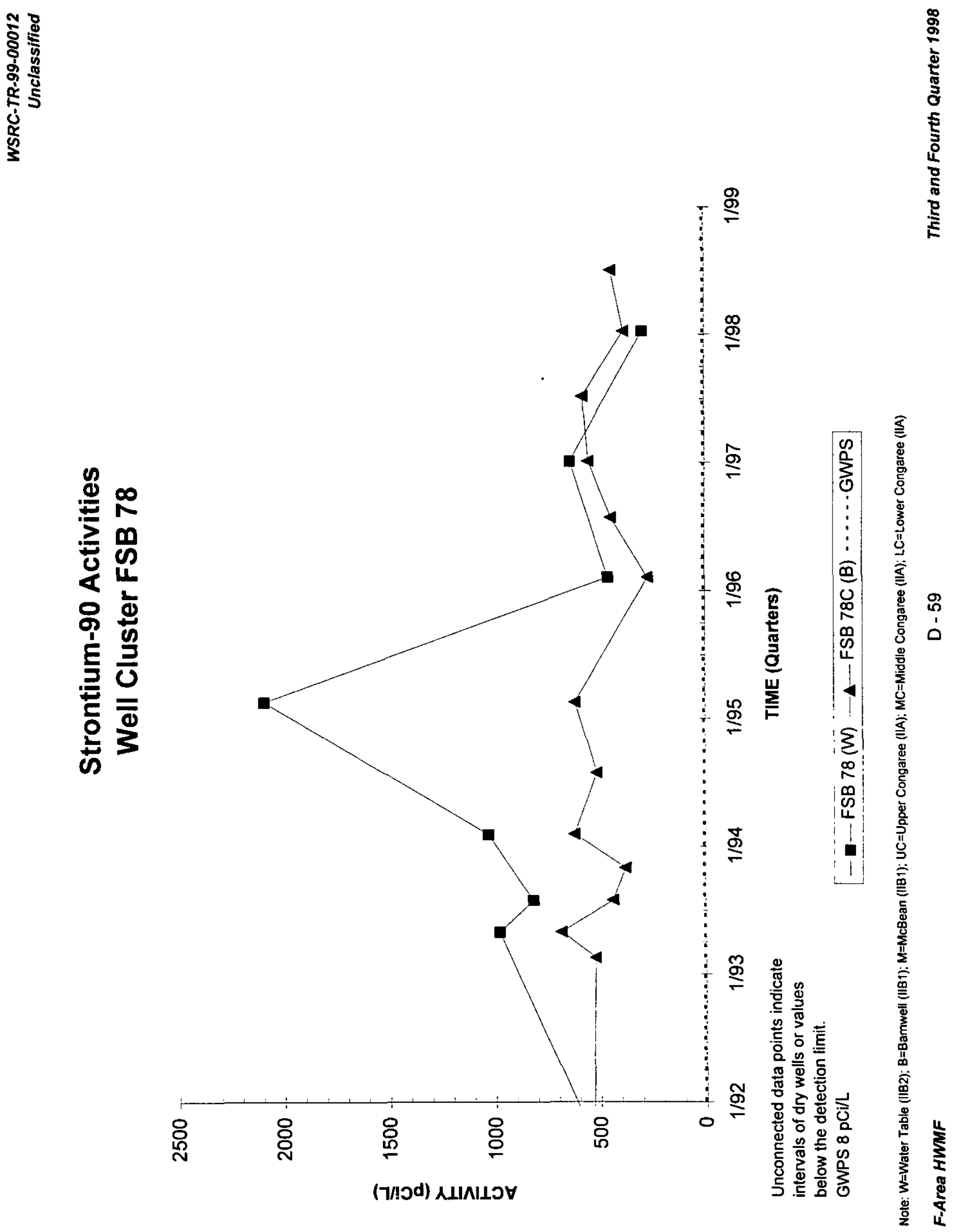


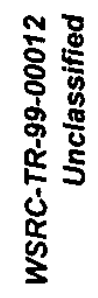

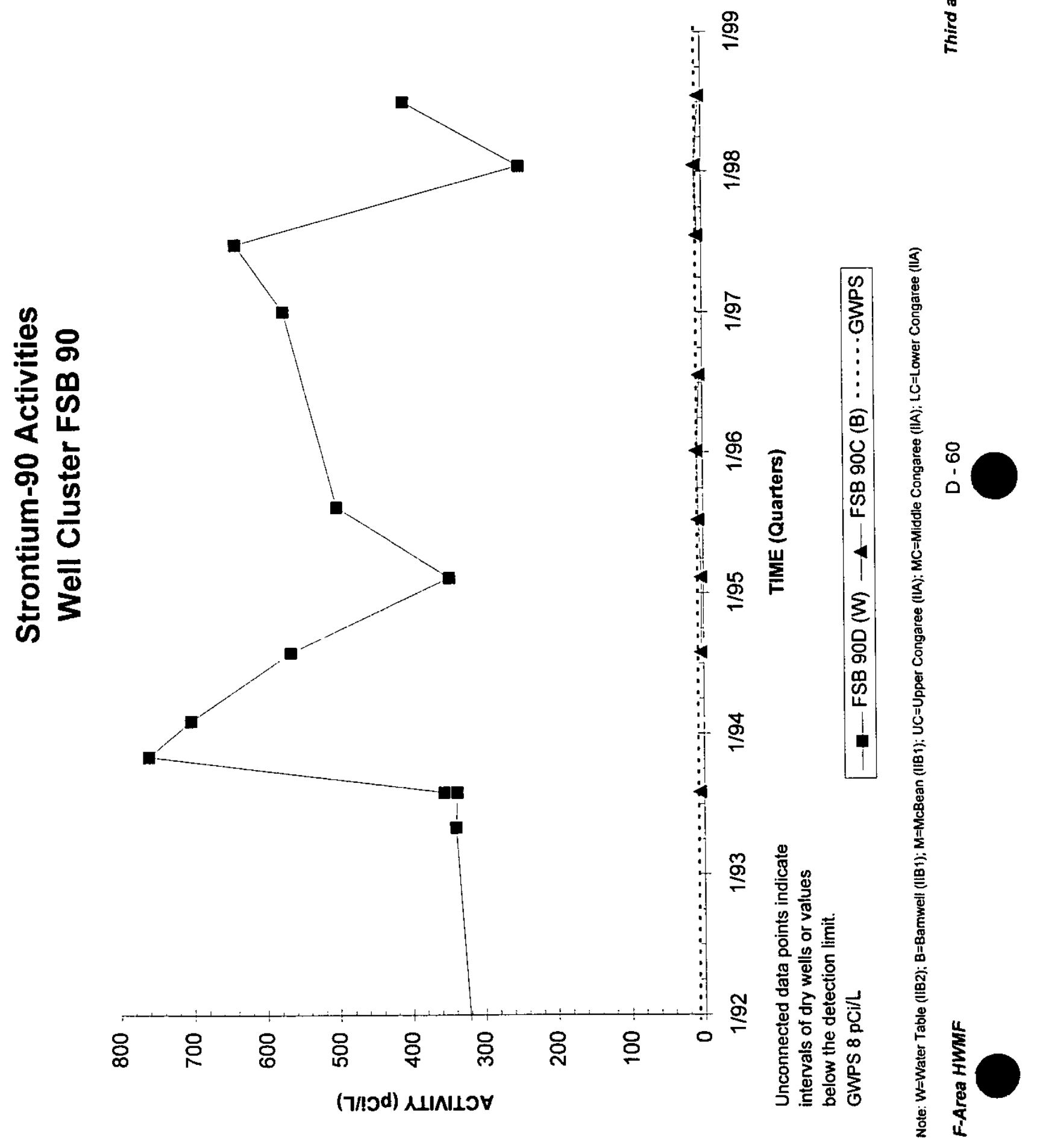



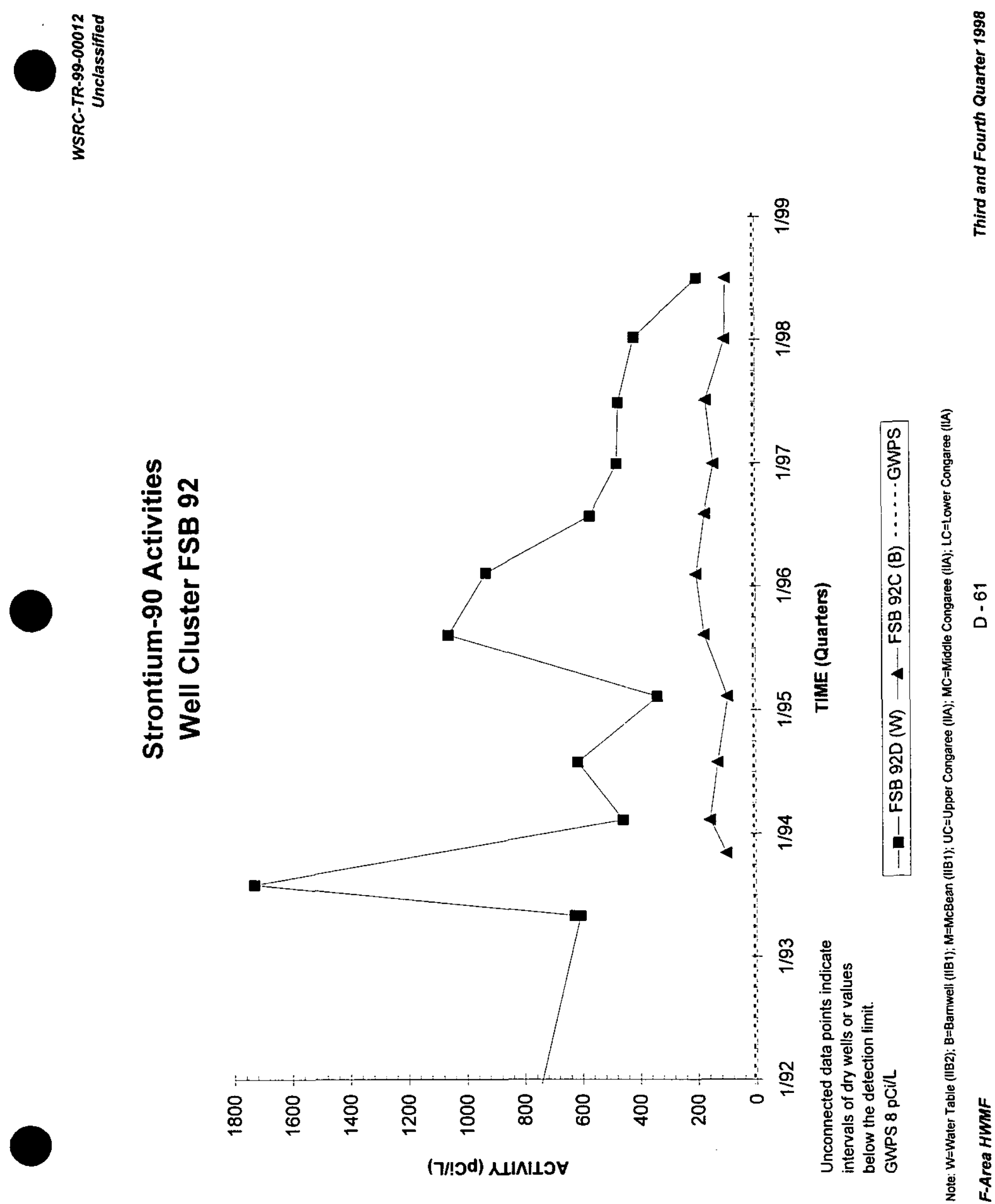

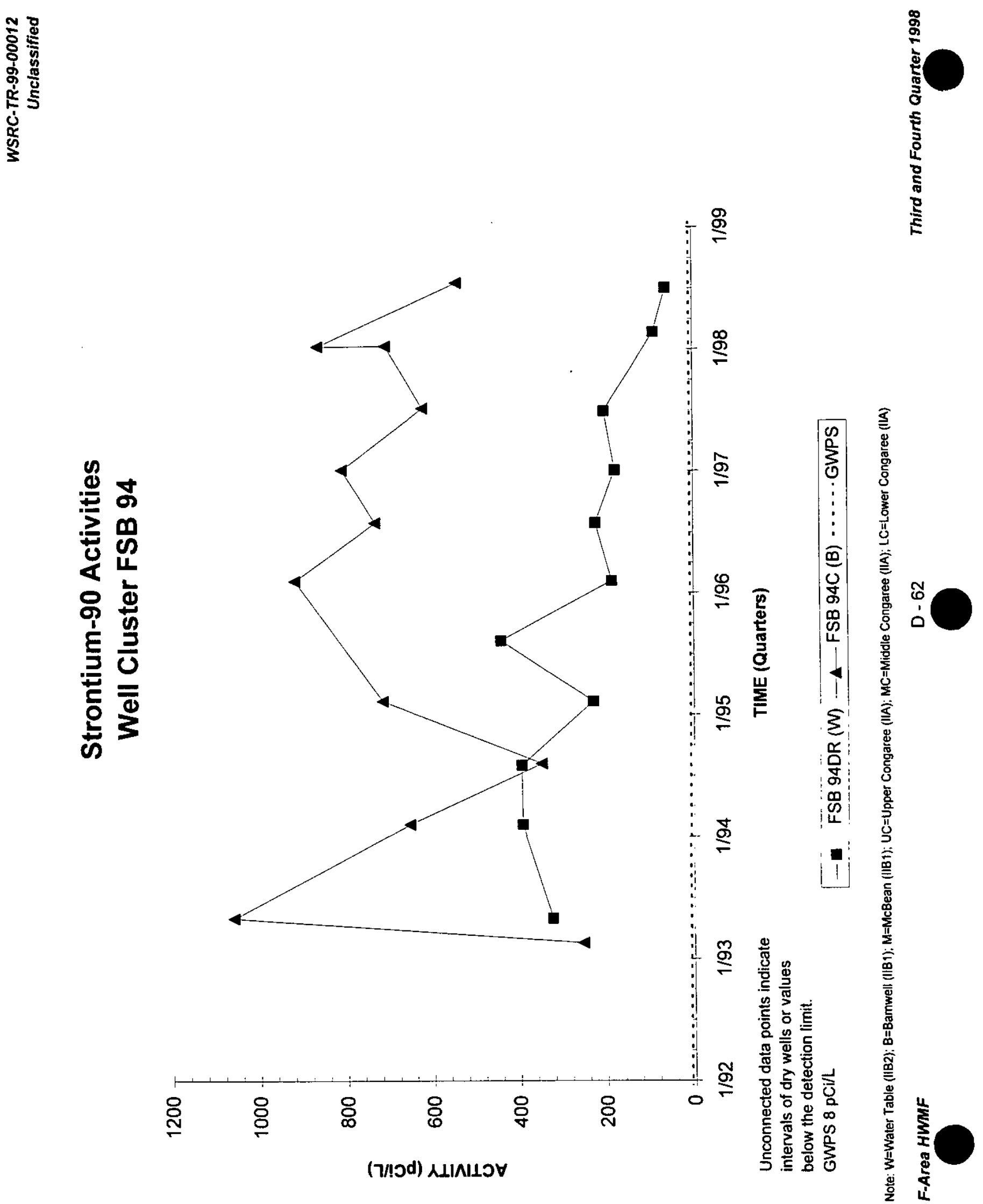

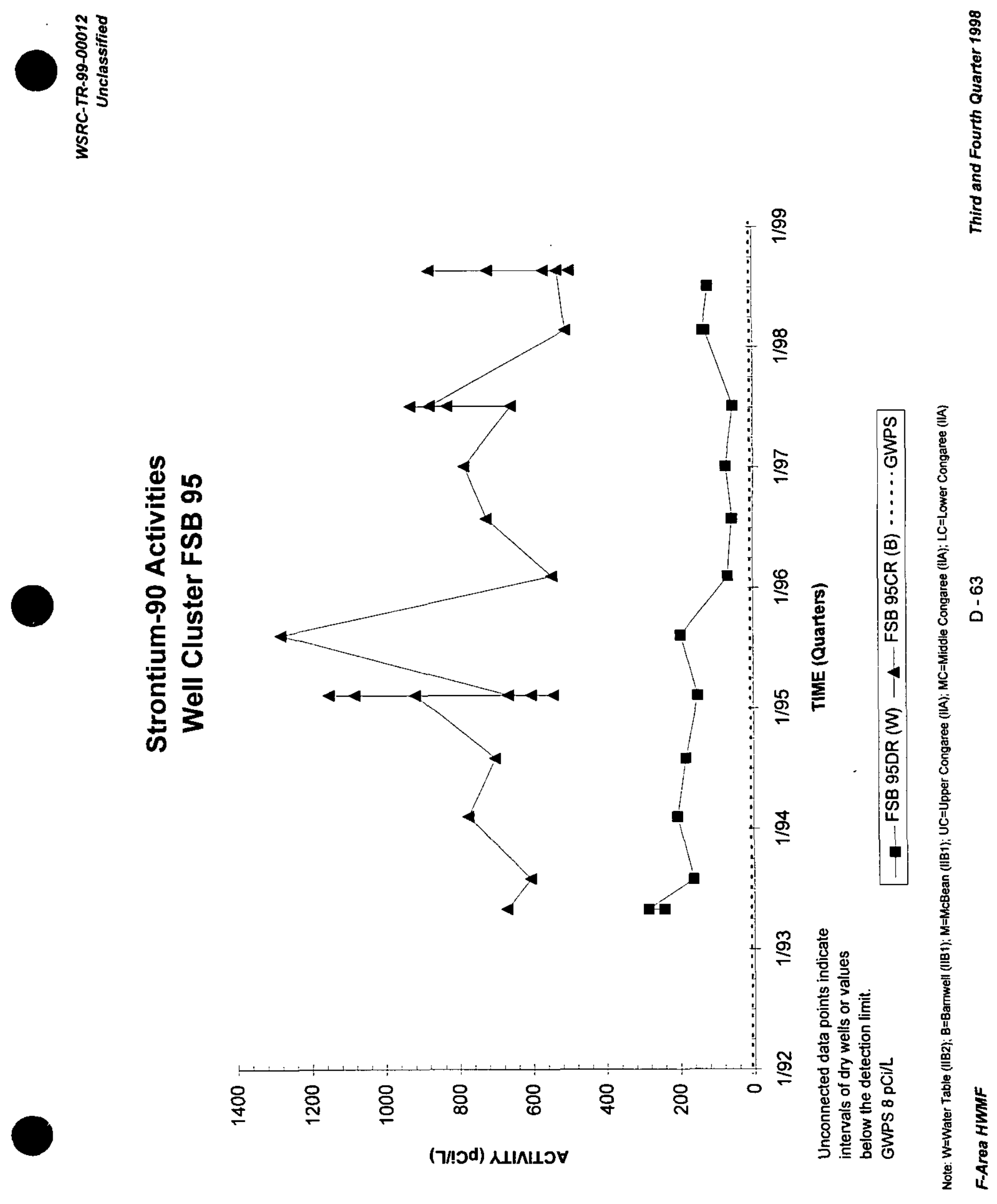


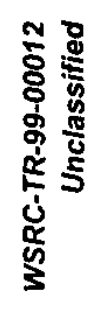

:
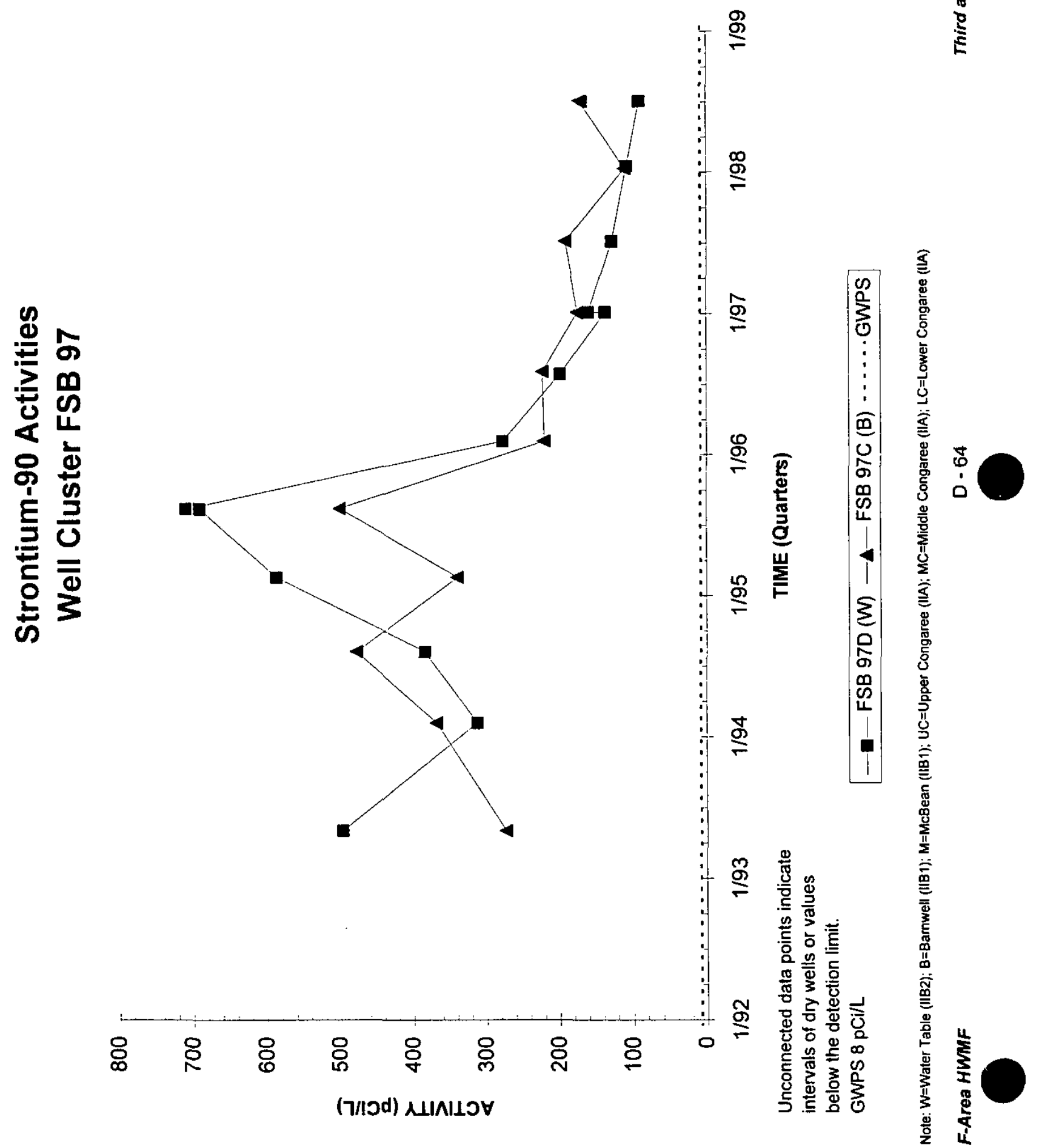


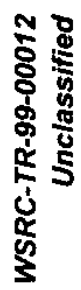

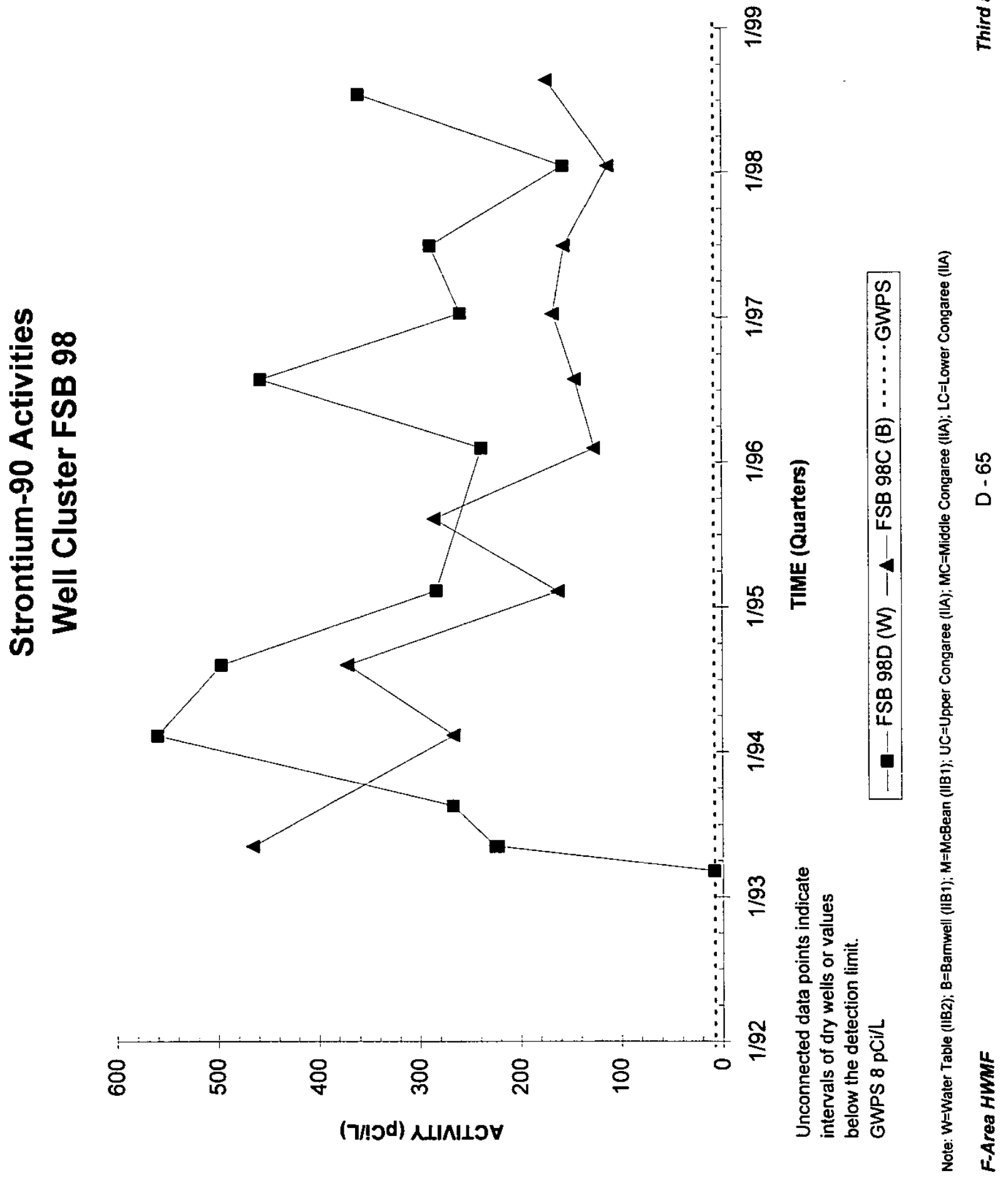



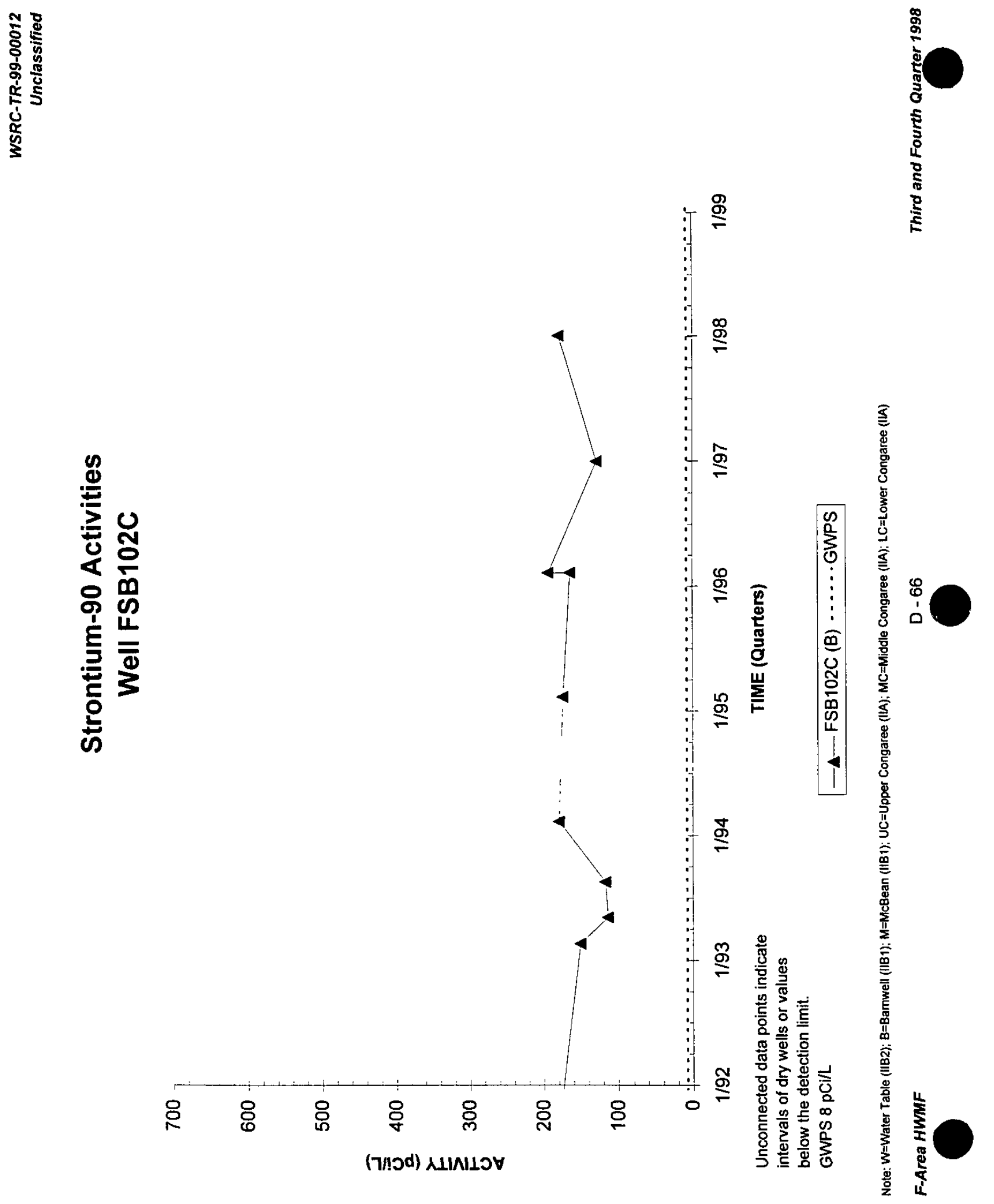

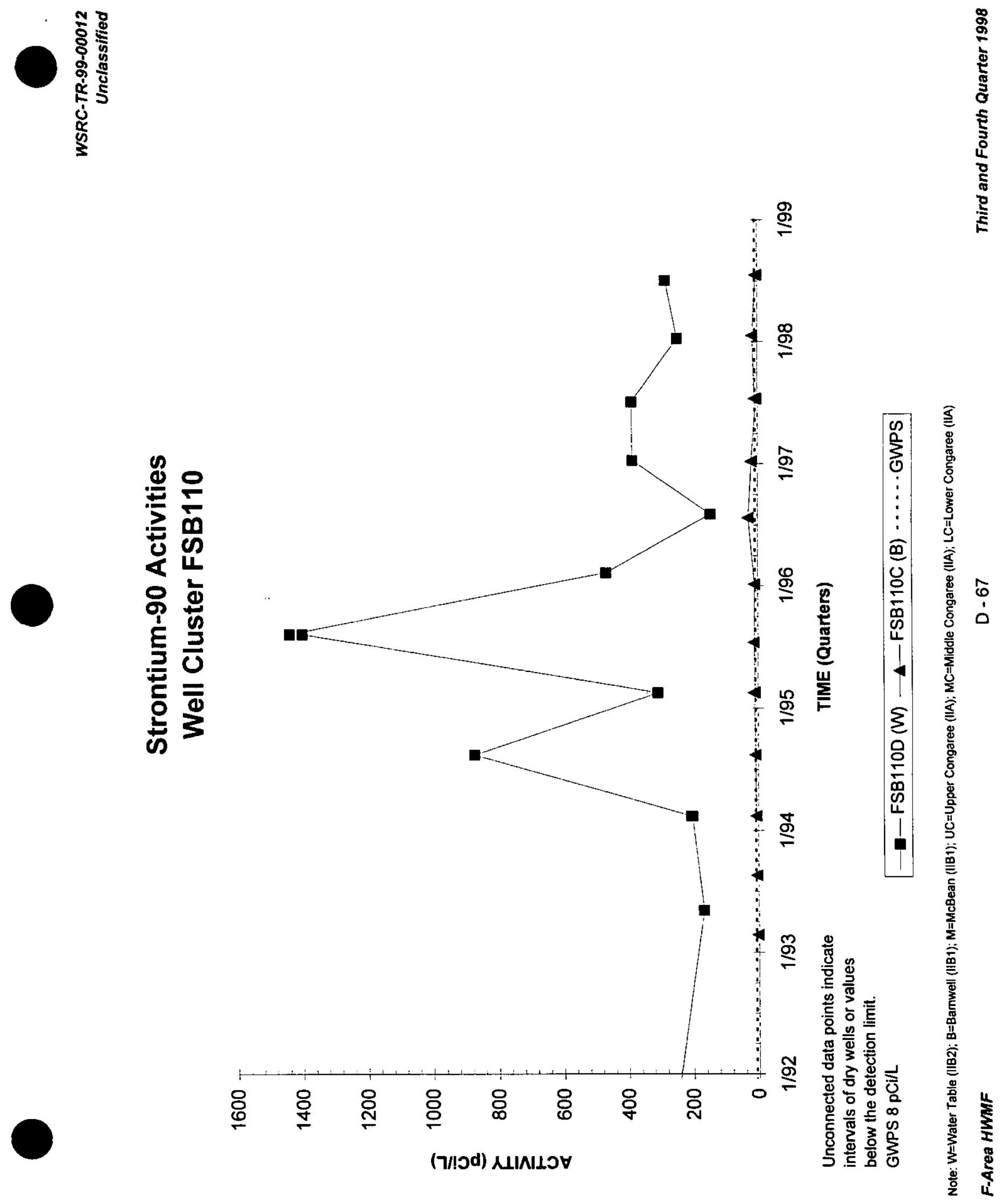


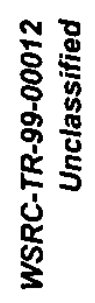

:
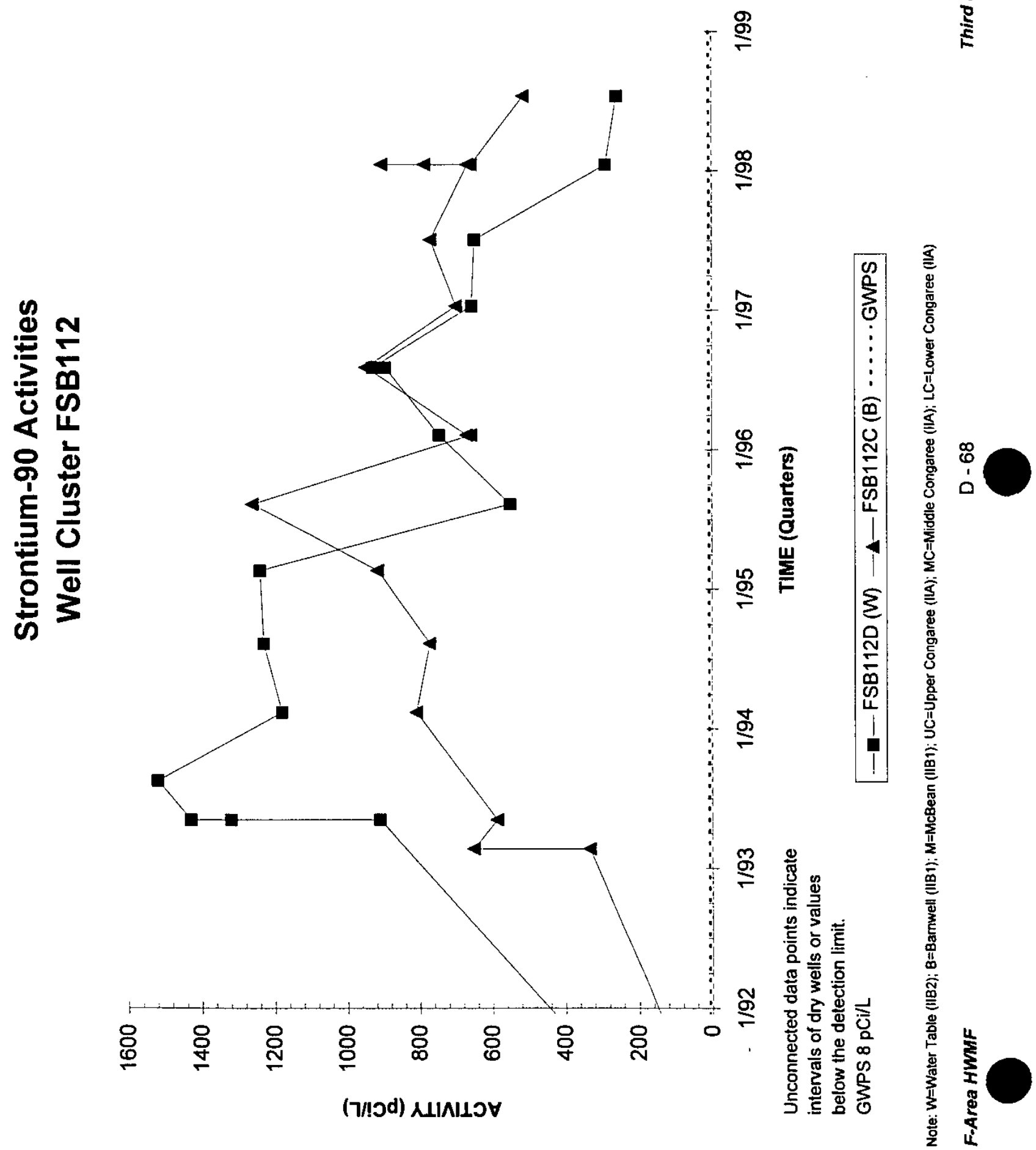


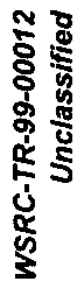

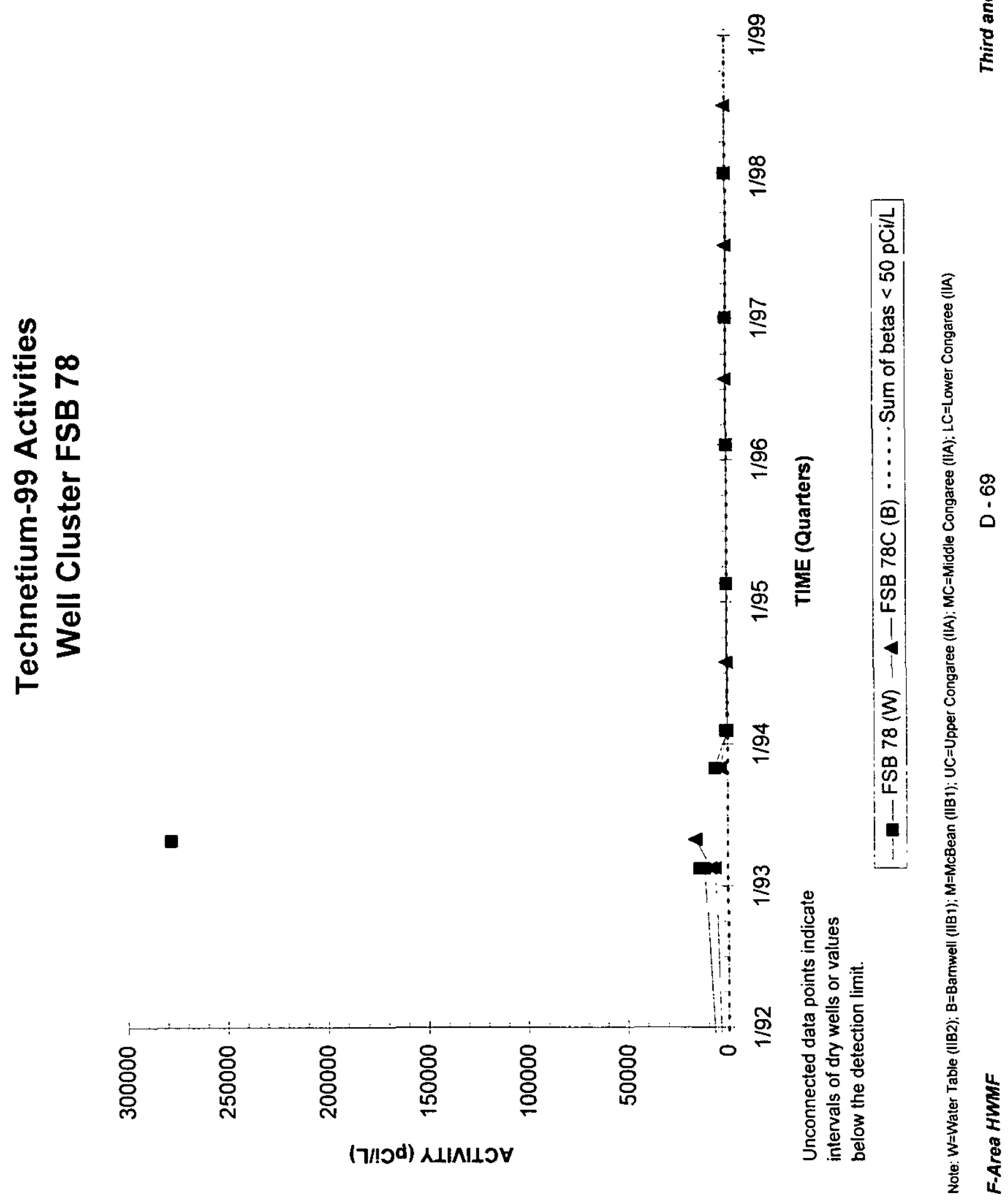



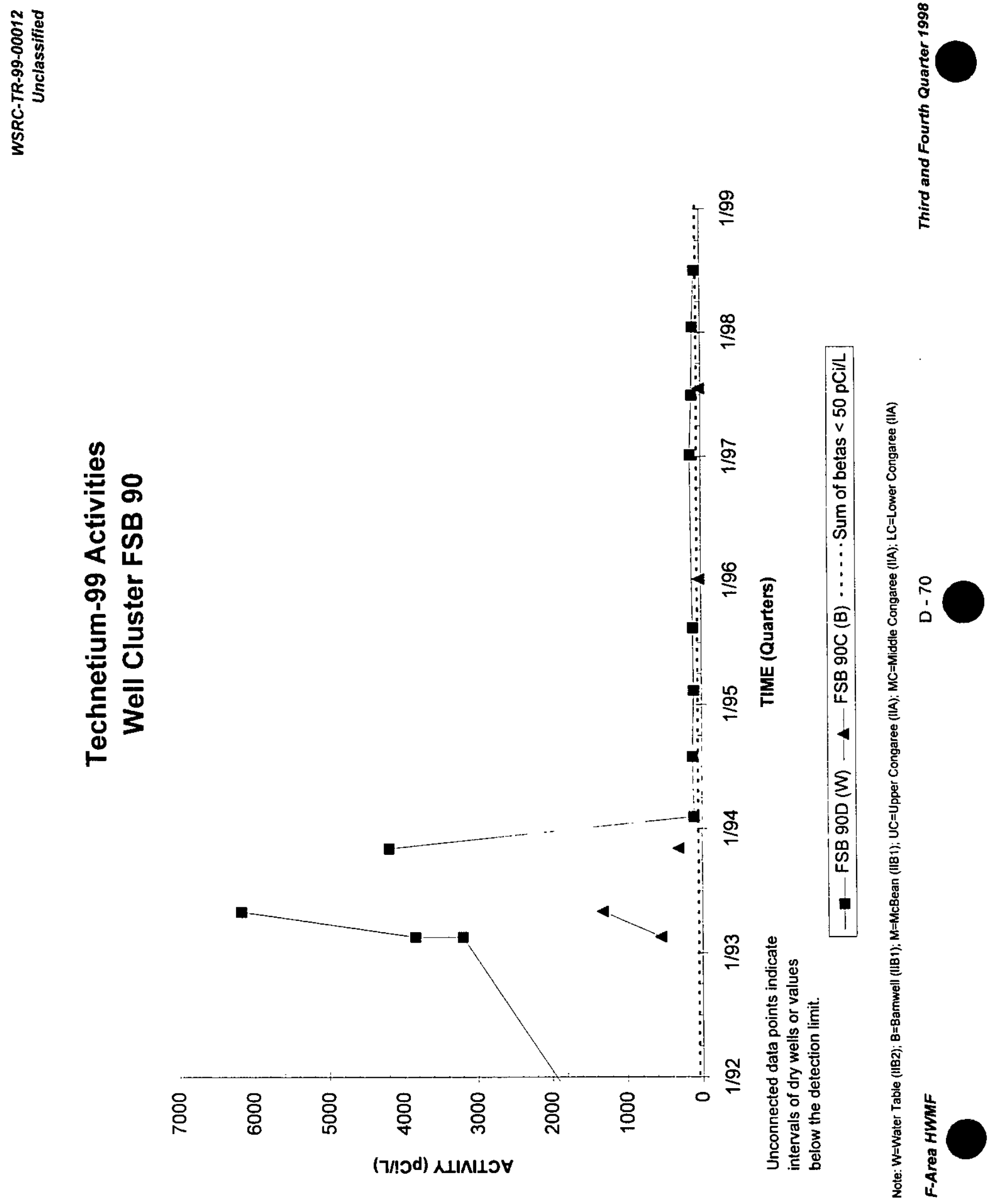

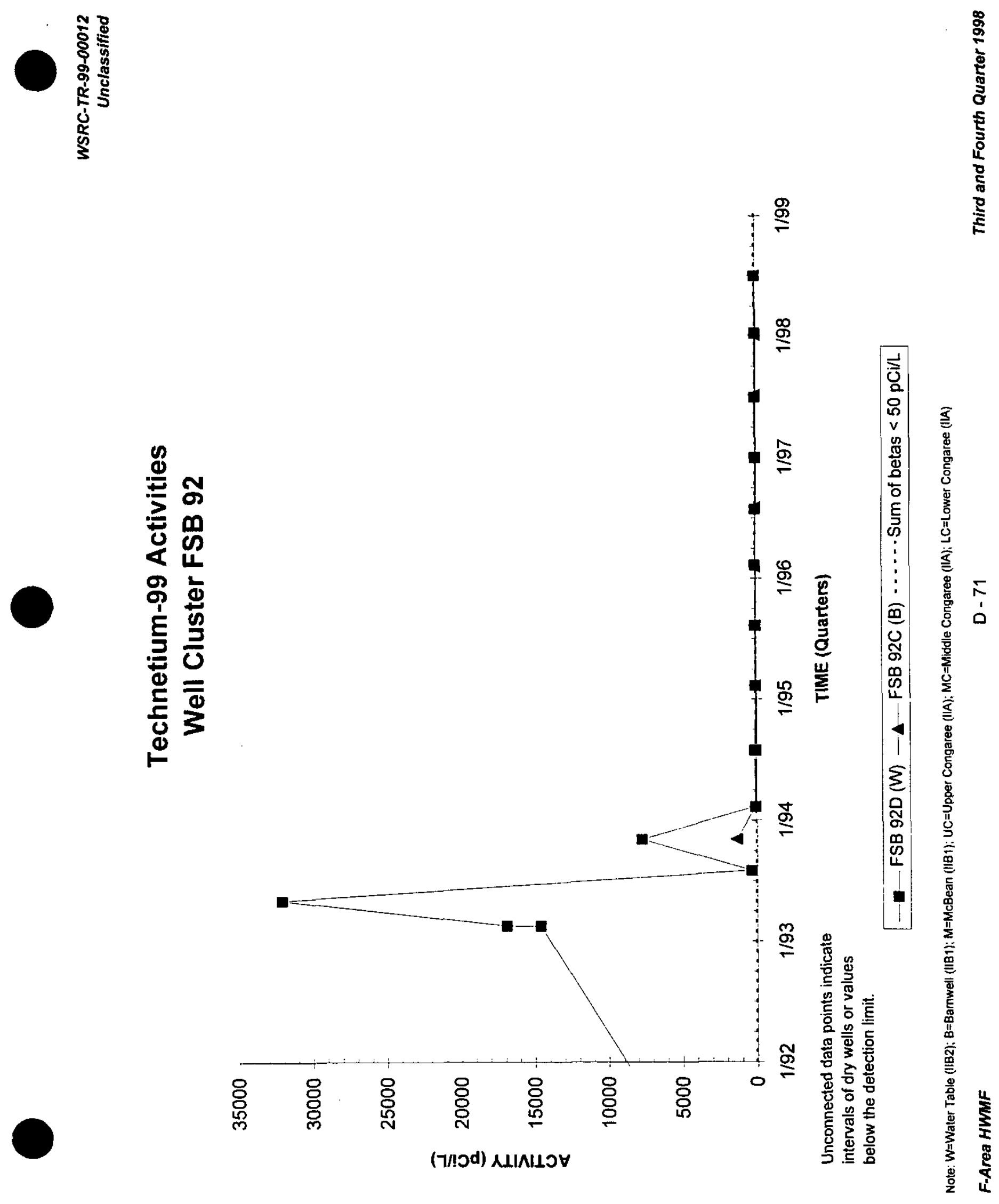

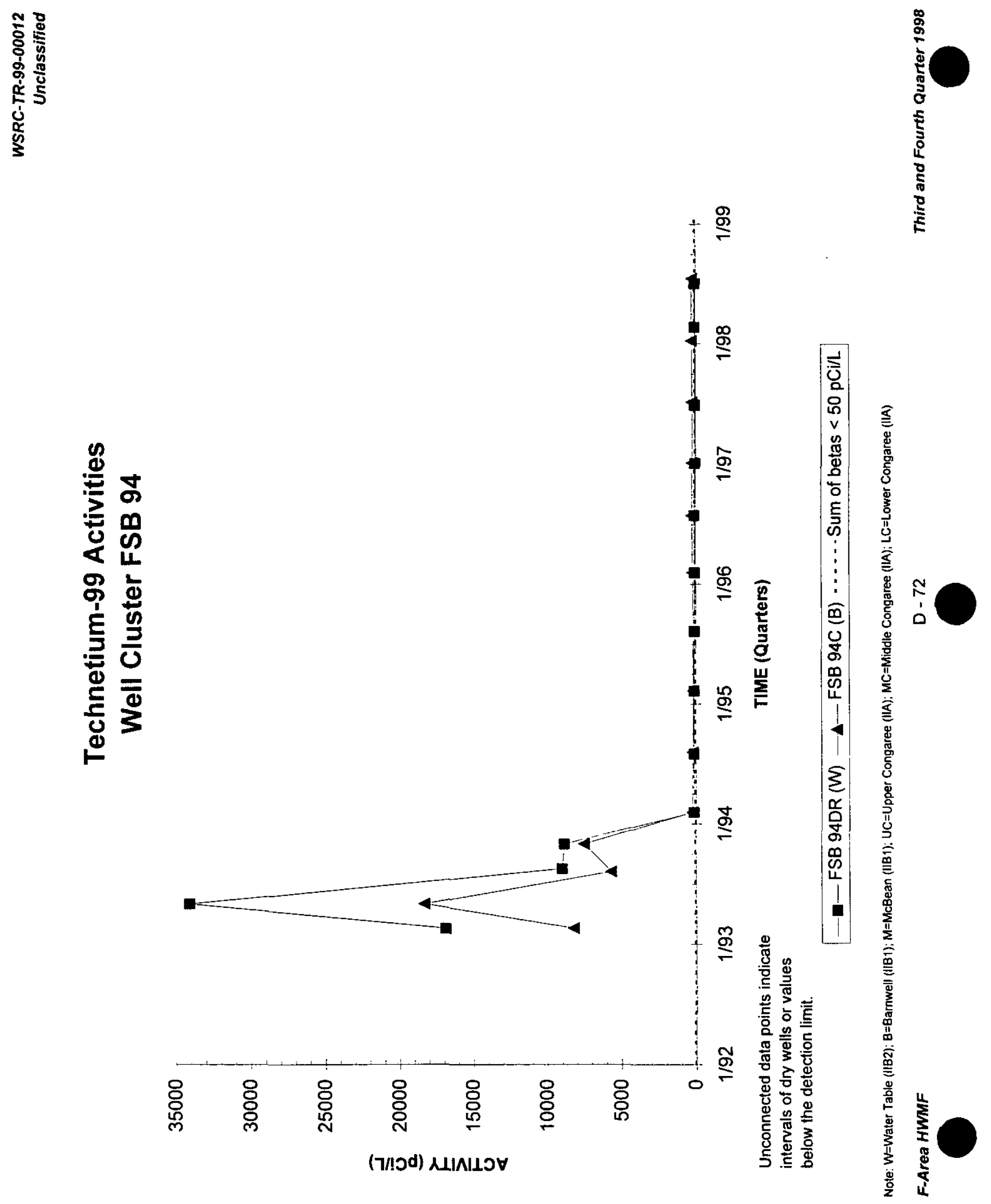

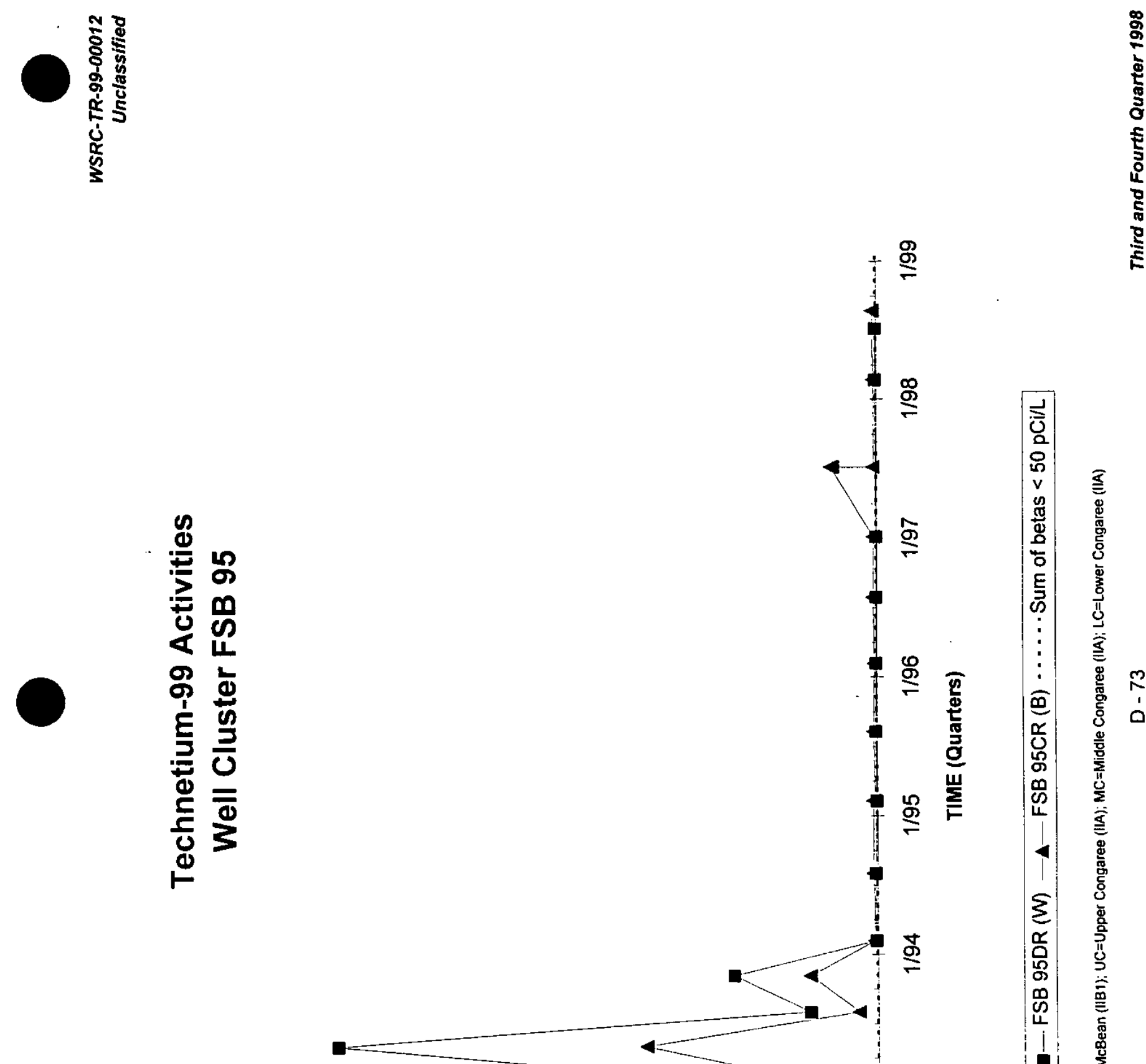

ڤิ

$\stackrel{m}{g}$

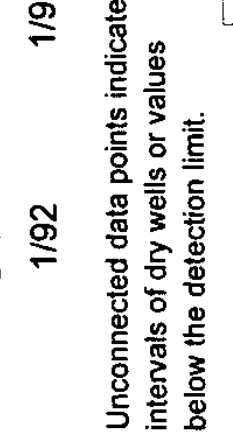

$$
\text { (7/!Od) 시IO }
$$




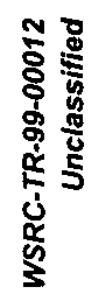

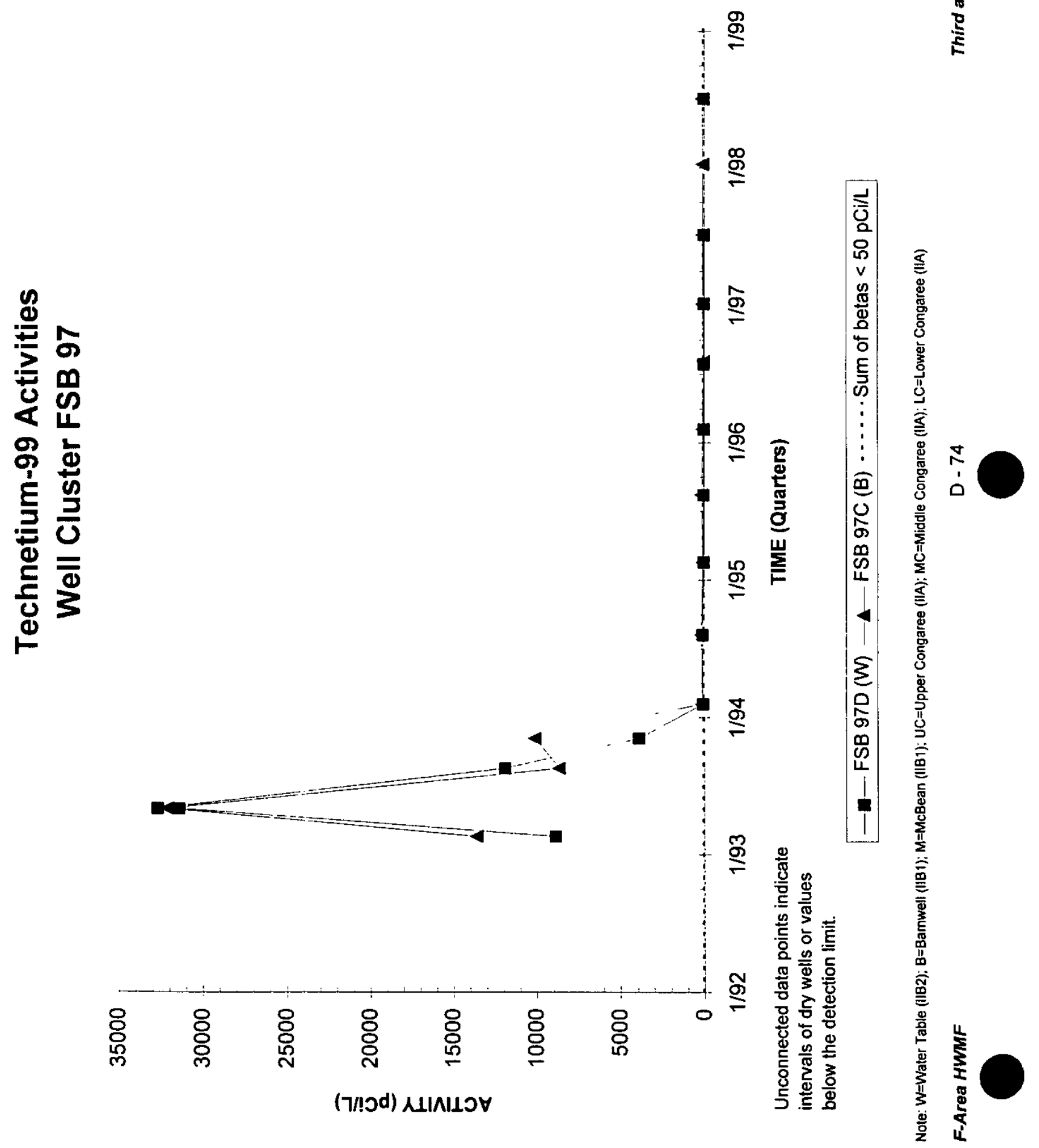



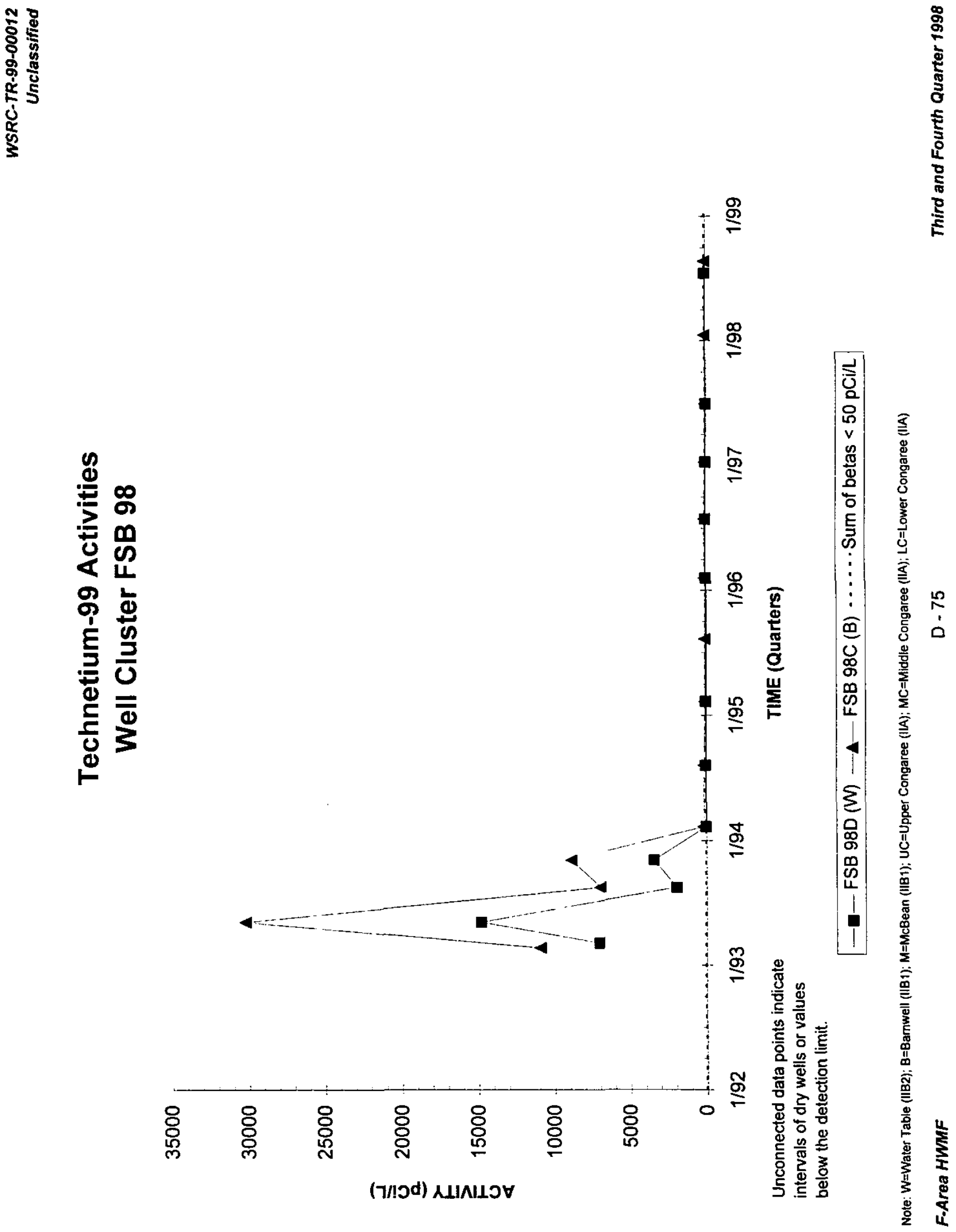

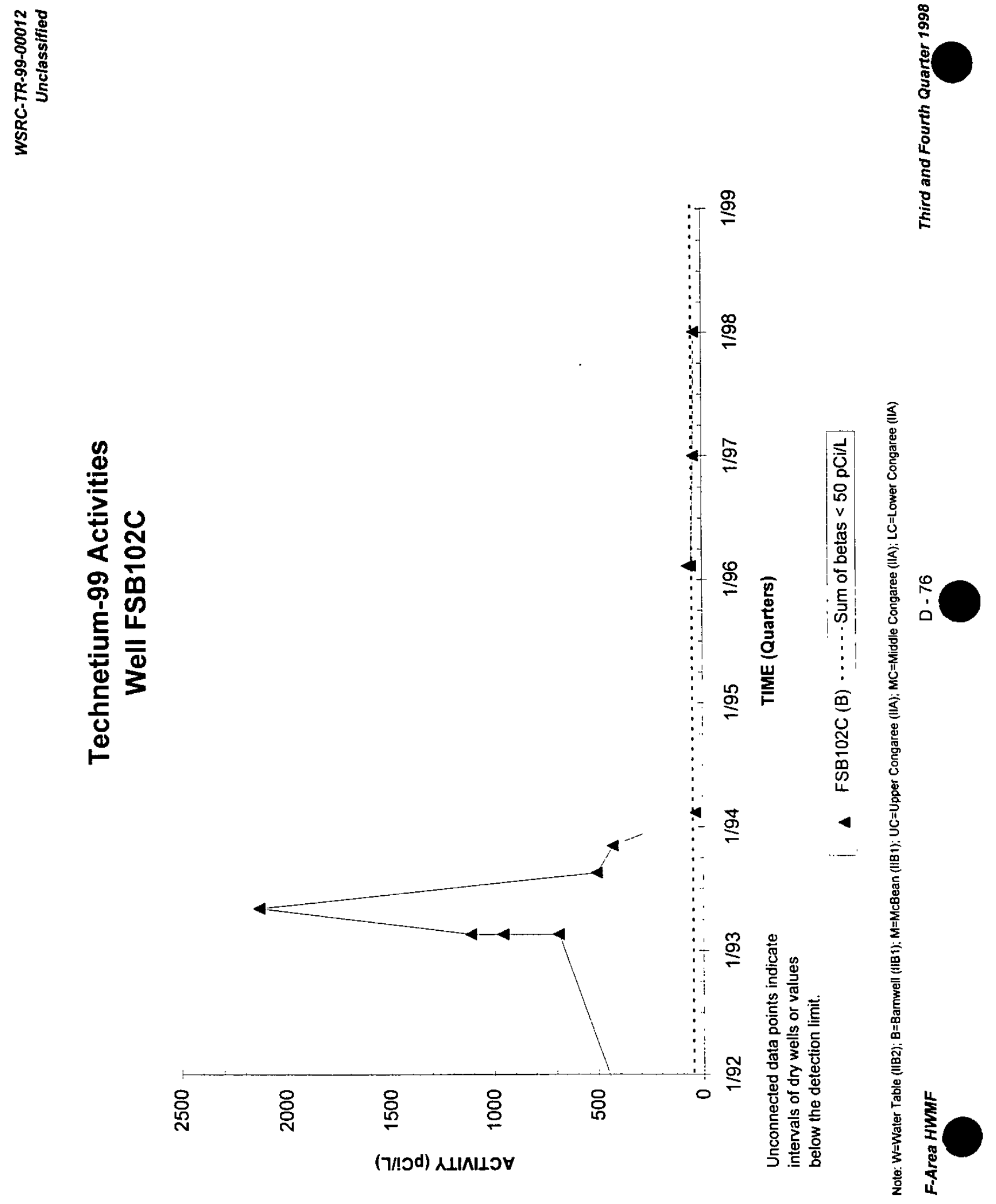

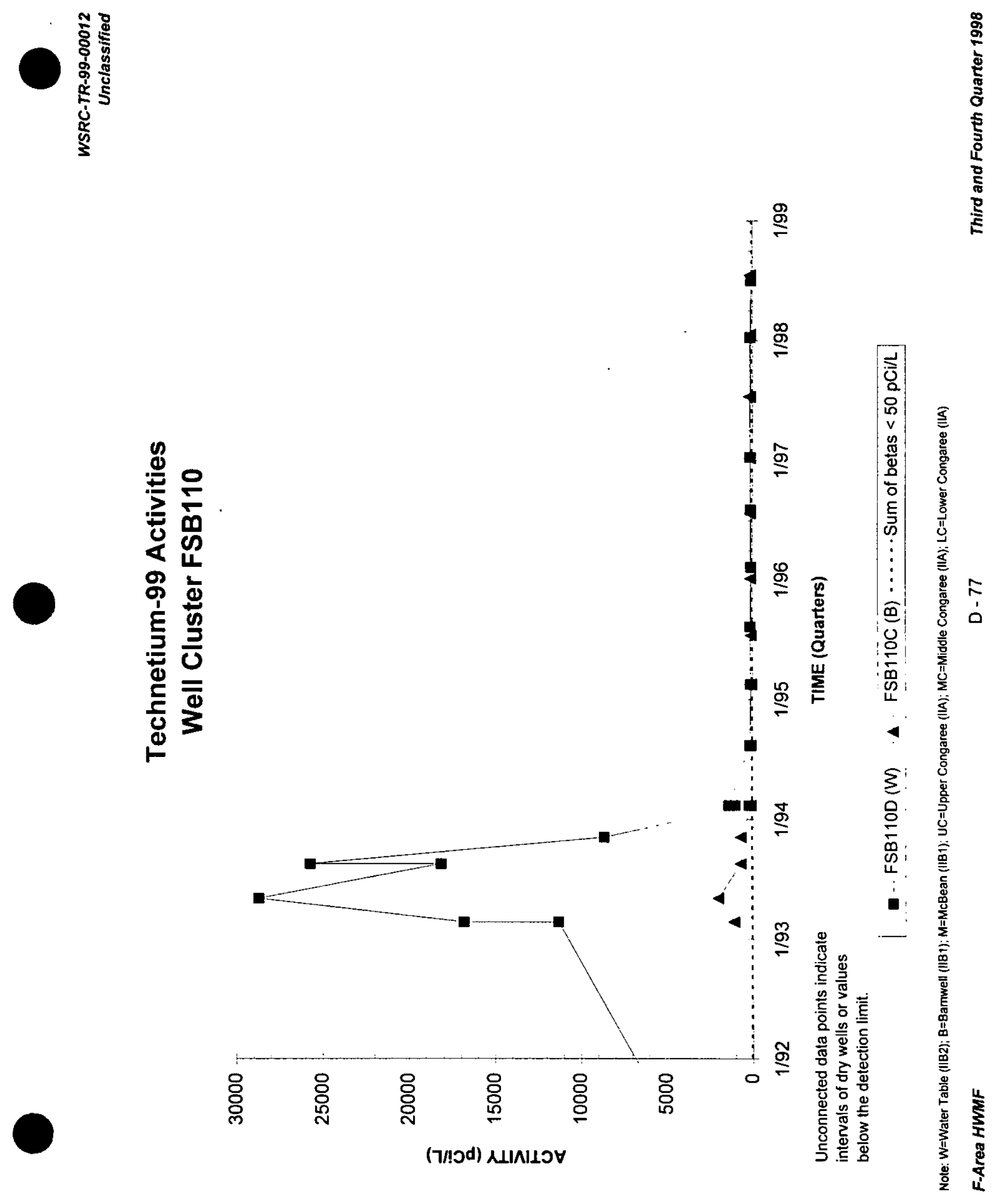


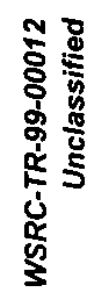

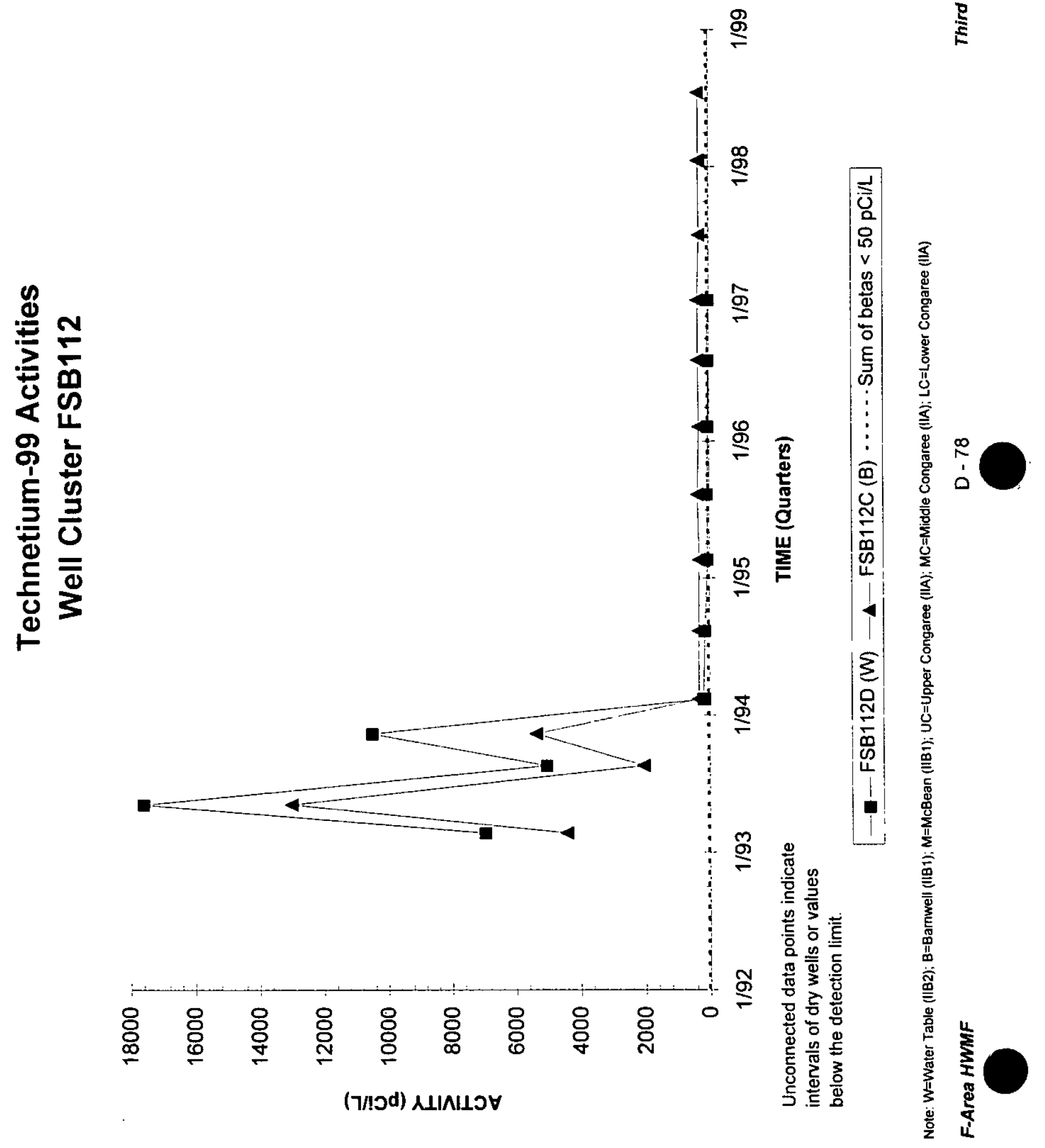




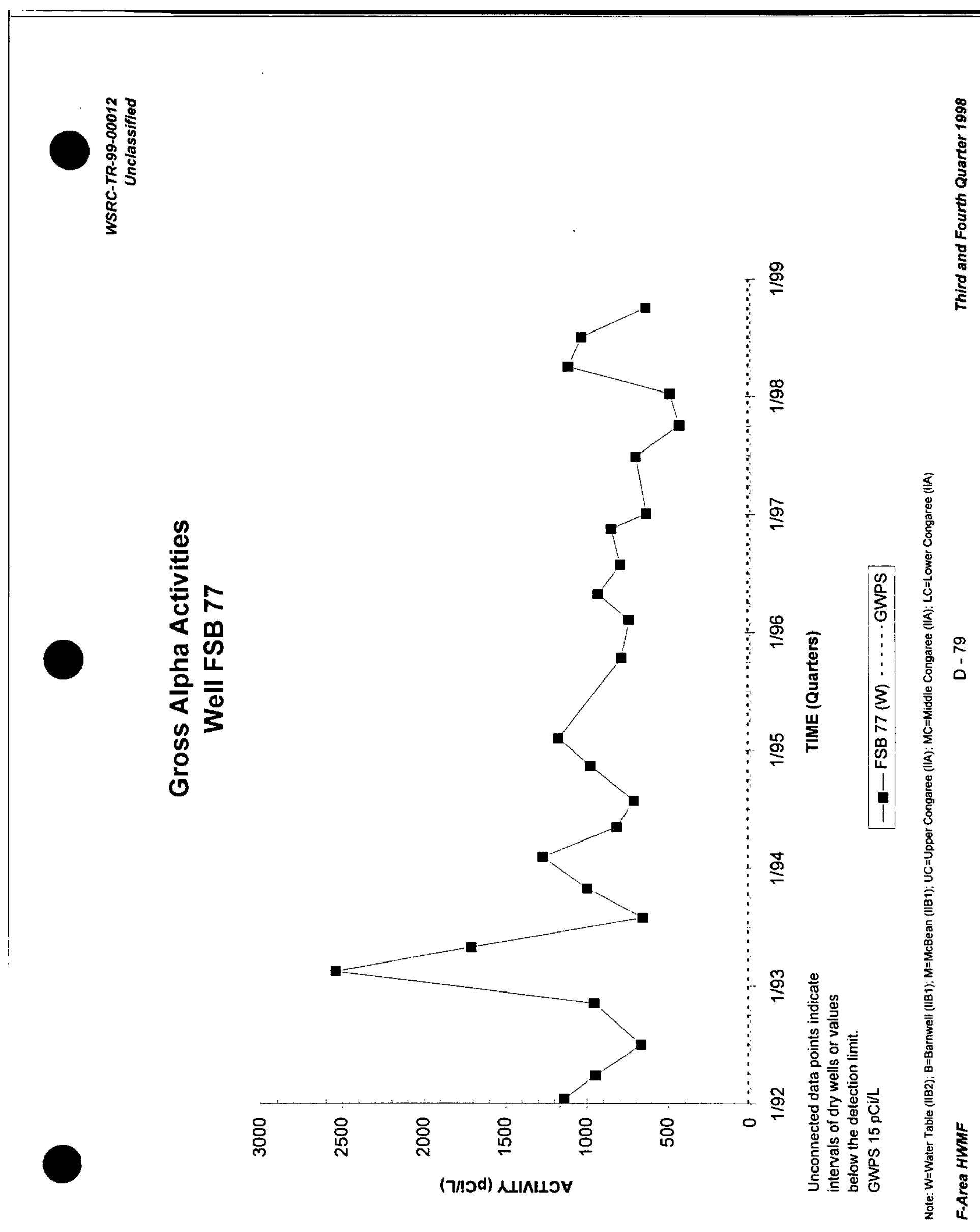



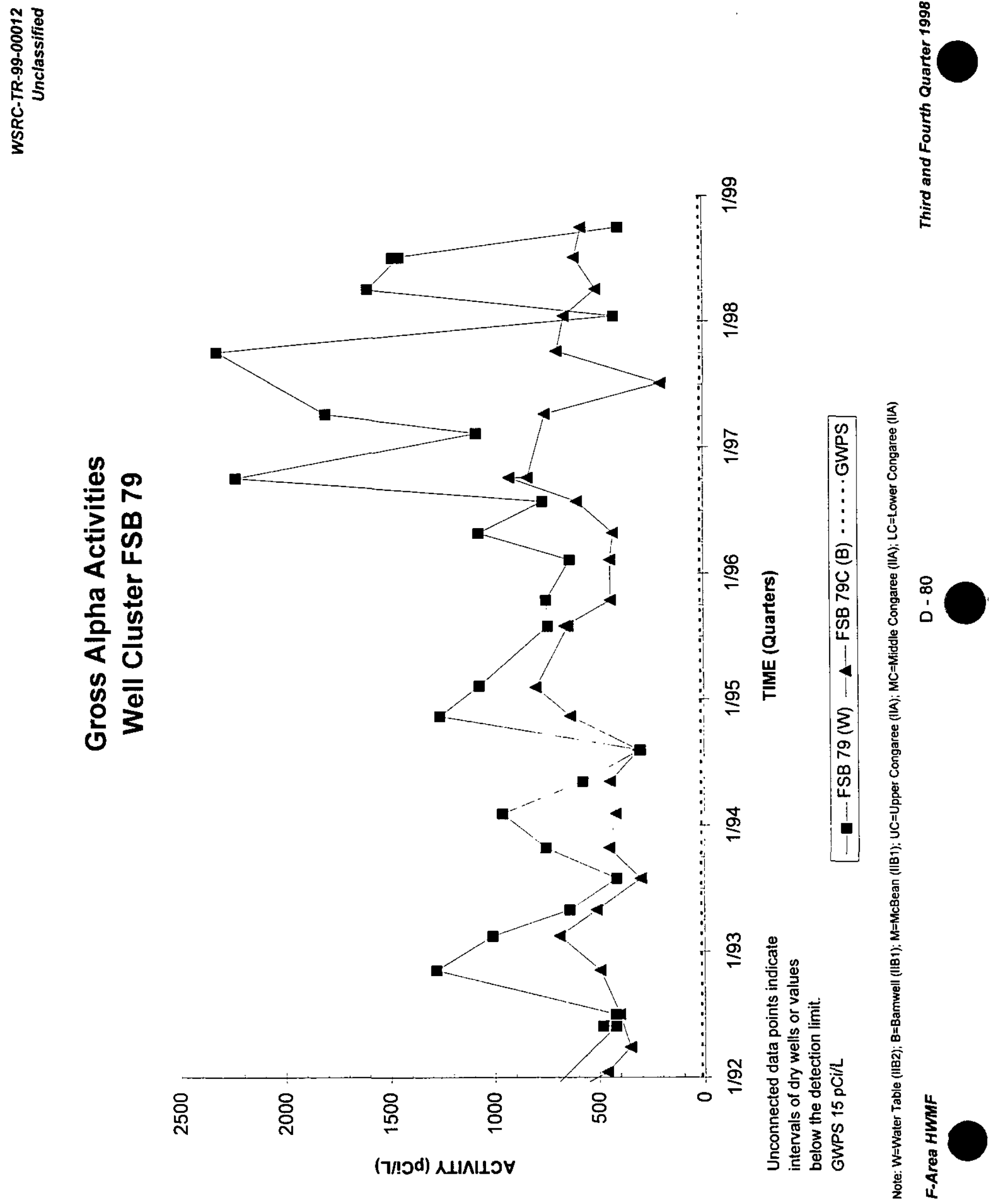

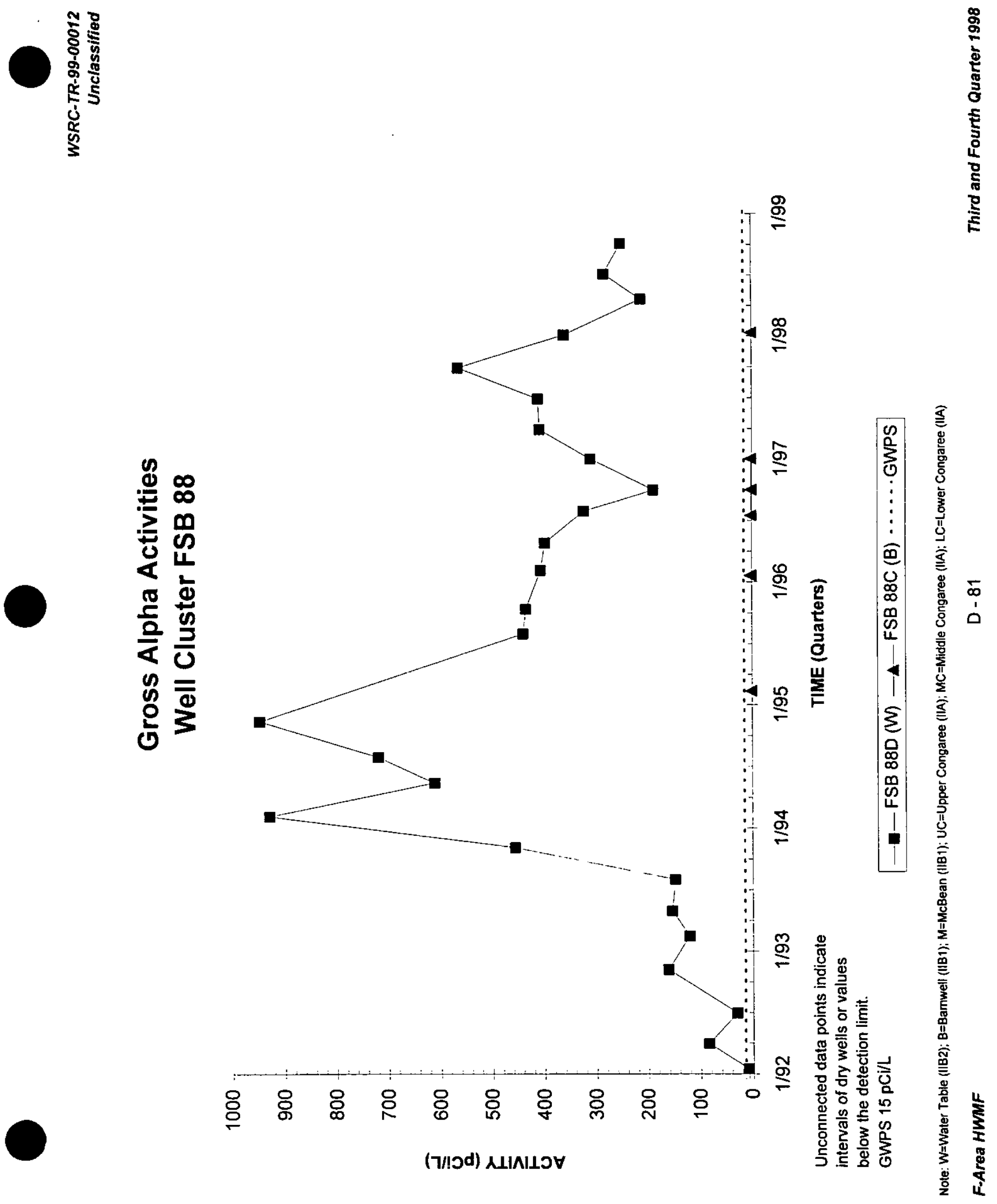

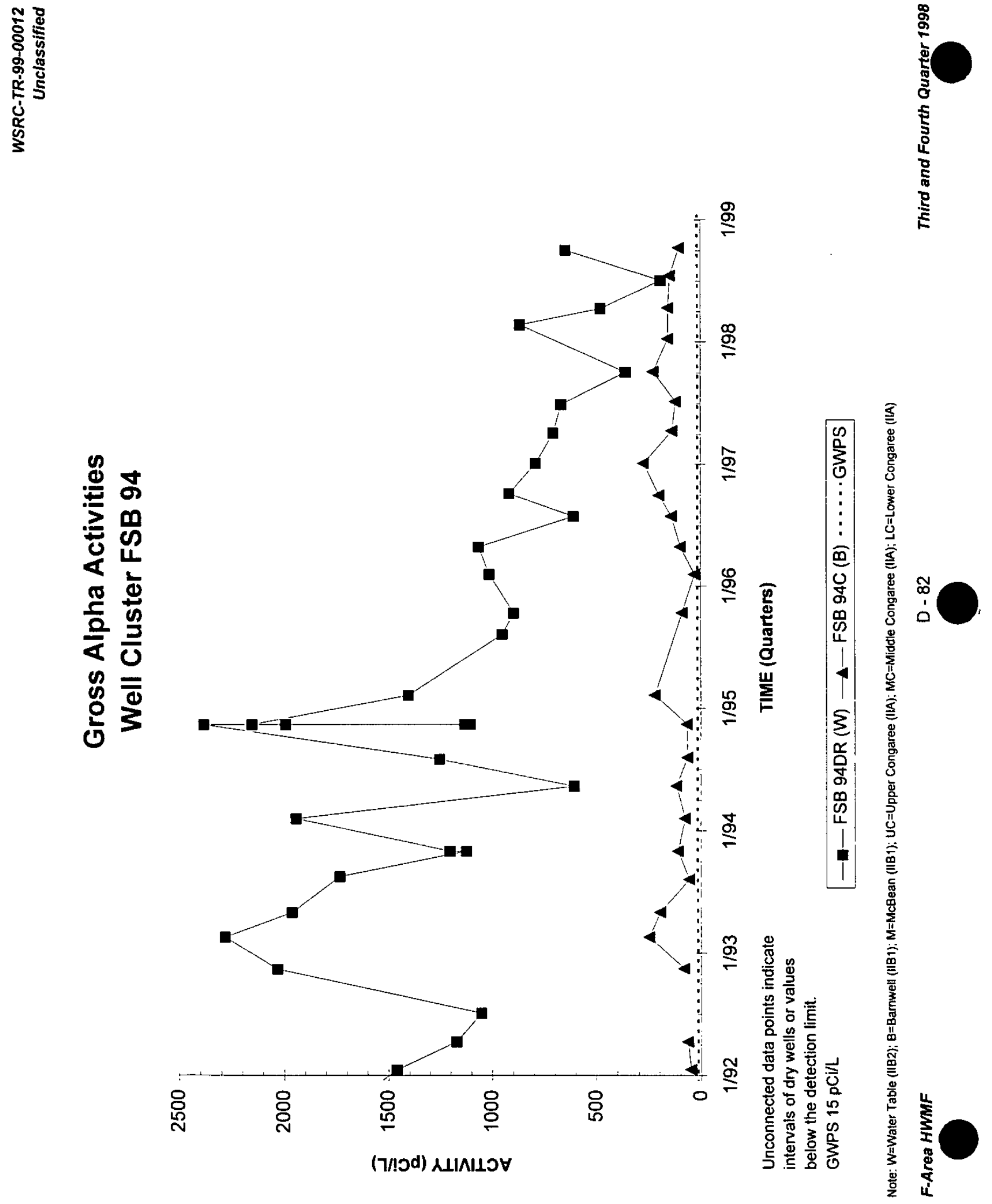


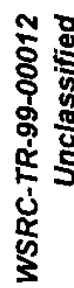

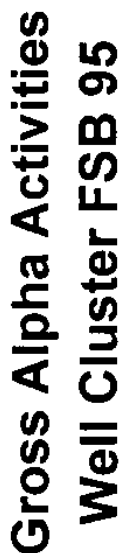

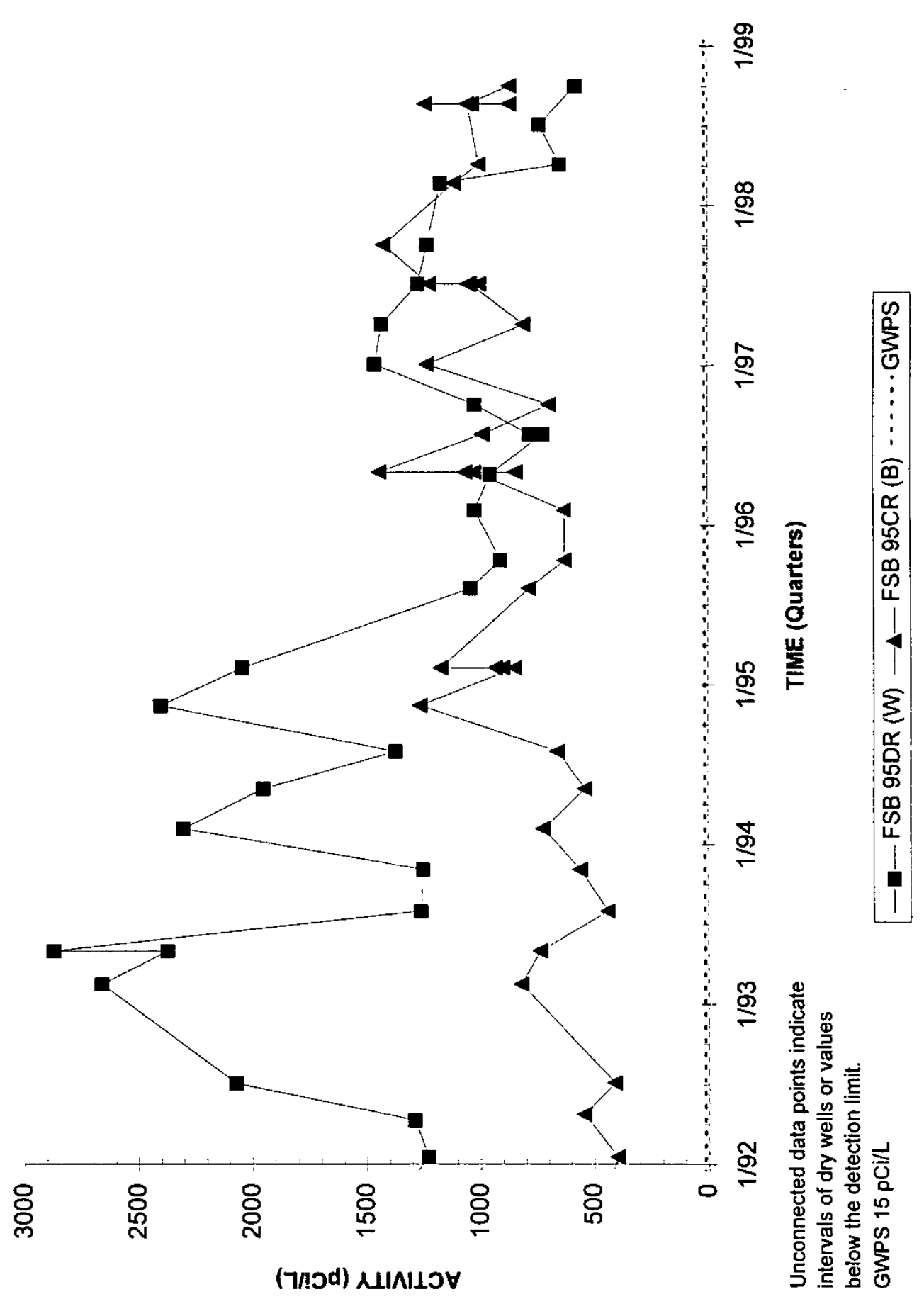



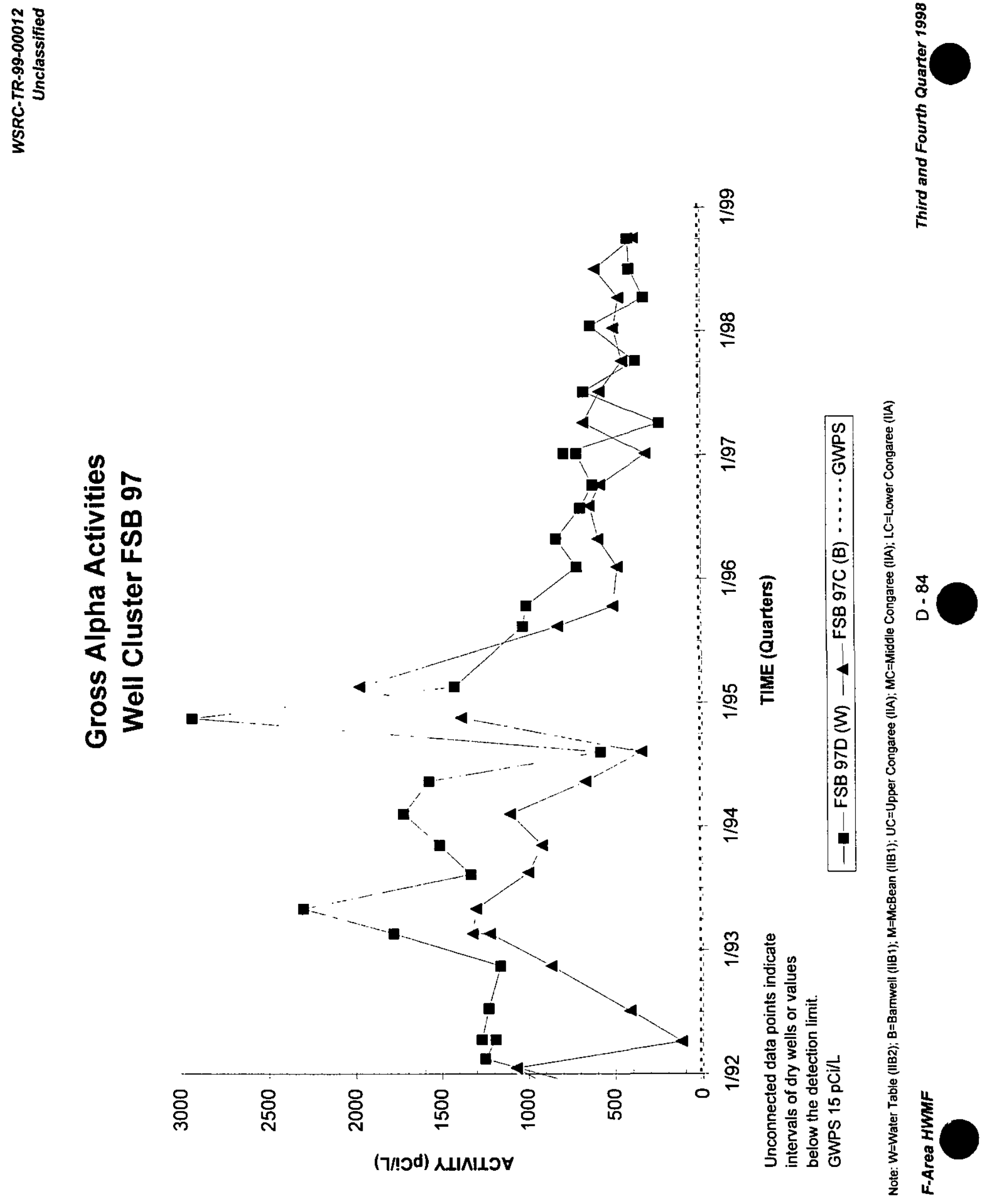


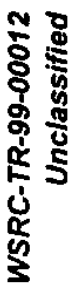

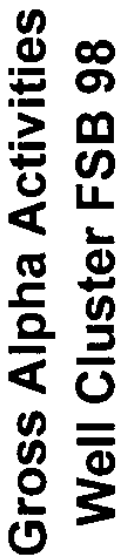

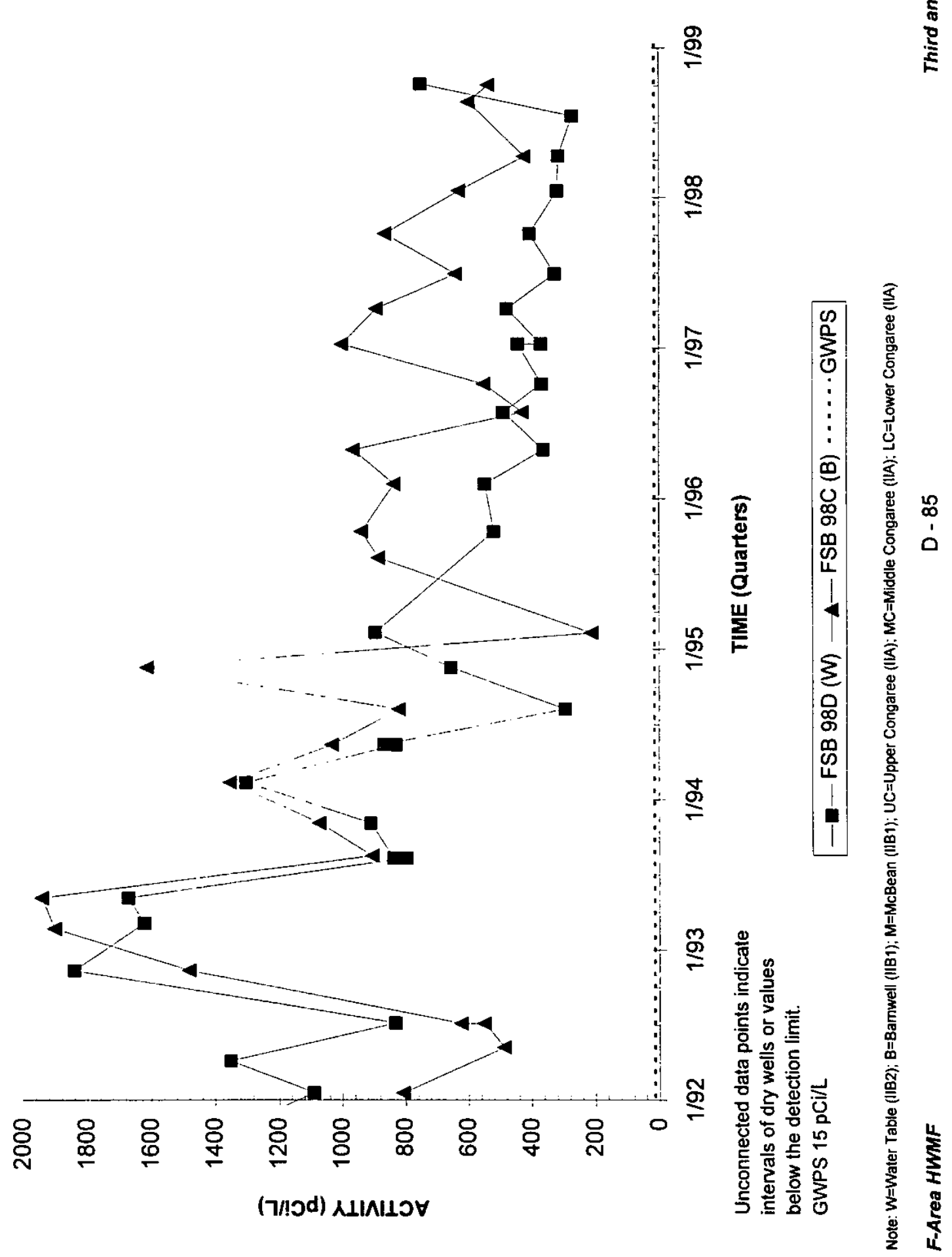



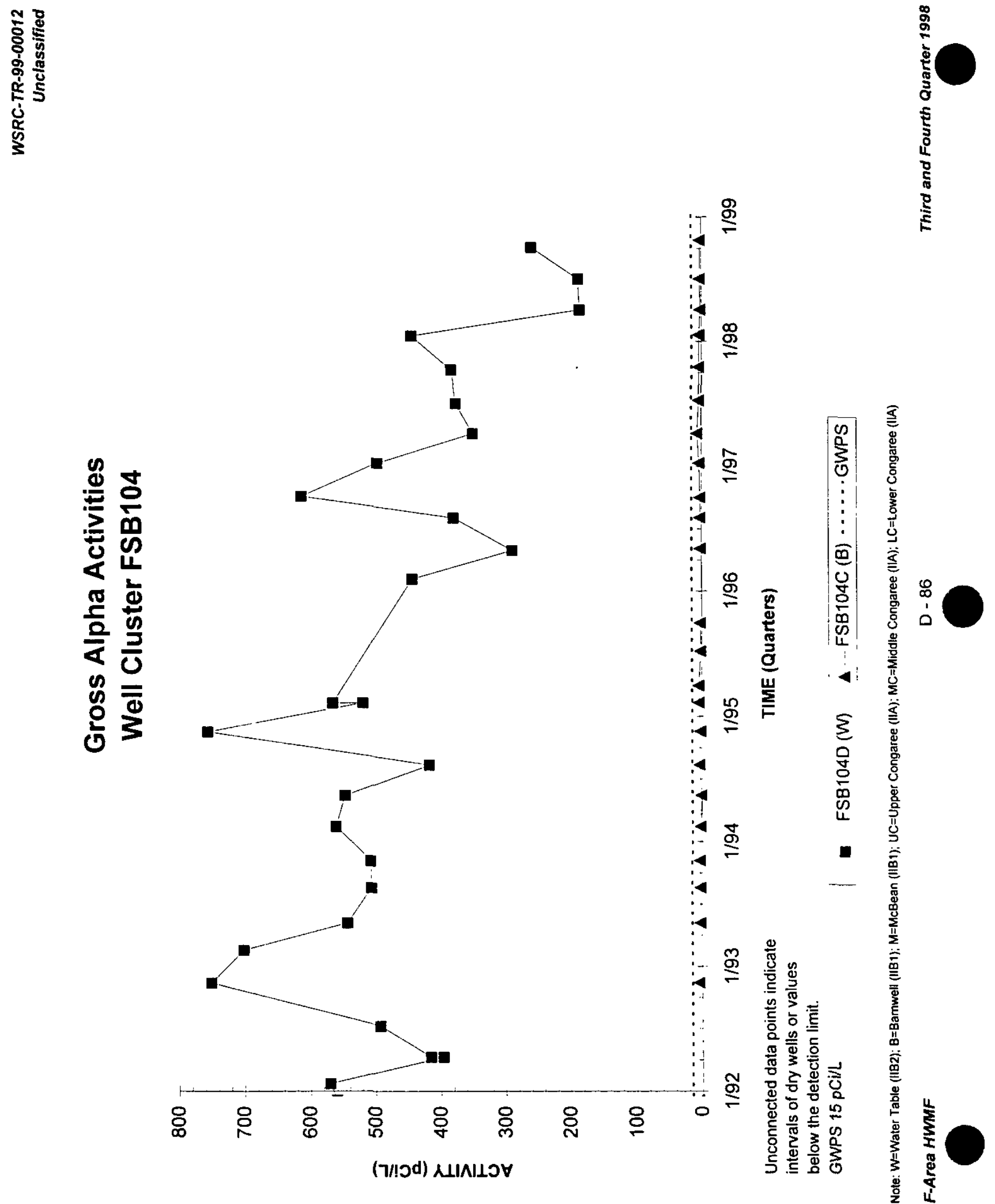

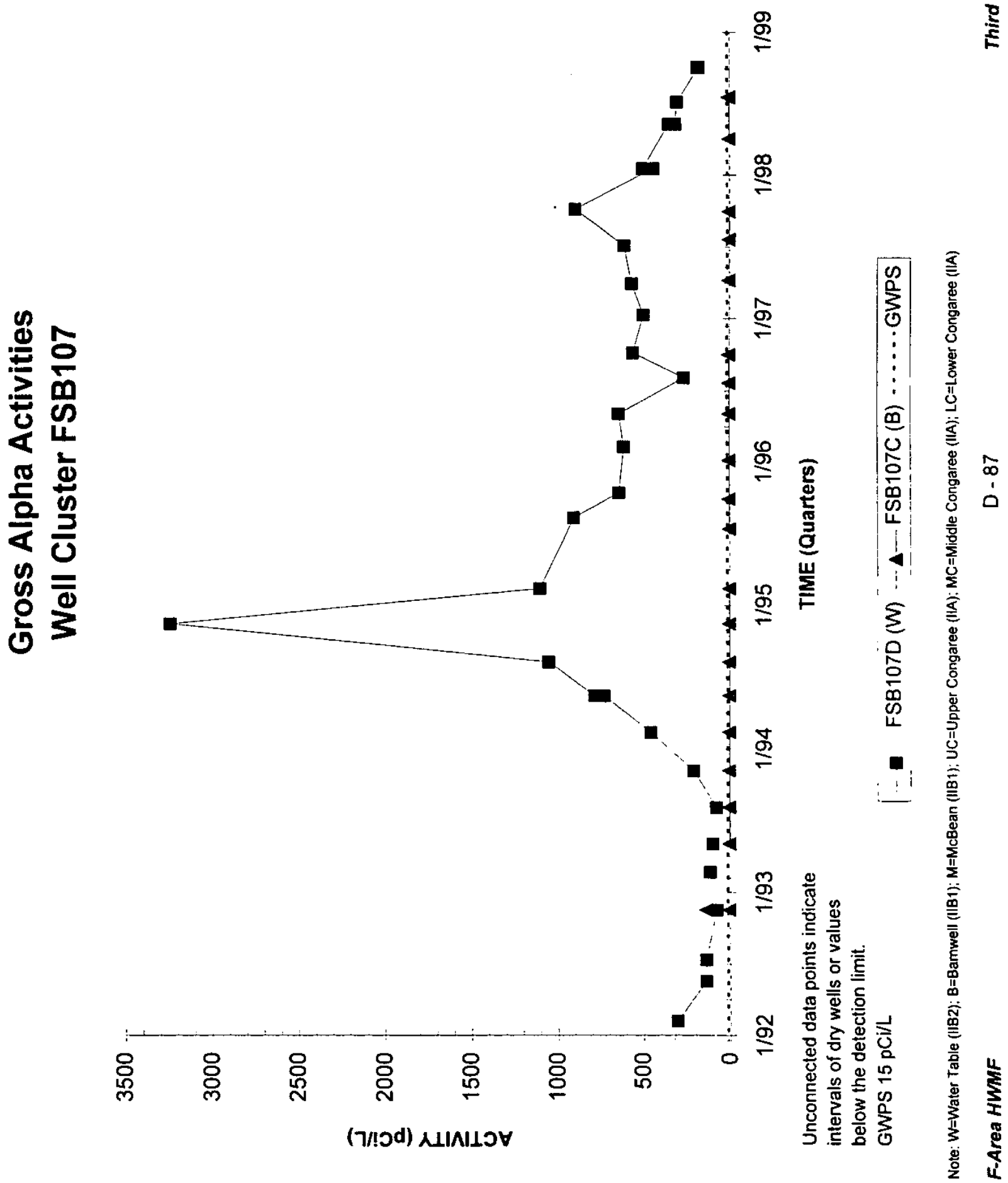

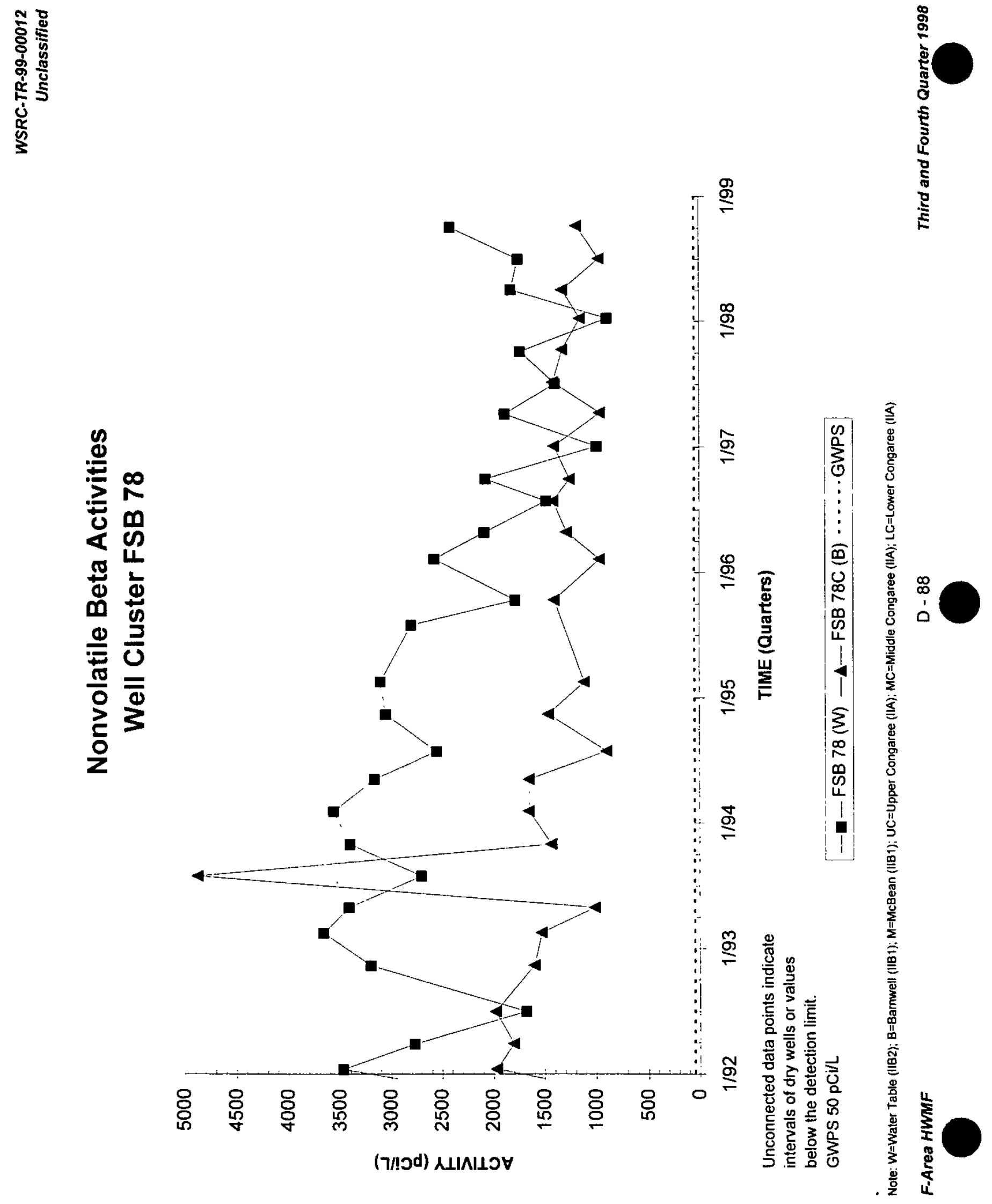

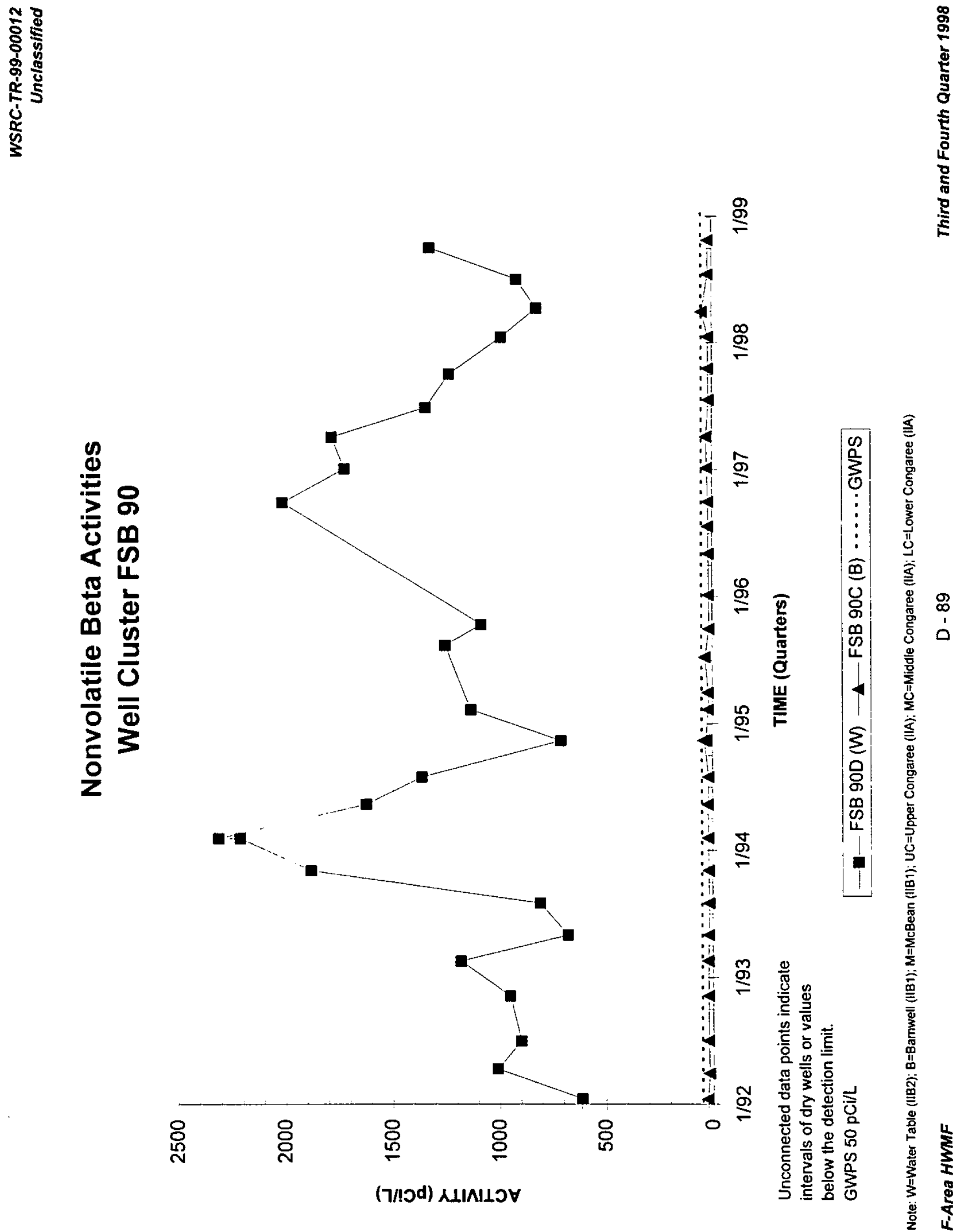

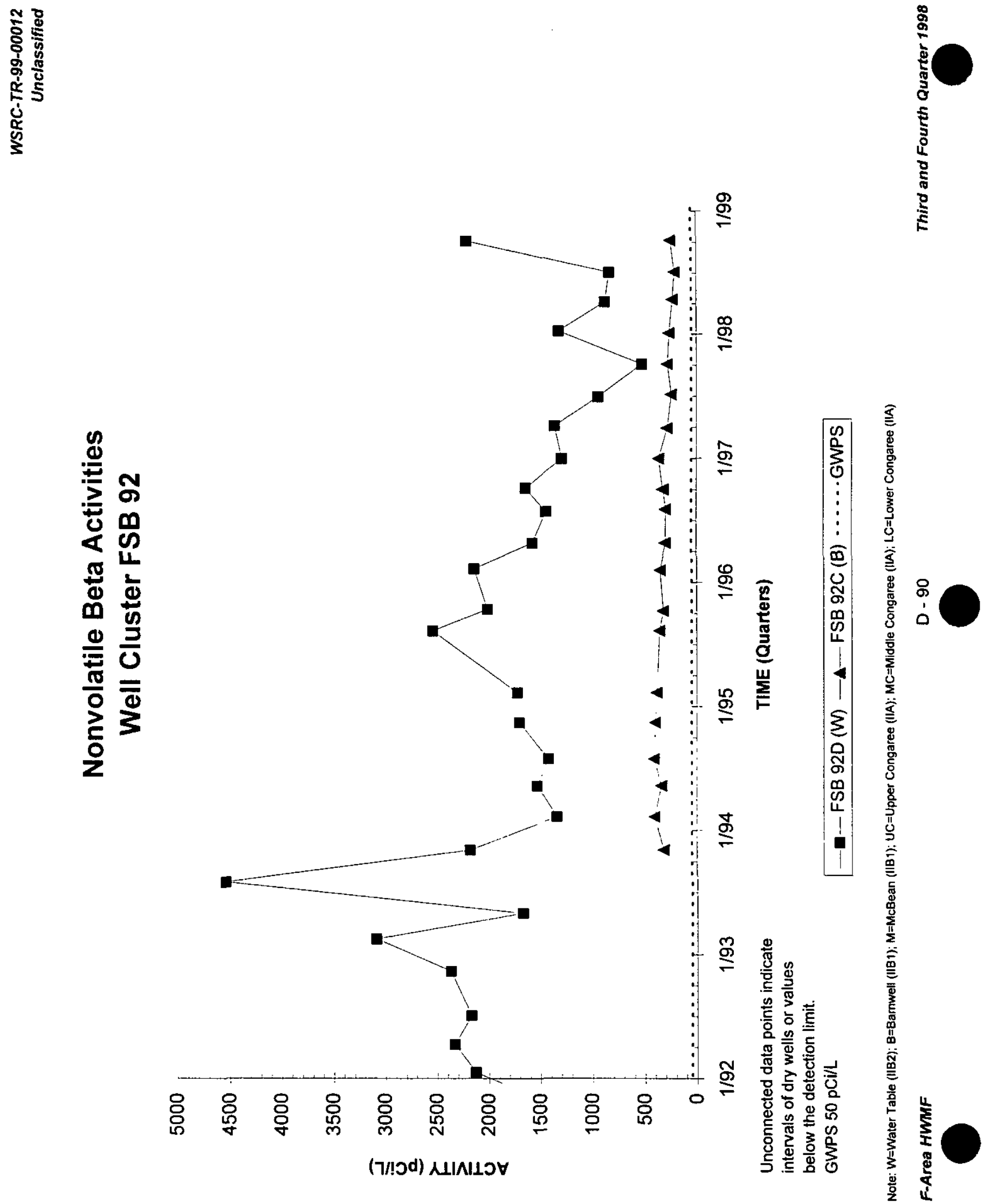

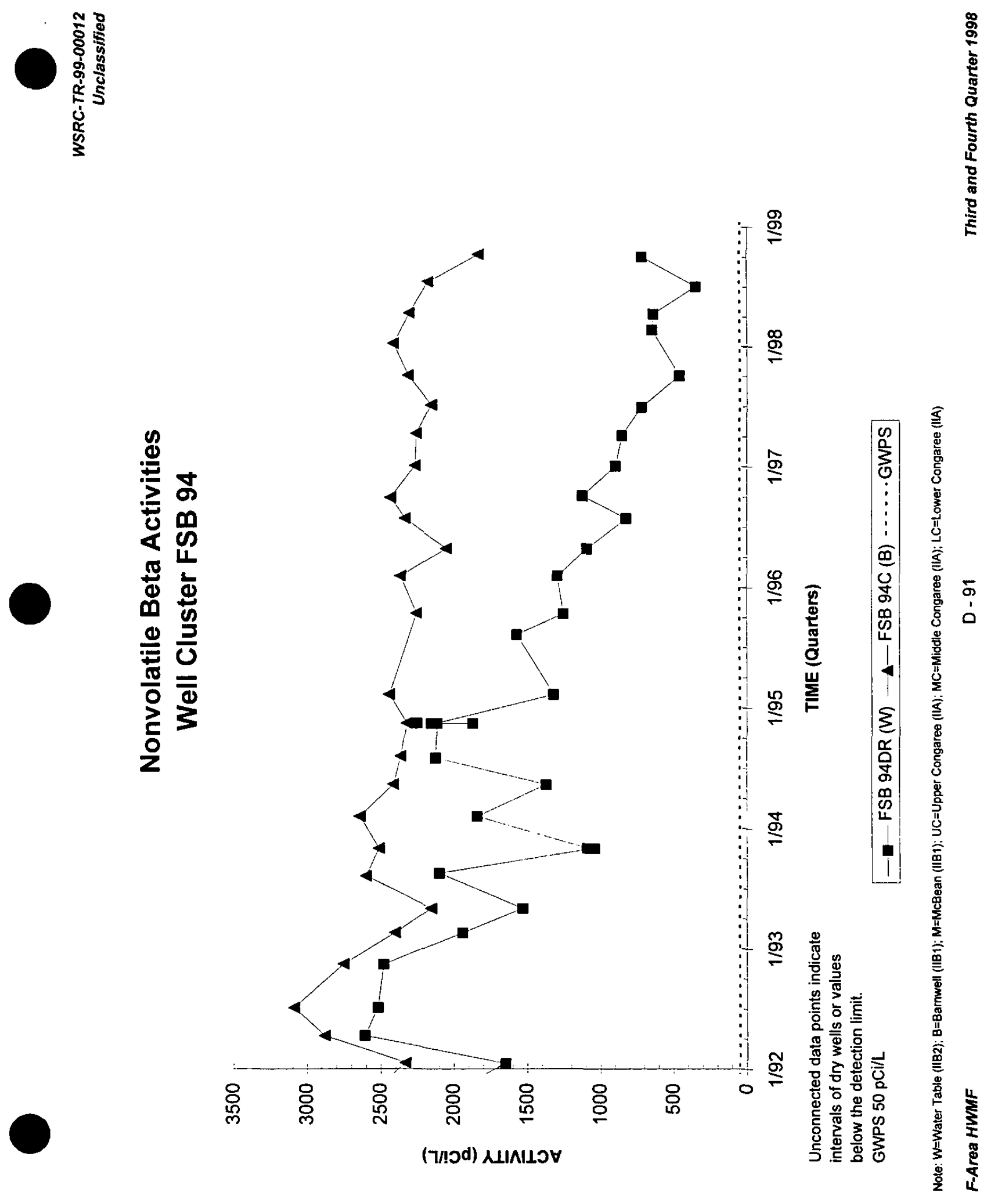

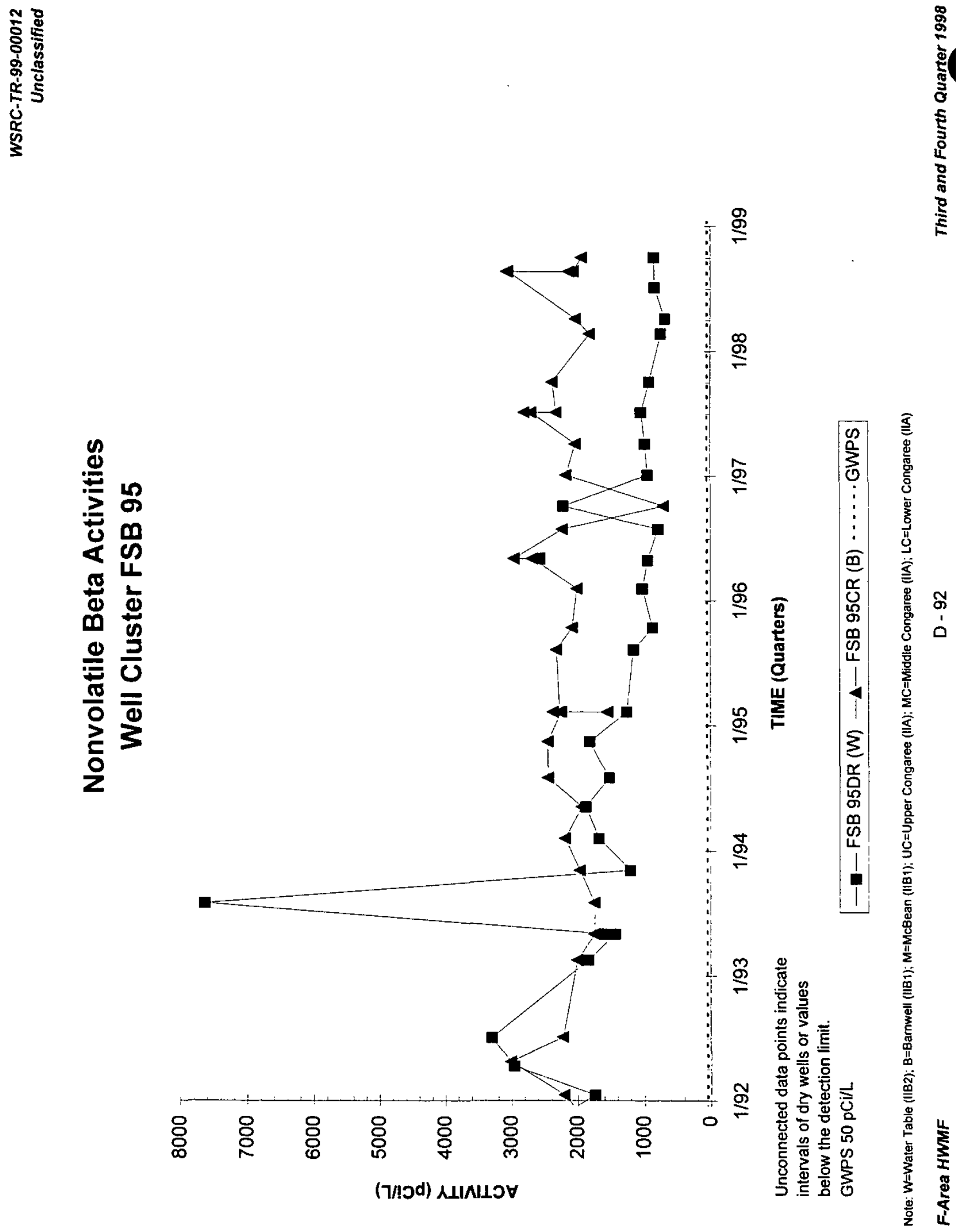

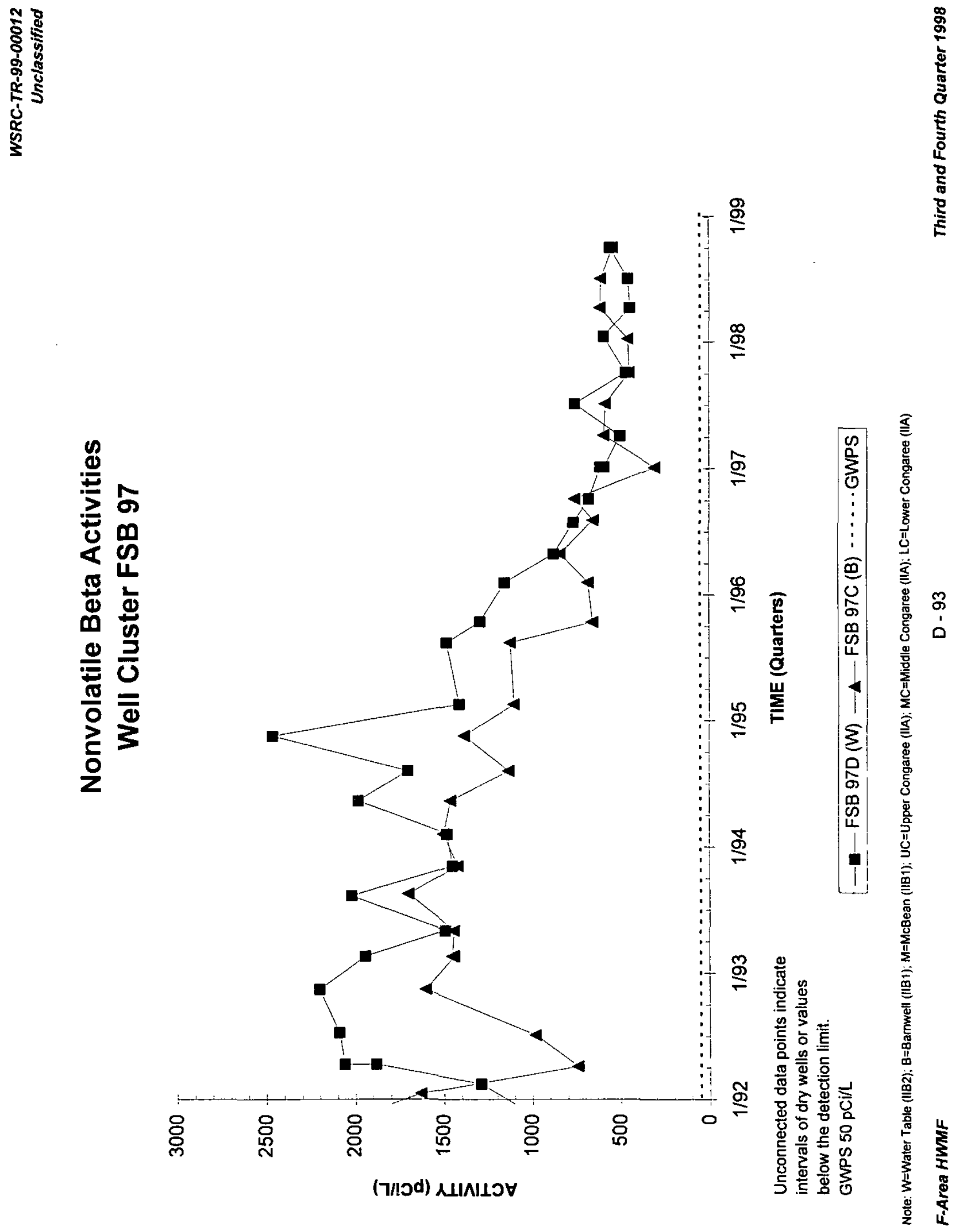


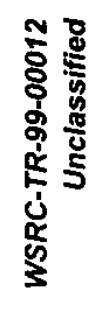

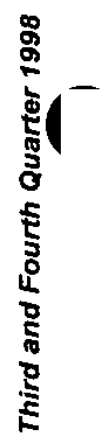

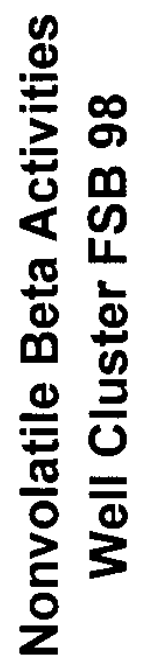

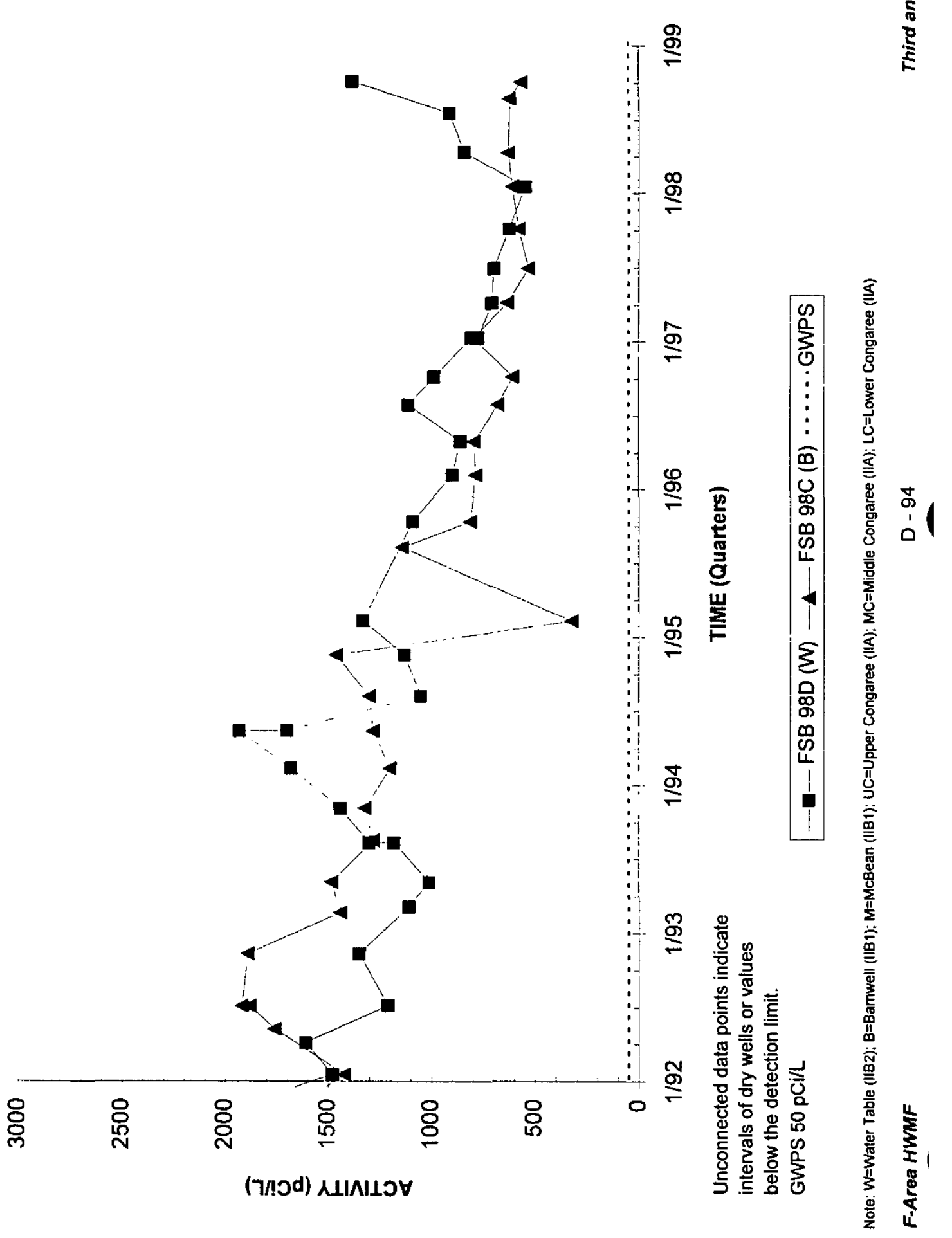



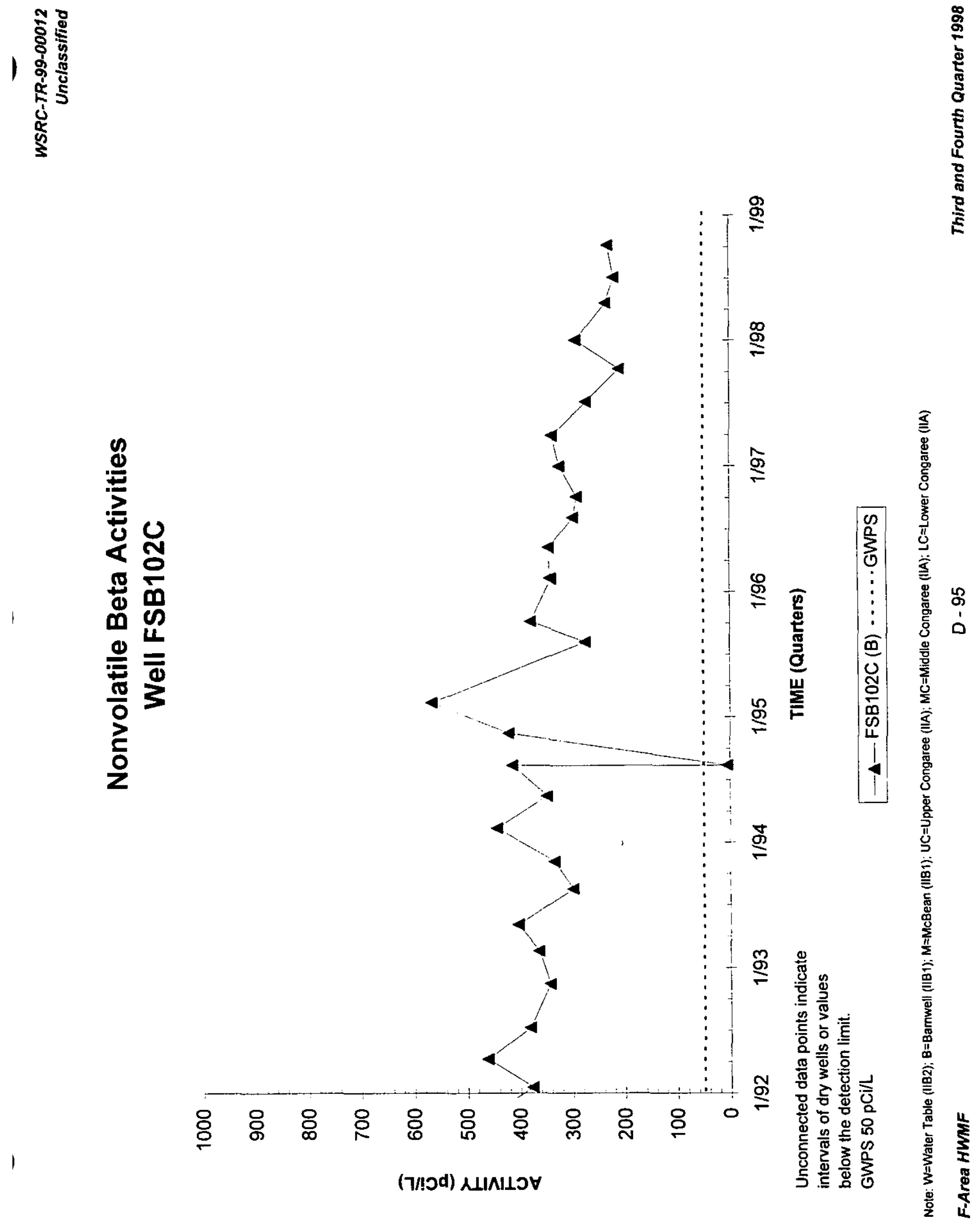

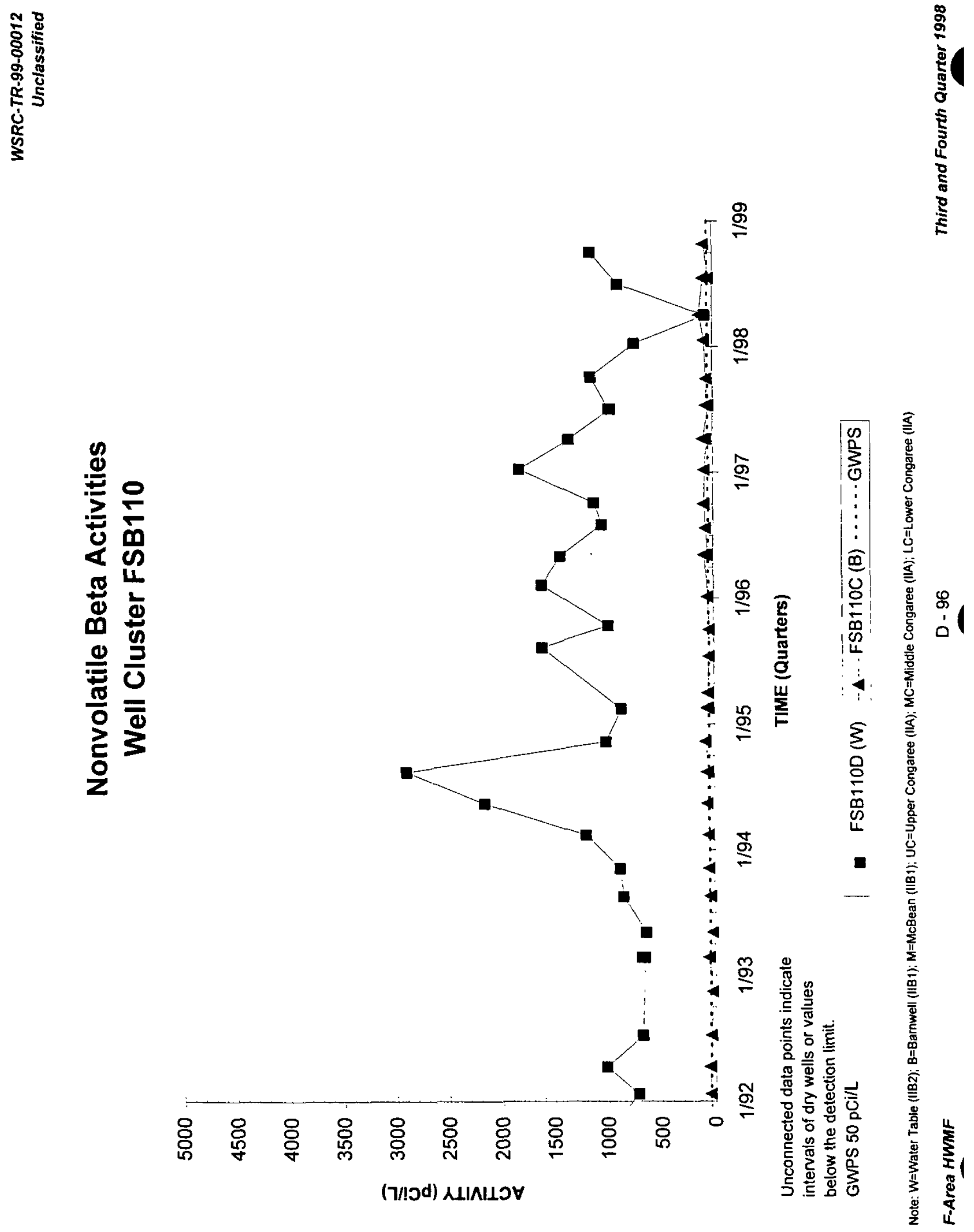

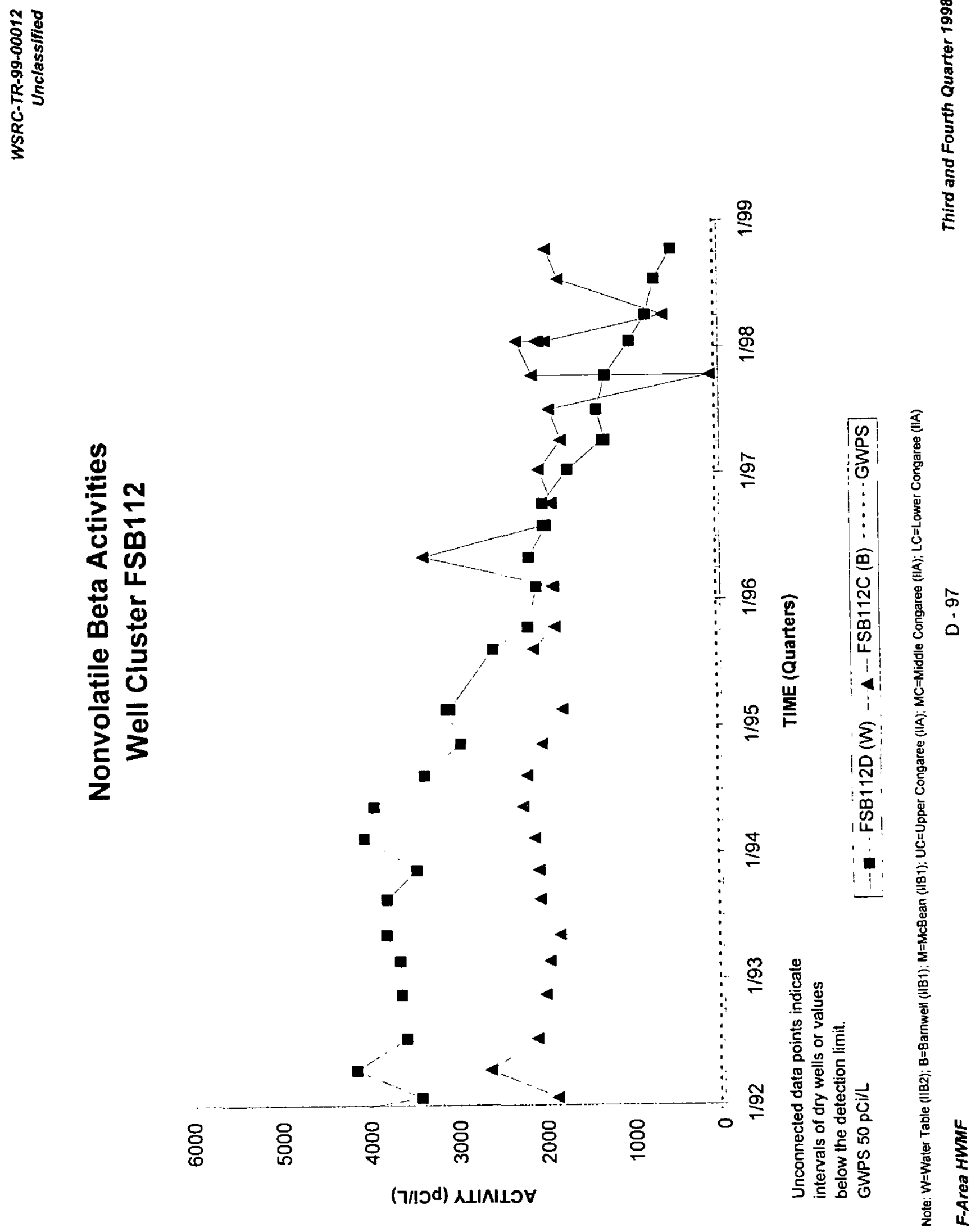
WSRC-TR-99-00012

Unclassified

THIS PAGE LEFT BLANK INTENTIONALLY. 
WSRC-TR-99-00012

Unclassified

1

\section{Appendix E}

\section{Hydrographs}


WSRC-TR-99-00012

Unclassified

THIS PAGE LEFT BLANK INTENTIONALLY. 


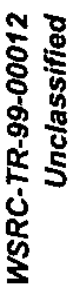
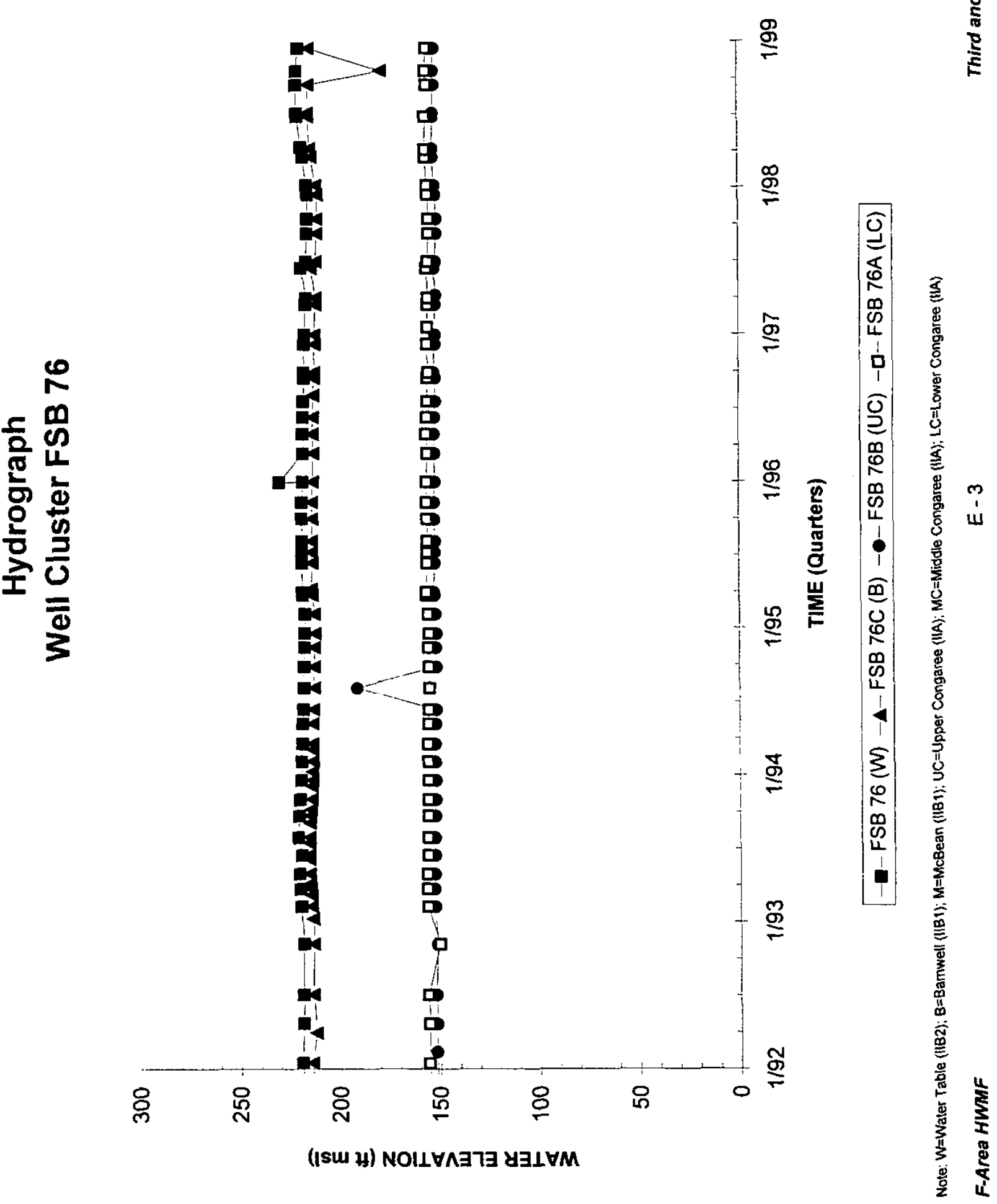


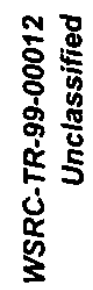

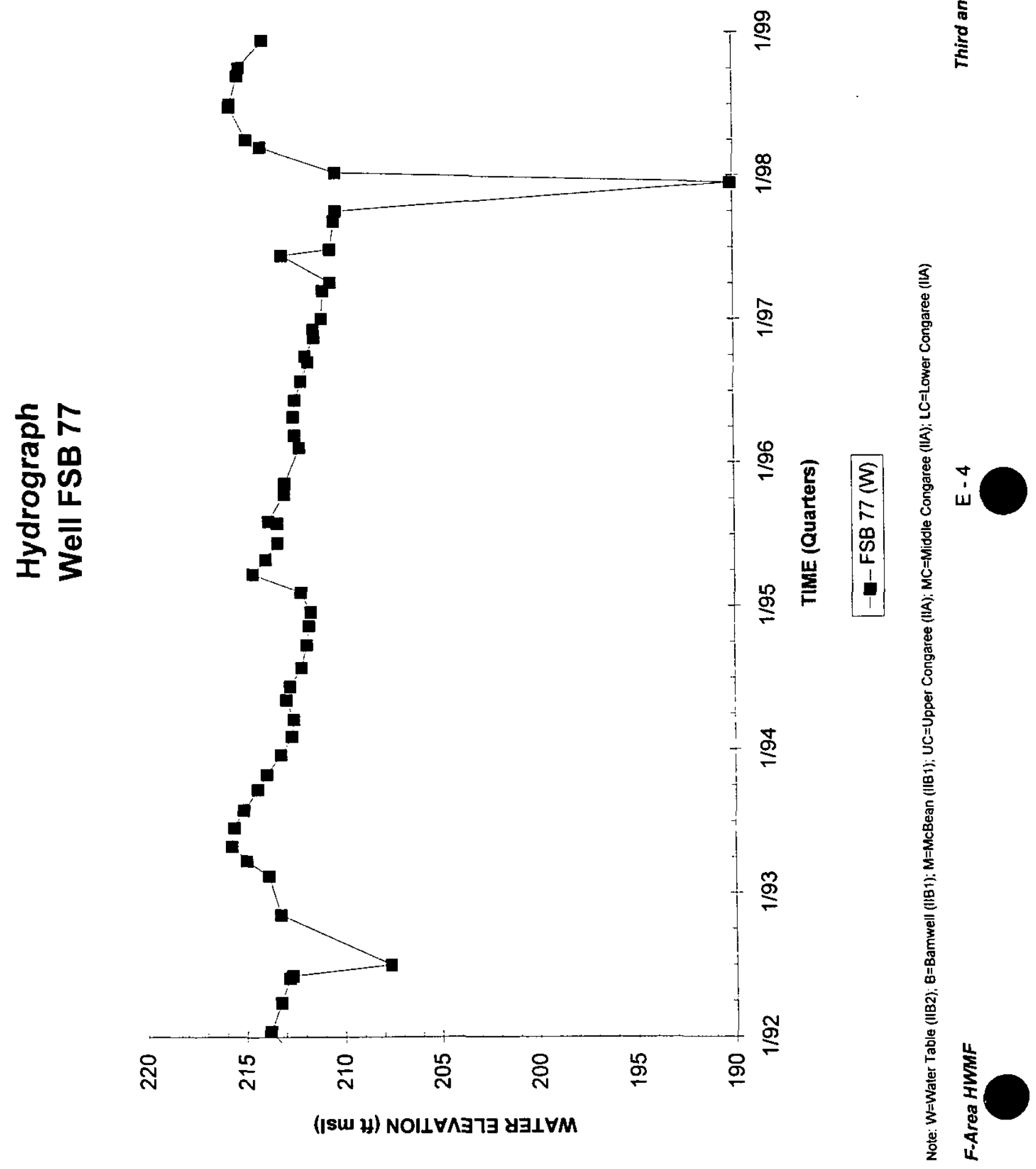




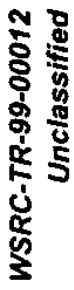

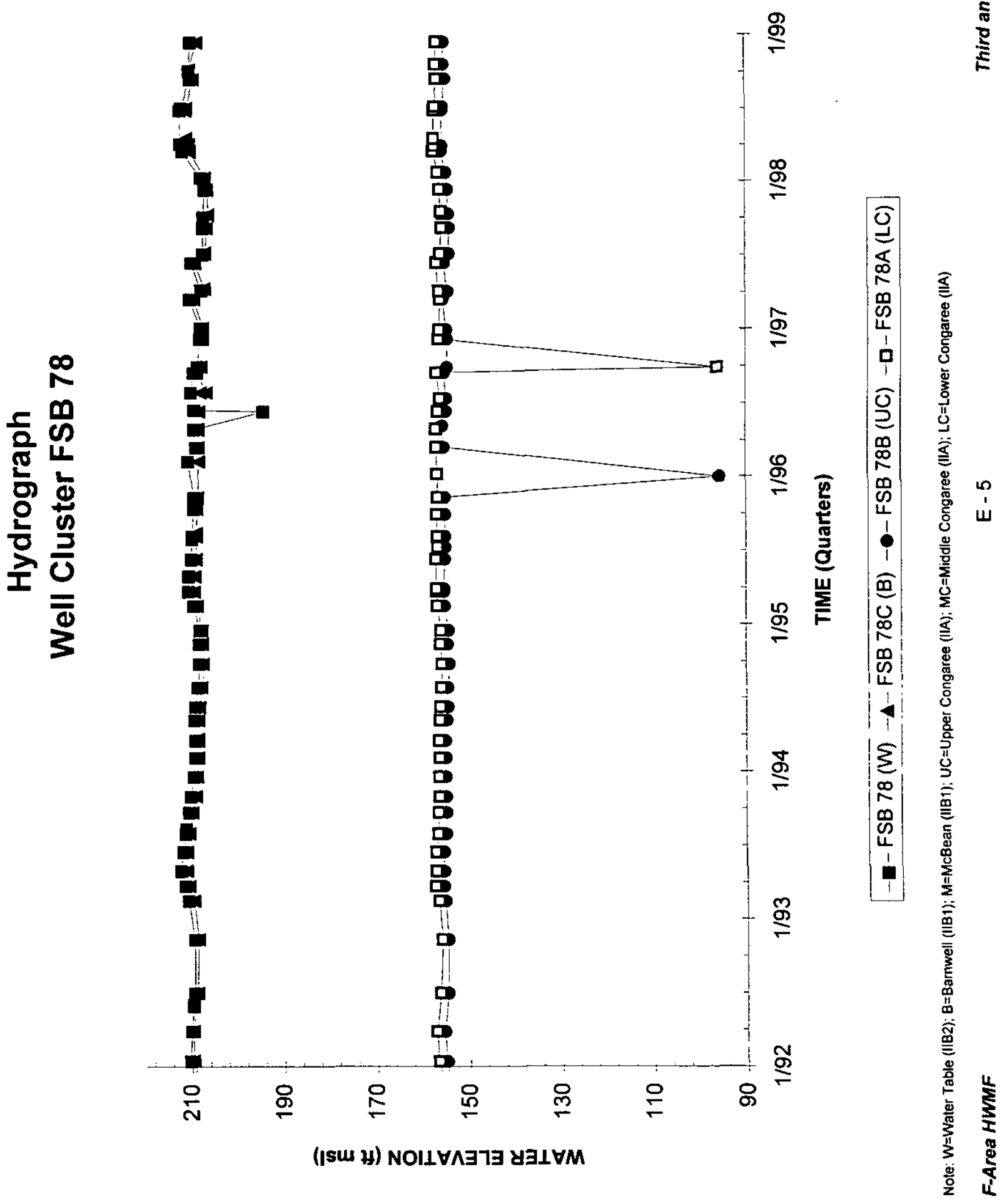



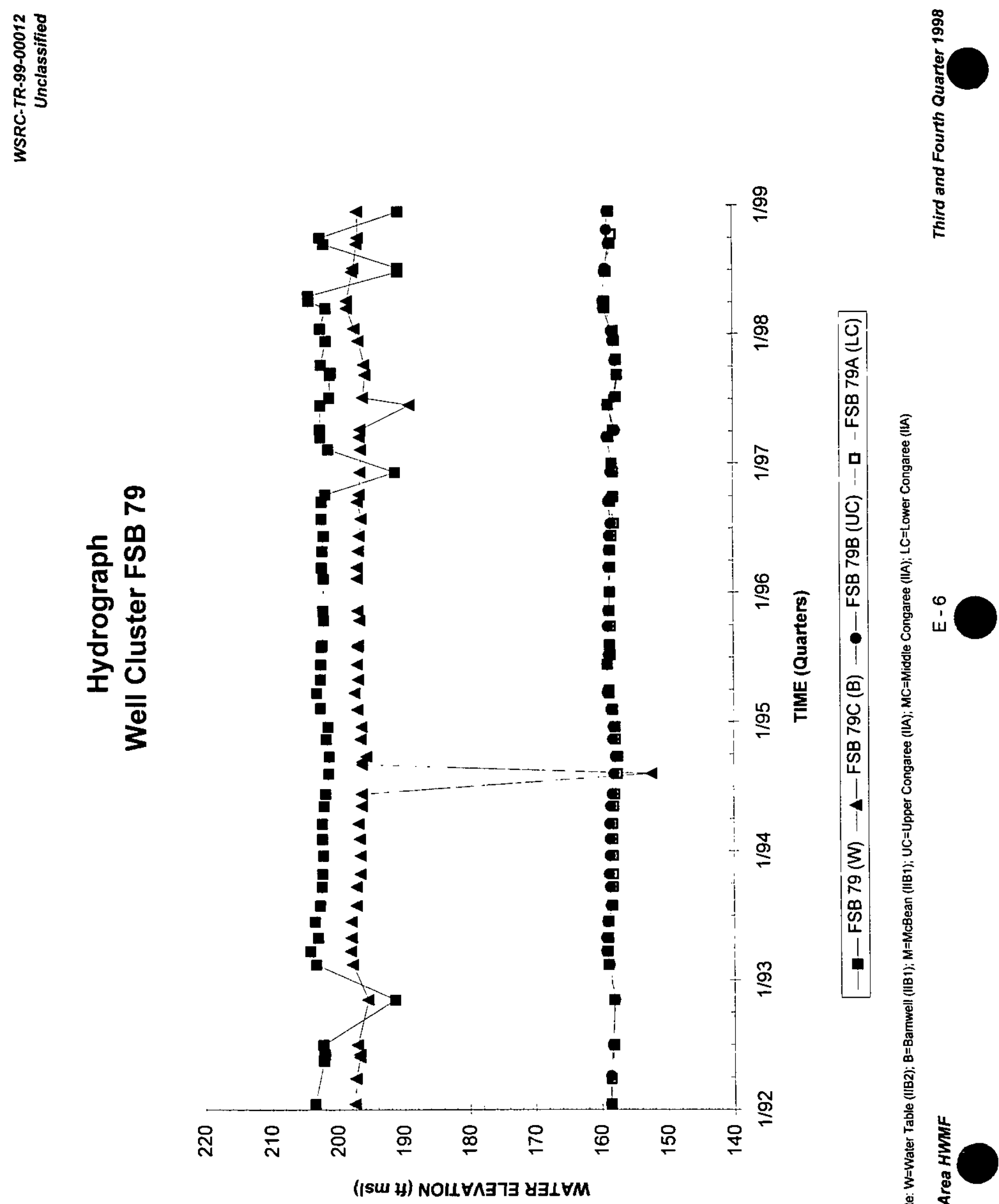


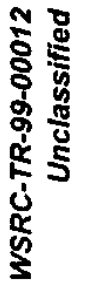
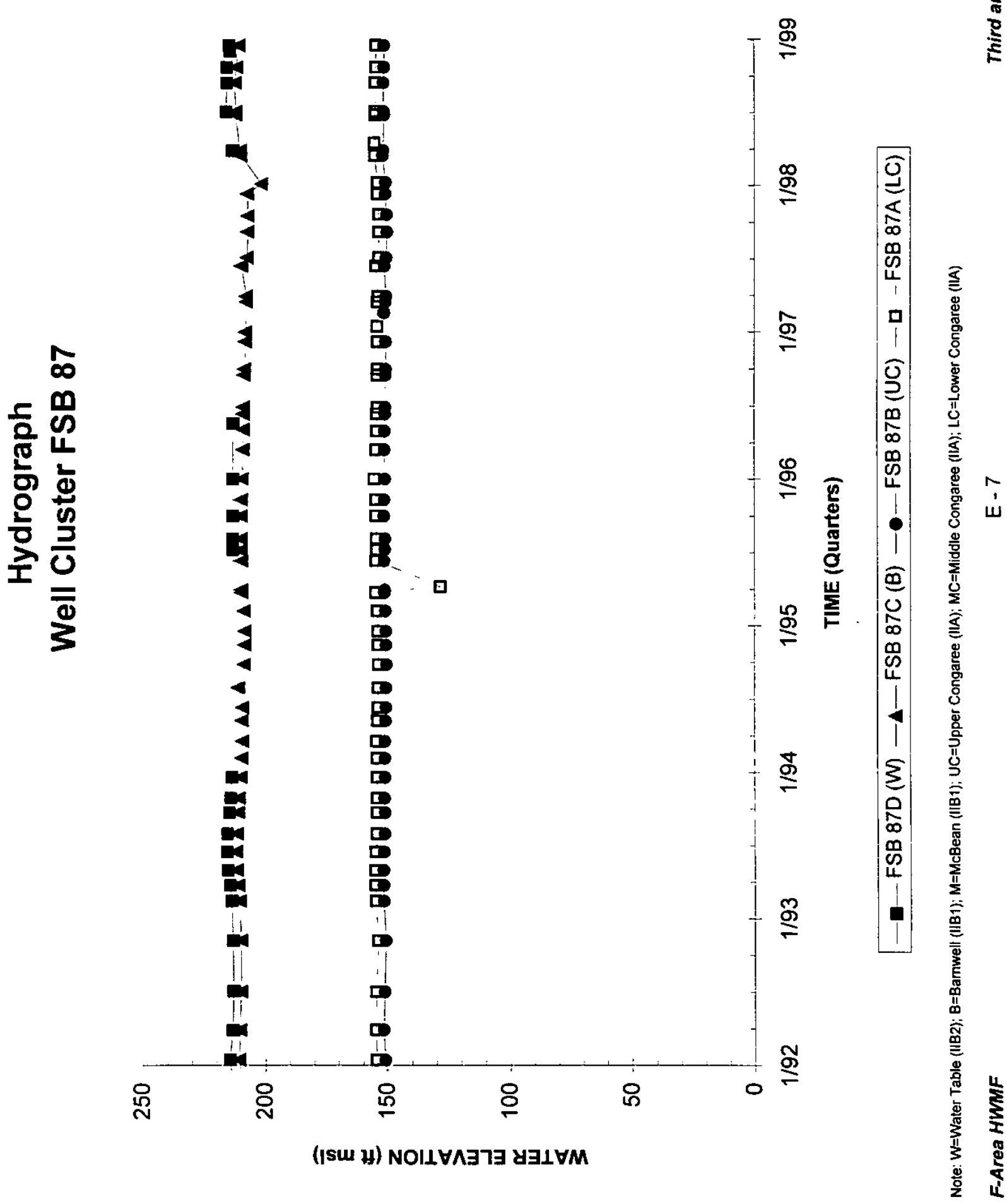


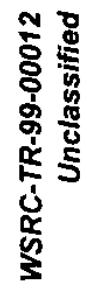
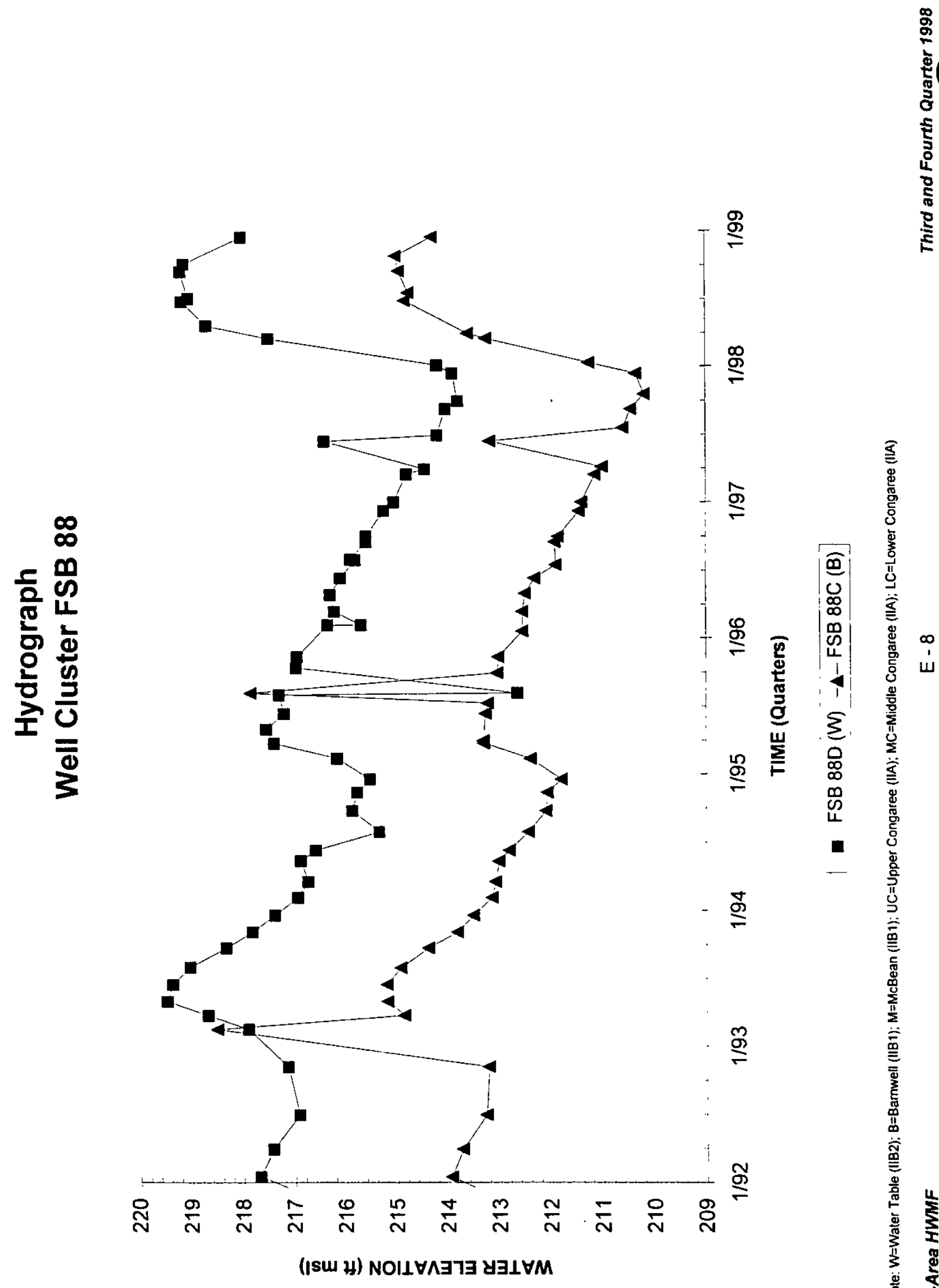

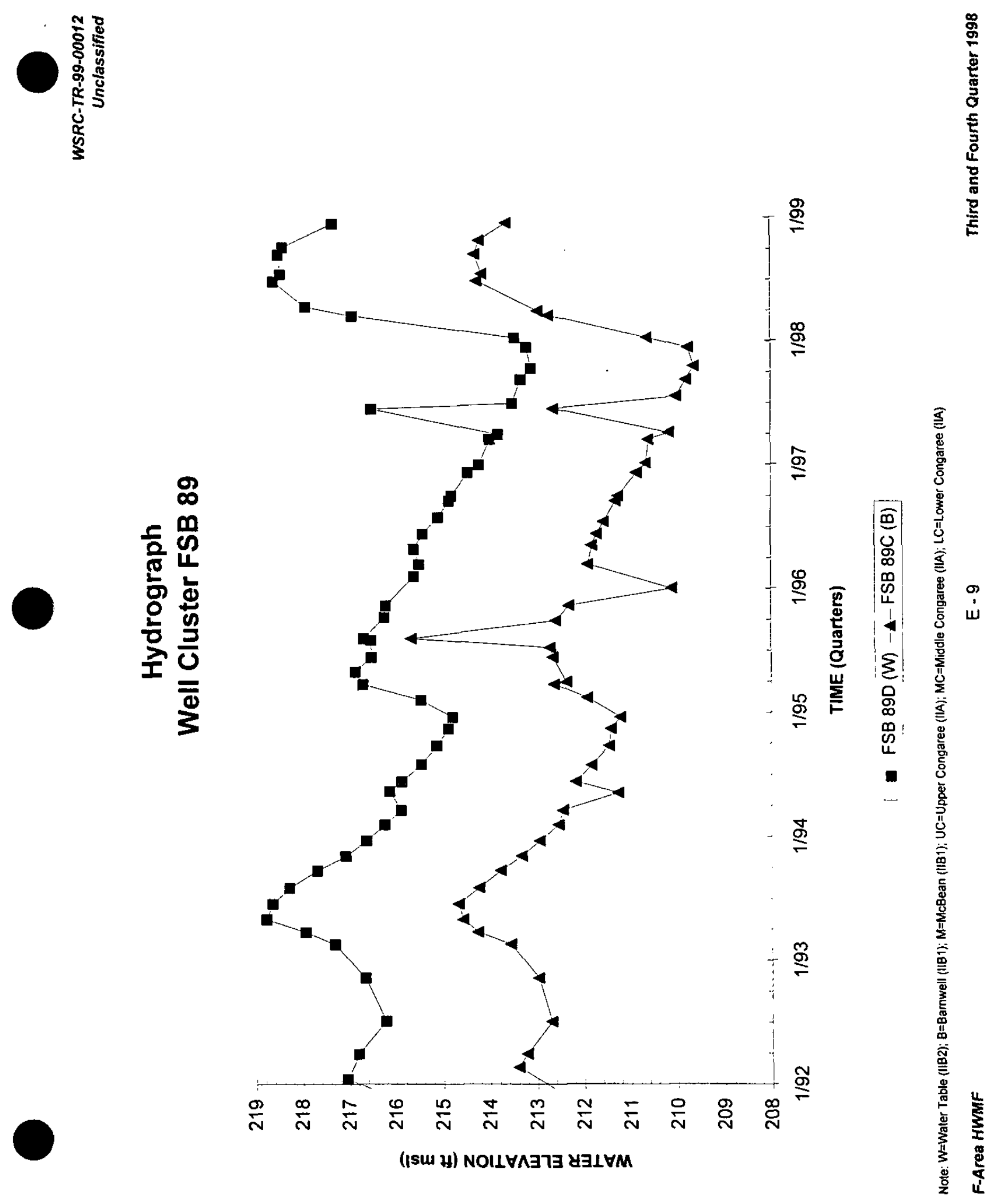

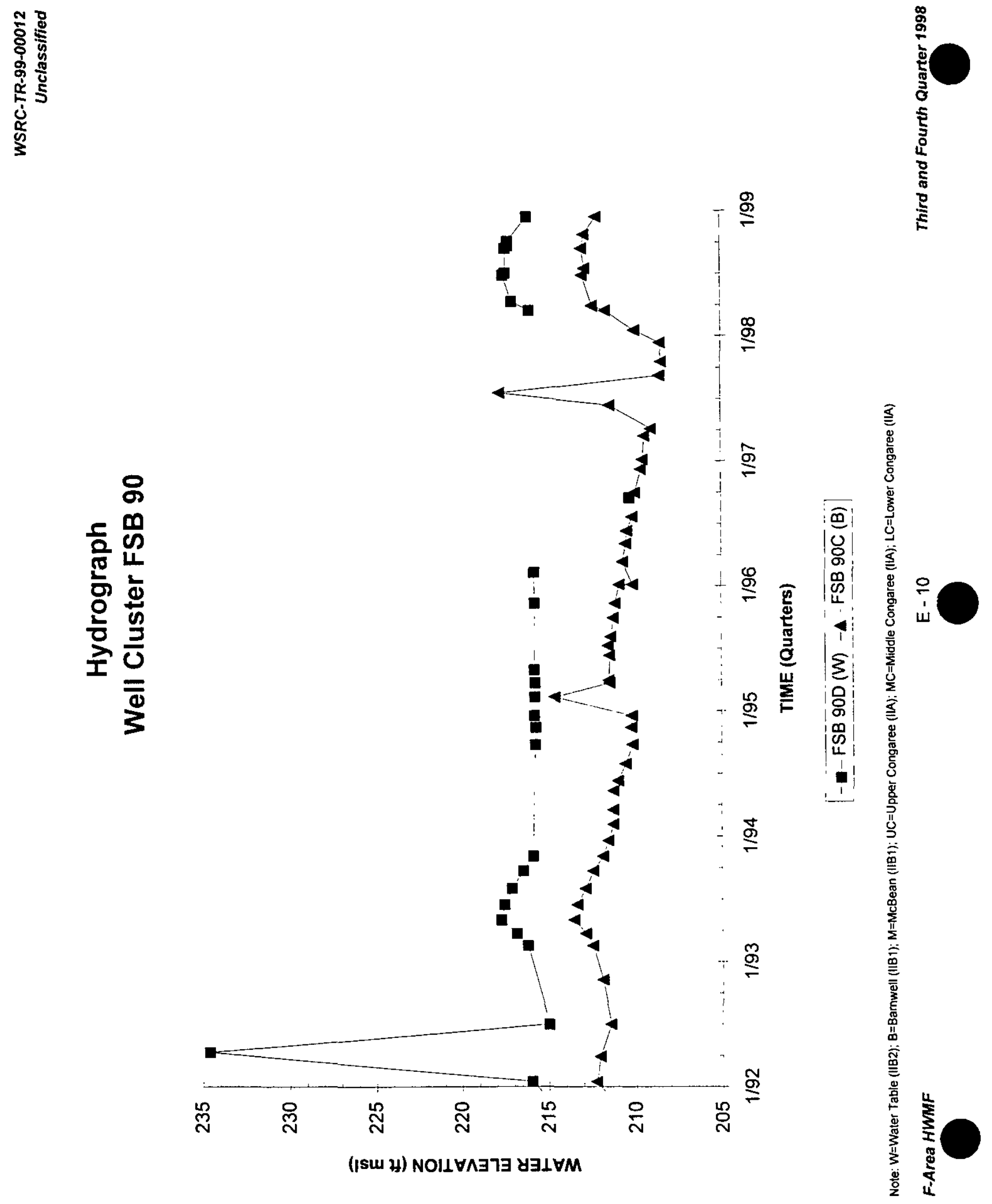


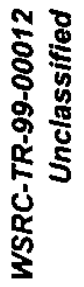

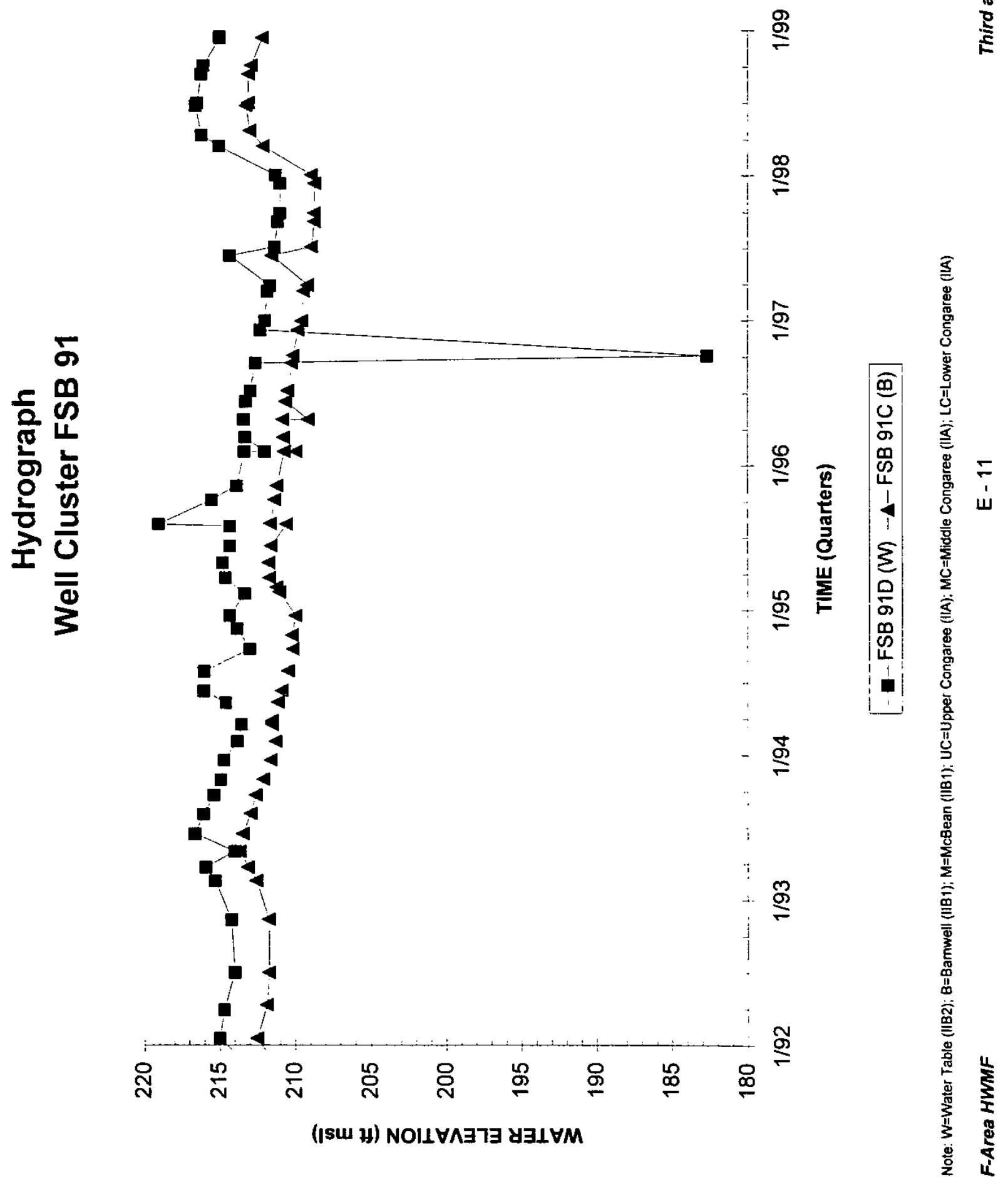



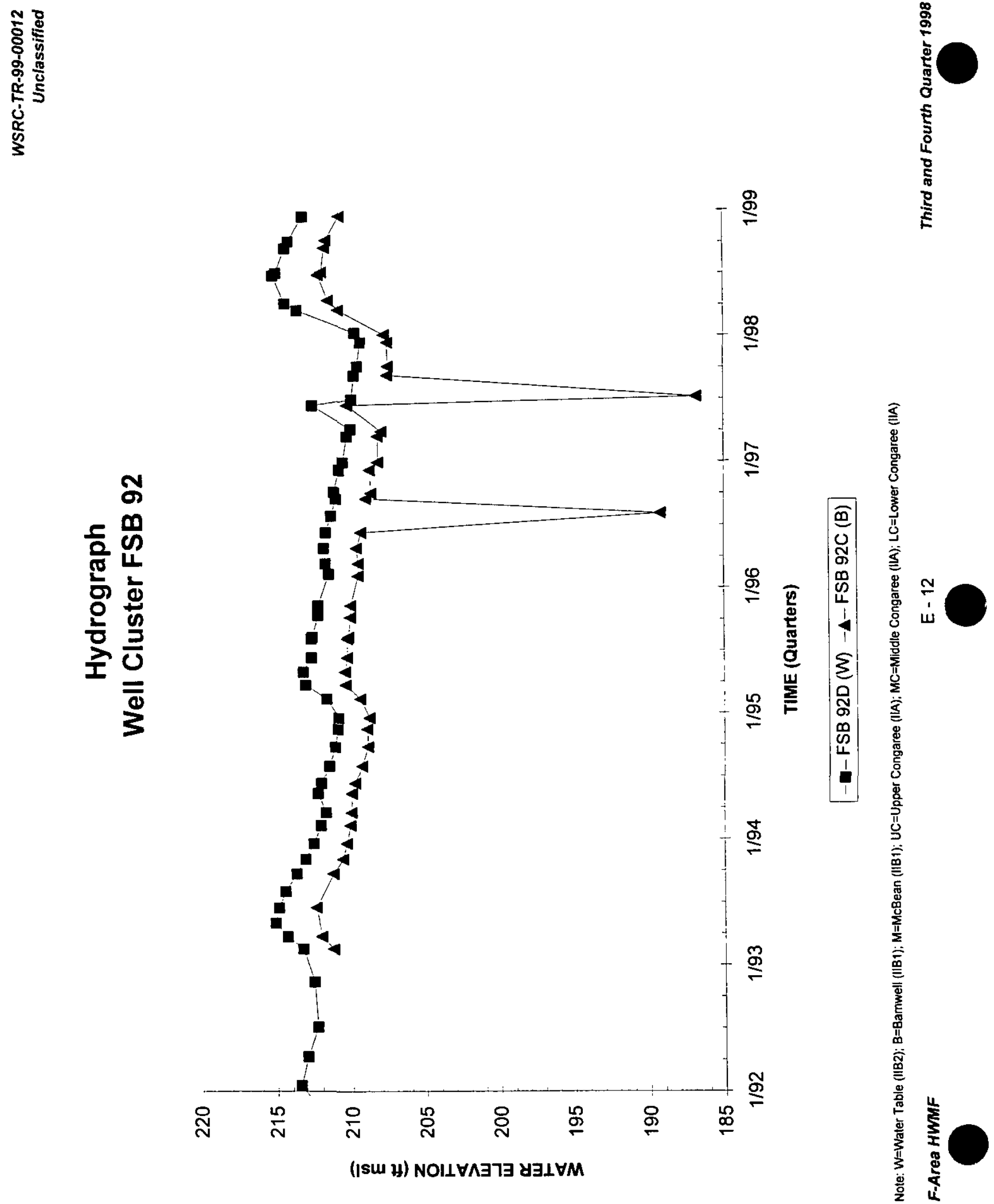


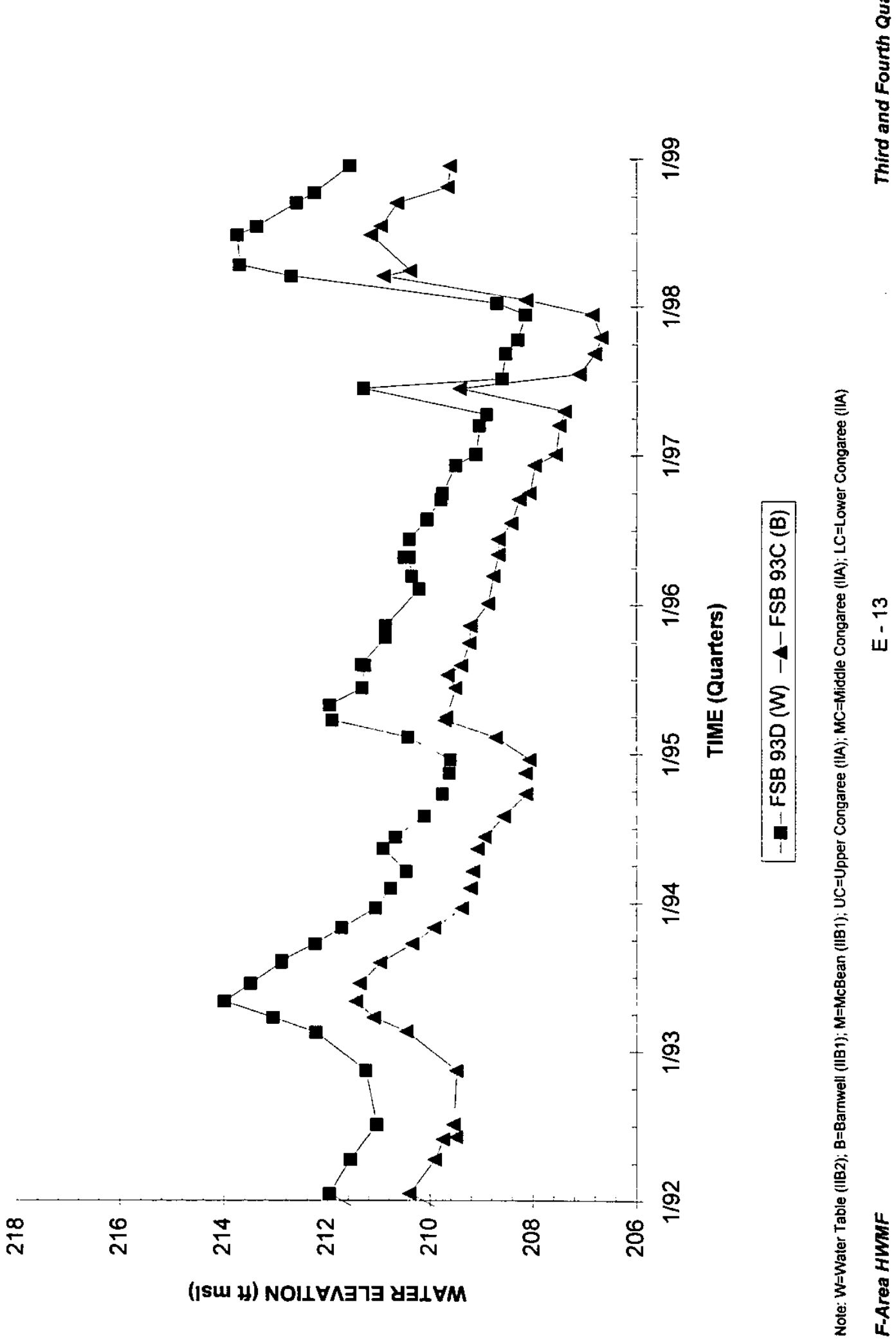




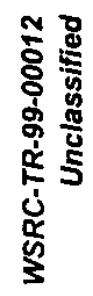

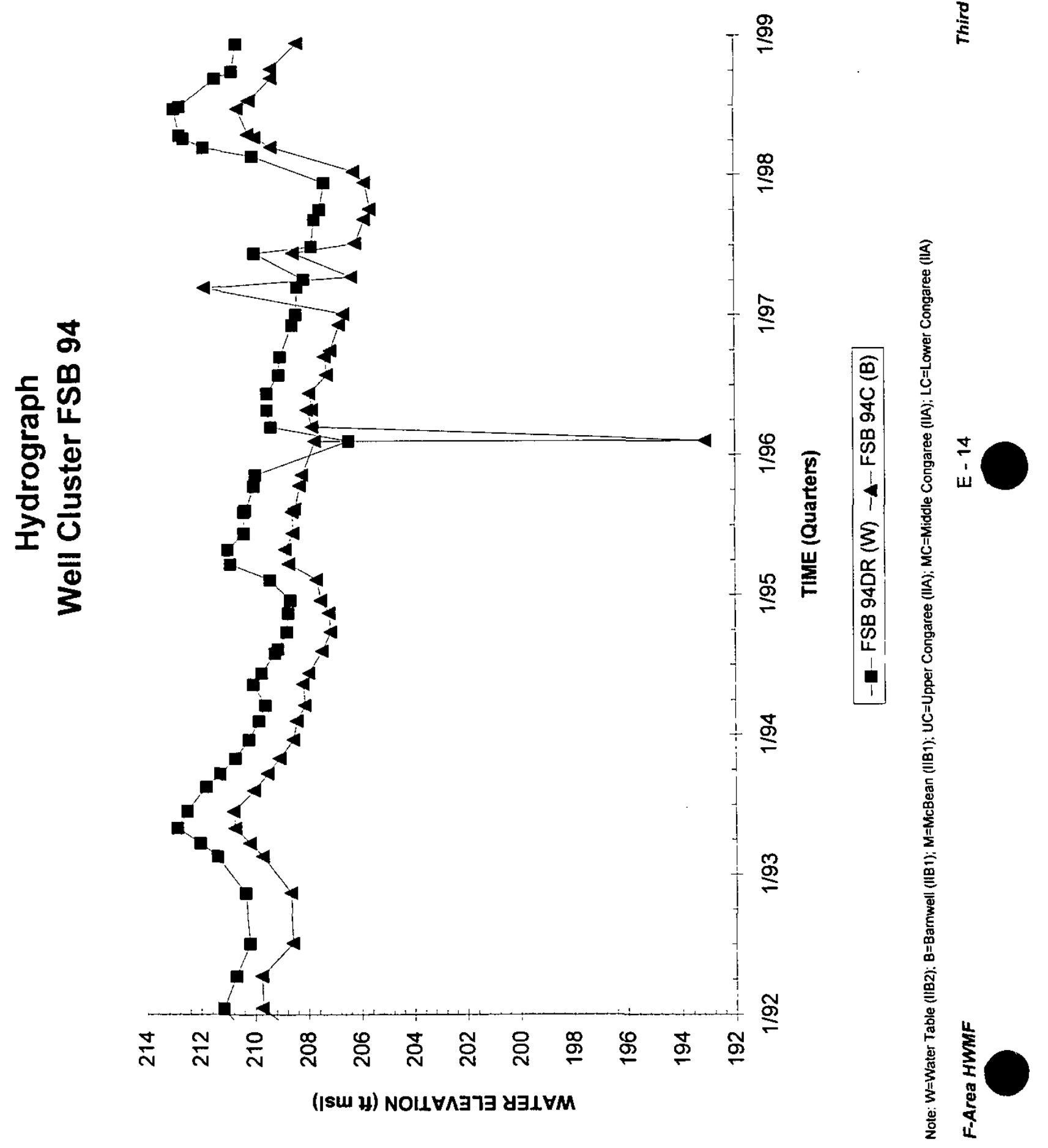



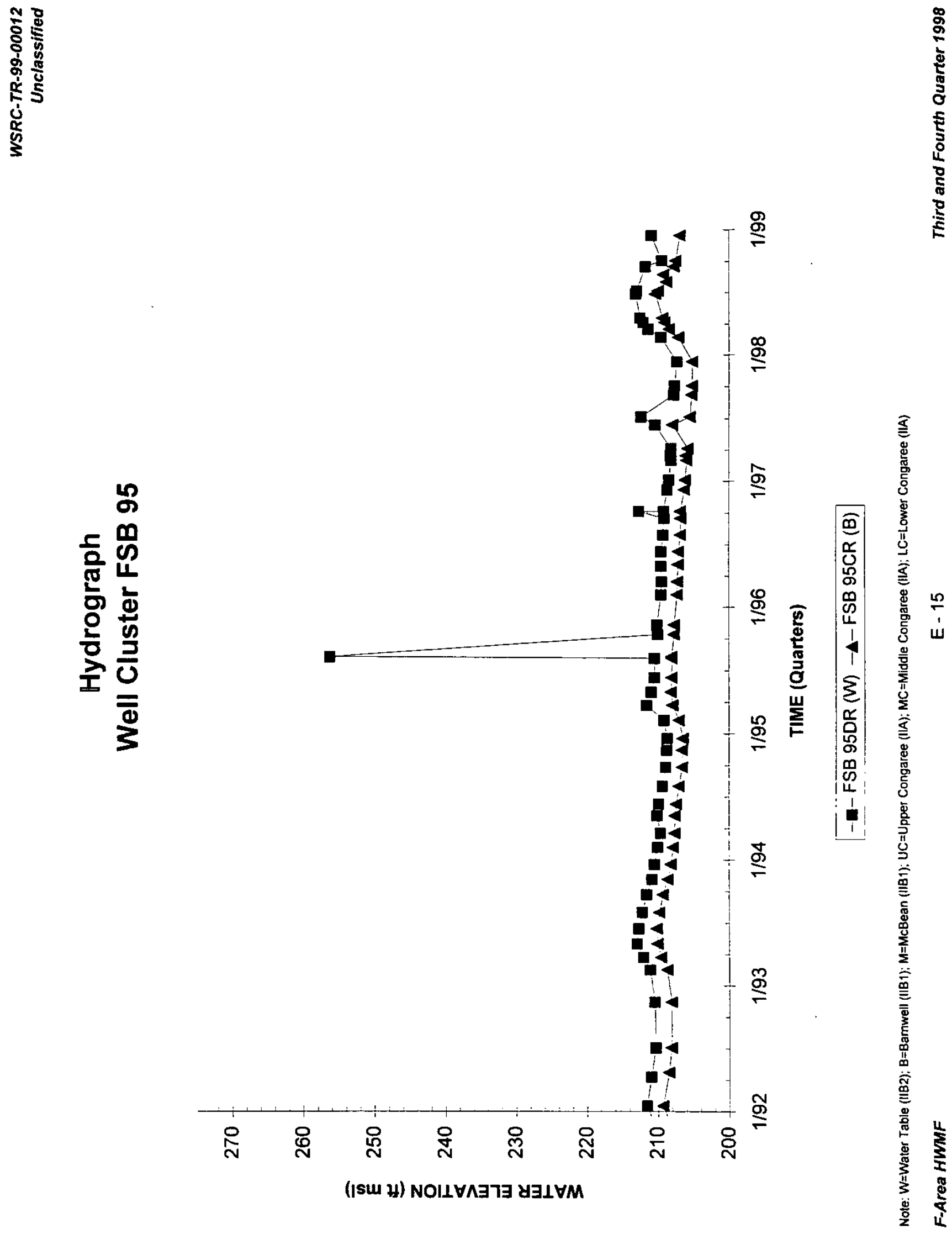

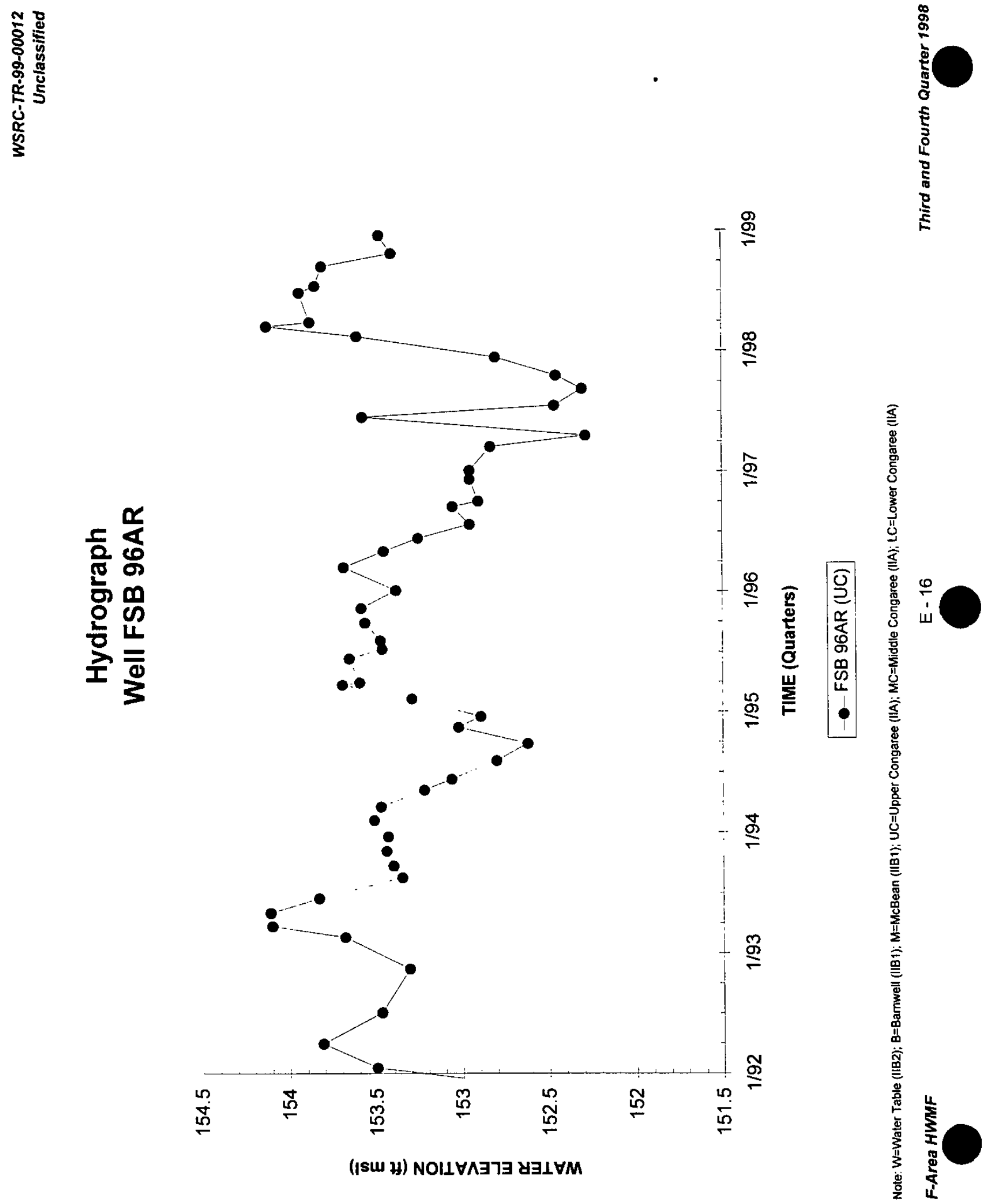

\% 


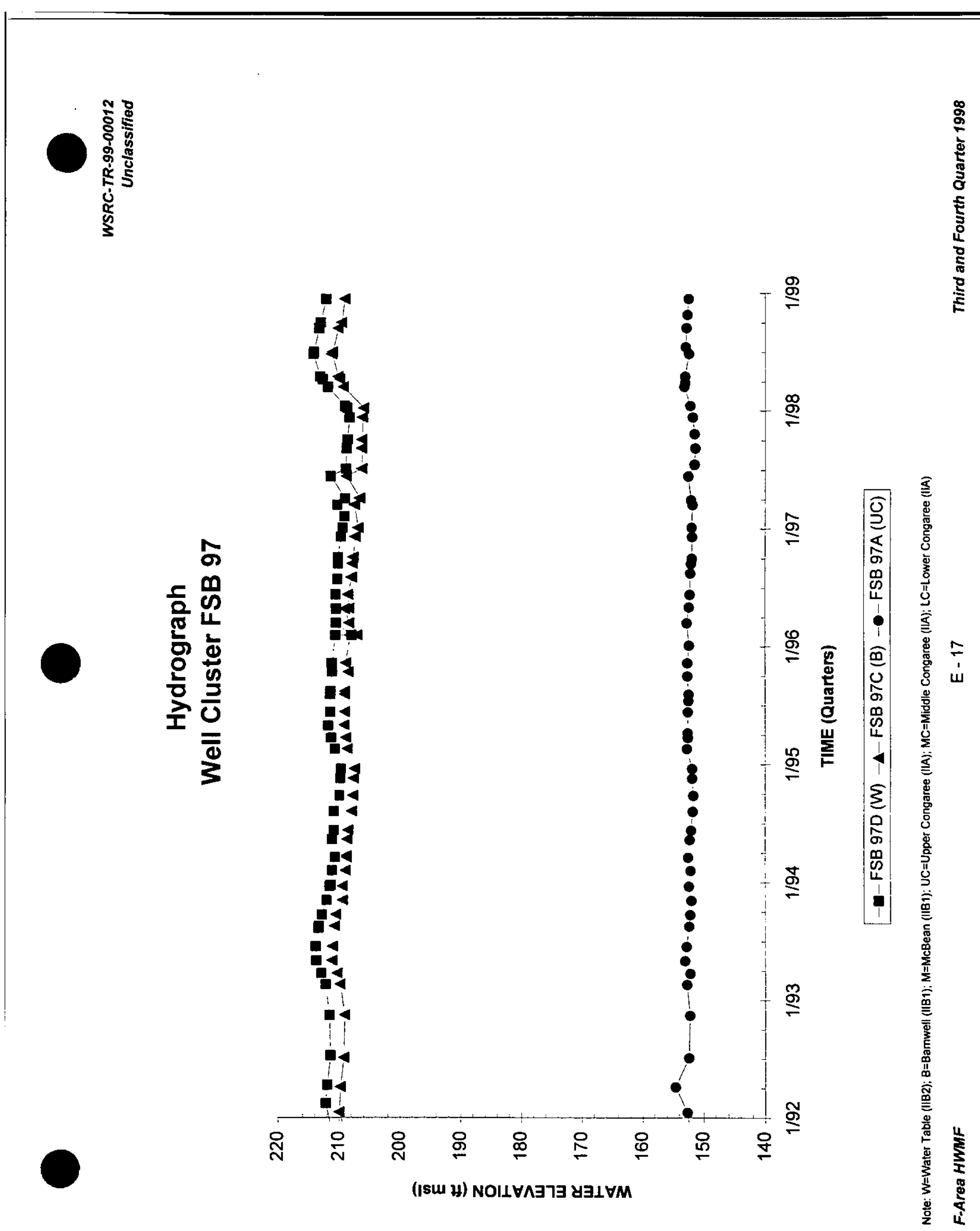




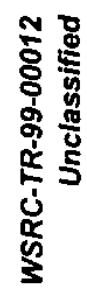
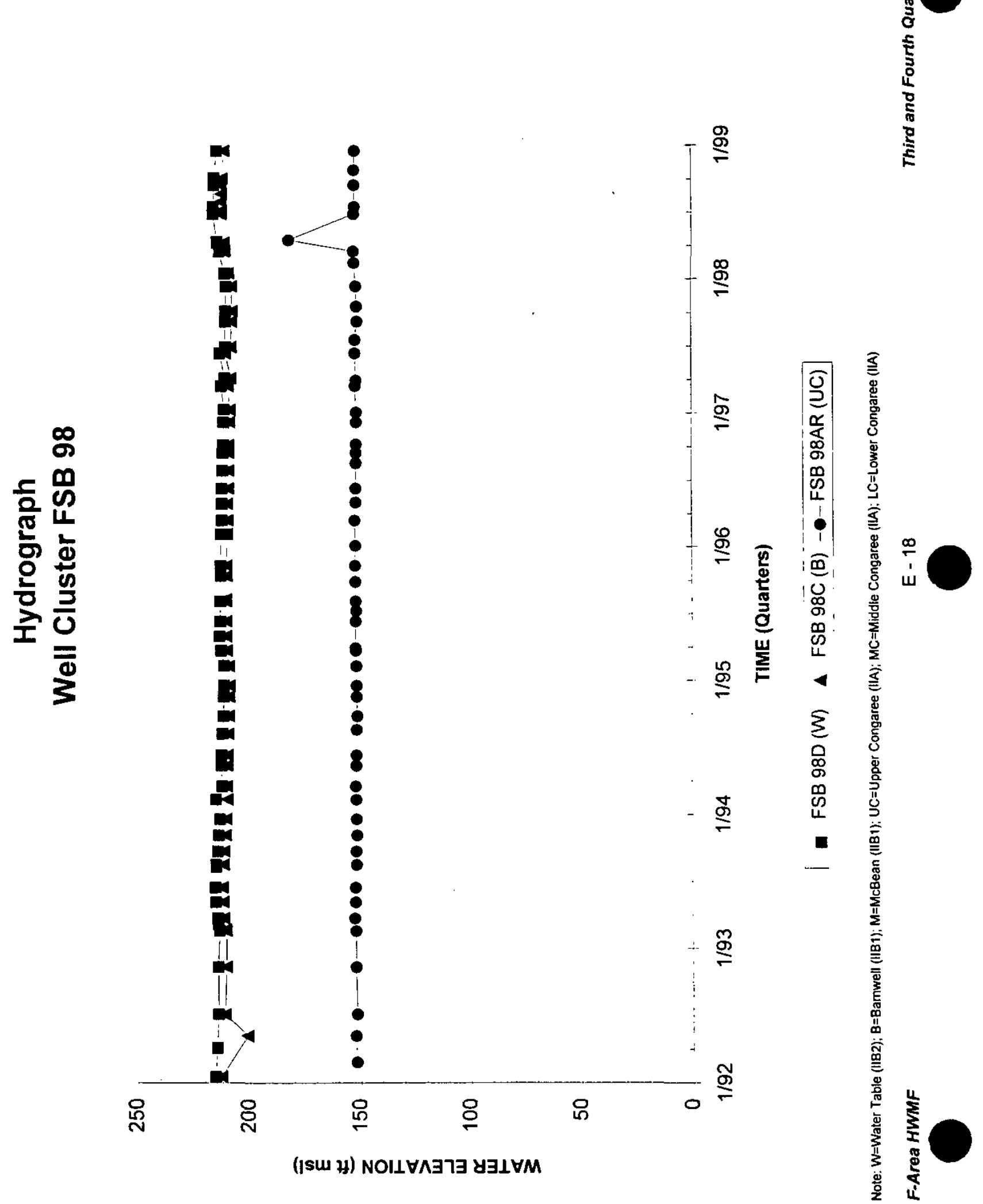

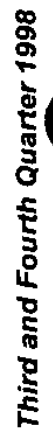




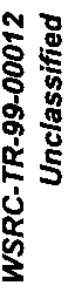

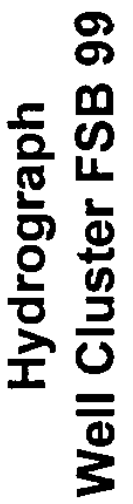

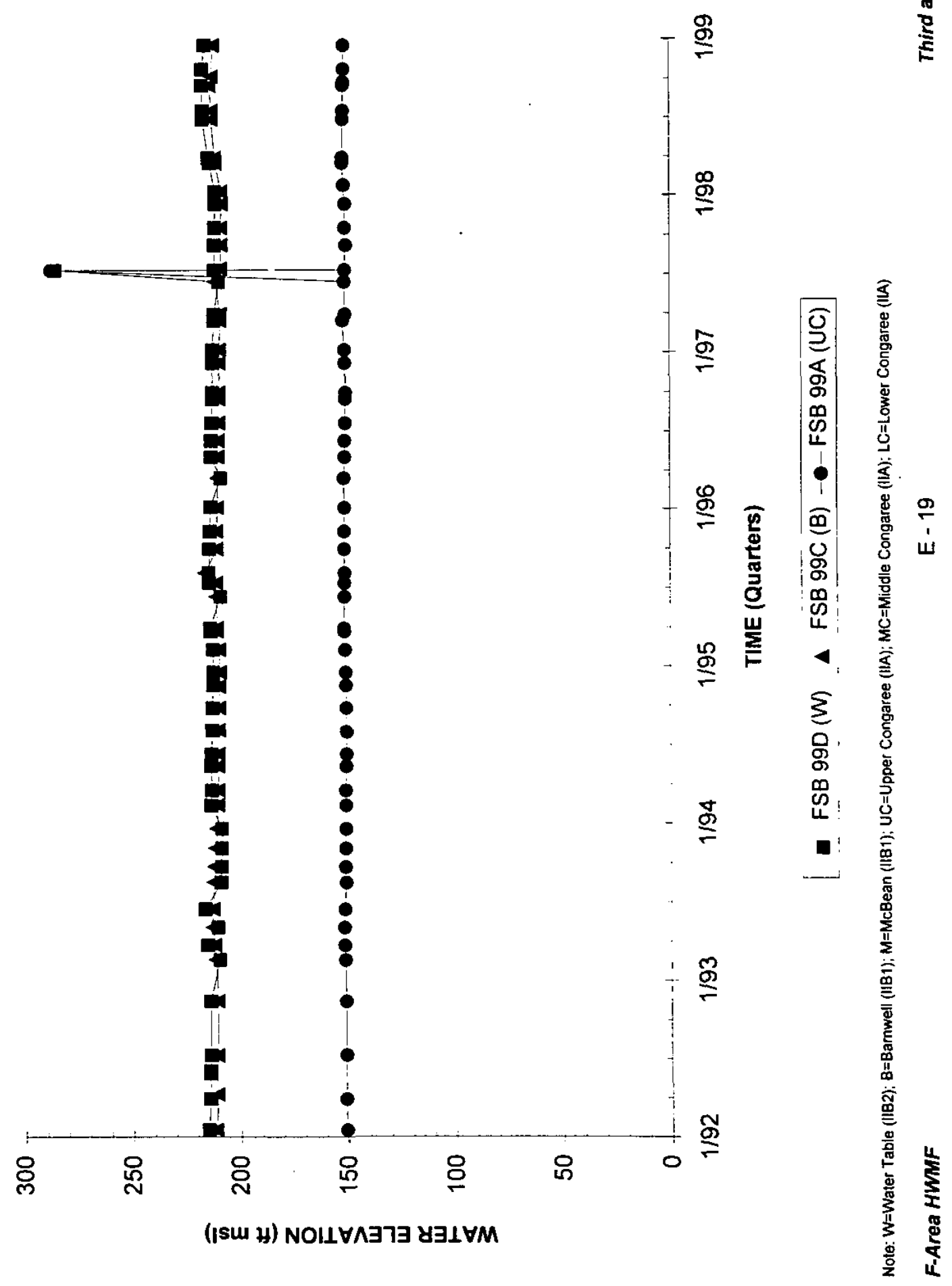



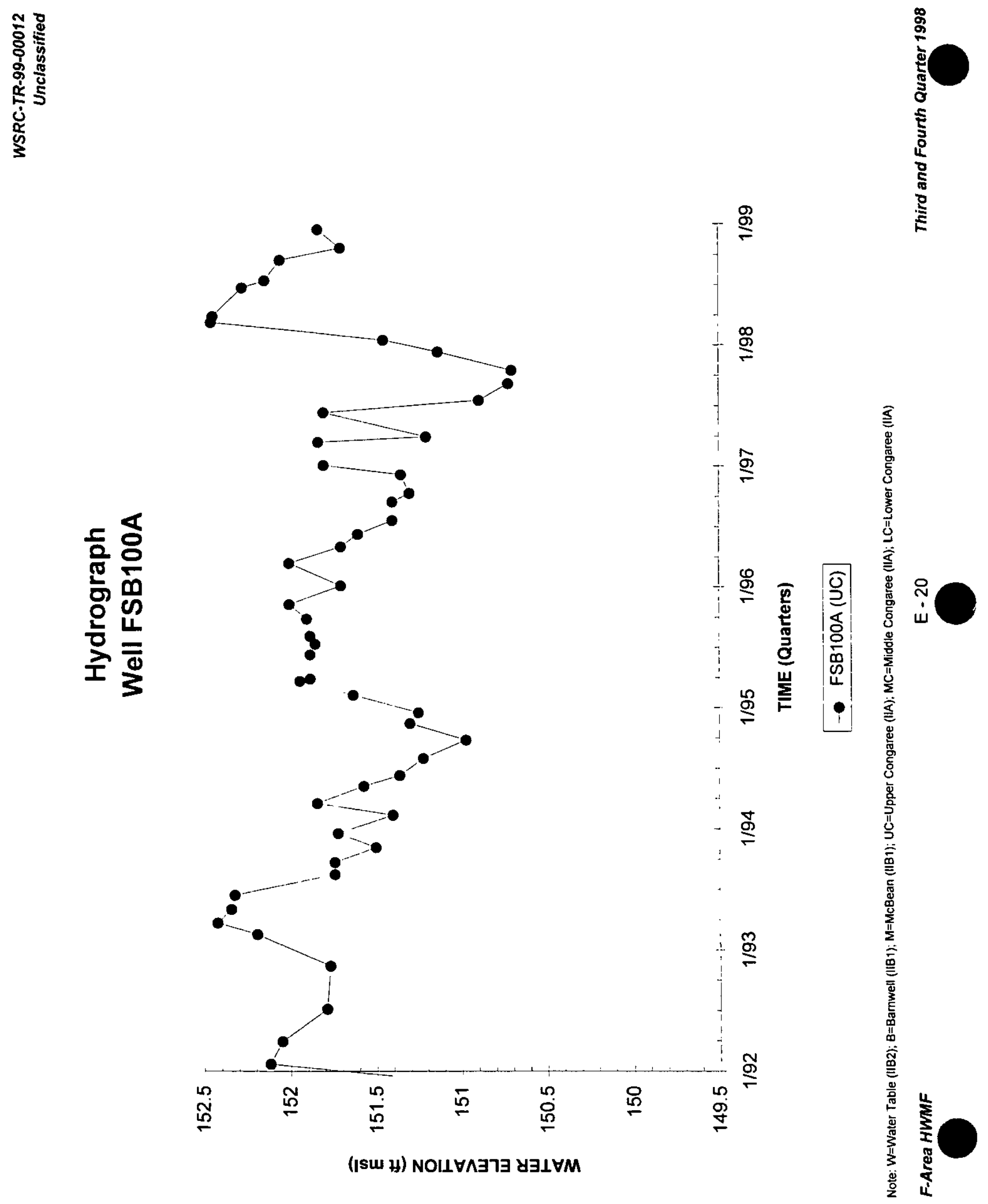


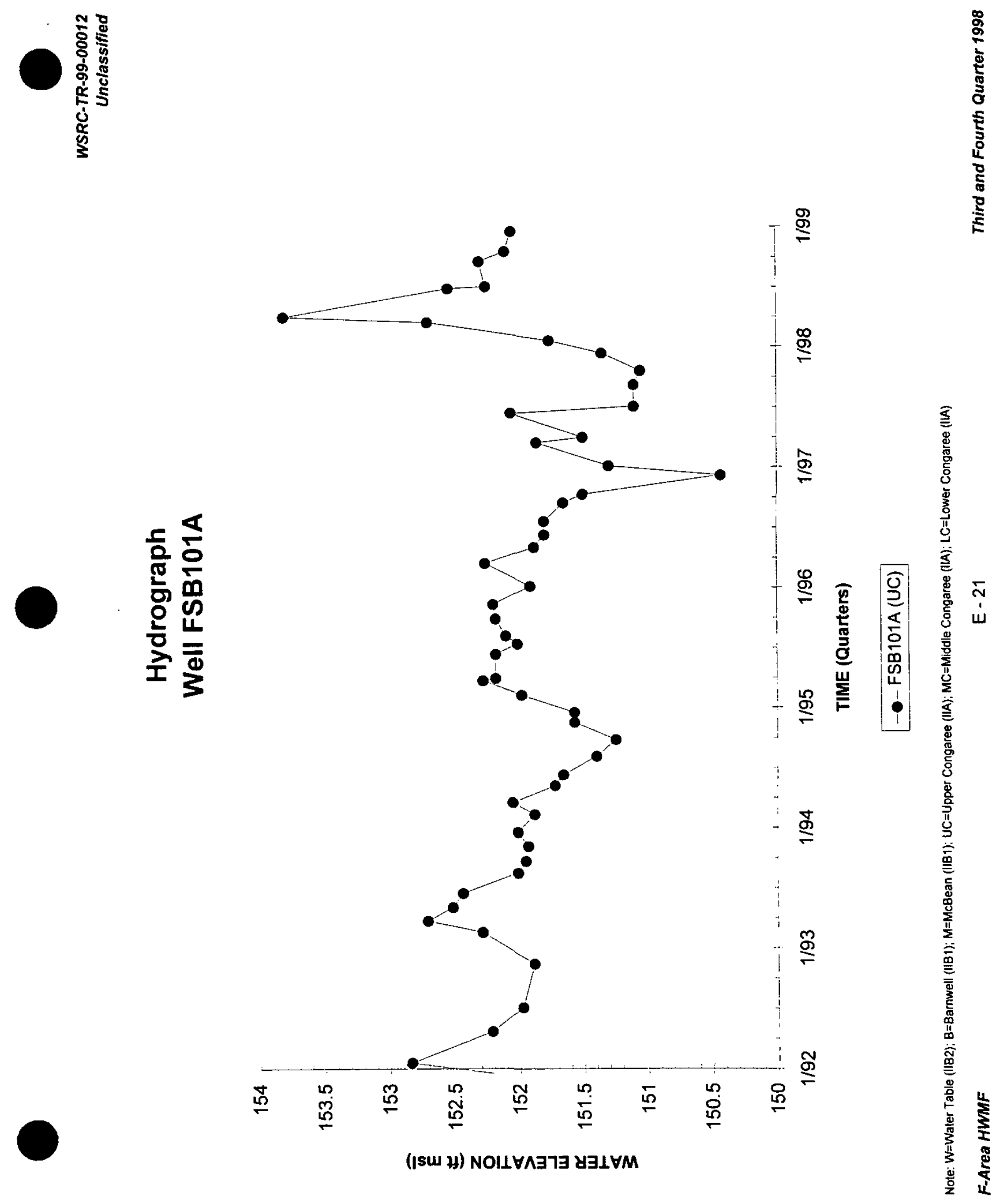




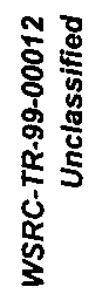

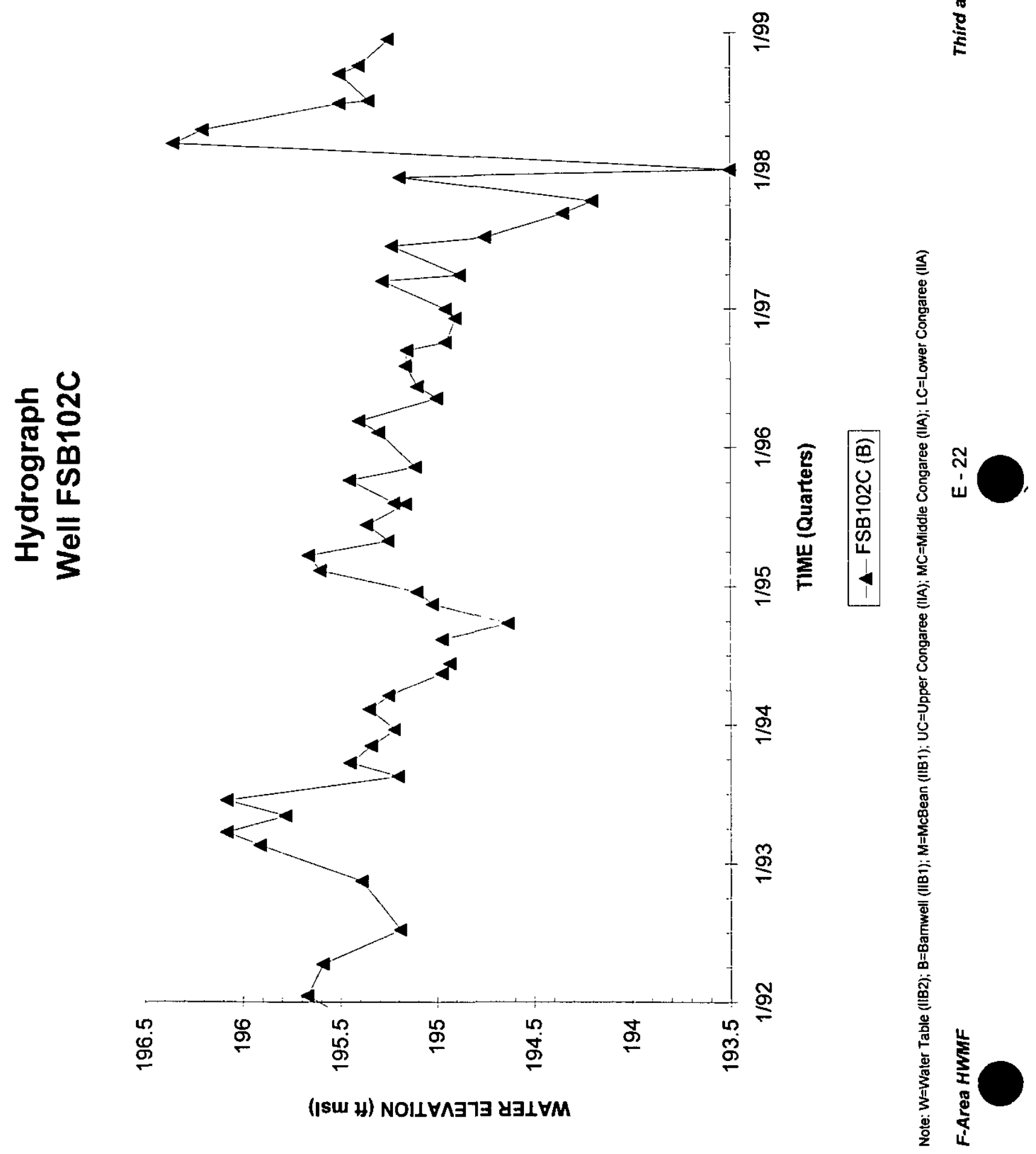




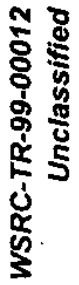
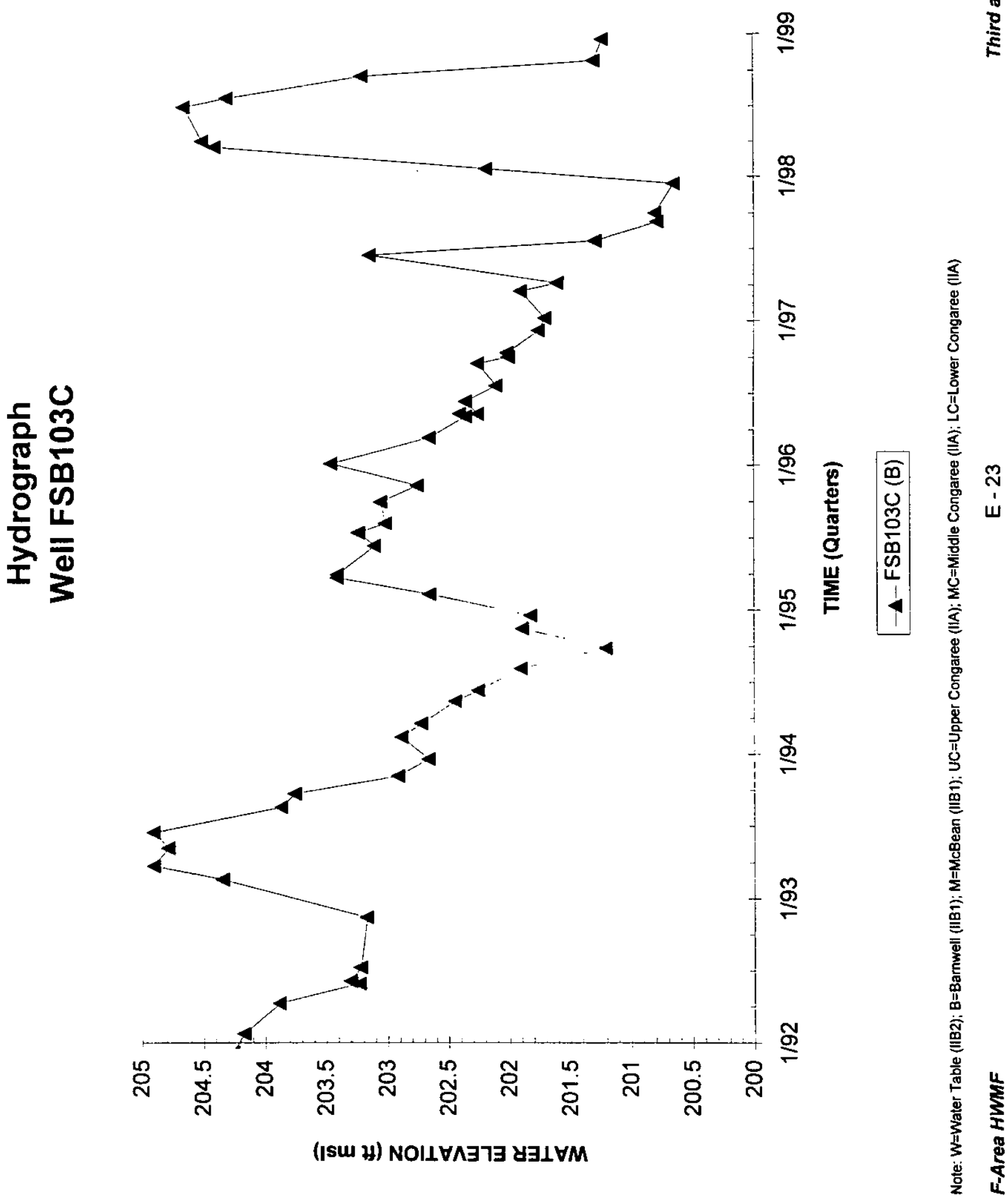

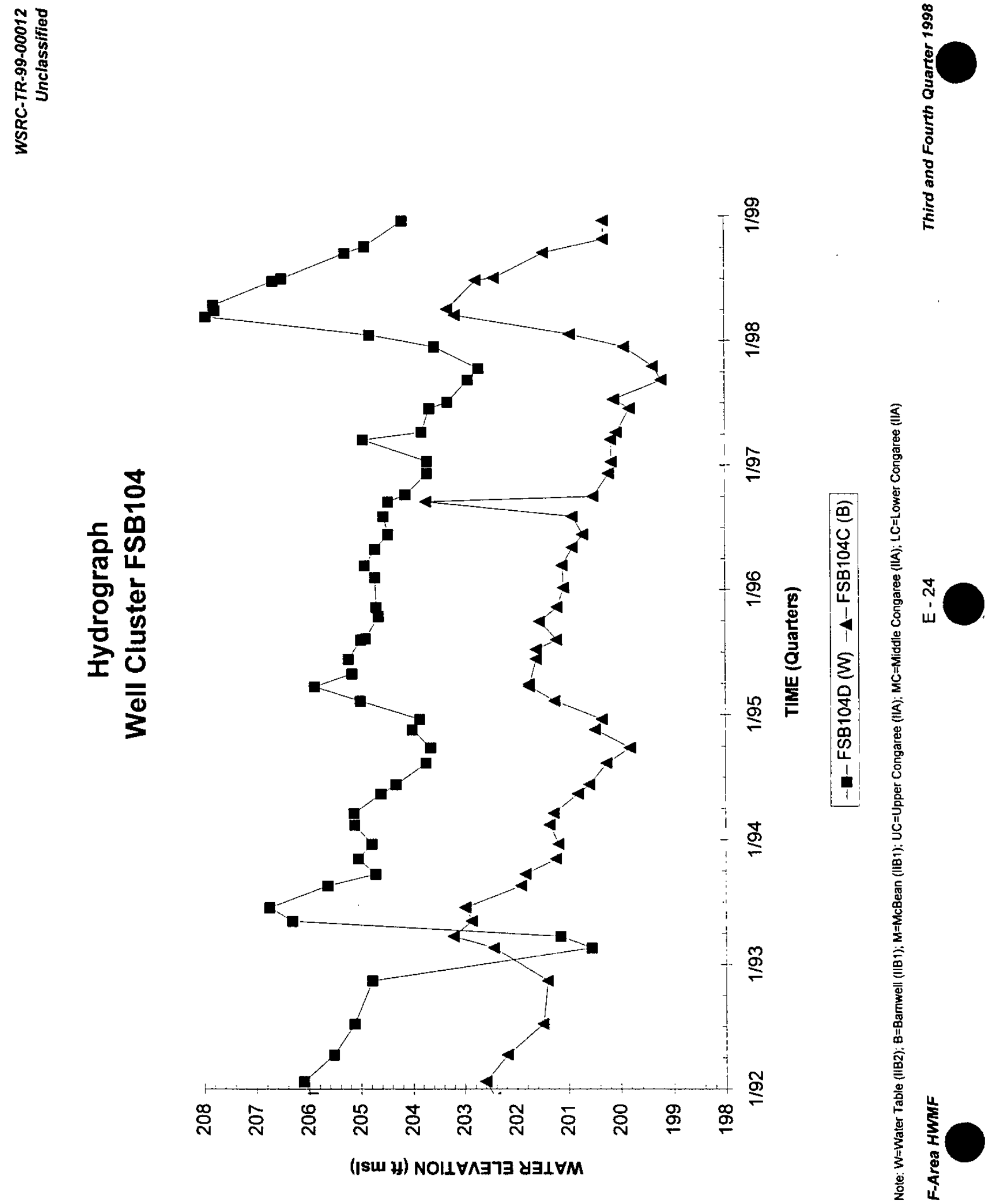

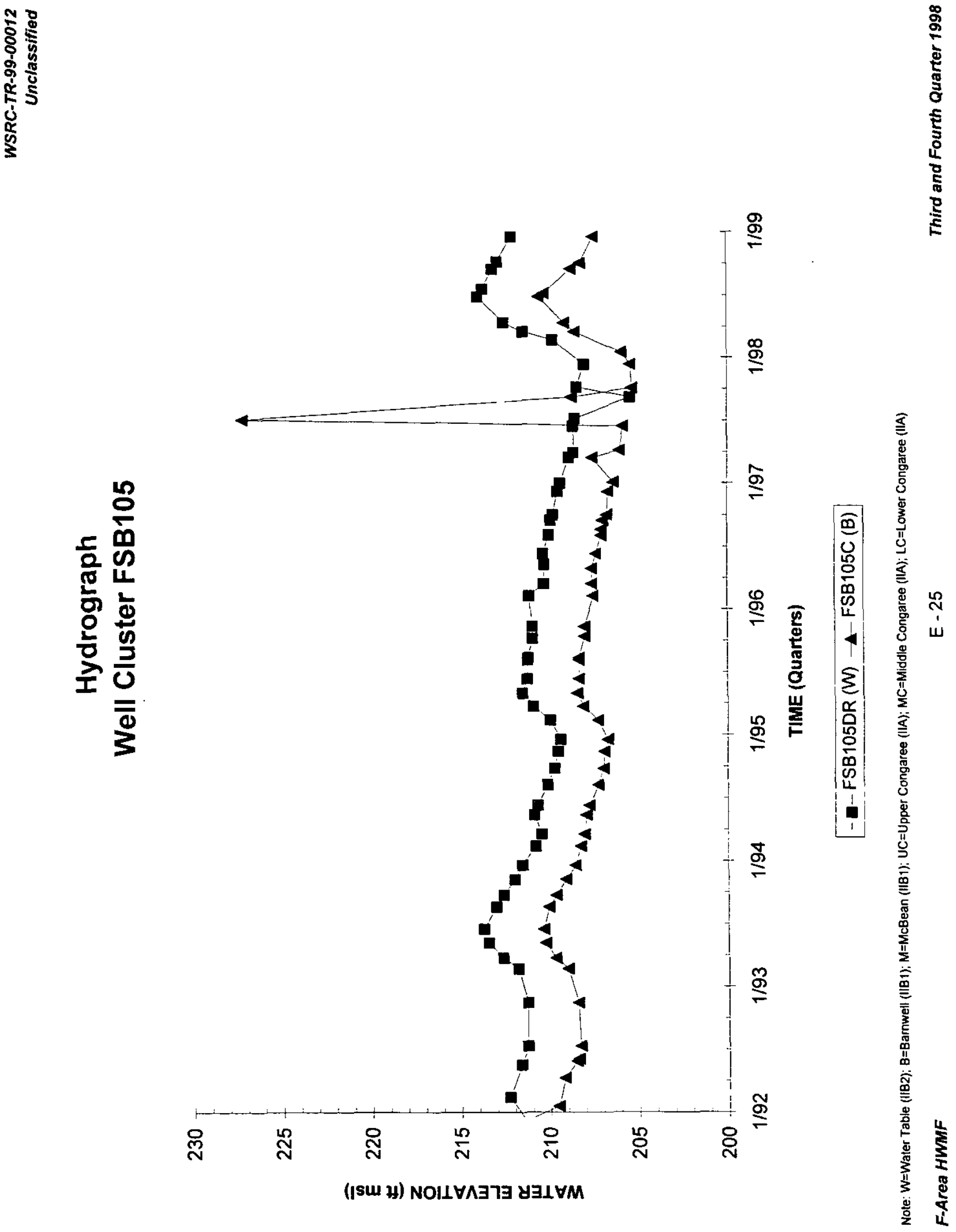


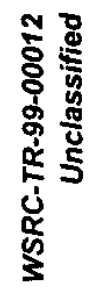
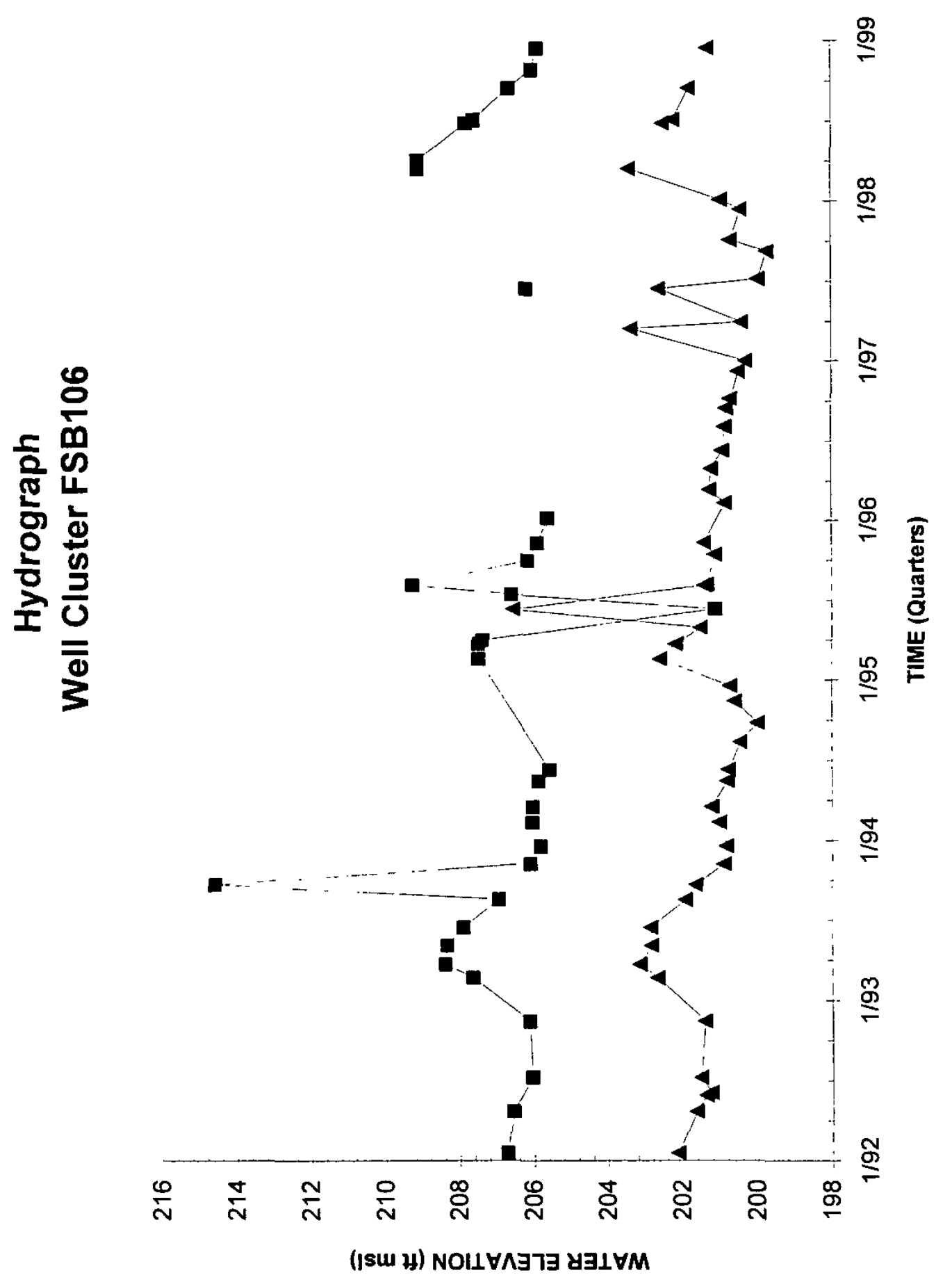

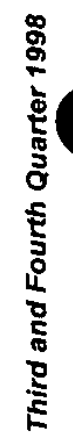

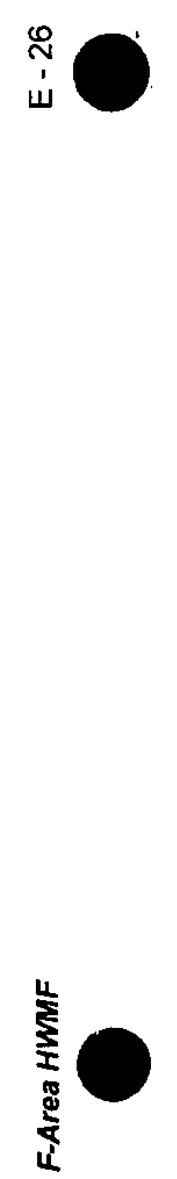




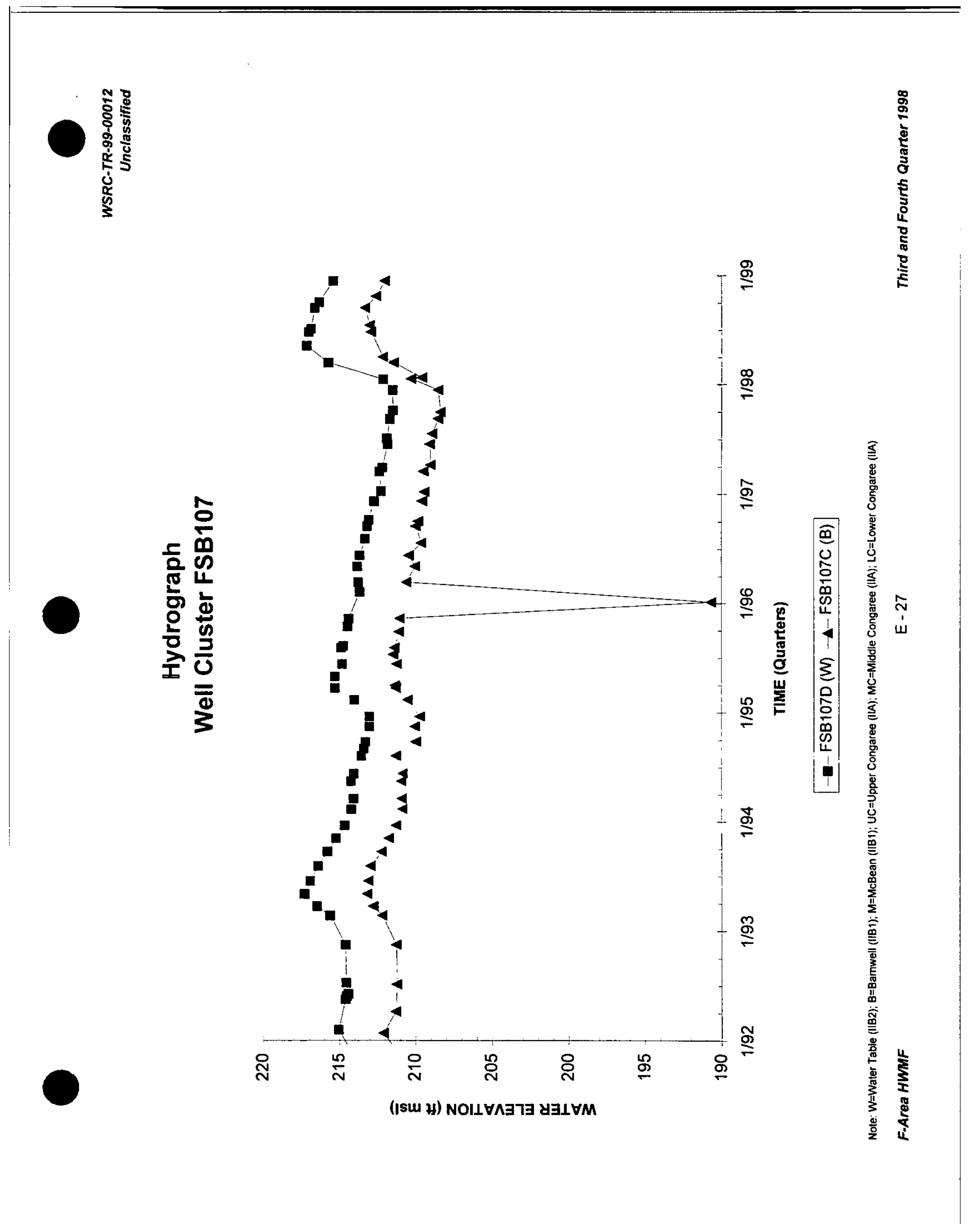




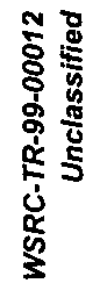
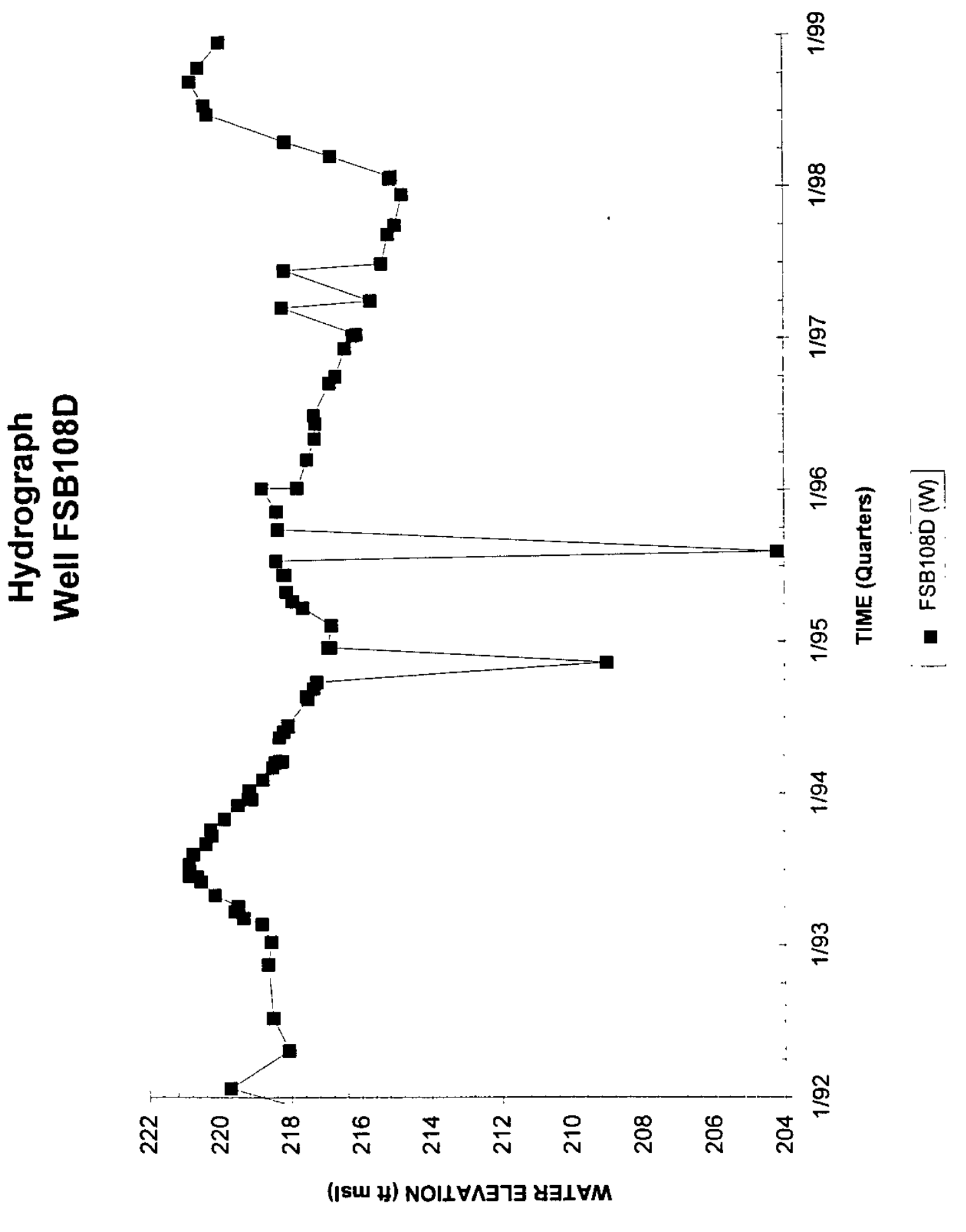

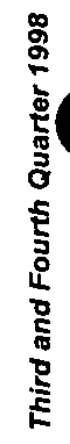

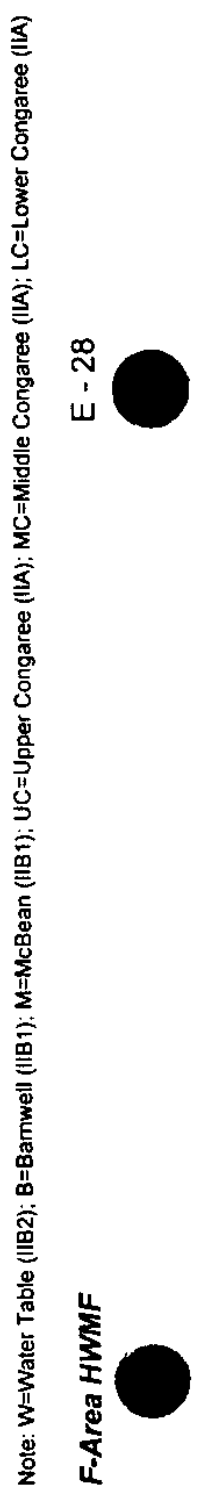




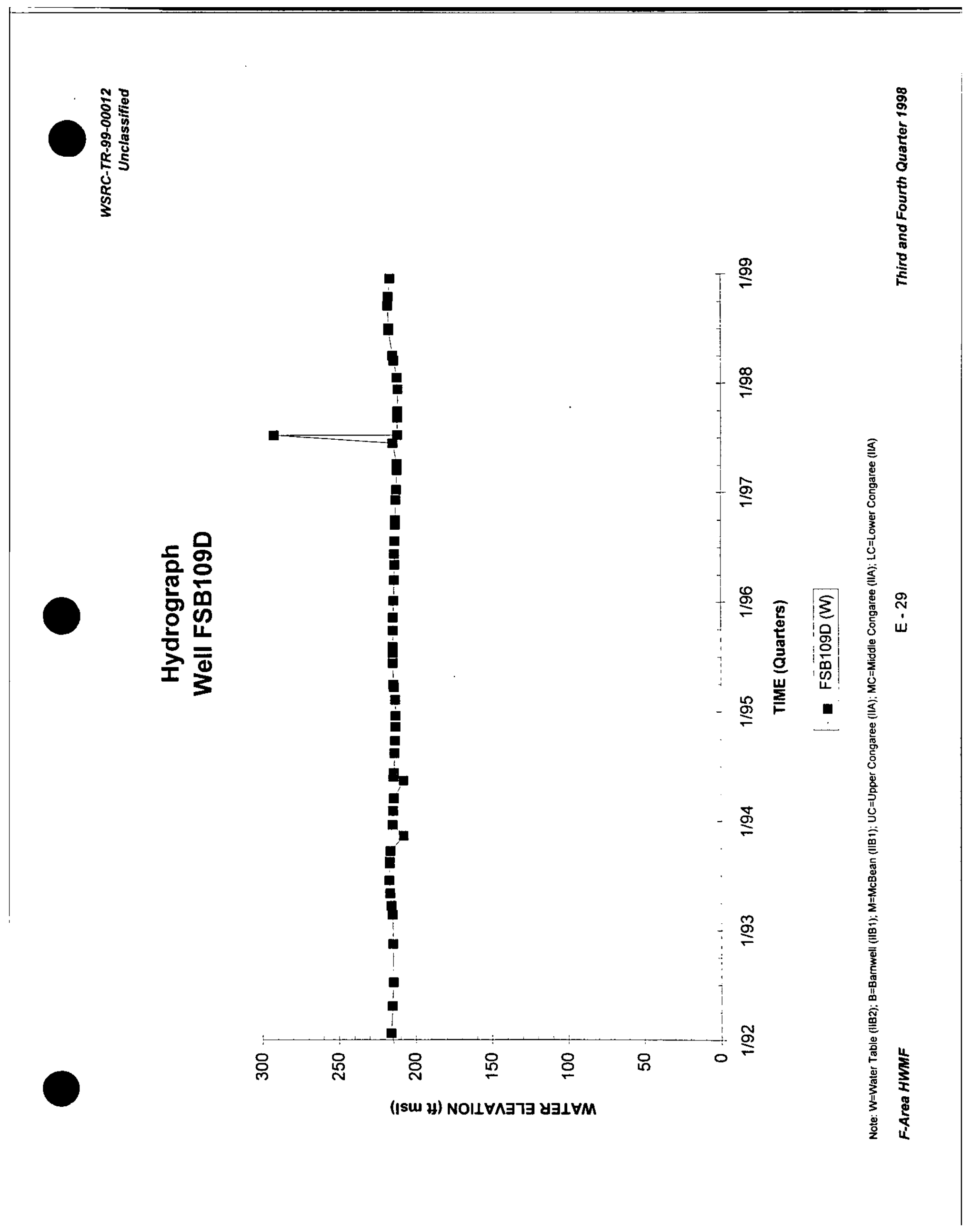




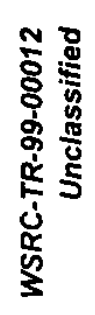

\%

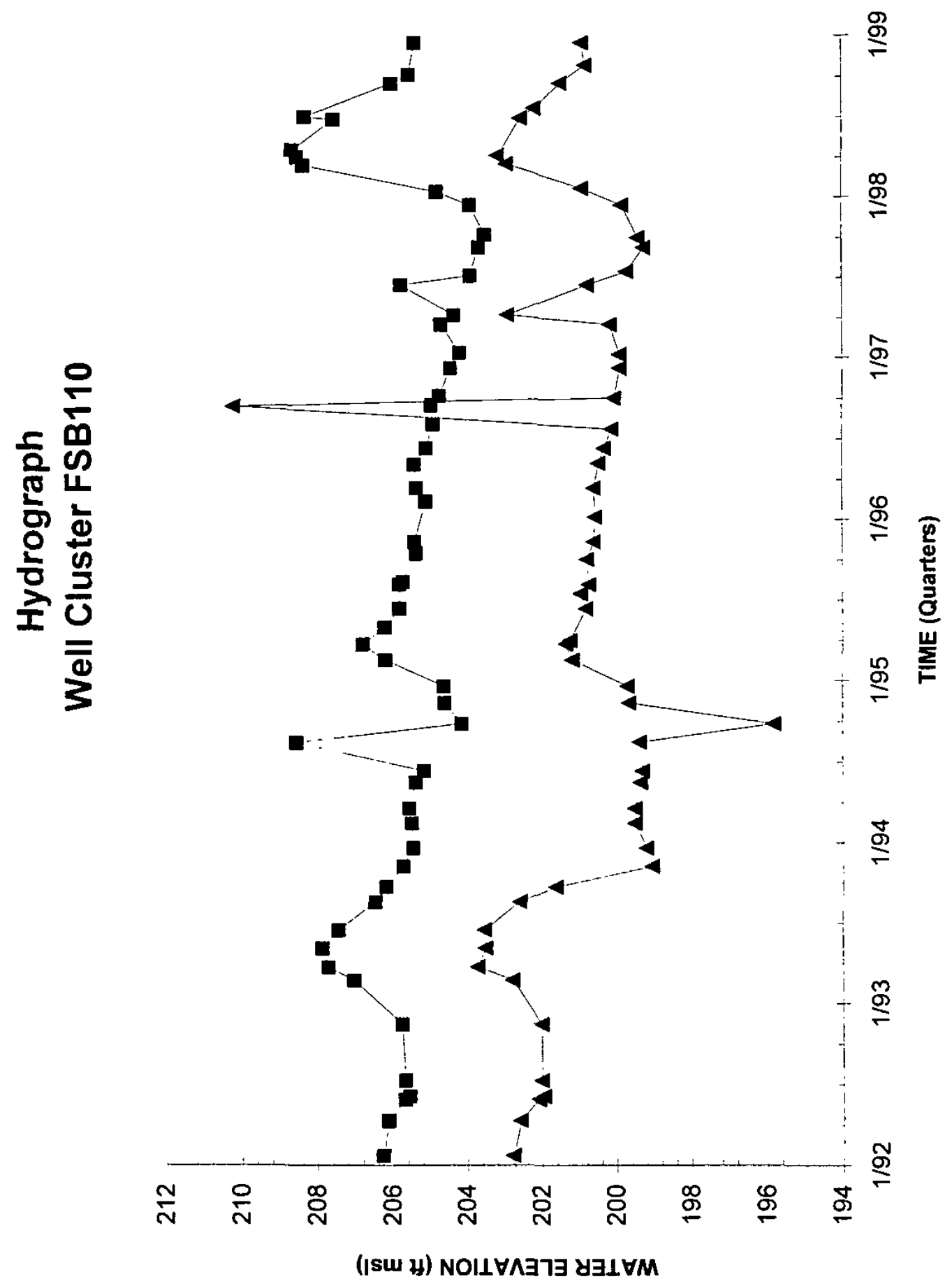




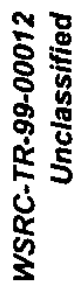

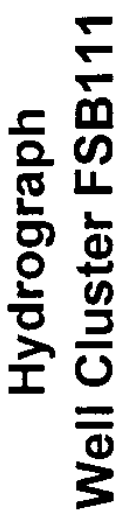

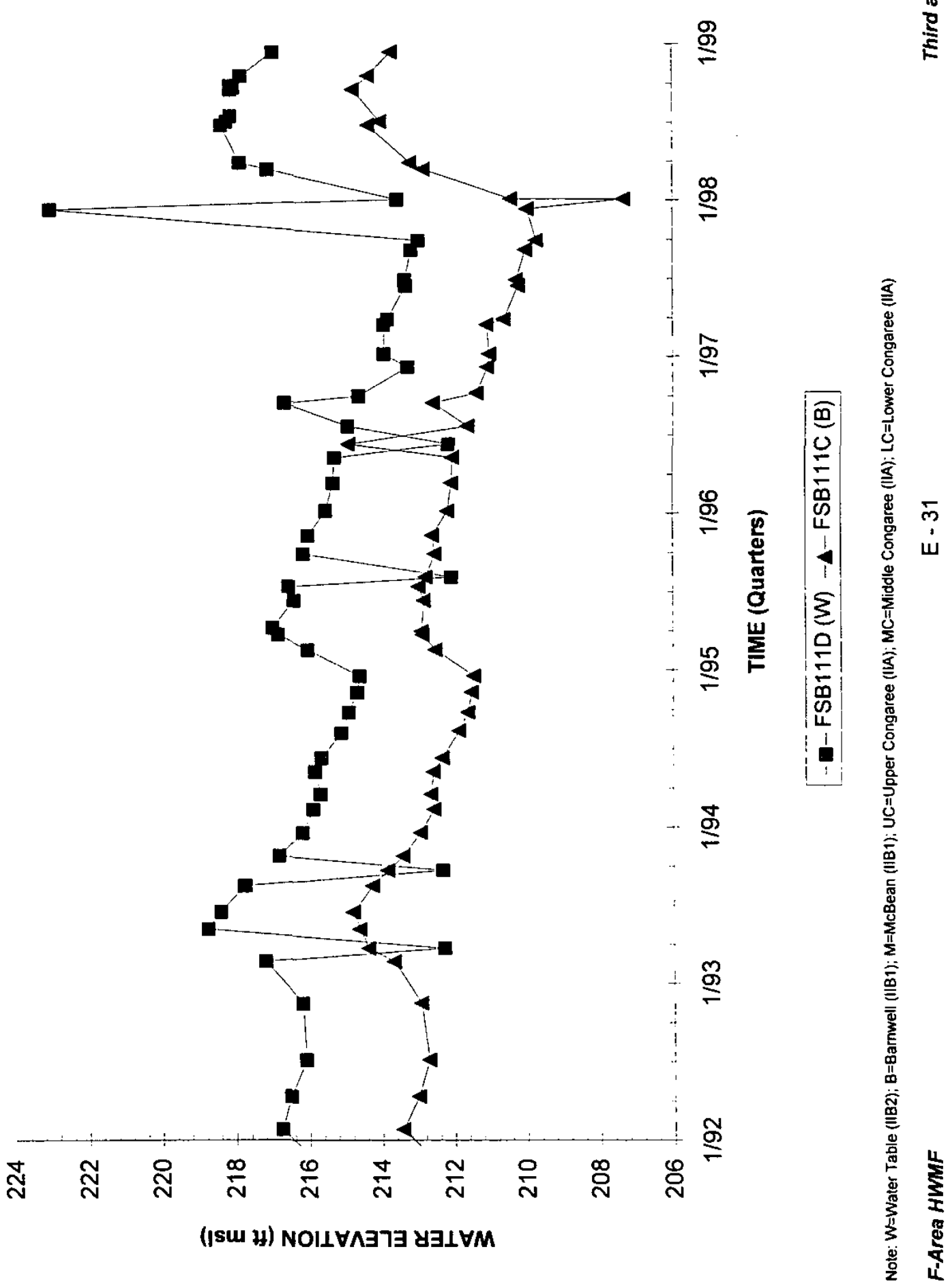




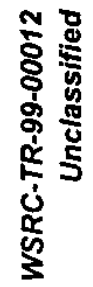
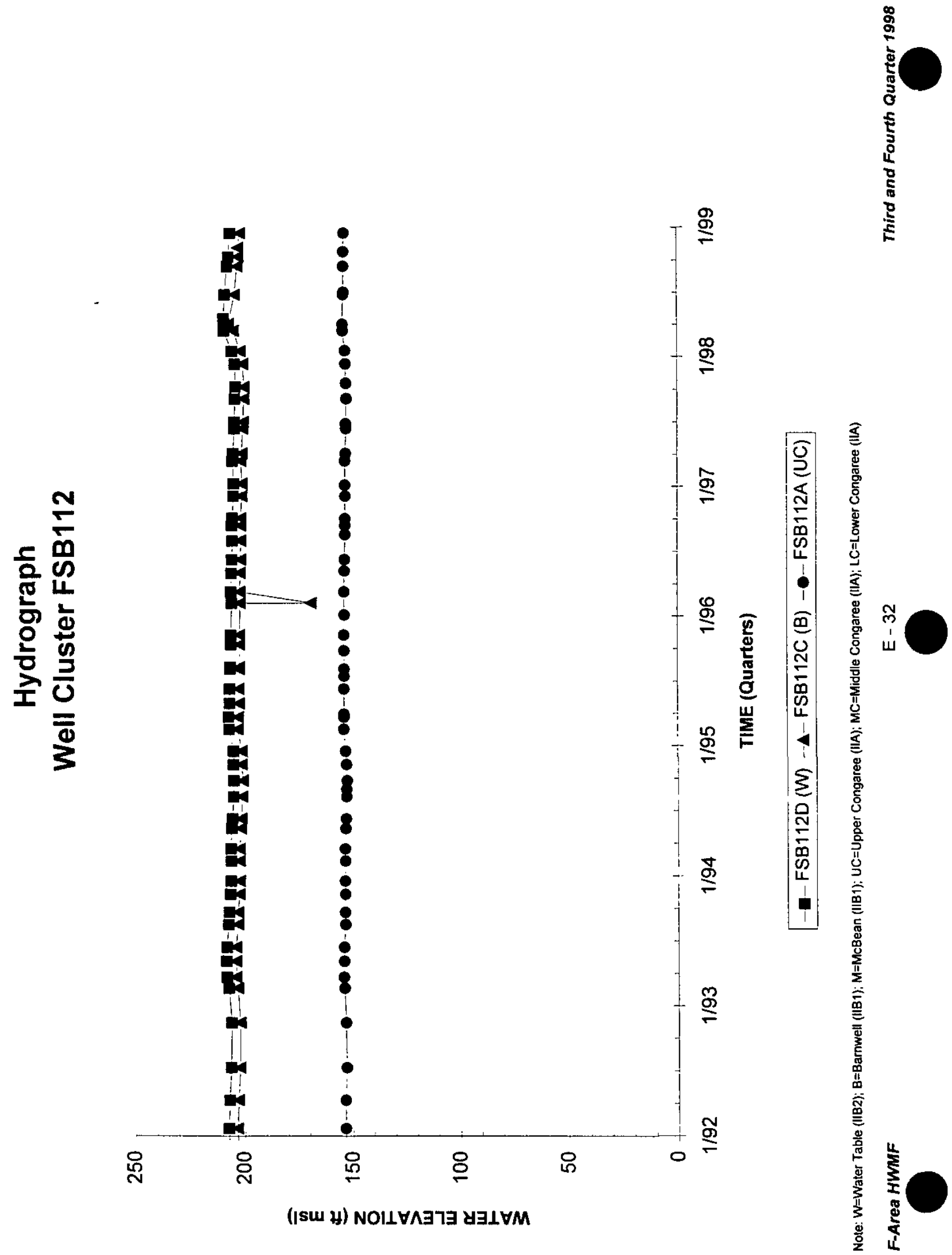


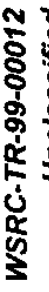

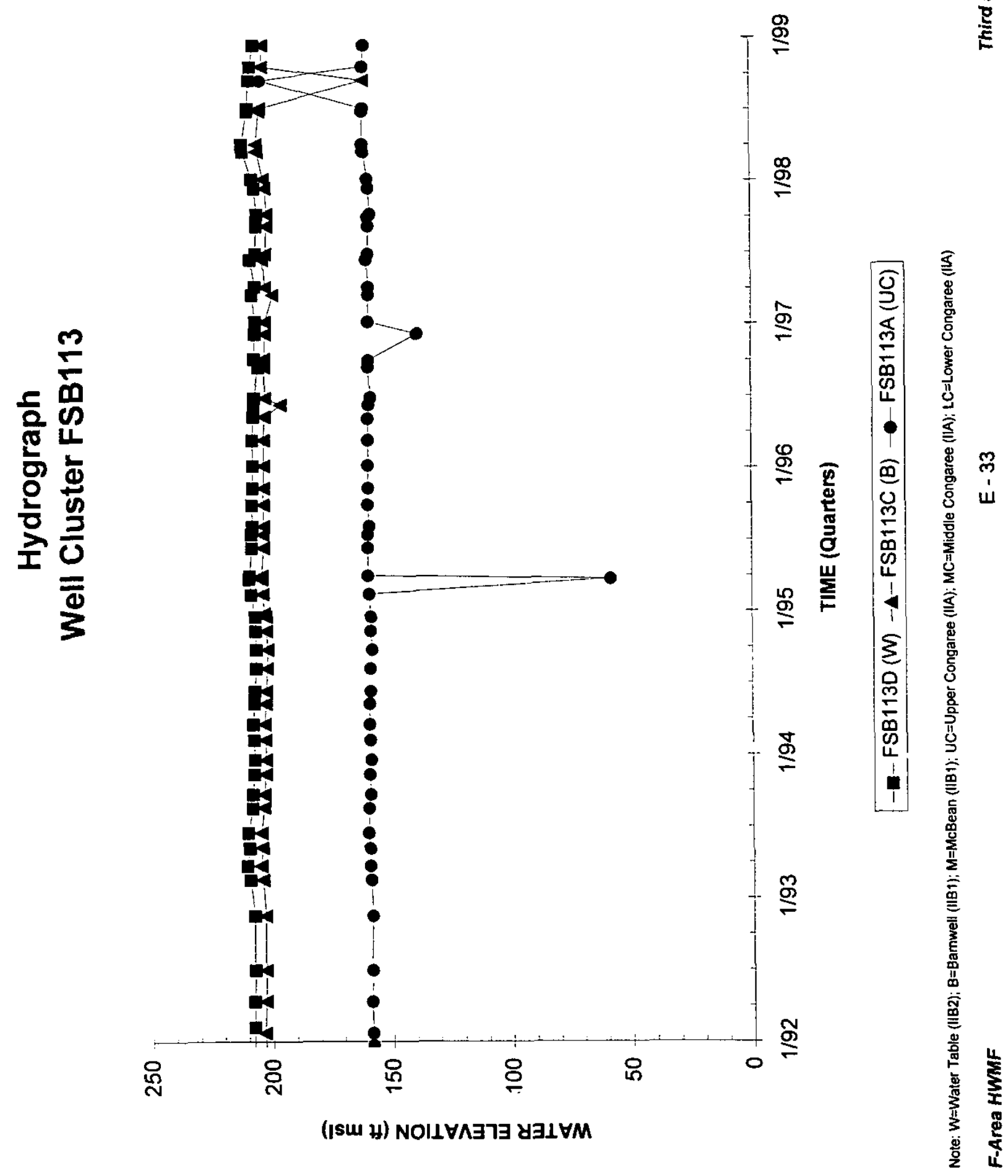



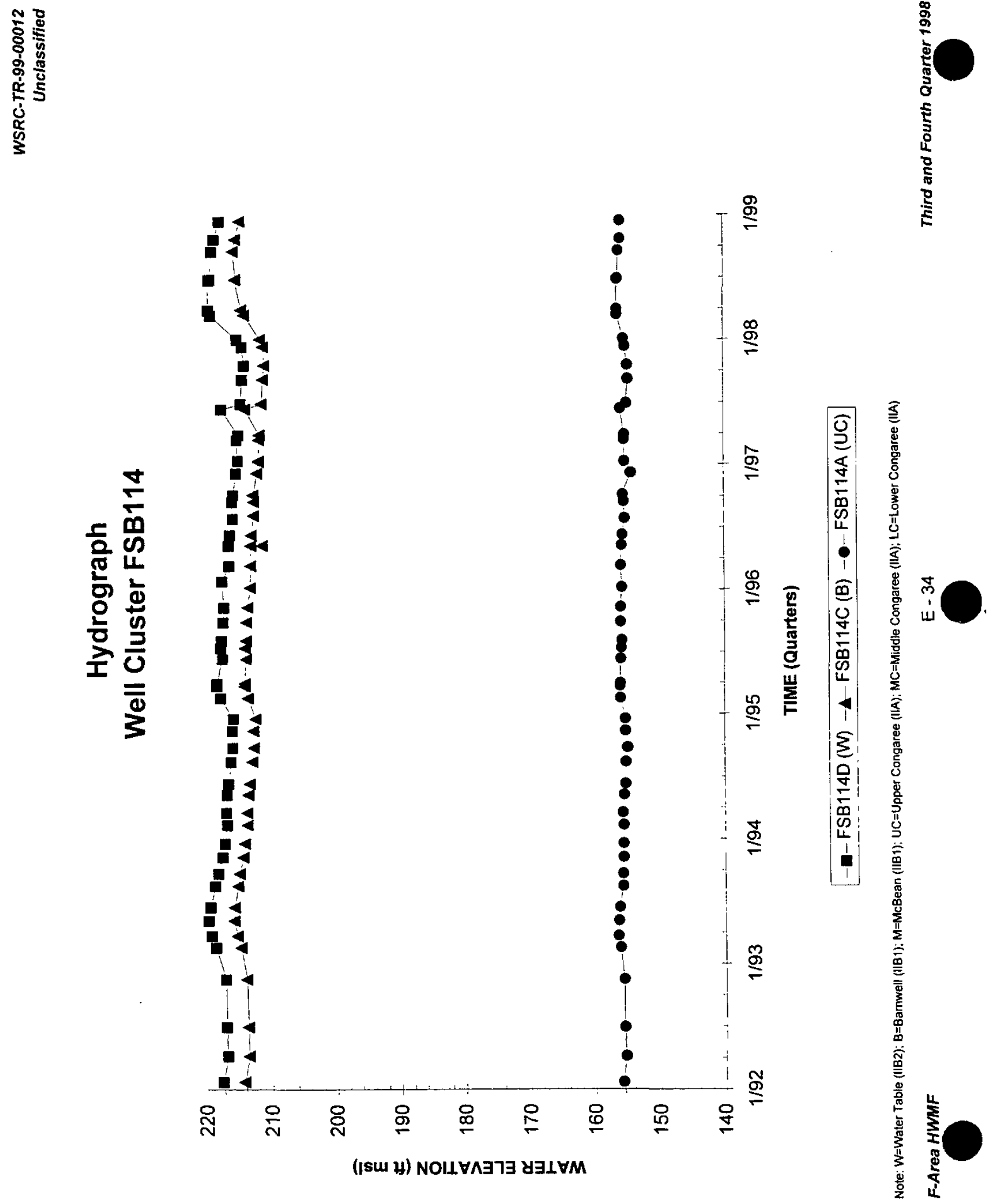


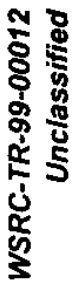
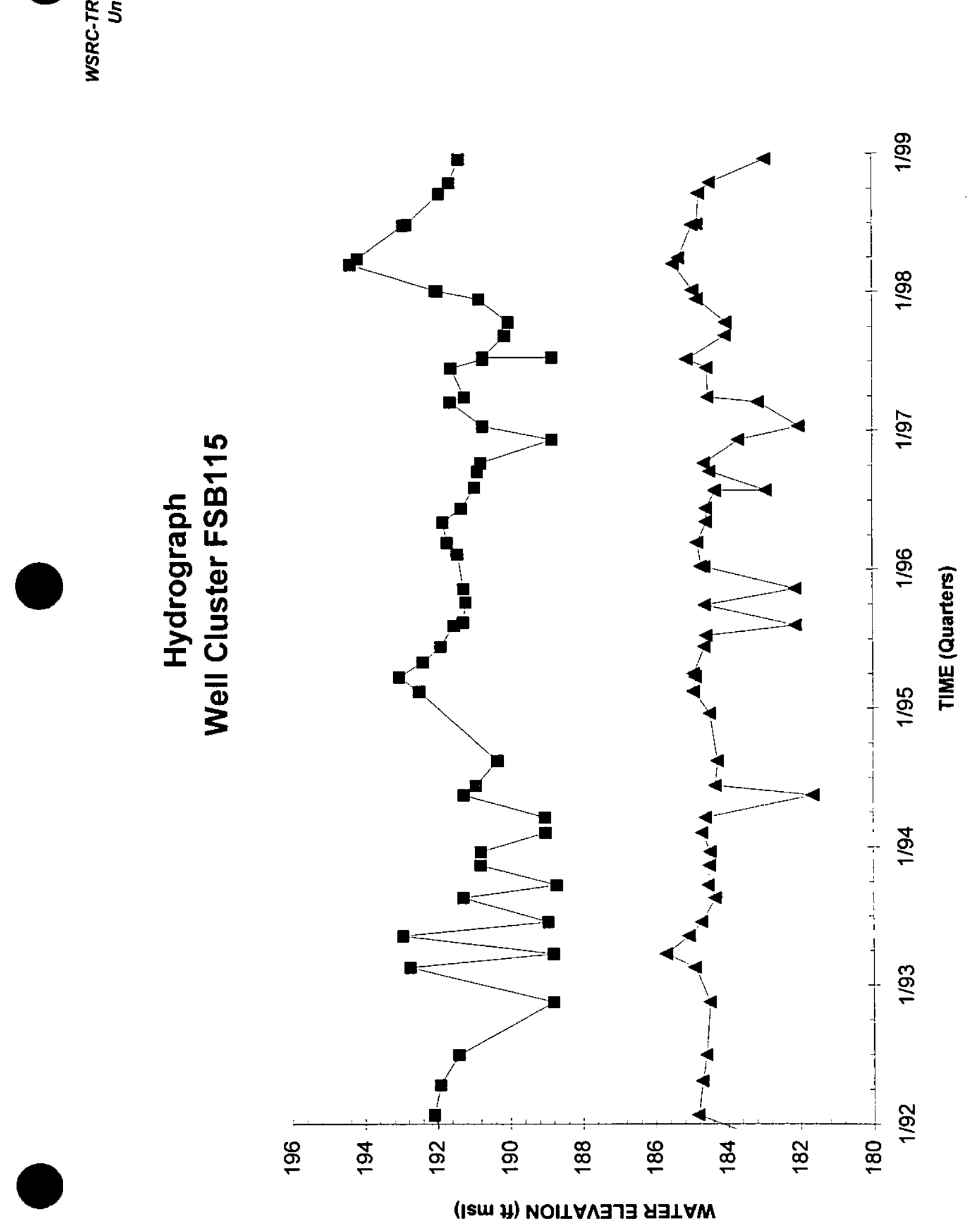


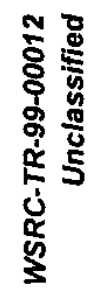

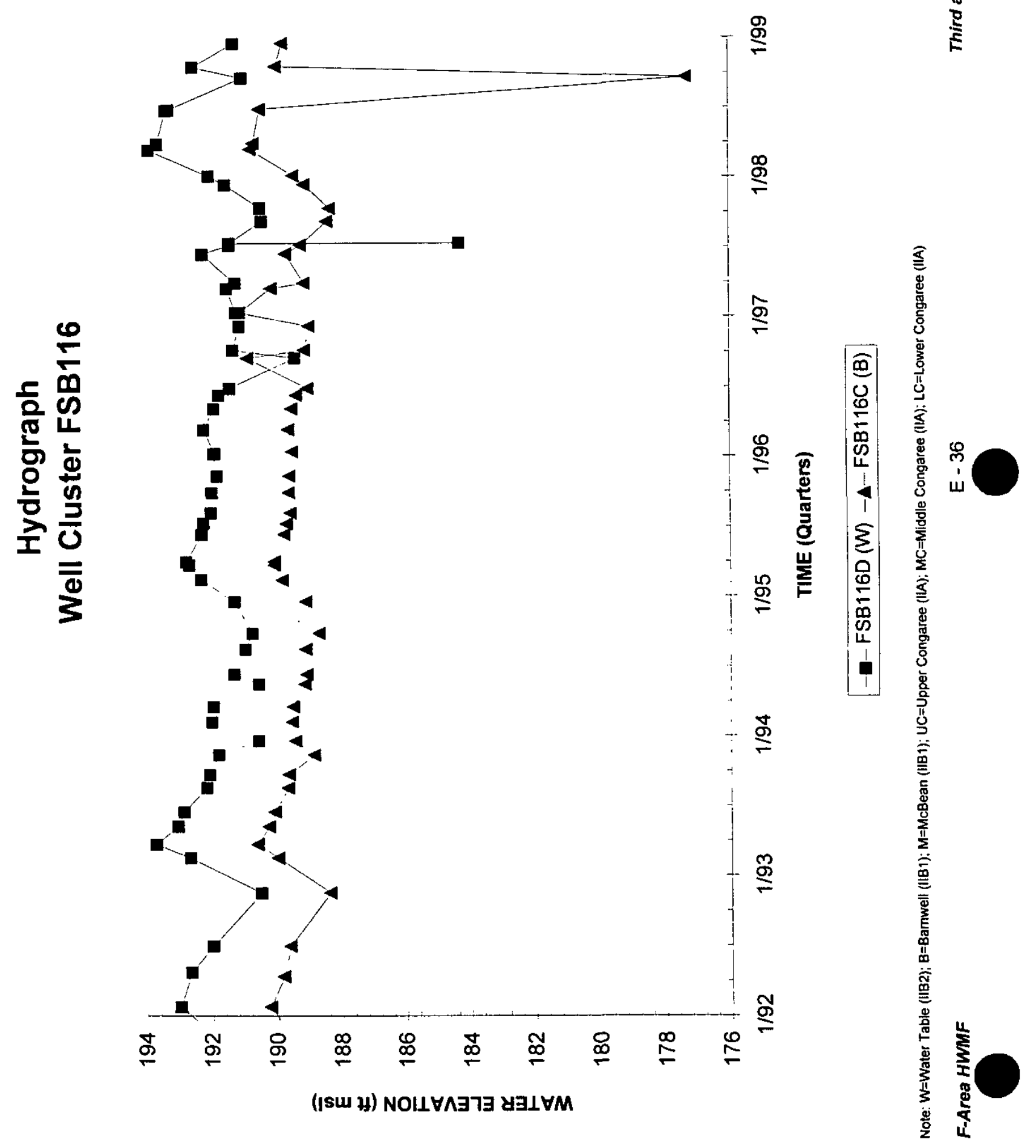



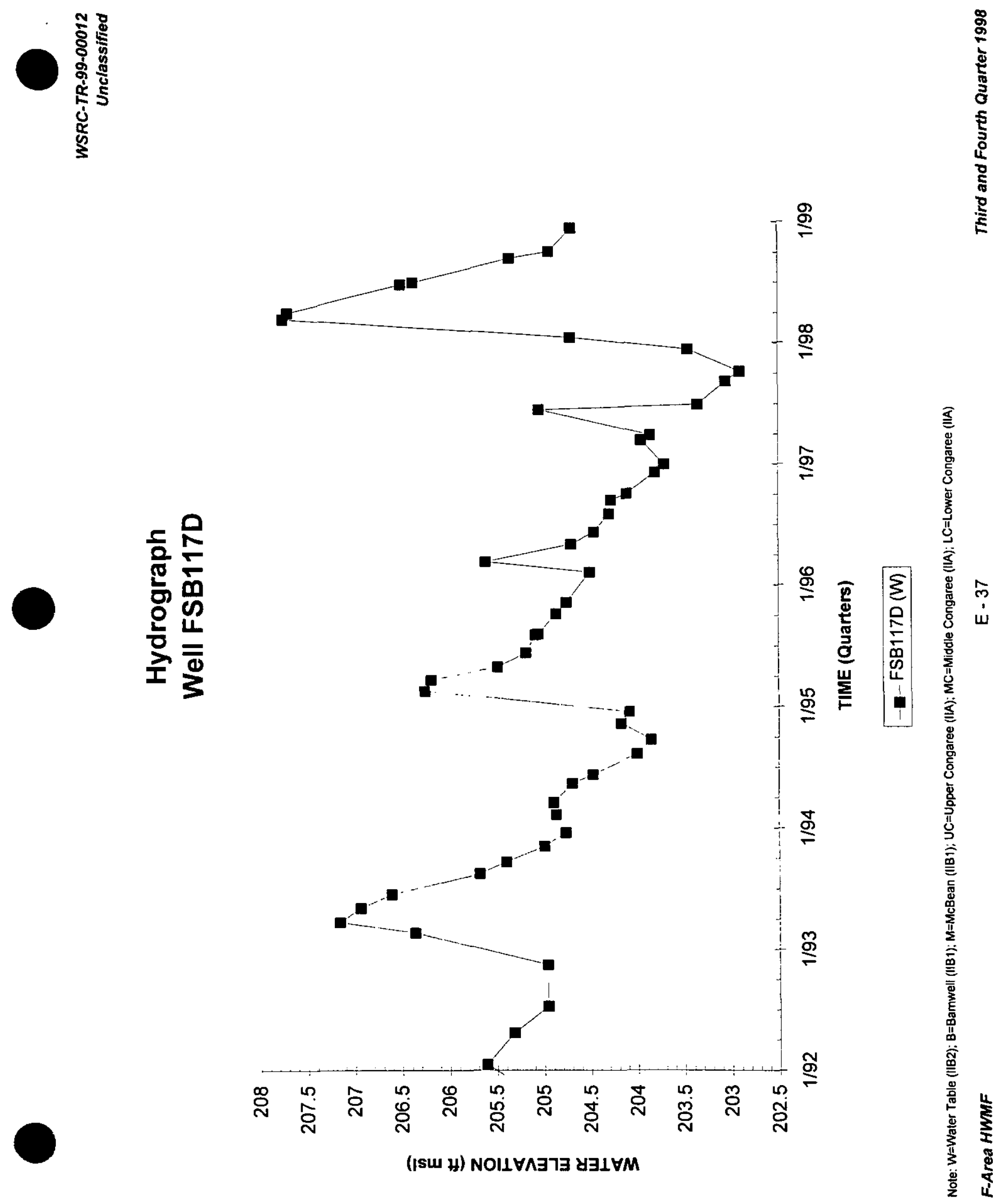


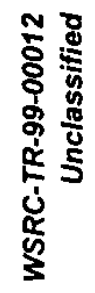
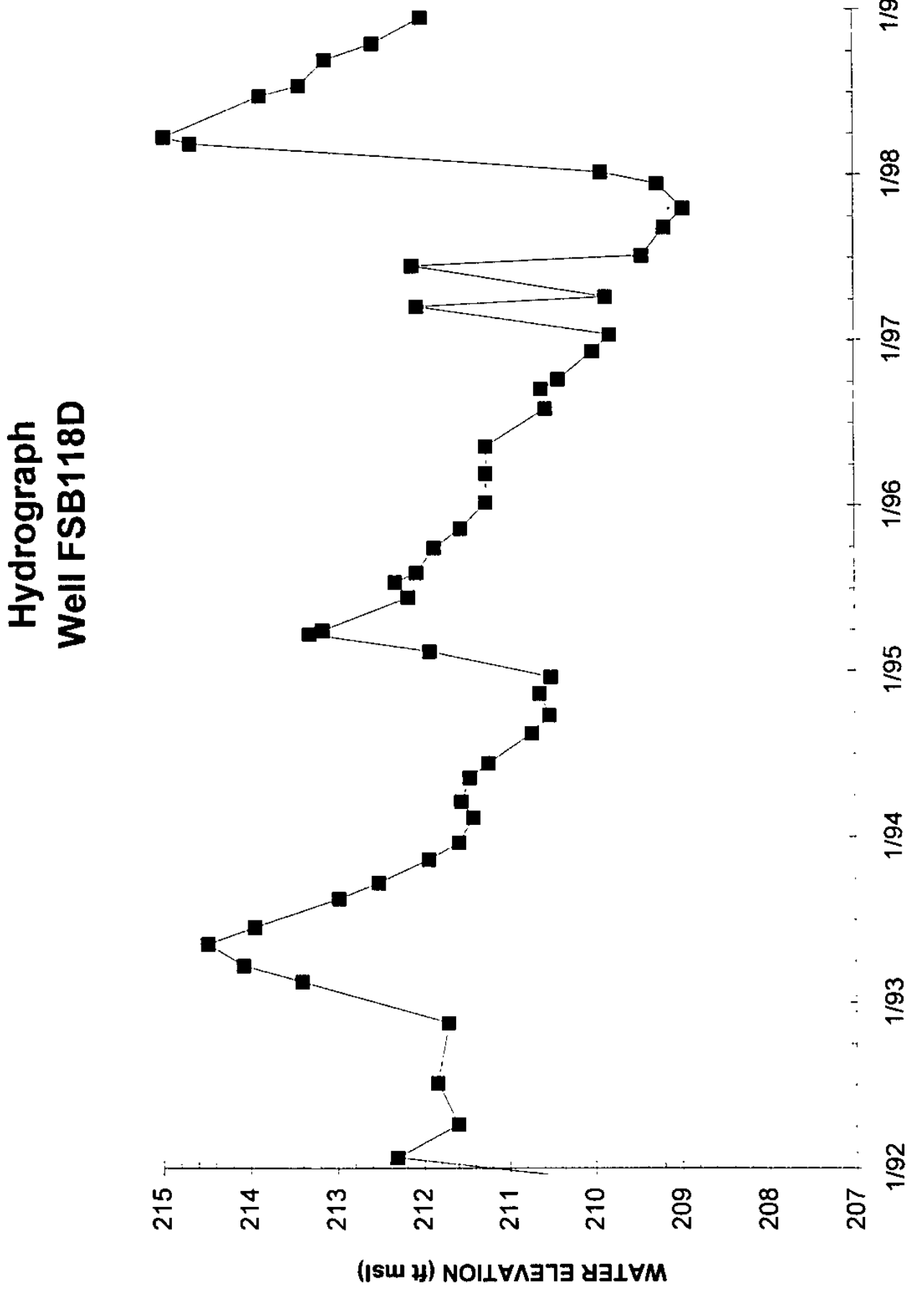


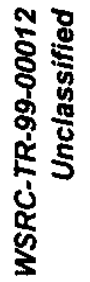

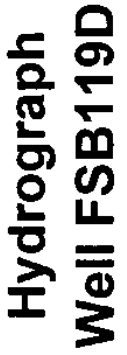

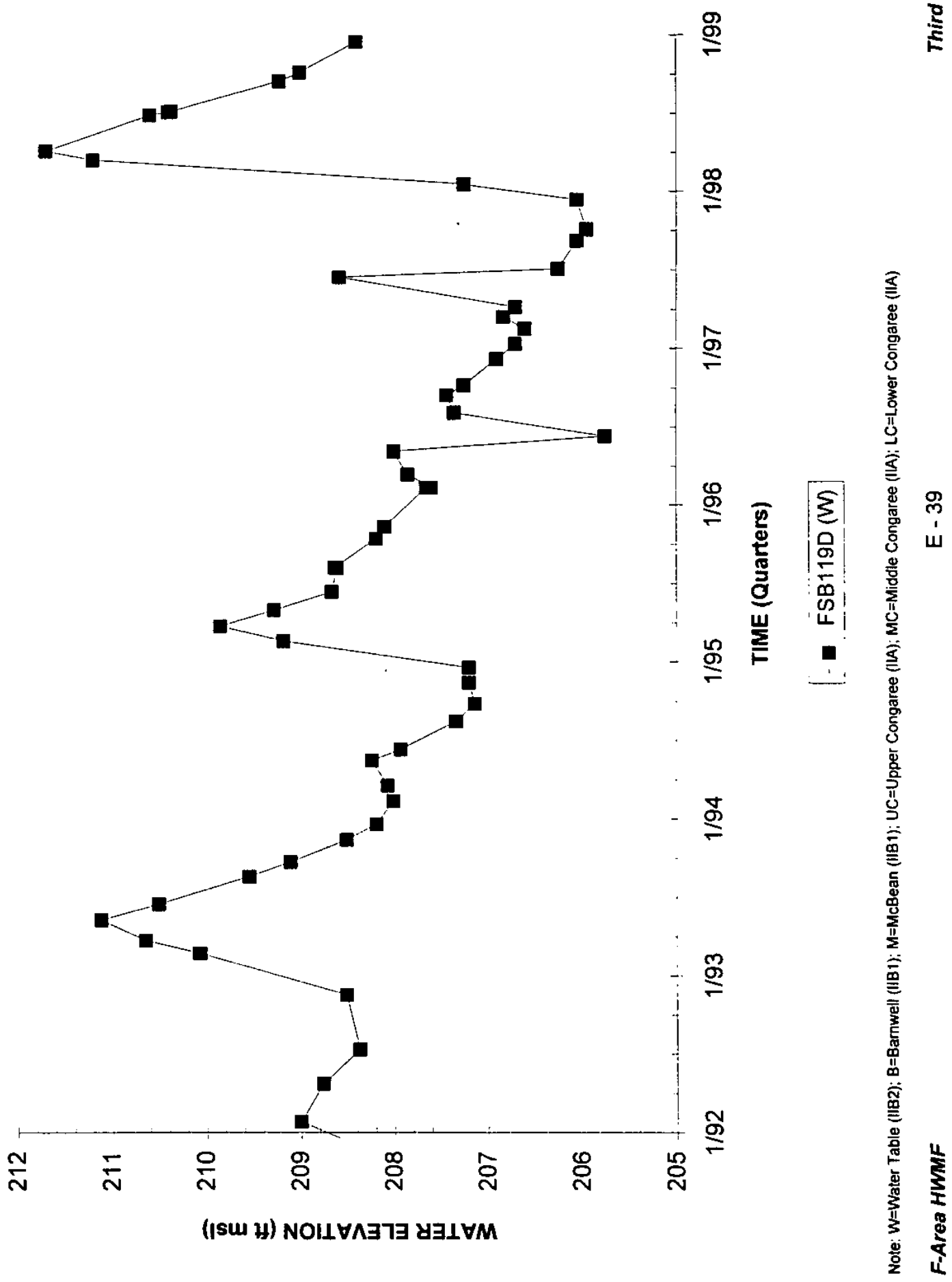




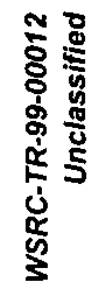

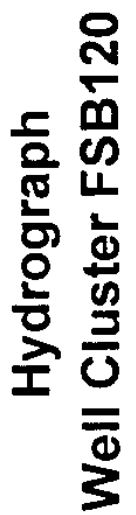
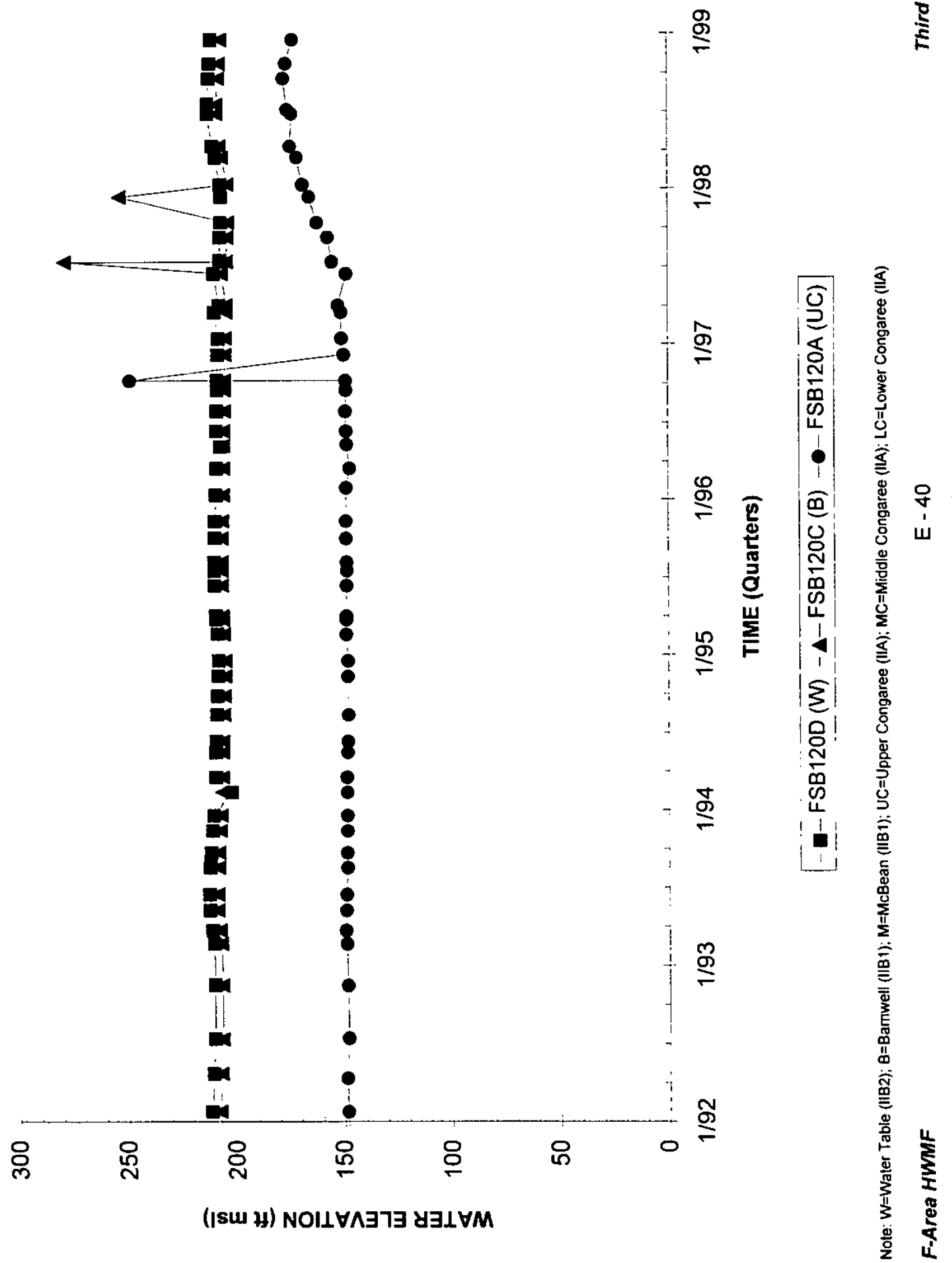


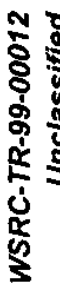
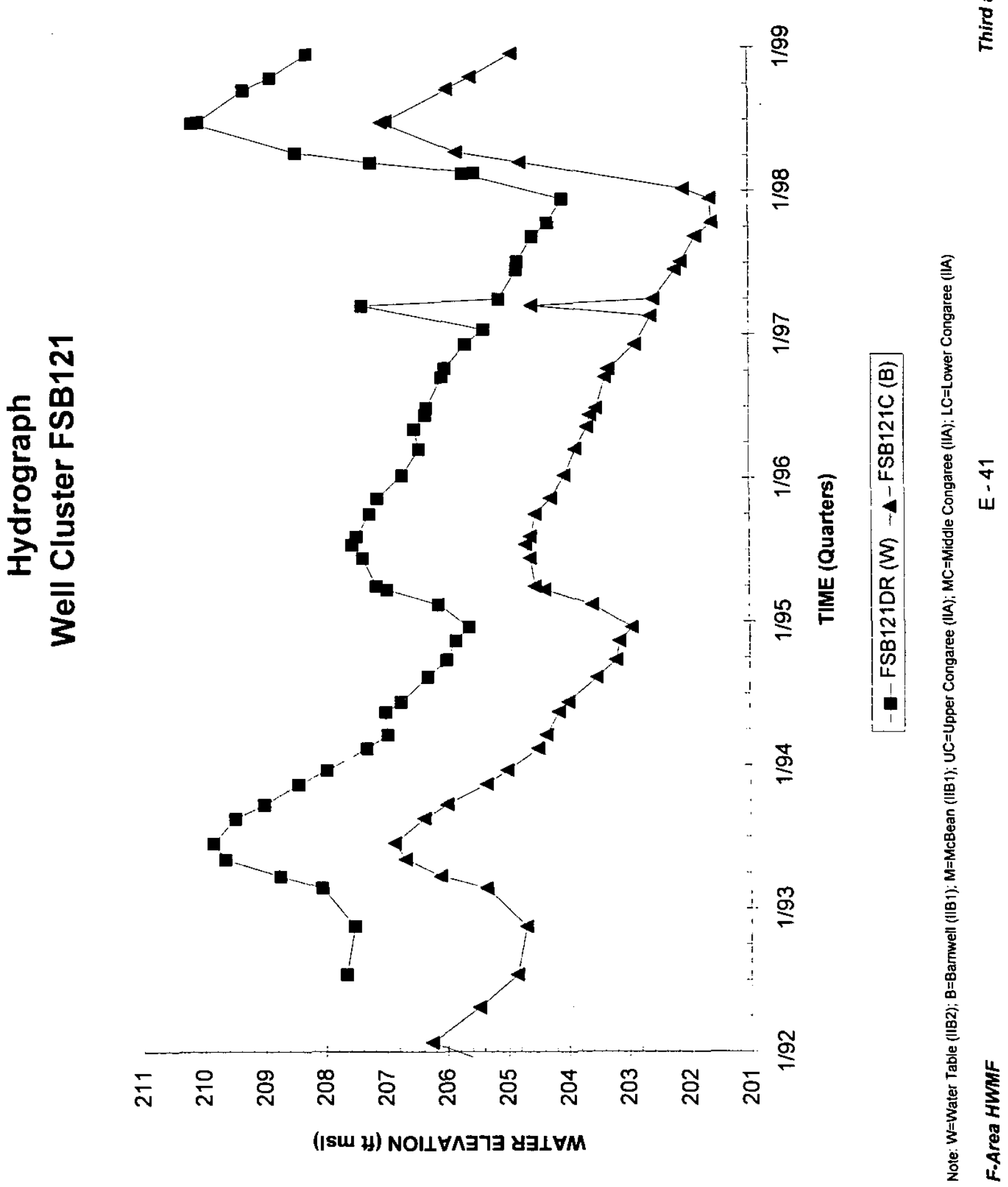


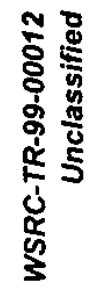
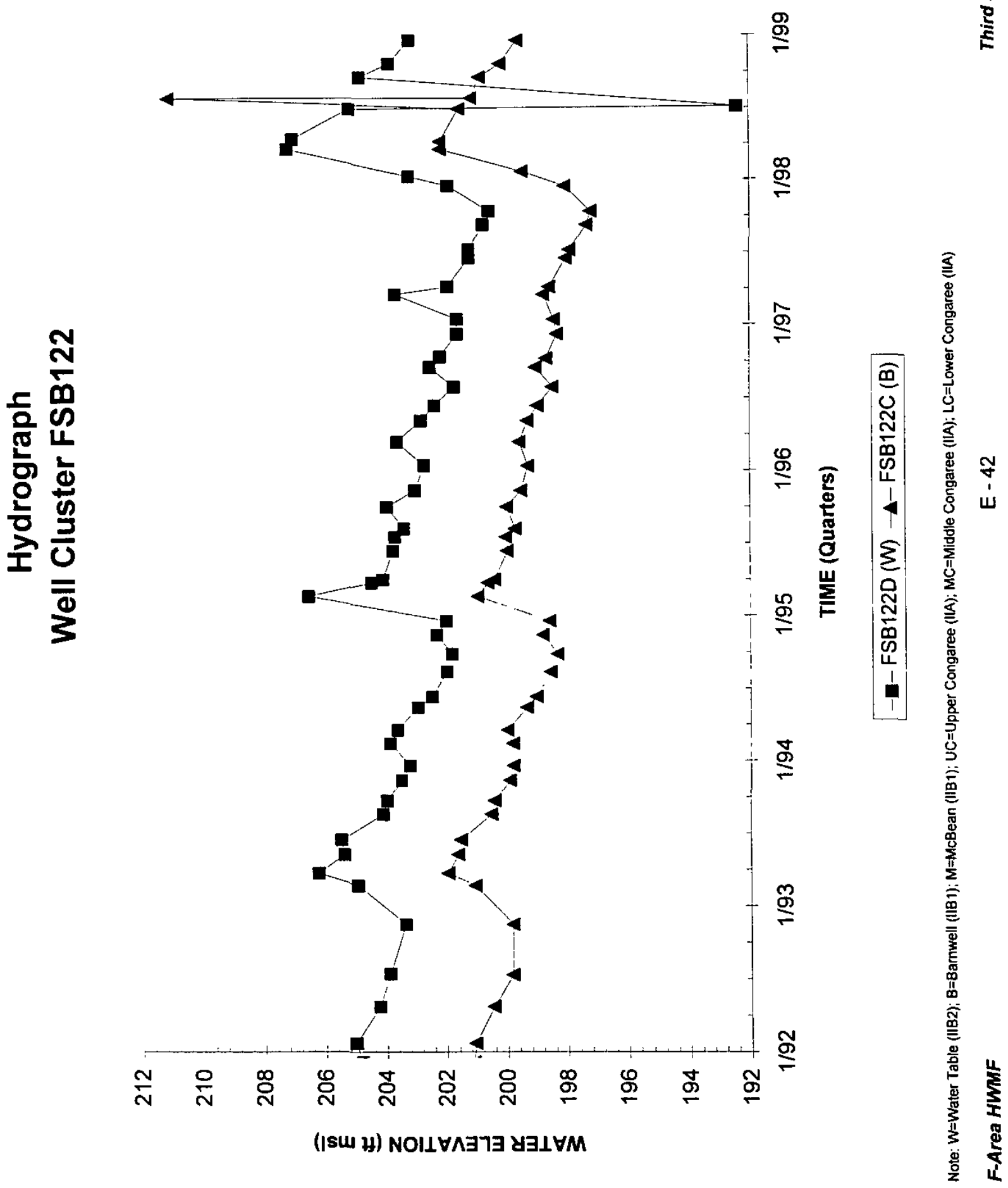


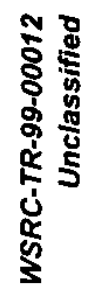
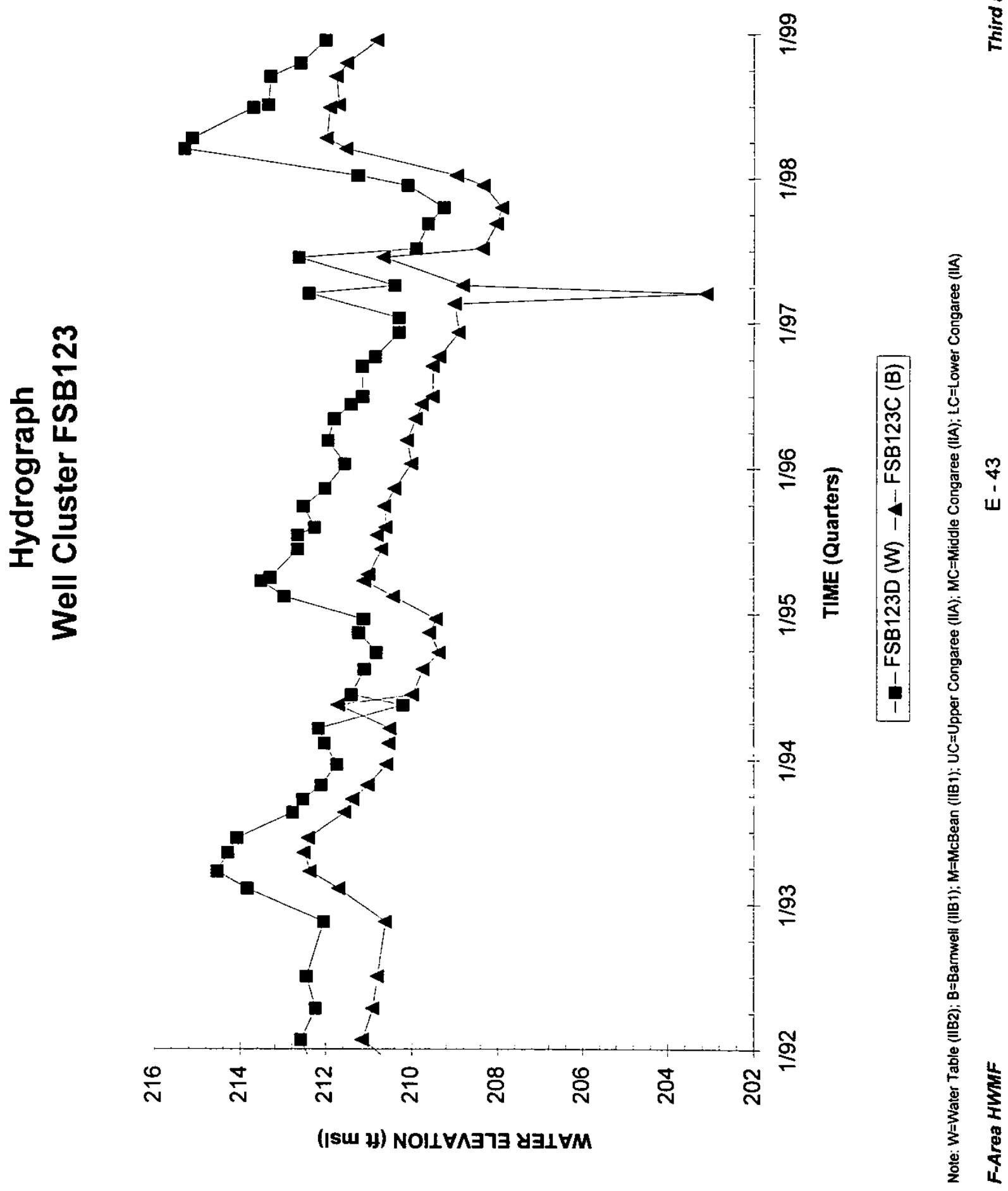

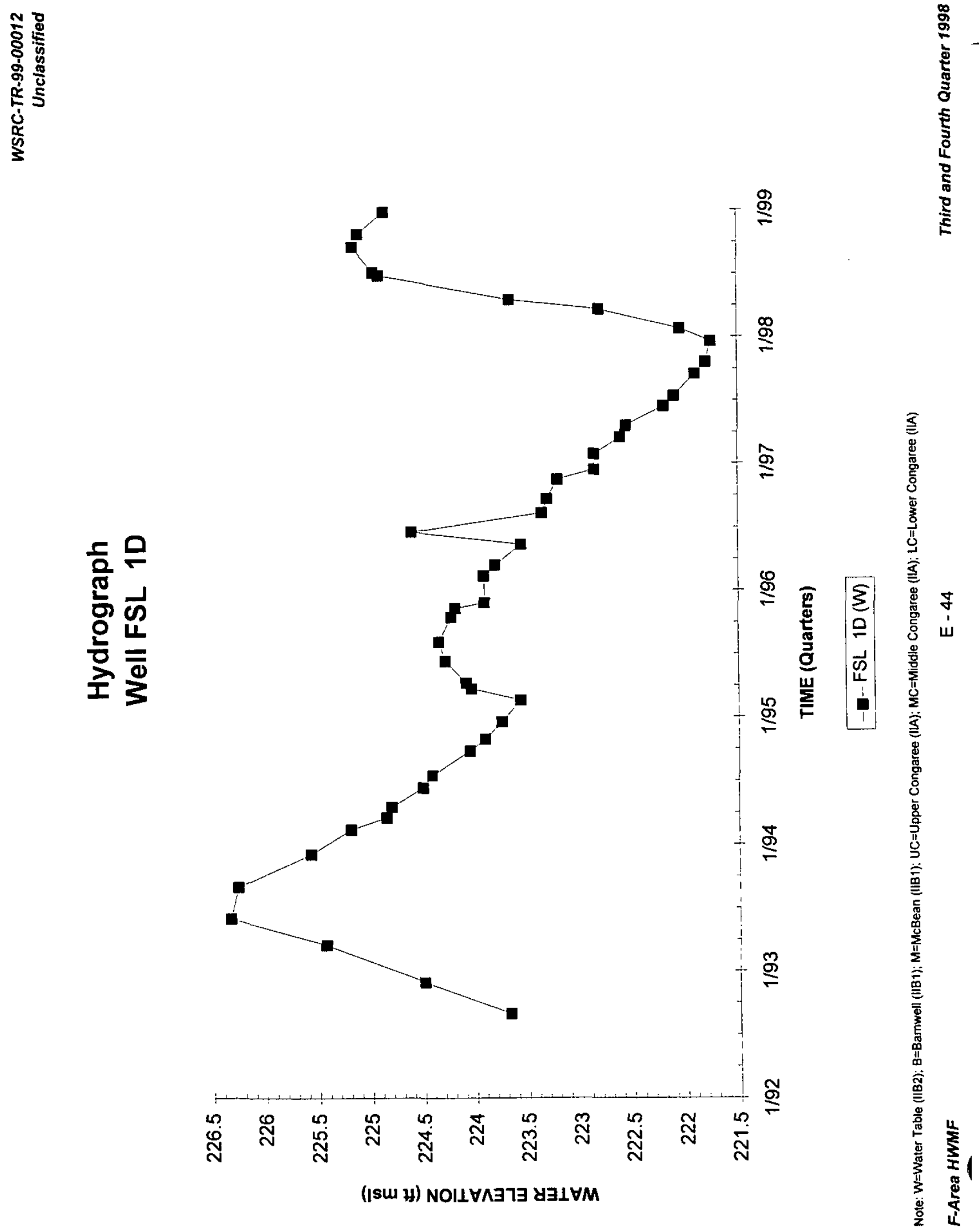

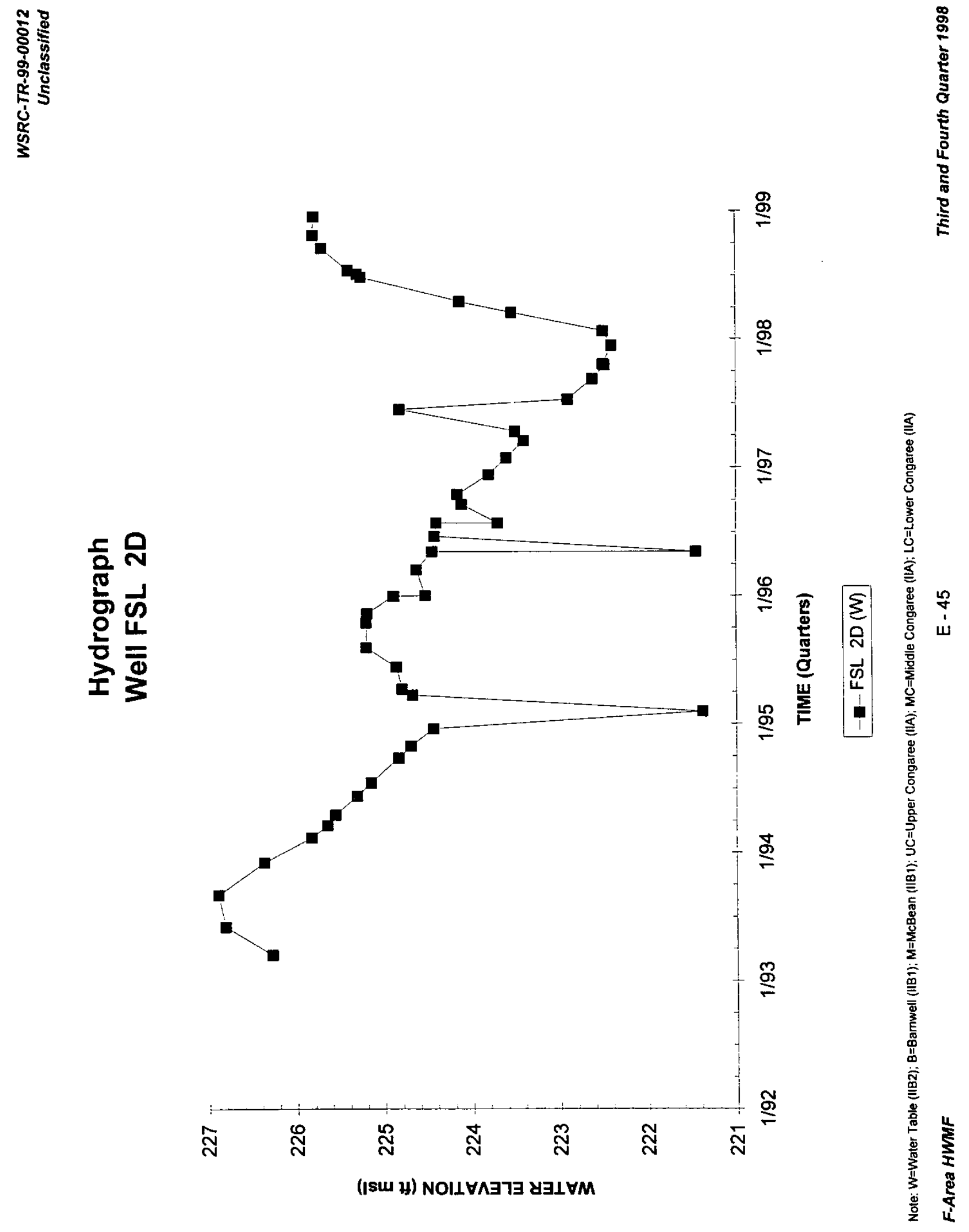

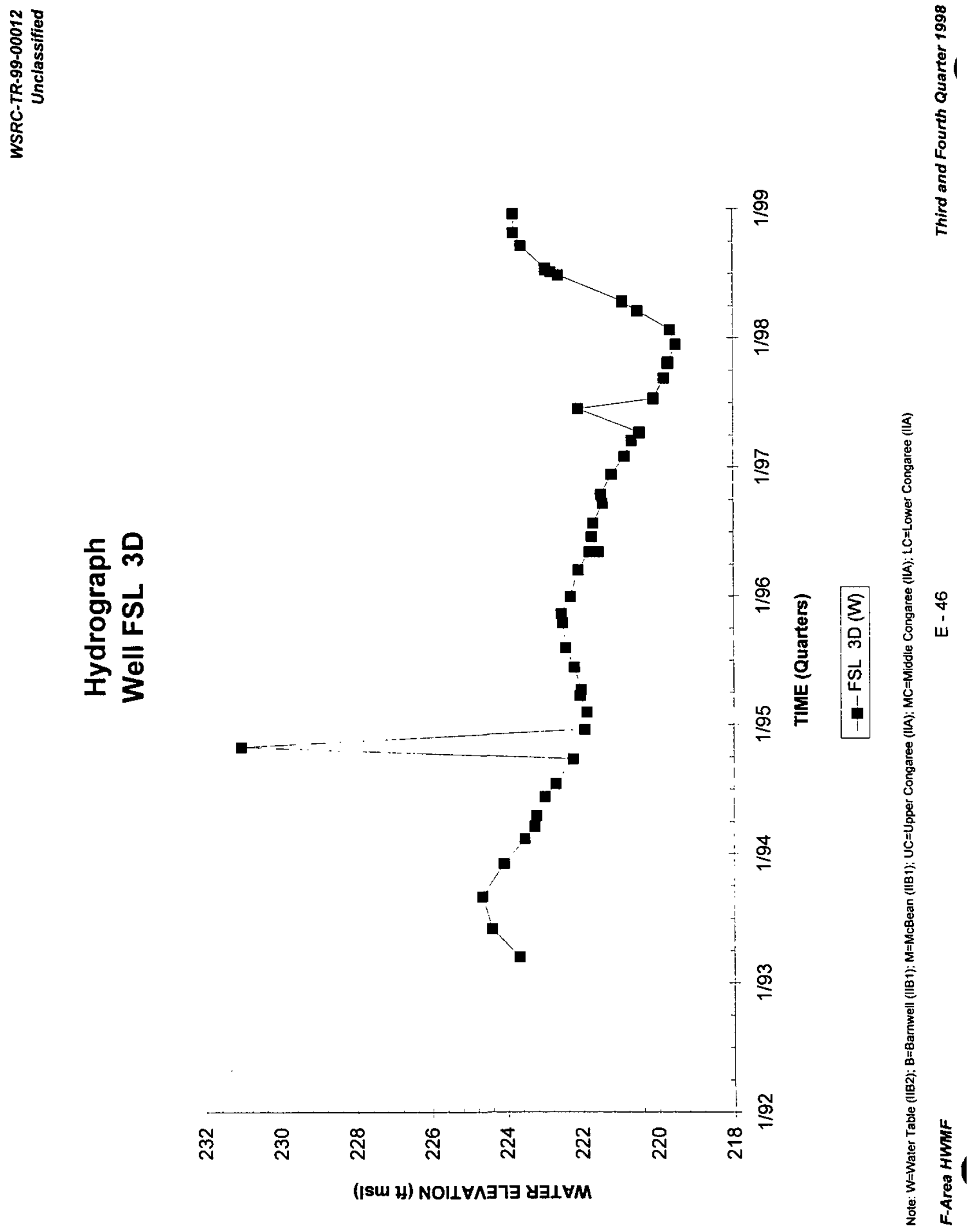

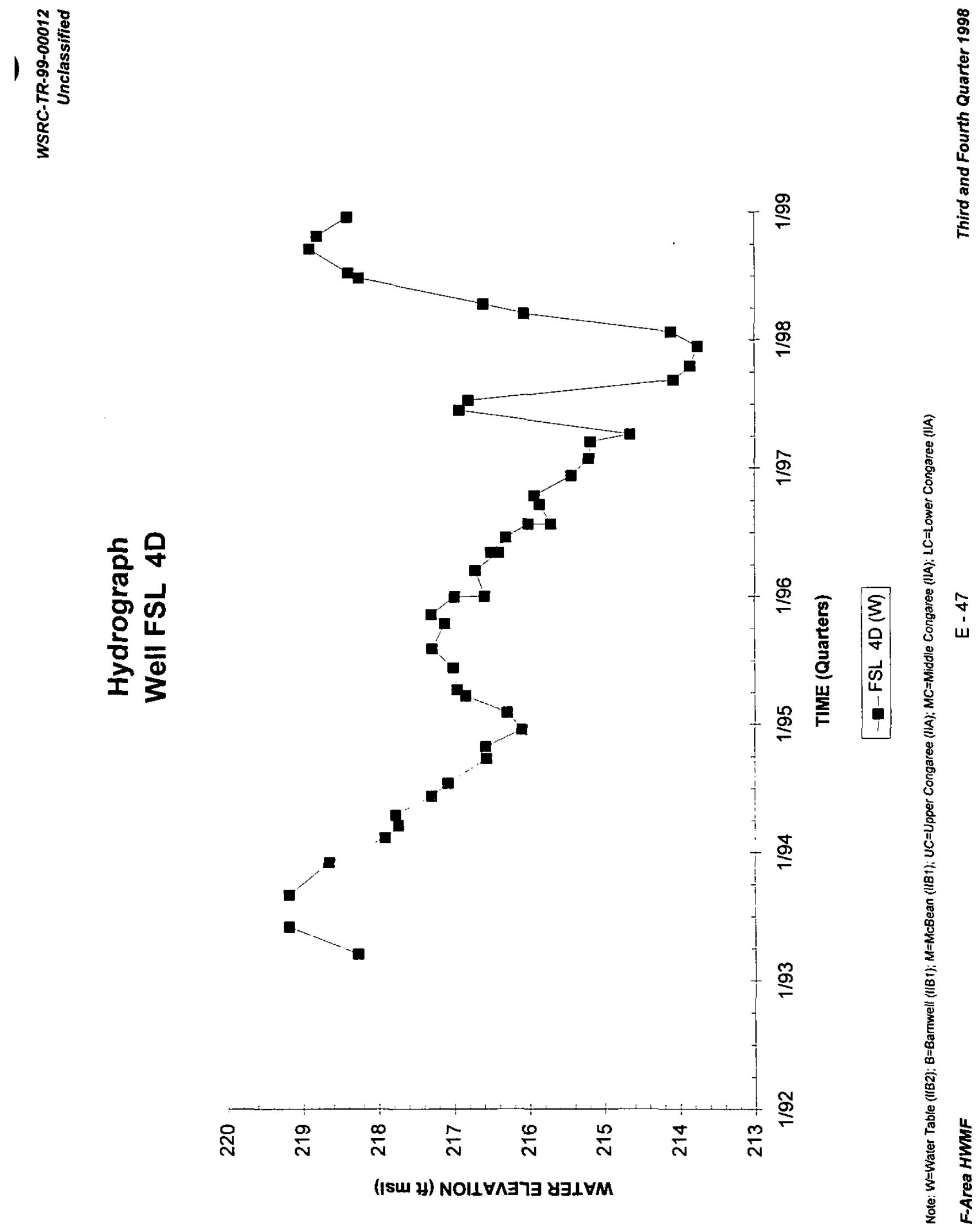

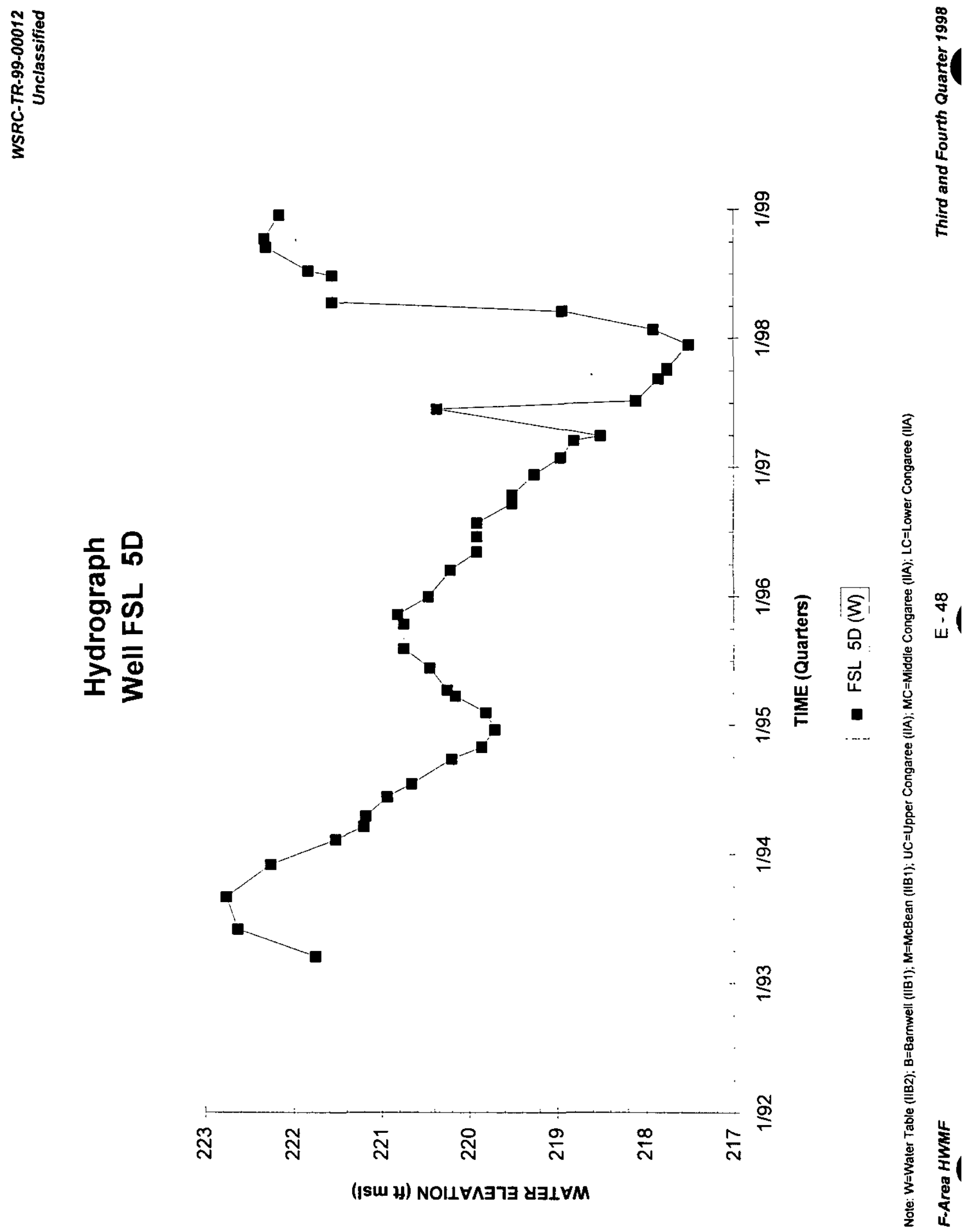

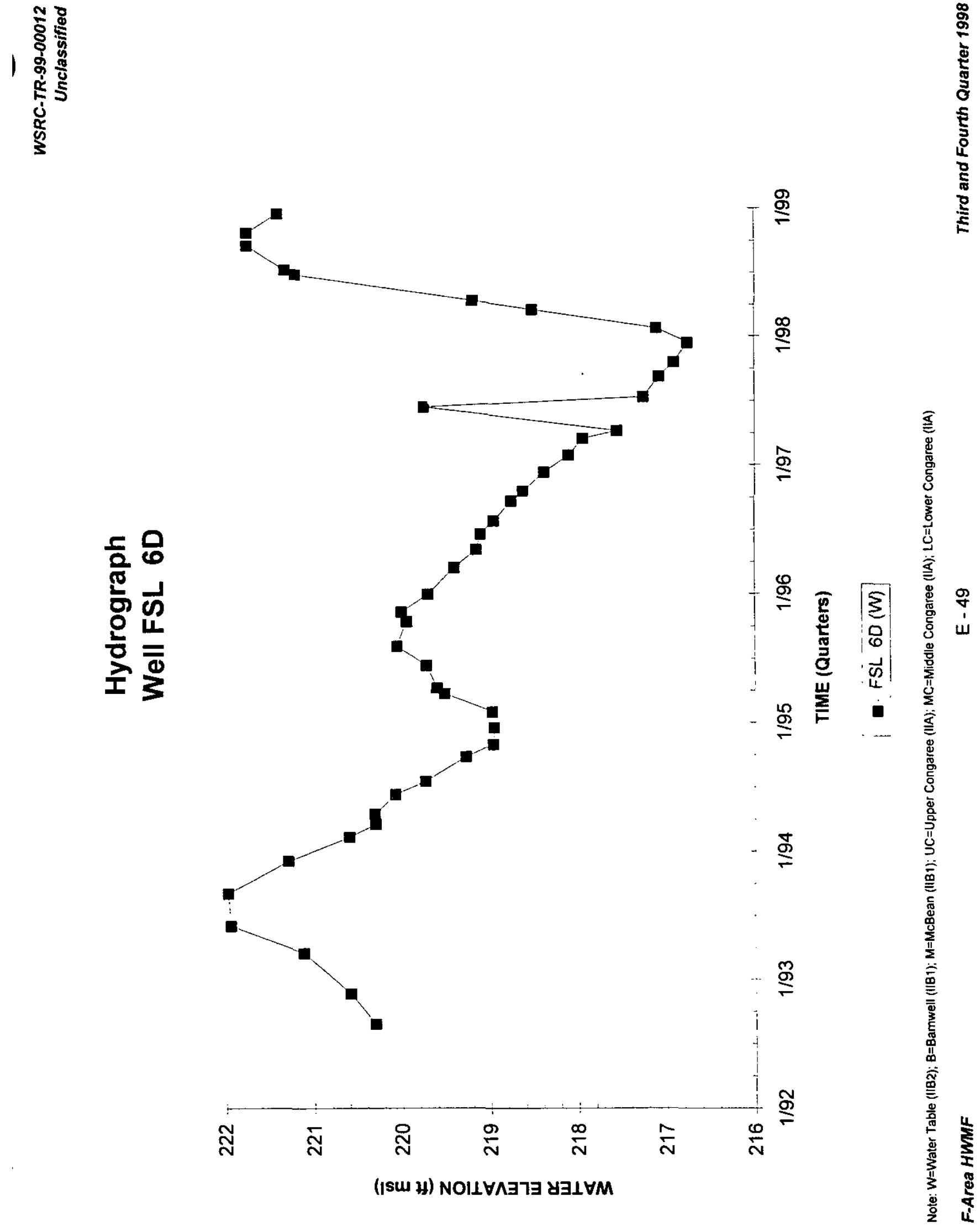


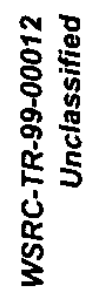

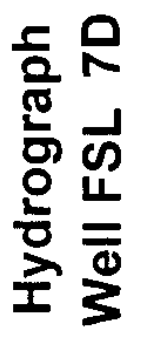

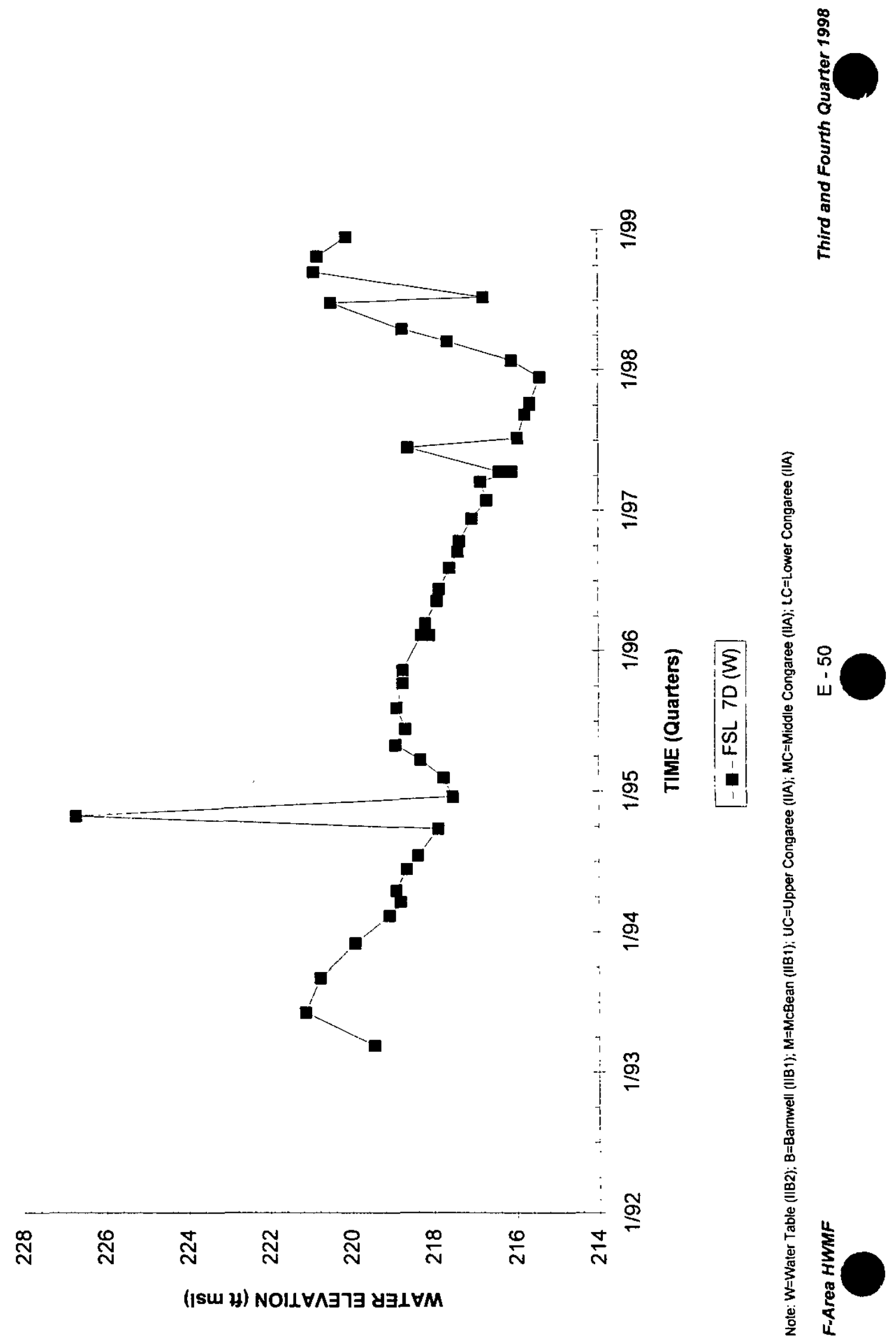




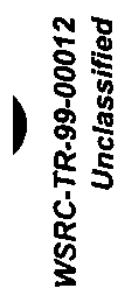

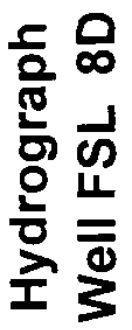

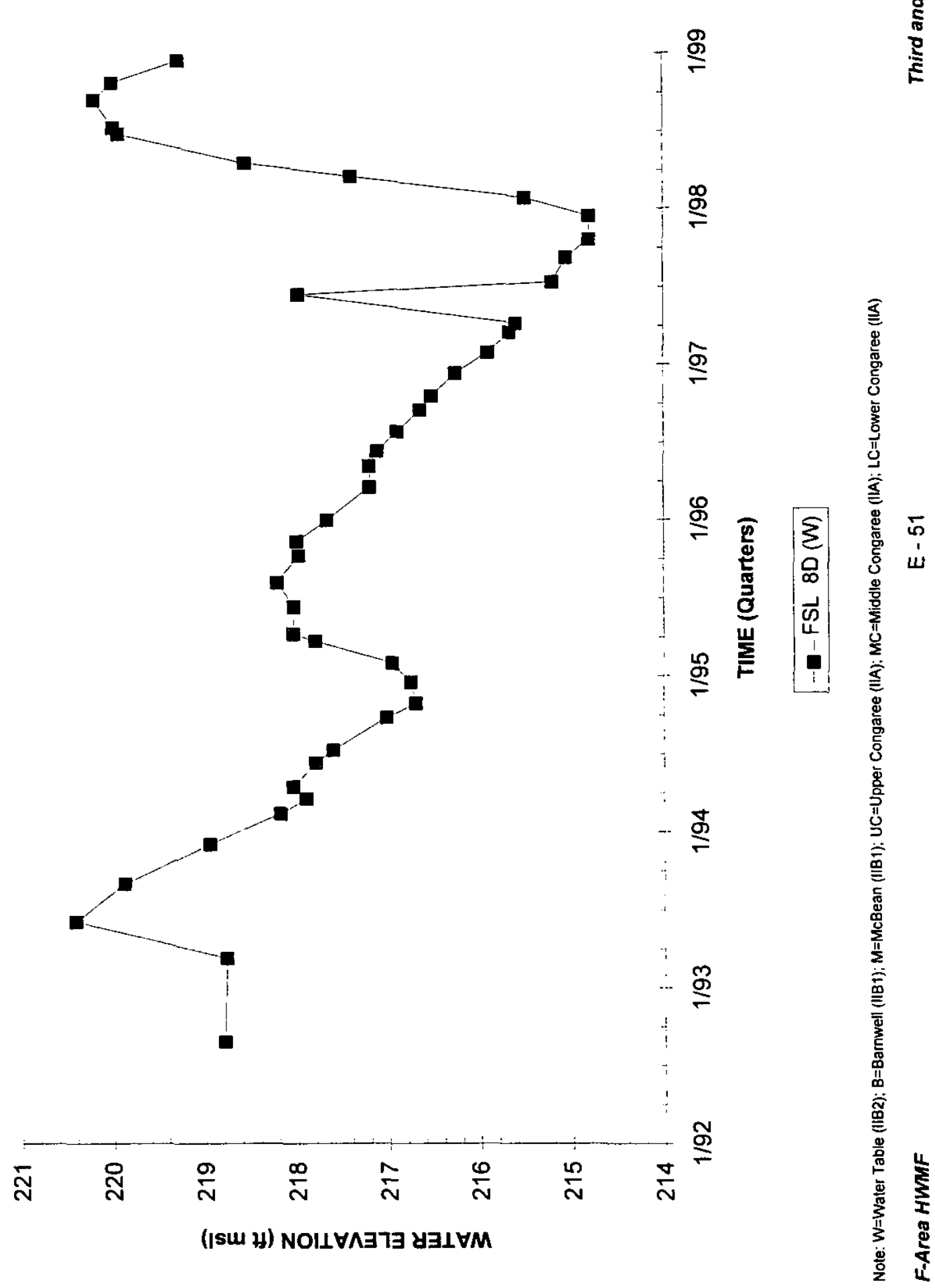



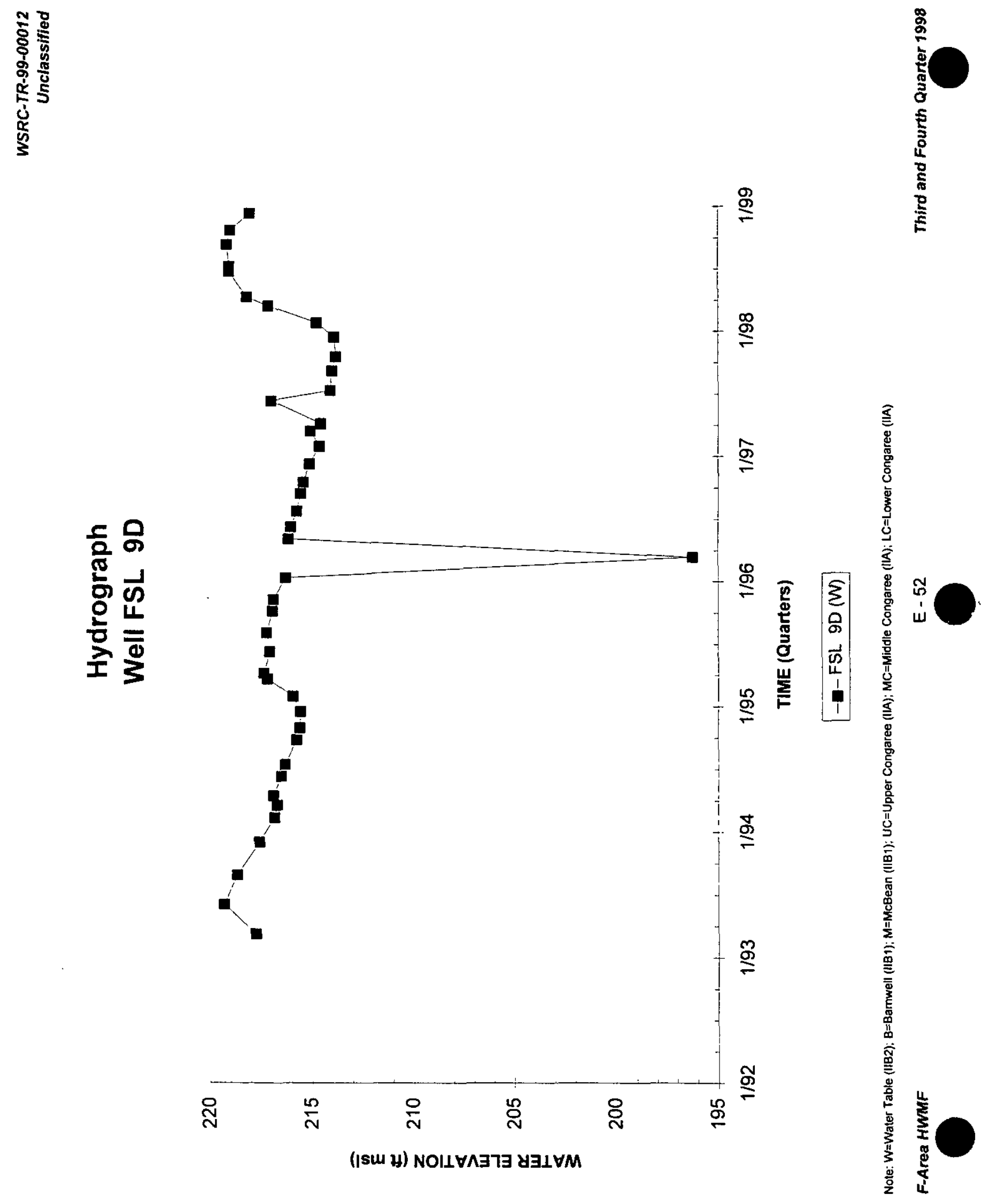


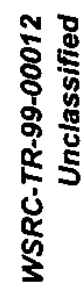

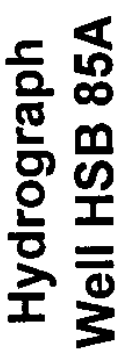
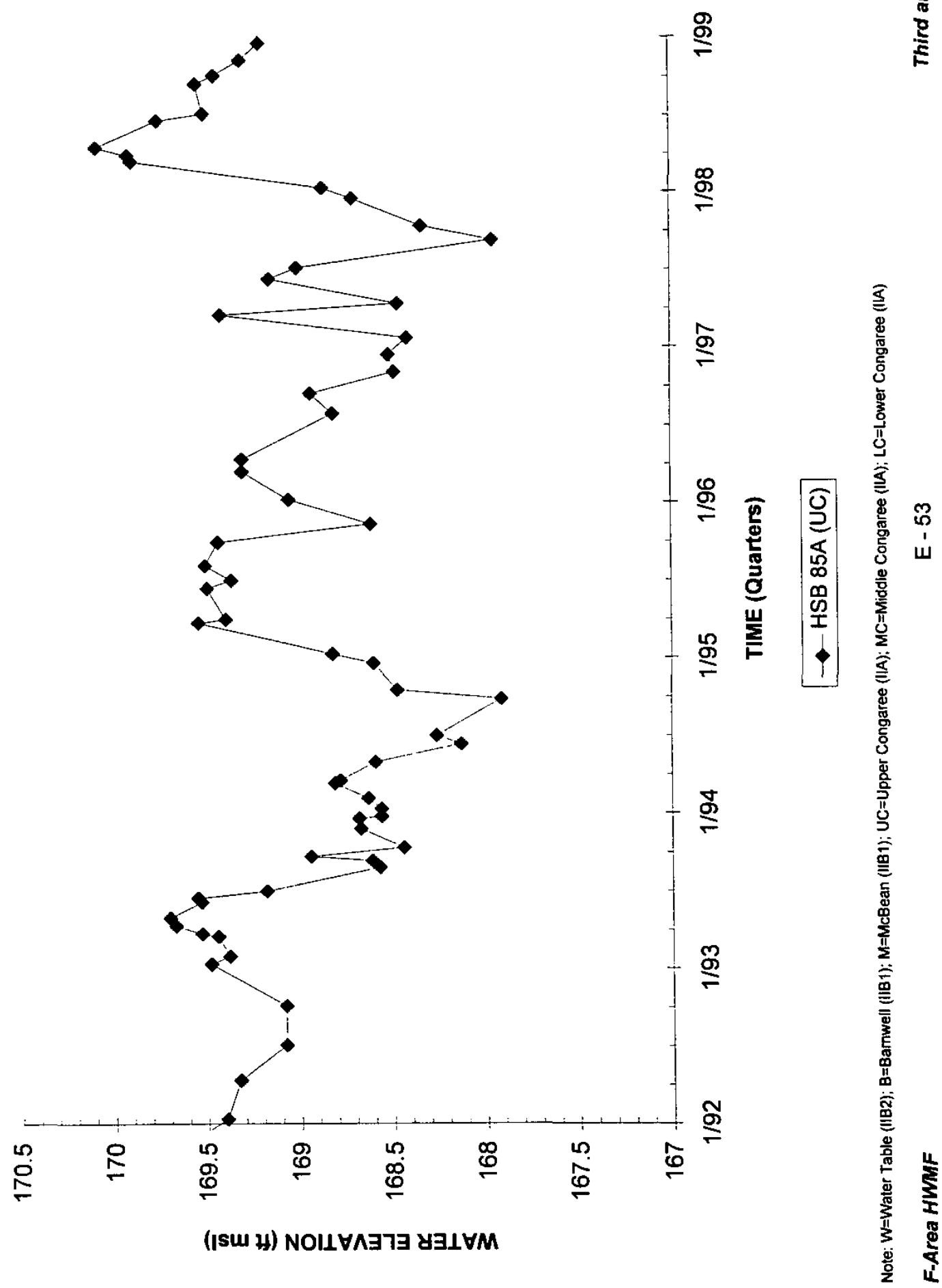


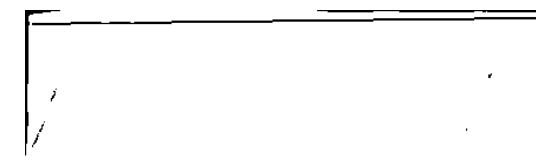

WSRC-TR-99-00012

Unclassified

THIS PAGE LEFT BLANK INTENTIONALLY. 